\title{
AN EMG-DRIVEN MODEL TO INVESTIGATE COCONTRACTION \\ OF LOWER EXTREMITY MUSCLES DURING GAIT
}

\section{DISSERTATION}

Presented in Partial Fulfillment of the Requirements for the Degree Doctor of Philosophy in the Graduate

School of the Ohio State University

By

Tasos Karakostas, M.S.

$* * * * *$

The Ohio State University

2001

Dissertation Committee:

Professor Mohamad Parnianpour, Adviser

Approved by

Professor Necip Berme

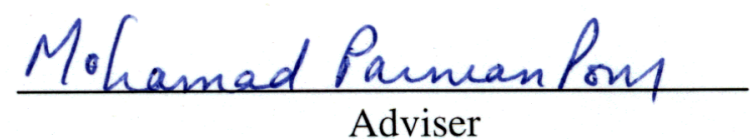

Professor William S. Pease Biomedical Engineering Graduate Program

Professor Peter M. Quesada (honored contributor)

Professor Sheldon R. Simon (honored contributor) 
Copyright by

Tasos Karakostas

2001 
ABSTRACT

A great challenge for the study of human motion is the development of accurate, non-invasive methods to calculate individual force-time histories during movement. In medicine, for many surgical decisions, muscle balance requires precise definition to avoid serious physician-caused errors. It was the purpose, therefore, of this study to introduce an EMG-driven neuromusculoskeletal model to predict cocontraction among individual musculotendon units that comprise the synergistic and antagonistic muscle groups involved in knee flexion/extension and ankle plantar/dorsi flexion during normal walking gait.

Description of the muscle's mechanical response was based on Hill's and Zajac's work, but incorporated individual muscle length, velocity, and excitation considerations for muscle contractions. Processed EMG represented the neural input to the muscle. A musculoskeletal model defining joint kinematics, muscle line of action and architecture of the left lower limb musculotendon units was developed by using SIMM and modifying its associated model. Muscle kinematics were then calculated in conjunction with three dimensional motion data. Computer simulations combined with a calibration procedure allowed the estimation of all model parameters.

Individual muscle force profiles were predicted for selected muscles of the lower limb using three subjects during normal walking. Model validation was performed against estimated net joint moments from inverse dynamics.

Estimated gains were within physiological range, and a good match was obtained between moment curves. Correlations ranged from 0.73 to 0.97 for the gait trials and RMS differences between 22.2 and $12.99 \mathrm{Nm}$ for the knee and ankle joints individually. Expressed relative to the peak measured moments during gait, the RMS differences ranged from 13.71 to 15.33 percent at the ankle, and 24.95 to 45.58 percent at the knee. 
The results were similar or better than those previously reported. Cocontraction indexes were higher during events in the gait cycle that required stability and control at the joint.

This model provides a solid foundation for further improvements that are discussed. The results support the feasibility of using the proposed model, as a potential solution to the interdeterminancy problem providing solutions to muscle forces involved in normal human movement, and a cocontraction index to assess muscle balance during gait. 
Dedicated to my family

my fiancé, my mother and

in the memory of my grandparents 


\section{ACKNOWLEDGEMENTS}

Although this project represents my work, half of the credit belongs to Mohamad Parnianpour, who has not only provided guidance throughout, but has worked long and awkward hours, at regular and, for an advisor, irregular places with me to identify solutions and new research concepts. He has provided support at every level, and one would consider himself fortunate just by meeting him in person, let alone have him as an advisor.

I am further in debt to the other two members of my committee, Necip Berme who has been available for guidance, flexible and understanding at times that it was necessary, and William Pease whose presence, contribution, and his electromyography lectures have been instrumental for the completion of the project. Along with them I wish to thank Peter Quesada and Sheldon Simon who were very important at the initial stages of this research. I am also in debt to Alan Litsky, my core Biomedical Engineering advisor, whose refreshing humor has always made things easier than they seemed to be as well as for his initial support and special arrangements he made when I first arrived in Columbus. Last, but not least, I am in debt to Deb Nicols whose financial and professional supports have allowed me to, not only complete my academic graduate research experience, but also to enter, concurrently, another professional arena.

Special thanks are due to the National Institute on Disability and Rehabilitation Research, Washington, D.C. grant (\#H133E30009) for supporting this research, in part, and to all of my subjects who had the patience and generosity to lent me so graciously their time.

A number of other people and entities are responsible for making the completion of this project possible. Among them is Mrs. Barbara Bostic, who graciously allowed access for 
me in the outpatient clinic where the isokinetic dynamometer was kept, and Hunger Orthopaedics for donating the base for my calibration brace.

Two individuals whose presence in my life and their moral support in completing this research have made them more than friends to me, but rather members of my family, Harris Miantzaridis and Jerald Greenspan. I am also grateful to Mr Andrew Pitonyak whose advice and will to share some of his computer programming knowledge has made my programming skills more efficient in implementing my algorithms.

Finally, above all, I am indebted to the people who are the foundation for the completion of this project as well as being responsible for who I have been, who I am, and who I am going to be. My spiritual teacher Mrs. Stela Basta who has taught me that there is always a bright side in everything and, at hard times, all I need to do is to tune my mind to this side to be persistent and to not give up. I thank her for being my mother at another level. My family (including Mr. Alexakos who was the first to support my studies in US by giving me as a Christmas present my one way airplane ticket to US in the 90's) who I owe them all the time that I have taken away from being with them while their neverending support and thought has always been with me. I just hope that I will be able to make up, eventually, for all the time I have missed being with them. Finally, I want to acknowledge the importance of the two people who really represent my past present and future. My mother who represents not only my ability to complete this project, but everything that $I$ am and stand for today, and my dearest Alexia whose support and assistance in completing this research was beyond vital, and the joy that she brings into my life and our future journey cannot be described by any words. 


\section{VITA}

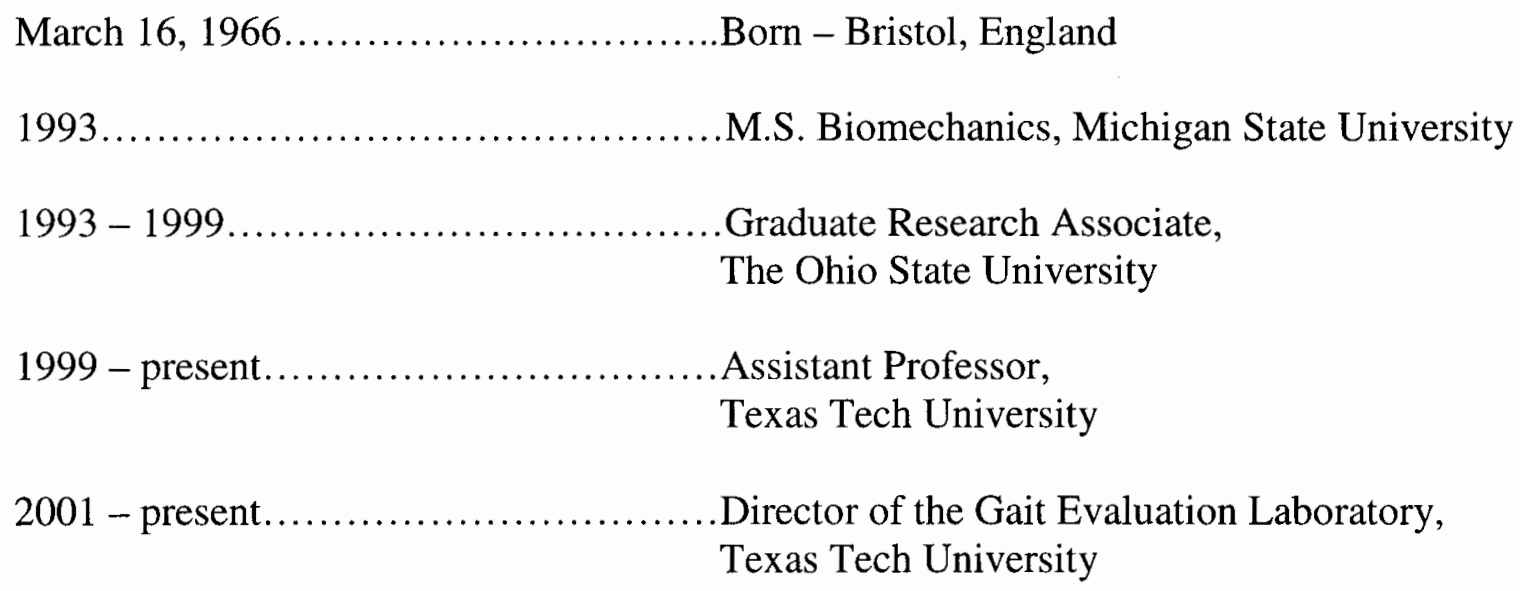

\section{PUBLICATIONS}

\section{Research Publication}

Dummer, G.M., DeYoung, L.L., Karakostas, T., Mattar, R., Neat, D.R., Sochacki, K.M. (1990): Curriculum development in adapted physical education. Michigan Journal of Health, Physical Education, Recreation, and Dance, Vol. 17, pp. 8-10.

Karakostas, T.: Biomechanics of the running gait of recreational runners who are blind. MSc. Thesis, Michigan State University, 1993.

Karakostas, T., Haubenstricker, J.L., Brown, E. (1994): Recreational runners who are blind: Examination of selected biomechanical variables. Engineering Systems Design and Analysis, Vol. 4, pp. 56-73.

Karakostas, T., Engin, A.E. (1996): An overview of the mechanical properties of spinal ligaments and injury mechanisms. The Journal of Rheumatology and Medical Rehabilitation, Vol. 7(1), pp. 45-56. 
Karakostas, T., Kamaleson, M., Quesada, P.M, Simon, S.R. (1996): Functional integrity of fasciocutaneous flaps: Biomechanical evaluation of selected kinetic variables. Engineering Systems Design and Analysis, Vol. 5, pp. 112-126.

Khalaf, K.A., Parnianpour, M., Karakostas, T. (2000): Surface responses of maximum isokinetic ankle torque generation capability. Journal of Applied Biomechanics, Vol. 16(1), pp. 52-60.

\section{FIELDS OF STUDY}

Major Field: Biomedical Engineering 


\section{TABLE OF CONTENTS}

\section{$\underline{\text { Page }}$}

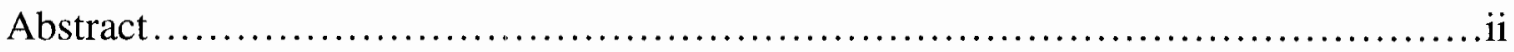

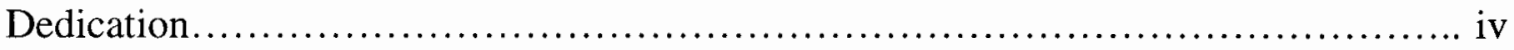

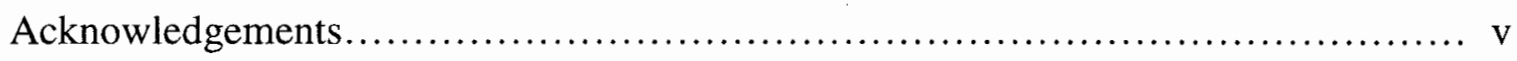

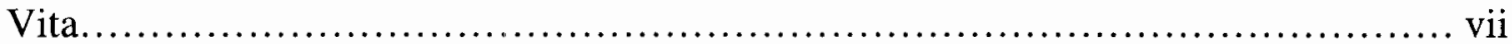

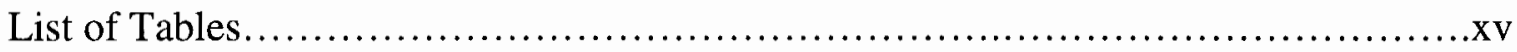

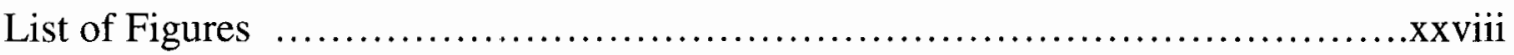

Chapters:

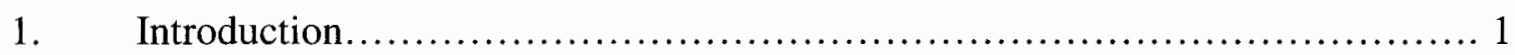

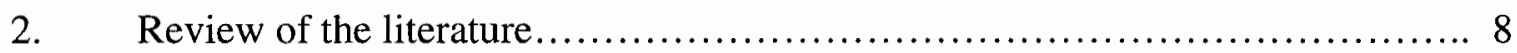

Modeling ...................................................... 12

Skeletal modeling ......................................... 13

Joint modeling ............................................ 18

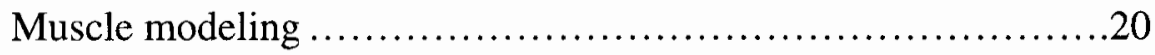

EMG signal demodulation ....................................... 28

Rectification ............................................ 28

Smoothing................................................. 29

Integration................................................. 29 
Root mean square 30

Further EMG signal processing....................................... 31

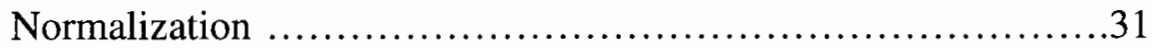

Option other than MVIC ......................................33

EMG-driven modeling .............................................

Cocontraction during gait and EMG-driven modeling ..............35

3. EMG-driven model development .......................................... 38

Introduction ..................................................... 38

Muscle modeling and parameters...................................41

The electromyogram........................................58

Processing of the electromyogram ..........................77

Normalization ............................................. 79

Calibration................................................... 81

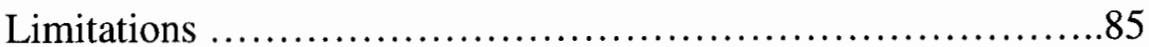

Planar Moments .........................................85

No series elastic component $($ SEC) $\ldots \ldots \ldots \ldots \ldots \ldots \ldots \ldots \ldots . \ldots 6$

No history of contraction effects $\ldots \ldots \ldots \ldots \ldots \ldots \ldots \ldots \ldots \ldots . . . \ldots 6$

Temperature effects ..................................... 88

Homogeneous muscle force generation.....................89

The physiological cross-sectional area ......................89

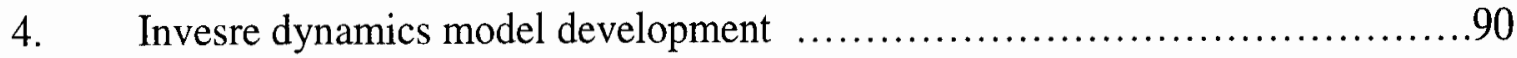

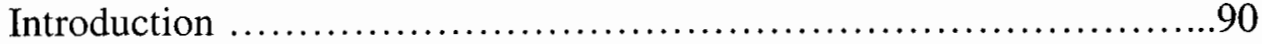


Model development ........................................91

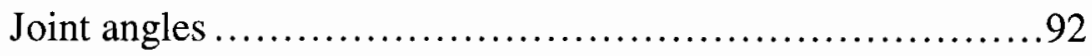

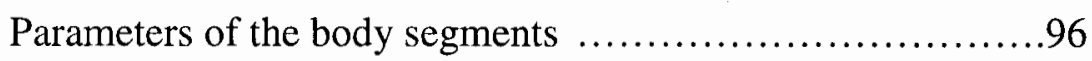

Estimations of the joint centers $\ldots \ldots \ldots \ldots \ldots \ldots \ldots \ldots \ldots . \ldots 9 . \ldots \ldots$

Force plate analysis.................................99

Net joint moments determination ........................ 102

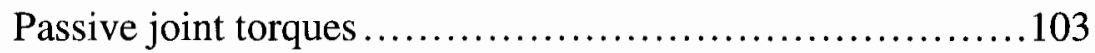

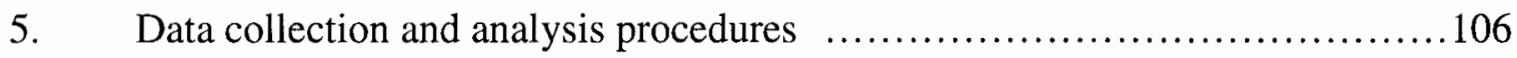

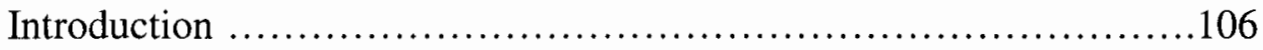

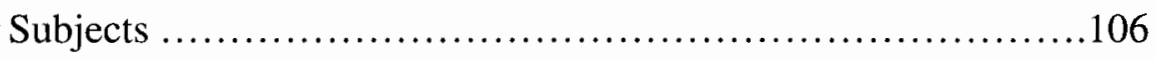

Informed consent procedures ........................... 107

Instrumentation and physical arrangement.......................107

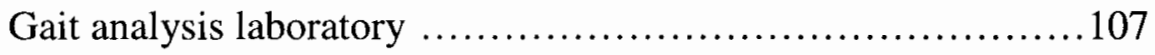

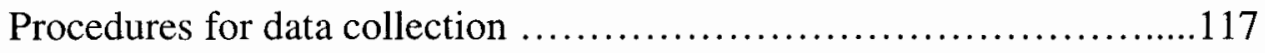

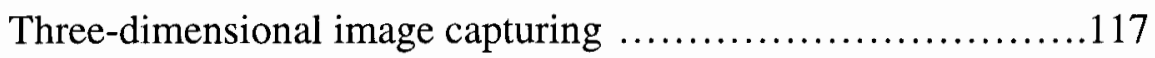

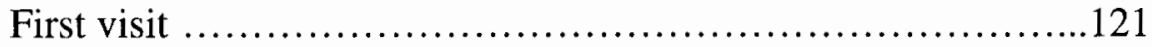

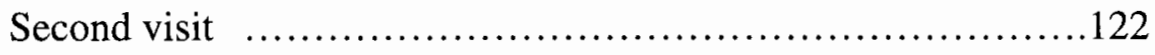

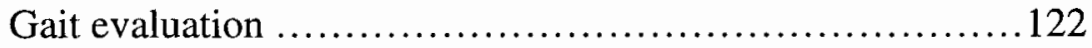

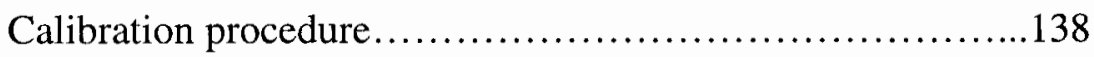

Feedback to the subjects............................ 168

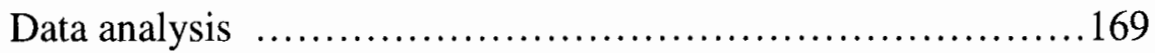

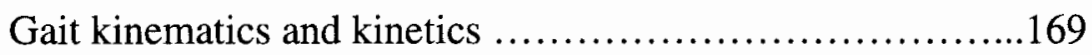


Electromyographic signals.

Estimation of the cocontraction index

EMG-driven model validation

Muscle activity assumptions

Overall model implementation and processing

6. Results and discussion .180

Inverse Dynamics

Kinematics

Joint angles

Kinetics

Kinetics output

EMG-driven model performance validation and results 188

EMG patterns 188

Model output 195

Gain 195

Correlations 199

Root mean square error .206

Muscle cocontraction .210

EMG-driven performance assessment .210

Model parameters

Muscle Architectural inputs .213

EMG patterns .215 
7. Conclusions.

List of references

APPENDIX A

Properties of each body segment

APPENDIX B

Sample input model file to Move3D

APPENDIX C

Sample anthropometric output from Move3D

APPENDIX D

Instantaneous joint center definition.

APPENDIX E

Move3D inverse dynamics analysis

APPENDIX F

Informed consent.

APPENDIX G

Tilt sensors calibration

APPENDIX H

Anthropometric information form

APPENDIX I

Simulation results from SIMM

APPENDIX J

Gait knee and ankle joint velocities. 
APPENDIX K

Gait knee and ankle ranges of motion.....

APPENDIX L

Gait knee and ankle moments

- APPENDIX M

Isokinetic knee and ankle results

APPENDIX N

Gait knee and ankle results

APPENDIX O

Optimum gait knee and ankle results

APPENDIX P

Comparison of the cocontraction methods .334 


\section{LIST OF TABLES}

Table

3.1. A summary of the relative characteristics of the two muscle modeling approaches used by Zajac's and Winter's groups

3.2 Physiological cross sectional areas (PCSAs) estimated by Hoy's (1990) and Delp's (1990) maximum isometric force data and the specific muscle tensions from the original studies used to obtain the maximum isometric muscle forces

3.3 Maximum contraction velocities used in the present project

3.4 Muscles to be monitored with surface EMG

3.5 Muscle innervation of lower extremity muscles of potential interest..... .75

3.6 Suggested grouping scheme of muscles not monitored, with respect to their innervation and activity, with muscles that were monitored

4.1 Segmental MOVE3D geometric shapes for the body segments of interest, and their mass distribution with respect to the total body weight on the basis of the anthropometric data reported by Dempster (1955)

4.2 Coefficients for the estimation of passive joint moments.

Passive joint moments represent the ligamentous and cartilaginous contributions to the net joint moment measured via inverse dynamics analysis.

5.1 Performance of the motion analysis system. A known distance between two markers was compared with the mean distance plus/minus the standard deviation to assess the over- or underprediction of the system respectively. Final results were presented in percent. Negative signs indicate under-prediction 
5.2 Performance of the motion analysis system. A known distance between two markers was compared with the predicted distance to assess the prediction error of the system. Final results were presented in percent. Negative signs indicate under-prediction

5.3 Ankle-knee positions of joint interactions during the gait cycle. At those positions one of the joints would be kept fixed (F) while the adjacent joint would go through its corresponding gait-specific dynamic range of motion (D) for the purposes of identifying the maximum joint moment and respective electromyographic signal (numerical values in degrees). The dynamic range includes the corresponding angle in the "Angle" column

5.4 Positions for the ankle-knee joint interactions tested 154

5.5 Positions for the ankle-knee joint interactions tested, and the respective joint ranges of motion during nearly isometric conditions. All values are in degrees. For the ankle joint, negative values indicate plantar flexion; positive values indicate dorsi flexion

5.6 Positions for the ankle-knee joint interactions tested, and the respective joint ranges of motion during isokinetic testing conditions. All values are in degrees. For the ankle joint, negative values indicate plantar flexion; positive values indicate dorsi flexion

6.1 Relative distance between certain markers throughout the gait cycle to test the validity of the rigid body assumption for the purposes of the inverse dynamics model

6.2 Summary of the statistical parameters of interest used to validate the EMG-driven model during the calibration trials. The parameters were the average and standard deviations of: (a) the Gain; (b) the correlation coefficient ( $\mathrm{r}$ ) between the measured and predicted moments;

(c) the determination coefficient; (d) the root mean square of the error (RMS);

(e) the normalized RMS with respect to the maximum measured moment value in the envelope (NRMS_Env); and (f) the normalized RMS with respect to the maximum measured moment value of all calibration trials for the joint (NRMS_MAX)

6.3 Summary of the statistical parameters of interest used to validate the EMG-driven model with a calibration-based global Gain (see text). The parameters were the average and standard deviations of: (a) the correlation coefficient $(r)$ between the measured and predicted moments; 
(b) the determination coefficient; (c) the root mean square of the error (RMS);

(d) the normalized RMS with respect to the maximum measured moment value in the envelope (NRMS_MOT); and (e) the normalized RMS with respect to the maximum measured moment value of all isokinetic calibration trials for the joint

6.4 Summary of the statistical parameters of interest used to validate the EMG-driven model with the calibration derived, joint-specific constant Gain. The parameters were the average and standard deviations of: (a) the correlation coefficient (r) between the measured and predicted moments; (b) the determination coefficient; (c) the root mean square of the error (RMS); (d) the normalized RMS with respect to the maximum measured moment value in the envelope (NRMS_MOT); and (e) the normalized RMS with respect to the maximum measured moment value of all isokinetic calibration trials for the joint

6.5 Summary of the statistical parameters of interest used to validate the EMG-driven model with a least squares estimated global Gain. The parameters were the average and standard deviations of: (a) the Gain; (b) the correlation coefficient (r) between the measured and predicted moments; (c) the determination coefficient; (d) the root mean square of the error (RMS); (e) the normalized RMS with respect to the maximum measured moment value in the envelope (NRMS_MOT); and ( $f$ ) the normalized RMS with respect to the maximum measured moment value of all isokinetic calibration trials for the joint

6.6 Summary of the statistical parameters of interest used to validate the EMG-driven model with a least squares estimated joint specific Gain. The parameters were the average and standard deviations of: (a) the Gain; (b) the correlation coefficient (r) between the measured and predicted moments; (c) the determination coefficient; (d) the root mean square of the error (RMS); (e) the normalized RMS with respect to the maximum measured moment value in the envelope (NRMS_MOT); and (f) the normalized RMS with respect to the maximum measured moment value of all isokinetic calibration trials for the joint

G.1 Voltage output data as a function of the tilt angle. The data reported by the manufacturer of the inclinometers were reported in millivolts $(\mathrm{mV})$, whereas the data at the OSU testing site are reported in volts $(\mathrm{V})$. The signal conditioner (SC) - tilt sensor (S) pairs that were used are reported also 
I.1 The ankle dorsi flexor muscle group moment (AnkDF_Sum) output as a function of the dorsi flexion joint angle (andf) using SIMM. The bolded values indicate the range of angles at which maximum muscle moment occurred. No plantar flexion cocontraction has been included in this table because the result would be a plantar flexor moment. No joint interaction data are being presented either, because the dorsi flexors are all monoarticular muscles

I.2 The ankle plantar flexor muscle group moment (AnkPF) SIMM output during plantar flexion as a function of the joint angle (anpf) and joint interactions with the angle at the knee. The knee positions tested were: neutral (K0), knee flexion at seven degrees (K7), knee flexion at twenty degrees (K20) and knee flexion at fifty five degrees (K55). The ankle plantar flexor muscle group moment output was obtained for the same conditions while assuming maximum antagonistic cocontraction (CoCon) from the dorsi flexors. Bolded values indicate the range of angles at which maximum muscle moment occurred. The hip was kept at 45 degrees.

I.3 The ankle knee extensor muscle group moment (Mkke) SIMM output during plantar flexion as a function of the joint angle (knex) and joint interactions with the angle at the ankle while assuming maximum knee flexor muscle group cocontraction (CoCon). The ankle positions tested were: neutral (A0), plantar flexion at ten degrees (A10P), plantar flexion at twenty degrees (A20P) and dorsi flexion at ten degrees (A10D). Bolded values indicate the range of angles at which maximum muscle moment occurred. The hip was kept at 45 degrees.

I.4 The knee flexor muscle group moment (MkkfA0) output as a function of the knee flexion angle (knfx) and joint interactions with the angle at the ankle using SIMM. Bolded values indicate the range of angles at which maximum group muscle moment occurred. No maximum knee extensor cocontraction has been included in this table because the result would be a knee extensor moment. The hip was kept at 45 degrees of flexion.

J.1 Angular velocities of the knee joint at different events during the gait cycle for six subjects (S). Peaks and maxima are approximated. 
J.2 Angular velocities of the ankle joint at different events during the gait cycle for six subjects (S). Peaks and maxima are approximated.

M.1 Isokinetic trials data for the ankle under different conditions (the various isokinetic conditions have been explained in the text) to identify the gain. Other parameters that have been estimated are: a) the correlation coefficient $(\mathrm{R})$; $b$ ) the coefficient of determination $\left(\mathrm{R}^{2}\right)$; c) the root mean square of the error (RMS); d) the normalized root mean square of the error with respect to the maximum moment in the trial (NRMS_EnvMax); and e) the normalized root mean square of the error with respect to the maximum moment of all trials (NRMS_EnvMax). The average values along with the standard deviations and the medians for each of the parameters have also been computed.

M.2 Isokinetic trials data for the knee under different conditions (the various isokinetic conditions have been explained in the text) to identify the gain. Other parameters that have been estimated are: a) the correlation coefficient (R); b) the coefficient of determination $\left(\mathrm{R}^{2}\right)$; $\mathrm{c}$ ) the root mean square of the error (RMS); d) the normalized root mean square of the error with respect to the maximum moment in the trial (NRMS_EnvMax); and e) the normalized root mean square of the error with respect to the maximum moment of all trials (NRMS_EnvMax). The average values along with the standard deviations and the medians for each of the parameters have also been computed.

M.3 Isokinetic trials data for the ankle under different conditions (the various isokinetic conditions have been explained in the text) to identify the gain. Other parameters that have been estimated are: a) the correlation coefficient $(\mathrm{R})$; b) the coefficient of determination $\left(R^{2}\right)$; c) the root mean square of the error (RMS); d) the normalized root mean square of the error with respect to the maximum moment in the trial (NRMS_EnvMax); e) and the normalized root mean square of the error with respect to the maximum moment of all trials (NRMS_EnvMax). The average values along with the standard deviations and the medians for each of the parameters have also been computed 
M.4 Isokinetic trials data for the knee under different conditions (the various isokinetic conditions have been explained in the text) to identify the gain. Other parameters that have been estimated are: a) the correlation coefficient (R); b) the coefficient of determination $\left(\mathrm{R}^{2}\right) ; \mathrm{c}$ ) the root mean square of the error (RMS); d) the normalized root mean square of the error with respect to the maximum moment in the trial (NRMS_EnvMax); and e) the normalized root mean square of the error with respect to the maximum moment of all trials (NRMS_EnvMax). The average values along with the standard deviations and the medians for each of the parameters have also been computed.

M.5 Isokinetic trials data for the ankle under different conditions (the various isokinetic conditions have been explained in the text) to identify the gain. Other parameters that have been estimated are: a) the correlation coefficient $(\mathrm{R})$; b) the coefficient of determination $\left(\mathrm{R}^{2}\right)$; $\left.\mathrm{c}\right)$ the root mean square of the error (RMS); d) the normalized root mean square of the error with respect to the maximum moment in the trial (NRMS_EnvMax); e) and the normalized root mean square of the error with respect to the maximum moment of all trials (NRMS_EnvMax). The average values along with the standard deviations and the medians for each of the parameters have also been computed.

M.6 Isokinetic trials data for the knee under different conditions (the various isokinetic conditions have been explained in the text) to identify the gain. Other parameters that have been estimated are: a) the correlation coefficient (R); b) the coefficient of determination $\left(\mathrm{R}^{2}\right)$; $\mathrm{c}$ ) the root mean square of the error (RMS); d) the normalized root mean square of the error with respect to the maximum moment in the trial (NRMS_EnvMax); e) and the normalized root mean square of the error with respect to the maximum moment of all trials (NRMS_EnvMax). The average values along with the standard deviations and the medians for each of the parameters have also been computed. .321 
N.1 Model validation results for the EMG-driven model for subject SA. The validation parameters included the global gain identified from the calibration trials, the correlation coefficients $(R)$, the coefficient of determination $\left(\mathrm{R}^{\wedge} 2\right)$, the root mean square of the error (RMS), the RMS expressed relative to the respective joint maximum measured moment in the envelope (NRMS_MOT), and the RMS expressed relative to the respective joint maximum measured moment from the calibration trials.

N.2 Model validation results for the EMG-driven model for subject SA. The validation parameters included the correlation coefficients (R), the coefficient of determination $\left(\mathrm{R}^{\wedge} 2\right)$, the root mean square of the error (RMSK), the RMSK expressed relative to the respective joint maximum measured moment in the envelope (NRMSK_Mot), and the RMSK expressed relative to the respective joint maximum measured moment from the calibration trials (NRMSK_KC). The gain implemented was identified from the calibration trials.

N.3 Model validation results for the EMG-driven model for subject SA. The validation parameters included the correlation coefficients $(\mathrm{R})$, the coefficient of determination $\left(\mathrm{R}^{\wedge} 2\right)$, the root mean square of the error (RMSA), the RMSA expressed relative to the respective joint maximum measured moment in the envelope (NRMSA_Mot), and the RMSA expressed relative to the respective joint maximum measured moment from the calibration trials (NRMSA_KC). The gain implemented was identified from the calibration trials 
N.4 Model validation results for the EMG-driven model for subject SC. The validation parameters included the global gain identified from the calibration trials, the correlation coefficients $(R)$, the coefficient of determination $\left(\mathrm{R}^{\wedge} 2\right)$, the root mean square of the error (RMS), the RMS expressed relative to the respective joint maximum measured moment in the envelope (NRMS_MOT), and the RMS expressed relative to the respective joint maximum measured moment from the calibration trials

N.5 Model validation results for the EMG-driven model for subject SC. The validation parameters included the correlation coefficients (R), the coefficient of determination $\left(R^{\wedge} 2\right)$, the root mean square of the error (RMSK), the RMSK expressed relative to the respective joint maximum measured moment in the envelope (NRMSK_Mot), and the RMSK expressed relative to the respective joint maximum measured moment from the calibration trials (NRMSK_KC). The gain implemented was identified from the calibration trials

N.6 Model validation results for the EMG-driven model for subject SC. The validation parameters included the correlation coefficients $(\mathrm{R})$, the coefficient of determination $\left(R^{\wedge} 2\right)$, the root mean square of the error (RMSA), the RMSA expressed relative to the respective joint maximum measured moment in the envelope (NRMSA_Mot), and the RMSA expressed relative to the respective joint maximum measured moment from the calibration trials (NRMSA_KC). The gain implemented was identified from the calibration trials 
N.7 Model validation results for the EMG-driven model for subject $\mathrm{SN}$. The validation parameters included the global gain identified from the calibration trials, the correlation coefficients $(\mathrm{R})$, the coefficient of determination $\left(\mathrm{R}^{\wedge} 2\right)$, the root mean square of the error (RMS), the RMS expressed relative to the respective joint maximum measured moment in the envelope (NRMS_MOT), and the RMS expressed relative to the respective joint maximum measured moment from the calibration trials.

N.8 Model validation results for the EMG-driven model for subject $\mathrm{SN}$. The validation parameters included the correlation coefficients (R), the coefficient of determination $\left(\mathrm{R}^{\wedge} 2\right)$, the root mean square of the error (RMSK), the RMSK expressed relative to the respective joint maximum measured moment in the envelope (NRMSK_Mot), and the RMSK expressed relative to the respective joint maximum measured moment from the calibration trials (NRMSK_KC). The gain implemented was identified from the calibration trials.

N.9 Model validation results for the EMG-driven model for subject $\mathrm{SN}$. The validation parameters included the correlation coefficients $(\mathrm{R})$, the coefficient of determination $\left(\mathrm{R}^{\wedge} 2\right)$, the root mean square of the error (RMSA), the RMSA expressed relative to the respective joint maximum measured moment in the envelope (NRMSA_Mot), and the RMSA expressed relative to the respective joint maximum measured moment from the calibration trials (NRMSA_KC). The gain implemented was identified from the calibration trials.

O.1 Model validation results for the EMG-driven model for subject SA. The validation parameters included the optimum global gain, the correlation coefficients (R), the coefficient of determination $\left(R^{\wedge} 2\right)$, the root mean square of the error (RMS), the RMS expressed relative to the respective joint maximum measured moment in the envelope (NRMS_MOT), and the RMS expressed relative to the respective joint maximum measured moment from the calibration trials 
O.2 Model validation results for the EMG-driven model for subject SA. The validation parameters included the optimum knee gain, the correlation coefficients $(\mathrm{R})$, the coefficient of determination $\left(\mathrm{R}^{\wedge} 2\right)$, the root mean square of the error (RMSK), the RMSK expressed relative to the respective joint maximum measured moment in the envelope (NRMSK_Mot), and the RMSK expressed relative to the respective joint maximum measured moment from the calibration trials (NRMSK_KC).

O.3 Model validation results for the EMG-driven model for subject SA. The validation parameters included the optimum ankle gain, the correlation coefficients $(R)$, the coefficient of determination $\left(R^{\wedge} 2\right)$, the root mean square of the error (RMSA), the RMSA expressed relative to the respective joint maximum measured moment in the envelope (NRMSA_Mot), and the RMSA expressed relative to the respective joint maximum measured moment from the calibration trials (NRMSA_KC)

O.4 Model validation results for the EMG-driven model for subject SC. The validation parameters included the optimum global gain, the correlation coefficients $(R)$, the coefficient of determination $\left(R^{\wedge} 2\right)$, the root mean square of the error (RMS), the RMS expressed relative to the respective joint maximum measured moment in the envelope (NRMS_MOT), and the RMS expressed relative to the respective joint maximum measured moment from the calibration trials

O.5 Model validation results for the EMG-driven model for subject SC. The validation parameters included the optimum knee gain, the correlation coefficients $(R)$, the coefficient of determination $\left(R^{\wedge} 2\right)$, the root mean square of the error (RMSK), the RMSK expressed relative to the respective joint maximum measured moment in the envelope (NRMSK_Mot), and the RMSK expressed relative to the respective joint maximum measured moment from the calibration trials (NRMSK_KC) 
O.6 Model validation results for the EMG-driven model for subject SC. The validation parameters included the optimum ankle gain, the correlation coefficients $(R)$, the coefficient of determination $\left(R^{\wedge} 2\right)$, the root mean square of the error (RMSA), the RMSA expressed relative to the respective joint maximum measured moment in the envelope (NRMSA_Mot), and the RMSA expressed relative to the respective joint maximum measured moment from the calibration trials (NRMSA_KC) .331

O.7 Model validation results for the EMG-driven model for subject SN. The validation parameters included the optimum global gain, the correlation coefficients $(R)$, the coefficient of determination $\left(R^{\wedge} 2\right)$, the root mean square of the error (RMS), the RMS expressed relative to the respective joint maximum measured moment in the envelope (NRMS_MOT), and the RMS expressed relative to the respective joint maximum measured moment from the calibration trials.

O.8 Model validation results for the EMG-driven model for subject $\mathrm{SN}$. The validation parameters included the optimum knee gain, the correlation coefficients $(R)$, the coefficient of determination $\left(R^{\wedge} 2\right)$, the root mean square of the error (RMS), the RMS expressed relative to the respective joint maximum measured moment in the envelope (NRMS_MOT), and the RMS expressed relative to the respective joint maximum measured moment from the calibration trials

O.9 Model validation results for the EMG-driven model for subject SN. The validation parameters included the optimum ankle gain, the correlation coefficients $(R)$, the coefficient of determination $\left(R^{\wedge} 2\right)$, the root mean square of the error (RMS), the RMS expressed relative to the respective joint maximum measured moment in the envelope (NRMS_MOT), and the RMS expressed relative to the respective joint maximum measured moment from the calibration trials 
P.1 Results from t-tests for pairwise comparisons. The three cocontraction estimation methods were compared (I. cocontraction on the basis of the normalized EMG; II. cocontraction on the basis the normalized EMG and the tension-length-velocity for each musculotendon unit; III. cocontraction on the basis of the individual musculotendon units). Bolded and italicized indicates significant differences at $\mathrm{p}<0.01666$, with $\mathrm{df}=100$ and $t=2.43$. These results are for the ankle joint of subject SA during the motion and KinCom calibration trials

P.2 Results from t-tests for pairwise comparisons. The three cocontraction estimation methods were compared (I. cocontraction on the basis of the normalized EMG; II. cocontraction on the basis the normalized EMG and the tension-length-velocity for each musculotendon unit; III. cocontraction on the basis of the individual musculotendon units). Bolded and italicized indicates significant differences at $\mathrm{p}<0.01666$, with $\mathrm{df}=100$ and $t=2.43$. These results are for the knee joint of subject SA during the motion and KinCom calibration trials

P.3 Results from t-tests for pairwise comparisons. The three cocontraction estimation methods were compared (I. cocontraction on the basis of the normalized EMG; II. cocontraction on the basis the normalized EMG and the tension-length-velocity for each musculotendon unit; III. cocontraction on the basis of the individual musculotendon units). Bolded and italicized indicates significant differences at $p<0.01666$, with $\mathrm{df}=100$ and $t=2.43$. These results are for the ankle joint of subject SA during the motion and KinCom calibration trials. 
P.4 Results from t-tests for pairwise comparisons. The three cocontraction estimation methods were compared (I. cocontraction on the basis of the normalized EMG; II. cocontraction on the basis the normalized EMG and the tension-length-velocity for each musculotendon unit; III. cocontraction on the basis of the individual musculotendon units). Bolded and italicized indicates significant differences at $\mathrm{p}<0.01666$, with $\mathrm{df}=100$ and $\mathrm{t}=2.43$. These results are for the knee joint of subject SA during the motion and KinCom calibration trials....

P.5 Results from t-tests for pairwise comparisons. The three cocontraction estimation methods were compared (I. cocontraction on the basis of the normalized EMG; II. cocontraction on the basis the normalized EMG and the tension-length-velocity for each musculotendon unit; III. cocontraction on the basis of the individual musculotendon units). Bolded and italicized indicates significant differences at $p<0.01666$, with $\mathrm{df}=100$ and $\mathrm{t}=2.43$. These results are for the ankle joint of subject SA during the motion and KinCom calibration trials

P.6 Results from t-tests for pairwise comparisons. The three cocontraction estimation methods were compared (I. cocontraction on the basis of the normalized EMG; II. cocontraction on the basis the normalized EMG and the tension-length-velocity for each musculotendon unit; III. cocontraction on the basis of the individual musculotendon units). Bolded and italicized indicates significant differences at $p<0.01666$, with $\mathrm{df}=100$ and $\mathrm{t}=2.43$. These results are for the knee joint of subject SA during the motion and KinCom calibration trials 


\section{LIST OF FIGURES}

Figure

3.1 Block diagram of the stages and parameters involved in the present project (for parameter explanations see text)

3.2 Block diagram of the stages and parameters involved in the present project

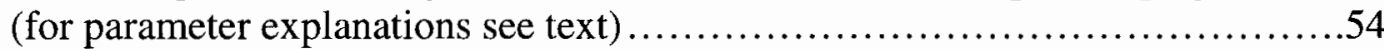

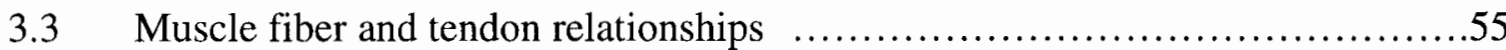

3.4 Zajac's muscle model implemented in SIMM. CE is the contractile element of the muscle, while the spring in parallel with the contractile element represents the passive component developed in the tension-length relationship of the muscle. The spring in series with the other two components represents the tendinous component of the musculotendon unit

3.5 Right ankle plantar-dorsi flexion of a normal subject during a complete gait cycle (A). Total dorsi flexion moment at the ankle when all dorsi flexors are maximally excited (top curve) and dorsi flexion moment generated when only the tibialis anterior and extensor digitorum longus are involved and they are maximally excited (B)

3.6 Right ankle plantar-dorsi flexion of a normal subject during a complete gait cycle (A). Total plantar flexion moment at the ankle when all plantar flexors are maximally excited (bottom curve) and plantar flexion moment generated when only the gastroc, soleus and peroneus brevis are involved and they are maximally excited (B)

3.7 Right knee flexion of a normal subject during a complete gait cycle (A). Total knee flexion moment when all knee flexors are maximally excited (top curve) and knee flexion moment when the sartorius and gracilis are not included (B) 
3.8 Right knee flexion of a normal subject during a complete gait cycle (A). Total knee extension moment when all knee extensors are maximally excited (bottom curve) and knee extension moment when the vastus intermedius is not included (B)

4.1 The four segments that comprised the left lower extremity were the pelvis, the thigh the lower leg and the foot. The dash lines and their associated markers show how each body segment was defined. The solid lines and their associated markers show how each segment was tracked

4.2 Global coordinate system (GCS) of the laboratory X,Y,Z. When a normal subject walks stands in the anatomical position each segment coordinate system (SCS) is aligned with the GCS. For the definition of each segment three non-collinear markers were placed allowing the construction of the SCS

4.3 Center of pressure for the left foot on the force plate for subject

P4 using the MOVE3D software

4.4 Passive ankle plantar/dorsi flexion moments.

The output is a function of position.

4.5 Passive knee flexion/extension moments. The output is a function of position

5.1 Laboratory layout showing the position of equipment .108

5.2 The raw (voltage) vertical component of the ground reaction force $(\mathrm{Fz}) \ldots \ldots \ldots 110$

5.3 The converted, in engineering units of Newton, vertical component of the ground reaction force $(\mathrm{Fz})$-and corrected for the sign

5.4 Diagram illustrating the hardware set up for the collection of data using the isokinetic dynamometer

5.5 Regression equation to convert the KinCom voltage positional data to angles. In the graph zero angle represents the position where the KinCom lever arm is perpendicular and points to the ground

5.6 Regression equation to convert the KinCom load voltage data to pounds

5.7 The tilt sensor

5.8 The linear portion of the signal conditioner (SC3) - tilt sensor pair (S8) voltage along with the regression equation that relates the response to the tilt 
angle

5.9 The linear portion of the signal conditioner (SC8) - tilt sensor pair (S4) ........116

5.10 Marker placement to define the body segments. Markers were placed on the first metatarsal head (L1M), the fifth metatarsal head (L5M), the lateral maleolus (LLML), the medial maleolus (LMML), ), the lateral tibial epicondyle (LLTE), the medial tibial epicondyle (LMTE), the lateral femoral epicondyle (LLFE), the medial femoral epicondyle (LMFE), the greater trochanter (LGRT), the left anterior superior iliac spine (LAS), and the right anterior superior iliac spine (RAS)

5.11 Joint angles of the hip (A) and of the knee (B) joints during a gait cycle of the first walking trial. The thick line represents the use of a marker at the greater trochanter landmark to estimate the specific joint angle. The thin line represents the use of a marker on the lateral femur, the lateral femoral stick, to determine the specific joint angle. Positive values indicate joint flexion. Negative values indicate joint extension

5.12 Joint angles of the hip (A) and of the knee (B) joints during a gait cycle of the second walking trial. The thick line represents the use of a marker at the greater trochanter landmark to estimate the specific joint angle. The thin line represents the use of a marker on the lateral femur, the lateral femoral stick, to determine the specific joint angle. Positive values indicate joint flexion. Negative values indicate joint extension

5.13 Joint angles of the hip (A) and of the knee (B) joints during a gait cycle of the third walking trial. The thick line represents the use of a marker at the greater trochanter landmark to estimate the specific joint angle. The thin line represents the use of a marker on the lateral femur, the lateral femoral stick, to determine the specific joint angle. Positive values indicate joint flexion. Negative values indicate joint extension

5.14 Targeting protocol for the purposes of tracking the body segments in space ....130

5.15 The original orientation of the laboratory coordinates. The $X$ represents the anterior-posterior, Y represents the mediolateral direction, and $\mathrm{Z}$ represents the vertical direction

5.16 The orientation of the laboratory coordinates after rotation, such that $\mathrm{X}$ represents the mediolateral direction, $\mathrm{Y}$ represents the anterior-posterior direction, and $\mathrm{Z}$ represents the vertical direction

5.17 Four anatomical landmarks were identified prior to collecting any calibration data. They were the left greater trochanter, the left lateral femoral epicondyle, 
the left lateral maleolus and another landmark under the left lateral maleolus. The left greater trochanter and left lateral femoral epicondyle were joined by a line. The left lateral femoral epicondyle and the last landmark were joined by another line

5.18 Set up for testing the knee joint; electrodes and thigh tilt sensor placement .....142

5.19 The modified strapping method of the foot on the isokinetic dynamometer such that the heel is not raised during plantar flexion

5.20 Maximum isometric force (A) and moment (B) response of the Dorsi flexors. Simulation output from SIMM. Negative angles indicate plantar flexion

5.21 Maximum isometric force (A) and moment (B) response of the Plantar flexors. Simulation output from SIMM. Negative angles indicate plantar flexion ......148

5.22 Maximum isometric force (A) and moment (B) response of the knee extensors. Simulation output from SIMM. Negative angles indicate plantar flexion .......149

5.23 Maximum isometric force (A) and moment (B) response of the knee flexors. Simulation output from SIMM. Negative angles indicate plantar flexion .......150

5.24 Ankle-knee joint interactions throughout a gait cycle. The time of the gait cycle is normalized to percent

5.25 Maximum isometric dorsi flexion moment for dorsi flexion concentric contractions simulated by SIMM. Maximum antagonistic cocontraction is not simulated because it would result to a plantar flexor moment

5.26 Maximum isometric plantar flexion moments for gait-specific joint interactions and ranges of motion simulated by SIMM. The conditions simulated represented plantar flexion joint angles while the knee was kept at zero, seven, twenty and fifty-five degrees of flexion (K0, K7, K20, K55) The first figure represents the plantar flexor output assuming no cocontraction (A). The second figure represents plantar flexor output assuming maximum antagonistic, dorsi flexor, cocontraction (B)

5.27 Maximum isometric knee flexion moments (Mkkf) for gait-specific joint interactions and ranges of motion simulated by SIMM. The ankle was held at neutral, ten and twenty degrees of plantar flexion (A10P, A20P), and ten degrees of dorsi flexion (A10D). Maximum antagonistic cocontraction was not simulated because it would result to a knee extensor moment. The hip was at forty-five degrees of flexion

5.28 Maximum isometric knee extension moments for gait-specific 
joint interactions and ranges of motion simulated by SIMM. The conditions simulated represented: (a) knee extension joint angles with no cocontraction (Mkke); and (b) knee extension joint angles with cocontraction (CoCon). During the simulations where cocontraction was implemented, the ankle was kept at neutral, ten degrees of plantar flexion, and ten degrees of dorsi flexion (A0, A10P, and $A 10 D$ respectively)

5.29a A superior view of the brace that was used for controlling the position of the ankle joint during isokinetic knee flexions and extensions

5.29b A sagittal view of the brace that was used for controlling the position of the ankle joint during isokinetic knee flexions and extensions

$5.29 \mathrm{c}$ A frontal view of the brace that was used for controlling the position of the ankle joint during isokinetic knee flexions and extensions

5.29d Placement of the brace on the subject in such a way that there was no interference with the electrodes placed on the gastrocnemius

5.30 Power spectral density analysis to identify the low pass cut off frequency for the electromyogram. For the medial gastrocnemius, most of the power of the signal (over 99.8 percent) is maintained between the second and third harmonics

5.31 Force produced on the isokinetic dynamometer during a pilot study trial (thin line). The force was corrected for gravity (thick line)

5.32 The corrected, for gravity, knee flexion moment (thick trace) during knee flexion on the isokinetic dynamometer, and the corrected moment for the moments generated by the passive structures (doted line)

6.1 Comparison of the kinematic output from the MOVE3D and from custom written code for the kinematics of the knee joint (trial from subject SNO05)

6.2 Comparison of the kinematic output from the MOVE3D and from custom written code for the kinematics of the ankle joint (trial from subject SNO05)

6.3 Knee joint moment during gait, estimated from inverse dynamics (o) and corrected ( + ) for passive contributions (trial from subject $\mathrm{SN} 005$ ) 
6.4 Ankle joint moment during gait, estimated from inverse dynamics (o) and corrected ( + ) for passive contributions (trial from subject SN005)

6.5 Selective recruitment (normalized EMG - NEMG) of knee flexor muscles and antagonistic cocontraction (from the bottom:

Medial Gastroc, Lateral Gastroc, Biceps Femoris short head, Semimembranosis/Semitendinosis, Biceps Femoris long head, Vastus Medialis, Vastus Lateralis, Rectus Femoris) for knee flexion at five degrees per second with the ankle at neutral.

Data from subject SC

6.6 Selective recruitment (normalized EMG-NEMG) of knee flexor muscles and antagonistic cocontraction (from the bottom: Medial Gastroc, Lateral Gastroc, Biceps Femoris short head, Semimembranosis/Semitendinosis, Biceps Femoris long head, Vastus Medialis, Vastus Lateralis, Rectus Femoris) for knee flexion at five degrees per second with the ankle at ten degrees of dorsi flexion. Data from subject SC

6.7 Selective recruitment (normalized EMG-NEMG) of knee flexor muscles and antagonistic cocontraction (from the bottom: Medial Gastroc, Lateral Gastroc, Biceps Femoris short head, Semimembranosis/Semitendinosis, Biceps Femoris long head, Vastus Medialis, Vastus Lateralis, Rectus Femoris) for knee flexion at five degrees per second with the ankle at ten degrees of plantar flexion. Data from subject SC

6.8 Normalized EMG history record for the ankle plantar/dorsi flexors of one subject (SC) during one full gait cycle \pm several frames added on either end of the trial. At the top the ankle joint plantar/dorsi flexion angle (in degrees) and moment $(\mathrm{Nm})$ have been included. Muscle definitions are included in the text

6.9 Normalized EMG history record for the knee flexors/extensors of one subject (SC) during one full gait cycle \pm several frames added on either end of the trial. At the top the knee joint flexion/extension angle (in degrees) and moment (Nm) have been included. Muscle definitions are included in the text

6.10 Effect of muscle length (A) and velocity (B) on the gain during knee extension isokinetic calibration trials (e.g., SNKE5CA0 implies: subject SN performing Knee Extension at 5 degrees per second concentrically with the Ankle at 0 degrees)

6.11 Output of the tilt sensor placed on the thigh during knee flexion. 
There is less than 0.7 degrees difference in the position of the thigh from the beginning of the knee flexion to the end

6.12 Distribution of the correlation coefficients between the measured and predicted joint moments for the purposes of validating the temporal consistency of the EMG-driven model.

The median $r$ value is 0.8

6.13A Comparison among the different types of output relative to the measured moment (for SC003) depending on the gain implementation procedure (see text for definition of the various gain implementation procedures) 208

6.13B Comparison among the different types of output relative to the measured moment (for SC003) depending on the gain implementation procedure (see text for definition of the various gain implementation procedures)

6.14 Cocontraction index at the ankle during a full gait cycle using three different cocontraction index algorithms (A). The respective kinetic and kinematic data, along with the predicted moment are also shown (B)

6.15 Cocontraction index at the knee during a full gait cycle using three different cocontraction index algorithms (A). The respective kinetic and kinematic data, along with the predicted moment are also shown (B)....

6.16 Two different strapping techniques for the ankle joint tested on the isokinetic dynamometer. On the top, the person's ankle was strapped according to the manufacturer's specifications allowing the heel to come off the supporting platform. On the bottom the ankle was stabilized such that the heel could not come off.

6.17 Temporal effect among the predicted and measured moments for the knee. Predicted moments were determined under two different kinds of gain implementation procedures

6.18 Temporal effect among the predicted and measured moments for the ankle. Predicted moments were determined under two different kinds of gain implementation procedures

7.1 Block diagram of the stages and parameters involved in the future project.....234 
D.1 Part of the lower extremity rigid link segment model representing the left lower leg (shank) and foot (anterior view). Marker A represents the medial maleolus, marker B represents the lateral maleolus, $\mathrm{C}$ represents the anterior shank marker, $D$ is the first metatarsal head

E.1 The foot and shank during the push of phase of a gait cycle for the left leg. The ground reaction force is represented by $\mathrm{F}$. The targets that were placed on the foot and shank to track their motion can also be seen. Target A was placed on the calcaneus, B was placed on the first metatarsal head, C was placed on the fifth metatarsal, D was placed on the tibial tuberosity, target $\mathrm{E}$ was placed on the shank, and $\mathrm{F}$ was placed on the posterior shank in line with $\mathrm{E}$

E.2 a) The foot and the targets placed on it along with the resultant ground reaction force; b) the SCS located at the $\mathrm{cm}$ of the segment and the instantaneous joint center of rotation for the ankle $\left(\mathrm{J}^{\mathrm{A}}\right)$ has been determined; $\mathrm{c}$ ) a free body diagram with the kinetic information at the foot ( $\vec{F}$ is the external ground reaction force, $\vec{R}$ is the reaction force acting at $\mathrm{J}^{\mathrm{A}}, \vec{W}$ represents the weight of the foot, and $\bar{M}$ is the joint moment)

E.3 a) The shank along with the SCS located at the center of mass $(\mathrm{cm})$ of the segment and the instantaneous joint center of rotation for the knee joint $\left(\mathrm{J}^{\mathrm{K}}\right)$ have been determined; $b$ ) a free body diagram with the kinetic information at the shank $\left(\vec{R}^{A}\right)$ and $\vec{R}^{K}$ are the reaction forces acting at the ankle joint, applied at the distal end of the shank segment, and at the $\mathrm{J}^{\mathrm{K}}$, respectively, $\vec{W}$ represents the weight of the shank, $\vec{M}^{A}$ and $\vec{M}^{K}$ are the reaction joint moment at the ankle, $\bar{M}^{A}$ applied at the distal end of the shank, and the joint $\vec{M}^{A}$ moment at the knee, respectively) .281

G.1 Set up connections for the tilt sensor - signal conditioner pair

G.2 The set up with the isokinetic dynamometer and the tilt sensor to verify the voltage as a function of the tilt angle

G.3 (A and B) Manufacturer output of the tilt sensor (S) with its paired signal conditioner (SC), in millivolts $(\mathrm{mV})$ as a function of the tilt angle. 
G.4 (A and B). Output of the tilt sensors (S) with its paired signal conditioner (SC), in volts (V) as a function of the tilt angle when the testing took place at OSU....

K.1 Kinematic data for all three trials of subject SN for the knee and ankle joints. The data are consistent.

K.2 Kinematic data for all three trials of subject SC for the knee and ankle joints. The data are consistent.

K.3 Kinematic data for all three trials of subject SA for the knee and ankle joints. The data are consistent.

L.1 Joint moment for all three trials of subject SN for the knee and ankle. The data are consistent with a little variability at the knee. The information is expressed in percent of the data points processed.

L.2 Joint moments for all three trials of subject SC for the knee and ankle. The data are mostly consistent. The information is expressed in percent of the data points processed.

L.3 Joint moment data for all three trials of subject SA for the knee and ankle. The data are consistent with a little variability at the knee joint. The information is expressed in percent of the data points processed. 


\section{CHAPTER 1}

\section{INTRODUCTION}

Human body movements, such as walking, involve the coordination of many segments. Such coordination is achieved by the complex interaction of the neurological, muscular, and skeletal systems. Within this order the muscle acts as a transducer, transforming neural signals into force, which in turn interacts with skeletal linkages to produce an output. The ability to measure individual muscle forces can provide insight to some of the mechanisms responsible for the control and generation of movement, such as cocontraction, and it can provide a means to measure the body's response to movement.

However, it is commonly agreed that one of the greatest challenges in the study of human motion is the development of accurate, non-invasive methods to calculate individual muscle force-time histories during normal movement. Furthermore, in the field of medicine it is widely advocated that in surgical decisions for the management of such orthopedic conditions as cerebral palsy muscle balance must be precisely defined if serious physician-caused errors, such as incorrect tendon lengthenings or transfers, are to be avoided (Gage, 1991; Gage and Koop, 1995). Procedures that satisfy the desire to achieve a measurement of muscle balance by non-invasive means would be more attractive.

Consequently, a direct measure of individual muscle forces could provide a better understanding in such areas as the movement strategies involved in the control of the skeletal linkages, the effect of mechanical loading on bone, and the mechanisms of musculoskeletal injuries. Furthermore, the ability to estimate individual muscle forces during human locomotion, could assist medical decisions and assess medical interventions for the management of various orthopedic conditions; it would also be 
valuable in the development of orthotics, prosthetics, and in the use of Functional Electrical Stimulation (Yamaguchi, 1989a). In general, the ability to ascertain quantitative information about the force generating performance of individual muscles would be useful for the rehabilitation therapists, the prosthetists, the orthopedic surgeons, and the researchers involved in the study of human motion.

However, muscle force cannot be easily obtained. The human musculoskeletal system is mechanically redundant. More muscles are acting at a joint than are required to satisfy joint motion equations. Therefore, unique solutions to muscle forces cannot be obtained by classical equations of mechanics. Furthermore, the force output of an individual muscle, in addition to being a function of the neural input, it also depends on the inherent mechanical, physiological and structural properties of the muscle.

Literature indicates that there have been various attempts to identify unique solutions to muscle forces (Davy and Audu, 1987; Galea and Norman, 1983; Hardt, 1978; Hof et al., 1987; Hughes, 1991; Nussbaum and Chaffin, 1998; Patriarco et al., 1981; Pedotti et al., 1978; Pierrynowski, 1982; Pierrynowski and Morrison, 1985). One approach involves working backward from the net joint moments. Such investigations have included geometric apportioning, muscle reduction, optimization, and physiological models. One common characteristic among these investigations is the requirement of a number of assumptions about the neural input to the muscle and the lack of their quantitative validation. However, qualitative attempts for validations have been made. Qualitative assessment has traditionally involved the comparison of the timing of the predicted force history curves to the timing of the muscle activity from electromyography records.

Another approach to identify unique solutions to muscle forces involves the use of some measure of the neural input and a deterministic muscle model to provide a forward solution to muscle force (Olney and Winter, 1985; White and Winter, 1993). Processed electromyography (EMG) has most often been used to represent neural drive to a muscle (Hof and Berg, 1981a; Hof and Berg, 1981b; Hof and Berg, 1981c, 1981d; Redfern, 1988; White and Winter, 1993). However, most models that involve a forward solution are based on joint kinematics (Olney and Winter, 1985). The investigator must contend 
with the complex interaction of length, velocity, and moment arm changes of a number of muscles. Therefore, muscle simplification has been traditionally required (Bouisset, 1973; Olney and Winter, 1985). In addition, the parameters of the elements of the model need to be estimated by iteration or generated independent of the physical and mechanical properties of the muscle (Olney and Winter, 1985; White and Winter, 1993).

A more appropriate approach would involve the adequate representation of the muscle architecture, a means of measure of the neural drive combined with a record of the muscle's contraction history during the movement, and a mathematical representation of the muscle response. Such an approach has been termed by Dr. Hatze as "Neuromusculoskeletal Modeling" (Hatze, 1981a). To the best of this author's knowledge, literature is very limited in Neuromusculoskeletal Modeling approaches for the purpose of quantifying muscle cocontraction during human gait. The only such modeling attempt appears to be limited by the assumptions and the mathematical representation of the muscle response, the procedures used to construct individual lower limb anthropometrics and, therefore, by the estimation of the muscle length histories and the respective muscle moment arms (White and Winter, 1993).

In addition, it should be emphasized that the ability to implement any such modeling approach to investigate pathologic gait presupposes its successful implementation and validation with normal population first. Such an approach allows for the identification of the most important parameters so that a clinical protocol can be established with the minimum requirements.

\section{Purpose}

The purpose of this project was to propose, construct and validate an EMG-driven neuromusculoskeletal model using computer based analysis to estimate cocontraction, i.e., muscle balance and imbalance (Gage and Koop, 1995), among the individual musculotendon units that comprise the synergistic and antagonistic muscle groups involved in knee flexion/extension and ankle plantar/dorsi flexion during normal gait. 


\section{Primary objective}

A more accurate measurement of cocontraction would be achieved based on the estimated muscle tendon forces. Therefore, the primary objective of this work is to assess the feasibility of predicting the lower limb musculotendon unit forces involved in knee flexion/extension and ankle plantar/dorsi flexion during gait using a combination of EMG, kinetic, and kinematic information from gait analysis for individuals who are normal, i.e., healthy with no prior history of neuro-orthopaedic disorders.

\section{Secondary objectives}

In order to answer the primary objective the following secondary objectives will need to be accomplished:

i.) Evaluate the feasibility of using surface electromyography for direct use in EMG-driven modeling.

ii.) Construct a calibration methodology to identify model parameters specific to an individual subject by controlling the joint angle, the joint velocity and the respective joint interactions between the knee and the ankle joints.

iii.) Evaluate different alternative methods and identify the most appropriate one for processing via normalization of the dynamic EMG signal to predict the force time histories of the lower limb musculotendon units of interest during gaiu.

iv.) Create a model for the appropriate estimation of joint moments from the kinematic and kinetic data collected during the walking trials.

v.) Minimize the potential error in estimating individual muscle moment arms and joint moments from the variacility in the anthropometrics among different subjects.

vi.) Determine the accuracy of the motion analysis system in locating the position of the body segments in space.

It is oftentimes desirable to address the goals of an investigation with a research, project-specific, hypothesis. Consequently, the following hypothesis would have to be addressed in order to answer the primary objective: "Given kinematic trajectories of the 
body segments, ground reaction forces and dynamic surface electromyography, the EMG-driven neuromusculoskeletal model will predict specific musculotendon unit forces and moments which will closely match the timing and magnitude of the respective joint moments determined with inverse dynamics. The predicted musculotendon unit forces will allow the determination of cocontraction on the basis of kinetic output."

\section{Delimitations}

i.) Inputs to the model representing measured and simulated parameters, such as: EMG, force-lengths and force-velocities relationships, calibration gains and muscle kinematics data, were restricted to the range of motion observed during normal mature walking gait.

ii.) Calibration constants and results were subject-specific, i.e., they were specific to the subject being evaluated and they were specific to the musculotendon units of interest acting at the knee and ankle joints.

\section{Limitations}

i.) The net joint moments that were obtained from the inverse dynamic analysis were subjected to the assumption of rigid link segment modeling.

ii.) Only one of the two lower extremities, the dominant, was studied electromyographically due to the limited number of channels available for the monitoring of the musculotendon groups of interest. Consequently, interactions and compensations between the dominant and the non-dominant legs could not be investigated.

iii.) Measurements of the musculotendon lengths and velocities may not reflect accurately the behavior of the individual muscle fibers.

iv.) When moment arms were estimated for the muscles crossing the knee joint, although the knee model implemented in this project accounted for rolling and gliding between the two articulating surfaces, it did not account for the range that the two articulating surfaces were not in contact. The instantaneous center of rotation was assumed to be the instantaneous point of contact 
between the two articulating surfaces. Its path as a function of the joint angle was prescribed (Yamaguchi and Zajac, 1989b).

v.) The anthropometric data pertaining to the muscle morphological characteristics for each subject, such as physiological cross sectional area (PCSA), pennation angle, resting muscle fiber length, fiber type distribution in each muscle, and tendon slack length, were all adopted from the literature, and, therefore, they were not subject specific.

vi.) Some parameters, such as temperature and contraction history, that may have an effect on the predictions on the model for the conditions for which the model is used, were not accounted for.

vii.) Output of the model focused on sagittal plane predictive moments.

viii.) The model accounted for non-fatiguing and smooth, i.e., non ballistic, movements.

\section{Assumptions}

i.) Each segment in the skeletal model was assumed to be rigid.

ii.) Joint moments determined using inverse dynamics were assumed to be accurate and to reflect the true net joint moments required for the movements. Thus, they were considered to be an adequate and appropriate reference for comparing the output predicted moments from the EMGdriven model.

iii.) Each muscle length was assumed to be a straight line between its effective proximal and distal attachments.

iv.) For a given level of excitation, the isometric force generation potential of a muscle was directly proportional to its PCSA.

v.) The neural drive to each of the musculotendon units of interest was assumed to be adequately represented by the respective surface electromyograms, when those were processed in the manner that it was selected for the purposes of this modeling approach. 
vi.) The surface electromyogram was believed to represent the overall muscular activity.

vii.) Muscle force was assumed to be uniformly generated throughout the entire muscle volume during a contraction.

viii.) A linear force-EMG relationship was assumed to exist between these two parameters over the isometric force production range, i.e. for the isometric case.

ix.) The muscle simplification, i.e., apportioning required for the validation procedure included the total force contribution of all muscles with potential to contribute to a joint moment.

x.) The calibration procedure would capture the synergistic and antagonistic behavior among the musculotendon units of interest.

xi.) During the calibration, synergist muscles were working in the same region of their muscle force-length relation for the range of motion consistent with gait.

xii.) During the calibration, synergist muscles not monitored, were excited equivalently and were contributing in proportion to their relative PCSA. 


\section{CHAPTER 2}

\section{REVIEW OF THE LITERATURE}

It has often been stated that in surgical decisions for management of cerebral palsy muscle imbalance must be precisely defined. Furthermore, "specific information regarding the degree of contraction and the source of power for movement must be present if serious iatrogenic (physician-caused) errors are to be avoided" (Gage and Koop, 1995). However, such a goal presupposes the development of methods and techniques that allow the determination of muscle balance among agonists and antagonists in normal individuals first. Nowadays, since this type of information is not feasible to attain accurately with the current scientific approaches, it is approximated by gait analysis.

There are two primary types of output from every gait analysis evaluation: (a) a kinetic, and (b) a kinematic. Kinematic evaluation is concerned with the description of motion. It involves the determination of position, velocity and acceleration in space of the body segments investigated. The kinetic output in a gait evaluation describes the ground reaction forces, the ground reaction moments, the joint moments responsible for the observed motion, and joint powers (Nordin and Frankel, 1989). The two output components, kinetic and kinematic, are linked by the activity of the musculotendon units which contract to output forces that provide ambulation and stability.

The underlying principles involved in collecting the ground reaction forces are established in the current literature (Antonsson and Mann, 1985; Cavanagh, 1981). So are the underlying principles involved in collecting the kinematic information by computing the centroids of "passive" markers located on anatomical landmarks of the body segments of interest (Shapiro, 1978; Winter, 1990). 
Once the three-dimensional position of each marker in each frame of interest has been determined, filtering and smoothing of the marker trajectories takes place for the purposes of reducing the effect of noise when the derivatives are to be calculated (Hatze, 1981b; Woltring, 1986). However, even the residual noise is often claimed to be a source of error when inaccurate investigational results are obtained (Moran, 1994). Therefore, there is a need of identifying methods for reducing the noise. The position and orientation of each body segment can be calculated next. Each segment must have at least three noncolinear markers in order to determine its three-dimensional position (Soutas-Little, 1991). The three-dimensional segmental positions can be converted into angular trajectories of the joints formed by the moving segments (Grood and Suntay, 1983; Soutas-Little and Verstrate, 1990).

The activity of the musculotendon units, which contract to provide ambulation and stability, is monitored by electromyography. An electromyogram (EMG) represents the temporal and spatial superposition of motor unit action potentials that can be recorded, intramuscularly or on the surface of the skin (with the body acting as a volume conductor), when multiple motor units are active (Perry and Bekey, 1981).

Because in this investigation EMGs were recorded, by means of surface electromyography using biopotential electrodes, silver-silver chloride (White and Winter, 1993; Winter, 1990), some related issues identified by previous works need to be highlighted. The advantages of the silver-silver chloride electrodes are: low half-cell potential and high stability during motion. Half-cell potential can introduce a significant amount of DC offset noise in the measured signal. From an electrode design stand point, the two most important characteristics of the EMG signal are its amplitude and frequency content (Webster, 1992). The amplitude is a function of muscle size, electrode location, and electrode geometry (De Luca, 1997). Skin impedance, as well as electrode site location and preparation also affect the amplitude and frequency (Gerleman and Cook, 1992; Lamb and Hobart, 1992; LeVeau and Andersson, 1992; Redferen, 1992; Soderberg, 1992; Soderberg and Knutson, 2000). The EMG signal frequency ranges from about 5 to $25 \mathrm{~Hz}$ at the low end (10 Hz is an acceptable low-end threshold), to several kilohertz at the upper end (Webster, 1992; Winter, 1990). However, it has been 
reported that the muscle tissue acts as a distance dependent low-pass filter (De Luca, 1997). Consequently, the average power of the surface EMG is lower than that of an indwelling electrode. Winter (1990) found that the bulk of the power in surface EMGs lies between 20 and $200 \mathrm{~Hz}$.

An amplifier is also required for the recording of a clean and undistorted EMG. Undistorted implies that the signal is evenly amplified throughout the frequency range of the signal. Clean EMG refers to a signal free of noise and artifact. The biggest problem in measuring biopotentials is noise and artifacts. Environmental noise, $60 \mathrm{~Hz}$ interference, on the surface of a human being is typically several orders of magnitude larger than the biopotentials being measured. However, the environmental noise on the body is common mode, i.e. it has the same value throughout the body (Gerleman and Cook, 1992; LeVeau and Andersson, 1992; Soderberg, 1992). Therefore, the most important property of any biopotential amplifier is that it is a differential amplifier with a high common mode rejection ratio (CMRR) (Webster, 1992). With a high CMRR amplifier and proper grounding, environmental noise can be brought under control.

Another class of noise is inherent to the signal or recording equipment and cannot be controlled by differential amplification (Gerleman and Cook, 1992). The most prevalent of this type of noise is Johnson or thermal noise which is inherent to resistive devices and its frequency content is considered white, i.e. it contains all frequency bands. To reduce thermal noise the values of the resistive elements used in amplifier design should be chosen reasonably small. However, reducing the resistive elements increases the current demands in the amplifiers, which is also undesired. Therefore, there needs to be a compromise in order to keep both thermal noise and current requirements to a minimum. Another method of reducing thermal noise is to limit the bandwidth of the signal to only that of interest.

One of the biggest source of error introduced by biopotential electrodes is motion artifact (Gerleman and Cook, 1992; Soderberg, 1992; Winter, 1990). Electrodes are usually covered in an electrolyte gel and placed on the skin. This creates a skinelectrolyte interface and an electrolyte-skin interface. During gait, these interfaces are constantly being altered due to the accelerations of the leg and the mass of the electrodes. 
This relative motion between the skin and the electrode results in a motion artifact noise which has frequency content between DC and $20 \mathrm{~Hz}$ (Webster, 1992).

Because of all the above-mentioned sources of errors, it is commonly agreed that dynamic EMGs can only provide objective evidence of specific muscle groups activities. In other words, the times when the muscle is actively contracting (on) or not (off) can be estimated fairly accurately, although investigators need to be cautious that crosstalk between muscles is not present (LeVeau and Andersson, 1992; Redferen, 1992; Soderberg, 1992). Furthermore, it is advocated that when pathologic gait is being investigated, dynamic EMGs can be useful in isolating the cause of a particular abnormality when combined with the rest of the kinematic and kinetic gait output (Perry et al., 1997).

The reason that, if EMGs are to be effective, they need to be used in combination with other gait analysis tools is because the timing of the muscular activity with the mechanical events is critical. The mechanical events are correlated with the onset of force of the contracting muscle. However, it has been documented that there is a temporal disassociation between the EMG and force signals, which is typically due to the electromechanical delay (EMD) (Herzog et al., 1995). It has been observed that the EMD is different between muscles (Cavanagh, 1974; Perry and Bekey, 1981), different among individuals (Ralston et al., 1976), and different at the onset compared to the end of the activity (Komi et al., 1987; Ralston et al., 1976). However, at the initiation of an activity, EMD values around $40 \mathrm{~ms}$ seem to be commonly reported.

Initial tension levels in the muscle also have an effect on the delay times seen at the onset. If the muscle is held at a baseline tension level before a step increase in force, then the delay time between the rise in EMG and the rise in force will be shorter. This is due primarily to the mechanical slack being taken out of the muscle (Redferen, 1992).

However, although all the limitations of the EMGs have been well documented, scientifically substantiated, and they have lead orthopedic surgeons like Dr. Gage (1995) to make statements of the type at the beginning of the current section, one should not forget the technological advancements with respect to the recording of the EMGs. New EMG collection systems are currently in the market which can account for almost all of 
the afore-mentioned sources of error with the result being a relatively clean, noise free signal. The Gait Analysis Laboratory at Ohio State University has such a system. Thus, the quantitative use of the EMG should be more feasible.

To this point, the primary type of information that is obtained with a gait evaluation and issues related to the quality of the recorded EMGs have been summarized. However, since the ultimate goal is to learn about the internal mechanisms that underline the observed movement, the information collected externally needs to be combined with modeling approaches that allow insight in the workings of the ambulatory musculoskeletal structure. Consequently, the remainder of this section will focus on the current methodologies that are used to obtain and quantify the parameters necessary to assess muscle imbalance during gait, and it will point out some of their limitations, which substantiate the need for this study.

\section{Modeling}

The methodology of relating selected parameters of a system in order to quantify its performance is referred to as modeling. A model is a simplification of the actual system so that it can be analyzed mathematically. For the investigation of gait, the system that is simplified is the human musculoskeletal system. Gait models, therefore, attempt the simplification of the complicated process of walking to one which can be analyzed mathematically, and, consequently, solved with the use of a computer (Yamaguchi, 1989a). The first step in modeling gait is the construction of a skeletal model that is used to determine the joint moments from the primary kinetic and kinematic information recorded during a gait evaluation (skeletal modeling). Then, the dynamics, and force production characteristics of the musculotendon units of interest need to be modeled in order to investigate the effect of the specific musculotendon units of interest (muscle modeling). Finally, investigation of the specific effects of the musculotendon units of interest involved in the joint moments estimated, involves the specific modeling of the joints spanned by the musculotendon units of interest (joint modeling). It should be noted that skeletal and joint modeling approaches could be combined.

In general, there are two approaches to modeling human movement. One approach considers the natural course of events going forward in time, and it is referred to as direct 
dynamics analysis. The natural course of events has commands originating at the central nervous system to the muscles, i.e., the muscular system, which, in turn, develops forces and exerts movements on the body segments, i.e., the skeletal system (Zajac and Gordon, 1989b). The second approach proceeds opposite in direction to the natural course of events, and it is referred to as inverse dynamics analysis. The inverse dynamics analysis starts from the quantitative observations of the movement trajectories, and uses dynamic musculoskeletal modeling to determine the joint moments and muscle forces that must have existed in order for the observed motion to take place (Zajac and Gordon, 1989b). The inverse dynamics analysis is the one that is currently used for clinical gait analysis applications.

With the inverse dynamic analysis, one can observe the dependence of, and express the output segmental torques as a function of the trajectory dependent terms (acceleration of the segmental mass, inertia, gravity and externally applied forces). To obtain the values of each of the parameters needed, different approaches and models are used. Furthermore, since the output of the analysis is in the form of joint torques rather than muscle forces and activations, different modeling approaches, most often optimal control methods, are used to distribute the tensile muscle forces among the redundant set of muscles at each instant of time. However, appropriate distribution of the joint torques to the musculotendon units involved, presupposes knowledge of the musculotendon units dynamics. Therefore, a complete model of the human movement, in general, and of gait, in particular, should include a musculotendon modeling component.

\section{Skeletal modeling}

Construction of a skeletal model to study gait is a function of the need to closely represent the specific movement. To really model gait, many of the characteristics of normal walking should ultimately be included in a 3-D model. The characteristics of normal gait have been referred to as the determinants of gait (Saunders et al., 1953), and they include: (1) pelvic rotation, (2) pelvic tilt, (3) knee flexion during stance, (4, and 5) knee and ankle interactions, and (6) lateral displacement of the pelvis. The "unifying principle" behind each of these determinants was the concept that essentially human walking involved the translation of the center of gravity through space with the least 
vertical fluctuation, i.e., along a pathway that required the least expenditure of energy. It was suggested that the lack of only one of the primary determinants of gait could be compensated for by the other five without severely affecting the appearance of the gait pattern. However, compensation for the loss of two of the determinants resulted into a threefold increase in energy consumption. Of the six determinants, loss of knee flexion was found to be the most detrimental.

Many gait models have been attempted over the years, each specific to the questions posed by the individual investigators. The majority of the gait models in the literature have been two-dimensional models restricted in the sagittal plane (Tumer et al., 1995). Limitation of motion in the sagittal plane requires simplification of gait. The variability in each model is a function of the different assumptions introduced by each researcher to achieve the simplification of walking. Several researchers have looked at the swing phase of the gait cycle. Mochon and McMahon (1980) modeled only the swing phase of gait using a body that is represented by three segments: the stance leg, the swing leg, and the shank. The assumption they made was based on Basmajian's work (1976) which implied that the swing phase is only ballistic in nature. A similar assumption for the swing phase was made by Mena et al. (1981), i.e., the swing leg is a compound pendulum. The swinging side alone was represented by three segments. They attempted to determine the relative contribution of specific swing phase variables. It was found that the three variables necessary to accurately represent the swing phase were thigh initial angular velocity, a passive (ligamentous) knee constraint, and an active moment applied to the foot to maintain toe clearance.

Other researchers have focused on the support phase of gait. Pandy and Berme (1988b) simulated the motion of the lower extremity using applied joint moments as inputs. They concluded that intermittent inputs of simple torque functions, such as linear ramps and steps, were successful in synthesizing the walking motion. In addition, the double support phase has been the focus of the work of some others. Ju and Mansour (1988) pointed out the additional difficulty in simulating the double support as opposed to simulating the single support due to the presence of closed loops kinematic constraints and/or ground reaction forces. Furthermore, attempts have been made to model the entire 
walking cycle too (Onyshko and Winter, 1980). They implemented a seven-segment model, three segments for each leg and one for the head, arm, and trunk. The simulation was accomplished by formulating two different mathematical models, each representing the single and double support phases respectively. The model was driven by a specific set of initial conditions and joint moments found from gait analyses. The works of other investigators who have tried to construct two-dimensional models of gait have employed other methods such as optimal control (Collins and O'Connor, 1991; Yen, 1987).

Restriction of human movement to the sagittal plane has been the most common simplification. It reduces the number of degrees of freedom substantially, particularly at the hip, where two 3 DOF joints are often replaced by a single 1 DOF joint. In addition, the complexity of the dynamic equations of motion is significantly reduced (Onyshko and Winter, 1980). However, two of the limitations of the complete gait models restricted into sagittal plane, is that pelvic tilt, and pelvic rotation, two of the determinants of gait, are neglected. The strong contractions of the hip abductors and adductors, if included at all, become evident only as minor contributions to the hip flexion/extension moment (Yamaguchi, 1989a).

Several attempts of three-dimensional models exist also. Compared to sagittal plane models, the three-dimensional ones are less of a simplification of the actual motion. In particular, they allow the hips to be more accurately described (Delp, 1990; Yamaguchi, 1989a). Instead of the common 1 DOF pin joint to represent the two hip joints, possessing $3 \mathrm{DOF}$ each, both joints may be modeled independently during the support and swing phases of gait (Pandy and Berme, 1988b).

Seireg and Arvikar $(1973,1975)$ solved for joint and muscular forces, plus the inertial and gravitational moments acting on each segment, to determine the 3-D quasistatic equilibrium of a linked system of seven rigid bodies (feet, shanks, thighs, and trunk). A single body for the head, arms, and trunk was utilized, as in most other modeling studies, which presumably is valid when arm swing is not excessive (Cavagna and Kaneke, 1977). The action of 31 forces on each side of the body was included to represent muscle tensions. At the sites where the line of action of the muscle force was interrupted by a bony structure, the line of action was changed to both wrap about the 
interference and impose a tangential force upon it. Since the positions of each link during the gait cycle were previously known, they could solve for the instantaneous torques at each joint using the inverse dynamic method, and for the muscle forces using static optimization. During double-support, the forces under the feet were approximated as linear ramps during the transfer of body weight from one leg to the other.

Initial modeling of the hips and ankles with 3 DOF each, in four segment models was restricted, due to computational limitations, to static optimization approaches when solving for the muscle forces of the lower extremities during walking, i.e., solutions found at discrete time-independent steps (Hardt, 1978; Hardt and Mann, 1980). Conclusions of this work, which focused on the stance leg, included the observation that muscles cannot be modeled as simple ideal unidirectional force actuators since the central nervous system must consider their dynamic and static properties when devising appropriate control strategies. Rather, the characteristics of muscular action, such as moment arms, pennation angles, tension-length and force velocity properties, passive (elastic) elements, and cost functions (in optimization studies) should be accounted for more accurately.

Several researchers have developed gait models using idealized mechanical elements, such as springs and dashpots (Pandy and Berme, 1987a, 1987b, 1988a, 1988b; Siegler et al., 1982). Siegler and his coworkers attempted to simulate the support phase of gait using ideal mechanical elements. Their model was composed of a mass supported by two massless straight legs in which a spring and a damper were combined to represent the mechanical properties of the musculoskeletal system. Simulations of the stance phase were comparable to experimental data with particular reference to the ground reaction forces. The conclusion of this work was that the dynamic characteristics of gait are primarily the result of inertial effects. Pandy and Berme tried to determine the elements of gait most critical to the production of realistic horizontal and vertical ground-reaction forces during single-leg support. Their model possessed five of the six determinants of gait (Saunders et al., 1953), and it was concluded that knee flexion in combination with ankle and knee interactions dominate the dynamic response of the lower extremity during single support. 
Calderale and Sceflo (1987) developed a 9-segment model with 31 muscles per limb to model gait using the concept of effective origins and insertions to ensure that the muscles are maintained within a reasonable volume of the leg. Apkarian et al. (1989) employed a four segment, three joint model to investigate the kinematics of the lower limb. Each of the three joints had three DOF since it was believed that sagittal plane kinematics do not fully describe gait, particularly the pathological gait. Yamaguchi (1989) developed a seven segment, eight DOF dynamic model to study the feasibility of restoring unassisted gait to paraplegics via functional neuromuscular stimulation. The model was developed by prioritizing the DOF in relation to including as many of the determinants of gait as possible. Due to computational restrictions, however, significant degrees of freedom were not included, and as a result, inaccurate active moments were obtained, especially with respect to hip adduction. Even more recently, Moran (1994) constructed a seven segment 13 DOF model with the goal of modeling the pathological gaits of children with cerebral palsy and to determine the optimal pattern of internal muscular forces during gait. It was concluded that the muscle force predictions were determined by the accuracy of the input kinematic data, which, in turn, were not felt to be of high precision due to the insufficiency of current motion analysis methodologies.

The concerns of specific 3-D gait models have often been argued to be the lack of segments such as feet (Hardt and Mann, 1980; Siegler et al., 1982) or trunk (Apkarian et al., 1989; Calderale and Scheflo, 1987; Delp et al., 1990b; Pandy and Berme, 1987a) or the lack of more than one of the gait determinants and adequate degrees of freedom (Yamaguchi, 1989a). In addition, it has been argued that the majority of the existing models are bilaterally symmetric and normal-sized. However, when they are implemented to study pathological gait, most times the pathological system that is modeled, a child with cerebral palsy for example, is neither. Consequently, there exists the need for subject specific modeling. Furthermore, distribution of the joint moments to the musculotendon units that span the joints of interest requires specific modeling of the respective musculotendon unit dynamics and of the respective joints. The former provides the force, whereas the latter provides the moment arm needed for the respective musculotendon unit to partially contribute to the total estimated joint force. 


\section{Joint modeling}

Each determined joint torque includes two components: a passive torque component, and an active torque component. Many of the gait models have stressed the inclusion of passive joint torques (Audu and Davy, 1985; Mena et al., 1981). These torques model the physiological constraints, i.e., bony and ligamentous structures, of the joints at the extremes of motion. Audu and Davy (1985) have suggested equations to determine the passive torques as a function of the joint angle and velocity. Yamaguchi (1989a) suggested that some adjustments to the constants provided by Audu and Davy (1985) may be needed to reduce the laxity in the joints.

The active joint torque is the result of the contributing musculotendon units producing tension, and their respective moment arms $\left(\vec{M}=\sum \vec{p} \times \vec{F}\right)$. The determination of the moment arms of the contributing musculotendon units can be accomplished in different ways (An, 1984). Since the musculoskeletal geometry determines the moment arm, and, therefore, the moment about a joint, some researchers advocate modeling of the specific joints with the proximal and distal attachments of the musculotendon units that span the joint (Hoy et al., 1990; Soutas-Little, 1991). The moment arm is determined as the cross product between the vector connecting the joint center to the origin (insertion) of the musculotendon unit, and the unit vector connecting the origin (insertion) to the insertion (origin) of the musculotendon unit. These investigators use actual origin and insertion coordinates, except when there exist effective ones.

The actual origin and insertion data are obtained from either of two sources: other anthropometric studies from which the data are scaled to the individual subject (Brand et al., 1982; Olney and Winter, 1985; White and Winter, 1993), or imaging techniques, like magnetic resonance imaging (MRI) (Rugg et al., 1990) and computer tomography (CT) with 3-D reconstruction (Nemeth and Ohlsen, 1985). In the most recent investigations there seems to be a trend of using imaging (MRI and CT) information to build subject specific musculotendon models, especially when pathological cases are being investigated (Arnold, 1996; Arnold et al., 1997; Green, 1992; Moran, 1994). The reason for this trend appears to be that scaling procedures that have been developed in the past 
seem to generate undesired errors when the joint centers and the origins and insertions of the muscles are being mapped to a subject (Olney and Winter, 1985). Some research groups have even started to apply dynamic MRI techniques to investigate specific joint parameters of interest that will allow accurate computation of the moment arms (Sheehan et al., 1996).

An alternative technique, but at the same time one that can be used in conjunction with the one in the previous paragraph, to determine musculotendon units moment arms, is based on the principle of virtual work (An et al., 1983; Spoor et al., 1990). This procedure is also known as the tendon and joint displacement method where these two parameters alone are used to determine the musculotendon unit moment arm. Its advantage lies on the fact that it does not require prior knowledge of the center of rotation or of the location of the point of application of the moment arm at the tendon. The concept of tendon and joint displacement method was recently advanced to the "partial velocity" method (Delp, 1990, 1995). This method provides one algorithm, a consistent technique, to compute the moment arms of muscles crossing all types of joints even though the mechanics of these joints differ considerably.

Another procedure used to determine the moment arm is the direct load measurement. There is a known input force, the output moment is measured, and with these two parameters known the moment arm length can be determined. Grood et al. (1984) studied in vitro the moment arm of rectus femoris at the knee joint with this method. Smidt (1973) applied a similar method to the knee in vivo. To define the output moment which is measured by a load cell together with its moment arm, the axis of movement must be known. If the load cell is applied as far as possible to the assumed axis of movement, the error introduced by a false positioning of the axis may be reduced. Unless a triaxial load cell is used, it has to be placed in the plane of movement which is, therefore, predefined. In other words, one assumes that the axis of movement does not change its attitude.

Many investigators have modeled the hip and ankle joints as ball and socket or frictionless revolute joints with $n$ DOF each (Delp, 1990, 1995; Hoy et al., 1990; White et al., 1989; Yamaguchi, 1989a). The moment arms of the musculotendon units of 
interest that span these joints are then determined with either of the methods outlined above. However, the pinpoint joint assumption is not accurate at the knee joint. At the same time, accuracy of the computed extensor moment arm is critical at the knee since large forces are exerted across small extensor moment arms. Consequently, small absolute errors in moment arm yield large errors in extensor moment of force. Several models exist in the literature trying to minimize those errors (Engin and Tumer, 1993; Tumer and Engin, 1993; Yamaguchi and Zajac, 1989b). While each model is specific to its purpose, the one model that is related to gait is that of Yamaguchi and Zajac (1989). The limitation of this model, apart from being planar, is that it is not based on equilibrium conditions. However, authors who have implemented the model for ambulation simulations claim that the extensor moment and moment arms results of their simulations agree with results reported in the literature.

\section{Muscle modeling}

It was mentioned earlier that the active component of the joint torque is the result of two components. The involved musculotendon units which produce tension or muscle force, and their respective moment arms $(\vec{M}=\vec{p} \times \vec{F})$.

Muscle force can be developed in either, or in combination of two ways: active contraction and passive stretch beyond the muscle's resting length or, at the microstructural level, beyond the muscle fiber's resting length $\left(\ell_{o}^{M}\right)$. The force developed during the active contraction is a function of both the length and the velocity of contraction of the muscle fibers. A plot of the tensile force produced by a stimulated muscle at different fixed lengths (i.e., with the velocity $=0$ ) is referred to as the isometric tension-length curve, and it represents the relationship between muscle force and muscle length. This curve includes a passive component which develops once the length exceeds the resting length. The passive component, in general, is modeled as a parallel elastic (PE) element (Zajac, 1989a). Subtracting the passive contributions from the isometric tension-length curves results in a range of length-dependent curves of active force development. The active tension-length relationship of the muscle can be scaled linearly with activation such that at maximum muscle activation, the active muscle force is the maximum isometric force the specific muscle can generate (Zajac, 1989a). 
In muscle modeling, the active force curves are thought of as encapsulating the interaction among different mechanical components. An undamped mechanical spring represents the length-dependent, and velocity independent shortening characteristic of the active muscle (Hill, 1938, 1950, 1953; Wilkie, 1956). This activation-dependent component is referred to as the series elastic (SE) element. Together with the PE, they comprise the elastic force-producing elements of the muscle. In addition, there is a forceproducing or "contractile component" (CE) of the muscle, working in parallel with a velocity-dependent damping element (DE). This model arose from Hill's suggestion that stimulation always results in maximal force development, but this force is dissipated due to viscous resistance during shortening (Hill, 1922).

It should be noted, that force cannot be actively developed if the length of the muscle is less than half or greater than 1.5 times $\ell_{o}^{M}$ (Zajac, 1989a). The maximum of active tetanic force appears to be developed at or near $\ell_{0}^{M}$ which theoretically arises due to the maximum number of crossbridges between the actin and myosin filaments (Gordon, 1966b; Huxley, 1974). In addition, this resting or optimal muscle fiber length has been confirmed to coincide with optimal sarcomere lengths ranging between 2.0 and $2.5 \mu \mathrm{m}$ (Gordon, 1966b). This optimal sarcomere length for mammalian muscle has been found to be between 2.5 and $2.6 \mu \mathrm{m}$, after correcting for the series elastic elements and for the difference between optimal lengths for twitch and tetanic contractions (Close, 1972).

Consequently, it becomes apparent that the force developed by a muscle is, partly, a function of its fiber lengths, which, in turn, are a function of the activation level of the respective muscle and, of the angle at the joint, or joints, that the muscle crosses. Thus, a number of researchers have tried to describe force-joint angle and joint moment-joint angle relationships (Chapman, 1978; Fidelus, 1968; Gleeson and Mercer, 1996; Goslin and Charteris, 1979; Hatze, 1981c; Ismail and Ranatunga, 1978; Jorgensen and Bankov, 1971; Kaufman et al., 1991b; Khalaf et al., 2000; Komi, 1973b; Marsh et al., 1981; Moffroid et al., 1969; Olney and Winter, 1985; Osterning et al., 1983; Rothstein, 1987; Sale et al., 1982; Scudder, 1980; Wilkie, 1950; Winter, 1987; Winter et al., 1981). However, there has been a lot of variability in the relationships that have been identified. 
The reason is often ascribed to the complex interaction of moment arm and structural differences between synergist muscles as well as to the fact that synergist muscles may be working at a different part of their respective tension-length relationship for a given angle.

On the other hand similar tension-length relationships have been observed for single muscle fibers and isolated whole muscle preparations (Abbot and Wilkie, 1953; Bahler et al., 1967; Bahler, 1968; Close, 1972; Gordon et al., 1964; Gordon, 1966b; Hill, 1953; Mashima et al., 1972; Phillips and Petrofsky, 1980; Rack and Westbury, 1969; Wilkie, 1950). Consequently, since similar tension-length curves are observed for most mammalian skeletal muscles, it has been felt possible to generate a generic, nondimensional curve representing the tension-length relationship, that can be scaled to any skeletal muscle. Such a dimensionless curve can be obtained by normalizing muscle fiber length, equivalent to muscle length, by $\ell_{o}^{M}$, and active force by $F_{o}^{M}$ (Zajac, 1989a). The issue then is the estimation of $F_{o}^{M}$. Evidence indicates that the maximum developed stress in muscles of the same subject or of a species is fairly constant. However, that constant has been found to be highly variable in different individuals and species with mammalian muscle (Granata, 1993; Granata and Marras, 1995; Hoy et al., 1990). Theoretically then, once the constant muscle stress has been identified measurements of average muscle cross sectional area can be used to estimate $F_{o}^{M}$. Yamaguchi (1989a) has suggested that the maximum developed stress across a wide spectrum of muscles and mammalian species is approximately $29.43 \mathrm{Ncm}^{-2}$.

In addition to the active tension-length relationship, the active force produced by a muscle is also dependent on the rate at which a muscle fiber changes length (Zajac, 1989a). This is referred to as the force-velocity relationship of the muscle, and it distinguishes between actions, i.e., when the muscle is shortening, concentric contraction, and when the muscle is lengthening, eccentric action. During concentric activity the force is less than that observed during isometric contractions, while the opposite is true for muscles that are being lengthened. One could think of it as if the muscle's contractile unit acts in parallel with a velocity-dependent fluid DE. The force-velocity relationship has been identified empirically by Hill (1938). For an eccentric contraction, the 
maximum normalized force produced is between 1.1 and 1.8 times that of an isometric contraction. Along with the normalization of the force by the $F_{o}^{M}$, muscle fiber velocities are also divided by maximum velocity of contraction, or maximum shortening velocity, to express contraction velocity as a normalized quantity (Zajac, 1989a).

In a similar manner, as the active tension-length relationship of a muscle can be scaled by the activation level, it has been found that a family of parallel tension-velocity curves can be obtained for different muscle fiber lengths (Bahler, 1968; Matsumoto, 1967; Petrofsky and Phillips, 1981). However, since contraction velocity appears to be proportional to myosin ATPase activity only (Close, 1972; Faulkner et al., 1980), it has been accepted that maximum velocity of contraction, i.e., velocity of contraction at zero load, is proportional to the rate of hydrolysis of ATP by myosin only, i.e., proportional to the rate of formation and braking of crossbridges between actin and myosin (Close, 1972; Phillips and Petrofsky, 1981). Consequently, the maximum velocity of contraction is independent of the number of myosin-actin bridges formed per se, and, therefore, it is independent of the muscle fiber length (Gordon, 1966b; Huxley, 1957; Petrofsky and Phillips, 1981). Furthermore, it has been shown that the maximum velocity of contraction appears to be constant for muscle fiber lengths of approximately $0.75 L_{o}$ to $1.2 L_{o}$ (Edman, 1979). Velocities of contraction for greater muscle fiber lengths have been related to intra-muscle fiber passive elastic elements (Close, 1972; Edman, 1979; Phillips and Petrofsky, 1981). Velocities of contraction for shorter muscle fiber lengths have been related to intra-muscle fiber passive resistance to shortening (Edman, 1979). Thus, maximum velocity of contraction for normal ranges of fiber length excursions should be independent of fiber length (Chapman, 1985).

It can be concluded, therefore, that if the maximum velocity of contraction is independent of the muscle fiber length (Chapman, 1985), then the parallel tensionvelocity curves observed at different muscle lengths are a function of the muscle's activation level. However, Zajac (1989a) has proposed that when the muscle fiber acts on the ascending region of the isometric tension-length relationship, then the muscle fiber length, along with the activation level of the muscle, determine the tension-velocity relationship of the muscle fiber. Furthermore, the work of Spector and his collaborators 
(1980), as well as that of Clafin and Faulkner (1985) who showed that a difference of as high as 1.6 times may be present when maximum velocity of contraction is estimated for isolated fast muscle fibers versus whole muscle preparations (the latter representing the accumulated muscle fiber behavior in the muscle), have shown that muscle architecture has an effect on the maximum velocity of contraction. Maximum velocity of contraction has been shown to be dependent on fiber type and, therefore, on the motor unit type associated with the respective type of muscle fiber (Faulkner et al., 1980). However, Zajac (1989a) has argued that maximum velocity of contraction in mixed fiber muscles can always be assumed to be a constant. This view has been discussed in "EMG-driven model development" section.

Thus, it can be concluded that, given the specific architecture of a specific muscle, its tension-velocity response will be a function of the activation level when the muscle operates at the flat region of its tension-length relationship. The tension-velocity response will be a function of the activation level and of the fiber length if the muscle operates at the ascending portion of its tension-length relationship (Zajac, 1989a). It is of particular importance for the present project to note that the magnitude of both the forcelength and force-velocity curves are assumed to be scaled linearly by activation, defined as the percentage of active fibers in the muscle (Zajac, 1989a). In order to determine the force for a given length, velocity and activation, one must identify a point shared by the force-length and force-velocity curves (scaled by activation).

The importance of the effect of tendon as part of the observed behavior of the musculotendon units of interest, especially if those units act at the ankle and knee joints has recently been established (Hoy et al., 1990; Zajac, 1989a). Thus, its inclusion, as a distinct element, in the overall musculoskeletal response to any kind of perturbation, such as gait, which is a control-gravity activity, is also important when physiologically realistic models are being constructed. Since knee flexion-extension is one of the determinants of gait and since most of the ambulatory power generated during gait originates from the plantar flexors (Simon and Ryan, 1992), muscular control of the knee and ankle are critical for gait. Consequently, the behavior of tendon is a desirable property in any model that seeks to investigate the musculoskeletal intricacies involved in 
gait. The effect of tendon has sometimes been included in gait models as part of the SE element in parallel with the PE element (Hof et al., 1983). In this particular study the significance of the Achilles tendon was identified as an elastic energy storage medium which stored energy right after foot strike and returned it during the push off phase in the gait cycle. Other studies, however, have involved its inclusion as a separate component, in series with the contractile element and the PE element (Bobbert et al., 1986; Pandy and Zajac, 1989; Zajac, 1989a). In these studies the SE element has been lumped into the tendon elastic response.

Although it is desirable to include the tendon characteristics as part of the overall behavior of the musculotendon unit, the mechanical properties of the tendon that have been identified in the literature are functions of its structure as well as functions of the tests performed and testing procedures (Haut, 1991). Moreover, the structure, and, therefore, the properties, are influenced by the age, species, diet, pregnancy, use of drugs, and level of activity (Carlstedt and Nordin, 1989; Haut, 1991). Consequently, it may be difficult to formulate a generic model of tendon of high precision.

However, since the muscular forces relative to their maximum strengths are not too high during walking, and because the upper limit for physiologic strain in tendons and ligaments is from 2 to 5 percent for jumping and running (Fung, 1981), a large amount of tendon stretch relative to muscle fiber length is not anticipated during gait for most of the muscles (Zajac, 1989a). Consequently, only the lower range of stresses and strains need be considered, which involve a "toe" and a linear region (Haut, 1991). Zajac (1989a) has modeled the strain in the "toe" region logarithmically as a function of stress. Then, the strain was approximated by a linearly increasing function with a slope equal to the elastic modulus $E^{T}=1200 \mathrm{MPa}$. Strain at $F_{o}^{M}$ was 3.3 percent, which is far below the ultimate tendon strain (Zajac, 1989a). For the tendon force to be obtained instantaneously as a function of its length, it had to be assumed that it was perfectly elastic, energy conservative, and massless. The tendon stress-strain curve was expressed in terms of its normalized force $\vec{F}^{T}=\frac{F^{T}}{F_{o}^{M}}$ (Zajac, 1989a; Zajac et al., 1986b). It should also be pointed out that in a musculotendon unit model, the tendon portion of the model distorts the 
force-length curve such that it has a wider ascending and narrower descending region under certain conditions, i.e., when the ratio of tendon slack length to optimum muscle fiber length is large (above 3) (Zajac et al., 1986b). Values provided by Zajac (1989a) indicate that the ratio for the plantar flexors is approximately in the order of 10 , while the ratio for the muscles working about the knee joint is approximately five. Thus, the effect of tendon at the knee and ankle should be included in a physiologically-based gait model.

The musculotendon unit force is equivalent to the tendon force due to the fact that the muscle and tendon are elements in series (Zajac, 1992). Consequently, muscle fiber shortening as a function of the activation level, tension-length and tension-velocity relationships results in tendon lengthening until equilibrium with muscle tension is reached along its axis. Consequently, by including the effect of tendon in the contraction dynamics, Zajac (1989a) has allowed the development of the musculotendon contraction dynamics, i.e., the force developed by the musculotendon unit is a function of its activation level as well as a function of the tendon length.

So far I have indicated the relationships between tension, length, and velocity in the activated muscle and tendon. However, in recent musculotendon unit modeling it is felt desirable to represent not only the mechanical, but the architectural properties too (Zajac, 1989a). Consequently, models are based upon two sets of parameters: one which is independent of the specific muscle (includes the generic tension-length-velocity and passive curves), and one that is musculotendon specific. There are four parameters in this second set which make the generic curves architecturally-specific to the musculotendon unit of interest: $F_{o}^{M}, \ell_{o}^{M}, \alpha$ (muscle fiber pennation angle) and $\ell_{r}^{T}$ (tendon slack length). Technological advances have allowed investigators to construct computer models which allow the implementation of all the parameters and the relationships discussed to this point. Some of these are commercially available and they are used in research practice including the present one (Delp, 1995; Delp et al., 1990b).

Once all the parameters have been implemented and the specific force-length, and force-velocity relations have been defined, these relations need to be scaled by the muscle activation level to identify the force produced by the musculotendon units of interest, and, consequently, its contribution to the joint moment. However, there seems to be a lot 
of controversy in the literature about the way the activation level is estimated, and, therefore, there is a lot of controversy about the estimated activation level values.

In general, the estimation of the individual or synergistic muscle group contributions as well as the antagonistic muscle contributions to net moments during dynamic activity has been pursued by two approaches. One approach uses optimal control theory to solve the muscle redundancy problem and to generate movement patterns from the perspective of the neuromotor system (Zajac and Winters, 1990; Zajac and Gordon, 1989b). The use of optimal control theory to solve the muscle redundancy problem is referred to as inverse or static optimization and it is accomplished by the use of a cost function or penalty function which time-independent. The use of optimum control theory to generate movement patterns is referred to as forward or dynamic optimization and it is accomplished with the use of a performance criterion integrated with the system dynamics in a time-dependent manner (Anderson and Pandy, 2001; Winters, 1995). In this field of research the use of neural networks currently seems to be a trend. However, the results of several investigators who applied those methods to gait have not been very encouraging (Crowninshield and Brand, 1981a; Davy and Audu, 1987; Moran, 1994; Patriarco et al., 1981; Pierrynowski and Morrison, 1985; Thunnissen, 1993). Inconsistencies in the relation of predicted muscle activation and the pattern of force-time histories to empirically derived EMG patterns have been reported, irrespective of the cost or performance function used (Moran, 1994).

The second approach to estimating the individual or synergistic muscle group contributions as well as the antagonistic muscle contributions to net moments during dynamic activity has been with the use of EMG-driven models. I will attempt to discuss, in a form of summary, the general characteristics of these models as well as their advantages. Reasons which advocate the use of these models include that: (a) they are not limited by the constraints of an optimization objective function; (b) muscle coactivity and antagonistic forces can be accurately represented via measurement; (c) coactive variability within and among subjects is measured directly; (d) physiologic coefficients predicted by the model can be used for instantaneous validity checking; and (e) accuracy may be documented through direct comparison of measured and predicted joint moments. 
The main characteristic of the EMG-driven models is the quantitative inclusion of the EMG signals in the model. The EMG signal is used to represent a measurement of the neural drive, and, therefore, a measurement of the excitation level of the muscle (Hof and Berg, 1981a; Olney and Winter, 1985; Patla et al., 1982). The logic behind this assumption is that "(a) the neural drive is the output, the 'final common pathway', of the central nervous system (CNS), via the motoneurons which includes the contributions of the higher centers, local circuitry, and sensory feedback pathways; (b) information transfer at the neuronal level is unidirectional (as opposed to bi-directional within the biomechanical system); and (c) this intermediate signal, the neural drive, is loosely tied to EMG activity, which can be experimentally measured" (Winters, 1995). Consequently, a model only for muscle dynamics is required rather than for neural dynamics too. However, it has been argued that this approach possesses the inherent limitations common to deterministic, open loop systems. Using the Hill structure as base, both quasi-static and dynamic models can be developed. It should be noted that the representation of the EMG as the excitation level of the muscle involves processing of the raw signal with any of the demodulation techniques available in the time domain. Demodulation techniques include rectification, smoothing or low pass filtering, integration, and root mean square (Gerleman and Cook, 1992; LeVeau and Andersson, 1992).

\section{EMG signal demodulation}

Explanation of the different processing methods of the EMG signal is beyond the scope of this review since those have been well documented (Gerleman and Cook, 1992; LeVeau and Andersson, 1992). However, a summary of the physiological significance of each of the methods is felt to be appropriate.

\section{Rectification}

This is the first process that the raw signal undergoes. Negative values are either eliminated or turned to positive. Full wave rectification, i.e., negative values turned to positive, with zero offset is preferred because it maintains the total energy of the signal. 


\section{Smoothing}

It is a form of low pass filtering to eliminate high frequency noise. Then one can better relate the EMG signal to the contractile features of the muscle (Winter, 1990). In addition, smoothing has been associated with the concept of linear envelope introduced by Winter (1990) where the signal is full wave rectified and then low-pass filtered. The effect of the low pass filter response is to average the variation that occurs in the rectified signal. The output of the linear envelope detector represents a moving average of the rectified signal following its peaks and valleys. Consequently, it provides a profile of the myoelectric activity over time representing with its amplitude proportionally the number of motor units firing, their firing rates and the amount of cancellation from superposition, while the width of its amplitude represents the duration of motor units firing. Consequently, the profile follows the increase or decrease of the muscular activity and the changes in the state of muscular contraction as a function of the motion and position of the joint and segments involved (Close et al., 1960). However, the low pass filtering may introduce a phase lag which is a function of the selected smoothing time constant. If the time constant however is selected carefully, it can resemble the rise time characteristics of the tension produced by the musculotendon unit monitored. Variations of the low pass filtering used can be a Butterworth or a critically damped filter that have been found to much the characteristics of the twitch (Fuglevand et al., 1993; MilnerBrown et al., 1973a; Winter and Patla, 1997), and their cut-off frequency represents the time constant linked to the rise time of the muscle twitch response (Hof and Berg, 1981a, 1981b, 1981c, 1981d). These will be discussed later under the "EMG-driven Model Development".

\section{$\underline{\text { Integration }}$}

The integrated EMG (IEMG) has been related to muscular force more often than any other form of the processed EMG (Bigland and Lippold, 1954; Bouisset and Goubel, 1971; Thorstensson et al., 1976). It is equivalent to calculating the area under the rectified EMG-time curve, and consequently, it determines the total amount of electrical activity displayed during a given interval of time. The IEMG is sensitive to the amplitude, duration, as well as frequency of the action potentials, and, therefore, it 
represents the number of active motor units and their frequency of firing. In addition, it is propagation velocity independent.

However, the quality of the IEMG is a function of the integrator window (level or time reset), which to the best of my knowledge is subjective. The effect, then, is that the IEMG may not be able to account for artifacts and noise in the signal and if the signal is of long duration, the IEMG will rise continuously providing an ambiguous representation of the physiological parameters involved in the generation of the signal. (Falconer and Winter, 1985).

\section{$\underline{\text { Root Mean Square }}$}

The root mean square (RMS) value of the EMG signal does not require prerectification for processing. Its amplitude is sensitive to the number of active motor units, their rate of firing, and the cross-correlation between the motor units. It is not sensitive to the superposition and, therefore, the resulting cancellation from motor units, or their synchronization. Its amplitude is inversely related to the velocity of propagation. Furthermore, while it has been shown that the RMS value contains more relevant information than the IEMG and the mean rectified EMG, it has also been shown that it is proportional to the mean rectified EMG, but its relationship to the active motor units and their respective firing rates is the square root of the amplitude (DeLuca and Van Dyke, 1975).

By taking a moving window mean of the squared signal and then the square root of the amplitude, the RMS provides a linear envelope, or a moving average over time. However, like the linear detector technique, the averaging window, can introduce a phase lag, which, if selected carefully for each musculotendon unit of interest can match the characteristics of the twitch.

Redfern (1988) used EMG-RMS very effectively to investigate moments at the ankle joint. DeVries (1968) determined the efficiency of electrical activity as a physiological measure of the functional state of muscle tissue, using the RMS values as an indication of myoelectric activity. The force and RMS values were linearly related, but the slopes of the lines were different for subjects of different strengths. Another basic study was completed by Lawrence and DeLuca (1983), who investigated whether the 
normalized EMG from surface electrodes versus normalized force relationship varies in different human muscles and whether this relationship depends on training and rate of force production. They used RMS values because the RMS more completely represents motor unit behavior during muscle contraction. Consequently, it can be concluded that, in general, the output form of the RMS is similar to the linear envelope.

\section{Further EMG signal processing}

\section{Normalization}

Many investigators proceed to further processing procedures of the demodulated EMG signal, such as normalization, to estimate the muscle excitation level (Granata, 1993; Granata and Marras, 1995; Marras and G.A., 1990, 1993). The reasoning underlying normalization is that the myoelectric signal amplitude is an indirect measure of the contractile activity of a muscle and its produced force. However, there does not appear to exist a one-to-one relationship between the two. Consequently, a standard of reference or a form of force calibration needs to be established if any comparisons among subjects, muscles or activities are to take place. In addition, normalization can account for the arbitrariness of the EMG signal and its artifacts, due to slight changes in electrode location, change in tissue properties, or change in tissue temperature. The absolute values of microvolts could give an inaccurate comparison of muscle function during different activities. Therefore, a normalization procedure made at each specific testing time for each subject tested could account for such arbitrariness.

One of the most common methods for normalizing the EMG signal is expressing it relative to a reference contraction. Such a contraction is usually an isometric maximal voluntary contraction (MVC or MVIC). The normalized electromyograms then represent a percent of the MVIC (Woods and Bigland-Ritchie, 1983). The use of the MVIC is based on the idea that, if fatigue is not present, there exists a monotonic relationship between the activation level of the muscle and the tension it produces. However, the form of the EMG-tension relationship has been the subject of considerable debate in this field of study. Several researchers have shown a linear or a near linear relationship 
between the myoelectric signal and the force produced (Bigland and Lippold, 1954; Hof, 1984; Hof and Berg, 1977; Lippold, 1952; Milner-Brown and Stein, 1975; Perry and Bekey, 1981; Woods and Bigland-Ritchie, 1983). The MVIC has not always been showing strong repeatability, i.e., it may vary from time to time, and thus, there is always the concern whether an individual exerts a maximum effort (De Luca, 1997). However, it has been argued that it is an acceptable way for referencing the electromyograms (Viitasalo and Komi, 1975). Furthermore, it should be mentioned that recent evidence indicate that the modulation and amplitude pattern of the EMG signal under maximum isometric contraction testing conditions is specific to the contraction state of the muscle, i.e., resisting versus applying load (Karakostas et al., 1995).

In addition, it has been shown that the relationship between EMG and force of a specific muscle depends to a large degree on the physiological makeup of the respective muscle. The motor unit recruitment pattern for each muscle is known to be different (Solomonow et al., 1986). As well as depending on the predominant motor units, a single muscle may exhibit linear or nonlinear EMG-force relations as the recruitment strategy or the task varies (Solomonow et al., 1986). Woods and Bigland-Ritchie (1983) found that muscles with near uniform fiber type composition had a linear relationship between EMG and force, but muscles with mixed fiber type composition had nonlinear relationships. Lawrence and DeLuca (1983), for example, found that the first dorsal interosseous muscle had a linear EMG-force relationship, but the biceps brachii and deltoid had a nonlinear relationship. In general, the number of active motor units increases with increasing force at low force levels, but the firing rate increases at higher force levels (Milner-Brown and Stein, 1975). Slow motor units tend to become active later and continue to fire at higher force levels. The EMG pattern detected at the electrode depends on the number of and firing rate of motor unit action potentials detected in the vicinity of the electrode, the amplitude of the motor unit action potentials, their distance relative to the electrode, and the amount of cancellation occurring from superposition (De Luca, 1979). Consequently, investigations have concluded that intrasubject comparisons are more precise than comparisons across individuals. For the same muscle, the EMGforce relationship appears to have small intrasubject variation, but large intersubject 
variation (Yang and Winter, 1983). Comparisons made on the same subject, therefore, appear to be more valid.

When dynamic activities, such as gait, are studied, a further complication arises, making normalization difficult. The EMG-force relationship found in isometric contractions does not remain the same when muscles are allowed to change length as they contract (Inman et al., 1952). This is the result of the inter-relationship between the length-tension and force-velocity muscle characteristics, and because of the changes in location of firing motor units relative to the surface electrodes. It has been suggested that if a MVIC is used as the reference contraction, the investigator must realize that in many cases the myoelectric signal will give an overestimation of the maximum force (Chaffin et al., 1980). To avoid the overprediction of muscle force, several researchers compute a gain factor, and they try to reduce the EMG levels by a ratio of the dynamic to static EMG levels, removing any velocity artifact in the signal (Granata, 1993). Other investigators devise a calibration procedure where they look at the EMG as a signal where all modulating parameters, such as the muscle physiological make up, are embedded in it and they derive regression equations that would avoid the overestimation of the muscle forces (Van Dieen and Visser, 1999).

\section{Options other than MVIC}

There is some research which suggests that reliability of the normalized EMGs is improved when the reference contractions are submaximal contractions (Perry and Bekey, 1981; Yang and Winter, 1982, 1983). The basis of such evidence appears to lie with the potential non-linearity of the EMG-force relationship. Thus for the purposes of inter- and intra-comparisons some researchers have used submaximal reference contractions. Submaximal contractions are also more likely to be more consistent with the level of contractions used to accomplish a certain motor task under investigation such as gait. Yang and Winter $(1983,1982)$ found that submaximal contractions were more reliable than MVIC and, therefore should be more reliable to use. Some researchers use reference electromyograms from specific tasks, i.e., reference task which may resemble to a certain extend the motor task under investigation. Such an approach was used by Winkel and Bendix (1986) who used three different reference tasks to obtain myoelectric 
signal reference values for the purposes of comparing the different seated work tasks studied. Bobet and Norman (1984), used the isotonic form of the unloaded walking activity to produce the reference contractions in order to investigate the loaded walking activities of interest.

The muscle antagonistic activity, muscle synergy and especially the muscle substitution, of particular importance in pathological situations, complicate the EMGforce relationship when complex motor tasks are being investigated (De Luca, 1997). Synergists and antagonists may be coactivated. Thus the production of the generated force will probably be shared among the synergists, while additional force will need to be generated to overcome the antagonistic effect.

In spite of the complex factors that affect the EMG-to-force relationship, it has been observed that the EMG potentials and contractile responses sum in a nonlinear manner such that the overall relationship between them remains roughly proportional (MilnerBrown and Stein, 1975). Consequently, solution to the indeterminacy problem when muscles co-act and individual muscle forces are of interest can still be obtained (Hof, 1984; White and Winter, 1993).

\section{EMG-driven modeling}

The significant work of Winter's group in the field of EMG-driven modeling should be noted, in particular as it relates to gait analysis (Falconer and Winter, 1985; Olney and Winter, 1985; White and Winter, 1993). In their most recent effort the neural drive, or the excitation level of the musculotendon units of interest was determined by incorporating individual musculotendon kinematics, whereas in the past, measures of the neural drives to individual muscles had been incorporated with joint kinematics. A critically damped, second-order Butterworth filter with cutoff frequency at $2.5 \mathrm{~Hz}$ was selected to represent the transfer function relating the processed EMG to muscle force, while Hof and Van den Berg (1981a,b,c,d) have used different constant for different muscles. They assumed a linear relation between the level of excitation, linear envelope EMG, and isometric force. 
A very interesting feature of this investigation was related to the computation of the gain for individual muscles. One parameter necessary to calculate individual muscle gains was the determination of the physiologic cross sectional area (PCSA) of the muscles that spanned a particular joint. PCSA of a muscle of interest was normalized with respect to the PCSAs of all the active muscles at the joint of interest that had the properly signed moment arm. However, absolute values were defined by data reported in the literature. Another very interest feature of this investigation was related to the way one of the limitations of the surface EMG procedure was addressed when the model was validated. Model validation required the comparison of the joint moments computed by inverse dynamics and those estimated by the model. Since surface EMG, by the nature of the procedure, make it impossible to monitor all the muscles that span a joint, and, therefore, to compute their force and moment contribution, muscles that were not monitored, were grouped and their PCSAs were added to those that had a common function.

The results of the study appeared to be encouraging, with exception of those reported during the eccentric contraction phase, and those pertaining to the knee joint. However, there is good reason to believe that the poor results are related to the assumptions made in modeling, such as the grouping, inaccuracies in the validation method and estimation of muscle lengths, as well as inaccuracies in the subject estimated PCSAs from the literature. Furthermore, an overall review of the work of Winter's group indicates that some of the errors obtained are likely to be due to the mapping of their musculoskeletal model to the subject (White, 1989). The results of the study could have been used to investigate cocontraction at the ankle knee and hip. However, no such attempt was reported.

\section{Cocontraction during gait and EMG-driven modeling}

It was indicated earlier in this section that the purpose of all the modeling procedures is their application to the human movement so that a better understanding of the workings of the system is attained and a methodology for identifying and quantifying cocontraction is achieved. However, the main problem in EMG-driven models with respect to their application in the study of normal and pathological gait is the need for the 
calibration of the tension-length, and tensio-velocity relationships. Such calibrations require some sort of controlled testing on such devices as isokinetic dynamometers.

Several other researchers have pointed out the importance of cocontraction, and especially the importance of quantifying cocontraction, especially for the motor performance of individuals with pathologies such as cerebral (Harrison and Kruze, 1987; McCubin and Shasby, 1985). Falconer and Winter (1985) used EMG to develop an isometric model that estimated the relative cocontraction between the antagonistic muscles, soleus, and tibialis anterior, acting about the ankle. The model was subsequently implemented in dynamic gait to investigate cocontraction. The model produced a measure of the relative cocontraction (cocontraction index) between the two muscles. It could not estimate, however, the torque contributions by the muscle groups. Although this model was limited, it did show that significant levels of cocontraction occur about the ankle during gait and must be taken into consideration. Most other investigations into processed EMG-muscle force relationships have assumed that no antagonistic or synergistic activity occurs during isometric contractions. If this assumption is not valid, the resulting estimations of the EMG-muscle force relationships would be affected. Furthermore, any changes in the amount of antagonistic and synergistic muscle activity between trials of a study would have an even greater effect, causing increased variance in any calculated model parameters. This error would occur even if the processed EMG-muscle force relationships for the individual muscles were totally stationary.

Redfern (1988) examined the isometric torque-processed EMG relationships of the soleus, gastrocnemius, and tibialis anterior during both plantar and dorsi flexion. They proposed a method for calibrating the EMG-torque relationships of the three muscles while taking their cocontractive nature into consideration. From their results, they showed significant synergistic and antagonistic activity during torque production in both the plantar and the dorsal directions. The individual components of torque about the ankle created by each muscle were predicted from the EMG data recorded. The predicted resultant torque was estimated by summing the respective individual components of each muscle. Their results indicated that significant antagonistic activities occur, even during 
simple isometric torque production. Redfern (1988) estimated that a $15 \%$ increase in the slopes of the EMG-torque calibrations occurred for the muscles acting on the ankle when cocontraction was taken into consideration instead of assuming no antagonistic activities. However, although there is the need, no attempt has been made to quantify cocontraction with respect to the forces computed during normal gait while accounting for all musculotendon unit parameters.

The most recent effort involved the work by Damiano and her collaborators (2000) for the musculotendon units acting at the knee joint. The investigators collected reference contraction EMG data from one leg which they used to normalize the EMG data from both legs. The reference EMG data were collected during the isometric case. Cocontraction was obtained by taking the ratio of the time varying dynamic normalized EMG of the antagonist during the trial to the time varying EMG of the agonist, and then finding the mean ratio for the trial. Prior to estimating the cocontraction ratio, a weighing factor was used to weigh the normalized electromyograms from each muscle. The weight factor was the degree of muscle weakness compared with normal pediatric strength values. For gait, the magnitude of cocontraction was computed by measuring the area of EMG overlap between the linear envelopes of agonist and antagonist knee muscles. However, the model did not account for the architectural characteristics of the muscle, and, therefore, cocontraction was not precisely quantified.

Recent technological advancements have made possible the construction of software that allows the interaction of neuromusculoskeletal modeling parameters including the individual musculotendon characteristics (Delp, 1990, 1995; Delp et al., 1990b). In addition, there has been expressed the desire to incorporate such efforts in clinical practice (Perry, 2000). The present project used that software as the initial stepping stone for the attainment of its input parameters in order to ultimately quantify cocontraction. 


\section{CHAPTER 3}

\section{EMG-DRIVEN MODEL DEVELOPMENT}

\section{Introduction}

The block diagram in figure 3.1 provides a conceptual framework for the suggested research project and the overall modeling approach for achieving the goals of the investigation. The primary theoretical phases of the project are represented by the blocks in series, after the "Noraxon" blocks to the "Active Moment Predicted", which reflects the summation of the moments that are generated by the individual musculotendon units.

Indirect inputs to the theoretical phases include motion analysis data from the gait study, which are used to determine the lengths, the velocities, the moment arms, and the maximum forces, as a function of length and velocity, of the musculotendon units of interest. Gait analysis information was collected using the subject population, and the collected data were processed to provide gait trajectories and force plate ground reactions, which, in turn, were subjected to inverse dynamics analysis to compute net joint moments. The net joint moments were separated into active joint muscle moments and passive moments.

Direct inputs to the theoretical phases included electromyographic (EMG) processed data from the gait analysis. Processed EMG data from the gait evaluation, combined with the calibration data determined the activation level of the muscle. The calibration data constituted another aspect of the theoretical phases of the model that additionally was used to estimate the average time variant gain $(G(t))$ of the musculotendon units of interest (Figure 3.2).

Another direct input to the theoretical phases is the normalized tension-length and tension-velocity relationship of the musculotendon units. The last input to the theoretical phases is the parameter information, i.e., the musculoskeletal geometry such as muscle 


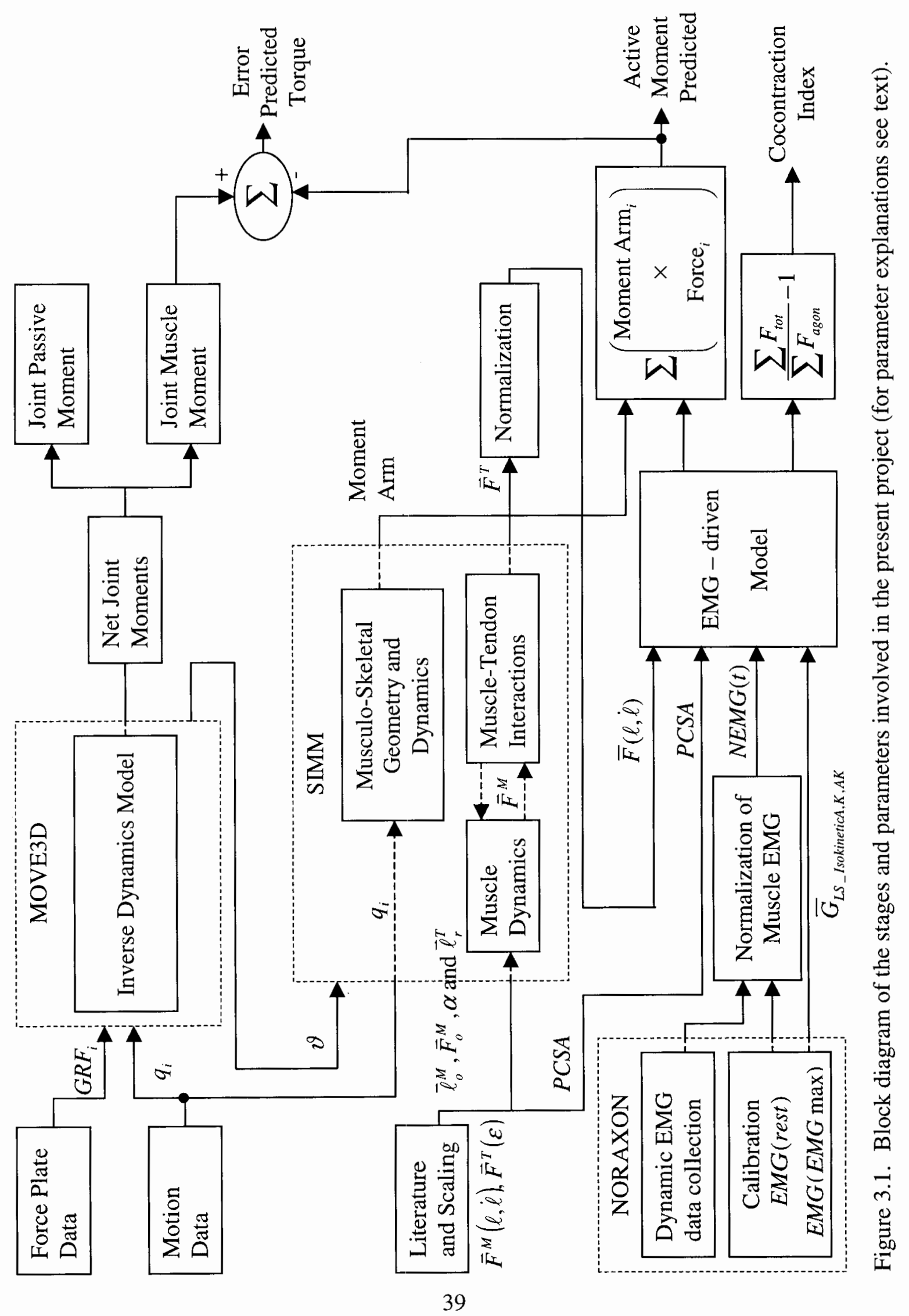




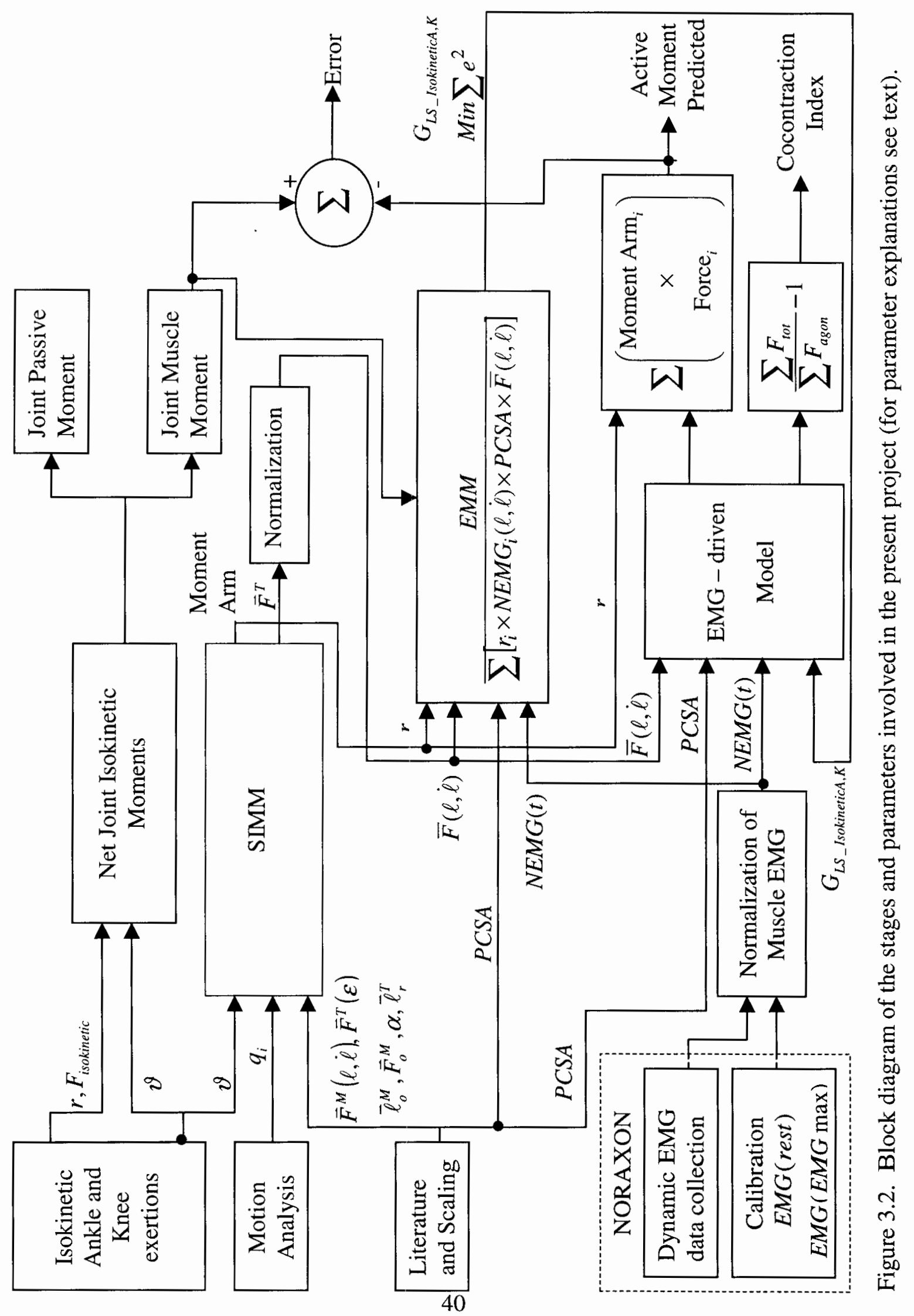


origins and insertions, muscle optimum fiber length, maximum isometric force, pennation angle, PCSA, and tendon slack length, which were supplied by the literature.

The goal of all these was to determine muscular moments so that the sum of all the predicted muscle moments acting about a specific joint matches, as close as possible, the respective active net joint moment determined with inverse dynamics analysis. If this is accomplished, then the ultimate output generated by the theoretical procedures is indicated at the bottom of figure 3.1, i.e., a cocontraction index. If the final output is zero, then there is no antagonistic cocontraction. Therefore, efficiency is maximum in performing the desired movement. However, cocontraction increases with antagonistic muscle activity. The higher a cocontraction index, the lesser the efficiency in performing the respective movement. Consequently, the more the difficulty, and, therefore, the greater the energy expenditure will be of an individual to perform the desired movement. In individuals with pathologies, such as children with mild cerebral palsy, the cocontraction index may be expected to be relatively high at certain joint velocities and joint angles. In children with severe cerebral palsy the cocontraction index may be expected to be much higher. The threshold value over which an individual is not capable of performing the desired lower extremity movements remains to be investigated.

\section{Muscle modeling and parameters}

The EMG-driven model that will be used in the present project to calculate the tensile force produced by each musculotendon unit, $i$, is described by the following components: (a) a gain factor, $G$, which represents the maximum muscle force per unit physiological cross sectional area; (b) the activation level of the muscle, $N E M G_{i}$, determined by the normalized EMG; (c) the physiological cross sectional area, $P C S A_{i}$, of the musculotendon unit of interest; and (d) the force-length and force-velocity relationships, $\bar{F}(\ell, \dot{\ell})$ of the musculotendon units (Figure 3.1).

$$
\text { Muscle_Force }{ }_{i}=G \times N E M G_{i}(t) \times P C S A_{i} \times \bar{F}_{i}(\ell, \dot{\ell})
$$

The following discussion will focus on the attainment of each of the components of the EMG-driven model. However, firstly it is necessary to justify and point out the significance of the suggested muscle modeling approach. 
Muscle modeling incorporating the quantitative contribution of EMG in the current literature (of the past ten years from the time that this project was constructed and completed), especially as it relates to gait, is being dominated by two schools of thought. From a qualitative point of view one school of thought has constructed a generic model which aspires application to a variety of motor tasks, Zajac's group. The other approach involves a muscle model that is more gait specific, Winter's group. Furthermore, Zajac's group models the muscle at the fiber, a microstructural, level, i.e., the muscle fiber is considered to be the representative unit that reflects, firstly, in the absence of a series elastic element in the fiber level, the scaled behavior of the individual sarcomeres in series, and secondly, therefore, the behavior of the muscle. Winter's group, on the other hand, approaches muscle from a macroscopic perspective, i.e., models the muscle as a whole.

From a mathematical point of view, one school of thought, models the musculotendon unit dynamics with differential equations because its specific purpose is the forward simulation of human movement by accounting for the behavior of the tendon, encapsulating Hill's (1938) series elastic component (SEC), that contributes dynamically to the musculotendon kinematics, and ultimately, to the final musculotendon force predicted, Zajac's group (Zajac, 1989a). In doing so, the musculotendon unit is allowed to behave under different activation and musculotendon unit length changes conditions either as a spring (force output is directly related to its length), or as a dashpot (force output is directly related to the rate of length changes), or as a central nervous system controlled force generator (force is directly related to the neural input). The other school of thought takes a more algebraic approach in modeling the musculotendon unit dynamics by treating the tendon as a stiff spring, Winter's group (White and Winter, 1993). The implication of such an assumption is that musculotendon unit length changes of each actuator do not need to be partitioned to contractile element and series elastic component length changes. The advantage of such an approach is that the researcher does not need to account for any length changes that may take place within each crossbridge, and, consequently, the potential for energy storage in the SEC (White and Winter, 1993). A summary of the characteristics and the differences between the two scientific 


\begin{tabular}{||l|l||}
\hline \multicolumn{1}{|c|}{ ZAJAC'S GROUP } & \multicolumn{1}{c|}{ WINTER'S GROUP } \\
\hline Modeling of the muscle fiber & Modeling of the muscle as a whole \\
\hline Differential equations & Algebraic equations \\
\hline $\begin{array}{l}\text { Distinct passive component (Parallel } \\
\text { Elastic Element) }\end{array}$ & No Parallel Elastic Element \\
\hline $\begin{array}{l}\text { Non-dimensional, scaled to all muscles, } \\
\text { curves representing the musculotendon } \\
\text { unit properties: }\end{array}$ & $\begin{array}{l}\text { Individual, specific to each subject } \\
\text { musculotendon unit curves to represent } \\
\text { each muscle: } \\
\text { Active muscle force-length } \\
\text { relationship }\end{array}$ \\
$\begin{array}{l}\text { Passive muscle force-length } \\
\text { relationship }\end{array}$ & $\begin{array}{l}\text { Active muscle force-length } \\
\text { relationship } \\
\text { Muscle force-velocity relationship } \\
\text { Tendon force-length relationship }\end{array}$ \\
\hline $\begin{array}{l}\text { Muscle fiber pennation angle, constant } \\
\text { roes not account for muscle fiber type } \\
\text { distribution in each muscle }\end{array}$ & $\begin{array}{l}\text { No muscle fiber pennation angle } \\
\text { Accounts for fiber type distribution in } \\
\text { each muscle }\end{array}$ \\
\hline $\begin{array}{l}\text { Do not use gain (incorporated, however, } \\
\text { in the inclusion of maximum isometric } \\
\text { force); in that sense gain is a constant } \\
\text { without allowing for inter-subject } \\
\text { variabilities }\end{array}$ & $\begin{array}{l}\text { Use gain, a non constant, allowing, } \\
\text { therefore, for inter-subject variabilities; } \\
\text { however, it does not guarantee constant } \\
\text { gain per subject }\end{array}$ \\
\hline $\begin{array}{l}\text { Do not use the physiological cross } \\
\text { sectional area (incorporated, however, } \\
\text { with the use of maximum isometric force) }\end{array}$ & Use the physiological cross sectional area \\
\hline $\begin{array}{l}\text { Normalized EMG can be used to represent neural drive, i.e. muscle activation level } \\
\text { Zajac's model can be modified to incorporate the advantages of both approaches }\end{array}$ \\
\hline
\end{tabular}

Table 3.1

A summary of the relative characteristics of the two muscle modeling approaches used by Zajac's and Winter's groups.

approaches is presented in Table 3.1.

Another distinct difference between the two main, for the purposes of this study, muscle modeling schools of thought, is that Zajac's group models the passive component of a muscle as a parallel elastic (PE) element (Zajac, 1989a), whereas in Winter's group the PE component, for the purposes of investigating gait, is not included on the basis of evidence by Bahler et al. (1968). It was advocated that the contribution of the PE element to the contribution of force by individual muscles is minimal in normal gait since 
there is little PE element force contribution up to 1.2 times muscle rest length. The assumption here was that the normal working length of the intact human muscle falls within a range of $73 \%$ to $120 \%$ of its normal resting length where parallel elastic forces are not present. It should be pointed out that the range reported is really the normal working range of the fiber length rather, than that of the whole muscle. Consequently, such an assumption can only be true if it is believed that external observations of the musculotendon kinematics represent the kinematics of the individual muscle fibers. However, since evidence indicates that in cases of isometric contractions, where the muscle length is assumed to be constant, there may still be muscle fiber shortening, the accuracy of the above assumption may be questionable. In addition, when movements that involve coactivation of agonist and antagonist muscles are being observed, the accuracy of the above assumption becomes even more controversial. Furthermore, it has been suggested that, especially with regard to the gait of individuals with spasticity, in general, and more specifically, with regard to the gait of individuals with cerebral palsy (CP), the passive elements surrounding each muscle appear to have an increased thickness, and to be shorter (Simon and Ryan, 1992). Consequently, the inclusion of the $\mathrm{PE}$ element in a muscle model that strives to be versatile on the grounds that it may, in the future, aim in studying pathological gait is very important. Furthermore, its separate inclusion is vital, if its effect as a distinct parameter is to be investigated.

While both schools of thought use Hill's model (Hill, 1938), the model used by Zajac's group has the muscle active force-length, muscle passive force-length, muscle force-velocity and tendon force-length relationships incorporated in the model as four generic, non-dimensional curves easily scaled to any musculotendon unit, given certain parameters that will be described below. On the other hand, the model implemented by Winter's group uses only the muscle active force-length, and muscle force-velocity relationships. It is required that these relationships are provided for every muscle or muscle group of interest (White and Winter, 1993). These relationships, once the models of both White and Winter's and that of Zajac are implemented into code can be generated via computer simulations. However, obtaining these relationships as a function of muscle activation via computer simulations can only implemented using Zajac's model (Delp, 
1990, 1995; Delp et al., 1990b). White and Winter's model requires implementation of a calibration procedure involving isokinetic testing at six degrees per second (White and Winter, 1993). The tendon force-length relationship, as it was indicated earlier, is excluded by considering the tendon a stiff spring. Although, evidence indicate that such an approach would be appropriate for musculotendon units with very small tendons, and, therefore, minimum maximal strains (Van Soest and Bobbert, 1993; Van Soest et al., 1995), a more complete muscle modeling approach should allow for the investigation of the effect of the muscle passive force-length relationship, as well as for the implementation of the tendon force-length relationship. The later would seem to be of particular importance due to the dynamic interaction of the muscle and tendon components (Van Soest et al., 1995; Zajac, 1989a). In particular, in Zajac's model the fiber length and fiber velocity are not identical reflections of the musculotendon unit. They differ by the length of the tendon and its velocity, both of which are a function of its compliance. Thus, while White and Winter (1993) assume that muscle fiber length changes mimic changes in musculotendon length on the basis that the tendon is a stiff spring, Zajac (1989a) does not, thereby accounting for very compliant tendons.

Moreover, while for both models the important muscle-specific parameters required are, optimum fiber length $\left(\ell_{0}^{M}\right)$, maximum isometric force $\left(F_{0}^{M}\right)$, pennation angle $(\alpha)$, and tendon slack length $\left(\ell_{r}^{T}\right)$-in Winter's group the $\ell_{0}^{M}$, i.e., the optimum muscle length or muscle resting length, and $\ell_{r}^{T}$ are treated combined as $\ell^{M T}$ - contrary to Zajac's approach the model of Winter's group formally includes the physiological cross sectional area (PCSA) ${ }^{1}$ of the muscle, the fiber type distribution, and the gain, which represents the physiologic muscle-force per unit cross-sectional area. On the other hand, Zajac's model includes the additional effect of muscle pennation angle. It should be noted, however, that the effect of the PCSA can be reflected on the estimation of the $F_{0}^{M}$ (Close, 1972; Hoy et al., 1990; Lieber, 1992; Pierrynowski, 1982, 1995; Spector et al., 1980; Wickiewicz et al., 1983).

\footnotetext{
${ }^{1}$ Physiological cross sectional area, for the purposes of this project is defined as the total muscle area that is normal to a longitudinal axis parallel to the direction of the muscle fibers.
} 
The representation of the specific to each musculotendon unit fiber type distribution and pennation angle is important for a more accurate estimation of each muscle's output. Fiber type distribution in the muscle affects the maximum velocity of contraction (Vo) of the respective musculotendon unit (Claflin and Faulkner, 1985; Faulkner et al., 1980; Spector et al., 1980; White and Winter, 1993). Zajac's approach suggests that for all mixed-fiber muscles the Vo should be $10 \ell_{0}^{M}$ per second since that is, approximately, the maximum shortening velocity of fast muscle tissue. The basis of this argument is the assumption that fully activated mixed muscle exerts tension until the maximum shortening velocity of the fastest fiber type is reached. It is the speculation of this author that this may not be an appropriate assumption, since the implication would be that the tension developed at the higher velocities of contraction by a mixed muscle depends equally on its fast twitch fibers as well as on its slow twitch since the maximum velocity of contraction is the same for all muscle fibers. However, that would not be true as slow twitch muscle fibers would stop producing tension at their respective maximum velocities of contraction, which would be smaller than these of the fast twitch muscle fibers. Thus, an error would be introduced at the estimation of forces where contraction velocities approach the maximum velocities of contraction of the muscle's fast twitch fibers. White and Winter (1993), on the other hand, suggest the computation of the Vo as a function of the fiber distribution in the muscle.

Furthermore, recent evidence have linked the behavior of the tendon, often times referred to as a series elastic element (SEE), with the maximum velocity of contraction (Van Soest et al., 1995). In fact, when the effect of its removal from the musculotendon unit was investigated, it was determined that the effect was inversely related to maximum velocity of shortening. In addition, a high maximum velocity of contraction indicated that the musculotendon unit system was only slightly damped as a result of which phase shift and force amplitude loss, due to tendon compliance, would be underestimated (Van Soest et al., 1995).

The pennation angle of the muscle fibers with respect to the tendon is accounted for directly in Zajac's approach when the muscle force is being computed. However, with respect to the muscle kinematics, i.e., shortening of the muscle fibers and shortening of 
the musculotendon unit, it was determined that only highly pennated muscles, such as the soleus and the short head of the biceps femoris, have their musculotendon function being affected significantly. In musculotendon units with highly pennated muscle fibers the musculotendon unit length change is greater for any given muscle fiber length change. Thus musculotendon units with highly pennated muscle fibers shorten faster than the individual muscle fibers do (Zajac, 1989a). In other words, because in musculotendon units with fiber pennation angles higher that 20 to 30 degrees, the change in musculotendon unit length is greater for any change in muscle fiber length, the respective rates of changes will be different, with the rate of change of the musculotendon unit length being greater than that of the muscle fiber length by the cosine of the pennation angle (Zajac, 1989a). However, to the best of this author's knowledge, while the pennation angle during any motor task changes as a function of muscle length and activation (Fukunaga et al., 1997), Zajac's model considers it a constant.

Winter's group on the other hand does not implement the correction of the estimated forces for the pennation angle of the muscle fibers. However, when the PCSA is being estimated, which is used for the determination of the $F_{0}{ }^{M}$, the respective PCSA is corrected for the pennation angle of the muscle fibers and it is assumed to be constant also. Furthermore, the effect of the pennation angle for the musculotendon dynamics is not accounted for either.

Both modeling approaches require an estimated excitation level of the muscle. The activation level of the muscle is obtained from the electromyographic (EMG) signal. In Zajac's approach the muscle activation level is the result of the neural-excitation input signal, i.e., of the EMG. The two are related via an activation dynamics process, i.e., "EMG-to-activation process", represented by a first order dynamics transfer function including a bilinear differential equation. In the transfer function there is a rate or time constant which determines the activation dynamics relationship between the neural excitation and the muscle activation level. That relationship is linear with the amount of excitation, being the fastest when the muscle is fully excited. In White and Winter's approach the EMG, when processed and normalized appropriately, represents the excitation or activation level of the muscle. Appropriate processing of the EMG signal 
involved full wave rectification, a critically damped, second order low-pass filtering with a $2.5 \mathrm{~Hz}$ cut-off frequency and normalization to an EMG value from a maximum voluntary isometric contraction.

The gain factor for the model used by Winter's group is a constant for each muscle monitored, and it is estimated as the ratio of the force to the processed EMG level at a specific muscle length. Based on Komi's definition (1973b) a gain value of one is then obtained during maximum isometric contraction when the normalized force is plotted against the normalized EMG. Given such a definition, then, the gain does not appear to have any physiological meaning other than being a scaling coefficient factor that relates in a polynomial fashion the EMG and muscle length values to the generation of the respective musculotendon unit forces in the dynamic cases. Since a polynomial approach is implemented, the gain encapsulates different coefficients of a third order polynomial. For others, however, the gain, also a constant, represents a physiological parameter, such as the force per unit cross sectional area, i.e., muscle stress (Granata, 1995; Granata and Marras, 1993, 1994). Granata and Marras (1993), in their EMG-driven model for the spine determined the gain factor, also a constant, by accounting, not only for the length, but also for the velocity artifact components embedded in the dynamic EMG, and in the force-length and force-velocity relationships of the muscle, since it is well established that the modulation of the EMG and force is a function of the muscle length (Marras and Sommerich, 1991a), and velocity of contraction (Bigland and Lippold, 1954). A more appropriate approach might be implemented if one was able to disassociate the length and velocity artifact components embedded in the dynamic EMG from the force-length and force-velocity relationships. This artifact could be incorporated in a time varying gain and then modeled appropriately.

Consequently, although, each of these models has its merits and has made an important contribution in the muscle modeling literature, and, in particular, in the field of EMG-driven modeling, none was felt to be complete and adequate on its own for the purposes of this project. Therefore, a modified version of the muscle model by Zajac's group was implemented because it involves most of the important identified muscle parameters individually. The modified model incorporated the effects of the fiber type 
distribution and gain while at the same time it accounted for the effects of the muscle length and velocity components on the collected EMG. Since the musculoskeletal graphics software SIMM (Musculographics Inc.) includes Zajac's muscle model, and it can be modified to incorporate most of the required parameters at the initial stages of the model development, it was felt that its use was appropriate for the purposes of this project. Some of the output of SIMM was incorporated in the EMG-driven model. Consequently, along with the parameters of the EMG-driven model the various input components to SIMM also need to be discussed.

It should be mentioned here, however, that one of the limitations of using Zajac's model as it has been implemented in SIMM, is that the specific muscle parameters that are used, cannot be scaled to each of the subjects that participated in the study. Methods for scaling anthropometric muscle parameters have been reported in the literature (Pierrynowski, 1995). However, in the case of SIMM the muscle parameters, especially that of tendon slack length, have been optimized to agree with kinetic data and kinetic output from experimental studies that had taken place and had been reported in the literature by the time that the model was implemented into code (Delp, 1990; Hoy et al., 1990). On the other hand, implementation of the EMG-driven model suggested, can individualize all the input parameters via the estimation of the subject-specific gain through a calibration procedure. Consequently, since some of the output of SIMM was incorporated in the EMG-driven model, it is felt necessary, along with the parameters of the EMG-driven model, to discuss the various input components to SIMM also.

Maximum isometric force can be determined for each muscle by the function

$$
F_{0}=2.5 \times g \times P C S A
$$

where the value 2.5 is a constant in kilogram units for mammalian muscles for the maximum developed stress, i.e., force per unit area (Yamaguchi, 1997), and $g$ is the gravitational acceleration. The PCSA values can normally be obtained from literature information (Alexander and Goldspink, 1977; Brand et al., 1982; Freivalds et al., 1984; Friederich and Brand, 1990; Lieber, 1992; Pierrynowski, 1995; Wickiewicz et al., 1983). However, for the purposes of this project, since Zajac's model, as it was implemented in Delp's and Loan's SIMM software (Delp, 1990, 1995) was used, in combination with the 
lower extremity muscle architectural information (for PCSAs), based on Hoy's work (Delp, 1990; Delp et al., 1990b; Hoy et al., 1990), the PCSAs for the muscles of interest were computed on the basis of the maximum isometric force data provided, given the respective specific muscle tensions from the investigations that the original data were used. Since Hoy et al. (1990) and Delp (1990) optimized the specific muscle tensions of the investigations that were used for the purposes of computing maximum isometric muscle forces, it was felt by the present author that using the maximum isometric forces given, along with the original specific tensions from the respective studies, the optimum PCSAs would be obtained. Thus, the "optimized" PCSAs that were used in the present study and the respective maximum isometric forces are given in Table 3.2.

Combination of the two variables, PCSA and $F_{0}^{M}$, could provide the constant gain factor. However, although it would be reasonable to assume that the intrasubject gain as a physiological, subject-specific, parameter should be a constant value, an adequate modeling approach should allow for a variable gain among individuals. Intersubject gain variability should allow accounting for factors such as personal conditioning, training, and natural ability. Therefore, a methodology was developed to allow the estimation of individual gains. The procedure will be discussed under the calibration section.

Values for $\ell_{0}^{M}$ were also obtained from literature data (Alexander and Goldspink, 1977; Brand et al., 1982; Delp, 1990; Friederich and Brand, 1990; Hoy et al., 1990; Lieber, 1992; Pierrynowski, 1995; Wickiewicz et al., 1983) and they were implemented as suggested in SIMM with Delp's (1990) lower extremity model. Values for $\alpha$, the pennation angle, were obtained from the literature also (Alexander and Goldspink, 1977; Brand et al., 1982; Delp, 1990; Friederich and Brand, 1990; Hoy et al., 1990; Lieber, 1992; Pierrynowski, 1995; Pierrynowski and Morrison, 1985; White and Winter, 1993; Wickiewicz et al., 1983), and since, to the best of my knowledge, there are no studies to suggest that they are variable across individuals, especially those with such pathologies as cerebral palsy, they can be assumed to similar for all people.

The $\ell_{r}{ }^{T}$ parameter of the model was obtained from literature data also (Delp, 1990; Hoy et al., 1990). It is important to note here that both of these studies estimated $\ell_{r}{ }^{T}$ 


\begin{tabular}{||l|c|c||}
\hline \multicolumn{1}{|c|}{ Muscle } & Maximum Isometric Force (N) & $\begin{array}{c}\text { PCSAs } \\
\left(\mathrm{cm}^{2}\right)\end{array}$ \\
\hline Biceps Femoris (long head) & 717 & 23.90 \\
\hline Biceps Femoris (short head) & 402 & 13.40 \\
\hline Rectus Femoris & 779 & 12.77 \\
\hline Semimembranosis & 1030 & 16.88 \\
\hline Semitendinosis & 328 & 5.37 \\
\hline Vastus Intermedialis & 1365 & 22.37 \\
\hline Vastus Lateralis & 1871 & 30.67 \\
\hline Vastus Medialis & 1294 & 21.21 \\
\hline Medial Gastroc & 1113 & 37.10 \\
\hline Lateral Gastroc & 488 & 16.26 \\
\hline Extensor Digitorum Longus & 341 & 5.59 \\
\hline Peroneus Brevis & 348 & 5.70 \\
\hline Soleus & 2839 & 46.54 \\
\hline Tibialis Anterior & 603 & 9.885 \\
\hline
\end{tabular}

Table 3.2

Physiological cross sectional areas (PCSAs) estimated by Hoy's (1990) and Delp's (1990) maximum isometric force data and the specific muscle tensions from the original studies used to obtain the maximum isometric muscle forces.

such that model output data matched data reported in the literature. Definition of this parameter is very significant as, when it is normalized to $\ell_{0}^{M}$, the ratio determines the compliance of the tendon. A high ratio indicates that a tendon is very compliant, whereas a low ratio indicates that a tendon is stiff. The significance of such an indication determines the musculotendon unit contraction dynamics, i.e., its mechanical behavior (Zajac, 1989a). A stiff tendon has very little effect on the behavior of the muscle fiber contraction dynamics, i.e., the dynamic changes of the musculotendon unit as a whole can be reflected in the dynamic changes of the muscle fiber. Furthermore, assuming a low ratio, such as it was done by Winter's group, may lead in misleading assumptions about the function of the specific actuator during motor tasks such as gait. For example, for Winter's group the function of the vastus lateralis muscle during the initial phase of the gait cycle would be considered to be equivalent to that of a spring. Zajac, however, 
has shown that given the tendon's stiffness, a function of its normalized slack length, given a relatively low activation level during the activity (these two parameters determine the frequency bands within which a musculotendon unit acts as a spring or as a dashpot), and given the low frequency content of the motion of its musculotendon length, the musculotendon unit acts like a dashpot rather, than a spring. Thus $\ell_{r}^{T}$ is a very important parameter.

The effect of fiber type distribution was included in the model as the maximum velocity of contraction determined, not by the kinematic information, combined with Zajac's assumption that, maximum shortening velocity is the same for all muscles, if they are mixed fiber muscles, as suggested by the SIMM consultants (Loan, 1998; Zajac, 1989a). Maximum velocity of contraction was determined individually for each musculotendon unit of interest by accounting for the respective fiber type distribution as outlined by Winter (1993), and based on data presented by Hof and van den Berg (1981).

In other words, research supports that $V_{0}$ is proportional to the rate of crossbridge formation and breaking since it is a directly related to the rate of ATP hydrolysis by myosin at individual enzyme sites (Close, 1972; Kirchberger, 1990; Phillips and Petrofsky, 1980). Evidence that advocated such a thesis are related to the high correlation between contraction velocity and myosin ATPase activity (Close, 1972; Kirchberger, 1990; Phillips and Petrofsky, 1980). Consequently, $V_{0}$ has been considered an innate constant that depends on motor unit fiber type. A value of $V_{0}=\frac{10.0 L_{0}}{s}$ (where $L_{0}$ represents the optimum or resting fiber length, and $s$ represents time in seconds) was adopted for the gastrocnemius muscle that is a mixed fiber muscle with a 48.2:51.8 distribution between slow (Type I) and fast (Type II) motor units (White and Winter, 1993). That same value has been adopted for all muscles by Zajac (1989a). For the purposes of this project, however, that value was used as a reference for computing $V_{0}$ for the other muscles. The $V_{0}=\frac{10.0 L_{0}}{s}$ was multiplied by the ratio of the fast motor units of the musculotendon units of interest expressed relative to the fast motor units of 


\begin{tabular}{||l|l|c|}
\hline Joint & \multicolumn{1}{|c|}{ Muscle } & $\begin{array}{c}\text { Maximum Contraction Velocity } \\
\left(L_{0} / s\right)\end{array}$ \\
\hline Knee & & 6.51 \\
\hline & Biceps Femoris (1) & 6.57 \\
\hline & Biceps Femoris (s) & 10.0 \\
\hline & Gastrocnemius & 11.68 \\
\hline & Rectus Femoris & 9.65 \\
\hline & Semimembranosis & 9.65 \\
\hline & Semitendinosis & 9.68 \\
\hline & Vastus Medialis & 9.65 \\
\hline & Vastus Intermedius & 10.56 \\
\hline & Vastus Lateralis & 10.00 \\
\hline & & 4.01 \\
\hline & Gastrocnemius & 8.93 \\
\hline & Soleus & 5.40 \\
\hline & Peroneus Brevis & 10.88 \\
\hline & Tibialis Anterior & \\
\hline & Extensor Digitorum Longus & \\
\hline
\end{tabular}

Table 3.3

Maximum contraction velocities used in the present project.

the gastrocnemius. Percentages of the respective motor units for the muscles of interest were taken from the literature (Johnson et al., 1973; Pierrynowski and Morrison, 1985; White and Winter, 1993) because the reported $V_{0}$ estimated with the procedure outlined by White (1993) could not be verified. The $V_{0}$ values that were used in this project (see Table 3.3).

The individual musculotendon unit lengths and velocities were determined accurately by SIMM, which calculated them as the sum of the lengths of the line segments that define the muscle path, and as the sum of the velocities of the line segments that define the muscle path, respectively (Delp, 1990, 1995; Delp et al., 1990b). These two experimental parameters were the initial input to Zajac's muscle model implemented in SLMM. However, in order to determine the muscle length, the proximal and distal attachments of the musculotendon unit to the body segments need to be known. The coordinates of these points were determined from literature data and they were implemented as suggested in Delp's lower extremity model (Delp, 1990). 
The proximal and distal attachments of each musculotendon unit in SIMM were found on different body segments that are expressed in different reference frames. Therefore, the proximal and distal attachment points were expressed in a common reference frame. The frame of the proximal attachment was selected as the common reference frame (Delp, 1990). The different reference frames were related by the directional cosines (rotation matrices) as one segment moved with respect to its respective proximal segment. Then the origin and insertion were vectorally added and the square root of the sum of the squares was added to obtain the vector magnitude.

Obviously, the length of a musculotendon unit is dependent upon the angle/angles of the joint/joints it crosses. This was taken into account by the directional cosines. If the musculotendon unit length is considered as a straight line between the proximal and distal attachments, in some cases the muscle would have been placed outside of the normal boundaries of the segments (Delp, 1990; Yamaguchi and Zajac, 1989). This is especially true for the muscles crossing the knee. Therefore, effective origins and/or insertions were defined so that the muscle acted in a more accurate direction. Because the effective coordinates did not translate with respect to the actual coordinates, the distance between the effective and actual origin and the distance between the effective and actual insertion remained constant (Yamaguchi and Zajac, 1989). Therefore, changes in the length occurred only between the effective coordinates.

Given the muscle-specific parameters $\left(F_{0}^{M}, \ell_{0}^{M}, \ell_{r}^{T}, \alpha\right.$, and maximum velocity of contraction, which was adjusted to represent the fiber type distribution), the instantaneous muscle length and velocity of the musculotendon unit, and the activation, the musculotendon model by Zajac (1989a), implemented in SIMM, computed the force produced by any given muscle and tendon in series. The instantaneous musculotendon unit lengths and velocities were determined by the kinematics from the motion data collection. For the purposes of this step in the modeling procedure, the muscle forces were determined while keeping the activation level maximum.

The implementation of Zajac's model in SIMM has a means for verifying whether the correct force has been computed. Since the muscle and tendon are in series, colinear or at an angle $\theta$ (see Figure 3.3), the force developed by the muscle and the tension 
transferred by the tendon are related with the cosine of the angle $\theta$ (where $\theta$ represents the pennation angle) as:

$$
F^{T} / \cos \theta=F^{M}
$$

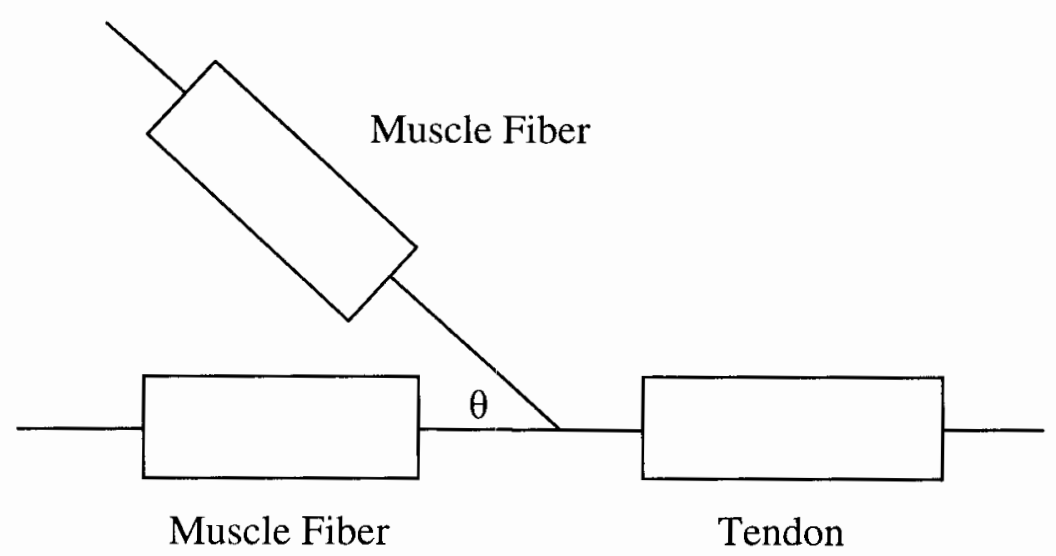

Figure 3.3. Muscle fiber and tendon relationships

Thus, the software ultimately tries to satisfy the above relation and determine the correct musculotendon unit force. More specifically, once the parameters previously mentioned have been entered in the model, an initial guess is made with regard to the lengths of the muscle fiber and tendon (I believe by assuming isometric conditions). The activation level scales the dimensionless active tension-length relationship first and then the tension-velocity relationship of the muscle fiber linearly (see Figure 3.4). In certain cases, i.e., when the muscle fibers are operating on the ascending or descending parts of the tension-length relationship, the muscle fiber length scales the tension-velocity alongwith the activation level of the muscle. From these relationships an initial muscle force can be computed by including the passive tension for the given fiber length.

Also, the corresponding tendon length should be $L^{T}=L^{M T}-L^{M}$. However, that relationship may not hold true because of the compliance of the tendon, i.e., assuming 

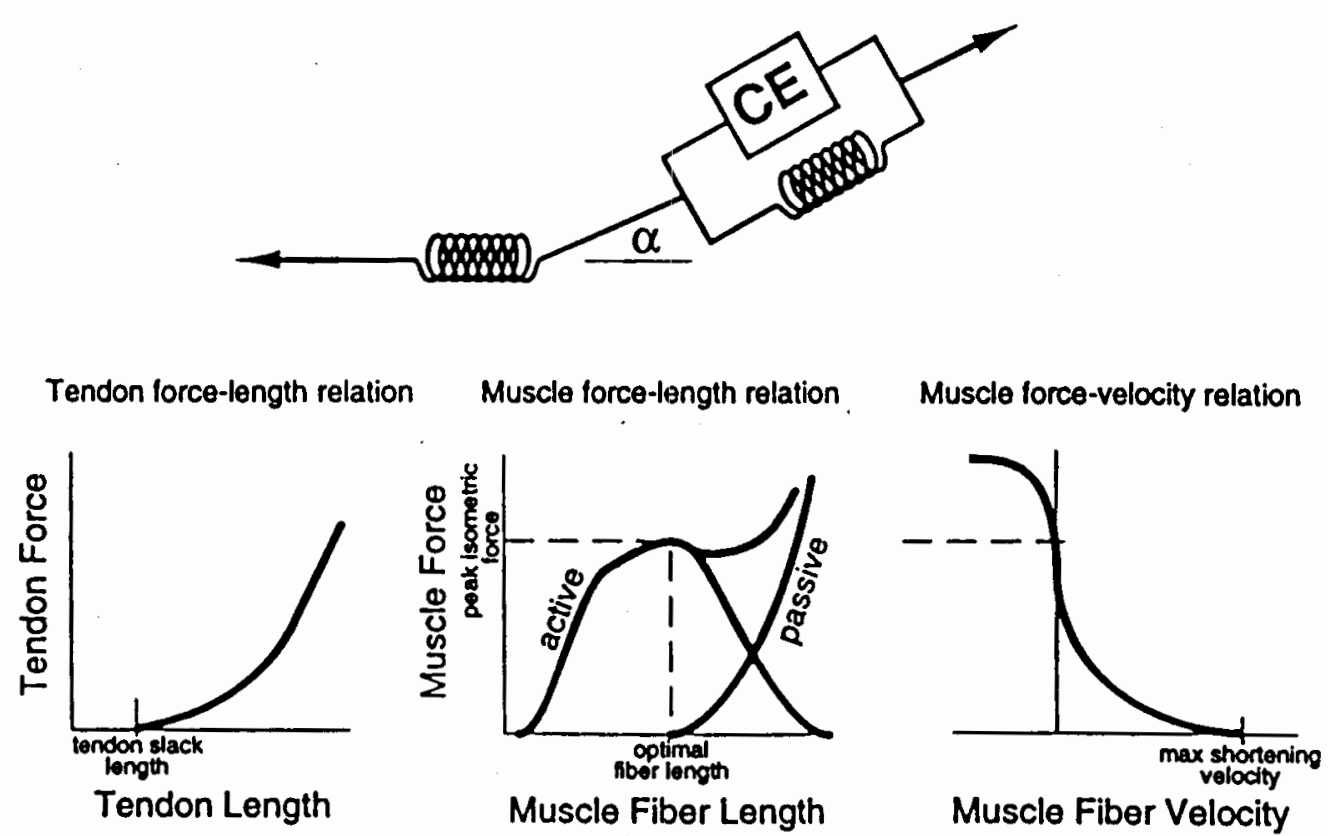

Figure 3.4. Zajac's muscle model implemented in SIMM. CE is the contractile element of the muscle, while the spring in parallel with the contractile element represents the passive component developed in the tension-length relationship of the muscle. The spring in series with the other two components represents the tendinous component of the musculotendon unit.

Figure has been reproduced with permission from Delp, S.L. and Loan J.P. (Delp, 1990).

that a musculotendon unit is placed at a given length and contracts, then, if the tendon is very compliant, the fibers will have to shorten more for stretching the tendon to develop a given high tension. The force by the muscle fibers has to be equal to the tendon tension.

And that tendon tension, given the tendon stiffness, will correspond to a specific tendon length. However, since the tendon stiffness has a reciprocal relationship to the tendon slack length, the tendon length estimated by $L^{T}=L^{M T}-L^{M}$ may not agree with the length estimated for it. Thus, the newly computed tendon length and its rate of change is fed back to the musculotendon contraction dynamics and combined with the musculotendon unit length and velocity determine the respective muscle fiber length and velocity which together with the activation level are used to compute a new muscle fiber 
force. That iteration is being repeated until the $F^{T} / \cos \theta=F^{M}$ relationship is satisfied. Once this relationship has been satisfied the respective muscle and tendon forces have been found. What is important to realize, however, is that the entire process is determined by the compliance of the tendon.

The force determined by Zajac's model, as implemented in this project, can be represented as $F(\ell, \dot{\ell}, 1)$. This force was normalized with respect to the maximum isometric force of that musculotendon unit. The maximum isometric force can be considered as $F\left(\ell_{0}, 0,1\right)$. Expressing the force output relative to the maximum isometric force provided the combined force-length-velocity relationships $\bar{F}=(\ell, \dot{\ell})$ that were used as input in the EMG-driven model.

The moment arm was also computed using the SIMM software. However, while the musculotendon parameters could not be scaled for the computation of musculotendon forces, the skeletal model implemented in SIMM could be scaled, along with the respective proximal and distal musculotendon unit attachments (see "Data Collection and Analysis Procedures" section). Consequently, individual subject-specific musculotendon moment arms could be determined.

Moment arm was determined using the partial velocity method (Delp, 1990). This method is based on the principle of virtual work, and it is also referred to as the tendon and joint displacement method (An, 1984; Hoy et al., 1990). It has been used by a variety of researchers to investigate the length of the moment arm of lower extremity muscles (Spoor et al., 1990), of muscles crossing the talocrural and subtalar joints (Klein et al., 1996), and of finger muscles (An et al., 1983). Using the formulation based on the virtual principle of work it is assumed that joints are frictionless and that ligaments in the plane of motion are not extensible. Then, the relationship between an externally applied moment $(T)$ at a joint and the tension it generates at the tendons $\left(\sum_{i=1}^{i=n} F_{i}^{M}\right)$ of the muscles that cross that joint is:

$$
T=\sum_{i=1}^{i=n} \frac{\partial x_{i}}{\partial \theta_{j}} \times F_{i}^{M}
$$


where $x_{i}$ represents the displacement of the tendon $i$ due to the angular movement of the joint in direction $j$. Thus, the partial derivative of the tendon displacement with respect to the joint angle represents the moment arm which is the slope of the tendon stretch to joint angle. In SIMM, this concept is implemented using the point of effective origin or insertion of the muscle $\left(P_{i}\right)$, instead of the tendon displacement:

$$
M A_{i}=\frac{\partial P_{i}}{\partial \theta_{j}} \cdot \hat{i}
$$

where $M A_{i}$ represents the moment arm of muscle $\mathrm{i}$, and $\hat{i}$ is a unit vector along the line of action of the muscle (Delp, 1990).

The advantage of this method lies on the fact that it is free of any geometrical considerations at the joint, i.e. it does not require prior estimation of the center of rotation or of the instantaneous center of rotation of the joint. Thus, it provides a consistent method, free of geometrical considerations, for the estimation of moment arms across any joint of interest. Consequently, moment arms can be determined analytically, given only muscle attachment coordinates and joint kinematics without the geometrical considerations of the system. However, the geometry effects become present via the proximal and distal attachments of the musculotendon units as well as via the joint kinematics.

\section{The electromyogram}

The last input to the EMG-driven model proposed, is the normalized EMG (NEMG), where the normalized amplitude is treated as a representation of the activation level of the muscle. The desire to treat NEMG in such a capacity is based on the observation that EMG, raw and processed, is related to the manifestation of muscle activity, i.e., the tension produced by the muscle. The clearest indication for the existence of such a relationship involves experiments where the length of the musculotendon unit is held at a constant length, i.e. during isometric experiments. During constant muscle length experiments an increase in muscle tension is accompanied by an increase in electromyographic activity (Hill, 1938). Furthermore, it has been established that the number of muscle fibers recruited, i.e., the number of motor units 
recruited and their rate of firing determines the amplitude of the EMG and the tension measured (Bigland-Ritchie, 1981). Thus, there is determinate relationship between EMG and force. The type of the relationship, however, has been the subject of many debates and many research studies.

The first step in investigating the relationship between the electromyogram and muscle tension is to make sure that the signal originating from the musculotendon unit of interest is of high fidelity. Recent reports in the literature have summarized the accumulated knowledge and respective issues on the topic for the past decades and have made recommendation regarding the best resolutions (Acierno et al., 1998; De Luca, 1997; LeVeau and Andersson, 1992; Redferen, 1992; Soderberg, 1992; Winter, 1997). In summary, the fidelity, i.e., for the purposes of this project, the amplitude, of the electromyogram recorded is related to:

a) The selection of the recording electrodes. The area and the shape of the electrodes determine the number of muscle fibers "seen" and, therefore, the number of motor units detected. Consequently, the amplitude of the electromyographic recording is a function of the electrodes selected;

b) The placement of the electrodes on the musculotendon units of interest. This issue refers to the orientation of the electrodes with respect to the orientation of the muscle fibers, which determines the detection quality of the conduction velocity of the signal (since the interdetection surface is fixed, the conduction velocity will be a function of the cosine of the angle between the fibers and the detection surfaces), the distance between the electrodes (assuming a differential bipolar configuration, which is used in the present study) to avoid shunting and signal attenuation, as well as the exact geometric location of the electrodes with respect to the geometric configuration of the muscle. Furthermore, this category may include skin-electrode preparation factors to reduce skin impedance and assist the conduction of the signal at the skin-electrode interface;

c) The placement of the electrodes is related to another issue that affects the signal amplitude, which is the cross talk. Placement of the electrodes at the edges of 
the muscle may result in contaminating the original signal from the musculotendon unit of interest with the signal from adjacent muscles;

d) Intervening tissues is another concern as they assume the role of a low pass filter. Thus adipose tissue between the electrode surface and the muscle as well as other tissues, such as connective, that may introduce additional space between the electrodes and the musculotendon unit of interest, thereby introducing a spatial filtering effect, will be an influence to the fidelity of the signal; and

e) The state of the electromyographic signal, i.e., whether it is stationary or dynamic, will also affect the fidelity of the electromyogram. Since the electrodes are fixed on the skin, their geometric relationship with the skin and with the recorded musculotendon unit fibers, under isometric conditions, is also fixed. Under dynamic conditions, while the geometric relationship between the electrodes and the skin remains fixed, the geometric relationship between the electrodes and the muscle fibers does not remain fixed. As tissues and, therefore, muscle fibers are allowed to move underneath the skin, additional cross talk issues may arise, motion artifacts maybe introduced into the signal, and additional motor units may come into or get out of the detection area while the force stays the same or increases, all affecting the amplitude of the signal.

In the current project, to account for these issues the recommendations brought forth by DeLuca (1997) have been followed (see "Data Collection and Analysis Procedures" section).

Beyond the issues that affect the fidelity of the signal in time, and therefore the EMG-force relationship, there are other physiological factors that are not technical in origin. It was mentioned earlier that the number of muscle fibers recruited, i.e., the number of motor units recruited and their rate of firing determines the existence of an EMG-force relationship. Because of the asynchronous recruitment nature of the motor units the relationship to force in the past has been shown to be non linear (Milner-Brown and Stein, 1975). However, when recruitment is synchronized the motor unit activity can result in a linear EMG-force relationship (Person and Libkin, 1970). 
The interplay between motor unit recruitment and firing rate has also been suggested as a factor that controls the EMG-force relationship. Motor unit recruitment has been suggested to precede firing at low levels of force generation, while the firing rate appears to take over at higher levels of force generation (Milner-Brown and Stein, 1975; Milner-Brown et al., 1973a; Milner-Brown, 1973b, 1973c). On the basis of these results recent research studies have demonstrated that when recruitment is the primary means of tension generation there was a quadratic increase in EMG with respect to the muscle tension, and a linear increase with firing rate (Solomonow et al., 1990). Furthermore, the same authors demonstrated that when recruitment results in less than the initial 50 percent of maximum force, a linear relationship between EMG and tension recorded can be assumed, while the rate of firing can account for the remaining tension recorded.

In addition, the muscle fiber distribution within each muscle affects the EMG-force relationship, since the motor unit recruitment patterns for different muscles are directly related to their respective muscle fiber compositions (Lawrence and De Luca, 1983; Woods and Bigland-Ritchie, 1983). Redfern (1992) in a review of the topic has provided a representative table with the studies that have found a linear and non-linear EMG-force relationships. One of the factors that the linearities and non-linearities were attributed to, was the fiber composition of the muscles. Muscles of uniform or near uniform fiber type composition had linear EMG-force relationships, whereas muscles composed of mixed fiber types had a curvilinear EMG-force relationship (De Luca, 1997; Woods and Bigland-Ritchie, 1983).

The effect of muscle length on the active tension-length relationship of the muscle has been well established (Hill, 1938). By extension, then, the effect of muscle length on the EMG-tension relationship has been investigated. Redfern (1992) in his review of the factors affecting the electromyographic signal indicated that a number of studies have demonstrated the dependency of the electromyographic signal on the muscle length (Grieves and Pheasant, 1967; Vrendenbregt and Rau, 1973). Thus, in order to derive musculotendon unit force from the electromyogram, the length-tension factor must be accounted for in the EMG modeling formulation. 
Previous EMG-assisted muscle modeling approaches for gait have implemented a modulating factor on the EMG that was related to the joint angle rather than the muscle (Hof and Berg, 1981a; Hof et al., 1987; Olney and Winter, 1985). The positional data were considered correlates of muscle length. However, Olney and Winter (1985) in their approach, because they were investigating both, the knee and ankle joints they implemented the single muscle equivalency concept (Bouisset, 1973). The joint angles and velocities were independent inputs to the model and at the implementation of each of the two variables an iterative approach was implemented that produced the best fit. However, the implication of this approach is that, although the resulting equations represent the combined muscle response to the conditions at which the equations were iterated, these conditions may not represent the physiological conditions under which each musculotendon unit functions. Thus, not only the results cannot be generalized across muscles and they do not consider the interaction between contraction variables and individual muscle architectural properties. Hof and Van Den Berg (1981a) were able to avoid the concept of muscle equivalency by using muscle simplification. While ignoring all plantar flexor muscles acting at the ankle, they derived individual torque-angle curves for the gastrocnemius and soleus muscles.

One of the advantages of models that accept joint kinematic inputs and treat those inputs as muscle length -and muscle velocity- correlates is that they reduce the number of measurements that are required, thereby simplifying the inputs to the model. However, the results, while representative of the compiled muscular activity, they may not represent the individual responses of the respective musculotendon units investigated. Thus, studies such as that of Baildon and Chapman (1983a, b) resulted in torque-angle and torque-angular velocity relationships that were different than individual musculotendon unit force-angle and especially force-velocity relationships reported in the literature. For the kinematic inputs to represent the individual musculotendon unit kinematics adequately, it must be assumed that changes in joint positions and velocities occur concurrently with the exact same changes in muscle lengths and velocities of contraction. However, the validity of such assumption presupposes that all musculotendon units involved in the generation of the measured moment do not change their mechanical 
parameters disproportionately with respect to each other, i.e., in terms of their moment arms, fiber lengths, and velocities of contraction, over the joint range of motion. Furthermore, it must be presupposed that the effect of changes and the effect of rate of changes in positions of adjacent joints on biarticular musculotendon units is either the same for all muscles (uniarticular and biarticular) crossing the joint of interest or it is negligible. Since none of the requirements that justify the above assumption seems to hold, in the general case, it can be concluded that joint kinematic input to represent the lumped to one muscle activity of many muscles working together may not be desirable if the purpose is to identify the individual contributions of each musculotendon unit involved in the production of the measured torque at a joint. However, it should be noted that the results of several studies that have investigated the ankle joint and have achieved a good fit between the measured and desired moments, indicate that these assumptions can be rendered justification for the ankle joint (Hof and Berg, 1981a, 1981b, 1981c, 1981d; Hof et al., 1987; Olney and Winter, 1985).

It should also be noted that the above assumptions and their associated implications could be avoided if modeling takes place at the individual musculotendon unit and especially at the microstructural level, i.e., the muscle fiber. Modeling at the individual musculotendon units for the purposes of predicting individual muscle forces in gait has been implemented by White and Winter (1993). They included a modulating factor to account for the effect of the muscle length on the estimated gain and electromyogram. The effect of muscle length was lumped in third and second order regression equations with different gain coefficients (White and Winter, 1993). Although their modeling approach did not account for the effect of muscle velocity of contraction, it did account for the effect of biarticularity. However, it has also been argued that when a calibration curve is determined at a set muscle length and contraction velocity (which was their approach), generalization of this relationship to other experimental conditions may produce substantial errors (Potvin et al., 1996; Van Dieen and Visser, 1999).

More, successful in modulating the EMG for the effect of not only the muscle length, but also the effect of the change of muscle length on the electromyogram has been research in the field of spine (Granata, 1993, 1995; Granata and Marras, 1993; Marras 
and Granata, 1997; Marras and Sommerich, 1991a; McGill, 1992; McGill and Norman, 1986; Nussbaum and Chaffin, 1998; Sparto, 1998; Sparto and Parnianpour, 1999). The modulating factors have been theoretical in nature, based on previous databases or directly experimental, usually correcting the predicted moment so that it fits the measured moment as a function of the erector spinae length. That relationship then is applied for all the musculotendon units of interest. One advantage that studies on spine research have over similar studies on gait is that the former do not deal with the issue of biarticularity very much. Thus predictions are prone to relatively less errors.

In the present study, although the effect of muscle length on EMG was not accounted for directly, a normalization was performed in the individual overall tensionlength-velocity relationships derived for each musculotendon units of interest, so that a linear relationship between EMG-tension could be assumed. It was mentioned in the previous section "Muscle modeling and parameters" that the tension-length-velocity relationship estimated for each musculotendon unit of interest was normalized to the respective maximum isometric force, $F_{o}^{M}$. Since previous research has determined that:

a) contraction velocity and the EMG of a muscle are independent of each other during maximum voluntary contraction (Komi, 1973a); and

b) when force is normalized with respect to the maximum force obtained from a maximum voluntary isokinetic contraction, then the NEMG is linearly related to force and it is independent of velocity (Komi, 1973b), i.e., for concentric contractions and assuming that inherent in the velocity effect is encapsulated the length effect:

$$
N E M G=k \cdot\left(\frac{F^{M}(\ell, \dot{\ell})}{F_{o}^{M}}\right)
$$

or given the relationships from the previous section "Muscle modeling and parameters", for each musculotendon unit of interest $i$ :

$$
N E M G_{i}=k \cdot \bar{F}_{i}(\ell, \dot{\ell})
$$

where $k$ relates the normalized EMG to the normalized force as the linear slope between the two variables. Some investigators refer to $k$ as the gain (White and Winter, 1993). However, for the purposes of this project, the gain $G$ represents the force developed per 
cross sectional area unit within each musculotendon unit of interest. Consequently, in other words, by normalizing the tension-length-velocity relationship to maximum isometric force for each musculotendon unit of interest as an input to the EMG-driven model, it might be assumed that a linear relationship between EMG and force was established.

While Komi's work (1973a, b) suggests that there is a contraction velocity above which a constant force can no longer be maintained, and, therefore, at that velocity the EMG will have reached its physiological maximum, other research has shown that the EMG-tension relationship is a function of the isokinetic contraction of the muscle, with the EMG increasing in a linear fashion with velocity when the force is kept constant (Bigland and Lippold, 1954). However, in that study only low velocities, relative to the maximum velocity of contraction, were used. Simulations have shown that in the limit, as the relative velocity, i.e., the ratio of contraction velocity to the maximum velocity of contraction, becomes much smaller than one, the EMG-tension relationship becomes linear -unfortunately the author of these simulations did not report the threshold value of the ratio, below which the EMG-tension relationship becomes linear (Granata, 1993). Thus, there does not appear to be a discrepancy among the studies, and it can be concluded that EMG is independent of velocity of contraction or it has very little dependency on the velocity of contraction by itself (Marras et al., 1986; Zajac, 1989a).

It can also safely be assumed that EMG is related to velocity of contraction via the effect that the later has on the muscle tension produced. In other words, there is the need for the EMG to be modulated for the effects of velocity on muscle tension. Such a modulation in the proposed model was achieved by including its effect on the force of each musculotendon unit of interest, and by then expressing that effect relative to the maximum isometric force. Previous gait EMG-assisted models have not accounted for the effect of velocity as a modulating factor on the EMG in the experimental stages of their model development (White and Winter, 1993). However, the velocity of contraction as a theoretical input implemented in their Hill-based modeling approaches was included. 
Contrary to the field of gait research, EMG-assisted modeling approaches for the spine have recognized the effect and the need for accounting for the velocity of contraction as a modulating factor of EMG (Granata, 1995; Granata and Marras, 1993; Parnianpour et al., 1990; Sparto and Parnianpour, 1998, 1999; Sparto et al., 1998). Regression equations have been used to describe the effect on the basis of previous databases or polynomials derived from experimental calibration trials. Thus, the velocity (and length) modified data are presented as the product of the normalized EMG and the modulation polynomial (Granata, 1995; Granata and Marras, 1993; White and Winter, 1993). As it has been indicated earlier, the tension-length-velocity affects have been accounted in the present modeling approach using the respective dimensionless relationships implemented in Zajac's model. The relationship was represented as:

$$
N E M G(\ell, \dot{\ell})=N E M G(t) *(\bar{F}(\ell, \dot{\ell}))
$$

where $\ell$ and $\ell$ were the specific time varying parameters based on the individual kinematics of each subject.

The effect of acceleration on the dynamic EMG has not been investigated extensively. Probably because the consensus appears to be that acceleration has no significant effect on EMG (Granata, 1993). With respect to its potential effect on muscles involved in gait, such as the soleus, it has been shown that the position and velocity variables account for most of the variance (approximately $85 \%$ ) observed (Yang et al., 1991). One factor that could increase the significance of acceleration as a parameter affecting the muscle output is inertia forces generated by the accelerated mass. However, if those inertial forces are considered part of the force output, acceleration has no significant effect (Granata, 1993).

In the above review and methodology to account for the non-linearities between the EMG-force relationship, the feasibility for implementing a linear EMG-force relationship was examined. Furthermore, since the EMG-force relation for submaximal levels of contraction is most often linear (Solomonow et al., 1990; White and Winter, 1993), and since muscle in normal ambulation can be expected to operate at a submaximal level, it was assumed, for the purposes of this study, that there existed a linear relationship between the surface electromyogram and the isometric force produced under non 
fatiguing contractions. Such linearity was assumed to hold true for all the musculotendon units of interest. The assumption was based on the belief that, considering the muscle at its microstructural level, if the series elastic component of the muscle is eliminated or it is assumed negligible, then the time varying contractile force will be a function of the muscle fiber length and of the muscle fiber velocity of contraction. In the isometric case, where the effects of length and velocity are eliminated, the time varying force output must be a function of the time-varying activation, or EMG input alone. Given that excitation can scale the active isometric length-tension relationship, and since the muscle fiber length is constant, the active muscle force output in an isometric contraction is proportional to the activation level, i.e., the electromyogram.

Another factor that affects the EMG-force relationship is the coactivation, which is presented as the relationship between agonists and antagonists, as well as the functional relationship among synergistic muscles that are being recruited for the accomplishment of a motor task such as walking gait (De Luca, 1997; Falconer and Winter, 1985; Granata, 1993; Hof and Berg, 1977; Redferen, 1992). Since cocontraction affects the internal moments generated at a joint, it also affects the EMG-tension relationship. Evidence indicates that effort, or exertion, level, and/or kinetic requirements result in increased cocontraction and may actually result in muscle substitution (Corcos et al., 1989; De Luca, 1997; Gottlieb et al., 1992). Falconer and Winter (1985) showed that highest cocontraction was observed during the weight acceptance in gait because the functional kinetic requirements of that gait event, i.e., stability, are the greatest compared to other gait events, such as toe-off or swing. Furthermore, cocontraction has appeared to be affected by joint position and velocity (Corcos et al., 1989; Gottlieb et al., 1992; Marras and Mirka, 1992; Yang et al., 1991).

Consequently, if one tries to define the EMG-force relationship for a specific musculotendon unit acting at a joint, assuming no antagonistic cocontraction or no synergistic activity, the results may be subject to significant error (Granata and Marras, 1995; Marras and Granata, 1997). Thus, research efforts either try to implement protocols that remove the effect of synergistic cocontraction (Lippold, 1952; Sale et al., 1982), or they try to implement protocols that account for the cocontractive nature of the 
muscles involved in the activity of interest individually (Redfern, 1988). Some authors on the other hand have accounted for the cocontractive and synergistic nature of muscles by treating synergists as a muscle equivalent (Redferen, 1992). However, synergists, on the basis of different nerve innervation may have different responses under different conditions. Finally it should be stated that Hoff and Van Den Berg (1977) concluded that while there exists a linear EMG-force relationship, the non-linearities that may be observed can be attributed to cocontraction.

In the present project the synergistic and antagonistic relationships among the muscles that are acting at the knee and ankle joints were accounted for by:

a) monitoring as many muscles as possible, and

b) should it be desired to account for the muscles that were not monitored, it was made certain that each of the muscles that was not monitored had a similar nerve innervation pattern with at least one of the muscles that was monitored.

However, since surface electromyography was used, it was impossible to monitor all the musculotendon units of interest, i.e., all the ankle plantar/dorsi flexors and the knee flexors/extensors. Therefore, some assumptions needed to be made.

Table 3.4 lists the muscles to be monitored. The effectiveness of the capability to monitor all the muscles of interest was based on the following procedure. Firstly, as many muscles as possible were monitored based on the work by White and Winter (1993). Secondly, the software SIMM was used to identify, during a gait cycle, the relative potential moment contribution of each individual musculotendon unit that spans the ankle or the knee joint, and ultimately to determine the moment output with all the muscles acting at the joint being active and the moment output without the muscles that were not to be monitored. It was assumed that each musculotendon unit was maximally activated throughout the entire cycle. Knowledge of the relative potential moment muscle contribution of each individual musculotendon unit that spans the ankle or the knee joint and especially knowledge of the maximum moment output with all the muscles acting at the joint being active and without the muscles that were not monitored, provided an idea of the maximum potential error that might be introduced when certain muscles could not be monitored (see Figures 3.5 through 3.8). Thirdly, the electromyographic 


\begin{tabular}{||c|c|c|c|c||}
\hline \hline Joint & \multicolumn{2}{|c|}{ Knee } & \multicolumn{2}{c|}{ Ankle } \\
\hline Muscle group & Flexors & Extensors & Plantar Flexors & Dorsi Flexors \\
\hline & Semimeb. & Vastus lat. & Soleus & Tibialis anter. \\
& Semitend. & Vastus med. & Medial Gastroc. & Ext. digit. long. \\
& Bicep. fem. (I) & Rectus fem. & Lateral Gastroc. & \\
& Bicep. fem. (s) & & Peroneus Brevis & \\
& Medial Gastroc. & & & \\
& Lateral Gastroc. & & & \\
\hline
\end{tabular}

Table 3.4

Muscles to be monitored with surface EMG.

data provided by Perry (1992) were examined. Assuming that there is a relationship between EMG and force, the EMG amplitudes, and areas under the EMG envelopes, of all the musculotendon units of interest were examined to identify their relative potential in producing force during the gait cycle from an electromyographic perspective. Finally, innervation (see Table 3.5) and the firing pattern, in terms of timing, of each of the musculotendon units of was examined to identify similarities among muscles with a common action during the gait cycle, i.e., knee flexion or knee extension, plantar flexion or dorsi flexion. Combination of the last two pieces of information allowed potential grouping of muscles that were not monitored with muscles that were monitored (see Table 3.6). Although the grouping scheme proposed was original, the approach was based on previous studies which suggested that the activity of the monoarticular muscles having a common function can be estimated by placing electrodes on a representative muscle (Hof and Berg, 1977; Olney and Winter, 1985; White and Winter, 1993).

Consequently, based on this investigation, it was determined that if of the dorsi flexors, the extensor hallucis longus and the peronius tertius could not be monitored, then assuming maximum contribution of all dorsi flexors in the dorsi flexion moment, a maximum of 14 percent error would be introduced. If of the plantar flexors, the deep plantar flexors, i.e., the flexor hallucis longus, the flexor digitorum longus, and the tibialis posterior, as well as the peroneus longus were not monitored, then assuming maximum contribution of all plantar flexors in the plantar flexion moment, a maximum 
of approximately 8.5 percent error would be introduced. Not monitoring the gracilis and sartorius of the knee flexors, and, again, assuming maximum contribution of all knee flexors in the knee flexion moment, would introduce a maximum of 5 percent error. Finally, inability to monitor the vastus intermedius among the knee extensors, and assuming maximum contribution of all knee extensors in the knee extension moment would introduce an error of approximately 25 percent. Since the muscles operate at no more than 25 to 50 percent of their maxima during normal walking gait, all the errors above may be expected to be almost insignificant, except for errors introduced by the vastus intermedius. For that muscle the grouping scheme proposed in Table 3.6 was adopted. Also, on the basis of the above analysis it was decided to monitor all biarticular knee flexor/extensor as well as ankle plantar/dorsi flexor muscles.

Furthermore, White and Winter (1993) grouped the PCSAs of the muscles not monitored to those monitored with a common function. For example, the PCSAs of the deep ankle plantar flexors were apportioned to soleus since they are single-joint muscles. However, the results of this investigation, contradict the adequacy of the procedures followed previously, i.e., the grouping and the lumping. With respect to the previous example, such a lumping would result in erroneous prediction of plantar flexor moment (White and Winter, 1993). A more accurate approach might be, based on the results provided by Perry, to group the firing pattern of the deep plantar flexors, in terms of timing, to the soleus, but, based on the results from SIMM, to apportion their PCSA to the lateral gastrocnemius, or, ideally, to Peroneus Longus, if it could be monitored, while obtaining their activation level with respect to the EMG signal obtained by the soleus divided by two.

However, in this study, it is suggested not to apportion the PCSA of each of the muscles that will not be monitored to any other muscle. Table 3.6 provides additional information about the grouping of the muscles not monitored with respect to their innervation and assumed timing and estimated activation level based on information obtained by SIMM and Perry (1992). 

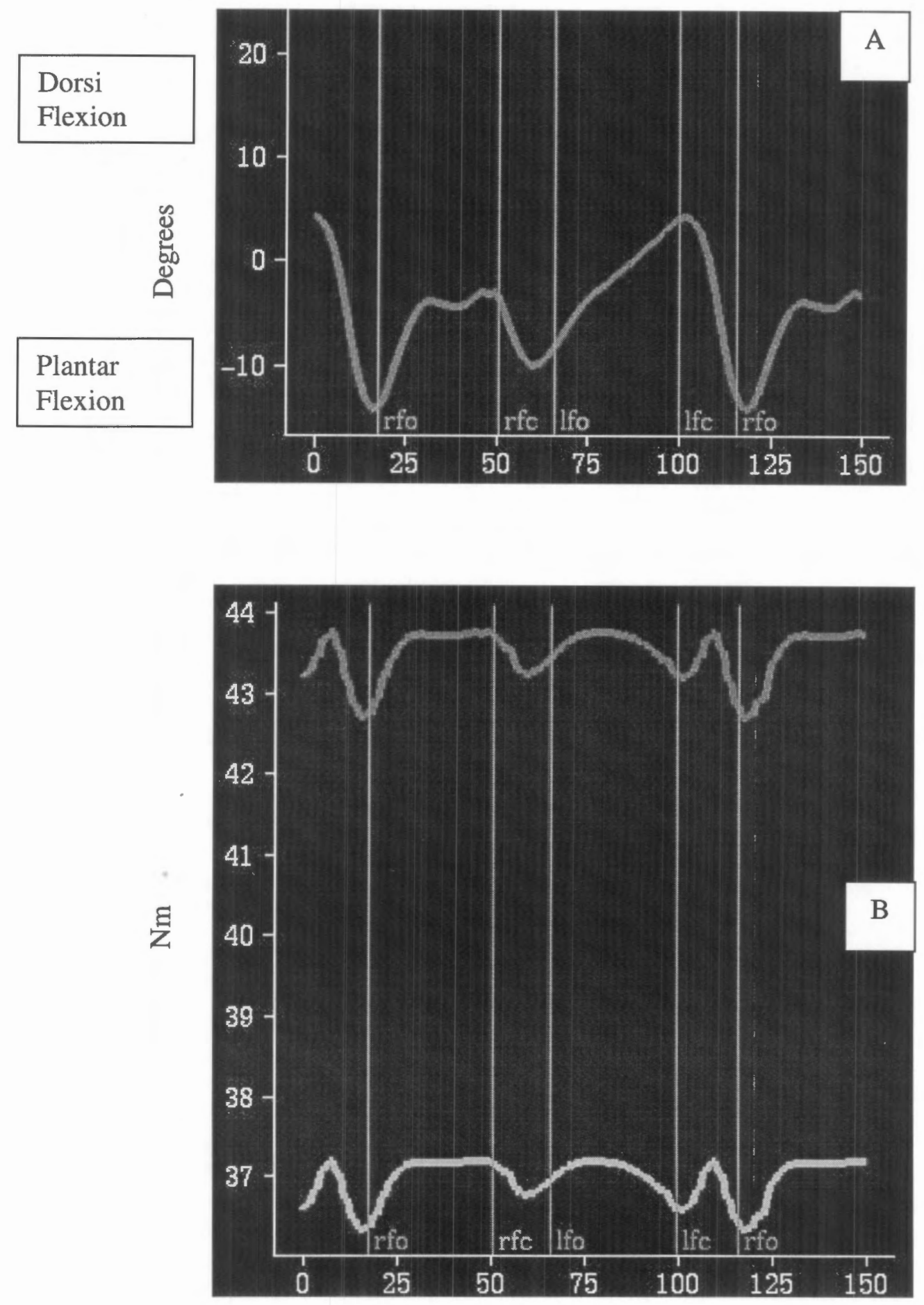

Figure 3.5. Right ankle plantar-dorsi flexion of a normal subject during a complete gait cycle (A). Total dorsi flexion moment at the ankle when all dorsi flexors are maximally excited (top curve) and dorsi flexion moment generated when only the tibialis anterior and extensor digitorum longus are involved and they are maximally excited (B). 

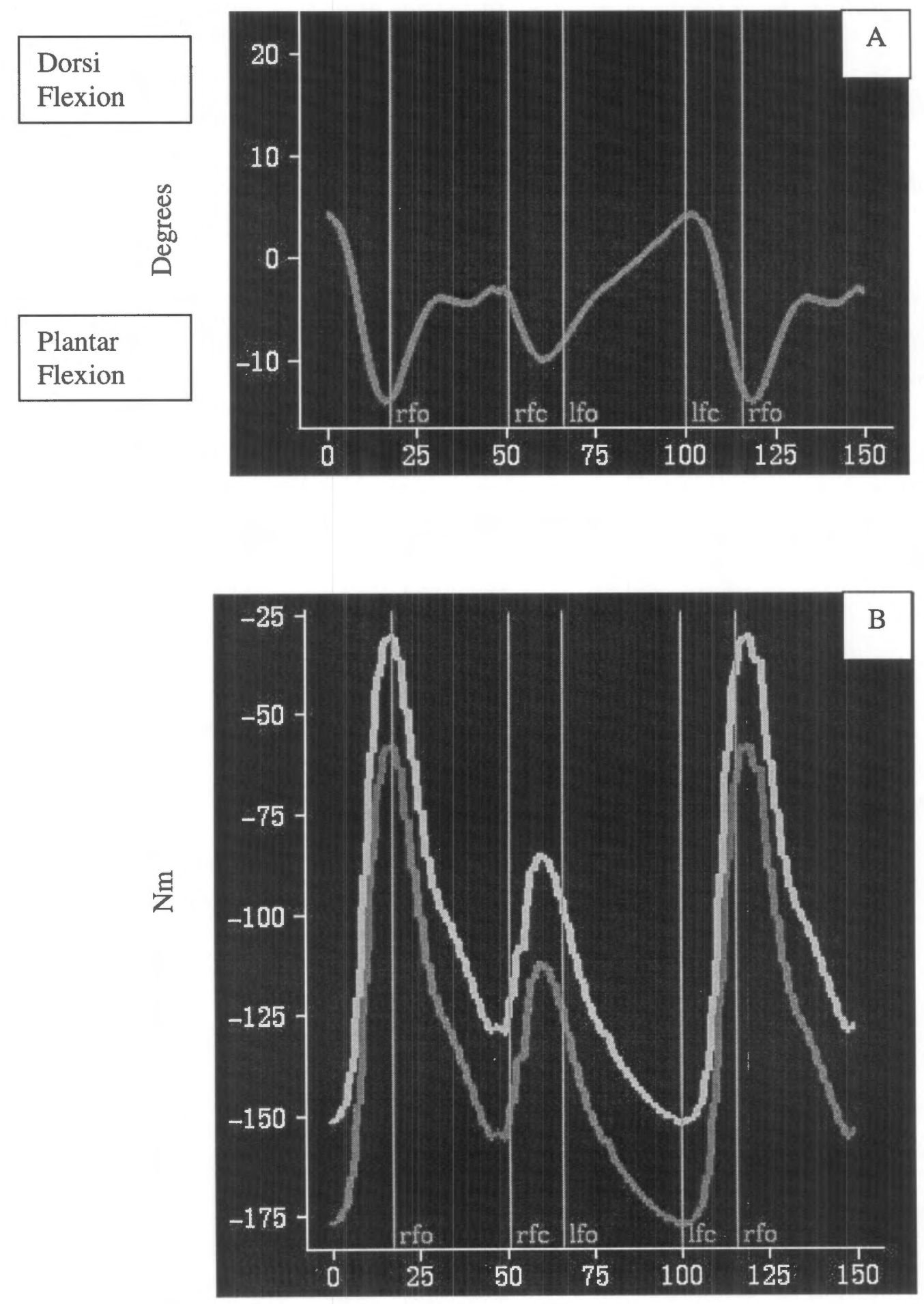

Figure 3.6. Right ankle plantar-dorsi flexion of a normal subject during a complete gait cycle (A). Total plantar flexion moment at the ankle when all plantar flexors are maximally excited (bottom curve) and plantar flexion moment generated when only the gastroc, soleus and peroneus brevis are involved and they are maximally excited (B). 

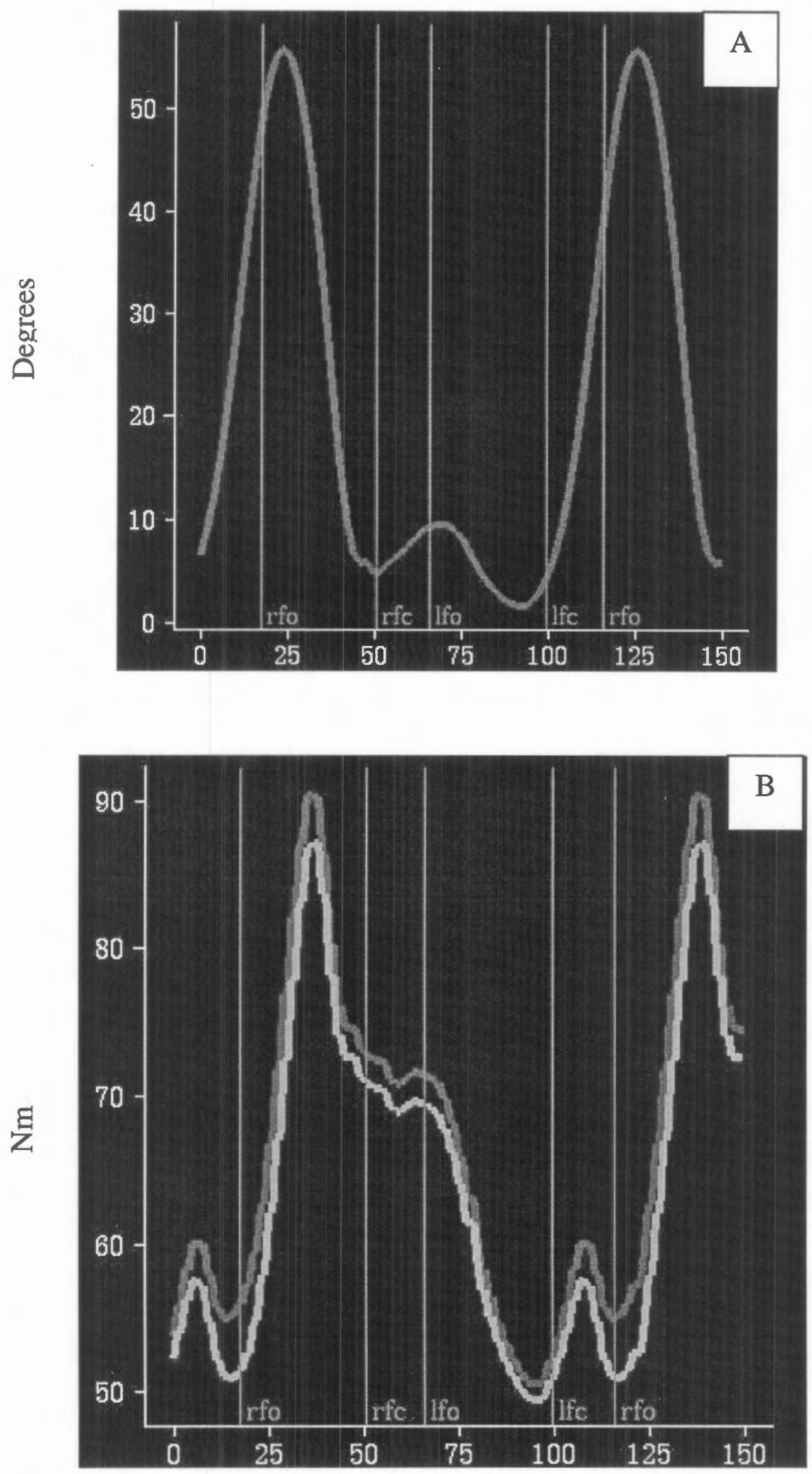

Figure 3.7. Right knee flexion of a normal subject during a complete gait cycle (A). Total knee flexion moment when all knee flexors are maximally excited (top curve) and knee flexion moment when the sartorius and gracilis are not included (B). 

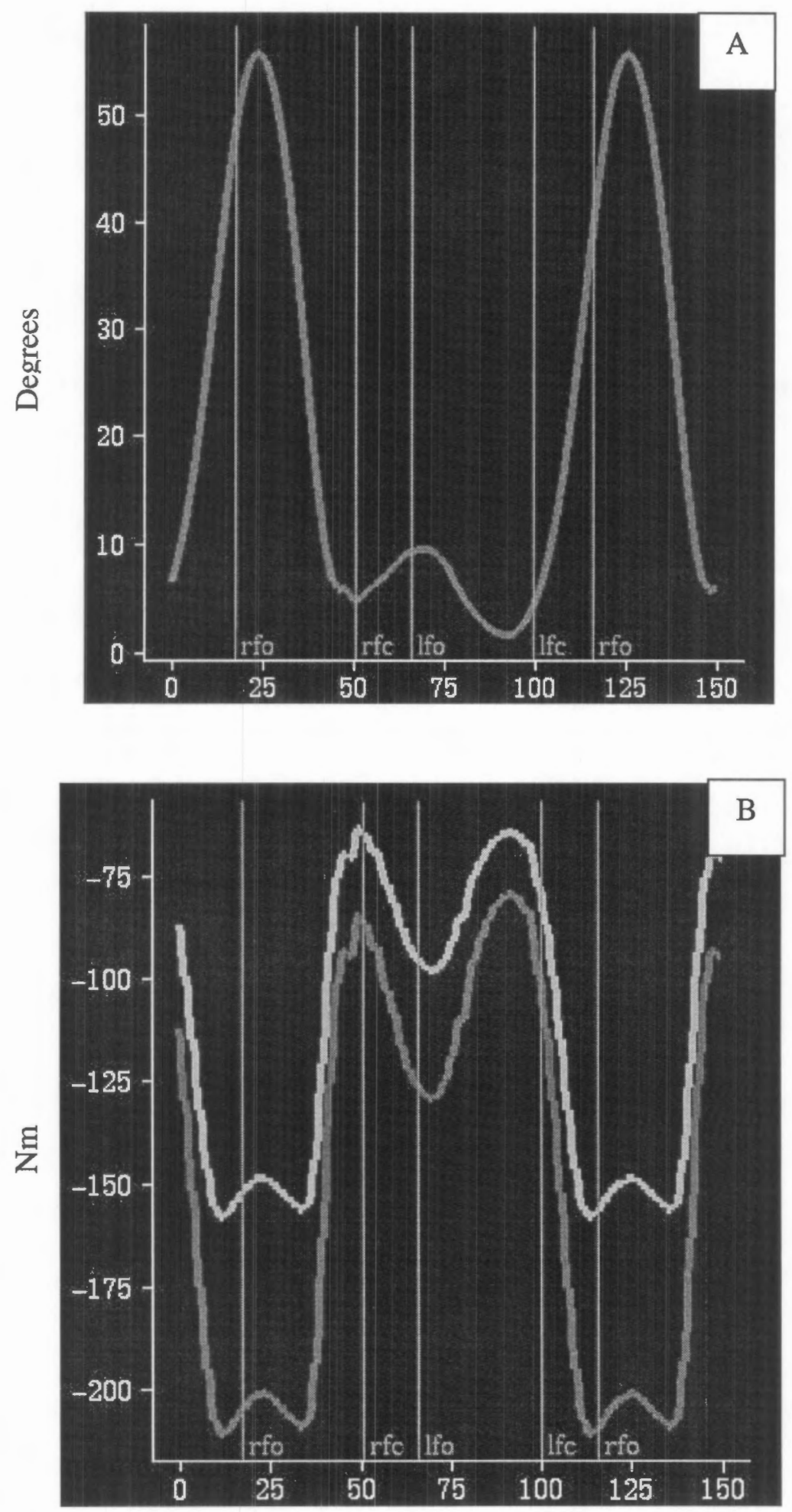

Figure 3.8. Right knee flexion of a normal subject during a complete gait cycle (A). Total knee extension moment when all knee extensors are maximally excited (bottom curve) and knee extension moment when the vastus intermedius is not included (B). 


\begin{tabular}{|c|c|c|c|}
\hline INNERVATION & LEVEL & $\begin{array}{c}\text { MUSCLES } \\
\text { MONITORED }\end{array}$ & $\begin{array}{l}\text { MUSCLES NOT } \\
\text { MONITORED }\end{array}$ \\
\hline Femoral Nerve & $\begin{array}{l}\mathrm{L} 2, \underline{\mathrm{L} 3}, \\
\underline{\mathrm{L} 4} \\
\text { (posterior } \\
\text { division) } \\
\mathrm{L} 2, \mathrm{~L} 3 \\
\end{array}$ & \begin{tabular}{|l|} 
Rectus Femoris \\
Vastus Lateralis \\
Vastus Medialis
\end{tabular} & $\begin{array}{l}\text { Vastus Intermedius } \\
\text { Sartorius }\end{array}$ \\
\hline $\begin{array}{l}\text { Sciatic Nerve } \\
\text { Tibial division } \\
\\
\text { Fibular } \\
\text { Peroneal } \\
\text { division }\end{array}$ & $\begin{array}{l}\frac{\mathrm{L} 5}{\mathrm{~S} 2}, \underline{\mathrm{S} 1}, \\
\mathrm{~L} 5, \underline{\mathrm{S} 1}, \\
\mathrm{~S} 2 \\
\mathrm{~L} 5, \underline{\mathrm{S} 1}, \\
\mathrm{~S} 2\end{array}$ & $\begin{array}{l}\text { Semitendinosus } \\
\text { Semimembranosus } \\
\text { Biceps Femoris (Long } \\
\text { Head) } \\
\text { Biceps Femoris (Short } \\
\text { Head) }\end{array}$ & \\
\hline $\begin{array}{l}\text { Deep Fibular } \\
\text { (Peroneal) Nerve }\end{array}$ & $\begin{array}{l}\underline{\mathrm{L} 4}, \mathrm{~L} 5 \\
\mathrm{~L} 5, \mathrm{~S} 1\end{array}$ & $\begin{array}{l}\text { Tibialis Anterior } \\
\text { Extensor Digitorum } \\
\text { Longus }\end{array}$ & $\begin{array}{l}\text { Extensor Hallucis Longus } \\
\text { Peroneus Tertius }\end{array}$ \\
\hline Tibial Nerve & $\begin{array}{l}\mathrm{S} 1, \underline{\mathrm{S} 2} \\
\frac{\mathrm{L} 4, \mathrm{~L} 5}{\mathrm{~S} 1} \\
\underline{\mathrm{S} 2}, \mathrm{~S} 3 \\
\mathrm{~L} 4, \mathrm{~L} 5\end{array}$ & $\begin{array}{l}\text { Gastrocnemius } \\
\text { Soleus }\end{array}$ & $\begin{array}{l}\text { Popliteus } \\
\text { Flexor Hallucis Longus } \\
\text { Flexor Digitorum Longus } \\
\text { Tibialis Posterior }\end{array}$ \\
\hline Obturator Nerve & $\underline{\mathrm{L} 2}, \mathrm{~L} 3$ & & Gracilis \\
\hline $\begin{array}{l}\text { Superficial } \\
\text { Fibular } \\
\text { (Peroneal) Nerve }\end{array}$ & $\frac{\mathrm{L} 5, \mathrm{~S} 1}{\mathrm{~S} 2}$ & $\begin{array}{l}\text { Fibularis (Peroneous) } \\
\text { Brevis }\end{array}$ & $\begin{array}{l}\text { Fibularis (Peroneous) } \\
\text { Longus }\end{array}$ \\
\hline
\end{tabular}

Underlining indicates primary innervation

Table 3.5

Muscle innervation of lower extremity muscles of potential interest. 


\begin{tabular}{|c|c|c|}
\hline Muscle to Group & Timing & Activation level \\
\hline $\begin{array}{l}\text { Ankle Plantar Flexors } \\
\text { Tibialis Posterior } \\
\text { Flexor Hallucis Longus } \\
\text { Flexor Digitorum Longus } \\
\text { Peroneus Longus }\end{array}$ & $\begin{array}{l}\text { Soleus } \\
\text { Soleus } \\
\text { Soleus } \\
\text { Soleus }\end{array}$ & $\begin{array}{l}\text { Lateral Gastroc } \\
\text { Lateral Gastroc } \\
\text { Lateral Gastroc } \\
\text { Lateral Gastroc }\end{array}$ \\
\hline $\begin{array}{l}\text { Ankle Dorsi Flexors } \\
\text { Extensor Hallucis Longus } \\
\text { Peroneus Tertius }\end{array}$ & $\begin{array}{l}\text { Tibialis Anterior }{ }^{1,2,3} \\
\text { Extensor Digitorum } \\
\text { Longus }^{4}\end{array}$ & $\begin{array}{l}\text { Extensor Digitorum } \\
\text { Longus } \\
{ }^{1} \\
\text { Tibialis Anterior }^{2} \\
\text { Extensor Digitorum } \\
\text { Longus }^{3} \\
\text { Extensor Digitorum } \\
\text { Longus }^{4}\end{array}$ \\
\hline $\begin{array}{l}\text { Knee Flexors } \\
\text { Gracilis } \\
\text { Sartorius } \\
\text { Popliteus } \\
\text { Knee Extensors } \\
\text { Vastus Intermedius }\end{array}$ & $\begin{array}{c}\text { Biceps Femoris (short head) } \\
\text { Not Accounted }^{4} \\
\text { Not Accounted }^{4} \\
\\
\text { Vastus Medialis }^{5,6} \\
\text { or Vastus Lateralis }^{6}\end{array}$ & $\begin{array}{c}\text { Biceps Femoris (short } \\
\text { head) } \\
\text { Not Accounted } \\
\text { Not Accounted }^{4}\end{array}$ \\
\hline
\end{tabular}

1: From Initial Contact (IC) to Loading Response (LR)

2: From Pre Swing (PS) to Mid Swing (MSw)

3: From Terminal Swing (TS) to IC

4: According to the assumption made by Perry (1992)

5: From IC to Mid Stance (MSt)

6: From TS to IC

Table 3.6

Suggested grouping scheme of muscles not monitored, with respect to their innervation and activity, with muscles that were monitored. 


\section{Processing of the electromyogram}

While there have been several methods proposed in the past for processing the EMG signal to relate it to force (see section "EMG signal demodulation" in "Review of the Literature"), for the purposes of this study the suggestions by Winter (1997) and White and Winter (1993) have been adopted. The muscle force response seen at the tendon of a muscle under tetanic contraction is related to the EMG signal as the summation of twitch responses where each twitch is considered the impulse response to an action potential, an impulse, by an $\alpha$ motor neuron. Although each twitch response may be thought of as a linear second-order system (Coggshall and Bekey, 1970; Crosby, 1978; Gottlieb and Agarwall, 1971; Winter, 1976), under isometric conditions it has been determined to have a second order critically damped response (Crosby, 1978; MilnerBrown et al., 1973a; Winter, 1976). Consequently, the musculotendon unit response, which represents the superposition of impulse responses, can be considered a second order critically damped system to the neural excitation, i.e., the EMG (Winter and Patla, 1997). The impulse responses of the system are a function of whether slow or fast twitch motor units are under consideration (Winter, 1997), and they represent a combination of chemical and mechanical factors associated with the contraction (Close, 1972; Gottlieb and Agarwall, 1971; Lieber et al., 1983).

Consequently, for the purposes of the present study, the EMG signal was first full wave rectified to represent the temporal summation of a series of impulses, where these impulses represented the motor unit action potentials, which, in turn represented the isometric force-generating activity at the cross bridges (Coggshall and Bekey, 1970; Crosby, 1978; Gottlieb and Agarwall, 1971; White and Winter, 1993; Winter, 1976). Then a low pass critically damped second order Butterworth filter was used to represent the EMG-tension relationship.

The choice of cut-off frequency is important as it is linked to the rise-time of the muscle twitch response and it affects the attenuation of the signal and of the noise (Redfern, 1988). Evidence from harmonic analyses indicates that the low pass cut-off frequency of the muscle twitch ranges between 1.7 to $5.0 \mathrm{~Hz}$ giving rise times between 30 and $150 \mathrm{msec}$, which are similar to the rise times reported for human muscle [(Bawa 
and Stein, 1976; Buchthal and Schmalbruch, 1970; Coggshall and Bekey, 1970; Crosby, 1978; Eberstein and Goodgold, 1968; Milner-Brown et al., 1973a; Stevens et al., 1980). The variability in the rise times, and therefore in the low pass cut-off frequencies are attributed to the variability in the motor units been modeled, i.e., fast twitch versus slow twitch (Fuglevand et al., 1993). In addition, the characteristics of the second order critically damped system match well the relaxation phase and the half relaxation phase of the muscle twitch (Eberstein and Goodgold, 1968; Milner-Brown, 1973c; Stevens et al., 1980).

With regard to the time constant used for filtering the rectified EMG previous researchers have used variable ones (Hof and Berg, 1981a, 1981b, 1981c, 1981d). Others, however, seem to have been equally successful by using just one (Olney and Winter, 1985; White and Winter, 1993). Consequently, while recognizing that an approach that might implement different time constants individualized to each specific muscle to smooth the rectified EMG, for the purposes of this study a low pass cut-off frequency of $2.5 \mathrm{~Hz}$ was used for all muscles (Olney and Winter, 1985; White and Winter, 1993). Then, assuming an average motor unit twitch response, the processed EMG was thought of encapsulating the muscle mechanical and physiological responses in isometric conditions via the filter characteristics. Assuming that the principle of superposition holds, i.e., twitches sum up in a linear manner to produce the measured force, the processed EMG can be assumed to be linearly related to the isometric moment $\left(M_{i s o}\right)$, i.e., isometric force after accounting for the effect of the moment arm $(r)$, with a linear gain factor $(k)$ representing the level of muscle excitation, i.e., the slope of the tension-EMG relationship (Hof, 1984; Winter et al., 1980). Thus:

$$
M_{i s o}=r \times(k \cdot N E M G)
$$

It should be noted here that this relationship does not account for conditions where fatigue is a factor (Granata, 1993; Marras and Granata, 1997), although, recently, attempts have been made to implement it in situations of fatigue (Sparto, 1998). Thus, in the present study care was taken in constructing the experimental protocol so that the subjects were rested during testing. Furthermore, the above equation was modified to 
account for dynamic situations by accounting for the muscle, or muscle fiber length, and velocity factors affecting the relationship (see previous sections "The electromyogram").

\section{Normalization.}

Measurement of the level of muscle excitation in this project was achieved by normalizing the electromyogram. Normalization, apart from providing the level of muscle excitation it also (De Luca, 1997; Mirka, 1991; Parnianpour, 1995):

a) provides a means of a generalized presentation of muscular activity for the purposes of comparison among muscle, individuals, and activities. Since there is not a one-to-one relationship between contraction and fore, the amplitude of the normalized EMG $(N E M G)$ is used as a standard reference for comparisons;

b) eliminates the arbitrariness of the processed EMG signal by eliminating the spatial filtering characteristics due to electrode placement, skin abrasion, skin and flesh impedance, muscle fiber density, changes in tissue temperature and electronic channel differences;

c) accounts for artifacts embedded in the EMG signal as functions of muscle length and velocity so that the relationship between EMG and force can be established for the musculotendon units of interest, i.e., the signal is scaled from zero to one in order to map the maximum force that can be generated;

d) allows the acquisition of reference EMG values as functions of the joint angle and change of the joint angle, where the joint angle and joint angular velocities may be thought of as muscle length and muscle velocity of contraction correlates;

e) allows the determination of resting baseline electromyographic levels which can be subtracted form the time dependent EMG recorded and the myoelectric maxima.

However, it should be noted that normalization may impose a limitation when pathological cases are under consideration. In its attempt to make similar the data from different subjects, it may suppress distinctions in the data that would be associated with abnormal or pathological cases (De Luca, 1997). 
There are various normalization methods, where the output of the normalized EMG determines the excitation level of a particular musculotendon unit (LeVeau and Andersson, 1992). For the purposes of this study, the processed dynamic EMG was normalized with respect to myoelecrtic maxima obtained at the length of the respective musculotendon units where maximum joint moment was generated as determined by computer simulations (see section "Calibration procedure"), and with respect to nearly isometric concentric contractions. The myoelectric maxima, normalization factors, were determined during a calibration procedure. Another two methods of normalization of the dynamic EMG are with respect to the peak dynamic EMG, mean dynamic EMG and maximum isometric EMG at different angles where then the dynamic EMG is being normalized at each dynamic position with respect to the maximum isometric EMG obtained for that position.

Several researchers have evaluated the various methods of normalizing the dynamic EMG (Allison et al., 1993; Burden and Bartlett, 1999; Knutson et al., 1994; Merletti and Lo Conte, 1997; Soderberg and Knutson, 2000; Yang and Winter, 1982, 1983, 1984). Knutson et al. (1994), and Soderberg and Knutson (2000) found best reproducibility from the MVIC normalization procedure, but they identified that there was always the concern of whether the subject actually performed the true maximum isometric contraction (De Luca, 1997). Yang and Winter $(1984,1983,1982)$, on the other hand found better correlation coefficients for EMG normalized to submaximal levels of maximum voluntary contraction. What appears to be a common outcome, is that most of the variability is usually identified during the eccentric phases of muscular contraction (Knutson et al., 1994; Olney and Winter, 1985; White and Winter, 1993).

Most recent evidence from the study of Burden and Bartlett (1999) indicate that the electromyographic maxima obtained from isokinetic and isometric maximum voluntary contractions are the most reliable means for normalizing the dynamic EMG signal. Those two methods produced the smallest root mean square difference when the normalized EMGs from each respective method were compared. Since the purpose of the dynamic mean and dynamic peak normalization methods is not to determine the percentage of the maximal activation capacity of the muscle required to perform isotonic 
contractions, output from these two methods cannot mirror the EMG increase which is in response to the increase in muscle force (Burden and Bartlett, 1999). Thus, for the purposes of this study a combination of the two most efficient methods, i.e., IMVC and isokinetic MVC for normalizing the dynamic EMG was implemented (see section "Calibration procedure").

\section{Calibration}

The purpose of the calibration procedure, combined with the normalization methods of the processed EMG was four fold:

a) to eliminate the arbitrariness of the processed EMG signal, i.e., the signal is scaled from zero to one in order to map the maximum force that can be generated;

b) to account for artifacts embedded in the EMG signal as functions of muscle length and velocity so that the relationship between EMG and force can be established for the musculotendon units of interest;

c) to obtain maximum EMG values as functions of the joint angle and change of the joint angle, i.e., muscle length and change of muscle length, and active state of the muscle for the purposes of normalizing the dynamic EMG signals recorded during gait; and

d) to establish resting baseline electromyographic levels which will be subtracted from the time dependent EMG recorded, and from the myoelectric maxima. The resulting normalized electromyographic signal, $N E M G_{i}$ for each musculotendon unit of interest $i$, will be:

$$
N E M G_{i}=\frac{E M G_{i}(t)-E M G_{i_{-} \text {resting }}}{E M G_{i_{-} \max }(\ell, \dot{\ell} \approx 0,1)-E M G_{i_{-} \text {resting }}}
$$

where $E M G_{i}(t)$ represents the time-dependent signal level from the musculotendon unit $i, E M G_{i_{-} \text {resting }}$ represents the resting baseline electromyographic signal for muscle $i$, and $E M G_{i_{-} \max }(\ell, \dot{\ell} \approx 0,1)$ represents the maximum electromyographic signal obtained for muscle $i$ as a function of the length at which it produces maximum moment about the joint of interest, under nearly isometric conditions and with maximum activation. 
In this project the gain, $G$, although under its definition as the physiologic muscleforce per unit cross sectional area, was desired to be a constant, it was expected that it might be time variant, $G(t)$. In other words, since gain, in essence, is a measure of stress it should have a constant value. More precisely, the gain for a given subject should be constant. However, due to differences between subjects in conditioning, training and natural ability, the inter-subject gains could be expected to be highly variable. However, even the intra-subject gain was expected to be variable with the present modeling approach because it was expected to be affected by the muscle length and velocity artifacts.

Consequently, although a constant gain value could be computed from the PCSA and the maximum isometric force a muscle can develop as determined by:

$$
F_{0}=2.5 \times g \times P C S A
$$

mentioned earlier, because of the inter-subject differences, it was thought that it would be more appropriate if it were actually measured. In addition, because of the muscle length and velocity artifacts, the intra-subject time varying gain was unlikely to have a constant value. Therefore, there was even bigger need to measure it in order to account for the muscle length and muscle velocity artifacts in the fututre.

Figure 3.2 provides the theoretical framework for calculating the time varying gain by estimating:

a) what could be thought of as a constant gain using maximum voluntary contractions under nearly isometric conditions at specific joint positions; the joint positions were selected after computer simulations indicated where the joint-muscle moment generated by all maximally activated muscles acting at the joint during normal gait was maximum $\left(G_{c}\right)$; and then

b) the time varying gain, $G(t)$, at different normal walking gait-like positions and velocities, to account for the artifact effect due to muscle length and velocity.

The gain, in general, can be computed by comparing the predicted moment generated by the modeled muscle forces, with the measured moment generated by the movement of the body segments about the joint axis of rotation (Figure 3.2). It is given as:

$$
\text { Gain }=\text { External Moment } / \text { Internal Predicted Moment }
$$


where the external moment is given either by the calibration device, such as an isokinetic dynamometer, or with the use of inverse dynamics, and the Internal Predicted Moment (IPM) is given by:

$$
I P M=G \times \sum\left[r_{i} \times N E M G_{i} \times P C S A_{i} \times F(\ell, \dot{\ell})\right]
$$

where $r_{i}$ represents the moment arm of the specific musculotendon unit $i$, and can be determined by SIMM, the $N E M G_{i}$ represents the ratio of the dynamic EMG to the normalizing factor defined earlier, and its value depends on the normalization scheme used; the PCSA obtained from literature data with the method outlined earlier, and, for this project, the force for the specific musculotendon unit of interest as a function of its length and velocity of contraction, $\bar{F}(\ell, \dot{\ell})$, obtained as outlined earlier.

The effectiveness and efficiency of the calibration method, and of the EMG-driven model was tested by assessing the time required by the subjects to complete the calibration (White, 1997). Consequently, it was important that the most significant aspects of the calibration procedure were identified so that the calibration time could be cut down to the minimum required, if any clinical implementation of the calibration was to be feasible. Then a protocol for clinical evaluations could be established. The effectiveness on the other hand, was related to the ability of accurately reconstructing the externally measured moments from the predicted muscle forces. This was one form of validation for the model (see section "EMG-driven Model Validation"). Moreover, for model adequacy checking, the predicted gain level should fall within the physiological ranges of 5.9 to $100 \mathrm{~N} \mathrm{~cm}^{2}$ (Kaufman et al., 1991a; Powell et al., 1984), although, as mentioned above, it should be expected to be highly variable among subjects, due to differences in individual conditioning and training levels as well as due to different genetic abilities, i.e., natural abilities. However, for the model to be valid, the gain predicted for a given subject should be constant throughout each of the experimental trials, unless muscle length and velocity of contraction artifacts are lumped in to it, as it is the case with the present modeling approach.

In order to solve for individual muscle forces from the calibration moment, two assumptions are most often implemented along with one observation. The observation is that there is an EMG-force relationship. However, the nature of this relationship, linear 
or non-linear, is the subject of much debate in the current literature (see discussion in section "The electromyogram"). The first assumption implemented is that only synergist muscle groups contribute to the calibration moment for a given joint. The second assumption implemented is that there is a direct relation between muscle force and physiological cross sectional area (Granata, 1995; White and Winter, 1993). While the present project adopted the second assumption, it was realized that there might be a significant amount of cocontraction.

One way to eliminate the antagonistic activity signal is to subtract it from the myoelectric signal of the reference and of the task (Lyons et al., 1983). Another way to deal with the cocontraction issue is to account for it. Redfern (1988) proposed in his dissertation a method for calibrating the EMG-torque relationships while taking coactivity into consideration. It was the activation level of each muscle that was related to the force. Initially, he determined the activation level of each muscle of interest (gastrocnemius, soleus, and tibialis anterior) from the processed and normalized EMG. The activation level was then smoothed by exponentially filtering it with the determined electromechanical time constant of the muscle. The electromechanical time constant was determined by normalizing the rise time in force for a contraction and the fall time in force in a relaxation. Then the relationships between the smoothed activation levels and the torque produced by the respective muscles were modeled as second order polynomials. The first and second order parameters of the polynomials were determined from two sets of calibration. While all three muscles were monitored at all times, in the first calibration set of tests, the biarticular gastrocnemius was positioned such that plantar flexion was the result of soleus activity alone. Consequently, only the monoarticular agonist and antagonist muscle groups were active. During the second set of calibrations the contribution of the gastrocnemius in plantar flexion was facilitated. The resultant moment was predicted as the sum of the relative contributions of the monitored muscles. The effects of muscle length artifacts and the force-length relationship of the musculotendon units of interest were all lumped in the activation level determined.

In the present project, however, the force as a function of muscle length and velocity of contraction are accounted for differently (see "muscle modeling and 
parameters" subsection). Furthermore, artifacts embedded in the EMG due to muscle length and velocity of contraction will be accounted for by using Newton's third law. At a joint, the sum of the individual muscle internal predicted moments must equal the measured external joint moment. However, due to the muscle length and velocity artifacts, such an equality might not be provable. Consequently, during the calibration, once the initial constant gain was determined, the relationship:

$$
\text { Gain = External Moment } / \text { Internal Predicted Moment }
$$

was used such that the gain was appropriately and automatically adjusted to satisfy this condition, i.e., to force the error to zero, when the joints of interest were at different positions first and when the joint positions changed with different velocities second. Then a gain was estimated in a least squares sense such that it accounted for the length and velocity artifacts. That gain was one of the input parameters to the model.

\section{Limitations}

\section{Planar Moments.}

The joints moments that were investigated in this study were the ones generated on the sagittal plane. Previous EMG-driven modeling approaches have attempted to investigate moments in the coronal and transverse planes, but they were not particularly successful. White and Winter (1993) and Olney and Winter (1985) although they monitored muscles that contributed moments in the coronal and transverse planes of the lower extremities joints of interest, they reported results for moments generated in the sagittal plane only. The reason was a very low signal to noise ratio in the other planes. The same was true for similar studies that investigated the lower back. Granata and Marras (1995) validated only extension moments for sagittally symmetric exertions due to low signal to noise ratios in the transverse and coronal planes. Their model, however, had the capability of simulating moments in all three planes. Consequently, given the experiences of these authors, the present EMG-driven model at its introductory stages focused only on the sagittal moments. However, the model, assuming input from musculotendon units that are responsible for coronal and transverse planes, has the capabilities of predicting moments in the respective planes. 


\section{No series elastic component (SEC).}

The effect of the series elastic component in the muscle was not included in the modeling procedure. The importance of the SEC has been investigated by several investigators in the past (Kovanen et al., 1984). Its effect on the mechanical properties of the skeletal muscle was found to be very significant, especially as it related to the ability of the slow muscles in maintaining posture, and with regard to its ability for storing elastic energy to perform dynamic work economically However, its separate inclusion in any EMG-driven modeling approach is very difficult (Olney and Winter, 1985; White and Winter, 1993). Zajac (1989a), whose muscle model was adopted for the initial stages of this modeling approach, did not include the effect of the SEC distinctly in his model. Its effect was rather lumped with the effect of the tendon, also in series with the contractile element. Some of the rationale behind such a decision includes that the effect of the tendon compliance dominates the behavior of the musculotendon unit, and therefore, the effect of the SEC can be neglected. In addition, inclusion of the SEC would result in an estimation of the fiber length disproportional to that of the sarcomere length. Thus, the fiber length would not be a scaled version of the sarcomere length because the sarcomere length would depend on the compliance of the SEC. The implication then is that the tension-length relation of the muscle fiber would not be directly proportional to that of the sarcomere. The muscle fiber velocity would not be a scaled version of the sarcomere velocity, either. Then, there may exist the potential for misinterpreting the direction of the change in length at points in the movement when there is a sudden change in the muscle excitation, such as when the movement is being initiated or even in the isometric case where the muscle fiber length will be assumed to be constant, but the sarcomere length will be shortening. Consequently, sarcomere and fiber velocities could even be opposite. Thus the potential error will be a function of the compliance of the SEC and of the rate of change of muscle excitation. Consequently, the SEC was lumped with the tendon compliance.

\section{No history of contraction effects.}

Mechanical events that precede muscle contraction affect the response of the muscle and its ability to produce force. It has been established that pre-stretching the 
muscle results in an increased force production, while shortening of the muscle and then performing a contraction results in a reduced force production (Abbot and Aubert, 1952; Abbot and Wilkie, 1953; Edman, 1993; Herzog and Leonard, 1997; Marechal and Plaghki, 1979; Meijer et al., 1997; Sugi and Tsuchiya, 1988). Most of these experiments implement a shortening or stretching of the muscle followed by a control isometric contraction to investigate the effect of pre-stretching or pre-shortening. With regard to the effect of history of speed of shortening some advocate that as the speed of shortening increases the amount of force reduction is decreased, and vice versa, (Abbot and Aubert, 1952; Marechal and Plaghki, 1979). Others have shown that if the speed of shortening remains constant then the speed of shortening has no affect on magnitude of force reduction (Herzog and Leonard, 2000). Edman and his collaborators (1978) observed that the plateau value of the force during stretching was proportional to both, the overlap of thick and thin filaments, and on the velocity of stretching. They further noted that shifts in the tension-velocity relationship associated with increased force after stretching are sarcomere length dependent. However, the mechanisms responsible for these phenomena are not yet clear, and there is a lot of debate in the current literature. Some have proposed that part of the explanation may lay in the existence of sarcomere length non-uniformities (Edman, 1993), or that they may be a function of the amount of attached cross-bridges (Ford et al., 1981). The increased force due to pre-stretching of the muscle has been found to be a function of the amount of stretch applied (Abbot and Aubert, 1952; Abbot and Wilkie, 1953; Sugi and Tsuchiya, 1988), but independent of stretching velocity for single muscle fibers (Sugi and Tsuchiya, 1988), but dependent on stretching velocity for whole muscles (Abbot and Aubert, 1952). The effect of pre-stretching of the muscle and its associated increased force has been suggested to be due to an increase in the average cross-bridge force (Ford et al., 1981).

The most recent attempts to investigate the effects of history of contraction on the muscle force produced under control isometric contractions, involve the simulations of physiologic-like situations, i.e. cycles of shortening-stretching and stretching-shortening (Herzog and Leonard, 2000). The results showed that each condition had a different effect in the control isometric forces produced following each of the dynamic muscle 
length change conditions. Shortening before stretching affected the force produced by the control isometric contraction as a function of the amount of stretch with respect to the stretch. In other words: (a) shortening that was equal to the amount of stretch had no positive or negative effect on the force produced by the control isometric contraction; (b) greater amount of shortening relative to the stretch, resulted in reduced force; (c) shortening smaller than stretching resulted in increased force. In the condition of lengthening before shortening, the effect on the magnitude of the force produced during the isometric control contraction was a function of the speed of lengthening. Slow rate of length change had no effect on the reduced force. Force was increased if lengthening speed was high. The authors observed that there were no modeling approaches in the literature that could account for all of their results. Furthermore, since this was the first study of its kind the results need to be treated with caution.

Consequently, although it may appear desirable to model a number of preceding contracture factors, these factors need to be defined more clearly before they can be modelled mathematically. It could also be expected that variable preceding events might introduce a hysteresis affect to the muscle responses that have already been modeled and for which tendon compliance may not account for.

\section{Temperature effects.}

It has been established that muscle response is sensitive to the operating temperature.(Binkhorst et al., 1977; Close, 1972; Edman, 1979; Katz, 1939; Partridge and Benton, 1981; Petrofsky and Lind, 1975; Petrofsky and Phillips, 1981). Since the interaction of acting and myosin is sensitive to temperature, so is the maximum velocity of contraction (Claflin and Faulkner, 1985; Spector et al., 1980; Zajac, 1989a). The tension-velocity relationship becomes flatter with increasing temperature (Binkhorst et al., 1977; Phillips and Petrofsky, 1980), although temperature has very little effect on the maximum isometric force (Bahler et al., 1968; Binkhorst et al., 1977; Petrofsky and Lind, 1975; Petrofsky and Phillips, 1981).

However, despite all these evidence there appears to be reluctance to implement temperature as a variable in any of the modeling approaches. The reason is that intraand inter-muscular temperature is very difficult to monitor and it will vary unpredictably 
as a function of the dynamic movement, muscle depth and superficial body fat (Partridge and Benton, 1981). Thus the relationships between these variables and temperature will be very complex since large, random, non-uniform thermal variations are found in the muscle, making the temperature a desirable but very difficult parameter to model.

Homogeneous muscle force generation.

In the present modeling approach, and in order to assume effective use of surface electromyography for the purposes of the study, a homogeneous force generation throughout the entire muscle volume was assumed (De Luca, 1997). On the basis of this assumption, it was possible to extrapolate from the signal detected by the electrodes to the entire muscle activity and to the entire force produced by the musculotendon unit if interest. Along with these assumptions, it was assumed that force was being produced by uniform sarcomere changes in length (Delp, 1995). However, it has recently speculated that force generation may be a function of non-uniform sarcomere length changes (Edman, 1993). Although such an observation was related to muscle fiber preparations for the experimental purposes of that study, if it represented the true physiological system, then the assumption of homogeneous muscle force generation may be subject to criticism.

The physiological cross-sectional area.

It was assumed, for the purposes of this study to be a constant. However, the absolute value of the muscle cross sectional measurement is really a representative of the muscle only at the muscle length at which the measurement was made (White and Winter, 1993). Thus, there may be the need to adjust the cross sectional area as a function of joint position or muscle length during any kind of dynamic muscle modeling. 


\section{CHAPTER 4}

\section{INVERSE DYNAMICS MODEL DEVELOPMENT}

\section{Introduction}

The methodology of relating selected parameters of a system in order to quantify its performance is referred to as modeling. A model is a simplification of the actual system so that it can be analyzed mathematically. For the investigation of gait, the system that is simplified is the human musculoskeletal system. Gait models, therefore, attempt the simplification of the complicated process of walking to one which can be analyzed mathematically, and, consequently, solved with the use of a computer (Yamaguchi, 1989a). The first step in modeling gait is the construction of a skeletal model that considers joint articulation characteristics. Such a model is used to determine the joint moments from the primary kinetic and kinematic information recorded during a gait evaluation. Then, the dynamics, and force production characteristics of the musculotendon units of interest need to be modeled in order to investigate their force output. Finally, investigation of the kinetic effect of the musculotendon units involved in the joint moments estimated, requires the combined implementation of the skeletal and muscular models.

In general, there are two approaches to modeling human movement. One approach considers the natural course of events going forward in time, and it is referred to as direct dynamics analysis. The natural course of events has commands originating at the central nervous system to the muscles, i.e., the muscular system, which, in turn, develops forces and exerts movements on the body segments, i.e., the skeletal system (Zajac and Gordon, 1989). The second approach proceeds opposite in direction to the natural course of events, and it is referred to as inverse dynamics analysis. The inverse dynamics analysis starts from the quantitative observations of the movement trajectories, and uses dynamic 
musculoskeletal modeling to determine the joint moments and muscle forces that must have existed in order for the observed motion to take place (Zajac and Gordon, 1989). The inverse dynamics analysis is the one that is currently used for clinical applications.

\section{Methods}

\section{Model Development}

Direct dynamic methods provide simulations of the natural course of events where neural controls serve as the inputs while movement trajectories serve as the outputs. Thus, direct dynamic models transform the neural controls to forces, torques, and ultimately to movement trajectories. This approach is generally represented mathematically in the following form:

$$
M \ddot{\bar{Q}}=\vec{T}+\vec{V}+\vec{G}+\vec{E}
$$

where one needs to solve for $\ddot{\bar{Q}}$. For the purposes of this investigation, the dynamic equation (4.1) will be reformulated to represent the inverse dynamic analysis approach in the form:

$$
\vec{T}=M \ddot{\bar{Q}}-\vec{V}-\vec{G}-\bar{E}
$$

where $\vec{T}$ is the $n \times 1$ output vector representing the joint torque corresponding to both, the active muscle torques and the passive, ligamentous, torque. $\mathrm{M}$ is the $n \times n$ "mass" matrix for the $\mathrm{n}$ degree of freedom (DOF) model containing the mass characteristics and instantaneous configurations of the body, and $\ddot{\bar{Q}}, \vec{V}, \vec{G}$, and $\vec{E}$ are all $n \times 1$ vectors. $\ddot{\vec{Q}}$ represents the accelerations of the segments, $\ddot{\bar{Q}}=\left(\vec{q}_{1}, \ldots, \vec{q}_{n}\right)^{\prime \prime}, \vec{V}, \vec{G}$, and $\vec{E}$ represent the torques due to inertia, gravity, and external (including ground reaction) forces respectively. Therefore, one can observe the dependence of, and express the output segmental torques as a function of the trajectory dependent terms grouped in the righthand side of the equation 4.2. The following sections will describe how to obtain the values of each of the parameters needed. 


\section{Joint angles}

The left lower extremity of each subject was modeled as a four-segment rigid link system. The four segments were the pelvis, the thigh, the shank (lower leg), and the foot (see Figure 4.1). Obviously, the assumption that body segments can be modeled as rigid bodies, which implies that the underlying skeleton's position in space as a function of time can be approximated by tracking the position of surface tissue in space as a function of time, is a source of error. Soft tissue motion artifact, inherent in all motion analyses of human subjects, is the result of soft tissue distribution changes during movement. It has been estimated that the anthropometric parameter errors introduced by the rigid body assumption are less than six percent (Hatze, 1980b).

Every two adjacent segments formed a joint. Each segment was defined by three or four non-collinear markers (Figure 4.1). Each segment was tracked by at least three noncollinear markers (Figure 4.1).

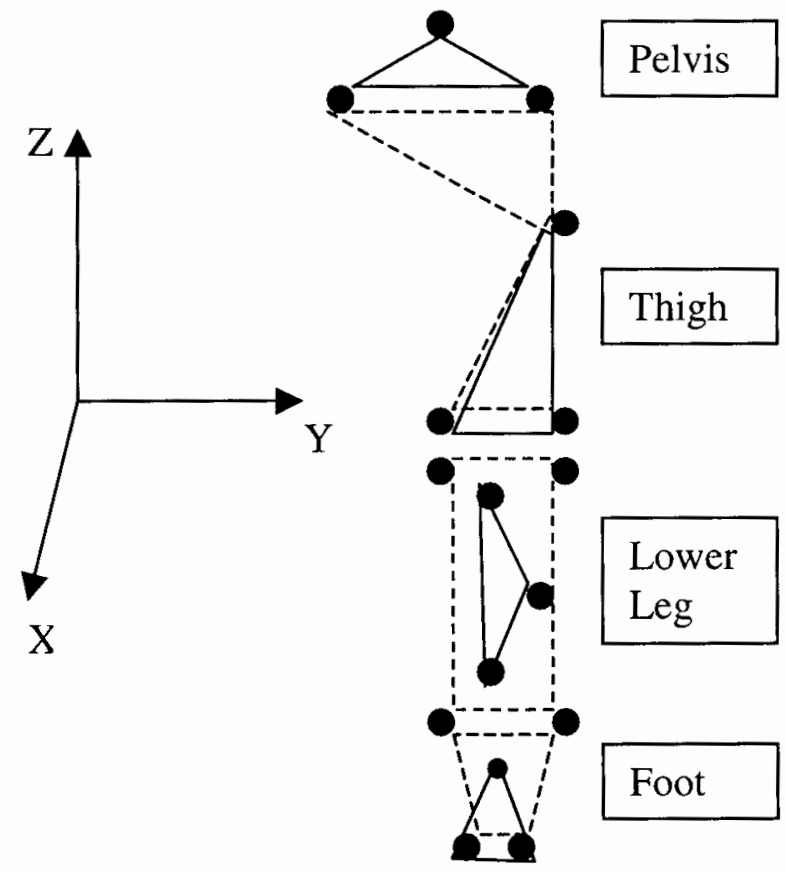

Figure 4.1. The four segments that comprised the left lower extremity were the pelvis, the thigh the lower leg and the foot. The dash lines and their associated markers show how each body segment was defined. The solid lines and their associated markers show how each segment was tracked. 
The three markers allowed the formation of a right-handed segment coordinate system (SCS) with respect to the fixed right-handed global coordinate system (GCS) of the laboratory. In the Gait Analysis Laboratory (GAL) of the Ohio State University (OSU), the $\mathrm{X}$ axis represented the posterior-anterior direction of the lab, the $\mathrm{Y}$ axis represented the medio-lateral direction of the lab and the $\mathrm{Z}$ axis represented the vertical direction of the lab.

The position of each marker in space and as a function of time was tracked by the motion analysis system, and it was expressed as coordinates in the fixed laboratory reference system:

$$
\vec{p}_{j}=X_{j}(t) \hat{i}_{X}+Y_{j}(t) \hat{i}_{Y}+Z_{j}(t) \hat{i}_{Z}
$$

where $\vec{p}_{j}$ was the position vector of marker $\mathrm{j}, X, Y, Z$ were each of the coordinates of the marker expressed as a function of time $t$, and $\hat{i}_{X}, \hat{i}_{Y}, \hat{i}_{Z}$ were the unit base vectors in the GCS that serve as pointers in the $X, Y, Z$ directions of the GCS respectively.

The position vectors for the markers of each segment were used to obtain each SCS, i.e., three lines, mutually perpendicular, attached to the segment, that remain at a fixed orientation to the specific segment. With respect to the settings in GAL at OSU, the $x$ segment axis was in the anterior direction, the y segment axis was in the medial-lateral direction, with positive $y$ directed to the left of the body segment, and the $\mathrm{z}$ segmental axis was directed on the superior direction or distal to proximal on the body segment (see Figure 4.2).

To form the right-handed SCS at least three markers were placed on each body segment such that two markers (e.g. markers P1 and P2) could define a segmental axis and the three markers (P1, P2, and P3) could form a segment anatomical plane (Cappozzo et al., 1995). Then a relative position vector between the two markers that defined the segmental axes (P1 and P2) was designated, and it was constructed by subtracting the coordinates of the one marker of the segment from the coordinates of the other. The magnitude of the position vector was then normalized to obtain a unit vector in the specific segmental coordinate direction. The unit vector was called axis3. Since it was assumed that each segment was a rigid body, the magnitude of the relative position 


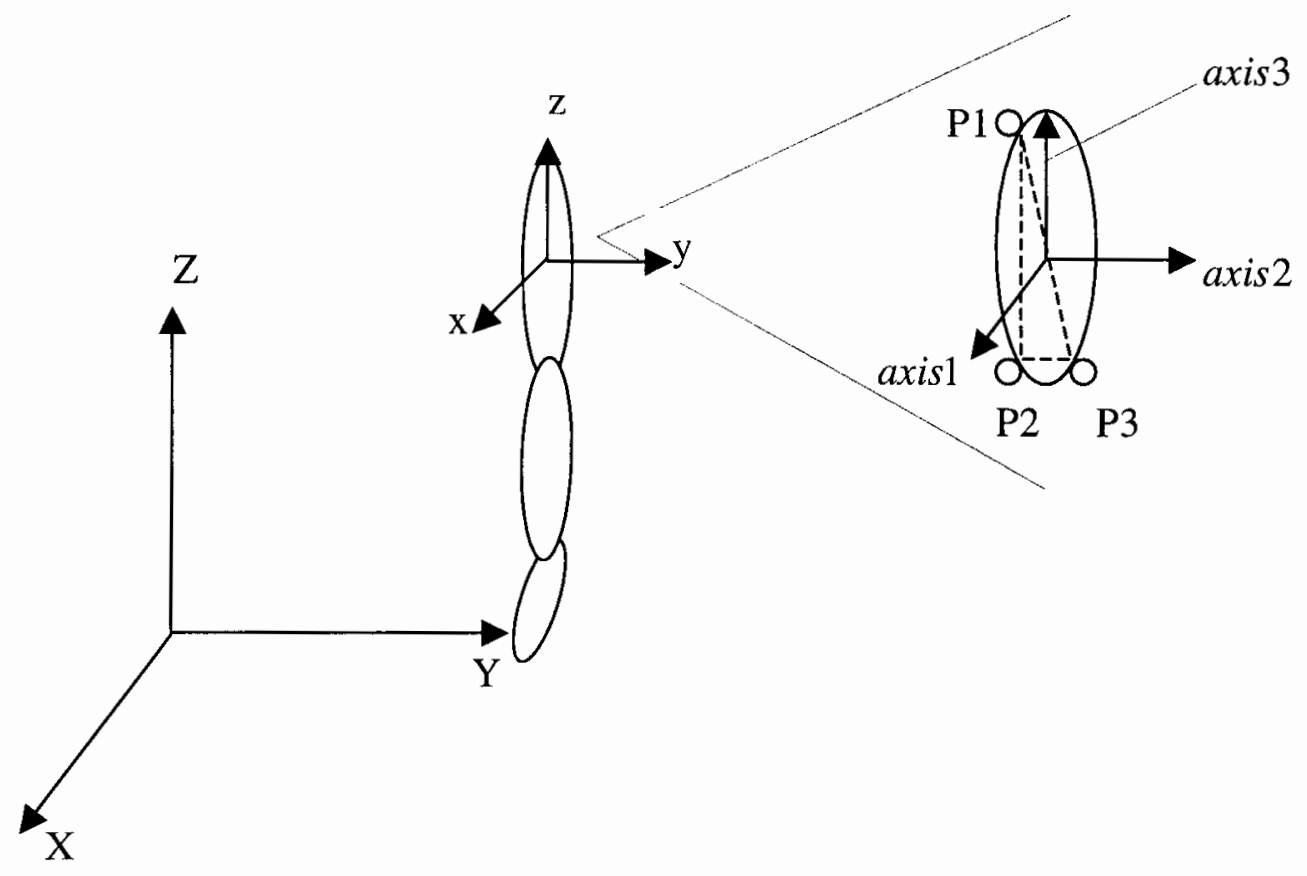

Figure 4.2. Global coordinate system (GCS) of the laboratory X,Y,Z. When a normal subject walks stands in the anatomical position each segment coordinate system (SCS) is aligned with the GCS. For the definition of each segment three non-collinear markers were placed allowing the construction of the SCS.

vector was assumed to remain constant. In addition, although the orientation of the unit vector in space changed, its relative orientation with respect to the body segment did not. To form a unit vector perpendicular to the frontal plane formed by P1, P2 and P3, axis 3 was crossed with the unit vector formed by the normalized difference between P2 and P3. The result of this cross product was labeled axis1. Finally, axis 3 was crossed into axis 1 to yield axis 2 , the final coordinate direction of the body segment. Mathematically this can be represented in the following procedure:

$$
\begin{gathered}
\text { axis } 3=\frac{P 1-P 2}{|P 1-P 2|} \\
\text { axis } 1=\frac{(P 3-P 2)}{|(P 3-P 2)|} \times \text { axis } 3 \\
\text { axis } 2=\text { axis } 3 \times \text { axis } 1
\end{gathered}
$$


Consequently, the position of a body segment was determined by the position vector to P3 (Spiegelman and Woo, 1987). The three-dimensional (3-D) orientation of the body segment was determined by the segmental base unit vectors (axis1, axis 2 , axis 3 or $\hat{i}_{x}^{s}, \hat{i}_{y}^{s}, \hat{\mathrm{i}}_{\mathrm{z}}^{\mathrm{s}}$, where $\mathrm{s}$ represents the specific segment), which represented a direction cosine matrix as a function of time (see Figure 4.2). With the subject standing in the anatomical position, all axes were aligned with the GCS. The origin of the SCS was located at the segment's estimated center of mass.

The three-dimensional segmental positions can be converted into angular trajectories of the joints formed by the moving segments (Grood and Suntay, 1983; Soutas-Little and Verstrate, 1990). However, it is important to note first that the determination of the orientation of the body segment is a function of the sequencing of three successive rotations that the body has undergone. These rotations are the Euler angles (Goldstein, 1980). Grood and Suntay (1983) determined the knee joint angle by first rotating about the medio-lateral $Y$ axis of the femur, yielding the flexion-extension angle. Then they rotated about what would be the original posterior-anterior axis of the tibia (axis $\mathrm{x}$, now intermediate axis $\mathrm{x}^{\prime}$ after the first rotation) to define the abductionadduction at the knee. The intermediate axis was called by Euler (Goldstein, 1980) the line of nodes and Grood and Suntay (1983) called it a floating axis. Finally, they rotated about what would be the original superior axis of the tibia (axis z, now $z^{\prime \prime}$ after the two rotations) to define the internal-external rotation at the knee. Soutas-Little and Verstrate (1990) determined motion at the ankle joint by first rotating about the medio-lateral axis of the tibia-fibula (shank) to define plantar-dorsi flexion. Then, rotation about the floating axis defined inversion-eversion. Medial-lateral rotation or internal-external rotation was defined about the superior axis of the foot.

However, it appears that different laboratories or motion analysis system manufacturers define the sequence of rotations in different orders, resulting in different joint angles (Soutas-Little, 1991). It should be noted, though, that following the method suggested by Grood and Suntay (1983) and Soutas-Little and Verstrate (1990), joint angles can be described by three angles independent of the order of rotation. In essence their method suggests the formation of a right-handed joint coordinate system (JCS) by 
the two SCS of the respective adjacent segments that form a joint. The JCS is formed by borrowing one axis of rotation from one segment, a second axis of rotation from the adjoining segment, and the third axis of rotation (floating axis) is obtained by crossing the two segmental axes. For the purposes of the kinematic and kinetic analysis in the present investigation a motion analysis software was used, developed and validated by the National Institutes of Health (MOVE3D). The software encapsulated all the theoretical concepts presented here except one. The software used the markers implemented for the definition of each body segment to generate the SCS, instead of using the markers implemented to track the segment in space. Joint velocities and joint accelerations were determined from the differentiation of the joint angular data.

\section{Parameters of the body segments}

The properties of each of the body segments of interest were required for the determination of the joint moments during the kinetic analysis, and for the location of the origin of the SCS. The body segment parameters that were needed included the mass of the segment, the location of the center of mass and the moments of inertia.

The software that was used for the purposes of the kinetic and kinematic analysis, MOVE3D, incorporated a geometric model for each of the body segments of interest. The foot, shank and thigh were all modeled as frusta of right cones. The pelvis was modeled as a cylinder (Table 4.1). Each body segment weight was determined from the total body weight of the subject and Dempster's (Dempster, 1955) anthropometric data (Table 4.1). On the basis of segmental weight and geometric shape the principal moments of inertia and center of mass for each body segment were determined (see Appendix A). The principal moments of inertia were determined about the principal axes of the segment located at its center of mass. Thus, the product moments of inertia were zero and, consequently, the rotational motions for each segment were decoupled.

Proximal and distal radii were required for all segments to determine each segmental length, necessary for determining the segmental properties (see Appendix B). For the foot and shank, two markers were used at each respective proximal and distal end of the segment, i.e., a proximal medial and a proximal lateral target, and a distal medial along with a distal lateral target. Consequently, proximal and distal radii were one-half 


\begin{tabular}{||l|c|c|}
\hline \multicolumn{1}{|c|}{ Segment } & $\begin{array}{c}\text { Mass } \\
\text { (Percent Body Weight) }\end{array}$ & Geometric Shape \\
\hline Foot & 1.45 & Cone \\
\hline Shank & 4.65 & Cone \\
\hline Thigh & 10.00 & Cone \\
\hline Pelvis & 14.20 & Cylinder \\
\hline
\end{tabular}

Table 4.1.

Segmental MOVE3D geometric shapes for the body segments of interest, and their mass distribution with respect to the total body weight on the basis of the anthropometric data reported by Dempster (1955).

the distance between the two targets at each respective end. The limitation using this method was that since the foot segment was assumed to be from the maleoli to the first and fifth metatarsal heads, the length of the cone might be an underestimation of the true length. Therefore, some minimum error should be expected in the location of the center of mass of the foot and in the estimated foot segment properties. For the thigh there was only one marker at the proximal end. Consequently, the radius for the proximal end had to be supplied manually. The radius was estimated from the distance between the left and right greater trochanteric markers divided by four (Kepple, 1997). Similarly, the distal radius for the pelvis was estimated from the distance between the left and right greater trochanteric markers divided by two (Kepple, 1997). All radii were corrected for the radii of the respective markers $(0.0125 \mathrm{~m})$ (see Appendix B).

\section{Estimation of the Joint Centers}

Estimation of the joint moments or torques requires the definition of the joint center of rotation or of the instant joint center of rotation for each of the joints of interest. Implementation of rigid body dynamics assumes that forces at a joint act through a single point. The point through which joint forces act is defined as the point where the resultant surface articular contact forces act. Alternatively, it may be considered to be the joint center of rotation in the absence of friction (Cappozzo, 1984).

The position of the joint center of rotation can be defined from anatomical considerations alone. In fact that is how some software manufactures define joint centers 
of rotation for the purposes of estimating joint moments (Vicon, 1997). For some joints, such as the hip, a joint center defined from anatomical measurements and modeled as a ball and half-socket joint may be adequate for the purposes of estimating joint moments (Davis et al., 1991). Thus, the femur is considered to rotate about the center of the spherical femoral head in movements relative to the pelvis. This point is considered to be stationary on both the pelvis and the femur. However, in the ankle and knee joints such an approach may not be appropriate. The knee, in particular, involves the femur rolling and sliding on the tibia (Wongchaisuwat et al., 1984). Consequently there is not one point that could serve as a hinge point. Alternatively, then, several researchers and most motion analysis software manufacturers define the geometric center of the joint as the joint center (Vicon, 1997; White and Winter, 1993), which provides a reproducible point for the analysis of the joint moments, although not the kinematic or rotational joint center. However, it can be realized that such an approach may introduce errors in the estimation of joint moments.

Consequently, accurate representation of joint moments requires the definition of a kinematic joint center. The position of the kinematic joint center of rotation in space can be determined as an instantaneous center of rotation for planar, 2-D, analysis, or as an instantaneous helical axis for 3-D analysis (Kinzel et al., 1972; Soudan and Audekercke, 1979; Woltring, 1986). The concept of an instantaneous axis of rotation is an extension of the axis of pure rotation (Spiegelman and Woo, 1987). In planar analysis the instantaneous axis of rotation is a line formed by the accumulation of points, where each point has zero velocity. This axis may pass through the rigid body or through the body extended (Spiegelman and Woo, 1987). The location of the center of rotation in planar motion can be solved in terms of the rotation angle and knowing the initial and final coordinates of two points on the object undergoing planar motion (Spiegelman and Woo, 1987). The knee joint has often been modeled as a joint with only sagittal plane motion (Yamaguchi and Zajac, 1989b). The instantaneous center of rotation or hinge point, then, is a point on the femur that coincides with a point on the tibia that has the same velocity.

In general motion, however, there will be an axis in space along which the motion will be described as a screw motion, i.e., the body rotates about this axis and translates 
along it relative to the other body. That axis will be the instantaneous helical axis or screw axis (Woltring, 1990). Recently, alternatives to the concept of the screw axis have been suggested without significant success (Halvorsen et al., 1999).

MOVE3D uses the concept of helical axes. The hip, knee and ankle joints were modeled, in MOVE3D, as six degree-of-freedom joints, i.e., three translations and three rotations were allowed about any axis. The instantaneous joint center of rotation is defined in MOVE3D as the intercept of the instantaneous helical axis with the sagittal plane (see Appendix D).

\section{Force plate analysis}

For the determination of joint moments both, measurement of external forces, such as the ground reaction force (GRF) acting on the lower extremity, and their application relative to the joint center of rotation are necessary. Such information was determined in the present research study from use of one force plate. The GRF is the resultant of all internal and external forces acting on the body while the foot is in contact with the ground, i.e., during the stance phase of gait (Winter, 1990). The force plate provided the three orthogonal components of the GRF ( $\mathrm{Fx}, \mathrm{Fy}$, and Fz). In the Gait Analysis Laboratory at OSU the Fx represented the fore-aft force, Fy represented the medio-lateral force, and Fz was the vertical component of the GRF. Furthermore, the force plate provided the moments ( $\mathrm{Mx}, \mathrm{My}$, and $\mathrm{Mz}$ ) produced by the components of the GRF about three orthogonal axes, a right-handed reference frame, with origin at the center of the plate.

In dynamics, all forces and moments acting on a rigid body can be replaced by an equivalent system, i.e., a single moment vector and a single force vector applied at a point on the top of the force plate (Soutas-Little, 1991). Combining the forces and moments the point of application of the GRF or the center of pressure (COP) can be determined. MOVE3D, including all the commercially available software for kinetic analysis of gait and, to the best of my knowledge, most gait analysis laboratories use one 
GRF component, Fz, and two moments, $\mathrm{Mx}$ and My, to determine the $\mathrm{COP}^{1}$ (Cavanagh, 1981) (see Figure 4.3):

$$
\begin{gathered}
C O P_{x}=-\frac{M_{y}}{F_{z}} \\
C O P_{y}=\frac{M_{x}}{F_{z}}
\end{gathered}
$$

However, it is important to note that while the point of application should be representing the position at which an equivalent resultant force is applied to the foot, the approach used above does not. While the resultant force is, theoretically, the summation of all the forces applied to the foot, the above procedure does not include the effect of the shear

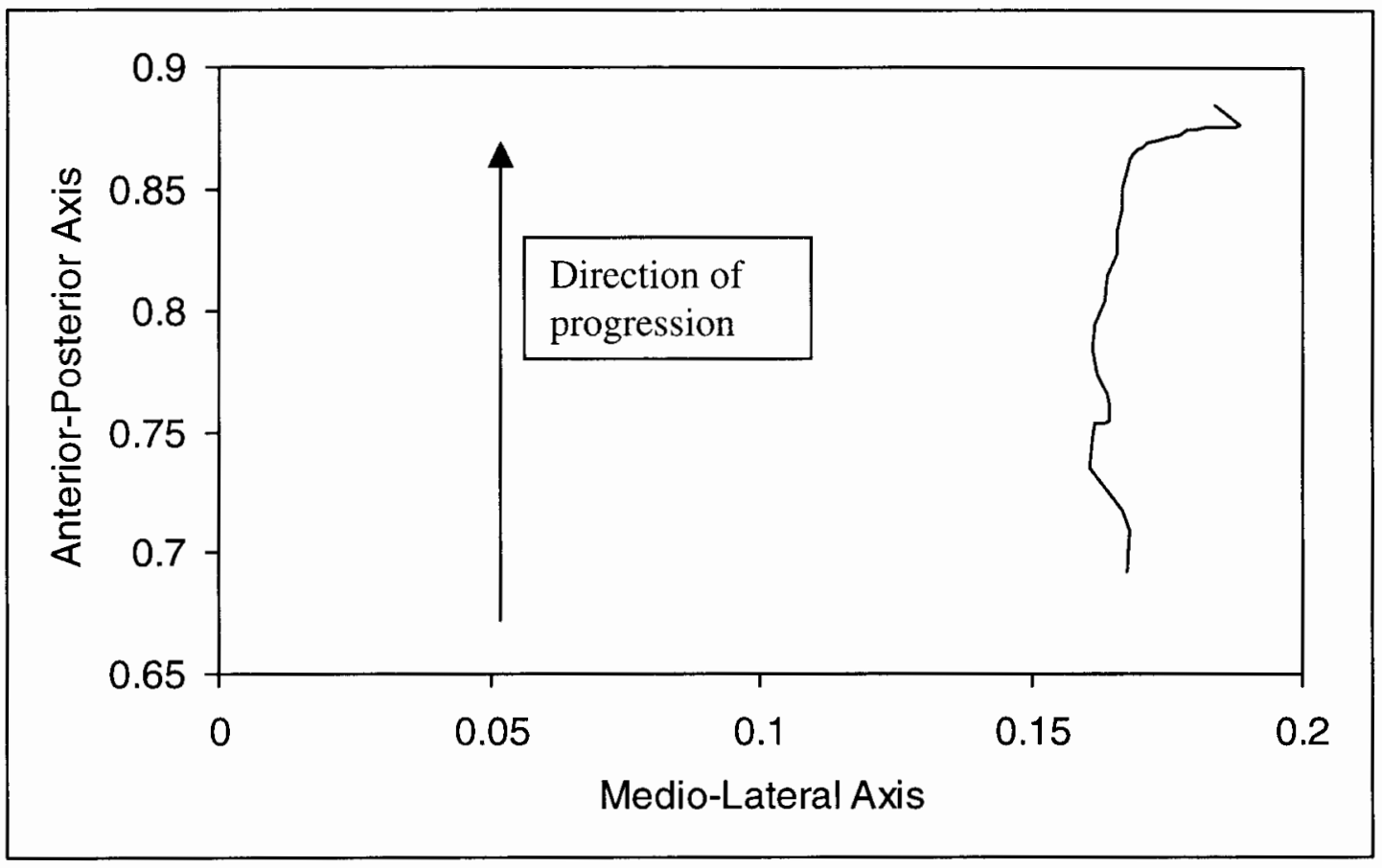

Figure 4.3. Center of pressure for the left foot on the force plate for subject P4 using the MOVE3D software.

\footnotetext{
${ }^{1}$ Finding the $C O P_{x}$ and $C O P_{y}$ precludes that the GRF has been corrected for the location of the force plate's geometric center of mass, provided by the manufacturer of the force plate.
} 
forces. The operative assumption here is the following: the force plate measures the three force components of the resultant GRF vector, Fx, Fy, and Fz, and the three moment components, $\mathrm{Mx}, \mathrm{My}, \mathrm{Mz}$ due to the applied force about the $\mathrm{X}, \mathrm{Y}$, and $\mathrm{Z}$ axes of the platform, plus any applied torques, $\mathrm{Tx}, \mathrm{Ty}$, and $\mathrm{Tz}$. Thus, the moment equations can be written as:

$$
\begin{aligned}
& M_{x}=F_{z} Y+F_{y} Z+T_{x} \\
& M_{y}=F_{z} X+F_{x} Z+T_{y} \\
& M_{z}=F_{x} Y+F_{y} X+T_{z}
\end{aligned}
$$

where $\mathrm{X}, \mathrm{Y}$, and $\mathrm{Z}$ are the positions of the applied loads relative to the respective axes. Parameter $Z$, in particular, is a constant representing the position of the applied horizontal loads above the origin of the platform. While walking, the ground is capable of applying a 3-D force, one normal and two frictional components, and, under normal conditions, torques about the anterior-posterior and medio-lateral axes cannot be applied, i.e., Tx and Ty are zero. Thus, only a 1-D torque can be applied by the ground to the foot while walking, about the vertical axis. Also, when estimating the location of the COP, the parameter $\mathrm{Z}$ can be considered to be zero since forces and moments are applied at the top of the force plate. Consequently, only equation (4.5) are needed to determine the location of the COP.

Some researchers advocate the above approach on the basis that since the $\mathrm{Fz}$ intersects the Fx and Fy components of the GRF on the force plate, and they all have a common origin, at the point of application of the resultant GRF vector, only the $\mathrm{Fz}$ component is needed to locate the COP (Davis, 1996). Others, however, believe that the above approach for the determination of the COP is not appropriate because a general force system is treated as being a parallel vertical force system (Soutas-Little, 1991). For the purposes of the current investigation, since we have used the MOVE3D for the inverse dynamics analysis, the COP will be determined by the method described previously with equations 4.5 . 


\section{Net joint moments determination}

Since all the parameters necessary for the inverse dynamics analysis are defined with the theoretical approaches outlined previously the net joint moments were determined using the six Newton-Euler equations of motion (the motion of each segment of the body was defined about its center of mass). The inverse dynamics analysis started from the most distal segment of the lower extremity, the foot where all external forces are known, and then, sequentially, it was applied to the more proximal segments:

$$
\begin{aligned}
& \sum F_{n X}=\left(m a_{c m_{X}}\right)_{n} \\
& \sum F_{n Y}=\left(m a_{c m Y}\right)_{n} \\
& \sum F_{n Z}=\left(m v_{c m Z}\right)_{n} \\
\sum M_{n x}= & {\left[I_{x x} \frac{d \omega_{x}}{d t}+\left(I_{z z}-I_{y y}\right) \omega_{y} \omega_{z}\right]_{n} } \\
\sum M_{n y}= & {\left[I_{y y} \frac{d \omega_{y}}{d t}+\left(I_{x x}-I_{z z}\right) \omega_{x} \omega_{z}\right]_{n} } \\
\sum M_{n z}= & {\left[I_{z z} \frac{d \omega_{z}}{d t}+\left(I_{y y}-I_{x x}\right) \omega_{x} \omega_{y}\right]_{n} }
\end{aligned}
$$

where $n$ represents the specific segment of interest, $m$ represents the mass of the segment, and $\frac{d}{d t} v_{c m}$ represents the acceleration of the center of mass of the segment in the three orthogonal axes of the GCS $(X, Y, Z)$. The forces are measured in the GCS. The $I_{x x}, I_{y y}, I_{z z}$ represent the inertial components of the segment $n$ about the segment principal axes; $\omega$, and $\frac{d \omega}{d t}$ represent the segment's angular velocity and acceleration, respectively. The moments are determined in the SCS since the inertia parameters are in the SCS.

To the best of my knowledge there exists no documentation about MOVE3D pertaining to the methodology that has been implemented for the inverse dynamics approach. In Appendix E, the method that I believe the author has used is outlined, on the basis of my experience with the software and my communications with him. 


\section{$\underline{\text { Passive joint torques }}$}

The net joint moments are a function of the muscle (active) and passive joint moments. For the purposes of this investigation, the moment produced by muscle contraction is the joint moment of interest. The muscle component of the net joint moment can be found by subtracting from the estimated net joint moment, the passive joint torque.

Passive joint torques encapsulate the constrains imposed on a joint's range of motion by the ligaments surrounding the joint, and its joint capsule. Furthermore, passive joint moments represent the damping effect of the cartilage and other viscoelastic structures in the joints. The mathematical formulation used to represent the passive torques are based on the work of Audu and Davy (1985), and Davy and Audu (1987) who constructed a model for the swinging leg during gait:

$$
\left|\vec{M}_{\text {passive }}(\theta, \omega)\right|=k_{1} e^{-k_{2}\left(\theta-\theta_{1}\right)}-k_{3} e^{-k_{4}\left(\theta_{2}-\theta\right)}-\beta \omega
$$

where $\vec{M}_{\text {passive }}$ is expressed in N-m, $\theta$ in radians, $\omega$ in radians per seconds.

The constants $\mathrm{k}_{1}$ and $\mathrm{k}_{3}$ roughly determine the magnitude of the passive torques while $\mathrm{k}_{2}$ and $\mathrm{k}_{4}$ control the slope and curvature of the torques. Adjustments to some of the $\mathrm{k}$ values were made by Yamaguchi (1989) and Green (1992) to reduce the laxity in the joints found with the values of Audu and Davy (1985), and Davy and Audu (1987). The angles $\theta_{1}$ and $\theta_{2}$ dictate the range of the passive torques, i.e. they represent the joint angles, while $\beta$ represents the damping coefficient. The values that will be chosen for the purposes of this project will be adapted from Audu and Davy (1985), Yamaguchi (1989) and Green (1992) (see Table 4.2).

It should be noticed that these torques model the physiological constraints of the joints at the extreme ranges of motion, which may not be seen in physiological gait, but they can certainly be part of the pathological gait. Each of the passive torques of the ankle and knee for the sagittal plane are represented in Figure 4.4 and Figure 4.5 respectively. The passive torques are a function of position only, i.e., angular velocity is zero. 


\begin{tabular}{||c|c|c|c|c|c|c|c|}
\hline Joint & $\mathrm{k}_{1}$ & $\mathrm{k}_{2}$ & $\mathrm{k}_{3}$ & $\mathrm{k}_{4}$ & $\theta_{1}$ & $\theta_{2}$ & $\beta$ \\
\hline Ankle plantar flexion $\left(30^{\circ}\right)$ & 9.0 & 5.0 & 2.0 & 5.0 & 0.524 & - & 0.943 \\
\hline Ankle dorsi flexion $\left(20^{\circ}\right)$ & 9.0 & 5.0 & 2.0 & 5.0 & - & -0.349 & 0.943 \\
\hline Knee flexion $\left(110^{\circ}\right)$ & 3.1 & 5.9 & 10.5 & 11.8 & -1.92 & - & 3.17 \\
\hline $\begin{array}{l}\text { Knee extension } \\
\left(0^{\circ} \text {, i.e., full extension) }\right.\end{array}$ & 3.1 & 5.9 & 10.5 & 11.8 & - & 0.0 & 3.17 \\
\hline
\end{tabular}

Table 4.2.

Coefficients for the estimation of passive joint moments. Passive joint moments represent the ligamentous and cartilaginous contributions to the net joint moment measured via inverse dynamics analysis.

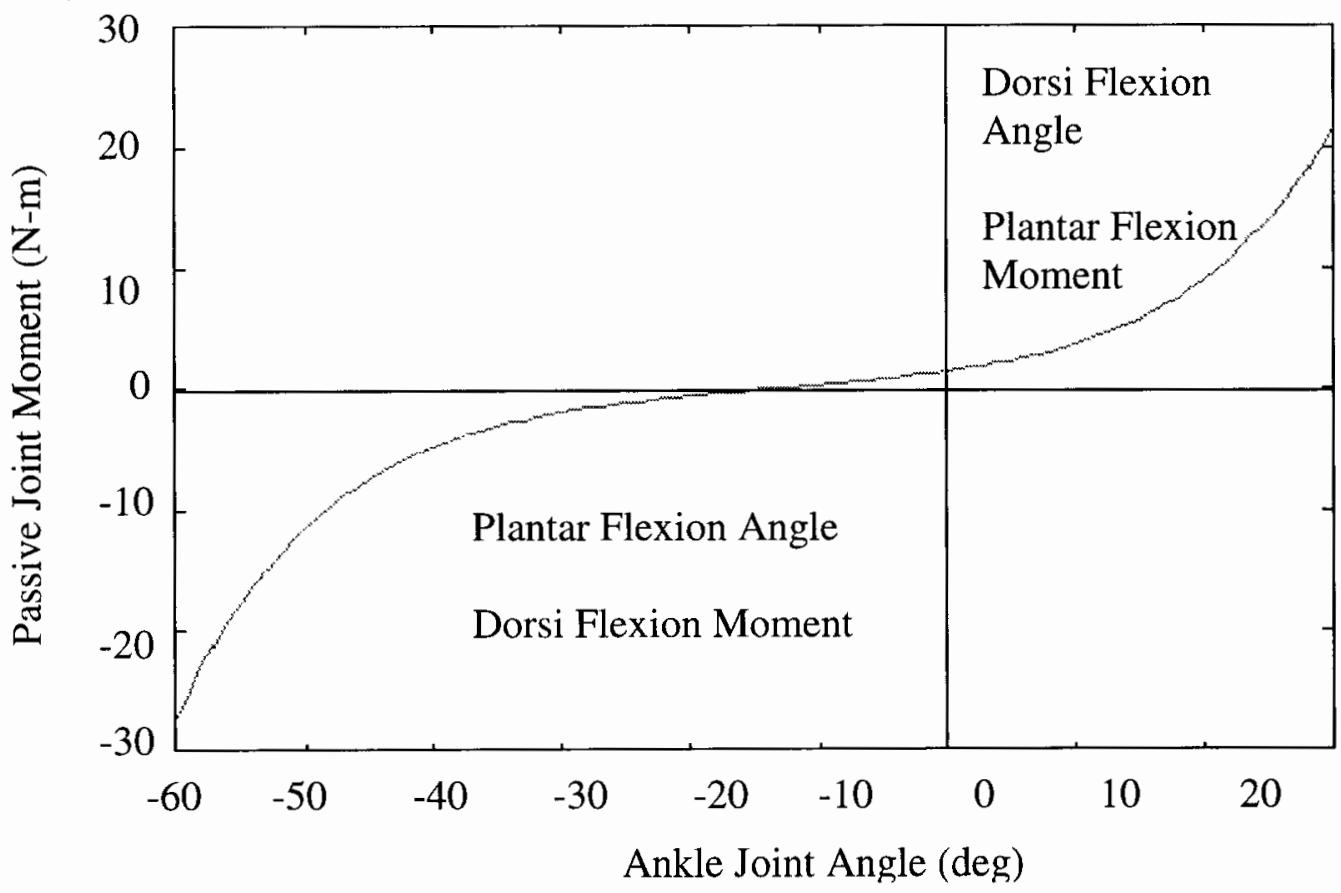

Figure 4.4. Passive ankle plantar/dorsi flexion moments. The output is a function of position. 


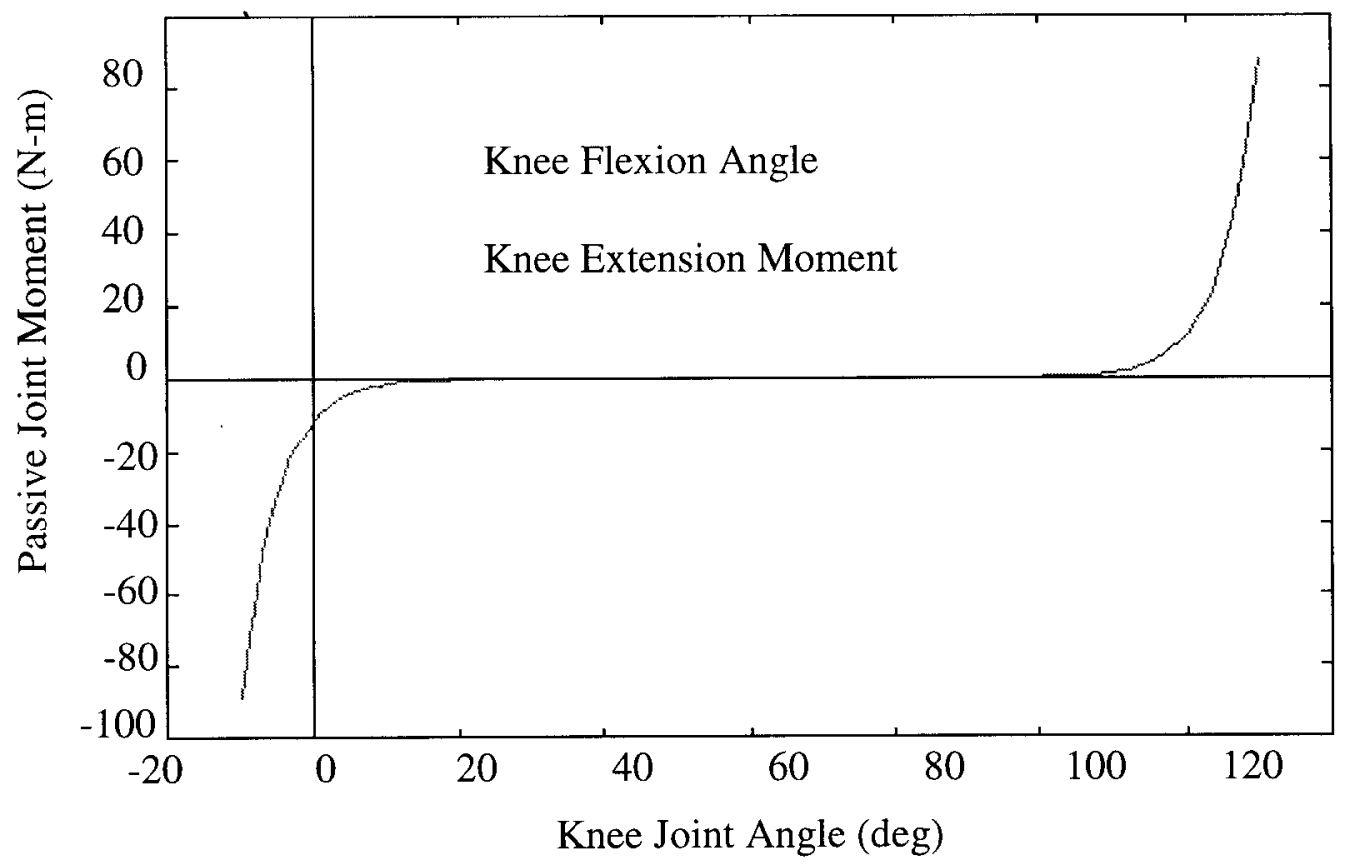

Figure 4.5. Passive knee flexion/extension moments. The output is a function of position. 


\title{
CHAPTER 5
}

\section{DATA COLLECTION AND ANALYSIS PROCEDURES}

\author{
Introduction
}

In the previous sections the inverse dynamics model and the EMG-driven model were developed. The sections that will follow aim in focusing on the evaluation of the accuracy of the measurement system that was used, and on the implementation and validation of the modeling approaches that were used. The procedures for collection of experimental data from normal subjects, the equipment used, and the analyses of the data acquired will also be described.

\section{$\underline{\text { Subjects }}$}

Three normal adult subjects whose age at the time of testing ranged between 24 and 34 years, participated in the present project. They were all required to demonstrate ability and adequate strength and co-ordination with their left lower extremity to operate an isokinetic dynamometer, KinCom. Furthermore, they all had range of motion at their left knee and ankle joints within the limits reported in the literature for normal adults during gait, i.e., from 60 degrees of knee flexion to full knee extension, and from 20 degrees of dorsi flexion to 30 degrees of plantar flexion, respectively (Kadaba et al., 1989; Ounpuu and Wyatt, 2000; Perry, 1992; Sutherland et al., 1994; Winter, 1987; Wu, 1995).

All subjects that were included in the study were free of injuries, free of orthopedic problems, like bone deformities, that could interfere with their ability to use the isokinetic dynamometer, or other disabilities, like blindness, that might interfere with their ability toambulate independently. In addition, all subjects were free of any drug treatment, at least six months prior to their selection in this investigation. In general, they were all free 
of any neurological or pathological disorders. Furthermore, because of concerns that have been reported about the effect of adipose tissue on the electromyogram (De Luca, 1997; Winter, 1997), the subjects that participated in the study were very athletically active individuals with minimum amount of observable adipose tissue in their lower extremities. Although the subjects involved in the study cannot be considered representatives of the entire population who are adult normals, they were believed to be typical examples of the respective population. Inclusion of the subjects in the study did not consider race or gender.

Furthermore, a pilot study on the different aspects of the data collection procedures indicated that data collection was likely to be a very lengthy process, in terms of time. Consequently, the subjects of this study were acquaintances of this investigator.

Informed consent procedures

The subjects of this study were initially informed about the investigation from this researcher. Additional information was provided when the subjects had questions about the experimental procedures and the nature of the study. They were all assured of confidentiality. They were also informed that they could withdraw from the study at any time. A consent form, approved by the human subjects committee at the Ohio State University (OSU), was constructed and presented to the subjects upon visiting the laboratory as proof of their willingness to participate in the study (see Appendix F).

\section{Instrumentation and physical arrangement}

\section{Gait Analysis Laboratory}

The layout of the testing environment of the Gait Analysis Laboratory at the OSU where the gait analysis was performed is presented in figure 5.1. The laboratory area included an anthropometric station for obtaining measures of the subjects' height, weight, and limb length. A $2 \mathrm{~m}$ free standing GPM (Martin type) anthropometer was used to measure the standing heights and the lower limb lengths of the subjects. The weight of each subject to the nearest pound was obtained with a scale (Health-o-meter scale, Continental Scale Corporation, Bridgeview, ILL) and then was converted to $\mathrm{kg}$. 


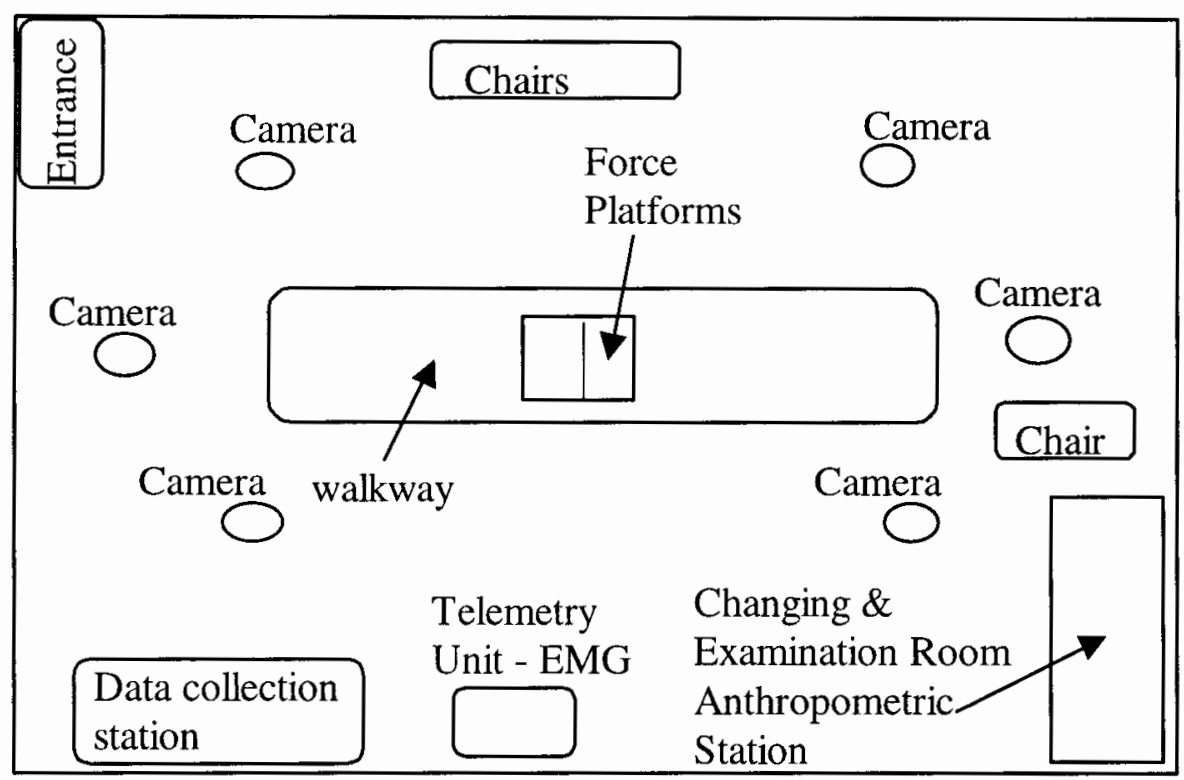

Figure 5.1. Laboratory layout showing the position of equipment.

Reflective self-adhesive body markers that can not harm the patient's skin were applied at the areas of interest. An approximately $15 \mathrm{~m}$ walkway, with two OR6-3 three dimensional force-torque dynamometers, force plates (Advanced Medical Technology, Inc. Newton, Massachusetts) set flush with the floor, positioned approximately $6 \mathrm{~m}$ from the starting line, were located in the middle of the laboratory area. Only one of the force plates was used in this study. The force plate used was connected on line with a datastation, part of the Oxford Metrics - 370 Vicon motion analysis system, which was, in turn, connected on line to a personal computer. The sampled data were changed to digital form by an-analog-to-digital (A/D) converter in the datastation and stored in the personal computer. The A/D board was a 12-bit 64 channel Oxford Metrics Limited card for the 370 Vicon System (Oxford Metrics Limited). The output was reading at a frequency of $1200 \mathrm{~Hz}$, and was recorded by six channels in millivolts for Fx, Fy, Fz forces, and Mx, My, Mz moments. The A/D process included the conversion of these values into engineering units of Newton, for force, and Newton-millimeters for moments. The conversion was accomplished by multiplying the raw force plate data (in millivolts) with a dynamometer-specific calibration matrix supplied by the manufacturer (see Figure 
5.2 and Figure 5.3 for the procedure). Equation 5.1 shows the calibration matrix that was implemented to convert the raw data collected by the force plate that was used for the purposes of this study, to their proper engineering units.

$$
\left[\begin{array}{l}
F x_{N} \\
F y_{N} \\
F z_{N} \\
M x_{N m m} \\
M y_{N m m} \\
M z_{N m m}
\end{array}\right]=\left[\begin{array}{rrr}
-3.043 & -0.018 & 0.013 \\
-0.001 & -3.092 & 0.000 \\
-0.127 & -0.121 & -11.743 \\
-1.560 & -0.007 & 0.002 \\
-0.006 & -1.571 & -0.005 \\
-0.006 & -0.002 & -0.779
\end{array}\right]\left[\begin{array}{l}
F x_{V} \\
F y_{V} \\
F z_{V} \\
M x_{V} \\
M y_{V} \\
M z_{V}
\end{array}\right]
$$

A black masking tape was taped to the floor at the beginning of the walkway to serve as a starting line. Six cameras using infrared light, part of the Oxford Metrics Limited -Vicon 370 motion analysis system- were positioned at six locations (Figure 5.1) to collect three dimensional motion data at $60 \mathrm{~Hz}$. A trained observer was positioned across the force plate of interest to determine whether there was a clean footfall in every trial, and to motivate, encourage, direct, and appraise the subject for his effort.

A sixteen channel telemetry unit (Telemyo, Noraxon USA, INC.) with $\mathrm{Ag} / \mathrm{AgCl}$ surface electrodes was used for the collection of electromyographic (EMG) data. The telemetry unit consisted of two transmitters, two receivers and two antennas (Yaggi) for ten channels each, of which, eight were dedicated to EMG signals. The recorded signal was first pre-amplified with a gain of 250 . Then it was passing through a high pass filter of $10 \mathrm{~Hz}(\mathrm{at}-3 \mathrm{~dB})$. Following the high pass filter the signal was amplified again with a gain of four and low pass filtered at $500 \mathrm{~Hz}$ (at $-3 \mathrm{~dB}$ ). Consequently, each channel's bandwidth was $10 \mathrm{~Hz}$ to $500 \mathrm{~Hz}$, operating at a radio frequency range of $904 \mathrm{MHz}$ to 926 $\mathrm{MHz}$. Each transmitter's lower cutoff filter was a first order highpass design, and the upper cutoff filter was a sixth order Butterworth lowpass design. The baseline noise was less than $1 \mathrm{uVolt}$ RMS. The common mode rejection ratio (CMRR) was a minimum of 85 $\mathrm{dB}$ throughout the operating range. The differential input impedance was 2 mega ohms $(\mathrm{M} \Omega)$, and the input bias current was \pm 2 nano-amperes $(\mathrm{nA})$. The transmitter's high level bipolar input voltage was $+/-2.5 \mathrm{~V}$. 


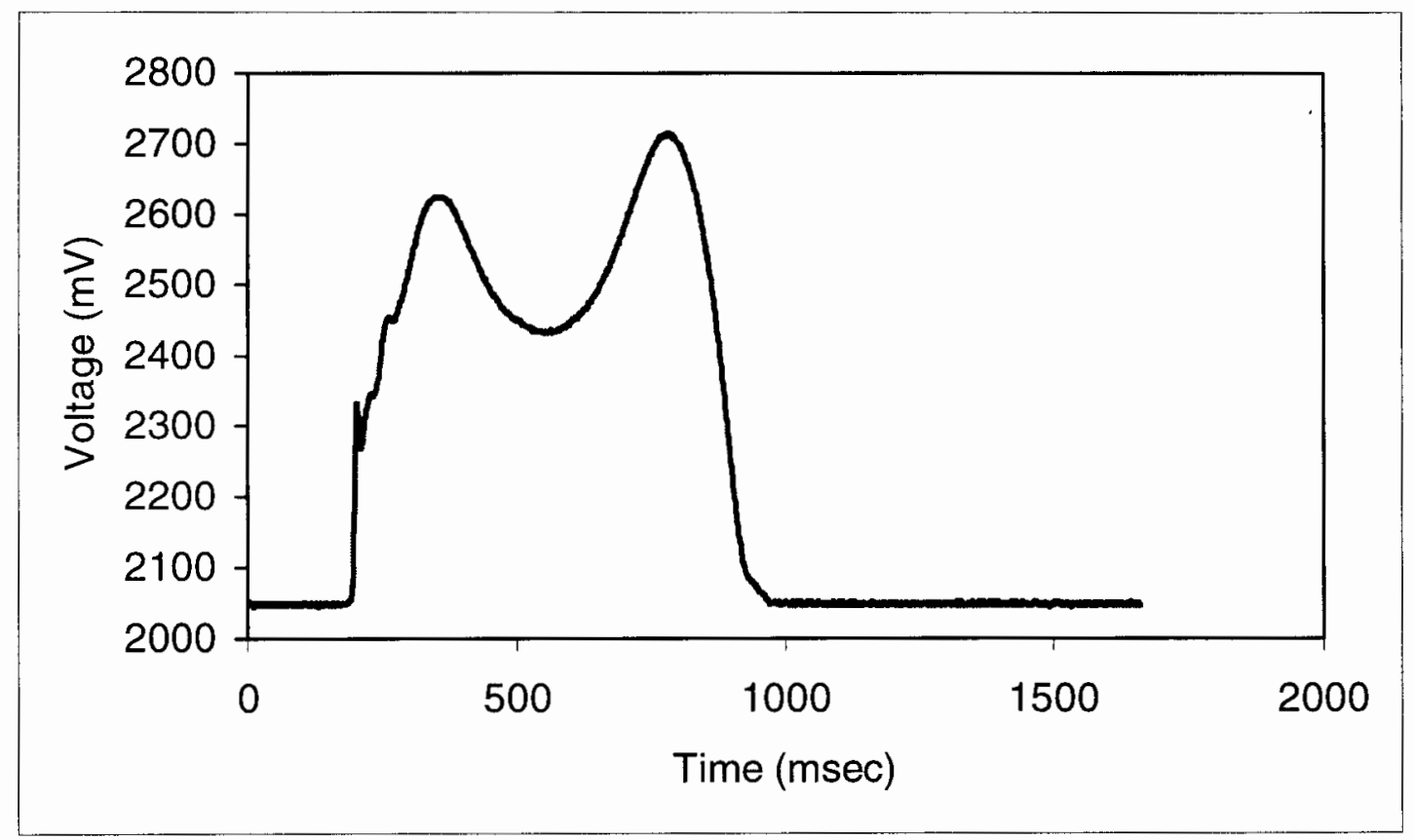

Figure 5.2. The raw (voltage) vertical component of the ground reaction force (Fz).

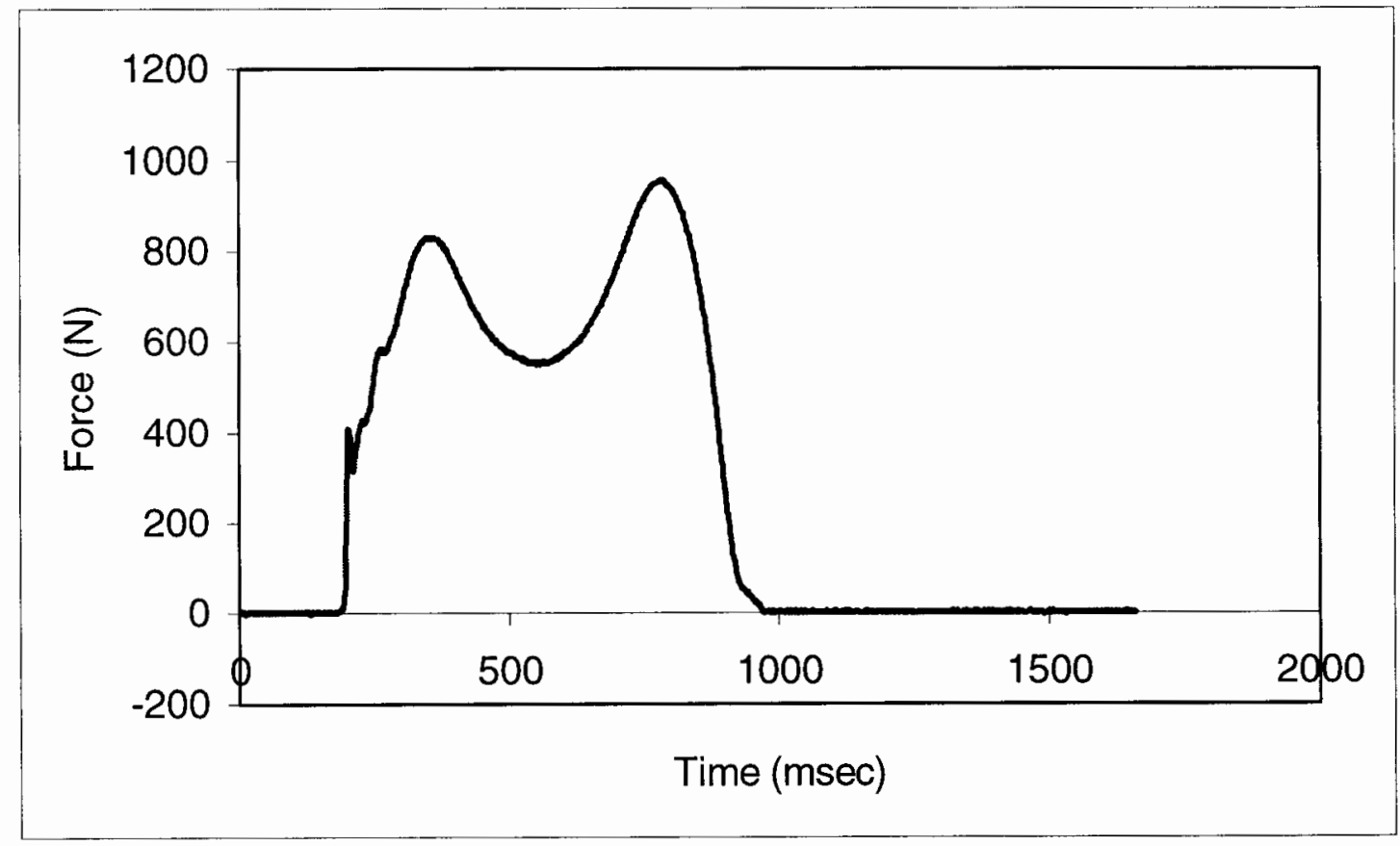

Figure 5.3. The converted, in engineering units of Newton, vertical component of the ground reaction force $(\mathrm{Fz})$-and corrected for the sign. 
The receiver's bipolar output voltage range was $+/-2.5 \mathrm{~V}$. At the receiver level the signal was amplified again with a gain of two. Consequently, the overall gain was 2,000 , fixed. The bandwidth of the receiver's amplifier was the same as that of the transmitter. Consequently, other than the amplification of the signal, it had no effect. The telemetry unit was positioned at the side of the walkway and outside the cameras' field of view. The antennas could, at all times "see" the transmitters. Surface, harmless, electrodes were positioned on the subject's skin to record muscular activity.

An additional component to the testing environment was a physical therapy room Neuropsychology building, Department of Rehabilitation Services, at OSU- with an isokinetic dynamometer, KinCom (KinCom, Chattex Corp., USA). Figure 5.4 shows the set up for the collection of the data using the isokinetic dynamometer. The positional and load analogue information was directed for concurrent storage with the EMG data to a personal computer where all information was $\mathrm{A} / \mathrm{D}$ converted using a 12-bit data acquisition Keithley megabyte card at 1000 samples per second, and stored for further processing. The A/D card accepted a bipolar voltage input range of $\pm 5 \mathrm{~V}$. However, the voltage output from the isokinetic dynamometer was $\pm 12 \mathrm{~V}$ for the positional output, and \pm 7 Volts for the force. Thus, a board was used to implement a voltage divider that reduced the maximum KinCom voltage output to less than five volts. The voltage dividers remained exactly the same for all experiments.

In addition, conversations with the manufacturers of the isokinetic dynamometer indicated that the nature of the experimental procedure would result in reversal of the load current directed to the $\mathrm{A} / \mathrm{D}$ card. The consequence of the reversal would be that current would leak back to the load cell. Such a leakage would result in uncontrollable jerky movements of the KinCom lever arm to which the subject's lower extremity would be attached for the purposes of collecting the data. Thus, leakage of the current back to the load cell would not only result in damaging the load cell itself, but it would also put the tested subject under physical danger. Consequently, a buffer was constructed by creating a unity-gain follower before the voltage dividers. To convert the load cell and lever arm (positional) voltages of the dynamometer to physical units, i.e., pounds and angles, in degrees, regression equations were derived (see Figure 5.5 and Figure 5.6). 


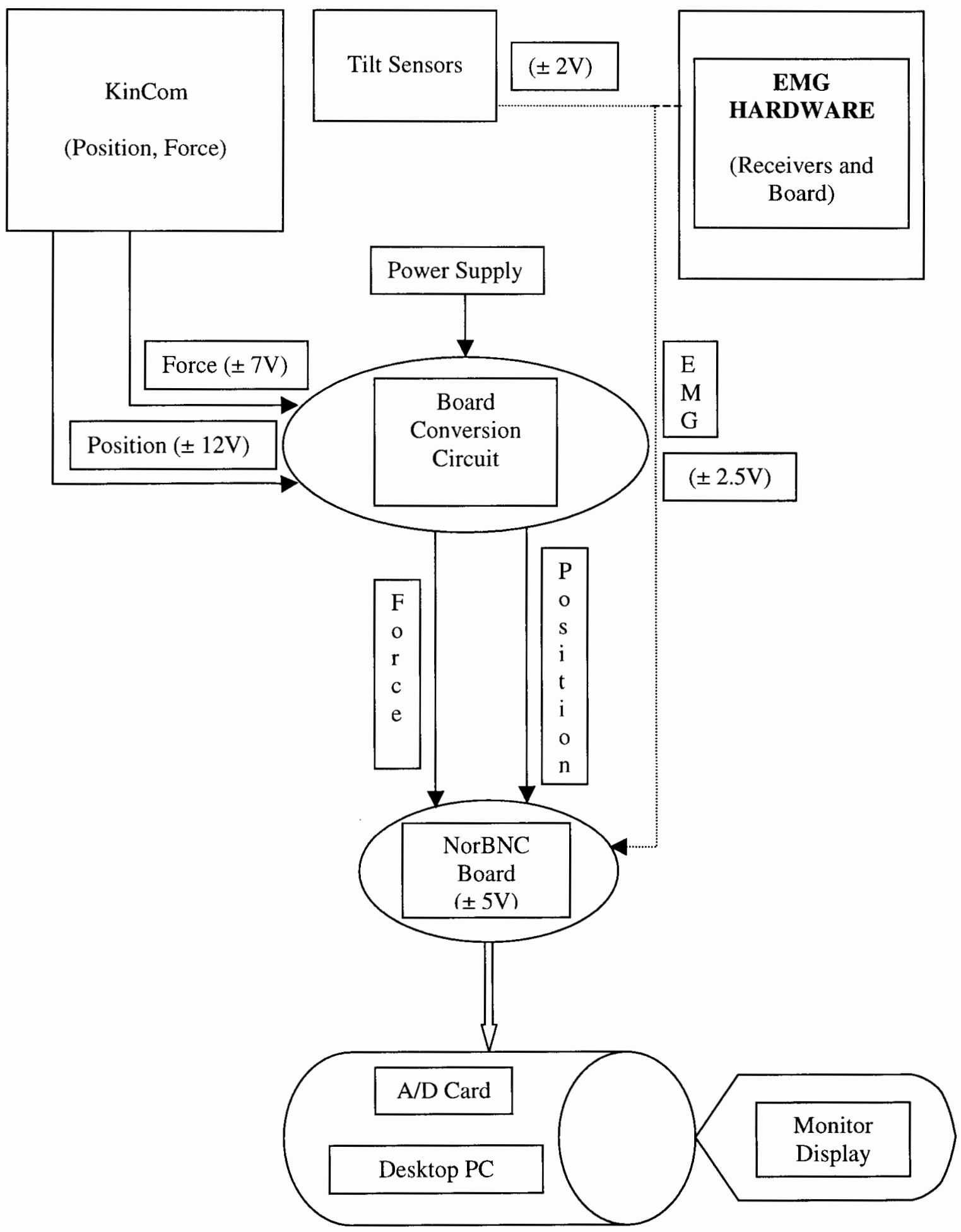

Figure 5.4. Diagram illustrating the hardware set up for the collection of data using the isokinetic dynamometer. 


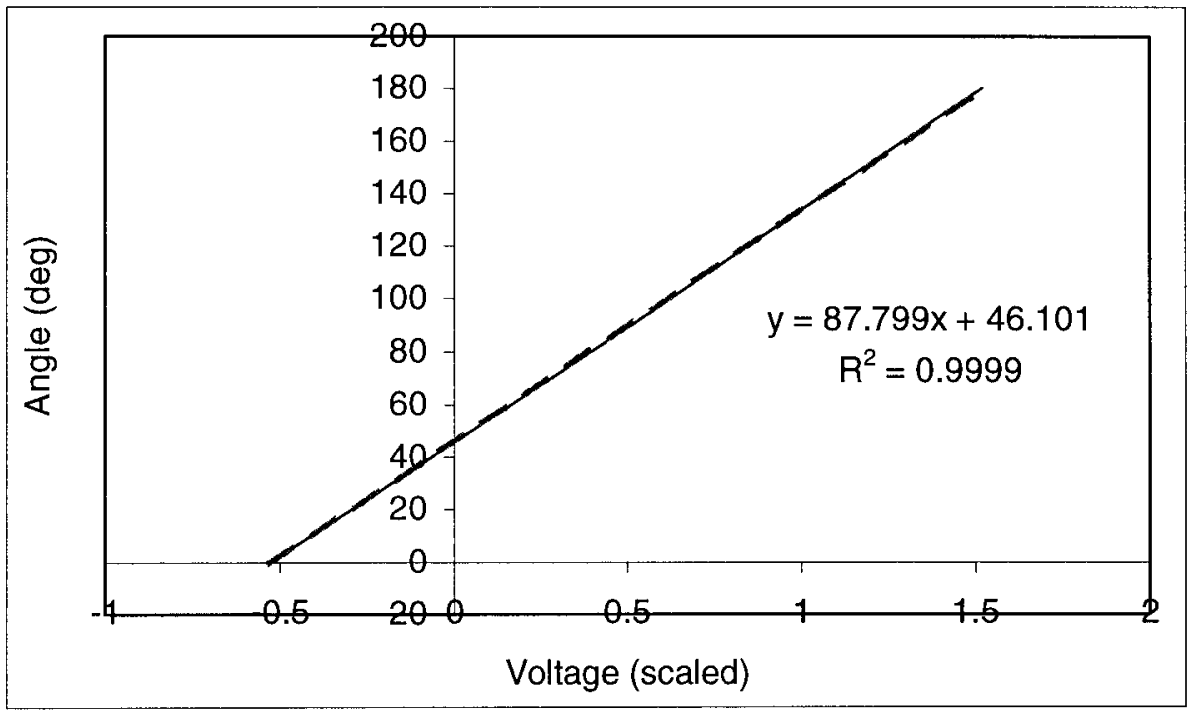

Figure 5.5. Regression equation to convert the KinCom voltage positional data to angles. In the graph zero angle represents the position where the KinCom lever arm is perpendicular and points to the ground.

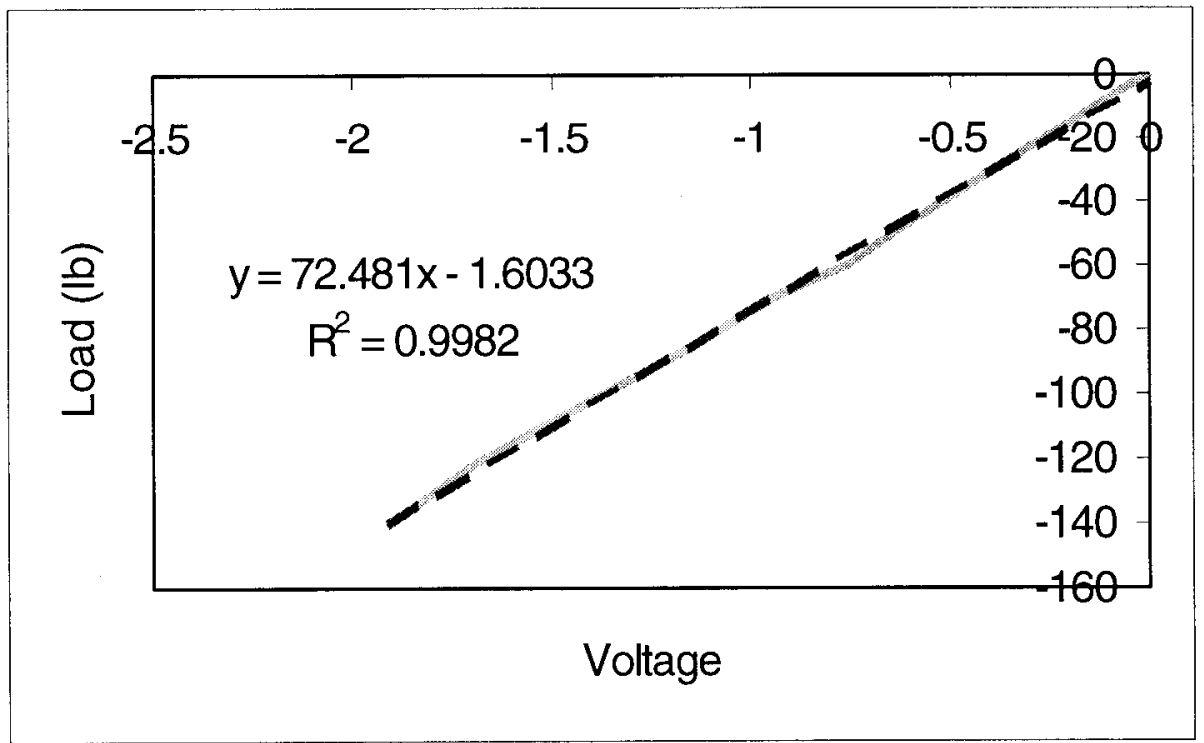

Figure 5.6. Regression equation to convert the KinCom load voltage data to pounds. 


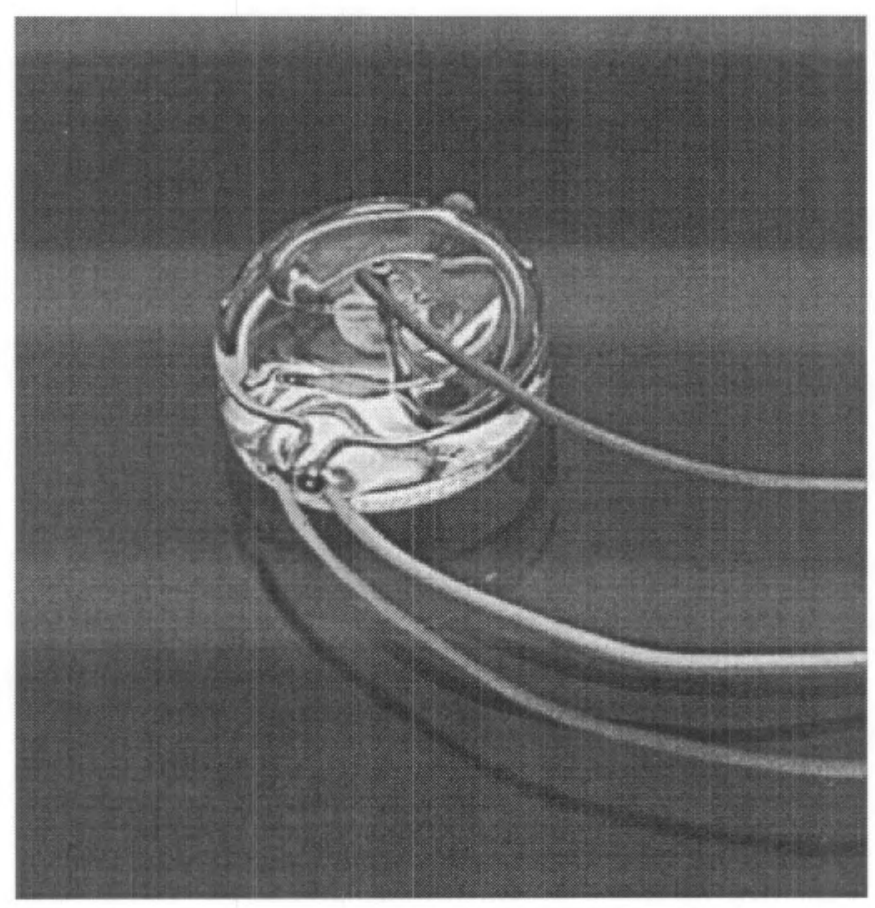

Figure 5.7. The tilt sensor.

Along with the isokinetic dynamometer two tilt sensors (S), inclinometers (Fredericks Company, Series 0725) were used to validate the KinCom positional input/output information. Each sensor consisted of two precision-molded glass halves, hermetically sealed together making a circular glass vial $(1.27 \mathrm{~cm}$ diameter) filled with partially conductive electrolyte liquid, and total mass of 2.8 grams (see Figure 5.7).

The liquid contacted three metal coplanar electrodes that were forming the legs of a Wheatstone bridge. When the sensor was level, an equal impedance existed to the common electrode, and a digital voltmeter would indicate a minimum or null, optimum. When the sensor was tilted the electrolyte was being redistributed submerging one of the outer electrodes more than the others. The electrolyte redistribution resulted in unbalanced impedance with respect to the common electrode, and, therefore, a different output voltage, when a signal conditioner was used (see below), as a function of the increasing or decreasing angle from the vertical. Furthermore, output included a phase that was indicative of the direction of tilt (see Appendix G, Figure G.3 and Figure G.4). 
A signal conditioner (SC) that was supplied by the manufacturer of the tilt sensor converted the sensor output into a signal that ranged 1.5 volts peak-to-peak. Thus, there was no compatibility issue with the Keithley megabyte A/D card. The signal conditioner incorporated a low-pass integrating filter that electronically dampened the output for noise in the 1 to $5 \mathrm{kHz}$ range. Prior to data collection the tilt sensor-signal conditioner pair that was used was calibrated (see Appendix G) and the linear response range of the conditioner-sensor was identified and used during data collection. In other words, during the data collection the tilt sensor was positioned such that the experimental range of motion would be encapsulated by the paired sensor's - signal conditioner's response. The output of the electrolytic-liquid tilt sensors has been previously validated (Parnianpour et al., 1997).

A conversion factor was established, in the form of a linear regression equation, i.e., a factor that represented accurately the change in voltage as a function of the change in angle, for the range of the linear response of the inclinometer - conditioner pair (see Figure 5.8 for the SC3-S8 pair, and Figure 5.9 for the SC8-S4 pair). The motivation behind the identification of such a conversion factor was the observation that, although the pattern of voltage response was similar for the manufacturer and OSU, the actual voltage values were different for the same angles (see Appendix G), i.e., although there is an implicit assumption of linearity, i.e., that the inclinometer produces voltage values that are linear to the angles measured, a zero millivolt measurement, however, may not correspond to a zero degree angle measured. A zero angle might correspond to several or several hundred millivolts.

After describing the involved hardware and its role in the data collection process with the isokinetic dynamometer, figure 5.4 that describes the hardware set up can be explained. The position and force analog signals from the KinCom first go to the board where the buffer and the voltage divider were implemented to assure that KinCom output was reduced to less than $\pm 5 \mathrm{~V}$ and was not leaking back to the KinCom due to current reversal. The converted signal then was directed to another board (NorBNC board) supplied by the EMG equipment manufacturer. Inputs to the board were all EMG signals and the output KinCom and tilt sensor signals. The tilt sensor and KinCom outputs were 


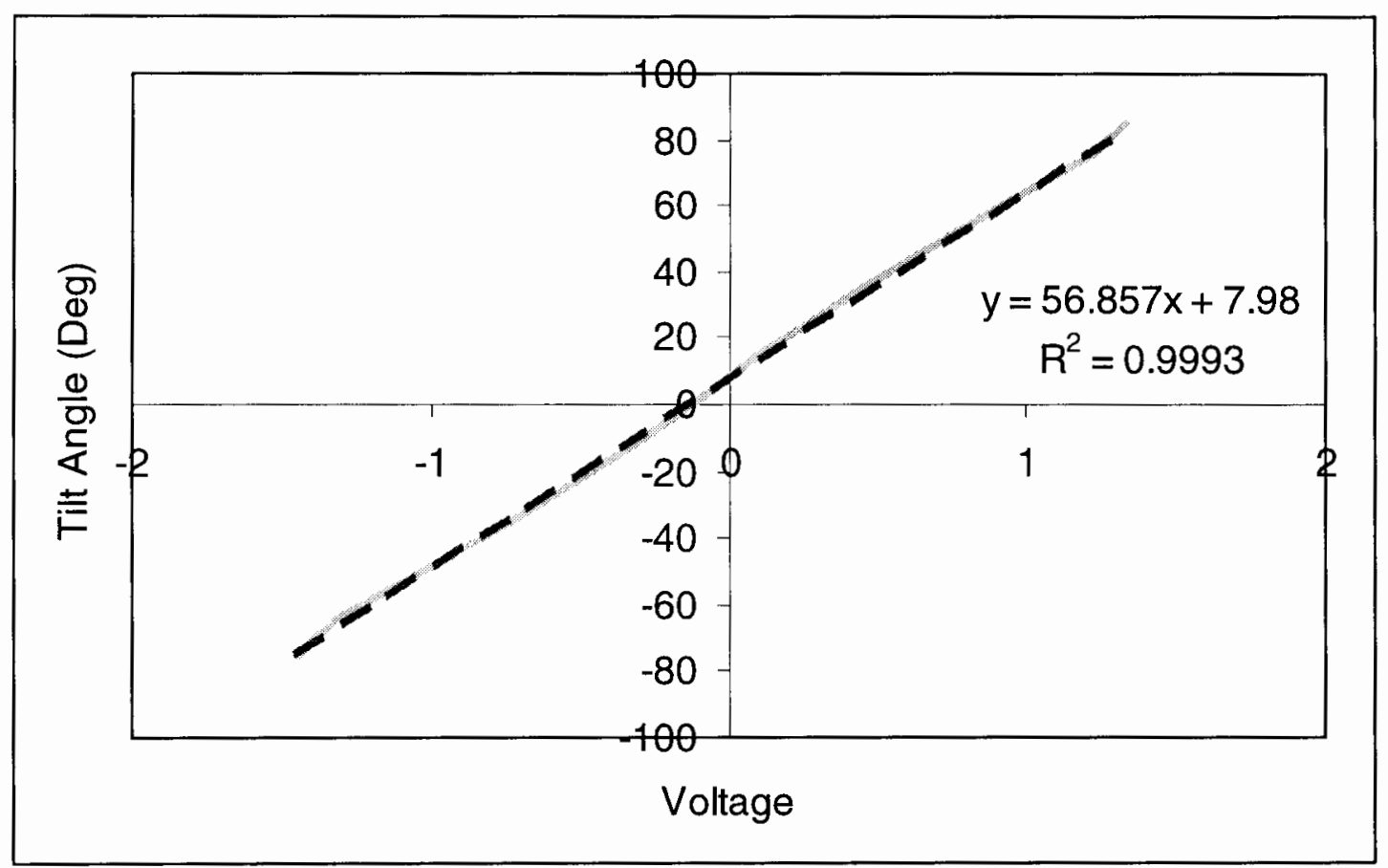

Figure 5.8. The linear portion of the signal conditioner (SC3) - tilt sensor pair (S8) voltage along with the regression equation that relates the response to the tilt angle.

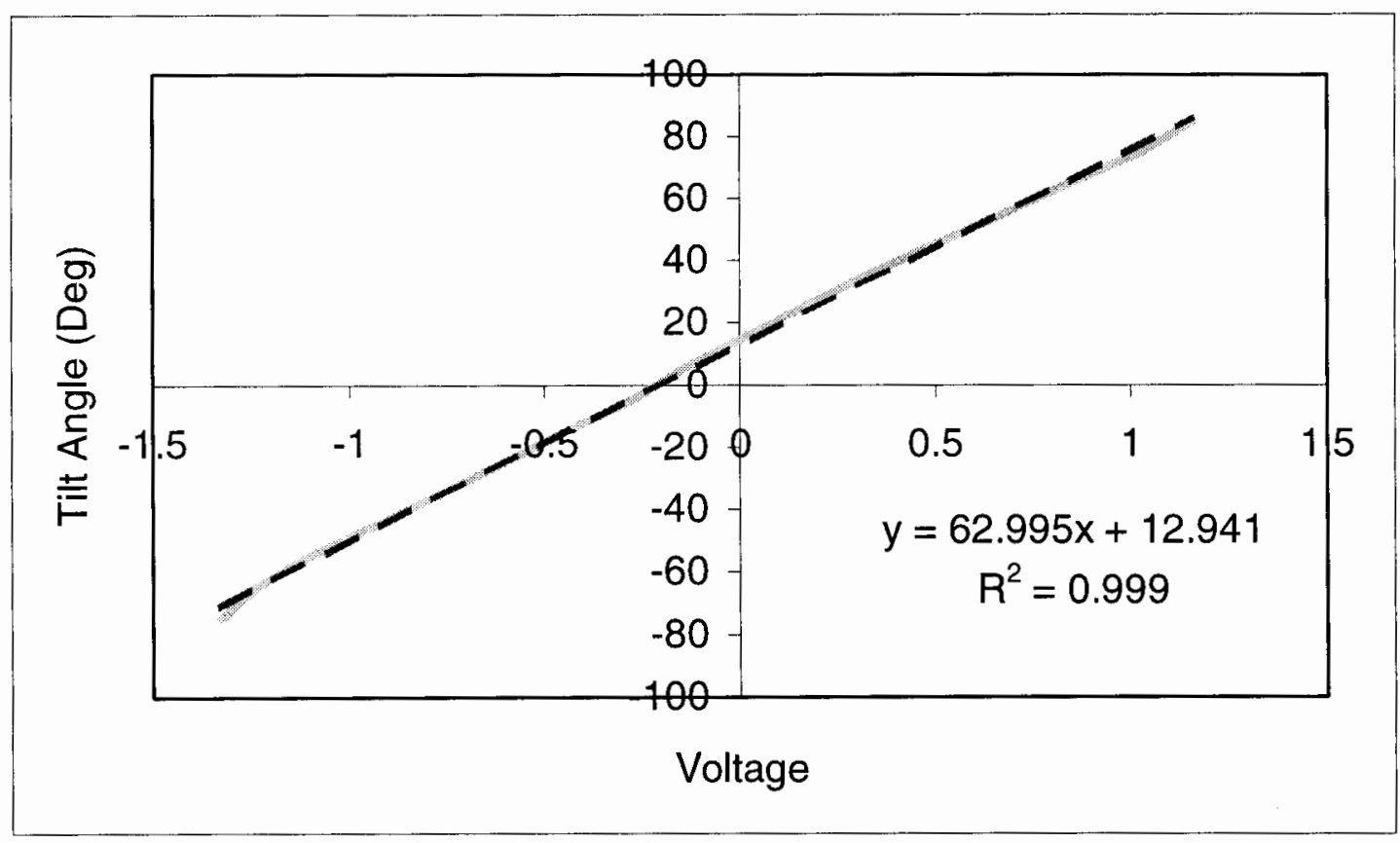

Figure 5.9. The linear portion of the signal conditioner (SC8) - tilt sensor pair (S4). 
external inputs to the board via its BNC connectors whereas the EMG signals were input via a 37-pin connector and an EMG-board interface cable. The NorBNC connector board was then connected with the A/D card via a 26-pin connector and interface cable. The A/D card was installed in a PC where all the raw data were stored for further processing.

\section{Procedures for Data Collection}

Subjects were tested at two sites of the Ohio State University campus in very close proximity. Gait evaluation took place at the Gait Analysis Laboratory of the University Hospitals. The calibration testing took place on the physical therapy site of the Department of Rehabilitation Services, at the OSU campus. Each subject visited the testing sites twice. However, since, just prior to the start of the data collection of this investigation, the gait analysis laboratory upgraded the motion analysis system, the accuracy of the upgraded motion analysis system needed to be tested.

\section{Three-dimensional Image Capturing Accuracy}

The accuracy of the motion analysis system was tested by assessing its ability to accurately reconstruct the three-dimensional location of two known markers in space at a fixed and known distance from each other. The known markers were placed at different locations within a calibrated volume. The calibration volume was constructed through a dynamic calibration procedure (Vicon, 1997). The calibration volume represented a rectangle that was approximately three meters in length, two meters wide, and two meters in height.

The two markers, at a fixed known distance from each other, were positioned at five different locations within the calibrated volume, such that it spanned its length and width. Data were collected for three seconds at 60 frames per second for each trial -location- of the two markers and the relative error between estimated distance and the known distance was computed.

The results from comparing the known and predicted distances can be seen in Table 5.1. The $X, Y, Z$ coordinates of each marker were represented as the respective means and standard deviations of all the frames collected. To estimate the potential over- 


\begin{tabular}{|c|c|c|c|c|c|}
\hline $\begin{array}{c}\text { Test } \\
\text { Trial }\end{array}$ & Markers & Mean & $\begin{array}{c}\text { Standard } \\
\text { Deviation }\end{array}$ & $\begin{array}{c}\text { Over-predicted } \\
\text { (in percent) }\end{array}$ & $\begin{array}{c}\text { Under-predicted } \\
\text { (in percent) }\end{array}$ \\
\hline & & & & Known =143.7 mm & Known = 143.7 mm \\
\hline 1 & Mkr 1 - 2 & 143.6 & 0.37 & 0.19 & -0.33 \\
\hline 2 & Mkr 1 - 2 & 142.3 & 0.26 & -0.79 & -1.16 \\
\hline 3 & Mkr 1 - 2 & 142.3 & 0.12 & -0.89 & -1.05 \\
\hline 4 & Mkr 1 - 2 & 142.1 & 0.073 & -1.06 & -1.16 \\
\hline 5 & Mkr 1 - 2 & 142.6 & 0.19 & -0.62 & -0.90 \\
\hline & & & & & -0.92 \\
\hline
\end{tabular}

Table 5.1

Performance of the motion analysis system. A known distance between two markers was compared with the mean distance plus/minus the standard deviation to assess the over- or under-prediction of the system respectively. Final results were presented in percent. Negative signs indicate under-prediction.

prediction of the system the predicted mean distance plus the respective standard deviation were normalized to the known distance between the two markers. To estimate the potential under-prediction of the system the predicted mean distance minus the respective standard deviation were normalized to the known distance between the two markers. The results were presented as percent.

It can be seen that the upgraded motion analysis system consistently underpredicted the known distance by as much as 0.9 percent, in average, which would correspond to $1.3 \mathrm{~mm}$. Such an error compares favorably with errors reported previously (Ayoub et al., 1970; Bergman, 1974; Cappozzo, 1983; Dapena et al., 1982; Pierrynowski, 1982; Shapiro, 1978; Van Gheluwe, 1974; Woltring, 1980). The last author reported reconstruction errors of 8 to $10 \mathrm{~mm}$ after filtering at $5 \mathrm{~Hz}$. Thus, from an initial evaluation the system appeared to be sufficient for the purposes of this investigation.

However, during movement, and especially during gait, the motion analysis system that was available for this investigation and its respective cameras could not collect three seconds worth of data for every gait event. Thus, the luxury of collecting 180 frames for every position of the lower extremity in space, which could, subsequently, be averaged to 
minimize the reconstruction error, was not available. Therefore, in order to obtain a more realistic performance of the system, sample frames were randomly selected for every trial and the distance between the markers was computed using equation 5.2. The results of reconstruction and the respective errors, for five trials are presented in Table 5.2.

$$
|M k r 1,2|=\sqrt{\sum(M k r 1-M k r 2)_{i}^{2}}
$$

where $\mid M k r 1,2) \mid$ represents the absolute distance between marker 1 and marker 2 and $i$ represents the direction.

It can be observed that, as it was observed before, the system was consistently under-predicting the distance between the two markers. The results show that underprediction can be expected to be less that 1.5 percent, i.e., less than $2.15 \mathrm{~mm}$. These values still compare favorably with previously reported results. Consequently, the upgraded system for the gait analysis laboratory was deemed to be appropriate for collecting the data for the purposes of this investigation. 


\begin{tabular}{|c|c|c|c|c|c|}
\hline $\begin{array}{l}\text { Trial }(\mathrm{T}) \\
\text { and } \\
\text { Video } \\
\text { Frame } \\
\end{array}$ & $\mathrm{X}$ & Y & $\mathrm{Z}$ & $\begin{array}{l}\text { Length Output } \\
\text { Known }=143.7 \\
(\mathrm{~mm})\end{array}$ & $\begin{array}{l}\text { Prediction Error } \\
\text { (percent) }\end{array}$ \\
\hline $\begin{array}{c}\text { T1 } \\
\text { Video \#34 } \\
\end{array}$ & & & & & \\
\hline Marker 1 & 987.094 & -506.625 & 79.406 & \multirow{2}{*}{143.55} & \multirow{2}{*}{-0.102} \\
\hline Marker 2 & 1109.719 & -474.188 & 12.188 & & \\
\hline $\begin{array}{c}\text { T2 } \\
\text { Video \#23 }\end{array}$ & & & & & \\
\hline Marker 1 & -405.563 & 90.844 & 76.406 & \multirow{2}{*}{142.00} & \multirow{2}{*}{-1.180} \\
\hline Marker 2 & -487.781 & -4.219 & 10.313 & & \\
\hline $\begin{array}{c}\text { T3 } \\
\text { Video \#14 } \\
\end{array}$ & & & & & \\
\hline Marker 1 & 68.719 & 69.375 & 78 & \multirow{2}{*}{142.25} & \multirow{2}{*}{-1.00} \\
\hline Marker 2 & -7.031 & -30.656 & 10.969 & & \\
\hline $\begin{array}{c}\mathrm{T} 4 \\
\text { Video \#41 } \\
\end{array}$ & & & & & \\
\hline Marker 1 & 1044.75 & 223.031 & 97.969 & \multirow{2}{*}{142.15} & \multirow{2}{*}{-1.072} \\
\hline Marker 2 & 1030.969 & 336.469 & 13.406 & & \\
\hline $\begin{array}{c}\text { T5 } \\
\text { Video \#52 } \\
\end{array}$ & & & & & \\
\hline Marker 1 & -366.281 & 390.656 & 78.469 & \multirow{2}{*}{142.47} & \multirow{2}{*}{-0.853} \\
\hline Marker 2 & -488.531 & 363.563 & 10.5 & & \\
\hline
\end{tabular}

Table 5.2.

Performance of the motion analysis system. A known distance between two markers was compared with the predicted distance to assess the prediction error of the system. Final results were presented in percent. Negative signs indicate under-prediction. 


\section{First Visit}

During the first visit, the subjects familiarized themselves with the Gait Analysis Laboratory layout by walking around the laboratory area while they were given a basic orientation. During the orientation, the subjects were given specific information about the nature and location of the testing equipment as well as the testing procedures. At the end of the orientation session at the Gait Analysis Laboratory some anthropometric data were collected (see Appendix $\mathrm{H}$ for the specific "Anthropometric Information Form"). Each subject's height lower limb length, knee and ankle widths were measured using the techniques described by Gordon, Chumlea Cameron, and Roche (1988), which is a standardized procedure for the purposes of gait analysis. Each subject's weight was also measured so that the ground reaction force (GRF), measured during the gait evaluation, could be expressed relative to the subject's body weight (BW). In addition, because in the recent past concerns have been expressed about the effect of adipose tissue on the electromyogram (De Luca, 1997; Winter, 1997), a basic skinfold measurement was taken for each subject to estimate their adipose tissue content using the Accu-Measure, body fat tester (Accu-Measure, Inc.).

Then the subjects walked through the tunnel to the adjacent testing site at the physical therapy room of the Department of Rehabilitation Services. An introductory orientation took place there too. During the orientation, the subjects were given specific information about the KinCom isokinetic dynamometer as well as the testing procedures. The subjects were also asked to sit on the KinCom seat for some practice trials with the left leg. The practice trials on the KinCom allowed the subjects to familiarize themselves with, understand and consolidate the concepts of concentric and eccentric contractions under isokinetic conditions. Furthermore, the practice trials provided an opportunity for the investigator to assess each subject's ability and coordination required operating the isokinetic dynamometer under different testing conditions. The preliminary testing involved isokinetic contractions at velocities similar to those used during the experimental data collection for each of the joints of interest and for some of the positions of interest. All practice sessions started with a few trials of passive warm up where the subject's segment was moved by the lever arm of the dynamometer with 
constant velocity without the need of any voluntary contraction on the part of the subject. Then, a few low velocity submaximal contractions were performed followed by maximum voluntary contractions (MVC).

\section{$\underline{\text { Second Visit }}$}

\section{Gait evaluation}

The second visit began with a gait evaluation at the Gait Analysis Laboratory of the OSU University Hospitals. The force plates and a volume within which the movement was captured were calibrated before each subject came to the laboratory. Each subject, upon arrival to the laboratory, changed to running shorts so that leg movements were not constrained and the entire lower leg could be visible. In addition, a light sleeveless top was worn so that arm swings were not limited. No shoes were used during the evaluation.

Initially, a set of calibration data was collected using two targeting protocols. The first was the standard protocol used in the gait analysis laboratory at OSU, and needed for processing using the Vicon Clinical Manager (VCM) gait analysis software (Vicon, 1998). The second protocol was the one decided as appropriate for the current and future purposes of this study. Consequently, prior to collecting data with the subject walking along the walkway, the subject was placed spherical retroreflective markers according to the OSU/VCM targeting protocol.

A calibration trial was performed initially. Markers for the calibration trial were placed as follows: a) a sacral stick target on the sacrum; b) bilateral markers on the anterior superior iliac spine (ASIS); c) bilateral markers on the lateral thighs using lateral femoral stick target; d) bilateral markers on the lateral shanks using lateral tibia stick targets; e) bilateral markers on the lateral malleoli; f) bilateral markers on the calcanei; and g) bilateral markers over the first metatarsal heads. For the collection of the first set of data during the calibration procedure, in addition to the markers identified in the previous sentence, a knee alignment device (KAD) was used bilaterally at the knee joint (provided by the manufacturer of the motion analysis system) to correct for offsets at the joints. The KAD was used according to the recommendations of the manufacturer 
(Vicon, 1997). Once the markers were positioned over the desired locations, one second set of image data were collected at $60 \mathrm{~Hz}$ with the subject standing in the anatomical posture at the center of the object field. The KAD, then was replaced by bilateral markers on the knee, placed just below the lateral femoral epicondyles, and image data were collected for another static trial to be processed using the VCM. The image capture that followed immediately the trial with the use of the KAD, completed the first part of the calibration procedure. The purpose of the first part of the calibration procedure was to scale the lower extremity model provided by Delp (1990) via the VCM translator, an add on module to the SIMM (Musculographics, Inc.) software.

The second part of the calibration procedure involved another standing calibration trial with the walking and calibration targeting protocols for the purposes of this study. After the first part of the calibration was completed, retroreflective markers were placed on specific anatomical landmarks to define the body segments and their parameters (for the theoretical development see Inverse Dynamics Model Development), as well as to track the segments during ambulation. The foot was defined by the first metatarsal head (L1M), the fifth metatarsal head (L5M), the lateral maleolus (LLML), and the medial maleolus (LMML). The lower leg, shank, was defined by the LLML, the LMML, the lateral tibial epicondyle (LLTE), and the medial tibial epicondyle (LMTE). The thigh was defined by the lateral femoral epicondyle (LLFE), the medial femoral epicondyle (LMFE), and the greater trochanter (LGRT). The pelvis was defined by the LGRT, the left anterior superior iliac spine (LAS), and the right superior anterior iliac spine (RAS) (see Figure 5.10).

The same marker set used to define body segments was used to create the segment coordinate systems (SCS). A different marker set was used, however, to track each body segment. Which anatomical landmarks are most desirable for the placement of markers over them, is a subject of considerable debate for the past two decades. The purpose is to target landmarks, which will allow implementation of the assumption that body segments can be modeled as rigid bodies. Thus, the position and motion of the underlying skeleton can be approximated by tracking the position and motion of the surface tissue. However, such an approach would necessitate the minimizing of the soft tissue motion error. 


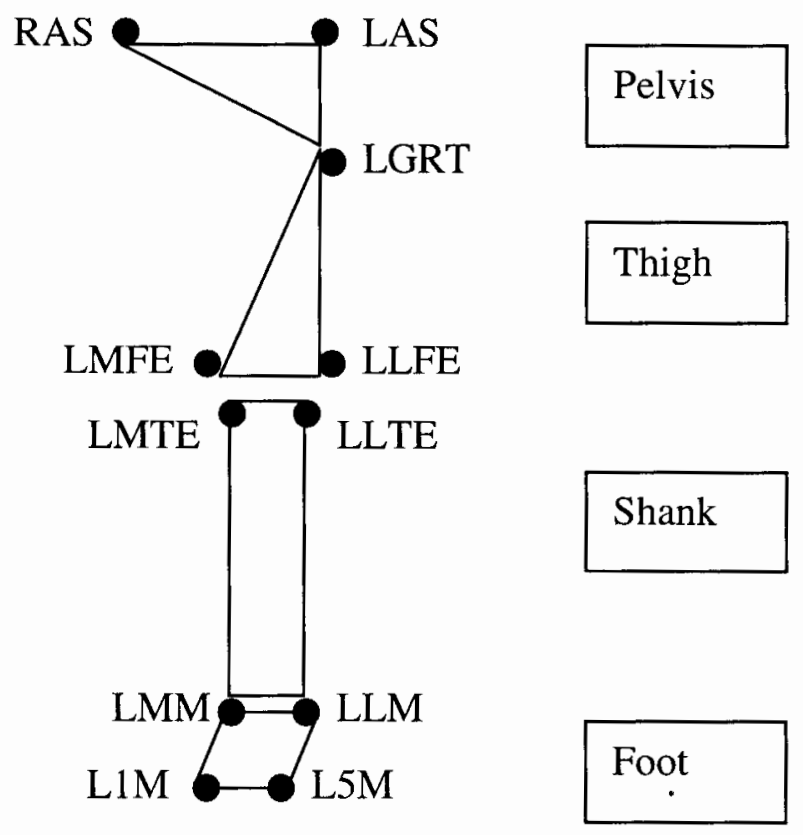

Figure 5.10. Marker placement to define the body segments. Markers were placed on the first metatarsal head (L1M), the fifth metatarsal head (L5M), the lateral maleolus (LLML), the medial maleolus (LMML), ), the lateral tibial epicondyle (LLTE), the medial tibial epicondyle (LMTE), the lateral femoral epicondyle (LLFE), the medial femoral epicondyle (LMFE), the greater trochanter (LGRT), the left anterior superior iliac spine (LAS), and the right anterior superior iliac spine (RAS).

To the best of my knowledge studies that have investigated the amount of error as a function of soft tissue movement, are very scarce. They have involved invasive techniques of putting pins in the bones and attaching markers to those pins. Then pin marker movement was compared with that of surface markers (Holden et al., 1994). Therefore, it is important to place markers at points where soft tissue movement is minimum. Some researchers suggest that targeting bony landmarks, where, incidentally, there is the least amount of intervening soft tissue between the marker and the bony landmark, should be avoided because those are the locations that introduce most soft tissue motion error (Andriacchi, 1999). Others, however, advocate the use of bony landmarks (Kadaba et al., 1989; Kadaba, 1990). For the purposes of the present investigation, there were certain 
criteria that were identified by this investigator for selecting each specific marker set to track the body segments:

a) selection of the anatomical landmarks used to track each body segment was a function of the ability of them being detected at any time by at least two cameras during the gait cycle;

b) furthermore, the markers used to track the body segments should introduce as little noise as possible due to motion artifacts;

c) two of the landmarks per segment could create an anatomical reference axis, such that when the person was standing, the anatomical axis could align with one of the axes of the global coordinate system (GCS);

d) three of the landmarks used per segment could create an anatomical plane;

e) there was relatively little intervening tissue between the marker placed on the skin and the bony landmark of interest under the skin; and

f) to comply with the proposed guidelines by the European motion analysis groups for standardization of motion analysis, i.e., computer aided movement analysis in a rehabilitation context (CAMARC) (Cappozzo et al., 1995).

To satisfy criterion (b) above, prior to finalizing the targeting protocol for tracking the position of the lower extremity in space, the implementation of a lateral femoral stick marker (LLFS), recommended by the personnel of the motion analysis system used in the Gait Analysis Laboratory at Ohio State University (Vicon, Oxford Metrics, Limited), as one of the markers for tracking the position of the thigh in space, was investigated against using a marker over the LGRT. Both markers were used simultaneously on a healthy normal subject along with the rest of the marker set that was thought to be effective for the purposes of this study. Three walking trials were collected from that subject. The positional data of each marker were processed to determine, initially, the position of the thigh in space, and, ultimately, the effect of each marker in determining the hip and knee joint angles.

The effect of using each marker on the respective output joint angles can be seen in Figure 5.11 through Figure 5.13 for each one of the trials. With either LGRT or LLFS markers, the knee and hip output joint angles were within the ranges previously reported 
for normal adults (Kadaba et al., 1989; Moran, 1994; Ounpuu and Wyatt, 2000; Winter, 1987; Wu, 1995). However, use of the one marker versus the other, produced slightly different results. When the LGRT marker was used, the hip joint angle was, in general, a little less than the one determined with the use of the LLFS. The biggest difference between the two marker approaches was seven degrees. When the knee joint angle is considered, the biggest difference between the two approaches was five degrees in favor of the LLFS during maximum knee flexion in stance, i.e., the maximum knee flexion angle during the stance phase of the gait cycle was greater when the LLFS marker was used. During the swing phase of the gait cycle, the maximum knee flexion angle was greater when the LGRT marker was used.

For the purposes of this investigation, the observation that was important, was that when the LGRT marker was used the trajectory of the joint angle determined was smoother than when the LLFS marker was used. The implication of this finding is of significance when angular velocities and accelerations are considered for the purposes of determining the net joint moments from inverse dynamics analysis. The noisier the positional output, the noisier would be the estimated velocities and acceleration. Thus, the error introduced in the estimation of net joint moments would be greater. Consequently, to justify the criteria set forth earlier, inclusion in the marker set of a target over the LGRT as one of the markers to track the position of the thigh in space, was believed to be a better choice, for the purposes of this investigation.

Thus, the protocol used for tracking the position of the foot included the placement of a marker on the calcaneous (LCAL), a marker on L5M, and a marker on L1M. The position of the lower leg (shank) in space was tracked by a marker on the tibial tuberosity (LTT), a marker on the anterior shank (LASH), half way between the length of the tibia, and a marker on the posterior shank (LPSH), in line with LASH. Two additional markers were used for determining the position of the shank, the LLML and LMML. The position of the thigh was defined by a marker on LGRT, a marker on LLFE, and a marker on LMFE. The pelvic orientation in space was determined by a placing a marker on RAS, a marker on LAS, and a marker on the basis of the sacrum (SACS) over the L5/S1 joint. The marker set used can be seen in Figure 5.14. 

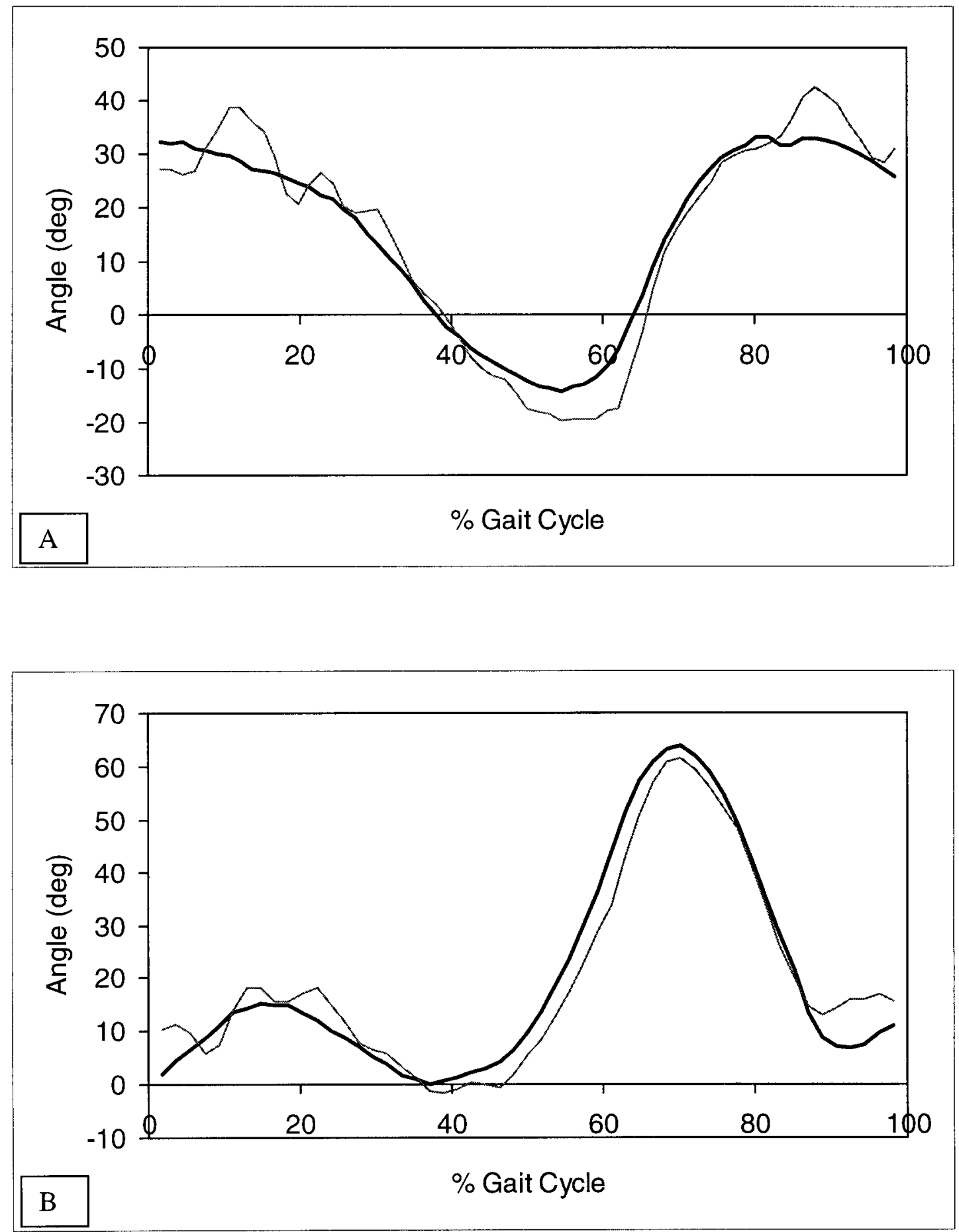

Figure 5.11. Joint angles of the hip (A) and of the knee (B) joints during a gait cycle of the first walking trial. The thick line represents the use of a marker at the greater trochanter landmark to estimate the specific joint angle. The thin line represents the use of a marker on the lateral femur, the lateral femoral stick, to determine the specific joint angle. Positive values indicate joint flexion. Negative values indicate joint extension. 

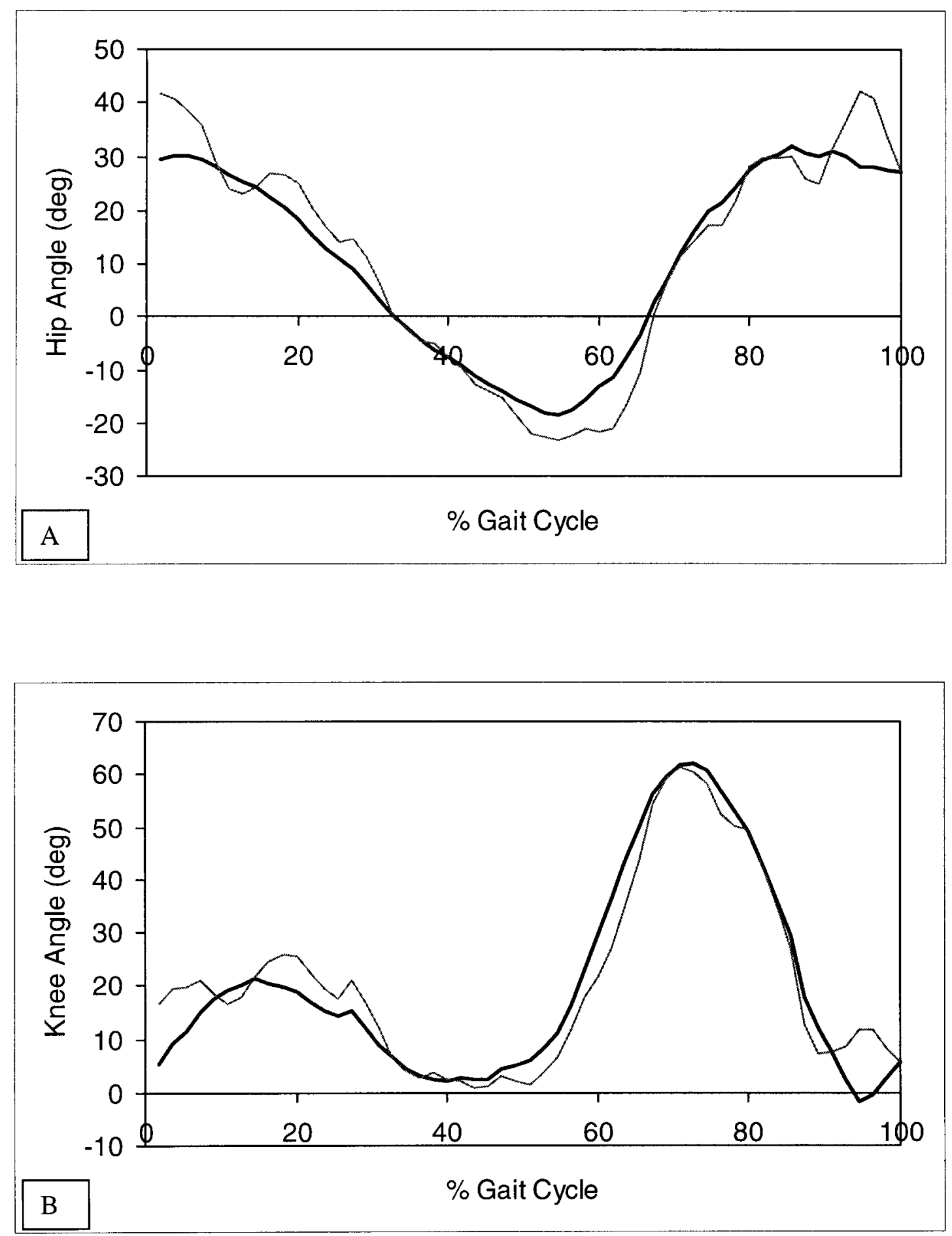

Figure 5.12. Joint angles of the hip (A) and of the knee (B) joints during a gait cycle of the second walking trial. The thick line represents the use of a marker at the greater trochanter landmark to estimate the specific joint angle. The thin line represents the use of a marker on the lateral femur, the lateral femoral stick, to determine the specific joint angle. Positive values indicate joint flexion. Negative values indicate joint extension. 

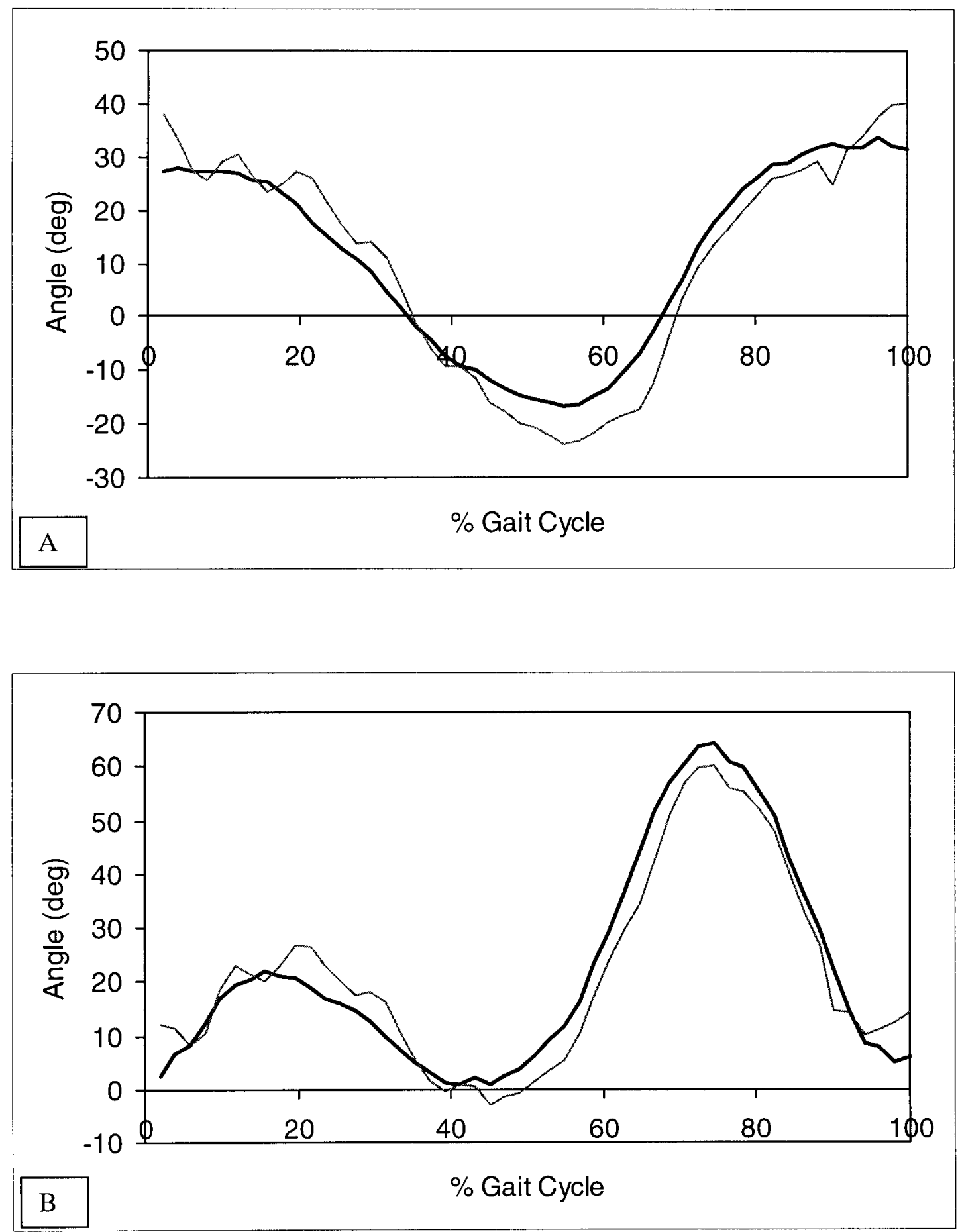

Figure 5.13. Joint angles of the hip (A) and of the knee (B) joints during a gait cycle of the third walking trial. The thick line represents the use of a marker at the greater trochanter landmark to estimate the specific joint angle. The thin line represents the use of a marker on the lateral femur, the lateral femoral stick, to determine the specific joint angle. Positive values indicate joint flexion. Negative values indicate joint extension. 


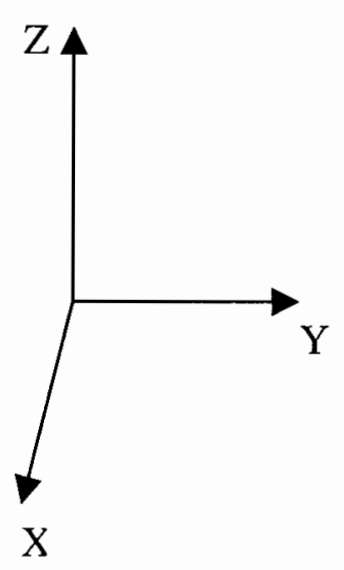

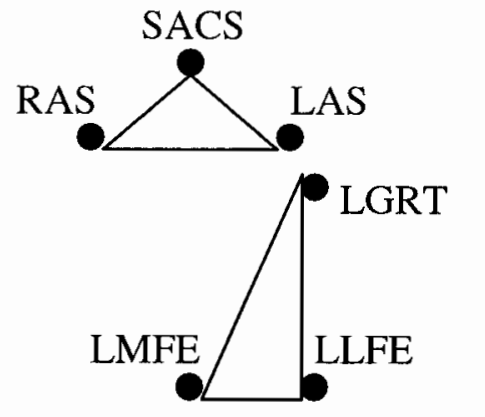
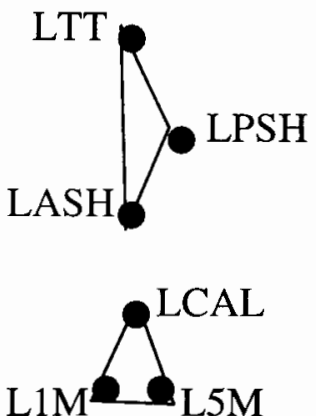

Pelvis

Thigh

Lower

Leg

Figure 5.14. Targeting protocol for the purposes of tracking the body segments in space.

For the purposes of analyzing the motion data, the fixed laboratory coordinate system needed to be rotated also. In the gait lab at OSU the laboratory system orientation has the $\mathrm{X}$ axis representing the anterior-posterior direction, the $\mathrm{Y}$ representing the medial-lateral direction, and the $\mathrm{Z}$ representing the vertical direction (see Figure 5.15). For the purpose of analyzing the data of the present investigation, however, the anteriorposterior direction was represented by the $\mathrm{Y}$ axis, the medial-lateral direction was represented by the $\mathrm{X}$ axis, and representation of the vertical direction did not change (see Figure 5.16). To rotate the laboratory coordinates a dynamic calibration structure was used (Vicon, 1997). The rotation performed to the laboratory was equal to the following rotation matrix $[\mathrm{R}]$ :

$$
[R]=\left[\begin{array}{rrr}
0 & -1 & 0 \\
1 & 0 & 0 \\
0 & 0 & 1
\end{array}\right]
$$




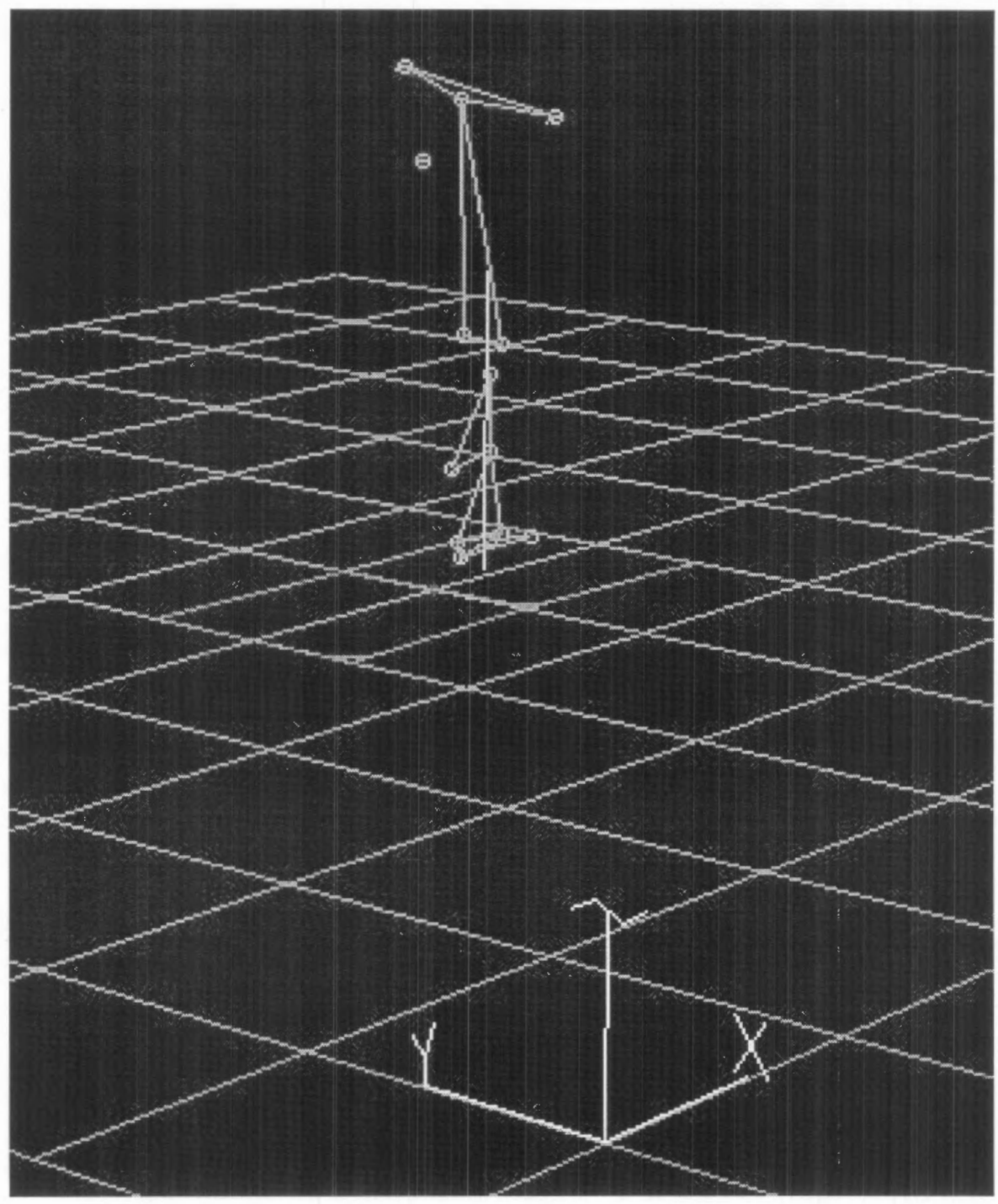

Figure 5.15. The original orientation of the laboratory coordinates. The $\mathrm{X}$ represents the anterior-posterior, $\mathrm{Y}$ represents the mediolateral direction, and $\mathrm{Z}$ represents the vertical direction. 


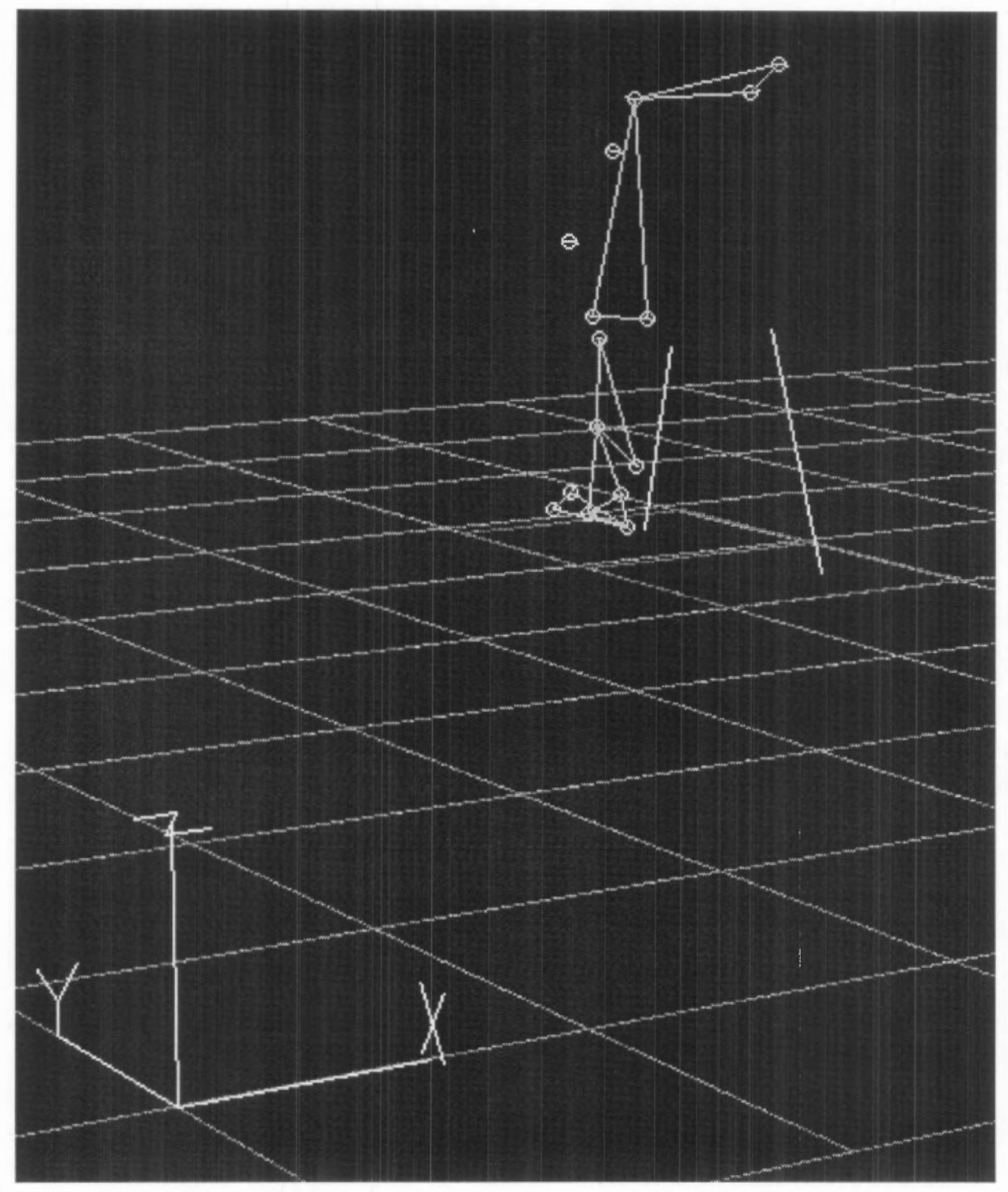

Figure 5.16. The orientation of the laboratory coordinates after rotation, such that $\mathrm{X}$ represents the mediolateral direction, $\mathrm{Y}$ represents the anterior-posterior direction, and $\mathrm{Z}$ represents the vertical direction. 
Consequently, once the first part of the calibration procedure was completed, and the laboratory coordinates were rotated, the combined protocols for: (a) the definition of the body segments of the lower extremity and their parameters, and (b) the tracking of the body segments in space, were used for the second calibration. The subject was asked to stand still with the feet parallel and in the direction of the anterior axis of the laboratory. From the calibration trial: (a) the body segments of interest were defined, and (b) the spatial relationship of the markers used to define the lower extremity body segments with the markers used to track the position of the respective segments in space was established.

Following the calibration, only the marker set used for tracking the body segments of interest in space was left on the left lower extremity. In addition, bipolar surface electrodes $(4 \mathrm{~mm})$ using a silver-silver chloride electrolyte system (Blue Sensor, Medicotest Marketing Inc.) spaced approximately 1-2 cm center-to-center were used to monitor simultaneously twelve muscles of interest. One of the advantages of the electrodes used was that, according to the manufacturer, they could stay on a subject's body without a degradation of the electrolyte solution for 48 hours. Thus, they were thought to be appropriate for the purposes of this study.

The placement of the electrodes with respect to the orientation of the muscle fibers has been a topic of debate for the past two decades. Some researchers advocate the orientation of the electrodes to be parallel to the direction of the muscle's fibers (Soderberg, 1992; White and Winter, 1993). Since the muscle fibers are oriented parallel to each other forming fluid filled channels that posses lower impedance, a parallel orientation of the recording electrodes with respect to the muscle fibers would allow for a recording with reduced impedance (Acierno et al., 1998). However, the issue of impedance is further complicated due to the skin and adipose tissues (Acierno et al., 1998; De Luca, 1997; Winter, 1997).

Other researchers advocate a recording orientation perpendicular to the orientation of the muscle fibers (De Luca, 1997; Hof and Berg, 1977). It has been shown that the amplitude of the motor unit action potentials is a function of the conduction velocity of the potentials (De Luca, 1997). The electromyographic signal amplitude, in turn, is 
proportional to the amplitude of the motor unit action potentials. Thus, if the interdetection-surface spacing, i.e., the length of the fibers that traverse the distance between the detection surfaces, was fixed, then the detected conduction velocity, encapsulated in the amplitude of the electromyogram, would be a function of the cosine of the angle between the fibers and the detection surfaces. Consequently, a recording orientation perpendicular to the orientation of the muscle fibers would seem to allow for the capturing of the maximum electromyographic amplitude possible.

Furthermore, in dynamic situations where the quantitative relation of the detected signal to the measured moments is desired, the stationarity of the signal is of prime importance. Thus, there is the need for placing the electrodes in such a manner that the detection surface of the signal follows the movement of the muscle fibers (De Luca, 1997). It is an extremely difficult task for anyone to predict the movement of the muscle fibers underneath the skin in dynamic situations, especially when impact is involve, such as in gait. Thus, on the basis of the information that has been offered above it was believed that, for the purposes of this study and given the spatial limitations involved in placing the electrodes over the musculotendon units of interest, the electrodes were placed as perpendicular as possible to the muscle fibers. Such an effort resulted in an actual placement of the electrodes at an angle to the orientation of the fibers and in an orientation that would be reasonable to assume that the muscle fibers would be more likely to move during the movement while still avoiding cross-talk from adjacent muscles. Partly, the muscle testing procedure at the time of placing the electrodes over the musculotendon units of interest was believed to have helped.

Two common electrodes were used as ground on bony surfaces. For the first set of eight muscles monitored, the knee flexors and extensors, the common ground electrode was placed over the patella. For the muscles acting at the ankle joint, excluding the medial and lateral gastrocnemius heads that were included among the knee flexors, the common ground electrode was placed on the tibia right below the LASH marker that was used to track the shank.

Electrode muscle site preparation is important for the collection of the electromyographic signals (Winter et al., 1980). Removal of the hair at the electrode-skin 
interface, along with removal of dead skin cells and oils results in decreased impedance at the skin-electrode interface. For the purposes of placing the electrodes on the subject's skin, two of the subjects, prior to coming in the laboratory for data collection had shaved their left lower extremity. For one subject the skin over the muscle sites of interest was shaved in the laboratory. Each muscle was palpated in order to locate its respective muscle belly. Although, because of the EMG data collection system used, minimum preparation was required prior to positioning the electrodes, the skin over the muscle site where the electrodes were going to be placed was still abraded with an abrasive conductive gel. Then paper alcohol soaked tissue was used to clean and rub vigorously the skin over the abraded muscle site prior to fixing the electrodes. Pre-test practice sessions by this investigator on subjects who did not ultimately participate in this study indicated that the muscle site preparation procedure used in this study resulted in a skin impedance of, in general, less than $450 \mathrm{ohms}$ when a volt-ohm-meter (Fluke 75 multimeter, John Fluke MFG. CO., INC) was used to measure it. Such reduced impedances following similar electrode muscle site preparations agree with those reported by Soderberg (1992).

The electrodes then were fixed at the center of the muscle belly of each muscle of interest, using the hypoallergenic harmless self-adhesive collar of each electrode. The exact locations of the electrodes on the muscles were based on the positions suggested by Delagi and Perotto (1981), Soderberg (1992), and LeVeau and Andersson (1992), which allowed for the implementation of De Luca's (1997) recommendation, i.e., that the electrodes need to be placed in the region halfway between the center of the innervation zone and the distal myotendinous junction. Exceptions to the above recommendations for electrode placement were the medial and lateral heads of the gastrocnemius, the extensor digitorum longus, the soleus, the short head of the biceps femoris, and the medial hamstrings (comprised by the semitendinosus and semimembranosus).

The electrodes on the gastrocnemii heads were placed horizontally according to the work by Hof (1977). The soleus, although Delagi and Perotto (1981) recommended that it is located on the medial aspect, electrodes were placed on the lateral aspect. The semitendinosus and semimembranosus were monitored as one muscle, the medial 
hamstring. Of the two muscles that comprise the medial hamstring, the semitendinosus was monitored to represent the activity of the medial hamstring muscles. The extensor digitorum longus was monitored at the lower third of the leg lateral to the tibia. Finally, the short head of the biceps femoris was monitored with longitudinal electrode placement, posterior and just medial to the biceps femoris tendon one to two flat finger widths superiorly to the tibio-femoral joint at the posterior aspect of the knee (White, 1997).

The small interelectrode distance restricted the pick up, detection area, of the electromyographic signal. Furthermore, by placing the electrodes in the center of the surface of the muscle belly, the distance that electrode pairs were away from adjacent muscles was increased. Consequently, the small interelectrode distance combined with the placement of the electrodes on the center of the muscle belly, reduced the potential for EMG "cross-talk". Moreover, when the EMG signal was quantified, although the possibility for "cross-talk" contamination might still exist for very low levels of contraction, the problem was assumed to be less significant, especially at higher levels of contraction (Hof, 1984). The cables coming off the electrodes were also fixed on each subject's lower extremity using self-adhesive hypoallergenic tape. Thus, the risk for introducing motion artifacts, i.e., low frequency noise, in the electromyographic signal was reduced.

At the time of electrode placement, muscle function testing was performed to assess the presence of cross-talk according to the following protocol developed for the purposes of this investigation.

i. Rectus Femoris: While sitting the subject was asked to flex at the hip with the knee relaxed;

ii. Vastus Lateralis and Vastus Medialis: While sitting on the floor with the lower extremity extended and relaxed, i.e., resting on the floor, the subject was asked to raise the heel off the floor with the extended and kept on the floor;

iii. Biceps Femoris (short head): From a standing upright position, the subject was asked to flex at the knee without moving the femur; 
iv. Biceps Femoris (long head): From the position above, the subject was asked to extend the hip; the activity of the short head of the biceps femoris remained the same, whereas that of the long head of the biceps femoris increased.

v. Medial Hamstrings (Semitendinosus and Semimembranosus): From a standing upright position, the subject was asked to internally rotate the tibia and flex at the knee;

vi. Soleus: The subject was sitting on a short chair that allowed the lower extremities to be flexed 60 to 70 degrees when the shank was perpendicular to the ground; the subject then was asked to raise only the heel off the floor;

vii. Lateral Gastrocnemius and Medial Gastrocnemius: From a standing upright position, the subject was asked to raise up on the toes;

viii. Fibularis (Peroneus) Brevis: The subject was sitting on a short chair that allowed the lower extremities to be flexed 60 to 70 degrees when the shank was perpendicular to the ground; the subject then was asked to raise the heel only off the floor once and evert twice;

ix. Tibialis Anterior: From a sitting position the subject was asked to dorsi flex;

x. Extensor Digitorum Longus: From the same sitting position as that used to test for the Tibialis Anterior, the subject was asked to dorsi flex once and then raise the four lateral digits of the lower extremity twice.

During the performance of function muscle testing, electromyograms were collected and displayed on the monitor of a PC in real time. The collected data were saved and once the testing for each muscle was completed the respective electromyographic record was displayed to assess the presence of cross-talk.

After the calibration procedures and the placement of the electrodes over the muscle sites of interest, electromyographic data were collected concurrently with the force plate and with the video cameras. The electromyographic data were transmitted via the two Noraxon transmitters, each contained in a beltpack which could be attached on a velcro elastic belt worn at chest height. Electromyographic and ground reaction force data were 
collected on five successful trials at $1200 \mathrm{~Hz}$ for the left lower extremity. A gain of two was used for the electromyographic signals, and a gain of one was used for the ground reaction force data. The positional data for these trials were captured by the six-camera $60 \mathrm{~Hz}$ VICON software system (Oxford Metrics, Oxford, England).

A trial was deemed successful when: (a) the entire foot of interest hit the force plate, and (b) the strides were natural. The trial was discarded and repeated if the investigator of the study observed lunging or short choppy steps, or if the foot of interest did not fall entirely on the force plate, or if the subject felt the stride was not natural. If there were any doubts as to the success of foot placement on the force plate, the trial was discarded and repeated also. The investigator of this study while operating the equipment he was observing the motion perpendicular to the plane of movement.

Practice walks were allowed, and stretching was permitted should the subject being tested found it necessary. It was emphasized to the subjects that they needed to walk naturally, at a self-selected pace, i.e., at a comfortable cadence, without targeting any of the equipment, i.e., primarily, the force plates. They were also informed that they could indicate any time that they might feel that their gait pattern was not natural. Additionally, the subjects were instructed to slow down after they passed a marker that indicated the end of the walkway.

\section{Calibration procedure}

After the gait evaluation, subjects walked through the OSU hospitals underground tunnel to the Department of Rehabilitation Services. Use of the tunnel allowed for a smooth transfer of the equipment, and especially of the PC, thereby reducing the risk of crashing the hard drive where all data were being stored. Furthermore, use of the tunnel accounted for unpleasant weather conditions. The employment of the calibration procedure after the gait evaluation was felt more appropriate because, by the time of the calibration, when the subjects performed maximum muscle contractions, an adequate warm up period had been allowed. In addition, fatigue was not a factor during the gait data collection session.

The purpose of the calibration procedure, combined with the normalization methods of the processed EMG, was four fold: 
a) to eliminate the arbitrariness of the processed EMG signal, i.e., the signal was scaled from zero to one in order to map the maximum force that could be generated;

b) to account for artifacts embedded in the EMG signal as functions of muscle length and velocity so that the relationship between EMG and force could be established for the musculotendon units of interest;

c) to obtain maximum EMG values as functions of the joint angle, i.e., muscle length, and active state of the muscle, for the purposes of normalizing the dynamic EMG signals recorded during gait; and

d) to establish resting baseline electromyographic levels, when the muscles were relaxed, which would be subtracted from the time dependent EMG recorded and from the myoelectric maxima.

Furthermore, it was felt important to structure the calibration procedure such that myoelectric maxima were collected under controlled conditions that resembled the walking gait. Similar studies in the past have traditionally used isokinetic dynamometers where the subject exerted voluntary contractions at one joint while the other joint was held at a constant position throughout the testing procedure (Olney and Winter, 1985; White and Winter, 1993). In other words, the interactions between the adjacent joints were never investigated. Contrary to these projects, the present study investigated the interactions observed during the gait cycle between the ankle and the knee. Such an approach aspired to simulate walking gait-like conditions on the isokinetic dynamometer. To this end it was extremely helpful that the manufacturers of the isokinetic dynamometer that was used for this study, provided additional equipment that allowed some of the interactions between the ankle and the knee joints to be investigated. For example, ankle dorsi-plantar flexion can be performed while maintaining the knee at different angles. Consequently, each subject of this investigation performed a number of near isometric and isokinetic joint torque trials.

The isokinetic dynamometer that was used for this part of the data collection was a KinCom servohydrolic dynamometer (KinCom, Chattecx Corporation). Twenty-four hours prior to each "second visit" data collection session the entire calibration procedure 
suggested by the manufacturer was performed. Because of the hydraulic nature of the dynamometer, and, therefore, the effect of temperature on its viscous components, no calibration was performed unless the dynamometer was turned on for a minimum of ten minutes. Then adjustments were made to the gains and resistors so that the angle, and velocity of the lever arm performance, as well as the performance of the load cell were as accurate as possible. The KinCom-hardware calibration was also performed one hour after the initial calibration procedure so that final adjustments could be made given the specific temperature of the dynamometer. It was observed that the adjustments necessary, after the dynamometer had been working for one hour, were minimum. The dynamometer was performing equally well when it had been working for ten minutes or one hour. Furthermore, during the data collection session, especially between testing conditions, the dynamometer was given adequate time (approximately five minutes) for cooling down. Also, after each maximum voluntary contraction, each subject was given two minutes rest. That subject resting time was an additional cooling down interval for the isokinetic dynamometer.

Prior to collecting any data on the KinCom the subject was drawn a line on the lateral aspect of the left lower extremity that was tested. First the anatomical landmarks of LGRT, LLFE, and LLM were identified and marked. In addition, with the subject sitting such that the left knee was flexed at $90^{\circ}$ and with the foot perpendicular to the shank of the lower leg, another anatomical landmark (ULLM) was marked at a distance of 1 to $1.5 \mathrm{~cm}$ directly below the LLM and approximately $1 \mathrm{~cm}$ distal, i.e., in the anterior direction. Then a line was drawn to join the LGRT and the LLFE. The LLFE and the ULLM anatomical landmarks were joined by another line (see Figure 5.17). The subject was also asked to warm up again prior to collecting any data to account for any potential cooling down during the set up of the equipment for the data collection. The supplementing warm-up consisted of five minutes on a bicycle ergometer at a self selected pace with no resistance, followed by a short period of stretching.

Just before the calibration tests, two tilt sensors were placed, one on the most proximal segment of the two that comprised the joint which was being evaluated, and the other on the distal segment. When the knee joint was being tested the tilt sensors were 


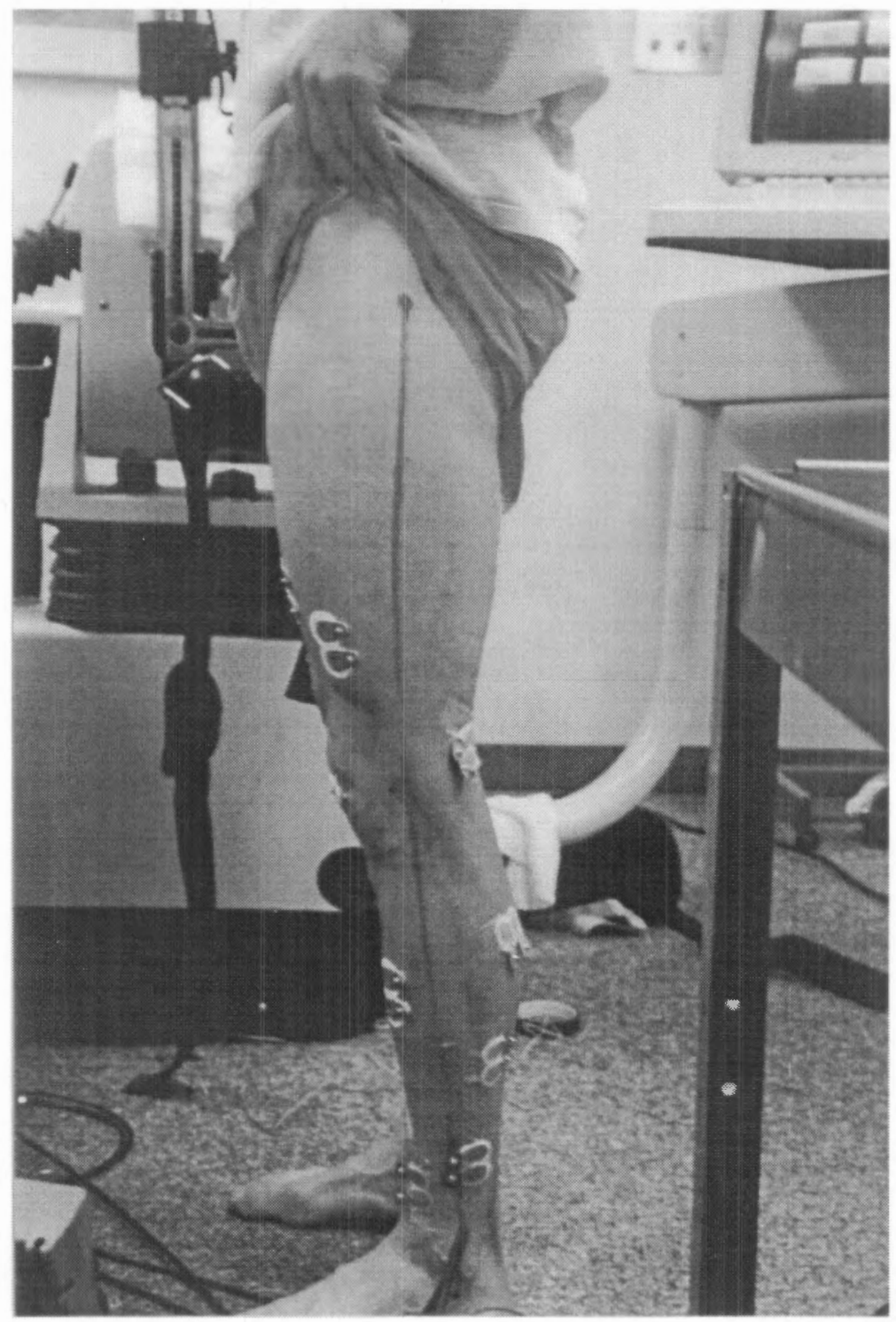

Figure 5.17. Four anatomical landmarks were identified prior to collecting any calibration data. They were the left greater trochanter, the left lateral femoral epicondyle, the left lateral maleolus and another landmark under the left lateral maleolus. The left greater trochanter and left lateral femoral epicondyle were joined by a line. The left lateral femoral epicondyle and the last landmark were joined by another line. 
placed on the line that was joining the LGRT and the LLFE, closer to the LLFE (see Figure 5.18) and the other on the line that was joining the LLFE and the LLM. The orientation placement of the tilt sensor was such that any movement of the thigh would fall within the linear response range of the sensor - signal conditioner pair. A similar approach was used for the sensor that was placed on the lateral shank when the ankle joint was being tested. The only difference was that one tilt sensor was placed on the lateral shank and the other on the foot, laterally. Then the subject was positioned on the KinCom to perform isokinetic knee flexion-extensions, and ankle plantar-dorsi flexions.

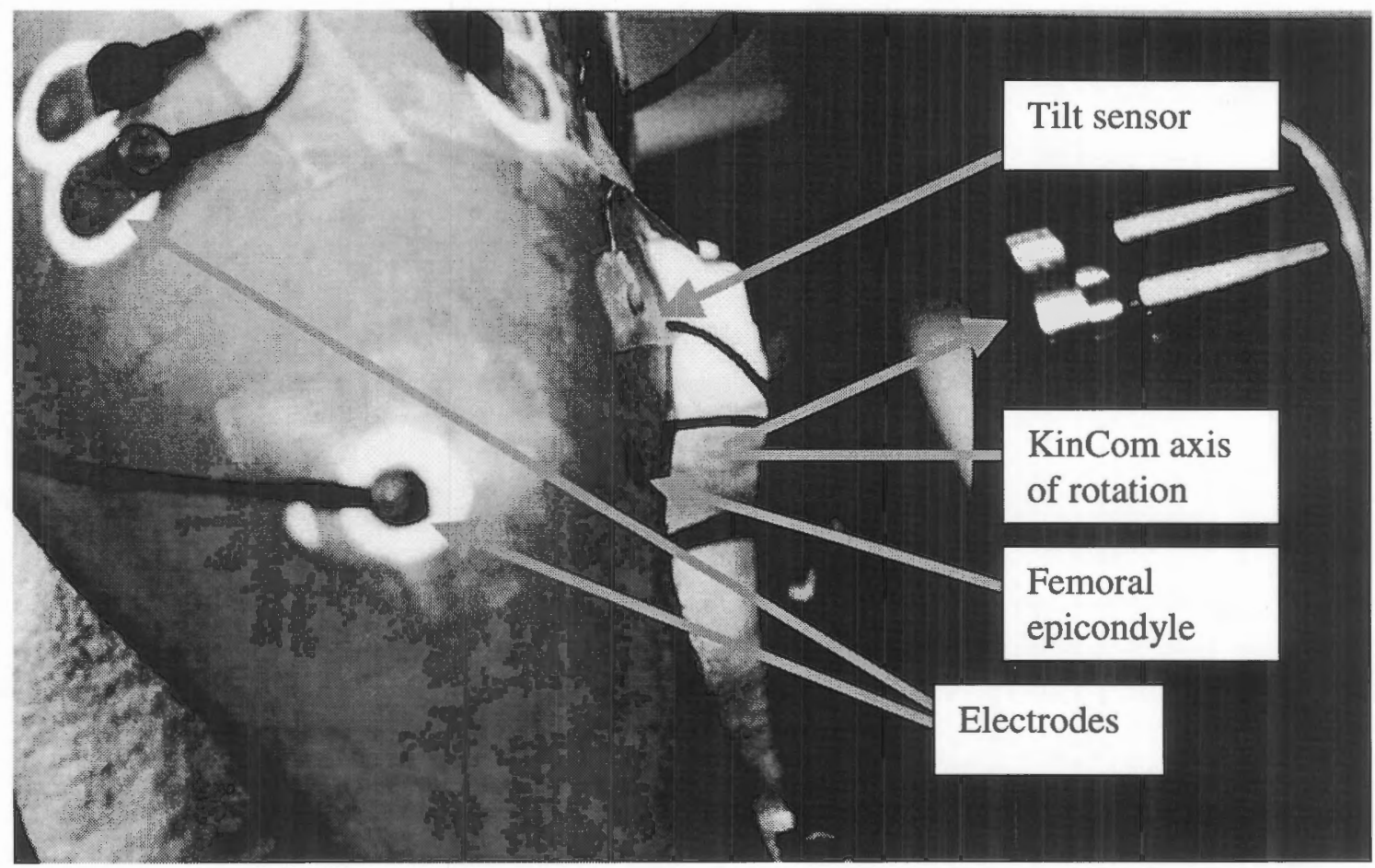

Figure 5.18. Set up for testing the knee joint; electrodes and thigh tilt sensor placement.

Before testing, as a safety precaution, each subject was given a safety switch of the KinCom. All subjects were instructed to press the safety switch at will in order to stop the testing, should there was, at any time, any discomfort during the calibration testing. 
For the purposes of this investigation, the KinCom seat settings were modified by creating an extra option that allowed sitting with the trunk at $45^{\circ}$ relative to the thigh. Such a position was felt to be important for accounting for the role of the rectus femoris muscle as one of the knee extensors (Simon, 1997). Furthermore, it has been reported that, when positioned on the isokinetic dynamometer for testing, sliding of the body is an issue (Dvir, 1996). Consequently, when the subject was sitting on the isokinetic dynamometer, he was strapped on the seat in such a way that the pelvis could not move. In addition, throughout the calibration testing, the subjects were instructed to grasp the sides of the "test table", i.e., the sides of the KinCom seat, and consciously stabilize the pelvis on the seat. Thus, every precaution was taken to prevent slipping forward during testing.

To establish resting baseline electromyographic levels, when the muscles were relaxed, and to observe the effect of the gravitational moments and of the parallel elastic component (PEC), a zeroing calibration was performed (White and Winter, 1993). Every joint of interest was placed at a position of maximum test excursion, i.e., the ankle was placed at a dorsi flexed position of 20 degrees, while the knee was placed at a position of complete extension. The subject then was instructed to relax. The segment was allowed to rotate through to its alternate joint excursion, i.e., ankle plantar flexion of 30 degrees, and knee flexion of approximately 60 degrees. The data recorded were considered to be a function of the gravitational forces and of the PEC.

In each test the proximal segment was strapped to the dynamometer bench with the joint center aligned as nearly as possible to the axis of rotation of the KinCom. The distal segment was secured to the load cell on the lever arm of the KinCom via the attachments provided by the manufacturer. At that time the moment arm with respect to the center of rotation of the dynamometer was determined. When the muscles crossing the knee were being tested, the anatomical axis of the joint about which flexion-extension occurred, was considered to pass through the femoral epicondyles (Clarkson and Gilewich, 1989). Consequently, the LLFE anatomical landmark was aligned with the axis of rotation of the KinCom (Kellis and Baltzopoulos, 1996). The femur was fixed in a modified manner that allowed the mid-portion of the femur to be free of contact with the KinCom seat. 
Furthermore, the arrangement allowed only for the distal part of the femur to be supported, allowing, therefore, no interference with electrodes monitoring the activity of the BFs and SEM muscles.

When the musculature crossing the ankle was being tested, the anatomical axis of the joint about which plantar-dorsi flexion was considered to pass just below the medial and lateral malleolli (Clarkson and Gilewich, 1989). Consequently, the ULLM anatomical landmark was aligned, as nearly as possible, with the axis of rotation of the KinCom. Results from preliminary, pilot, studies indicated that, when the ankle joint of five subjects was being tested, using the methodology indicated by the manufacturer of the isokinetic dynamometer, each subject raised the heel at the beginning of each plantar flexion maximum voluntary contraction (MVC) to achieve maximum plantar flexion force. The implication was that, perhaps, the recommendation of the KinCom manufacturer for the attachment of the foot on the isokinetic dynamometer was not as effective. Consequently, for the purposes of the present study, the attachment of the foot on the isokinetic dynamometer was modified such that the heel of the foot could not come off the KinCom ankle plantar - dorsi flexion attachment (see Figure 5.19).

However, in general, previous studies indicate that the KinCom axis of rotation does not always coincide with the segment line between adjoining joint centers (Kaufman, 1995, 1991b). Such an error can be introduced as a result of

movement of the proximal joint segment. Consequently, a servo-potentiometer reading slightly biased to the true joint angle may be obtained, compared to the subject specific lever arm angle. To compensate for this error, researchers in the past have placed the center of rotation of the KinCom lever arm $2 \mathrm{~cm}$ inferior and $2 \mathrm{~cm}$ posterior to the anatomical axis of the knee (Ghori et al., 1995) on the basis of the work of Tredinnick and Duncan (1988) who observed an inferior and posterior movement of the knee during KinCom isokinetic knee flexions and extensions. Other researches have implemented the concurrent use of electrogoniometers (Kaufman, 1995, 1991b). In this project the tilt sensors served as the means for correcting the error. The inclinometer located at the proximal segment, provided information about the superior-inferior 


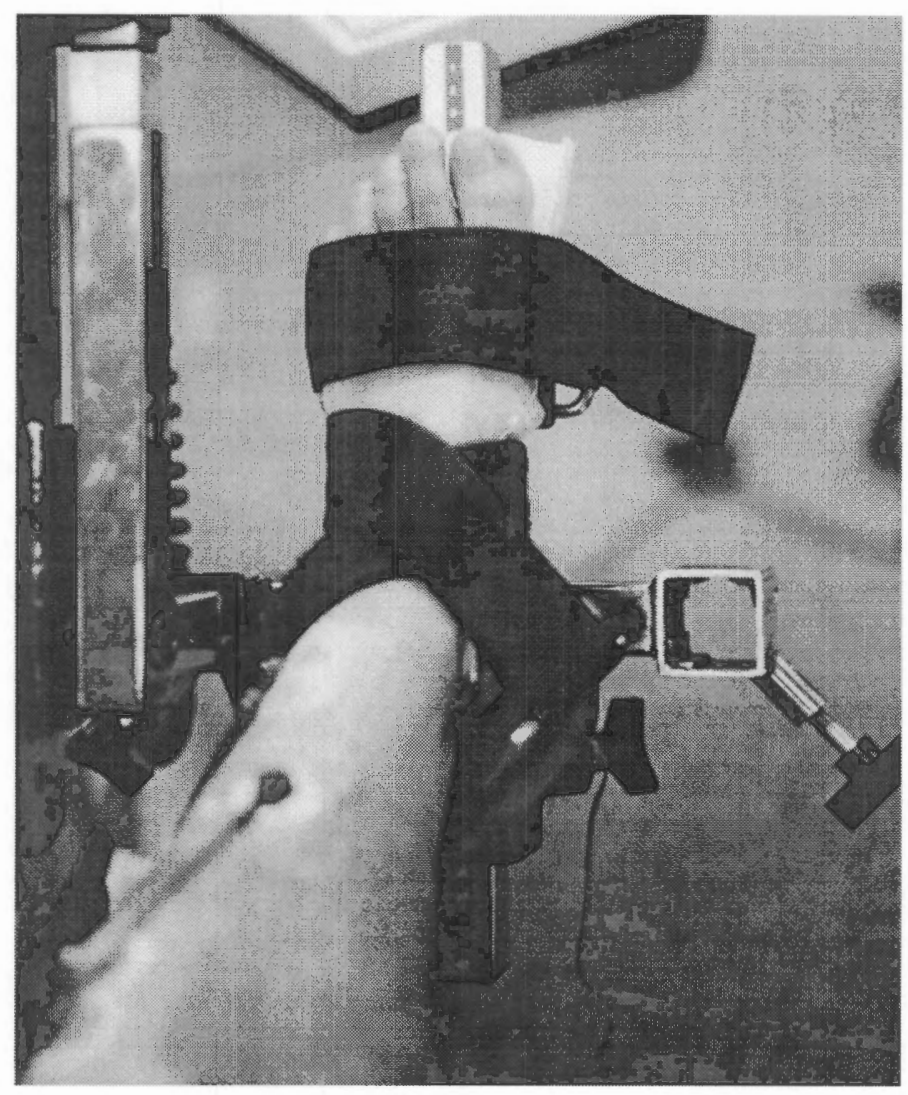

Figure 5.19. The modified strapping method of the foot on the isokinetic dynamometer such that the heel is not raised during plantar flexion.

movement of the segment, while combination of the output from both sensors allowed the estimation of the joint angle in the sagittal plane. Thus, assuming a significant superiorinferior movement of the proximal segment of the joint, a more accurate mapping of the EMG-Force-Joint Angle relationship could be established. Furthermore, pictures were taken for randomly selected trials at the beginning and end of the respective joint excursions.

To obtain EMG values corresponding to the maximum joint moment generated for the purposes of normalizing the dynamic EMG signals recorded during gait, surface EMGs for maximum voluntary nearly isometric contractions (nMVIC) were collected during concentric efforts. The knee and ankle joints were made to go through a range of angles at very low velocities (five degrees per second) where the specific muscle group of 
interest (i.e., knee flexors, knee extensors, ankle plantar flexors, and ankle dorsi flexors) was capable of generating maximum force or moment. The range of angles at which the knee and ankle joints were made to go through was determined by using the interactive musculoskeletal graphics software SIMM. The maximum potential of each muscle group of interest was plotted against the ankle and knee ranges of interest respectively (see Figures 5.20-5.23).

It was observed from the simulations that for all muscle groups maximum force was achieved outside the physiological joint range of motion reported for gait. Maximum dorsi flexion muscle force took place between 35 - 40 degrees of plantar flexion. Maximum plantar flexor muscle force took place between $22-36$ degrees of dorsi flexion. However, the physiological joint range of motion observed at the ankle during gait has been reported to be between 30 degrees of plantar flexion and 20 degrees of dorsi flexion (Gage, 1991; Kadaba et al., 1989; Kadaba, 1990; Perry, 1992; Sutherland et al., 1994; Winter, 1987). Maximum knee extensor muscle force took place between 66 - 76 degrees of knee flexion. Maximum knee flexor muscle force took place at 20 degrees of hyperextension. However, the physiological joint range of motion observed at the knee during gait has been reported being from between 0-10 degrees of knee flexion to 60 degrees of knee flexion (Gage, 1991; Kadaba et al., 1989; Kadaba, 1990; Perry, 1992; Sutherland et al., 1994; Winter, 1987). Consequently, since the joint ranges at which maximum muscle forces occurred, were outside the normal gait joint ranges of motion, it was deemed appropriate to use the respective information for the maximum joint moments. The maximum joint moments appeared to fall within the normal gait joint ranges of motion (see Figures 5.20 through 5.23). Furthermore, the observation that maximum muscle group forces and moments occurred over a range of joint angles, rather than at one specific position, defined the need for recording normalizing electromyographic maxima, during the experimental data collection, over a range of joint angles as opposed to placing the joint at an assumed position of maximum moment generation (White and Winter, 1993). Thus, during the calibration data collection procedure, it was thought to be more practical creating nearly isometric contractions by 

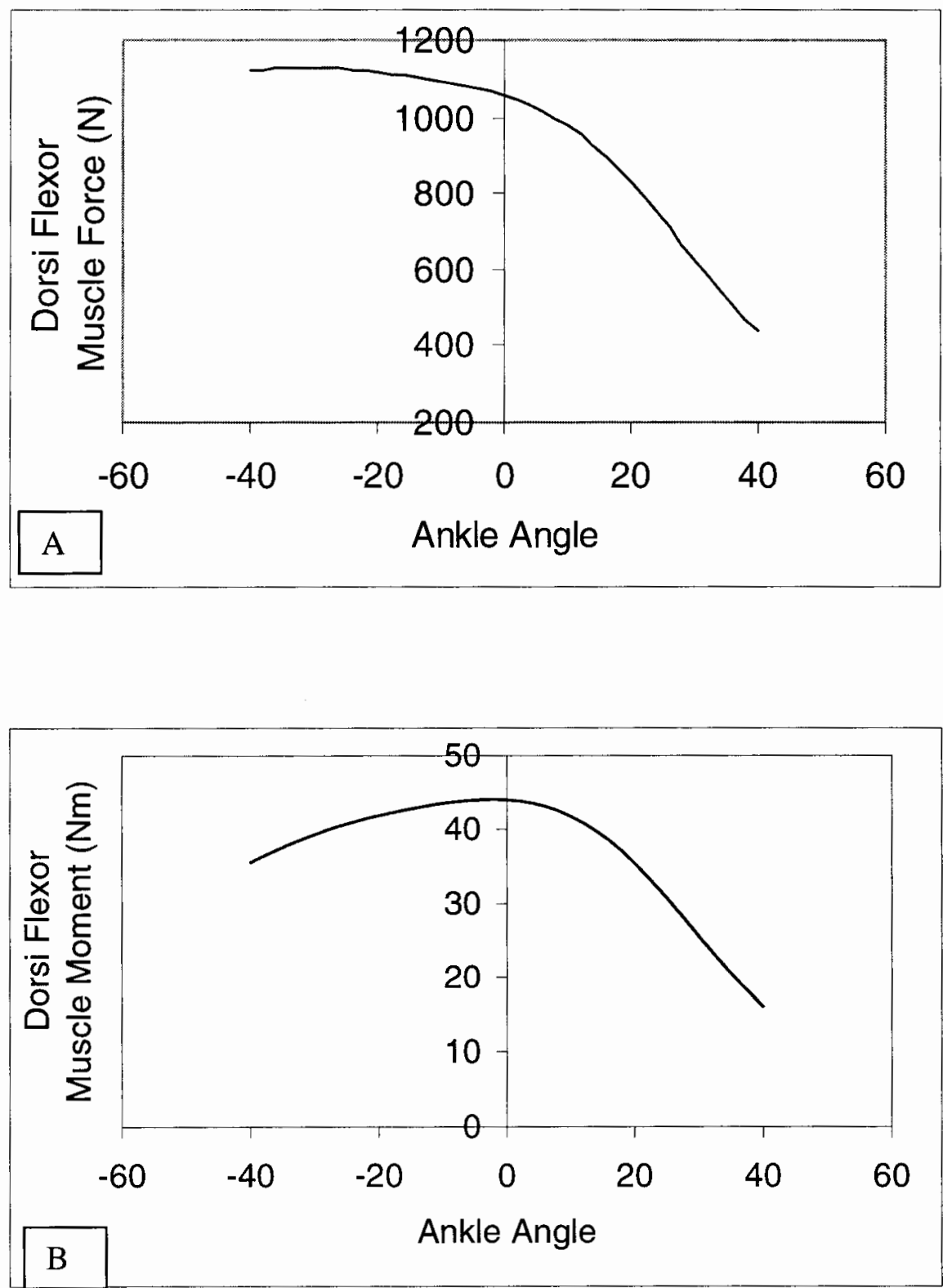

Figure 5.20. Maximum isometric force (A) and moment (B) response of the Dorsi flexors. Simulation output from SIMM. Negative angles indicate plantar flexion. 

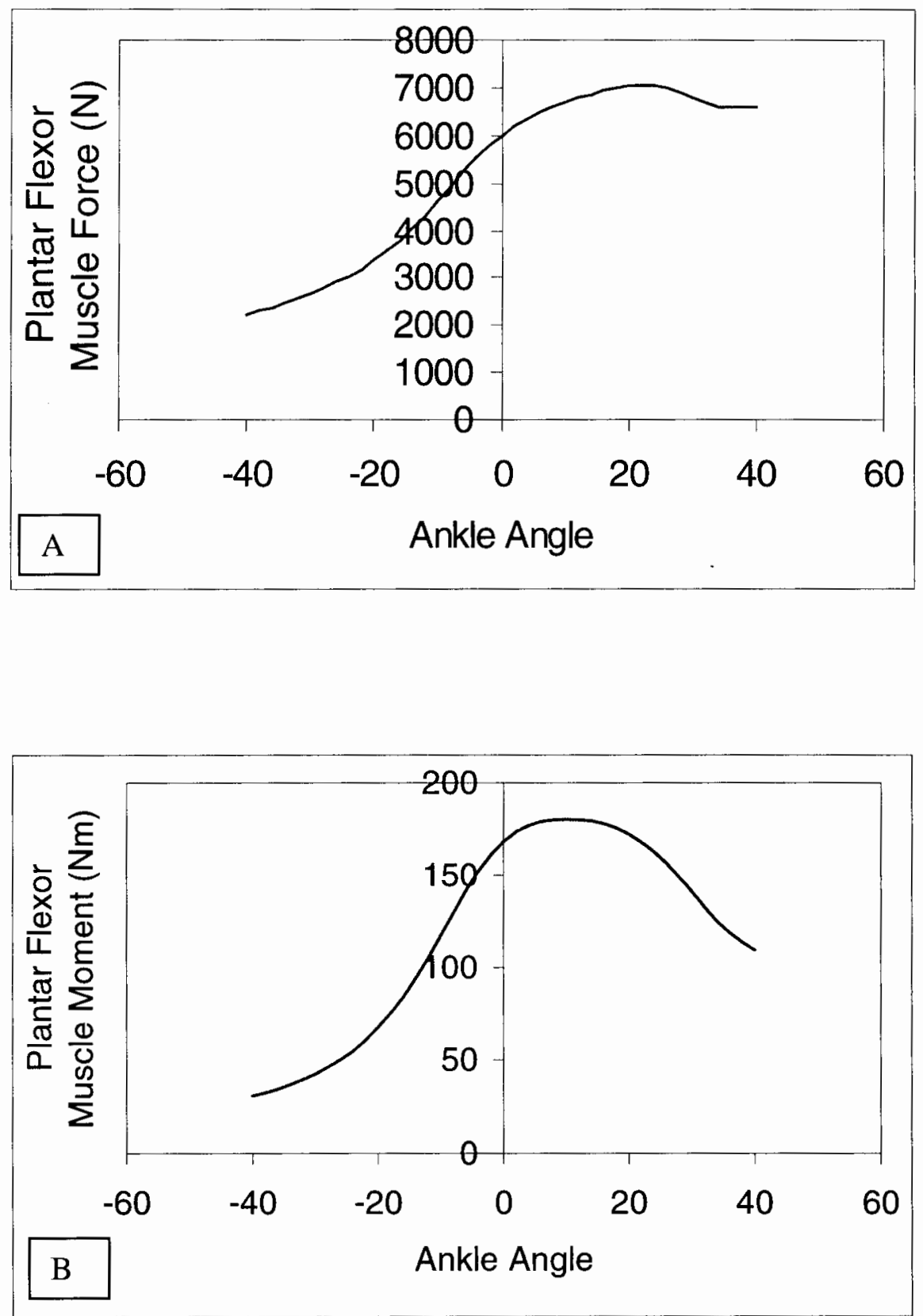

Figure 5.21. Maximum isometric force (A) and moment (B) response of the Plantar flexors group. Simulation output from SIMM. Negative angles indicate plantar flexion. 

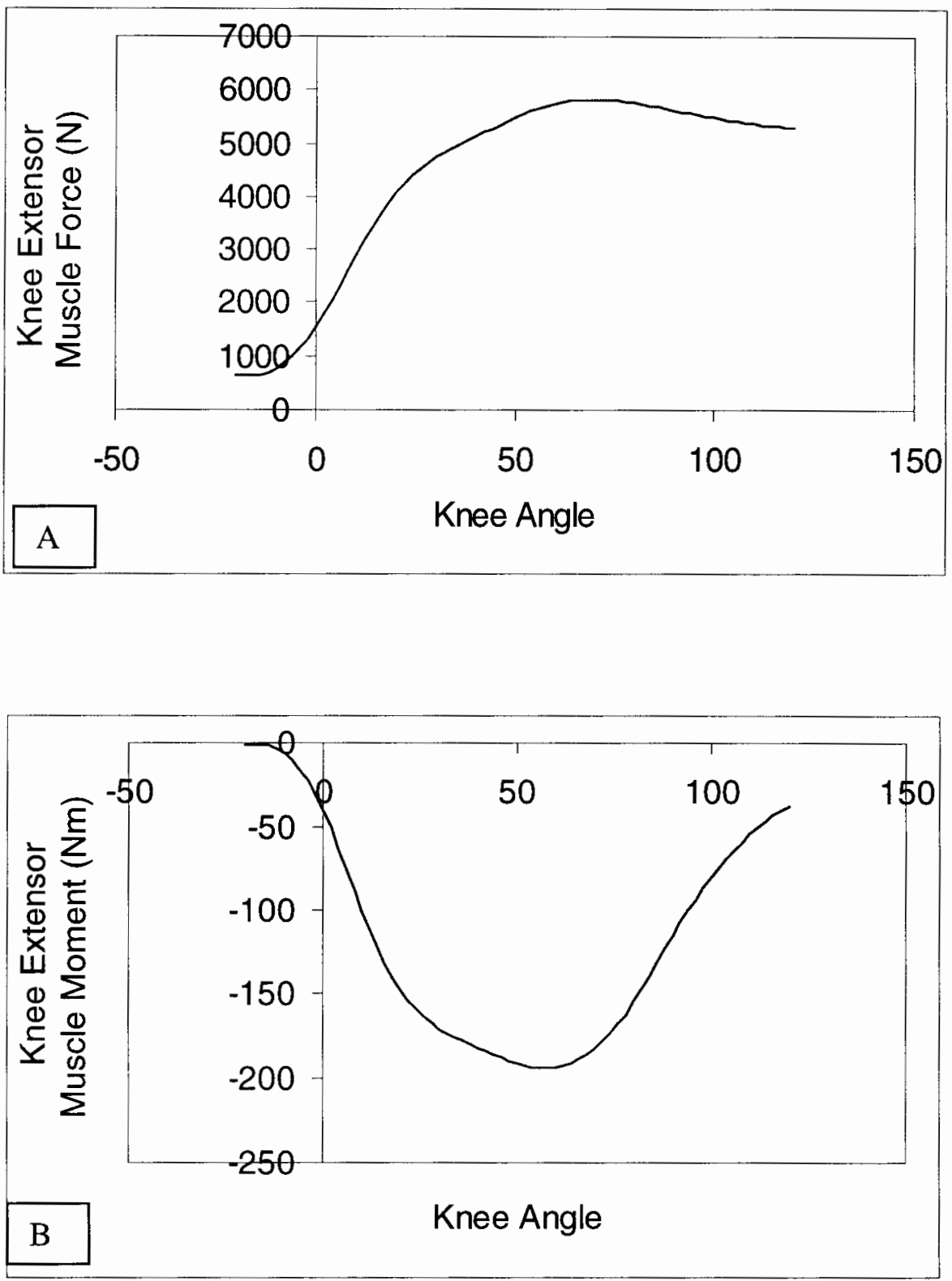

Figure 5.22. Maximum isometric force (A) and moment (B) response of the knee extensors group. Simulation output from SIMM. Negative angles indicate plantar flexion. 

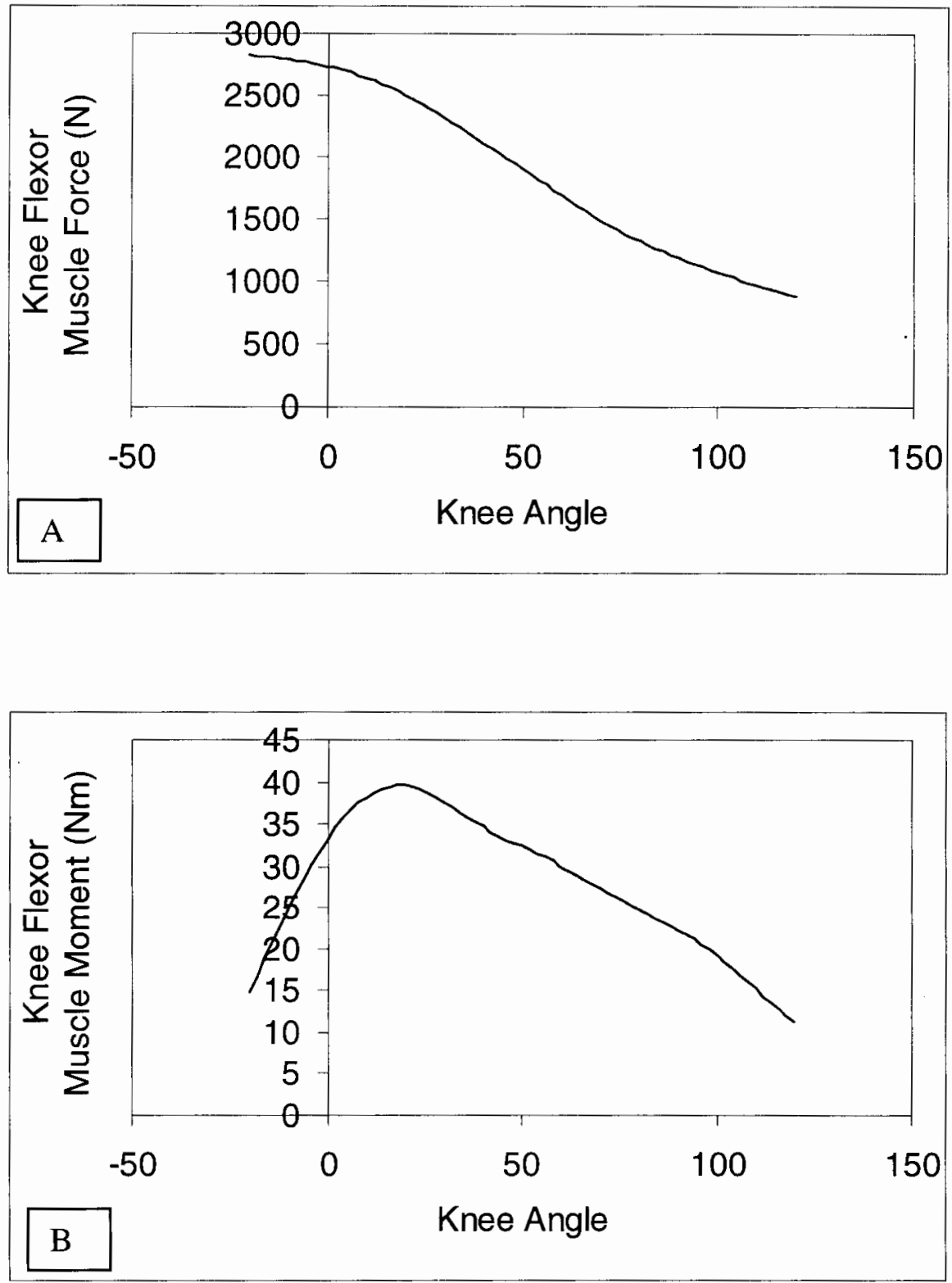

Figure 5.23. Maximum isometric force (A) and moment (B) response of the knee flexors group. Simulation output from SIMM. Negative angles indicate plantar flexion. 
having the joints of interest go through a pre-specified range of motion at very low angular velocities.

It was observed from the simulations that for all muscle groups maximum force was achieved outside the physiological joint range of motion reported for gait. Maximum dorsi flexion muscle force took place between 35 - 40 degrees of plantar flexion. Maximum plantar flexor muscle force took place between $22-36$ degrees of dorsi flexion. However, the physiological joint range of motion observed at the ankle during gait has been reported to be between 30 degrees of plantar flexion and 20 degrees of dorsi flexion (Gage, 1991; Kadaba et al., 1989; Kadaba, 1990; Perry, 1992; Sutherland et al., 1994; Winter, 1987). Maximum knee extensor muscle force took place between 66- 76 degrees of knee flexion. Maximum knee flexor muscle force took place at 20 degrees of hyperextension. However, the physiological joint range of motion observed at the knee during gait has been reported being from between 0-10 degrees of knee flexion to 60 degrees of knee flexion (Gage, 1991; Kadaba et al., 1989; Kadaba, 1990; Perry, 1992; Sutherland et al., 1994; Winter, 1987). Consequently, since the joint ranges at which maximum muscle forces occurred, were outside the normal gait joint ranges of motion, it was deemed appropriate to use the respective information for the maximum joint moments. The maximum joint moments appeared to fall within the normal gait joint ranges of motion (see Figures 5.20 through 5.23). Furthermore, the observation that maximum muscle group forces and moments occurred over a range of joint angles, rather than at one specific position, defined the need for recording normalizing electromyographic maxima, during the experimental data collection, over a range of joint angles as opposed to placing the joint at an assumed position of maximum moment generation (White and Winter, 1993). Thus, during the calibration data collection procedure, it was thought to be more practical creating nearly isometric contractions by having the joints of interest go through a pre-specified range of motion at very low angular velocities.

It was decided, therefore, to observe the isometric moment output of each muscle group of interest, i.e., dorsi flexor muscle group, plantar flexor, knee extensor and knee flexor muscle group, by simulating the respective concentric joint action that would 
correspond to the joint range of motion observed, for that joint, during gait. The adjacent joint would be kept fixed, i.e., if the ankle was being investigated, the knee joint would be fixed and the ankle would be made to go through its gait-specific joint range of motion. If the knee joint was being investigated, the ankle joint would be fixed, and the knee would be made to go through its gait-specific range of motion. The hip was fixed at all times at 45 degrees of flexion.

However, such an approach, where the joint being investigated was made to go through its gait-specific range of motion, while the adjacent joint of interest was being kept fixed at one position, would only be appropriate for investigating the monoarticular ankle dorsi flexors and, for the purposes of the present study, the knee extensors. The dual effect of the biarticular knee flexors and plantar flexors, i.e., the effect of the two gastroc heads, would not be accounted for. Thus, it was desirable to simulate gait-like conditions for the purposes of identifying the range of motion during which maximum knee flexion moment was generated as a function of the ankle position, and vice versa for the purposes of identifying the position at which maximum plantar flexion was generated as function of the knee flexion angle. Consequently, the interactions during the gait cycle between the knee and ankle joints in the sagittal plane were identified by plotting the respective joint ranges of motion against each other (see Figure 5.24).

Pairs of interactions were recorded at the peaks of each of the joints of interest where the derivative for the respective joint would become zero. The peak represented the position at which that joint would be fixed, while the adjacent joint would be made to go through its corresponding gait-specific range of motion in the sagittal plane. Also, the corresponding range of motion at the knee was investigated when the ankle joint was at neutral, i.e., when it was crossing from dorsi flexion to plantar flexion, and vice versa.

Eleven pairs of joint angle positions, interactions, were identified (see Table 5.3). In order, however, to simplify the number of interactions, and since on the basis of those identified interactions experimental testing was planned to be conducted on the isokinetic dynamometer, it was necessary to reduce the number of identified pairs to avoid subject fatigue during testing. The resulting joint interactions, which allowed the combination of the information presented in Table 5.3, in a reduced form, is presented in Table 5.4. 


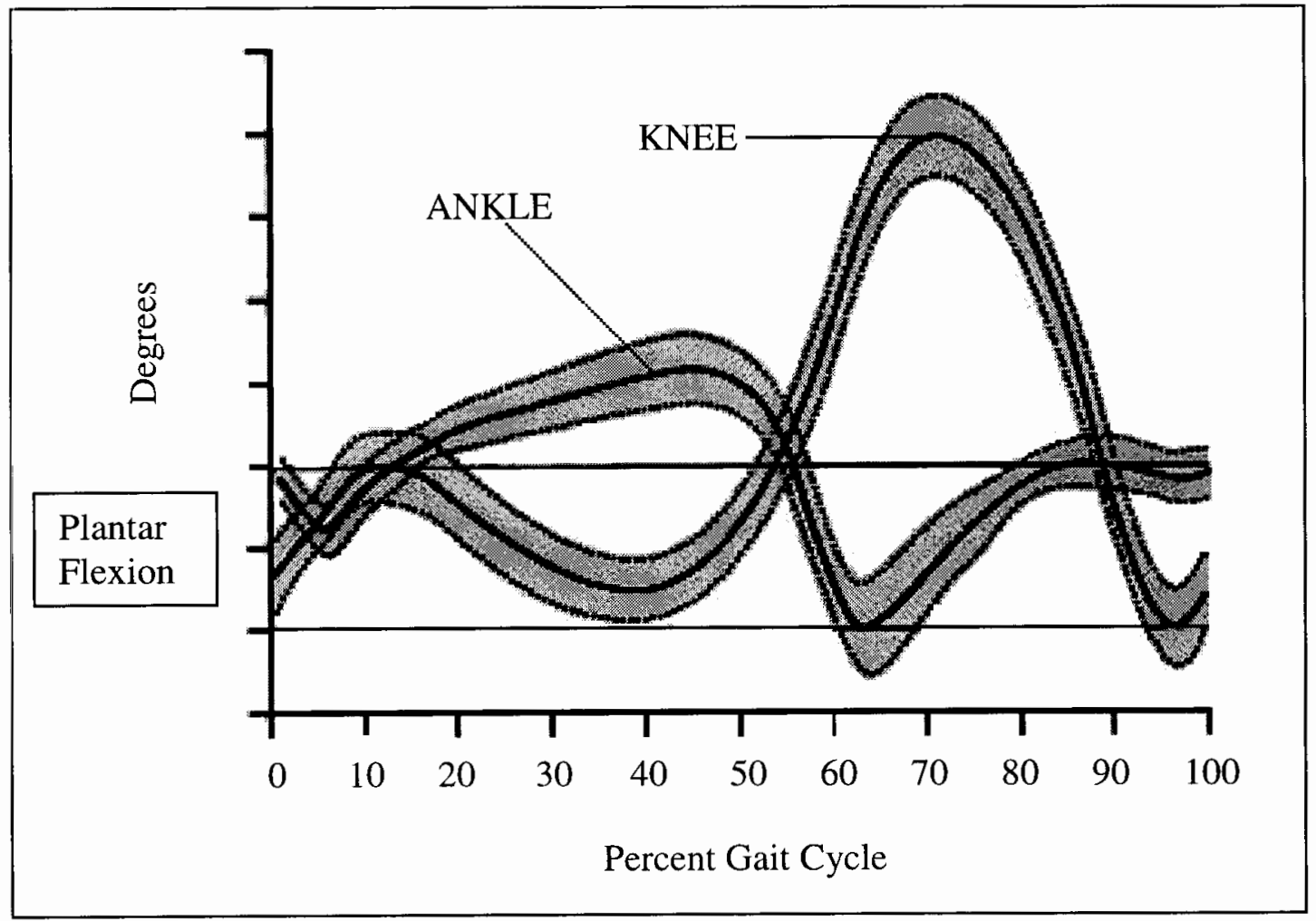

Figure 5.24 Ankle-knee joint interactions throughout a gait cycle. The time of the gait cycle is normalized to percent. 


\begin{tabular}{|l|c|c|c|c|c|c|}
\hline Joint & \multicolumn{3}{|c|}{ Ankle } & \multicolumn{3}{c|}{ Knee } \\
\hline & Angle & Condition & $\begin{array}{c}\text { Position or } \\
\text { Motion Direction }\end{array}$ & Angle & Condition & $\begin{array}{c}\text { Position or } \\
\text { Motion Direction }\end{array}$ \\
\hline & 1 & $\mathrm{~F}^{\mathrm{l}}$ and $\mathrm{D}^{2}$ & $\mathrm{PF}^{3}$ & 7 & $\mathrm{~F}$ & $\mathrm{Fl}^{4}$ \\
\hline & 8 & $\mathrm{~F}$ & $\mathrm{PF}$ & 15 & $\mathrm{D}$ & $\mathrm{Fl}$ \\
\hline & 0 & $\mathrm{~F}$ and D & $\mathrm{N}^{5}$ & 20 & $\mathrm{~F}$ and D & $\mathrm{Fl}$ \\
\hline & 10 & $\mathrm{D}$ & $\mathrm{DF}^{6}$ & 5 & $\mathrm{D}$ & $\mathrm{Fl}$ \\
\hline & 12 & $\mathrm{~F}$ & $\mathrm{DF}$ & 7 & $\mathrm{D}$ & $\mathrm{Fl}$ \\
\hline & 0 & $\mathrm{~F}$ & $\mathrm{~N}$ & 24 & $\mathrm{D}$ & $\mathrm{Fl}$ \\
\hline & 20 & $\mathrm{~F}$ & $\mathrm{PF}$ & 51 & $\mathrm{D}$ & $\mathrm{Fl}$ \\
\hline & 11 & $\mathrm{D}$ & $\mathrm{PF}$ & 60 & $\mathrm{~F}$ & $\mathrm{Fl}$ \\
\hline & 0 & $\mathrm{~F}$ and D & $\mathrm{N}$ & 20 & $\mathrm{~F}$ and D & $\mathrm{Fl}$ \\
\hline & 0 & $\mathrm{~F}$ and D & $\mathrm{PF}$ & 0 & $\mathrm{~F}$ and D & $\mathrm{Fl}$ \\
\hline
\end{tabular}

Table 5.3.

Ankle-knee positions of joint interactions during the gait cycle. At those positions one of the joints would be kept fixed (F) while the adjacent joint would go through its corresponding gait-specific dynamic range of motion (D) for the purposes of identifying the maximum joint moment and respective electromyographic signal (numerical values in degrees). The dynamic range includes the corresponding angle in the "Angle" column.

\begin{tabular}{|c|c|c|c|}
\hline $\begin{array}{c}\text { Knee Fixed Position } \\
(\text { degrees })\end{array}$ & $\begin{array}{c}\text { Ankle Action } \\
\left(\mathrm{DF}^{1}, \mathrm{PF}^{2}\right)\end{array}$ & $\begin{array}{c}\text { Ankle Fixed Position } \\
(\text { degrees })\end{array}$ & $\begin{array}{c}\text { Knee Action } \\
\left(\mathrm{KF}^{3}, \mathrm{KE}^{4}\right)\end{array}$ \\
\hline $0 \mathrm{KF}$ & $\mathrm{DF}$ and PF & 0 & $\mathrm{KF}$ and $\mathrm{KE}$ \\
\hline $7 \mathrm{KF}$ & $\mathrm{DF}$ and PF & $10 \mathrm{PF}$ & $\mathrm{KF}$ and KE \\
\hline $20 \mathrm{KF}$ & $\mathrm{DF}$ and PF & $20 \mathrm{PF}$ & $\mathrm{KF}$ \\
\hline $55 \mathrm{KF}$ & $\mathrm{DF}$ and PF & $10 \mathrm{DF}$ & $\mathrm{KF}$ and KE \\
\hline
\end{tabular}

1: Dorsi Flexion

2: Plantar Flexion
3: Knee Flexion

4: Knee Extension

Table 5.4.

Positions for the ankle-knee joint interactions tested. 
Initially, in order to not only identify the locations at which maximum muscle moments occurred, but also to investigate the effect of maximum antagonistic cocontraction on the location at which peak muscle moments could occur as a function of the joint interactions, while one joint was fixed at the positions identified in Table 5.5, the other joint was simulated going throughout the entire range of motion observed for that joint during gait under two conditions:

a) with the agonists fully activated only; and

b) with both, the agonists and antagonists fully activated concurrently.

The knee was made to flex and extend from zero to seventy degrees, while the ankle was kept fixed at different positions; the ankle was made to plantar and dorsi flex between thirty degrees of plantar flexion and twenty degrees of dorsi flexion. The results have been included in Appendix I.

It was observed that peak muscle group moments occurred over a range of joint angles rather than at one particular position even when joint interactions were considered. In addition, the range of angles at which peak muscle group moments occurred, during knee flexion and ankle plantar flexion, varied with the position of the adjacent joint. Thus, the calibration procedure needed to account for the effect of the biarticular gastroc heads. Furthermore, cocontraction also affected the range of angles at which maximum muscle group moments occurred (see Table I.2). The effect was more profound when joint interactions and cocontraction were observed together for the biarticular gastroc at the knee joint during knee extension (see Table I.3). The observation that peak muscle group moments occurred over a range of joint angles during the simulations of joint interactions, advocated the idea for recording normalizing electromyographic maxima over a range of joint angles using nearly isometric contractions, for practical reasons. Consequently, in light of these observations, the gait-specific range of motions and joint interactions, for the purposes of nearly isometric testing can be seen in Table 5.5. 


\begin{tabular}{|c|c|c|c|c|c|}
\hline $\begin{array}{c}\text { Knee Position } \\
\text { (degrees) }\end{array}$ & \multicolumn{2}{|c|}{ Ankle Action } & Ankle Position & \multicolumn{2}{c|}{ Knee Action } \\
\hline $0 \mathrm{KF}$ & -10 to 15 & 20 to -5 & 0 & $\mathrm{KF}^{3}$ & $\mathrm{KE}^{4}$ \\
\hline $7 \mathrm{KF}$ & -10 to 15 & 17 to -7 & -10 & 10 to 35 & 60 to 5 \\
\hline $20 \mathrm{KF}$ & -20 to 5 & 5 to -20 & -20 & 49 to 53 & - \\
\hline $55 \mathrm{KF}$ & -30 to 5 & -6 to -25 & 10 & 0 to 17 & 0 to 20 \\
\hline
\end{tabular}

1: Dorsi Flexion

2: Plantar Flexion

3: Knee Flexion

4: Knee Extension

Table 5.5.

Positions for the ankle-knee joint interactions tested, and the respective joint ranges of motion during nearly isometric conditions. All values are in degrees. For the ankle joint, negative values indicate plantar flexion; positive values indicate dorsi flexion.

All ranges of motion, apart from being gait-specific, they did not exceed twentyfive degrees. Consequently, on the basis of De Luca's (1997) recommendations and results, when the nMVIC were performed on the isokinetic dynamometer at its lowest possible velocity, approximately five degrees per second (dps), no fatigue effect was introduced in the electromyogram. The SIMM output was observed to identify the location of the expected maximum moment during the gait-specific ranges of motion for each of the muscle groups of interest (see figures 5.25 through 5.28), and to ensure that there was at least one gait-specific condition where the muscle group of interest could generate maximum moment as it was predicted by SIMM. The SIMM output was tested against the experimental results for all conditions and joint interactions tested. The EMG values of the muscles monitored for the respective muscle group of interest which corresponded to and were responsible for the respective maximum joint moment, were considered to be the maximum EMG values produced on the isokinetic dynamometer, and gait-like joint interactions specific. 


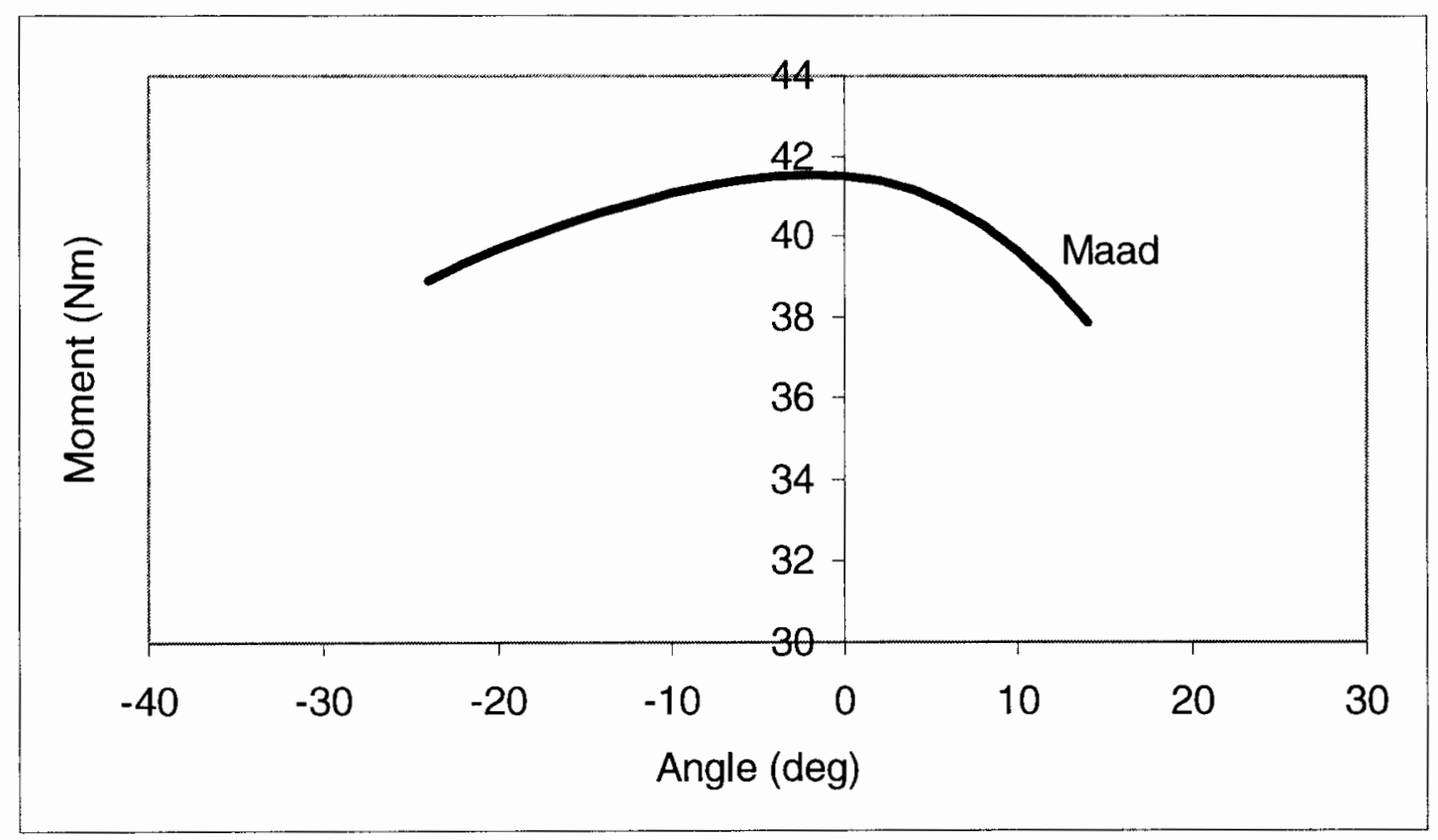

Figure 5.25. Maximum isometric dorsi flexion moment for dorsi flexion concentric contractions simulated by SIMM. Maximum antagonistic cocontraction is not simulated because it would result to a plantar flexor moment. 

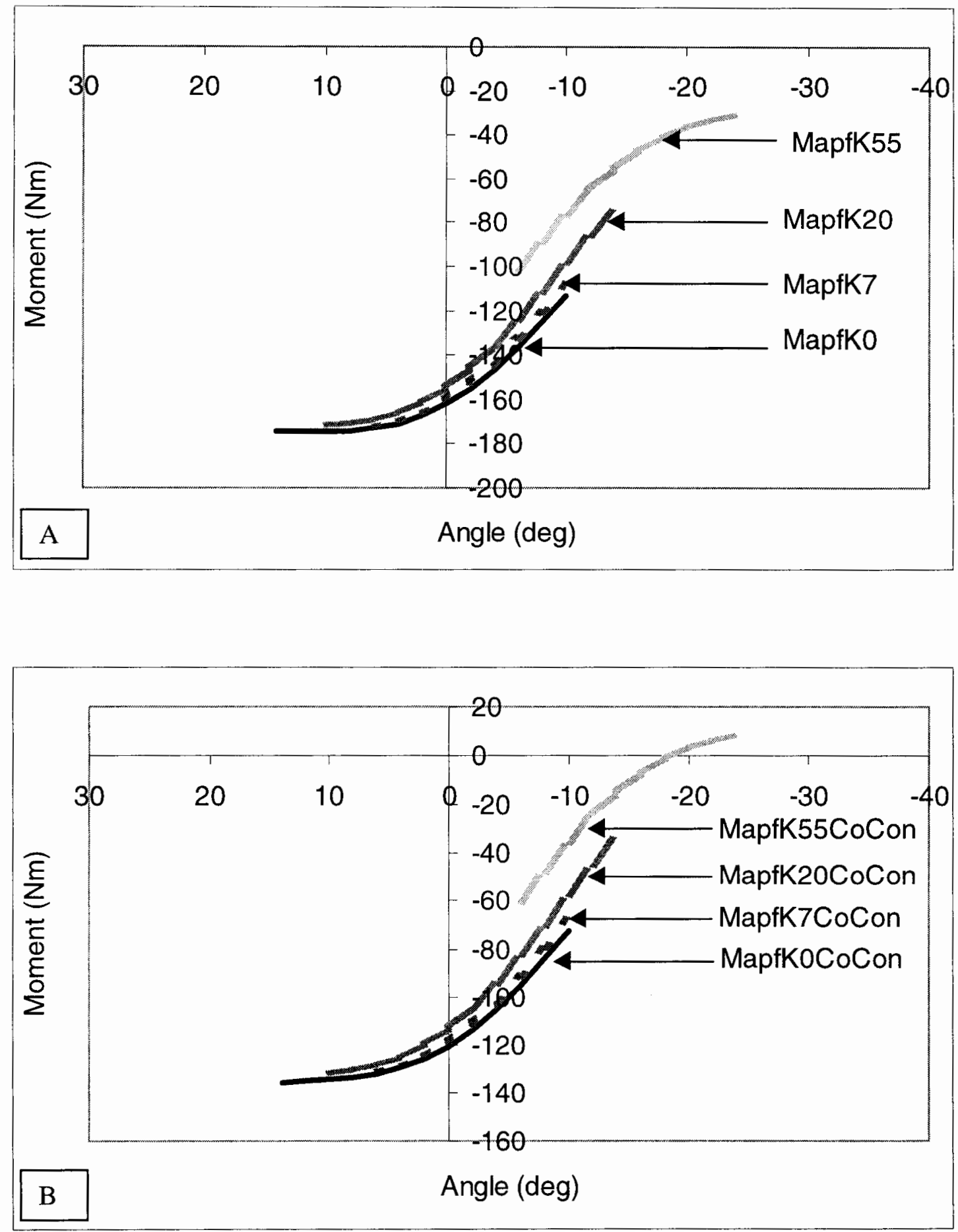

Figure 5.26. Maximum isometric plantar flexion moments for gait-specific joint interactions and ranges of motion simulated by SIMM. The conditions simulated represented plantar flexion joint angles while the knee was kept at zero, seven, twenty and fifty-five degrees of flexion (K0, K7, K20, K55) The first figure represents the plantar flexor output assuming no cocontraction (A). The second figure represents plantar flexor output assuming maximum antagonistic, dorsi flexor, cocontraction (B). 


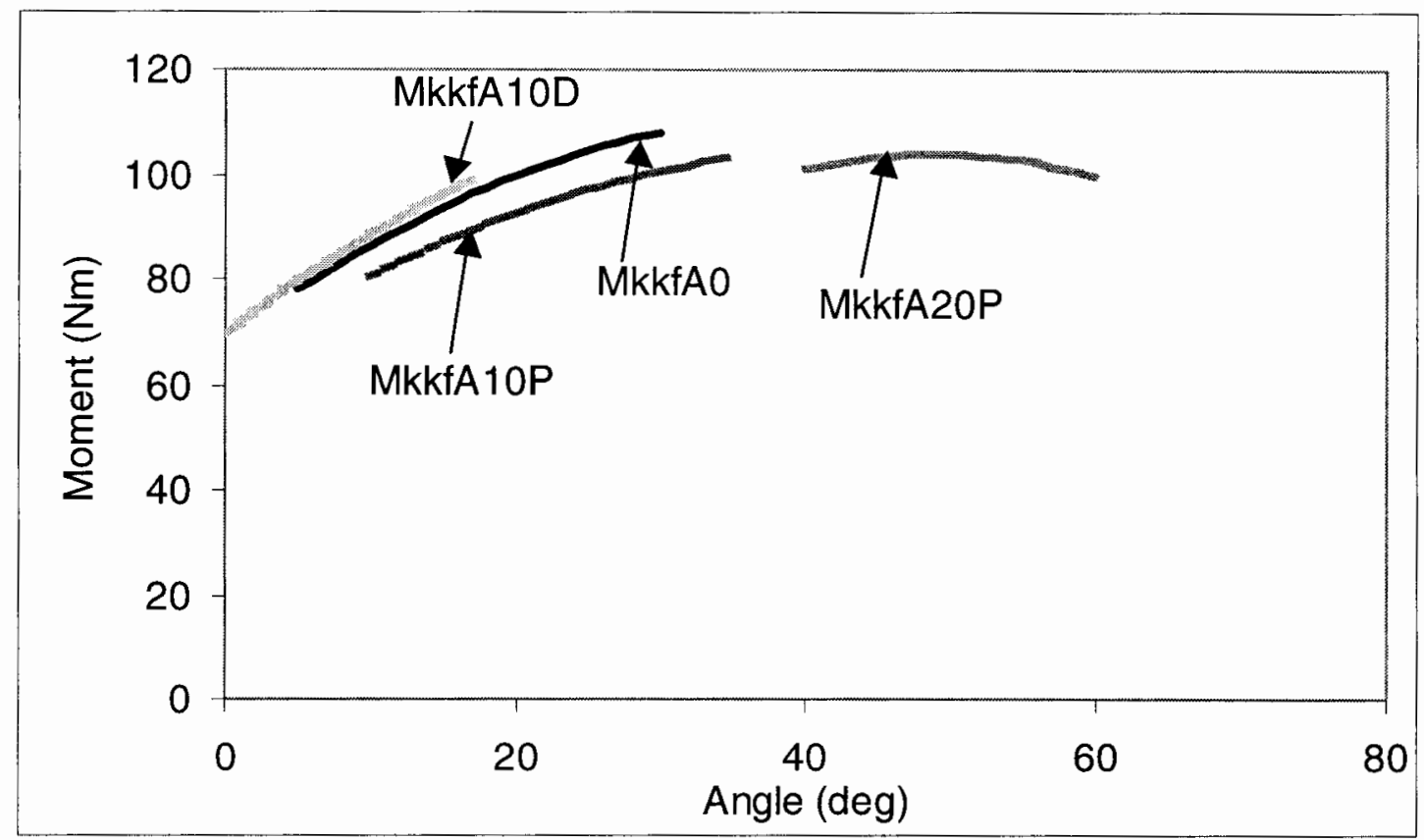

Figure 5.27 Maximum isometric knee flexion moments (Mkkf) for gait-specific joint interactions and ranges of motion simulated by SIMM. The ankle was held at neutral, ten and twenty degrees of plantar flexion (A10P, A20P), and ten degrees of dorsi flexion (A10D). Maximum antagonistic cocontraction was not simulated because it would result to a knee extensor moment. The hip was at forty-five degrees of flexion. 


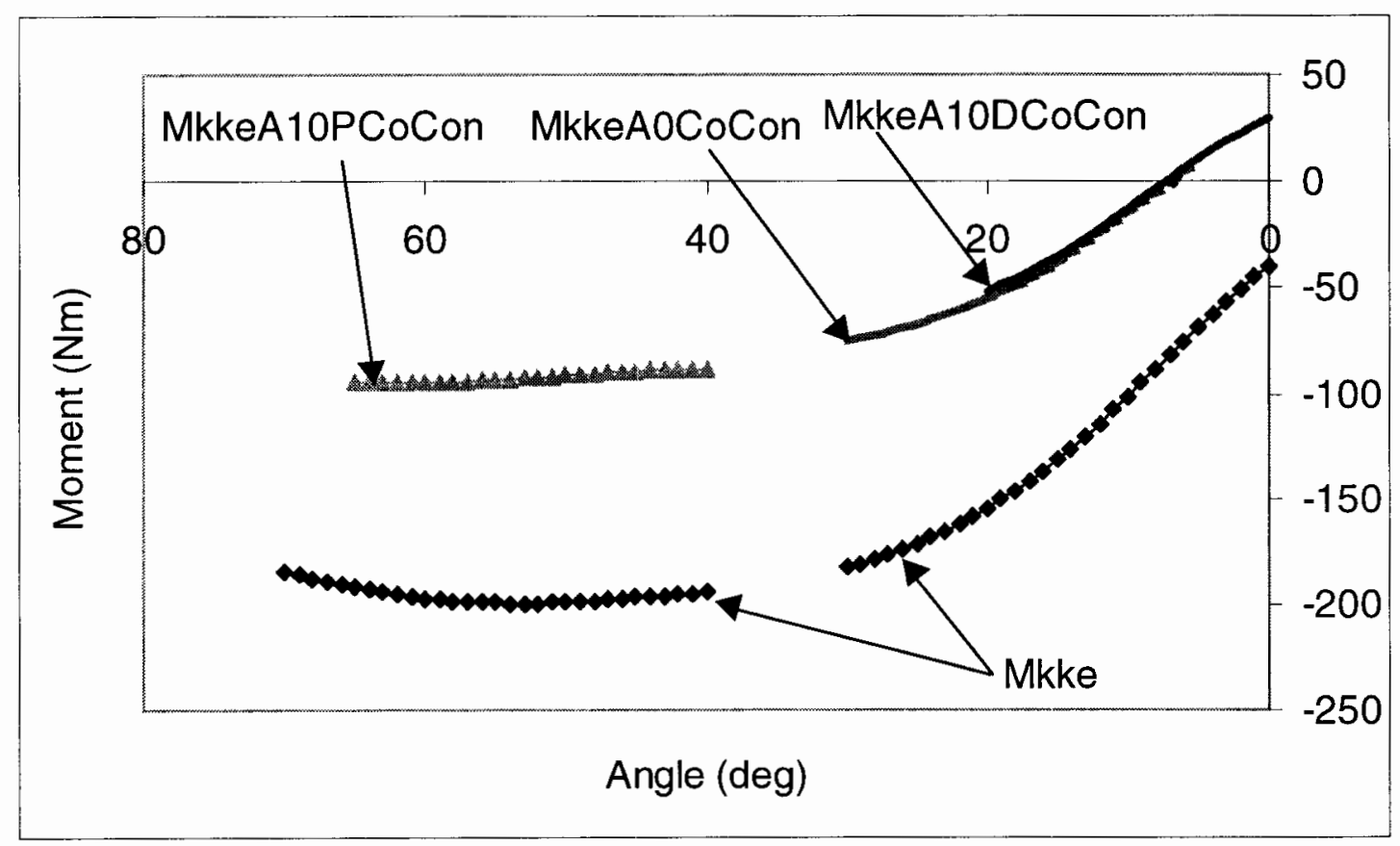

Figure 5.28 Maximum isometric knee extension moments for gait-specific joint interactions and ranges of motion simulated by SIMM. The conditions simulated represented: (a) knee extension joint angles with no cocontraction (Mkke); and (b) knee extension joint angles with cocontraction (CoCon). During the simulations where cocontraction was implemented, the ankle was kept at neutral, ten degrees of plantar flexion, and ten degrees of dorsi flexion (A0, A10P, and A10D respectively). 
To account for the combined effects of muscle length and muscle contraction velocity embedded in the EMG signals, dynamic surface EMG were also collected for isokinetic concentric contractions about the ankle and knee joints at different angular velocities. The testing procedure was the same as for the nMVIC, but the ranges of motion tested were larger. In other words, one of the joints of interest was fixed at the positions indicated in Table 5.6, while the other joint of interest was made to go through a larger range of motion compared to the nMVIC conditions, and at different velocities (see Table 5.6).

\begin{tabular}{|c|c|c|c|c|c|}
\hline $\begin{array}{c}\text { Knee Position } \\
\text { (degrees) }\end{array}$ & \multicolumn{2}{|c|}{ Ankle Action } & Ankle Position & \multicolumn{2}{c|}{ Knee Action } \\
\hline $0 \mathrm{KF}$ & -10 to 20 & 20 to -10 & 0 & $\mathrm{KF}^{3}$ & $\mathrm{KE}^{4}$ \\
\hline $7 \mathrm{KF}$ & -10 to 17 & 17 to -10 & -10 & 0 to 34 & 34 to 0 \\
\hline $20 \mathrm{KF}$ & -20 to 15 & 15 to -20 & -20 & 40 to 70 & 70 to 5 \\
\hline $55 \mathrm{KF}$ & -35 to 5 & 5 to -35 & 10 & 0 to 20 & 20 to 0 \\
\hline
\end{tabular}

1: Dorsi Flexion

2: Plantar Flexion

3: Knee Flexion

4: Knee Extension

Table 5.6.

Positions for the ankle-knee joint interactions tested, and the respective joint ranges of motion during isokinetic testing conditions. All values are in degrees. For the ankle joint, negative values indicate plantar flexion; positive values indicate dorsi flexion.

To determine the range of isokinetic velocities that were tested, data from the database of the gait lab for five normal adult subjects were examined. Six trials had been collected for each of the subjects except one that had five trials in the database. Joint velocities were determined from one full gait cycle for the joint interactions identified in Table 5.3 for the left and right lower extremities. Since original processing output did not include angular velocities, a program was written employing the relationship between 
power and work (see equation 5.4), which were data available as part of the original output, to determine the joint velocities:

$$
P=W \omega
$$

The output data have been included in Appendix J. It was observed that there was no consistency across subjects with respect to the joint velocities during various phases and events throughout the gait cycle, which included the joint interactions investigated in the present study (see Appendix J). Consequently, isokinetic testing angular velocities that spanned the ranges observed in Appendix $\mathrm{J}$ were selected on the basis of recommendations of other researchers who have implemented isokinetic testing with normal adults for the knee and ankle joints (Dvir, 1996; Evetovich et al., 1999; Ghori et al., 1995; Gleeson and Mercer, 1996; Goslin and Charteris, 1979; Kaufman, 1991b; Kellis and Baltzopoulos, 1996; Khalaf et al., 2000; Moffroid et al., 1969; Osterning et al., 1983; Rothstein, 1987; Scudder, 1980; Seger and Thorstensson, 1994; Westing, 1991; Westing et al., 1990). However, an upper velocity limit was imposed in the selection since the isokinetic dynamometer that was used for the purposes of the present study could allow angular velocities up to $250 \mathrm{dps}$. Thus, isokinetic knee flexions and extensions were tested at $60,120,190$, and 250 dps. Ankle isokinetic plantar and dorsi flexions were tested at $30,60,90$, and $120 \mathrm{dps}$. The order of velocities was selected randomly.

To test and simulate the gait-like knee and ankle joint interactions, i.e., to test the effect of the biarticular muscle groups involved in knee flexion-extension and ankle plantar-dorsi flexion with the hip joint fixed, two assisting devices were used. The first was a KinCom attachment, the "universal stabilizer", which was used to adjust the knee joint at different angles when the ankle was being tested. Determination of the joint angle at each of the fixed joint positions was accomplished via goniometry (Clarkson and Gilewich, 1989). The anatomical landmark that was used for aligning the center of rotation of the goniometer was the LLFE. Each of the axes of the goniometer was aligned with the respective lines drawn previously on the lower extremity of the subject; that between the LGRT landmark and the LLFE landmark, and the one between the LLFE and the LLM landmarks. 
Another assisting device that was used for stabilizing the joint that needed to be fixed was an ankle brace, which was custom-made for the purposes of this project. It was used for stabilizing the ankle at different angles when the knee joint was being tested. Determination of the joint angle at each of the fixed joint positions was accomplished via goniometry (Clarkson and Gilewich, 1989). However, the goniometer that was used for the ankle joint was a protractor that was attached on the brace (see Figure 5.29b). The anatomical landmark that was used for aligning the center of rotation of the brace was the ULLM.

The superior, sagittal and frontal views of the ankle brace can be seen in figures $5.29 \mathrm{a}$ through $5.29 \mathrm{c}$. The brace consisted of a plastic, polymer (polypropylene) foot base that made it tough enough and of minimum weight. The base was covered with hypoallergenic padding (see Figure 5.29a). At either side of the foot base there were two adjustable foot plates to prevent medio-lateral movement of the foot during knee flexion and extension (see Figure 5.29a and Figure 5.29b). At the back, there was an adjustable heel rest to ensure that the axis of rotation of the ankle could be lined up with that of the ankle joint (see Figure 5.29a). On either side of the brace two adjustable attachments were placed (see Figure 5.29b). The attachments were adjustable in the superior-inferior and antero-posterior directions such that the axis of rotation of the ankle joint could be brought in line with the axis of rotation of the brace in the sagittal plane. The brace was fixed on the shank by two longitudinal attachments on which four fixing, heightadjustable, supplemental devices were attached (see Figure 5.29c). These supplemental devices, after being attached on to the shank, they were connected via velcro straps (see Figure 5.29c). All connections and attachments were done with special care so that there was no interference with the recording electrodes (see Figure 5.29d). At the sides of the brace a protractor was glued so that the ankle angle could be monitored (see Figure $5.29 \mathrm{~b}$ ). The components of the brace were specifically designed in a way that, when assembled, they would not cause any damage to the protractor. The foot was kept on the foot plate at all times with the use of two velcro straps where by, one stabilized the heel and kept it on the foot plate (see Figure 5.29b and Figure 5.29c), and the other kept the rest of the foot on the plate (see Figure 5.29b and Figure 5.29c). The whole design of the 
brace, in order to be flexible and accommodating to the lower extremity sizes of all subjects that participated in this project was based on the anthropometric data from Pheasant (1996) for the United States adult male population.

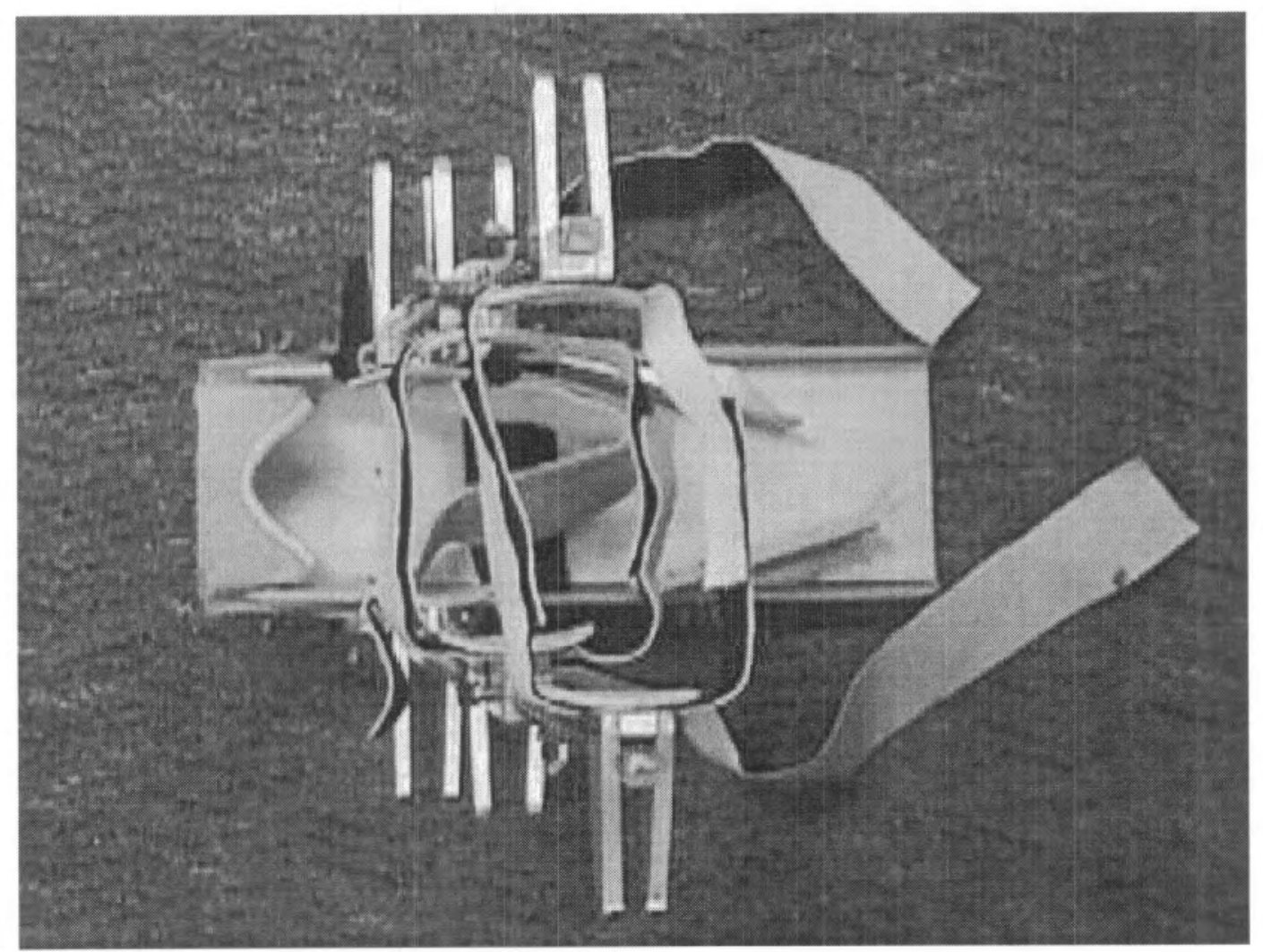

Figure 5.29a.

A superior view of the brace that was used for controlling the position of the ankle joint during isokinetic knee flexions and extensions. 


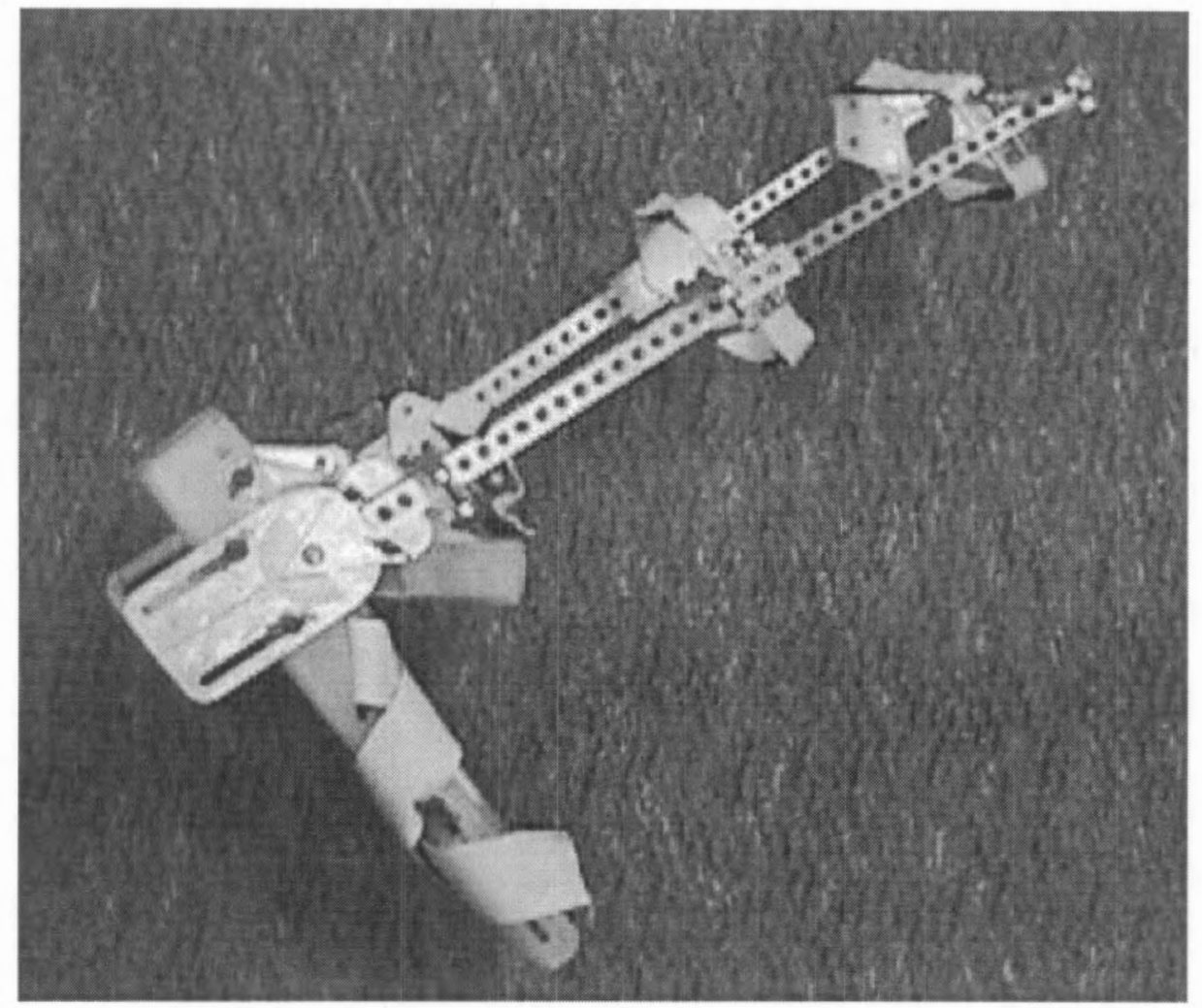

Figure 5.29b. A sagital view of the brace that was used for controlling the position of the ankle joint during isokinetic knee flexions and extensions. 


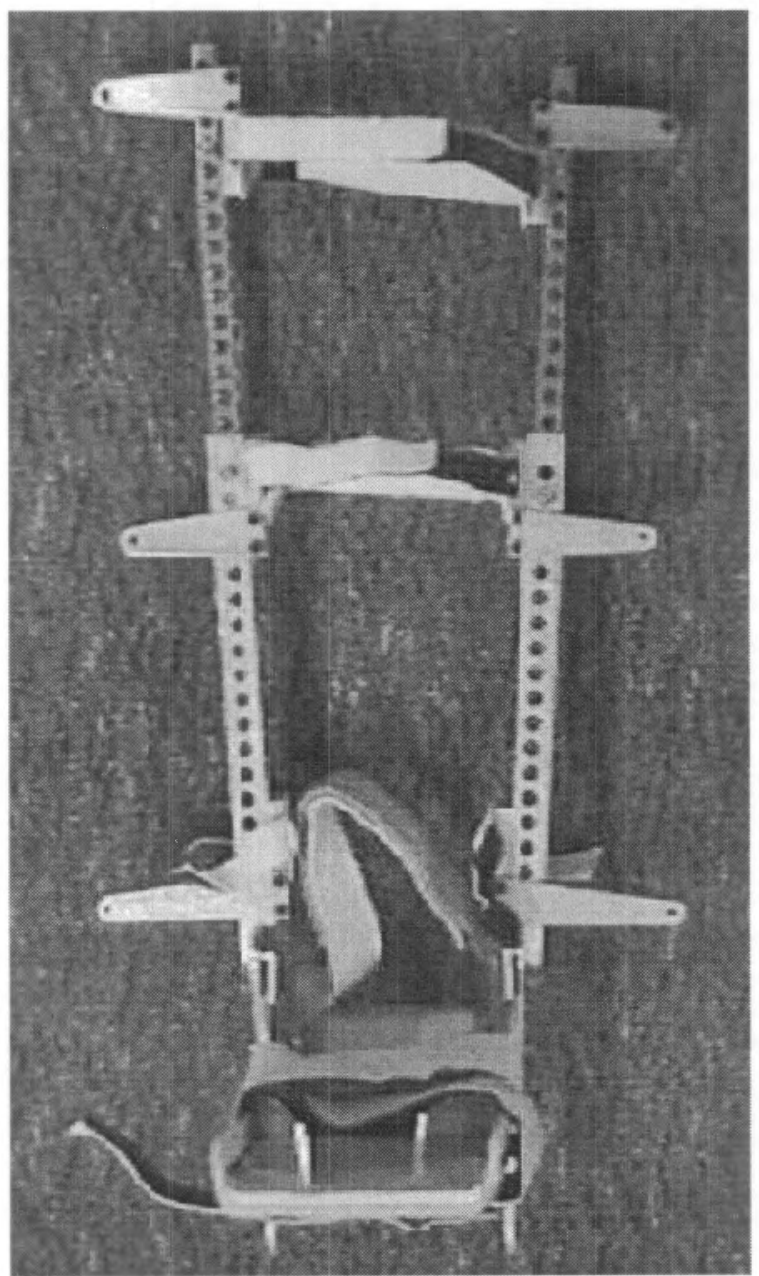

Figure 5.29c.A frontal view of the brace that was used for controlling the position of the ankle joint during isokinetic knee flexions and extensions. 


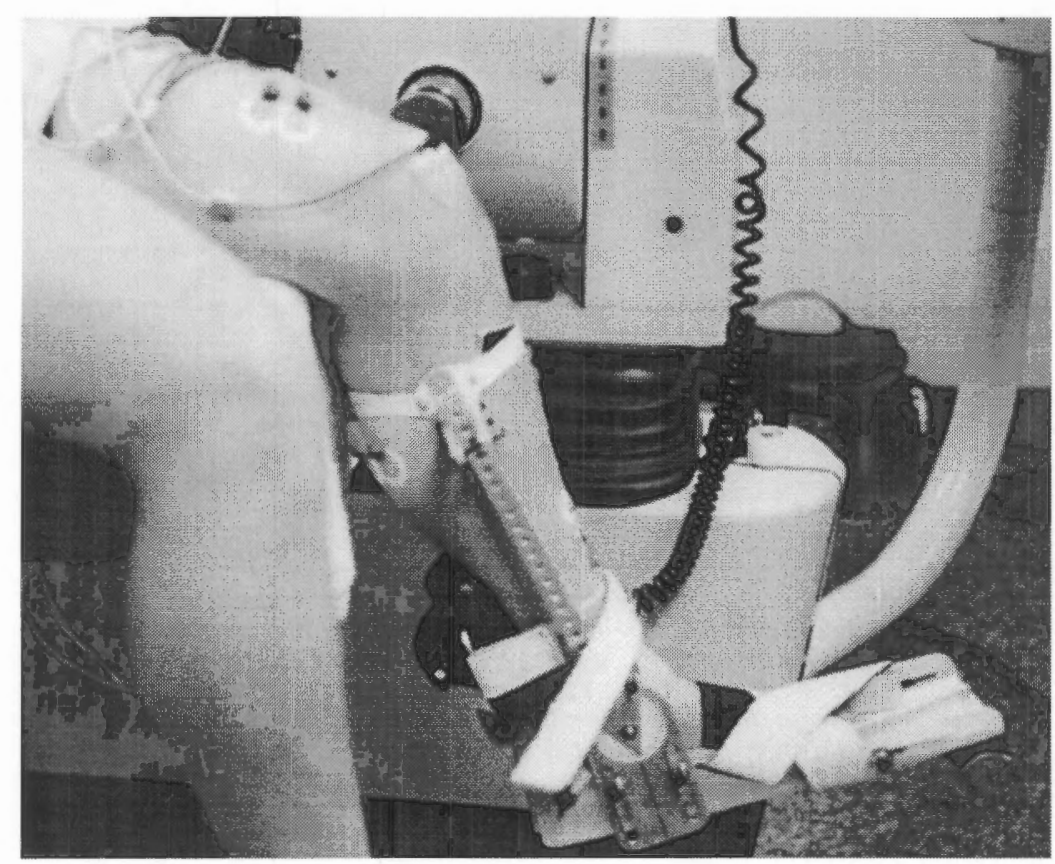

Figure 5.29d. Placement of the brace on the subject in such a way that there was no interference with the electrodes placed on the gastrocnemius.

For the use of the ankle brace to be effective, each subject who participated in the project was asked to provide feedback after each trial whether he felt his heel was touching the foot-rest-plate of the brace throughout the trial. If the subject was not feeling with his heel the foot-rest-plate of the brace throughout the trial, then the trial was discarded and repeated.

The KinCom pre-load force was set at $22 \mathrm{lbs}$ for the knee flexion and extension trials, and at $4.5 \mathrm{lbs}$ for the ankle and plantar flexion trials. Prior to any movement being allowed to commence, this force had to be applied by the distal segment of the joint that was being tested, i.e., by the segment that was attached to the KinCom load cell. Consequently, it was ensured that muscle tension was being produced prior to any movement, and, therefore, elctromechanical delays were being accounted for. Furthermore, the isokinetic dynamometer accelerations and decelerations at the beginning and the end of each trial were set at low to account for the electromechanical delays as well as for the acceleration and impact artifact issues reported by other researchers 
(Gransberg and Knuttson, 1983; Murray et al., 1982; Sapega et al., 1982; Winter, 1981). No KinCom impact artifact was observed during this protocol.

For each joint calibration isokinetic trial a silent period of one to three seconds was recorded at the beginning and the end of the trial, i.e., prior to its start and after its completion. During that silent period all EMG, tilt sensors and analog KinCom inputs were being recorded to establish any EMG and torque biases. The KinCom lever arm was placed at an angle of five degrees greater than the starting and ending angle of each trial to account for inertia effects over the joint range of interest.

Each subject was asked to perform two to three MVC through the joint range of interest while the researcher provided verbal motivation throughout each trial. Due to hardware limitations, one trial was stored of every two consecutive trials where the force output was similar, i.e., the second trial of the two similar consecutive ones was being stored for further processing.

Joint excursions spanned the ranges of motion and joint interactions observed during normal walking gait. At all times the anatomical position was defined as the zero degree joint position. Joint moments were determined from the force data and the moment arms measured at the beginning of each joint testing when the subject was sitted on the isokinetic dynamometer seat.

Feedback to the subjects

At the end of each test, the subject was given verbal feedback about the performance in terms of kinematics, based upon the immediate observations by the researcher. In addition, when the results of the study became available, the researcher contacted each subject and provided them with feedback about their walking technique. The researcher discussed with each subject the walking patterns observed for the general population, as they have been identified in the literature, and then, when deemed appropriate, suggestions were made for alterations in the subject's walking technique to improve efficiency in terms of the ground reaction forces experienced, and the walking posture exhibited. 


\section{Gait Kinematics and Kinetics}

The kinematic and kinetic data from the gait evaluation were processed using the standard National Institutes of Health (NIH) motion analysis software for VICON, C3D binary files, MOVE3D. A model was constructed as it is outlined in section "Inverse Dynamics Model Development" and it was inputted in the software (see Appendix B) as an input file. The output provided kinematic information about the subject's lower extremity positions in space. At the same time, coupled with the results form the force

plates, i.e., the ground reaction forces (vertical, antero-posterior, and medio-lateral) and moments, the same software, implementing an inverse dynamics approach, was used to output the net joint moments, of which, knee flexion-extension and ankle plantar-dorsi flexion were used for the purposes of this project. The mass of each of the body segments of interest, the three principal moments of inertia, the velocities and accelerations, of each segment required for the inverse dynamics approach, along with the COP of the resultant GRF, were all determined via MOVE3D (see Appendix C).

\section{Electromyographic Signals}

The raw EMG signals after the initial processing at the receiver and transmitter level, where they were bandpassed between $10 \mathrm{~Hz}$ and $500 \mathrm{~Hz}$, and an overall gain of 2,000 was implemented, they were A/D converted. After the A/D conversion, they were corrected for any DC offset that might be present, and then they were full wave rectified. From each of the calibration EMG signals base line noise was removed by subtracting the corresponding resting EMG level, determined during the non-force producing period at the beginning or at the end of each trial. The time interval for each analog channel, over which the base line noise was calculated, was determined by the researcher upon visual inspection of each signal. The data points over the resting EMG period were averaged and then subtracted from the respective channel.

The raw EMG obtained from the walking trials, after their initial processing at the hardware level, were also A/D converted and full wave rectified. Base line resting EMG level noise was removed by subtracting the resting EMG level obtained from the 
calibration trials that involved the respective muscles (only eight muscles at a time could be monitored during the calibration, whereas twelve muscles were monitored during the walking trials).

In addition, all EMG signals were filtered with a critically damped, second order low pass butterworth filter (Olney and Winter, 1985; White and Winter, 1993; Winter and Patla, 1997). The filter was bi-directional to correct for the phase shift. Adjustments were made to the filter coefficients internally when the code was produced using the Matlab software (The Mathworks Inc.) so that the order of the filter was maintained (Winter and Patla, 1997). For the cut off frequencies the inclination was to adopt the coefficients suggested by White and Winter (1993). However, prior to adopting the proposed coefficients, their suitability for the purposes of the present project was verified.

To verify the cut off frequencies previously proposed (White and Winter, 1993), a power spectral density analysis was performed for the gastrocnemius muscle that has 50 percent slow twitch and 50 percent fast twitch fibers. It was found that the signal retained over 99.8 percent of its power between the second and third harmonics (see Figure 5.30). Thus, the respective cut off frequency agreed with the suggestions of White and Winter (1993).

Consequently their suggested low pass cut off frequencies of $2.5 \mathrm{~Hz}$ for the motion EMG data and $3.0 \mathrm{~Hz}$ for the calibration EMG data were adopted. Furthermore, the results of the power spectral density analysis and the cut off frequencies proposed by White and Winter (1993), all fall within the optimal range of cut off frequencies reported in the past for a similar set of investigated muscles (Olney and Winter, 1985). 

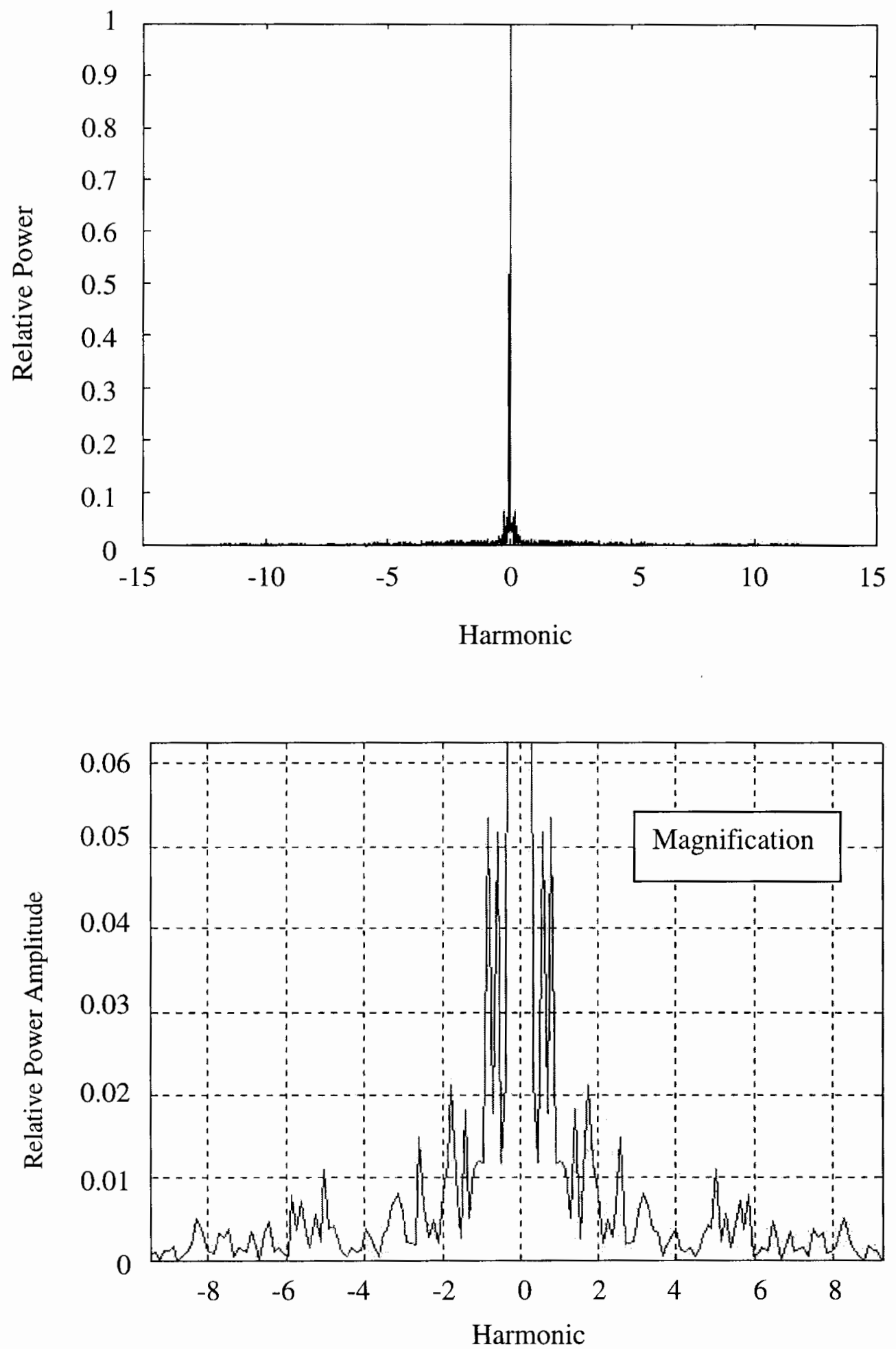

Figure 5.30. Power spectral density analysis to identify the low pass cut off frequency for the electromyogram. For the medial gastrocnemius, most of the power of the signal (over 99.8 percent) is maintained between the second and third harmonics. 


\section{Estimation of the Cocontraction Index}

The procedure for estimating the gain of the muscles that cross the specific joint of interest and the process for determining the individual muscle tendon forces that result in the respective internal predicted joint moment have been described previously (see section "EMG-driven model development". In general, five input parameters were required to produce the desired output: a) the externally measured joint moment; b) the individual muscle moment arms; c) the tension-length and tension-velocity relationships for each musculotendon unit of interest as a function of the respective joint angle and angular velocity; d) the individual physiologic cross sectional area of each muscle of interest; and e) the respective electromyographic signals relative to corresponding electromyographic signals (normalization factors) obtained during generation of the, externally measured, maximum joint moment in nMVIC.

Bias was removed from the joint torques generated during the calibration trials by firstly adding or subtracting the gravitational forces determined during the calibration trials, and, secondly, by subtracting the passive moments, adopting the approach of Audu and Davy (1985) for their estimation (see Figure 5.31 and Figure 5.32). During knee flexion, for some trials at the and of the range of motion there was a tendency to have an over shoot indicating a knee extension force. To correct for this response at the end of the knee flexion range of motion, any overshoot, resulting in a force sign opposite to that expected for the specific trial, was equated to zero force. The corrected force, then, was converted to units of Newton and then to moment using the measured moment arm (in meters). The moment was subsequently sampled over as many sample points, and averaged, as it took for every degree change in the angle. Finally it was corrected for the moment generated by the passive structures (see Figure 5.30). For each of the isokinetic calibration trials the corresponding angular velocity was derived from the positional data. Trials that were kept for further analysis were only those where the subject had achieved the desired velocity ( \pm 5 degrees per second) and maintained it for a range of consecutive joint angles. The rest of the trials were discarded. The net joint moments that were determined via inverse dynamics analysis were also corrected for the passive structures with the same approach. 


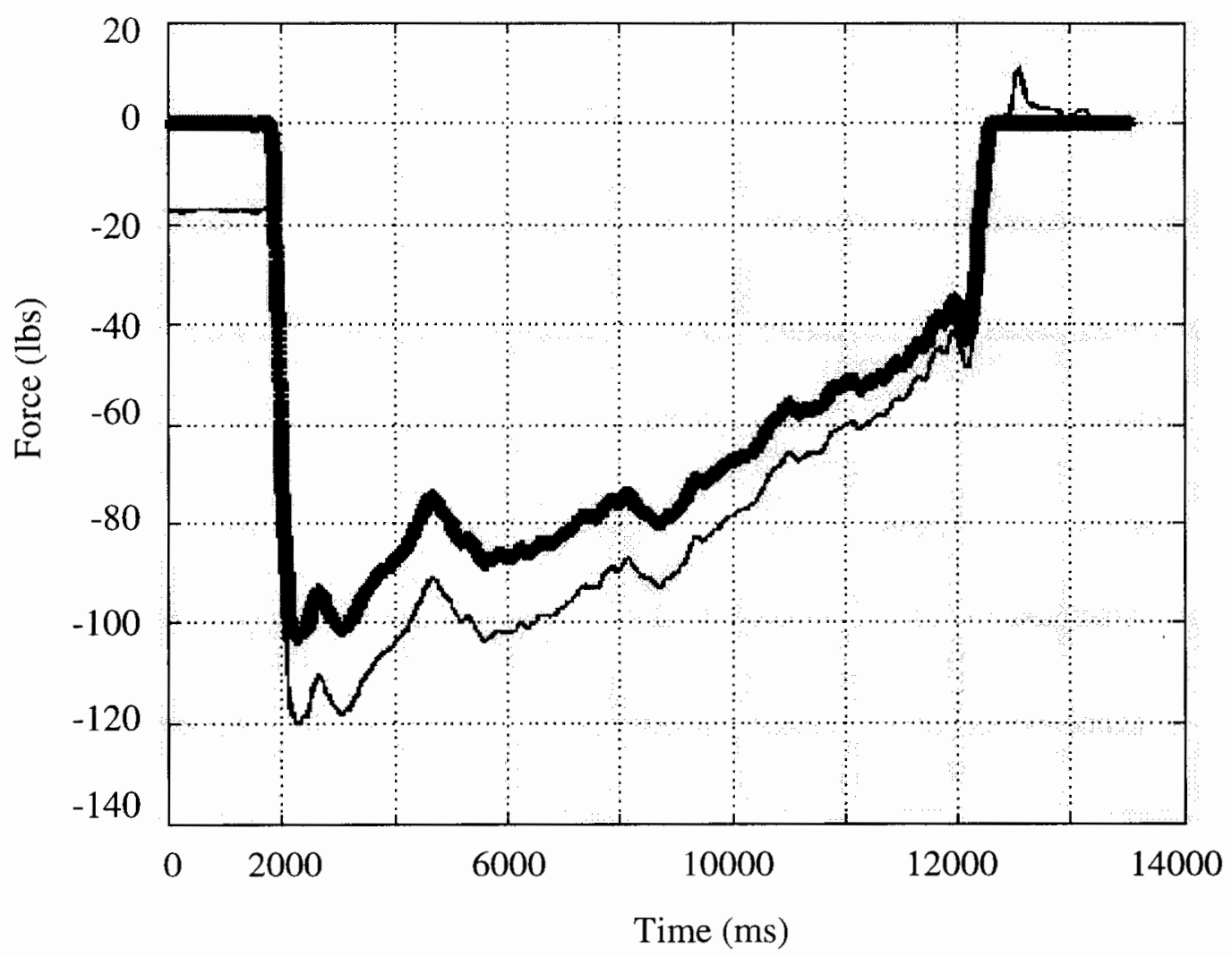

Figure 5.31. Force produced on the isokinetic dynamometer during a pilot study trial (thin line). The force was corrected for gravity (thick line). 


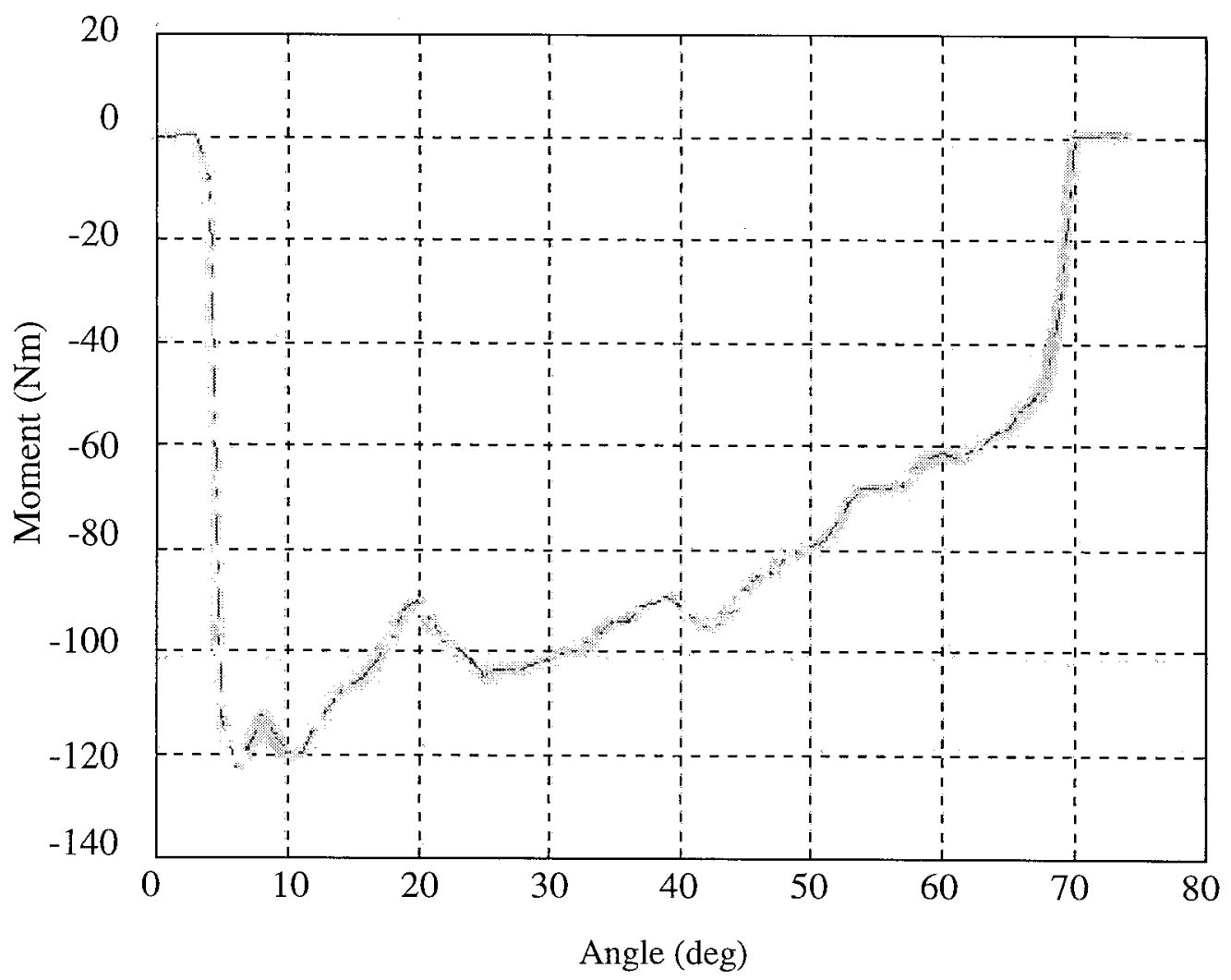

Figure 5.32. The corrected, for gravity, knee flexion moment (thick trace) during knee flexion on the isokinetic dynamometer, and the corrected moment for the moments generated by the passive structures (doted line).

The kinematic output from the MOVE3D was formatted to become input files to a software for interactive musculoskeletal modeling (SIMM, Musculographics, Inc.). Processing of the MOVE3D kinematic output via SIMM provided the musculotendon units moment arms of interest for the estimation of the respective muscle. Prior to the determination of the musculotendon units moment arms, the skeletal model of SIMM was scaled to match the anthropometric characteristics of the specific subject that data was collected for (see section "Gait Evaluation" under "Data Collection and Analysis Procedures". Thus, musculotendon moment arms were subject specific.

SIMM was also used to produce the maximum force output of each musculotendon unit of interest as a function of the kinematic, positional and derived velocity, input to the model. Each musculotendon unit force output was expressed relative to its corresponding 
maximum isometric force. Thus, the unitless tension-length and tension-velocity relationships for each musculotendon units of interest were obtained. The rest of the model inputs have been explained previously (see section "Aspects of the Model").

Once individual musculotendon forces were obtained then the cocontraction index was determined as it was indicated in figure 3.1:

$$
\text { Cocontraction_Index }=\frac{\sum F_{\text {tot }}}{\sum F_{\text {egon }}}-1
$$

where $\sum F_{t o t}$ represents the sum of all the individual musculotendon unit forces from the muscles that were active, and $\sum F_{\text {agon }}$ represents the sum of the individual musculotendon unit forces whose activity is responsible for the net joint moment that was determined via the inverse dynamics analysis.

Finally, t-tests were performed to compare three different methods of estimating the cocontraction index. The three methods were:

a) estimation of the cocontraction index from the electromyographic records alone;

b) estimation of the cocontraction index from the electromyographic records. corrected for the tension-length and tension-velocity relationships of each musculotendon unit of interest; and

c) estimation of the cocontraction index using the EMG-driven model proposed in this project.

\section{EMG-driven Model Validation}

The performance of the model was assessed by comparing the net sagittal muscle joint moment, determined from the inverse dynamics analysis and corrected for the passive structures (measured moment), with the sum of the predicted muscle moments, determined from the respective musculotendon moment arms crossed with the corresponding predicted musculotendon units forces, derived from the EMG-driven model. The degree of accuracy of the model was a function of the degree of "closeness" between the two curves, which was quantitatively assessed in the following ways:

1. By calculating the root mean square difference between the two curves (RMS). 
2. By expressing the joint moments RMS relative to the respective maximum, joint moment computed during the calibration trials and during dynamic walking (NRMS).

3. Through a correlation analysis of the two curves, computing the correlation coefficient ( $\mathrm{r}$ ).

4. By computing the coefficient of determination $\left(r^{2}\right)$.

5. Through a distribution analysis of the squared correlation coefficients.

6. By examining the predicted gain.

The predicted gain, which represents the subject's muscle force per unit area, should be falling within physiological limits. Literature reviews have indicated that those values can range from 10 to $100 \mathrm{Ncm}^{-2}$ (Kaufman et al., 1991a) or from 5.9 to $34.5 \mathrm{Ncm}^{-2}$ (Powell et al., 1984). Furthermore, the gain, as it represents the subject's muscle stress required to generate the necessary musculotendon forces for ambulation, it is relatively independent from the model's ability to simulate the measured net joint moment.

The correlation coefficient on the other hand provides a quantitative measure of the model's ability to simulate a predicted joint moment curve whose pattern is similar to that of the measured joint moment. In other words, it is a measure of the magnitude of the relationship between the predicted and measured joint moments. However, the correlation coefficient does not provide information with regard to the differences in magnitude between the measured and predicted moments.

The coefficient of determination represents a measure of the amount of variance in the measured moment that is predictable from the total predicted musculotendon moment. It quantifies the amount of variability in the measured moment that can be accounted for in the predicted moment (Lieber, 1994). High $\mathrm{r}^{2}$ values with high frequency density distributions, as reflected in the histograms, along with valid subject gains, indicate that the model accounts for the variability in the joint moment, imply that the model can accurately predict the dynamic behavior of the net joint moments, and it generates an accurate simulation of joint loads during walking. However, it too does not provide information with regard to the differences in magnitude between the measured and predicted moments. These differences can be quantified using the RMS error. 
The RMS error gives a measure of the magnitude of the error between the two curves. Such a measurement cannot be extracted from the gain and from the correlational statistics $\left(r, r^{2}\right)$. Consequently, all the measures mentioned above were needed to assess the validity of the model. However, for the above analysis to be valid, all muscles crossing the joint of interest must be considered.

\section{Muscle activity assumptions}

Some assumptions were made to account for some of the muscles that were not monitored. The Semitendinosus and Semimembranosus muscles were monitored as one muscle, i.e. medial hamstrings, for the purposes of collecting the EMG data. However, in the EMG-driven model they were treated differently, each having its own muscle parameters. The basis of this approach is the fact that they share the same nerve innervation, they both have the same function (Moore, 1992) and they have the same phasic activity during the walking gait (Perry, 1992).

The same approach was used for the Vastus Intermedius and its relationship to the Vastus Lateralis and Vastus Medialis that were monitored. However, of the two Vastii heads that were monitored, the phasic activity of Vastus Intermedius was mapped after the Vastus Lateralis. The action taken with the Vastus Intermedius was felt important since it is one of the three Vastii heads, are all single joint muscles, who dominate knee extension. Also, the Tibialis Posterior, using the same approach, and on the basis of the data presented by Perry (1992), was grouped, in terms of its phasic activity, with the Soleus (it has common innervation with the Soleus and the Gastrocnemius, although the branch of the nerve that innervates them is different), and, in terms of its level of activation, with the Gastrocnemius.

\section{Overall Model Implementation and Processing}

The overall implementation of the model and processing of the data at any stage of this project was very involving. Apart from the modeling that was done in the form of generating model input files for the MOVE3D (for the purposes of the inverse dynamics analysis) and for SIMM (a model input joint file was created, and it was associated with transformations of the kinematic output from MOVE3D so that the segments of each 
joint were allowed to rotate and translate about and along specific joint coordinate systems, and a model muscle file was generated to implement the musculotendon unit parameters, as was deemed to be appropriate) five programs were developed to achieve the goals of this project. A brief description of each stage according to the outline of figure 3.1 follows below:

i) The first program implemented all the signal processing procedures on the signals collected during calibration (EMG, tilt sensors, force, and position) and during gait (EMG). The output was the low passed filtered signals and converted to physical units (for the force, and position).

ii) The second program involved the mapping of the SIMM simulations with the data from the nMVIC calibration trials. The program was computing the dimensionless tension-length and tension velocity relationships for each musculotendon unit of interest, the constant velocity interval during the trial and the outputs were:

a) the maximum joint moment generated of all the nMVIC trials;

b) the joint position and interaction during which it occurred; and

c) the corresponding normalization factors for the rest of the EMGs (calibration and gait).

iii) The third program was for the rest of the calibration trials with the same inputs as in the second program (only for the rest of the calibration trials) but the output was different. The program was mapping the SIMM simulation data with the corresponding experimental data, the dimensionless tension-length and tension velocity relationships for each musculotendon unit of interest, the constant velocity interval during the trial and the outputs were:

a) the corrected for gravity and passive structures isokinetic moments;

b) the normalized EMGs;

c) the corresponding musculotendon unit lengths for the constant velocity interval; 
d) the corresponding unitless tension-length and tension-velocity of each musculotendon unit of interest specific to the trial; and

e) the corresponding constant velocity interval moment arms.

iv) The fourth program computed and outputted:

a) the predicted individual musculotendon unit forces;

b) the corresponding moments;

c) the root mean square error (actual and normalized);

d) the gain that would minimize the error; and

e) the cocontraction indexes on the basis of the three schemes identified earlier.

v) The fifth program interacted with the kinetic and kinematic output from MOVE3D, the walking gait EMGs and the corresponding normalization factors, the simulation SIMM output which was used to compute the dimensionless tension-length and tension-velocity relationships, and the scaled subject specific musculotendon moment arms. The output was:

a) the predicted individual musculotendon unit forces;

b) the corresponding predicted joint moments;

c) the root mean square error (actual and normalized);

d) the joint specific gain that would minimize the error;

e) the cocontraction indexes under each of the three schemes identified previously; and

f) a global gain for both the ankle and the knee joints that would minimize the overall error along with the corresponding predicted moment, root mean square error, and cocontraction indexes. 


\section{CHAPTER 6}

\section{RESULTS AND DISCUSSION}

The purpose of this study was to construct and validate an EMG-driven model to quantify cocontraction among the musculotendon units involved in knee flexion/extension and ankle plantar/dorsi flexion during normal walking gait. The previous chapters have demonstrated: a) the development of the EMG-driven model; b) the development of an inverse dynamics model, currently implemented via MOVE3D (NIH software), to validating the EMG-driven model output; and c) the procedures of data collection to estimate the model parameters and to facilitate the model output.

In this chapter the results for each model are presented and discussed for the three subjects. Whenever appropriate, outputs are presented as case studies. In the first sections, the results from the inverse dynamics analysis, which served the purpose of validating the output of the EMG-driven model (when that was implemented in walking gait), are presented and issues associated with them are discussed. In the later sections the results from the EMG-driven model are presented and the model performance is assessed via a) the gain estimations; b) the computations of the correlation coefficients

$(r)$ and the respective coefficients of determination $\left(r^{2}\right)$ between the predicted and inverse dynamics measured joint moments; and c) the respective root mean squares of the errors $(R M S)$ as well as the normalized root mean squares of the errors (NRMS). 
Inverse Dynamics

$\underline{\text { Kinematics }}$

\section{$\underline{\text { Joint Angles }}$}

The intra subject kinematic data were consistent and within the ranges reported in the literature for normal walking gait (see Appendix K). Accurate determination of the positional data is very important for the completion of the inverse dynamics analysis since on the basis of this information the joint angular accelerations, velocities and the respective accelerations of the centers of masses of the segments are being computed (Soutas-Little, 1991). Consequently, the kinematic output of MOVE3D was verified by writing code to complement the information presented in Chapter 4 . The output can be seen in figure 6.1 for the knee and in figure 6.2 for the ankle. It can be verified that the output from the code of this author and the output from MOVE3D is almost identical for both joints.

\section{$\underline{\text { Kinetics }}$}

\section{$\underline{\text { Kinetic Output }}$}

Similarly to the kinematic data, the intra subject kinetic output was generally consistent and, when normalized to the subjects body weight, it was within the ranges reported in the literature for normal walking gait (see Appendix L for the joint moments). Normalizing to individual body weight $(\mathrm{Nm} / \mathrm{BW})$ the peak ankle plantar flexor moment peaks ranged between 1.61 to $1.68(\mathrm{Nm} / \mathrm{BW}), 1.42$ to $1.54(\mathrm{Nm} / \mathrm{BW})$ and 1.23 to 1.83 $(\mathrm{Nm} / \mathrm{BW})$, for subjects $\mathrm{SN}, \mathrm{SC}$ and $\mathrm{SA}$ respectively. With regard to the first knee extensor peak, knee flexor peak, and second knee extensor peak the moments ranged between 0.57 to $0.7(\mathrm{Nm} / \mathrm{BW}),-0.39$ to $-0.43(\mathrm{Nm} / \mathrm{BW})$ and 0.19 to $0.42(\mathrm{Nm} / \mathrm{BW})$ for subject $\mathrm{SN}, 0.58$ to $0.89(\mathrm{Nm} / \mathrm{BW}),-0.24$ to $-0.31(\mathrm{Nm} / \mathrm{BW})$ and 0.19 to 0.26 $(\mathrm{Nm} / \mathrm{BW})$ for subject $\mathrm{SC}$ and 0.67 to $0.81(\mathrm{Nm} / \mathrm{BW}),-0.37$ to $-0.54(\mathrm{Nm} / \mathrm{BW})$ and 0.31 to $0.46(\mathrm{Nm} / \mathrm{BW})$ for subject SA respectively. All these results agree, and fall within the ranges of data provided by Meglan and Todd (1994) and Winter et al. (1995), although the plantar flexor moment of SA appear to fall at the lower end of the reported values in 


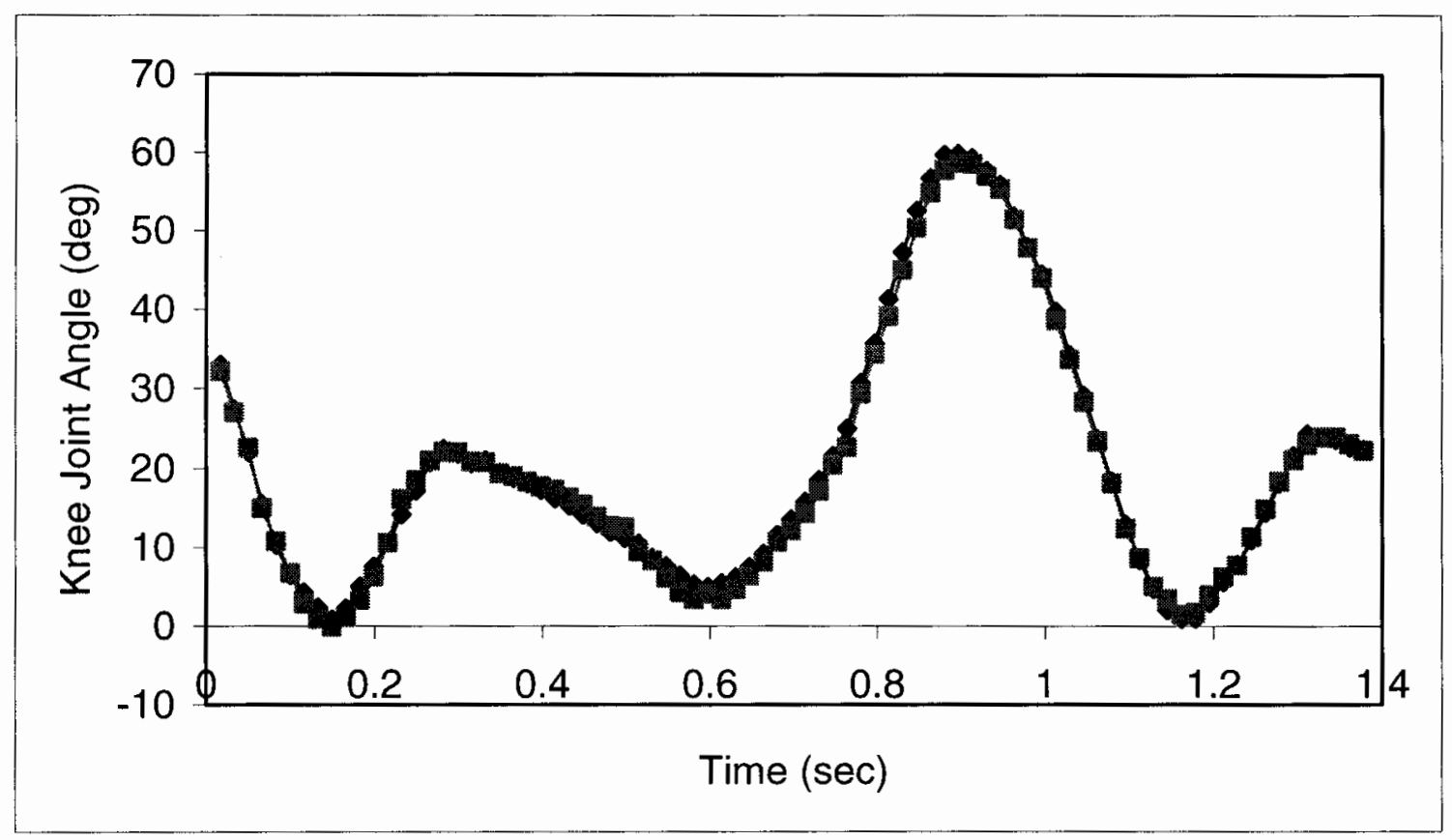

Figure 6.1. Comparison of the kinematic output from the MOVE3D and from custom written code for the kinematics of the knee joint (trial from subject SN005).

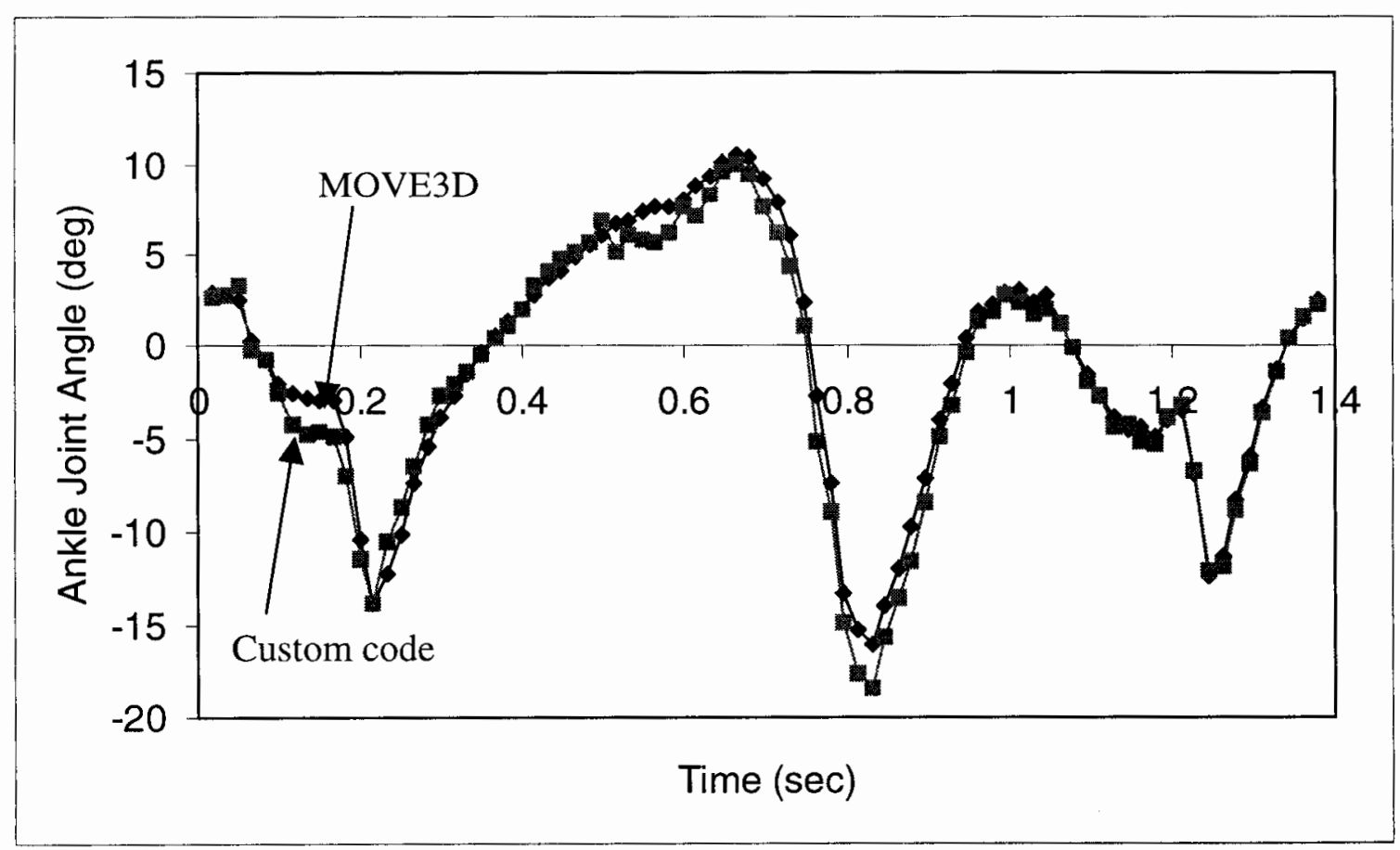

Figure 6.2. Comparison of the kinematic output from the MOVE3D and from custom written code for the kinematics of the ankle joint (trial from subject SN005). 
the literature. The reason might be that for that trial he walked a little slower than the rest of his trials. However, since velocity of walking could not be assessed instantaneously, and because he met the criteria for a successful trial outlined in "Data Collection and Analysis Procedures" the trial was deemed to be appropriate.

Once each net joint moment was obtained, the passive component was removed to obtain the net joint moment due to the muscles crossing the joint of interest alone (see Figure 6.3 for the knee and Figure 6.4 for the ankle). It is clear that the contribution of the passive structures to the net joint knee flexion/extension and ankle plantar/dorsi

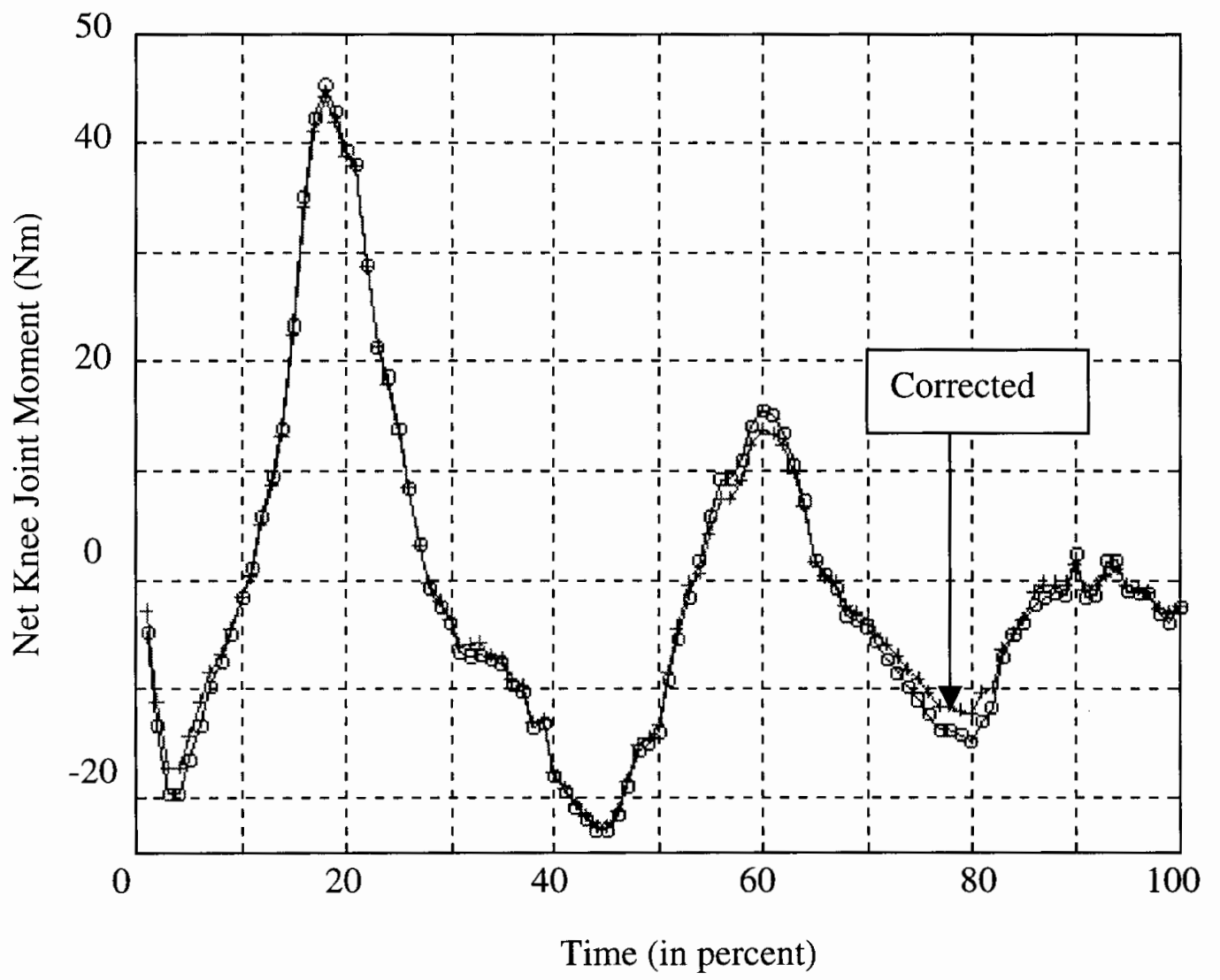

Figure 6.3. Knee joint moment during gait, estimated from inverse dynamics (o) and corrected (+) for passive contributions (trial from subject SN005). 

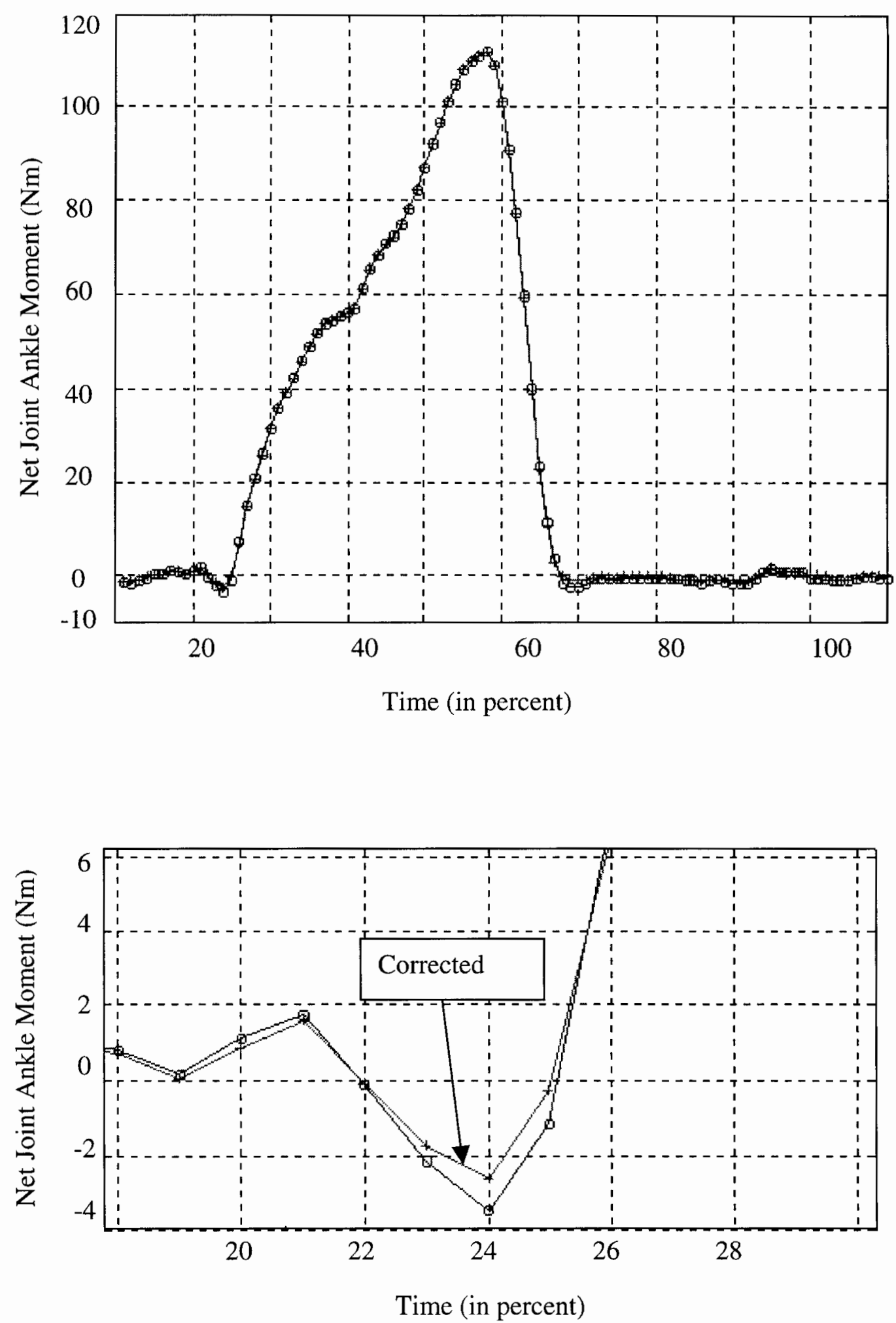

Figure 6.4. Ankle joint moment during gait, estimated from inverse dynamics (o) and corrected $(+)$ for passive contributions (trial from subject SN005). 
flexion moments is not significant. With respect to the present model, the reason may be that the transfer function representing the passive structures rises exponentially at the ends of the ranges of motion in the sagittal plane. Since sagittal knee and ankle joint range of motion during normal gait does not approach the extreme ranges of motion available to a normal individual, the contribution of the passive structures is not significant.

Furthermore, especially with respect to the knee, Lloyd and Buchanan (1996) have determined that during knee motion on the isokinetic dynamometer there is very little contribution from the passive structures to the joint moments at the knee in the sagittal plane. Their modeling approach determined that the passive tissues support primarily the external moments generated at the frontal plane, i.e., the varus/valgus moments, by as much as 83 percent, while the muscles accounted for the other 17 percent. Thus, the soft tissues were the prime means of achieving stability at the knee in the frontal plane. The muscles, by means of cocontraction and some specific muscle activation, accounted for the rest of the support, especially as the knee was becoming more extended. The results of their study were complemented by the investigation of Schipplein and Andriacchi (1991) who have shown that during gait patients with varus deformities optimize and achieve support of the excessive valgus moments by increasing their net external flexion/extension moments. It may be speculated, therefore, that at the knee joint the neuromuscular system has the specialized function of accounting primarily for the moments generated in the sagittal plane, while assisting support in other planes also. Such a speculation would seem to justify the focus of investigators who construct neuromuscular models for the knee on the sagittal plane.

In Chapter 4 where the inverse dynamics model was being constructed it was stated that one of the fundamental assumptions in the implementation of this model as well as in the implementation of the inverse dynamics model that was ultimately used to determine the net joint moments was that the body was assumed to be a series of linked rigid segments. Consequently, in the process of determining the joint moments the potential violation of that assumption was assessed by observing the relative distances of markers throughout the gait cycle for one subject who was not among the subjects participating in 
the study. Non-violation of the rigid segments assumption would imply minimum distance changes among the markers placed on the selected anatomical landmarks (see Table 6.1 for the results of this analysis for randomly selected markers placed on selected anatomical landmarks according to the targeting protocol proposed in Chapter 5).

\begin{tabular}{||l|l|c|c||}
\hline \multicolumn{1}{|c|}{ First Point } & \multicolumn{1}{|c|}{ Second Point } & $\begin{array}{c}\text { Relative Distance } \\
\text { (mean - in mm })\end{array}$ & Standard Deviation \\
\hline $\begin{array}{l}\text { Right Anterior } \\
\text { Superior Iliac } \\
\text { Spine }\end{array}$ & $\begin{array}{l}\text { Left Anterior } \\
\text { Superior Iliac Spine }\end{array}$ & 253 & 2.3 \\
\hline Greater Trochanter & $\begin{array}{l}\text { Lateral Femoral } \\
\text { Epicondyle }\end{array}$ & 360 & 2.7 \\
\hline $\begin{array}{l}\text { Medial Femoral } \\
\text { Epicondyle }\end{array}$ & $\begin{array}{l}\text { Lateral Femoral } \\
\text { Epicondyle }\end{array}$ & 115 & 4.9 \\
\hline Tibial Tuberocity & Anterior Shank & 131 & 2.7 \\
\hline Anterior Shank & Posterior Shank & 148 & 2.4 \\
\hline Medial Malelolus & Lateral Malelolus & 103 & 1.8 \\
\hline Calcaneus & Fifth Metatarsal & 196 & 4.0 \\
\hline
\end{tabular}

Table 6.1.

Relative distance between certain markers throughout the gait cycle to test the validity of the rigid body assumption for the purposes of the inverse dynamics model.

The standard deviations of this analysis indicate that marker movement during walking gait was relatively small. The largest variability appeared between the medial and lateral femoral epicondyles. Andriacchi, (1999) suggested that in his experience most motion artifact is obtained by markers that are placed on bony prominences. That may be the reason for the variability, as both, the medial and lateral femoral epicondyles are bony prominences. However, even this variability is less compared to the variabilities presented from other investigators (Soutas-Little, 1991). Since the above information represents raw data, when the effect of skin motion artifact is factored in the 
analysis, it may be observed that the rigid segment assumption, if it is violated, the effect is minimum. Confidence in the stability of each marker's position during movement is of particular importance since it affects the predicted joint centers about which the joint moments are being computed. It can be concluded, therefore, that the targeting protocol and the measurement system, given the results from the sensitivity analysis performed on the motion capture system (see chapter 5), provided commensurable accuracy for estimating the kinematic and kinetic outputs from the inverse dynamics model. Furthermore, accurate representation of the anatomical landmarks selected for targeting allowed appropriate scaling of the musculoskeletal model implemented later in the software for interactive musculoskeletal modeling (SIMM -Musculographics Inc.) for the estimation of subject-specific muscle moment arms.

An important observation here is that the calculated moment arms in SIMM are only a function of the joint kinematics which, in turn. take place about the instantaneous axis of rotation. However, when the net joint moments are being computed in MOVE3D, their determination is taking place about fixed joint centers of rotation, at least in the version of the software that was available at the time the analysis took place. Consistency between the computation of joint moments about the same joint center of rotation between SIMM (as the sum of all the muscle moments generated about the joint) and the inverse dynamics model is vital for compatibility between the outputs from the two approaches. The inverse dynamics model proposed in chapter 4 accompanied by the targeting protocol implemented in chapter 5 could allow the computation of the helical axes and the definition of the instantaneous joint center of rotation, with respect to the computation of the sagittal plane moments, as the piercing point of the axis to the sagittal plane.

Another parameter that may have affected the computed net joint moments is the estimation of the body segment parameters. Use of cadaveric tables (Dempster, 1955) and treating the body segments as geometric objects (Kepple, 1997), as it is done in MOVE3D, may have resulted in a misrepresentation of the masses and inertial properties of the foot, thigh and shank segments of the living subjects in this study. 
There are several reports in the literature for the segment parameters of living humans (McConville et al., 1980; Zatsiorsky and Seluyanov, 1985; Zatsiorsky, 1983). McConville and his collaborators (1980) established the relationship between human body size and its mass distribution properties. They used a combination of stereophotometric and anthropometric techniques to measure a group of 31 living male subjects and to ultimately provide 85 body size variables. They determined volume and moments of inertia for the body and body segments. Furthermore, they used the correlation between moments of inertia and body size, as well as the correlation between moments of inertia of separate parts and relevant dimensions of these parts to derive regression equations for predicting mass distribution characteristics for other humans. Zatsiorsky and Seluyanov $(1983,1985)$ determined masses and inertial properties of human body segments using radioisotope methods on 100 young healthy males. Initially (1983), they expressed segment masses and moments of inertia relative to respective weights and segment lengths; they also developed regression equations to predict segment masses and moments of inertia from subject height and weight. Later (1985) they provided regression equations for the prediction of segment masses and moments of inertia from other anthropometric variables.

Consequently, a new inverse dynamics model, as outlined in the chapter 3, could take advantage of these more recent developments. It could allow the integration of the regression equations developed by Zatsiorsky and Seluyanov $(1983,1985)$ for a more accurate estimation of the inertial properties of each body segment of interest. However, in the present study the computed net joint moments were within the ranges reported previously by others.

\section{EMG-driven Model Performance Validation and Results}

\section{EMG patterns}

Prior to laying out the results of the study that pertain to the validation of the model it is considered appropriate to emphasize some temporal observations. It appeared that throughout the calibration procedure there was selective recruitment of some muscles for similar contractions, i.e, not all synergists were activated in the same pattern for the same 
movement. An example of this can be seen in figure 6.5, figure 6.6 and figure 6.7 which represent knee flexion at an angular velocity of five degrees per second with the ankle at the neutral, ten degrees dorsi flexed and ten degrees plantar flexed respectively. The gastrocnemius muscle, when the ankle was at neutral was silent over the first ten degrees of the knee flexion calibration. It became an active knee flexor, however, for the remaining part of the knee flexion calibration range. For the same calibration knee flexion range, when the foot was positioned at ten degrees of dorsi flexion, both heads of the gastrocnemius were active. In addition, when the ankle was placed at ten degrees of plantar flexion, the gastrocnemius was again active. It can also be observed that some of the normalized EMG values appear to be higher than one. It can also be observed that cocontraction, although very little relative to the exertion effort, it was always present. This was true for almost all of the ankle calibration trials also.

Validation of the temporal aspect of the dynamic EMG signals recorded during the walking trials was achieved via comparison with other studies in which they have beenreported for gait (Rab, 1994). Figure 6.8 and figure 6.9 show the normalized EMG (NEMG) of the muscles crossing each joint of interest along with the respective joint kinematics and kinetics in the sagittal plane for subject SC. These results, i.e., the processed and NEMGs along with the kinetic and kinematic information, are representative of all three subjects and of all the walking trials recorded. 


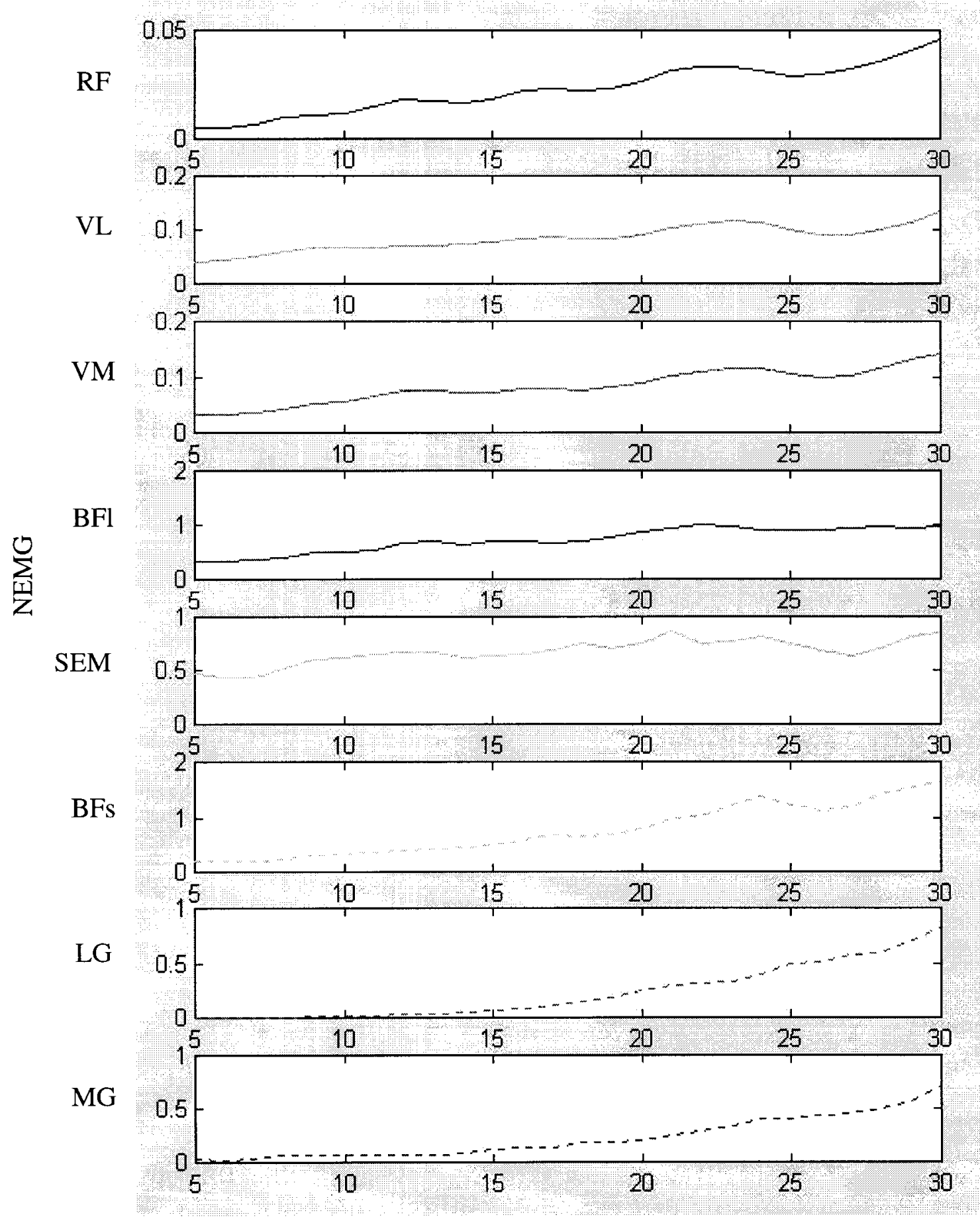

Angle (deg)

Figure 6.5. Selective recruitment (normalized EMG - NEMG) of knee flexor muscles and antagonistic cocontraction (from the bottom: Medial Gastroc, Lateral Gastroc, Biceps Femoris short head, Semimembranosis/Semitendinosis, Biceps Femoris long head, Vastus Medialis, Vastus Lateralis, Rectus Femoris) for knee flexion at five degrees per second with the ankle at neutral. Data from subject SC. 


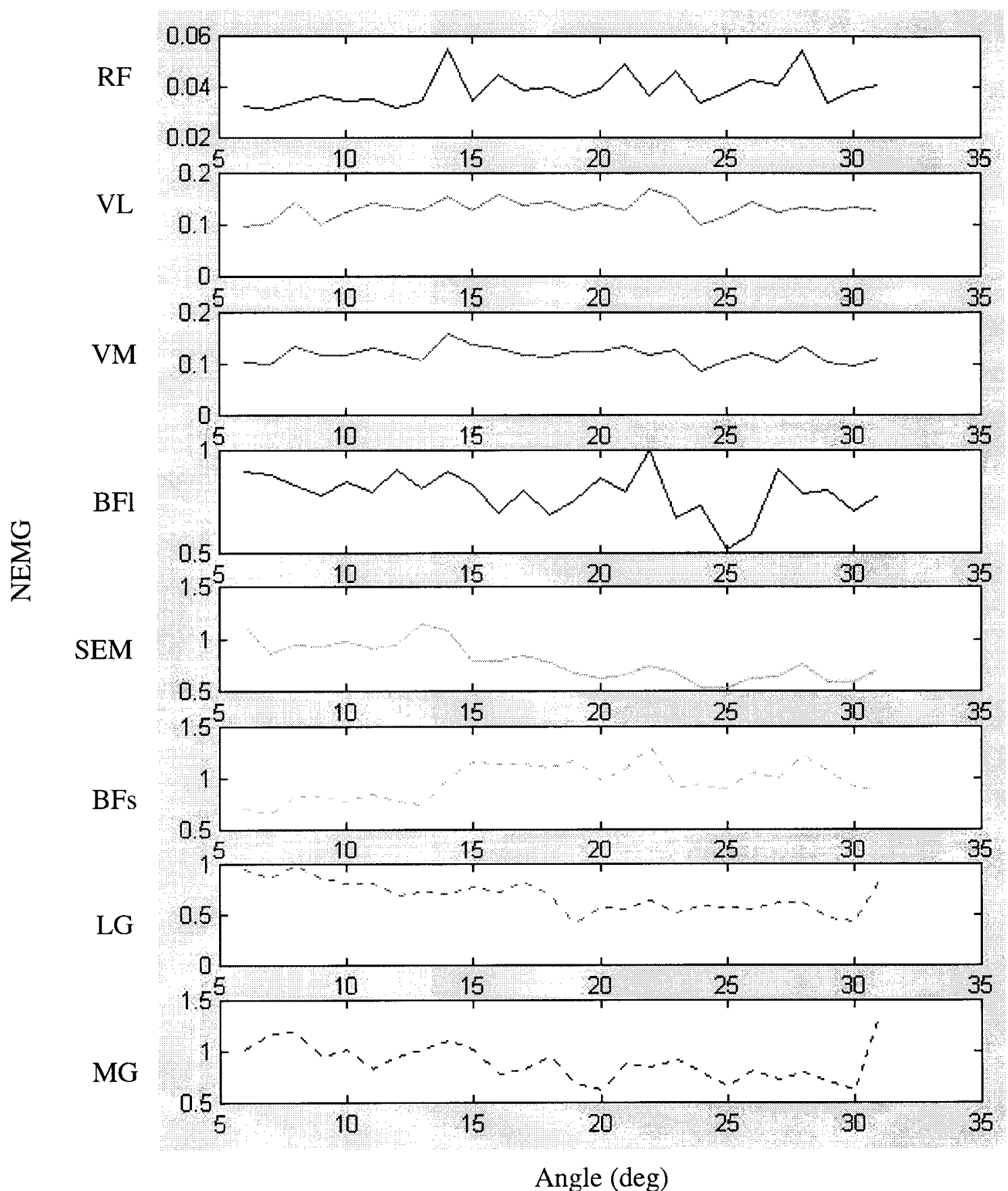

Figure 6.6. Selective recruitment (normalized EMG -NEMG) of knee flexor muscles and antagonistic cocontraction (from the bottom: Medial Gastroc, Lateral Gastroc, Biceps Femoris short head, Semimembranosis/Semitendinosis, Biceps Femoris long head, Vastus Medialis, Vastus Lateralis, Rectus Femoris) for knee flexion at five degrees per second with the ankle at ten degrees of dorsi flexion. Data from subject SC. 


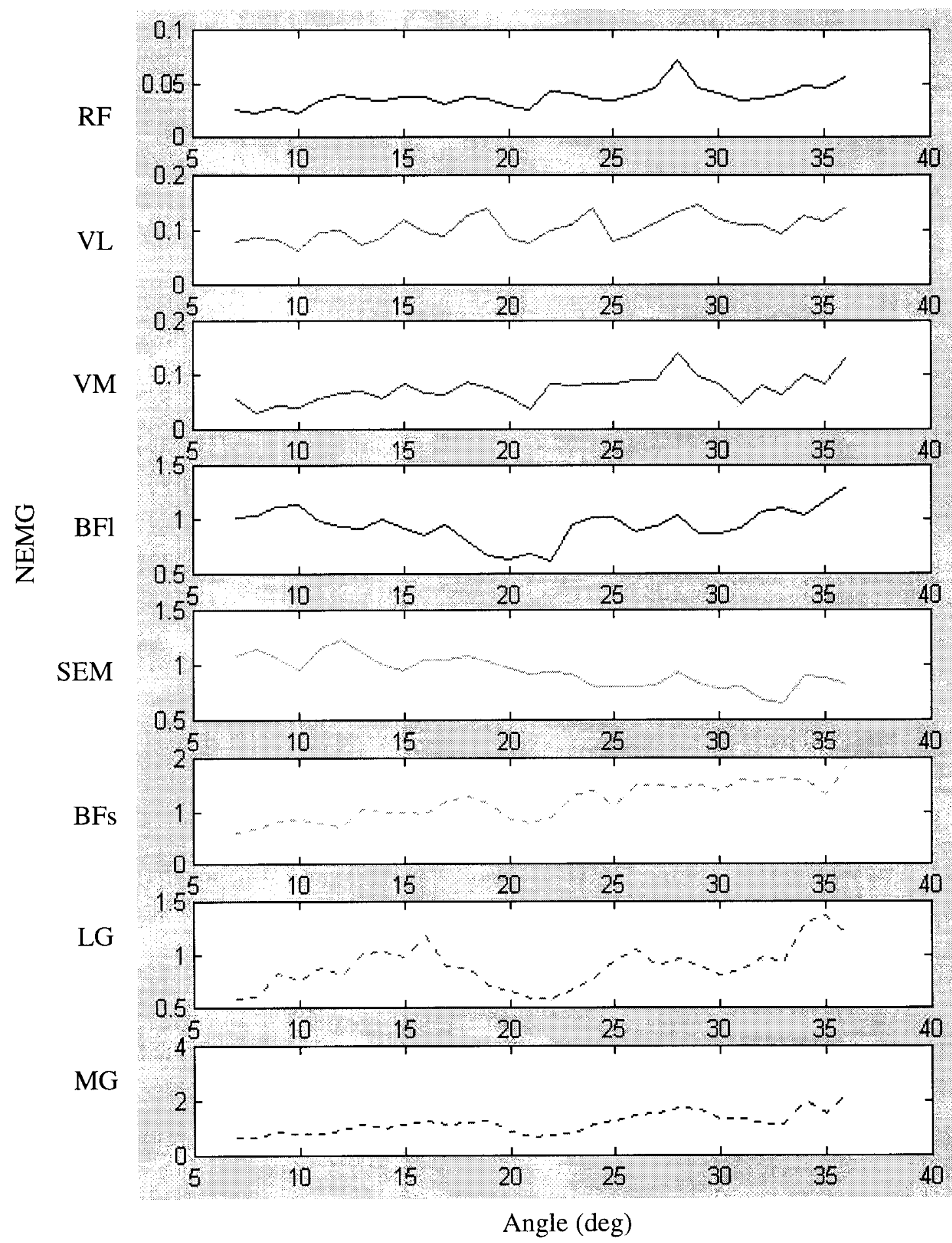

Figure 6.7. Selective recruitment (normalized EMG -NEMG) of knee flexor muscles and antagonistic cocontraction (from the bottom: Medial Gastroc, Lateral Gastroc, Biceps Femoris short head, Semimembranosis/Semitendinosis, Biceps Femoris long head, Vastus Medialis, Vastus Lateralis, Rectus Femoris) for knee flexion at five degrees per second with the ankle at ten degrees of plantar flexion. Data from subject SC. 


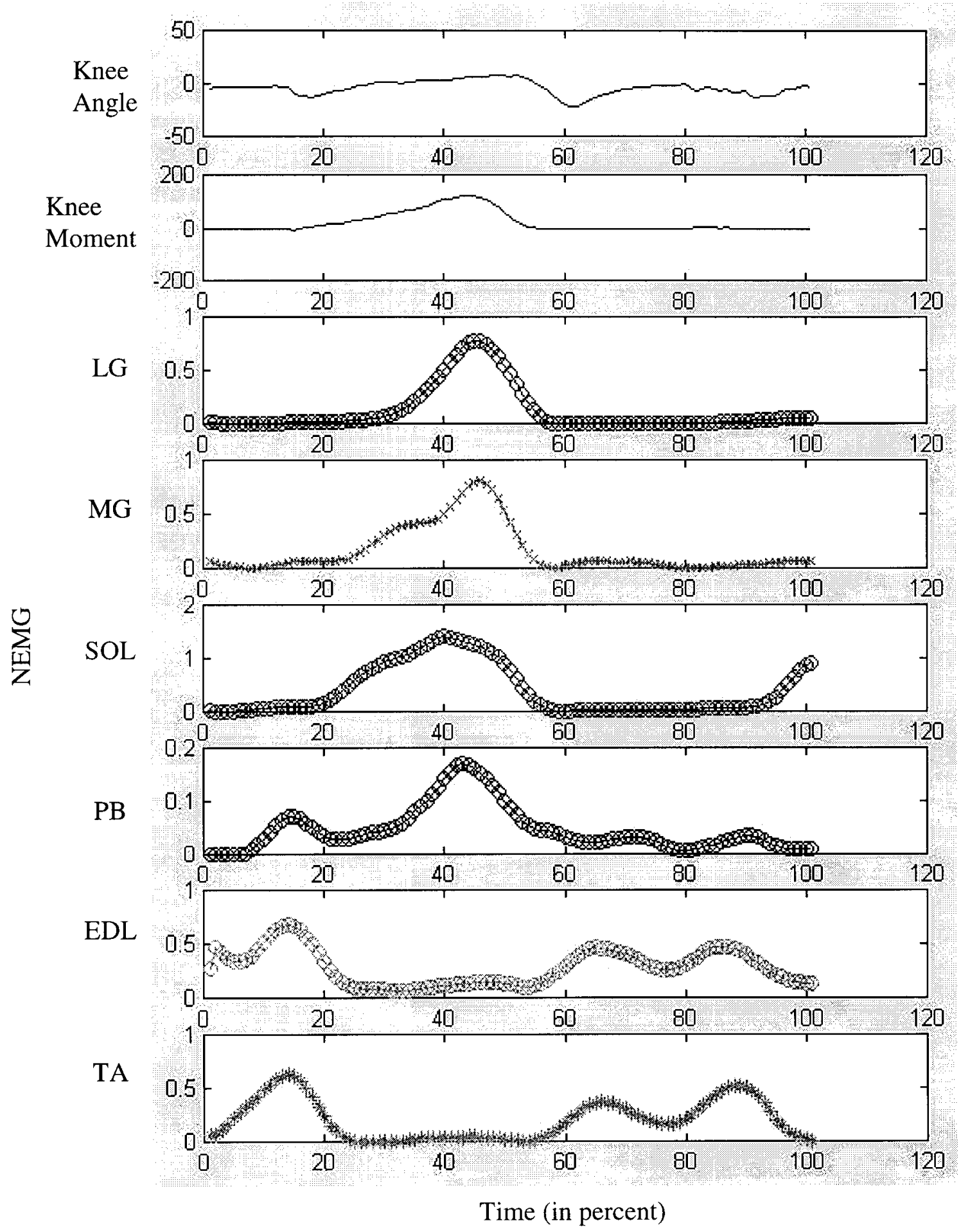

Figure 6.8. Normalized EMG history record for the ankle plantar/dorsi flexors of one subject (SC) during one full gait cycle \pm several frames added on either end of the trial. At the top the ankle joint plantar/dorsi flexion angle (in degrees) and moment $(\mathrm{Nm})$ have been included. Muscle definitions are included in the text. 


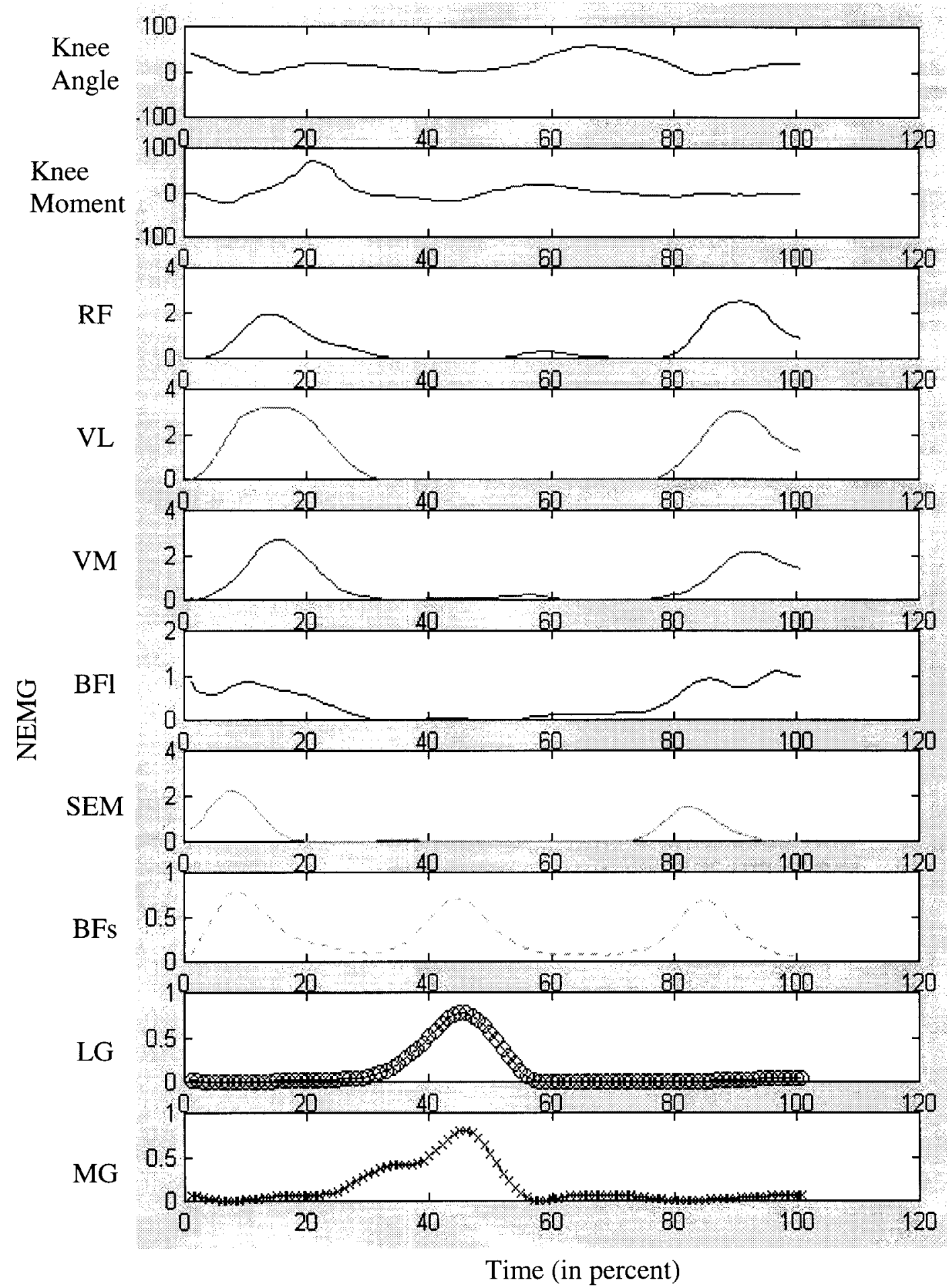

Figure 6.9. Normalized EMG history record for the knee flexors/extensors of one subject (SC) during one full gait cycle \pm several frames added on either end of the trial. At the top the knee joint flexion/extension angle (in degrees) and moment $(\mathrm{Nm})$ have been included. Muscle definitions are included in the text. 


\section{$\underline{\text { Model Output }}$}

All results with respect to the parameters studied have been included in Appendices $M$ through $P$ in detail. Because of the small number of individuals who participated in the study, here a summary will be presented for each subject separately. For the purposes of assessing and validating the model performance six parameters were computed. Among these six parameters, three were used for the purposes of comparing the performance of the model with previous EMG-assisted modeling attempts to investigate muscle force time histories during the walking gait (Hof et al., 1983; Olney, 1982; Olney and Winter, 1985; White and Winter, 1993).

\section{Gain}

To assess the physiological consistency of the model the gain for each subject was computed via the calibration procedure. One gain for each group of muscles that cross the joint of interest. The average calibration gains were between 10.64 and $23.18 \mathrm{~N} \mathrm{~cm}^{2}$ with standard deviations (SD) from 4.36 to 11.41 for the muscles crossing the ankle. For the musculotendon units crossing the knee joint the average gains ranged from 32.62 to $42.54 \mathrm{~N} \mathrm{~cm}^{2}$ with SD ranging from 20.76 to 24.74 . Thus, although the computed gains varied among subjects, these values fall within the physiologically acceptable boundary values of 5.9 to $100 \mathrm{~N} \mathrm{~cm}^{2}$ (Kaufman et al., 1991a; Spector et al., 1980). However, observation of the data in Appendix $\mathrm{M}$ indicates that there was an intra-subject variability among the computed gains also. It has been advocated that since the gain represents the physiological ability over the muscle to produce force per unit of its cross sectional area, it should remain constant if it is to be valid. In other words gain should not change from one exertion to the next (Granata, 1993; Granata and Marras, 1993, 1995; Marras and Granata, 1997; Marras et al., 1999). However, even more careful observation of the data in Appendix $\mathrm{M}$ for each individual subject, indicates that the gains for trials under the exact same conditions were, if not constant for most of them, at least very similar (see bolded trials in Appendix M). Thus the model was consistent and stable for tests of the same conditions, while it predicted physiologically valid gains.

To identify the source of intrasubject variability, the gains were plotted against velocity and against muscle length of the biarticular musculotendon units, which was 
affected by the angle at the adjacent joint (for a representative example see Figure 6.10). The results of this analysis indicate that there was a muscle length and velocity of contraction effect on the estimation of the gains. Such effects were not considered in the present model.

Also, with respect to the computed gains and the calibration trials, it should be mentioned that it can be observed from the calibration trials in Appendix $M$ that not all calibration condition trials have been represented in the tables. Trials where the subject was not able to achieve the predetermined joint angular velocity, or trials which would fit the definition by Redferm (1992) of a ballistic type of contraction were excluded. In other words, only smooth types of contraction were accounted for by the modeling approach and, therefore, were included in the calibration.

In addition to implementing the computed gain from the calibration trials in the model and letting it compute the individual musculotendon unit forces, gains were computed using least squares (LS), i.e., to estimate the best possible gain that would minimize the squared error between the measured and predicted moments. That allowed a direct assessment of the model's internal physiological validity. If the model could compute gains which were physiologically valid and minimized the error, then the physiological response of the model could be considered a valid one. If in addition the predicted LS gains were similar to those of the average calibration gains then the model could be deemed to have internal validity.

Results from this type of analysis have been included in Appendix O. Three types of gain were computed: (a) LS_ankle gain, (b) LS_knee gain, and (c) LS_global gain, a gain that would be considered universal for each subject and it could be applied to all musculotendon units of interest. Predicted gains for the ankle were very similar to the calibration gains for that joint. Predicted gains for the knee, however, were very different from the calibration gains for that joint. The LS_global gains were closer to the gains predicted for the musculotendon units that cross the ankle joint rather. One observation, 

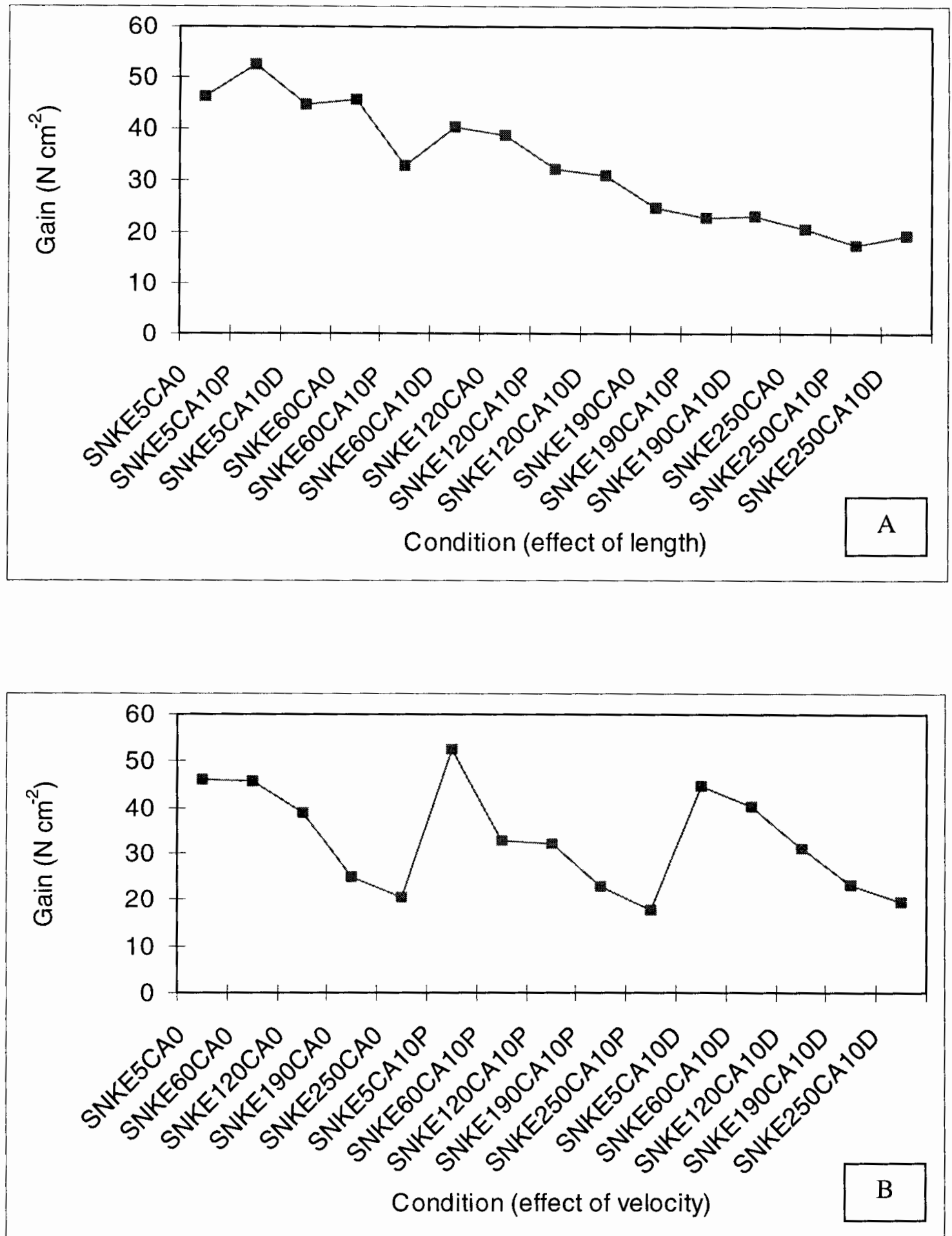

Figure 6.10. Effect of muscle length (A) and velocity (B) on the gain during knee extension isokinetic calibration trials (e.g., SNKE5CA0 implies: subject SN performing Knee Extension at 5 degrees per second concentrically with the Ankle at 0 degrees). 
however, was that all predicted LS gains (another way of referring to them is optimum gains, since they minimized the error) fell within the standard deviations of the predicted from the calibration gains for the respective joints.

In addition, since the calibration gain depends on the external measured moment, which, in turn, depends on the position of the limb, the measured moment is dependent upon the reliability and consistency in which the calibration device tracks the position of the moving segment while maintaining the adjacent segment fixed at one position (Osterning et al., 1983). While the tracking accuracy of the moving segment by the isokinetic dynamometer has previously been verified via the development of a regression transfer function, the position of the fixed segment is usually given very little consideration, while at the same time its movement is the reason for the incorrect estimation of the joint angle (Osterning et al., 1983). To account for such an error two tilt sensors were used to measure the relative angle at the joints of interest, especially of the knee. However, prior to start computing the relative angle between the segments, the need for doing so was validated by assessing the reliability of our fixation of the proximal lower extremity segment using the tilt sensor output from that segment (see Figure 6.11).

The results indicated that since there was virtually no movement of the thigh on the isokinetic dynamometer, our fixation technique was very appropriate, and, in light of these results, the tilt sensor outputs, although recorded, they were not used for the purposes of this study. 


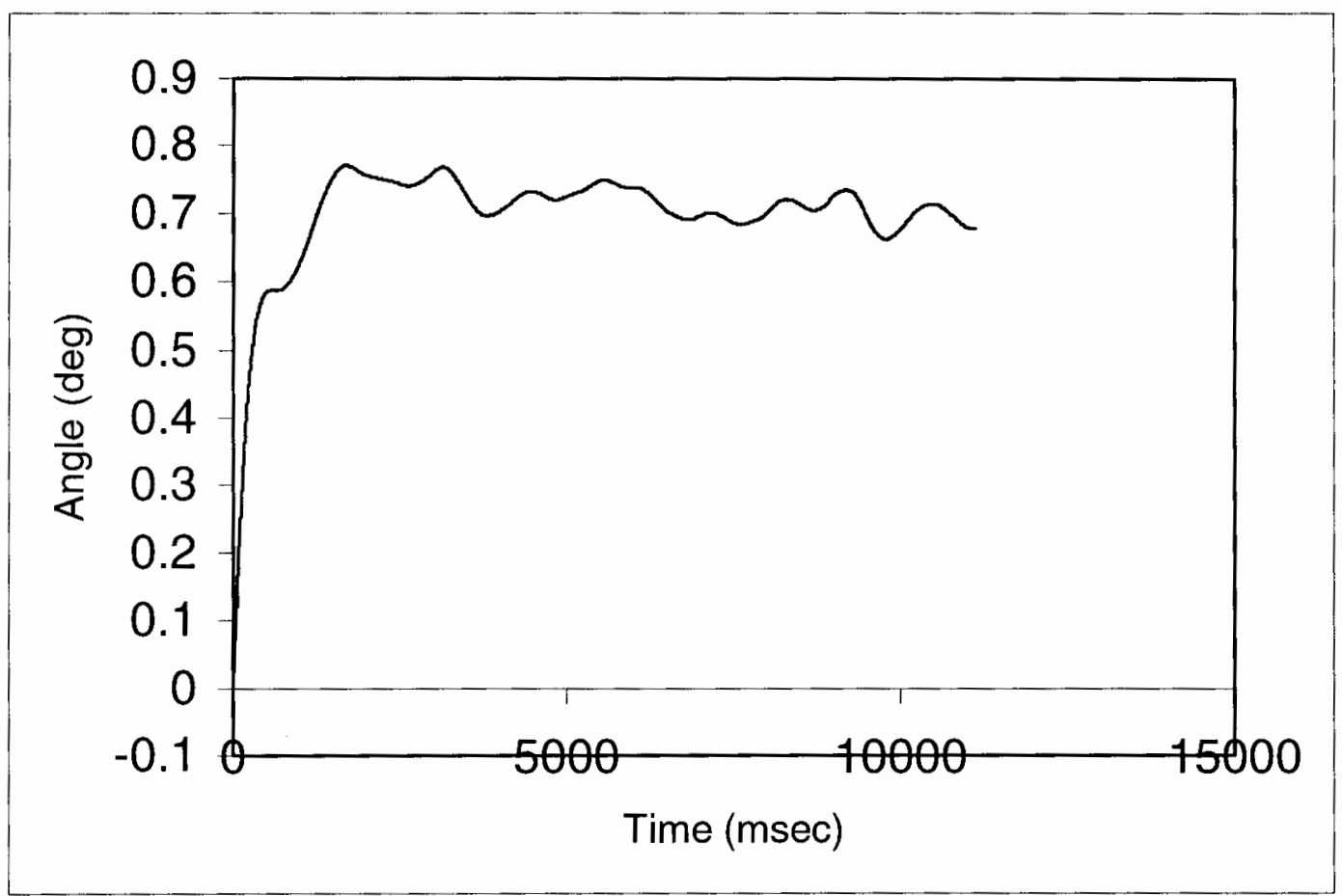

Figure 6.11. Output of the tilt sensor placed on the thigh during knee flexion. There is less than 0.7 degrees difference in the position of the thigh from the beginning of the knee flexion to the end.

\section{Correlations}

The temporal validation of the model was achieved by observing the correlation coefficients ( $r)$ and the coefficients of determination $\left(r^{2}\right)$ between the measured and predicted moments. All results for all trials, i.e., calibration and motion have been included in Appendices $M$ through $O$. The $r$ and $r^{2}$ are both measures of how well the model describes the data. The closer the $r$ values get to 1 indicate that the better a model is as a descriptor of the relation between the predicted and measured moments. On the other hand, $\mathrm{r}$ near 0 indicates that the model cannot predict the temporal variation of the measured joint moment. The $r^{2}$ in addition to being a measure of how well the model describes the measured moment, it takes into account the number of independent variables which reflects the degree of freedom. In other words $r^{2}$, as the coefficient of determination, indicates the percent of variation that can be predicted knowing the 
predictor variable. Thus, for the purpose of completeness, the $r^{2}$ values between the predicted and measured moments have also been computed and included in the results. However, for the purposes of comparison with previous studies the focus will be on the $r$ values.

The correlation coefficients for the gait trials were, in general, high (see Appendix $\mathrm{N}$, and Tables 6.2-6.6). All of them were above 0.8 and most were close to or above 0.9. Such performance indicates good temporal relationships between the predicted and measured moments for both joints of interest. The highest $r$ value for the ankle was 0.97 and for the knee 0.95 , while the lowest were 0.75 and 0.78 respectively. However the average correlation coefficient for each subject was not as high from the calibration procedure (see Table 6.2). The fact that the respective $r$ medians were consistently higher than the $r$ averages (see Appendix N), implies that it was only a few calibration trials that had an adverse effect on the estimation of the average $r$ value. Thus, to assess the temporal performance of the model a distribution of the correlation coefficients was performed (see Figure 6.12). The results indicate a very satisfactory performance of the EMGdriven model. Most of the times the model was able to predict the response of the measured moment, i.e., the shape of each pair of compared curves seem to match closely. That was true for both joints, especially for the ankle, for all movement trials as it can be inferred from the correlation coefficient values $(r)$ (see Figure 6.13 for a representative example). 


\begin{tabular}{|l|c|c|c|c|c|c|}
\hline \multicolumn{1}{|c|}{$\begin{array}{c}\text { Subjects } \\
\text { Parameters }\end{array}$} & \multicolumn{2}{c|}{ SA } & \multicolumn{2}{c|}{ SC } & \multicolumn{2}{c|}{ SN } \\
\hline Ankle & Average & $\begin{array}{c}\text { Standard } \\
\text { Deviation }\end{array}$ & Average & $\begin{array}{c}\text { Standard } \\
\text { Deviation }\end{array}$ & Average & $\begin{array}{c}\text { Standard } \\
\text { Deviation }\end{array}$ \\
\hline Gain & 10.65 & 4.72 & 23.18 & 11.41 & 13.92 & 4.36 \\
\hline$r$ & 0.71 & 0.23 & 0.72 & 0.25 & 0.69 & 0.29 \\
\hline$r^{2}$ & 0.55 & 0.29 & 0.57 & 0.33 & 0.55 & 0.30 \\
\hline RMS & 5.91 & 4.86 & 3.88 & 3.01 & 3.53 & 2.80 \\
\hline NRMS_Env & 30.67 & 24.98 & 18.21 & 15.50 & 16.43 & 6.41 \\
\hline NRMS_MAX & 17.04 & 13.03 & 13.31 & 15.56 & 10.78 & 7.45 \\
\hline Knee & & & & & & \\
\hline Gain & 35.06 & 21.50 & 32.62 & 20.76 & 42.54 & 24.74 \\
\hline$r$ & 0.71 & 0.22 & 0.67 & 0.31 & 0.78 & 0.19 \\
\hline$r^{2}$ & 0.54 & 0.28 & 0.54 & 0.35 & 0.65 & 0.27 \\
\hline RMS & 12.92 & 8.70 & 11.67 & 9.11 & 14.11 & 7.32 \\
\hline NRMS_Env & 19.58 & 10.77 & 11.59 & 8.55 & 16.80 & 8.02 \\
\hline NRMS_MAX & 13.81 & 9.11 & 9.23 & 7.11 & 7.78 & 3.59 \\
\hline
\end{tabular}

Table 6.2.

Summary of the statistical parameters of interest used to validate the EMG-driven model during the calibration trials. The parameters were the average and standard deviations of: (a) the Gain; (b) the correlation coefficient ( $r$ ) between the measured and predicted moments; (c) the determination coefficient; (d) the root mean square of the error (RMS); (e) the normalized RMS with respect to the maximum measured moment value in the envelope (NRMS_Env); and (f) the normalized RMS with respect to the maximum measured moment value of all calibration trials for the joint (NRMS_MAX). 


\begin{tabular}{|l|c|c|c|c|c|c|}
\hline $\begin{array}{c}\text { Subjects } \\
\text { Parameters }\end{array}$ & \multicolumn{2}{c|}{ SA } & \multicolumn{2}{c|}{ SC } & \multicolumn{2}{c|}{ SN } \\
\hline Ankle & Average & $\begin{array}{c}\text { Standard } \\
\text { Deviation }\end{array}$ & Average & $\begin{array}{c}\text { Standard } \\
\text { Deviation }\end{array}$ & Average & $\begin{array}{c}\text { Standard } \\
\text { Deviation }\end{array}$ \\
\hline Gain & 23 & - & 27.50 & - & 28 & - \\
\hline$r$ & 0.92 & 0.05 & 0.88 & 0.06 & 0.91 & 0.02 \\
\hline$r^{2}$ & 0.85 & 0.09 & 0.79 & 0.10 & 0.82 & 0.04 \\
\hline RMS & 57.57 & 3.77 & 10.47 & 2.46 & 62.95 & 11.84 \\
\hline NRMS_MOT & 67.23 & 6.54 & 19.88 & 4.30 & 51.28 & 11.01 \\
\hline NRMS_KC & 116.72 & 7.65 & 8.20 & 1.92 & 119.35 & 22.45 \\
\hline Knee & & & & & & \\
\hline Gain & 23 & - & 27.50 & - & 28 & - \\
\hline$r$ & 0.93 & 0.03 & 0.91 & 0.05 & 0.83 & 0.05 \\
\hline$r^{2}$ & 0.87 & 0.06 & 0.84 & 0.09 & 0.68 & 0.08 \\
\hline RMS & 9.98 & 2.73 & 16.15 & 7.49 & 15.07 & 3.58 \\
\hline NRMS_MOT & 19.15 & 5.30 & 13.84 & 6.53 & 30.67 & 5.54 \\
\hline NRMS_KC & 8.62 & 2.36 & 29.38 & 13.62 & 7.61 & 1.81 \\
\hline
\end{tabular}

Table 6.3.

Summary of the statistical parameters of interest used to validate the EMG-driven model with a calibration-based global Gain (see text). The parameters were the average and standard deviations of: (a) the correlation coefficient ( $r$ ) between the measured and predicted moments; (b) the determination coefficient; (c) the root mean square of the error (RMS); (d) the normalized RMS with respect to the maximum measured moment value in the envelope (NRMS_MOT); and (e) the normalized RMS with respect to the maximum measured moment value of all isokinetic calibration trials for the joint. 


\begin{tabular}{|l|c|c|c|c|c|c|}
\hline \multicolumn{1}{|c|}{$\begin{array}{c}\text { Subjects } \\
\text { Parameters }\end{array}$} & \multicolumn{2}{c|}{ SA } & \multicolumn{2}{c|}{ SC } \\
\hline Ankle & Average & $\begin{array}{c}\text { Standard } \\
\text { Deviation }\end{array}$ & Average & $\begin{array}{c}\text { Standard } \\
\text { Deviation }\end{array}$ & Average & $\begin{array}{c}\text { Standard } \\
\text { Deviation }\end{array}$ \\
\hline Gain & 10.65 & - & 23.18 & - & 14.00 & - \\
\hline$r$ & 0.92 & 0.05 & 0.91 & 0.05 & 0.91 & 0.02 \\
\hline$r^{2}$ & 0.85 & 0.09 & 0.84 & 0.09 & 0.82 & 0.04 \\
\hline RMS & 13.11 & 3.93 & 16.38 & 8.15 & 19.30 & 2.49 \\
\hline NRMS_MOT & 15.33 & 4.69 & 14.04 & 7.10 & 15.68 & 2.27 \\
\hline NRMS_KC & 26.59 & 7.98 & 29.79 & 14.82 & 36.60 & 4.72 \\
\hline Knee & & & & & & \\
\hline Gain & 35.06 & - & 32.17 & - & 42.50 & - \\
\hline r & 0.93 & 0.03 & 0.89 & 0.06 & 0.82 & 0.05 \\
\hline$r^{2}$ & 0.87 & 0.06 & 0.79 & 0.10 & 0.68 & 0.08 \\
\hline RMS & 20.20 & 2.53 & 12.99 & 2.06 & 22.23 & 6.05 \\
\hline NRMS_MOT & 39.02 & 8.81 & 24.95 & 5.57 & 45.58 & 11.61 \\
\hline NRMS_KC & 17.44 & 2.19 & 10.17 & 1.61 & 11.23 & 3.05 \\
\hline \multicolumn{7}{|l|}{} \\
\hline
\end{tabular}

Table 6.4 .

Summary of the statistical parameters of interest used to validate the EMG-driven model with the calibration derived, joint-specific constant Gain. The parameters were the average and standard deviations of: (a) the correlation coefficient ( $r$ ) between the measured and predicted moments; (b) the determination coefficient; (c) the root mean square of the error (RMS); (d) the normalized RMS with respect to the maximum measured moment value in the envelope (NRMS_MOT); and (e) the normalized RMS with respect to the maximum measured moment value of all isokinetic calibration trials for the joint. 


\begin{tabular}{|l|c|c|c|c|c|c|}
\hline \multicolumn{1}{|c|}{$\begin{array}{c}\text { Subjects } \\
\text { Parameters }\end{array}$} & \multicolumn{2}{c|}{ SA } & \multicolumn{2}{c|}{ SN } \\
\hline Ankle & Average & $\begin{array}{c}\text { Standard } \\
\text { Deviation }\end{array}$ & Average & $\begin{array}{c}\text { Standard } \\
\text { Deviation }\end{array}$ & Average & $\begin{array}{c}\text { Standard } \\
\text { Deviation }\end{array}$ \\
\hline Gain & 8.84 & 0.30 & 21.57 & 0.28 & 11.69 & 1.65 \\
\hline$r$ & 0.92 & 0.05 & 0.91 & 0.05 & 0.91 & 0.02 \\
\hline$r^{2}$ & 0.85 & 0.09 & 0.84 & 0.09 & 0.82 & 0.04 \\
\hline RMS & 12.72 & 2.85 & 17.71 & 7.15 & 17.68 & 2.83 \\
\hline NRMS_MOT & 14.86 & 3.46 & 15.17 & 6.29 & 14.31 & 1.94 \\
\hline NRMS_KC & 25.79 & 5.78 & 32.22 & 12.99 & 33.53 & 5.36 \\
\hline Knee & & & & & & \\
\hline Gain & 8.84 & 0.30 & 21.57 & 0.28 & 11.69 & 1.65 \\
\hline r & 0.93 & 0.03 & 0.88 & 0.06 & 0.83 & 0.05 \\
\hline$r^{2}$ & 0.87 & 0.06 & 0.79 & 0.10 & 0.68 & 0.08 \\
\hline RMS & 13.69 & 3.32 & 15.80 & 1.65 & 13.35 & 2.00 \\
\hline NRMS_MOT & 25.89 & 4.36 & 30.26 & 4.39 & 27.30 & 3.24 \\
\hline NRMS_KC & 11.82 & 2.87 & 12.37 & 1.29 & 6.74 & 1.01 \\
\hline
\end{tabular}

Table 6.5.

Summary of the statistical parameters of interest used to validate the EMG-driven model with a least squares estimated global Gain. The parameters were the average and standard deviations of: (a) the Gain; (b) the correlation coefficient (r) between the measured and predicted moments; (c) the determination coefficient; (d) the root mean square of the error (RMS); (e) the normalized RMS with respect to the maximum measured moment value in the envelope (NRMS_MOT); and (f) the normalized RMS with respect to the maximum measured moment value of all isokinetic calibration trials for the joint. 


\begin{tabular}{|l|c|c|c|c|c|c|}
\hline \multicolumn{1}{|c|}{$\begin{array}{c}\text { Subjects } \\
\text { Parameters }\end{array}$} & \multicolumn{2}{c|}{ SA } & \multicolumn{2}{c|}{ SC } \\
\hline Ankle & Average & $\begin{array}{c}\text { Standard } \\
\text { Deviation }\end{array}$ & Average & $\begin{array}{c}\text { Standard } \\
\text { Deviation }\end{array}$ & Average & $\begin{array}{c}\text { Standard } \\
\text { Deviation }\end{array}$ \\
\hline Gain & 12.69 & 1.30 & 11.25 & 1.40 & 16.96 & 1.91 \\
\hline R & 0.92 & 0.05 & 0.91 & 0.05 & 0.91 & 0.02 \\
\hline$R^{2}$ & 0.85 & 0.09 & 0.84 & 0.09 & 0.82 & 0.04 \\
\hline RMS & 12.63 & 2.85 & 16.82 & 7.41 & 17.65 & 2.83 \\
\hline NRMS_MOT & 14.76 & 3.44 & 14.42 & 6.51 & 14.28 & 1.94 \\
\hline NRMS_KC & 25.61 & 5.78 & 30.60 & 13.48 & 33.47 & 5.36 \\
\hline Knee & & & & & & \\
\hline Gain & 8.46 & 0.48 & 24.78 & 0.18 & 11.40 & 1.60 \\
\hline R & 0.93 & 0.03 & 0.89 & 0.06 & 0.82 & 0.05 \\
\hline R & 0.87 & 0.06 & 0.79 & 0.10 & 0.68 & 0.08 \\
\hline RMS & 12.91 & 2.81 & 12.53 & 2.62 & 12.62 & 2.12 \\
\hline NRMS_MOT & 24.44 & 3.49 & 23.61 & 1.28 & 25.79 & 3.32 \\
\hline NRMS_KC & 11.15 & 2.43 & 9.82 & 2.05 & 6.37 & 1.07 \\
\hline
\end{tabular}

Table 6.6.

Summary of the statistical parameters of interest used to validate the EMG-driven model with a least squares estimated joint specific Gain. The parameters were the average and standard deviations of: (a) the Gain; (b) the correlation coefficient (r) between the measured and predicted moments; (c) the determination coefficient; (d) the root mean square of the error (RMS); (e) the normalized RMS with respect to the maximum measured moment value in the envelope (NRMS_MOT); and (f) the normalized RMS with respect to the maximum measured moment value of all isokinetic calibration trials for the joint. 


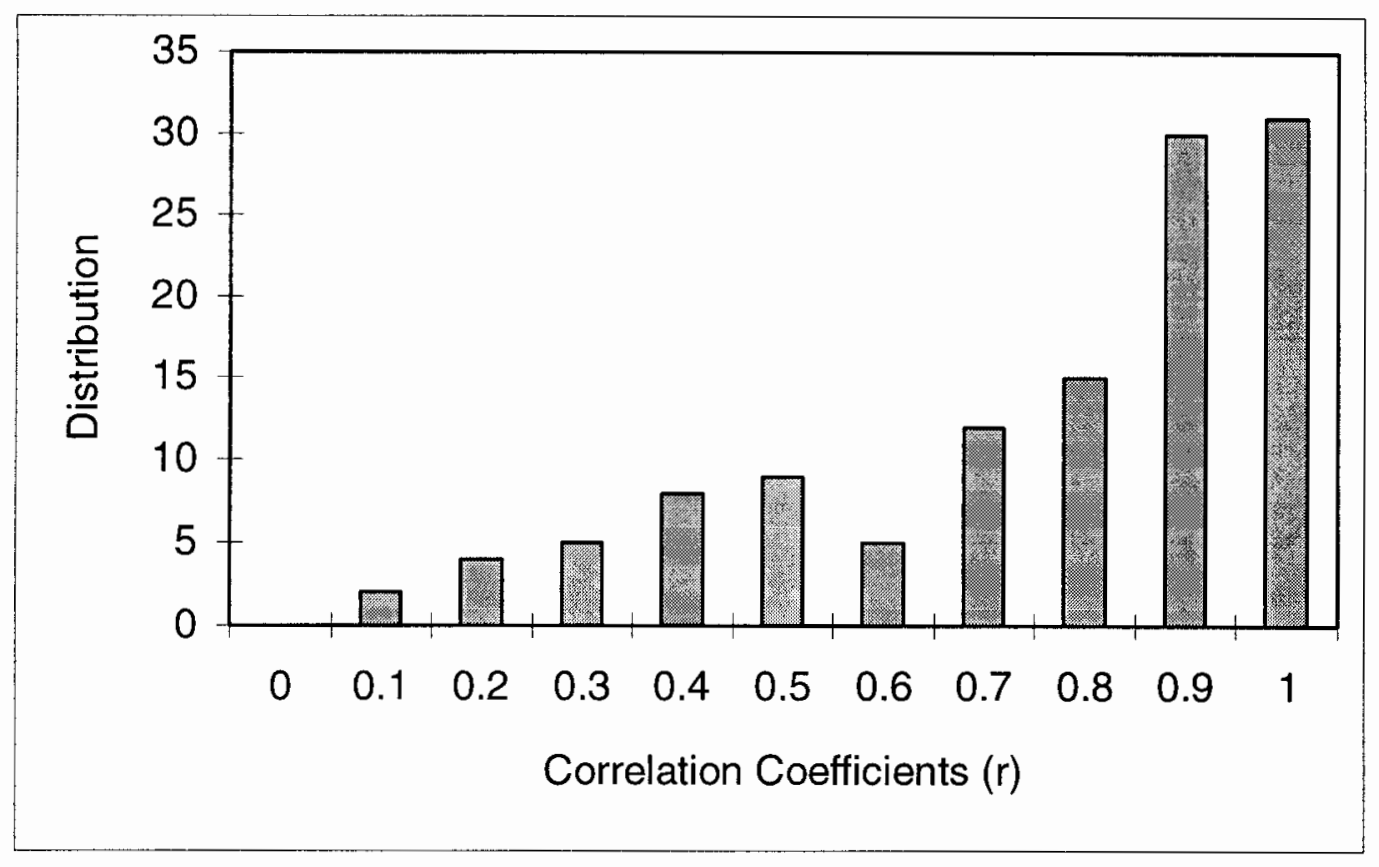

Figure 6.12. Distribution of the correlation coefficients between the measured and predicted joint moments for the purposes of validating the temporal consistency of the EMG-driven model. The median $r$ value is 0.8 .

\section{$\underline{\text { Root mean square error }}$}

The root mean square error and its variations provide a means for assessing the ability of the model to match, in terms of magnitude, the measured moment. The results of this analysis are included analytically in Appendices $M$ through $\mathrm{O}$, and they are summarized in tables 6.2 through 6.6. It can be seen that the error term was expressed in three different ways: (a) in terms of its absolute root mean square value, (b) relative to the maximum joint-specific moment in the measured net joint moment, and (c) relative to the maximum moment computed on the isokinetic dynamometer. The last two methods served as means for comparison with previous studies and as a way to identify whether maximum voluntary contraction exertions were performed on the isokinetic dynamometer.

In general, it can be observed that the resulting errors are a function of the gain implementation procedure. The gains computed and implemented in this study were: 
i) A joint-specific gain during the calibration procedure (Calib_JntGain);

ii) A global gain represented as the average of the two joint-specific Calib_JntGains to account for the biarticular muscles (CalibGlobGain);

iii) A global gain estimated using least squares in the gait trials (LS_ GlobGain); and

iv) A joint-specific gain using least squares (LS_JntGain).

Figure 6.13A and Figure 6.13B provide an example output for the predicted moments depending on the gain that has been implemented. For example, observation of the RMS error as a function of the LS_GlobGain and LS_JntGainK ,i.e., for the knee, and LS_JntGainA, i.e., for the ankle, indicates that the RMS error for the respective joint was reduced when the LS_JntGainK or LS_JntGainA was used versus the LS_GlobGain.

Furthermore, it was observed that when the CalibGlobGain was being implemented, the RMS was being reduced for the knee (relative to when the Calib_JntGainK was implemented). Thus, a gain for the knee closer to the numerical value of that for the ankle, improved the RMS error for the knee. In fact, when the optimum global gain, i.e., LS_GlobGain, was being computed its numerical value always approximated that of the ankle. Visual observations indicated that the optimum global gain as well as the optimum joint gain for the knee consistently, most of the times, underpredicted the measured moment at the knee (see Figure 6.13A). The CalibGlobGain always overpredicted the measured moment at the ankle, while the LS_GlobGain either matched it or underpredicted it. The LS_JntGain for the ankle always matched the peak of the measured ankle moment. It was also observed that consistently for the knee when the NRMSK_Mot and NRMCK_KC values were computed the former were bigger than the latter. The reverse, however, was true for the ankle joint. When the NRMSA_Mot and NRMSA_KC values were computed the _KC values were consistently higher. 

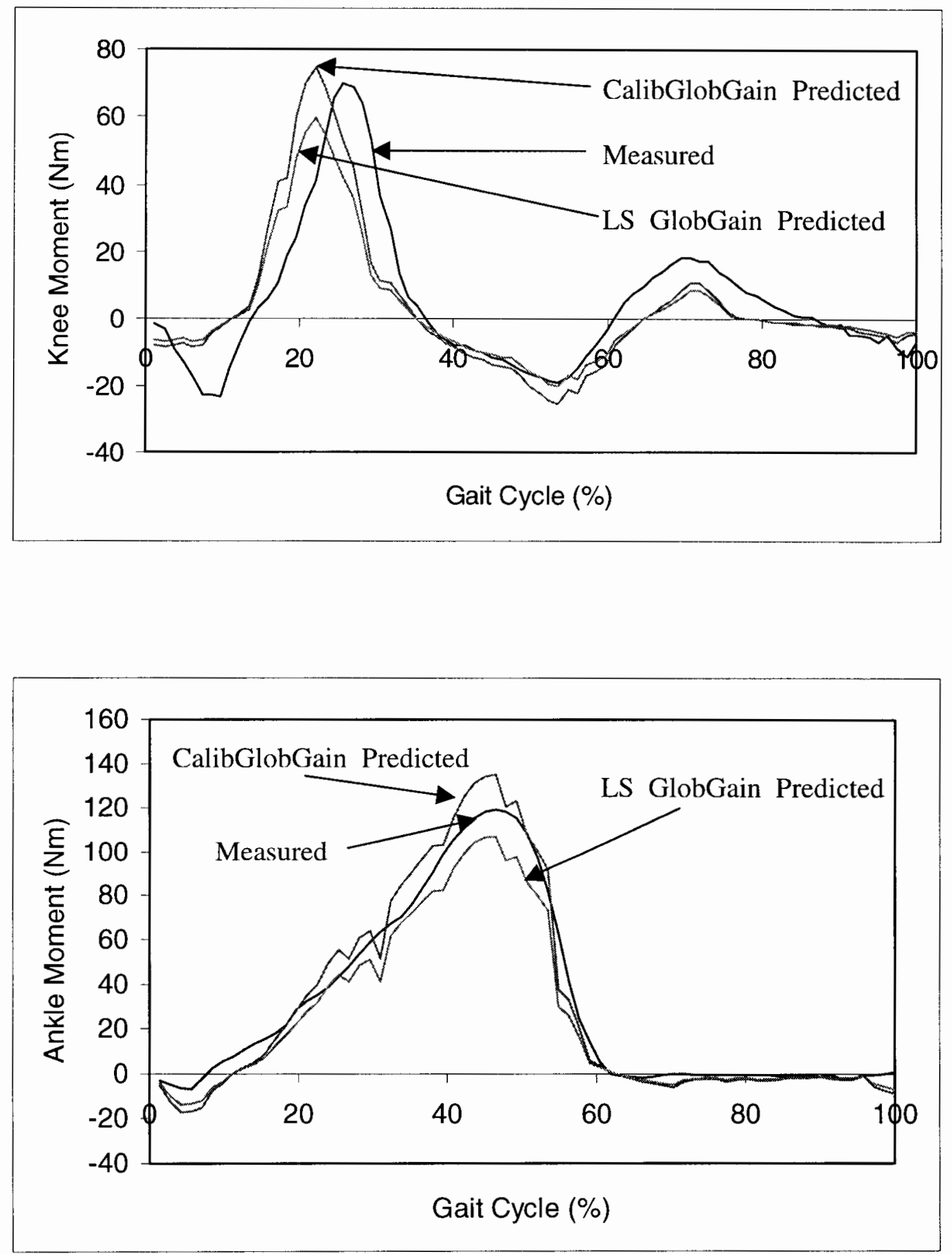

Figure 6.13A. Comparison among the different types of output relative to the measured moment (for SC003) depending on the gain implementation procedure (see text for definition of the various gain implementation procedures). 

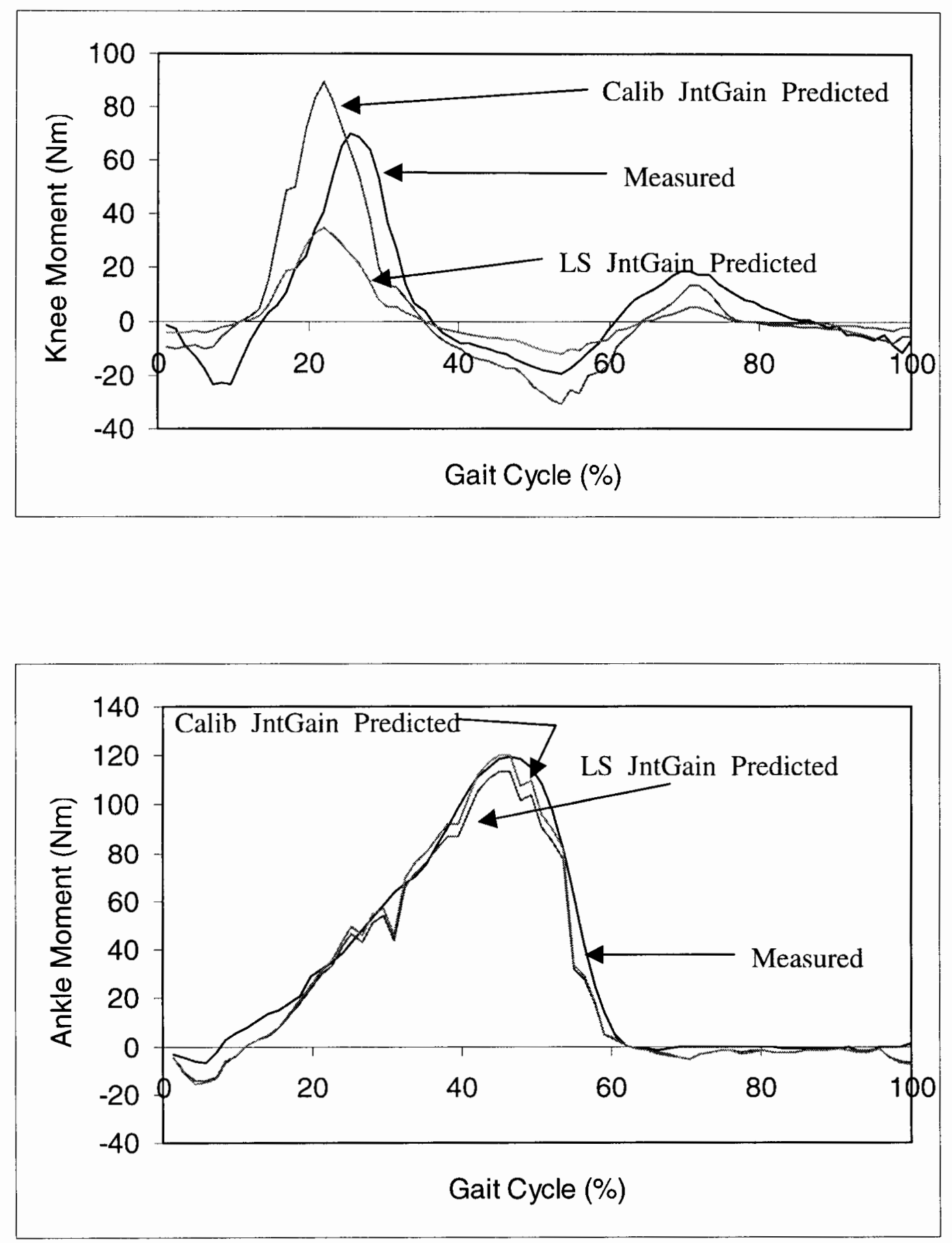

Figure 6.13B. Comparison among the different types of output relative to the measured moment (for $\mathrm{SC003}$ ) depending on the gain implementation procedure (see text for definition of the various gain implementation procedures). 


\section{Muscle cocontraction}

Figure 6.14 and figure 6.15 are examples of muscle cocontraction at the ankle and knee joints. The cocontraction index was computed by (a) using the EMG signals alone, (b) by using the EMG signals combined with the tension-length-velocity relationships of the musculotendon units, and (c) by using the predicted muscle forces from each of the musculotendon units involved. Muscle cocontraction output, the cocontraction index, appeared to vary among the methods that were implemented for its computation, although not always statistically significantly (at a $\mathrm{p}<0.0166, \mathrm{df}=100$ and $\mathrm{t}=2.43$ ). The results from the statistical analysis for the walking trials and from the isokinetic calibration trials are all included in Appendix P. Most of the times, the cocontraction index results did vary statistically significantly among the three methods used to compute it in the isokinetic calibration trials.

Significant amount of cocontraction occurred during the phases in the gait cycle that require stability, such as when the foot is being planted on the ground at the beginning of the gait cycle, or when maximum unloading occurs bringing the knee at its first knee flexion peak. Significant amount of cocontraction also occurred during the swing to control the forward motion of the lower extremity segments, such as after toe off when the foot needs to "clear" the floor in preparation for landing, or when the knee flexes to assist with the foot clearance and advance the knee forward.

\section{EMG-driven Performance Assessment}

In general, the present EMG-driven model appears to be a good predictor of individual musculotendon unit forces and, therefore, of cocontraction. The high potential of the model can be inferred by the within physiologic limits gains, the correlation distributions (see Figure 6.12) and the modeling assumptions and limitations within the perspective of the small number of subjects. Although the small number of subjects limits any reliable statistical conclusions, a high median correlation coefficient $(\mathrm{r}=.80$, from figure 6.12) appears to advocate that the model can determine very well the dynamic response, during walking gait, of the musculotendon units of interest acting at 

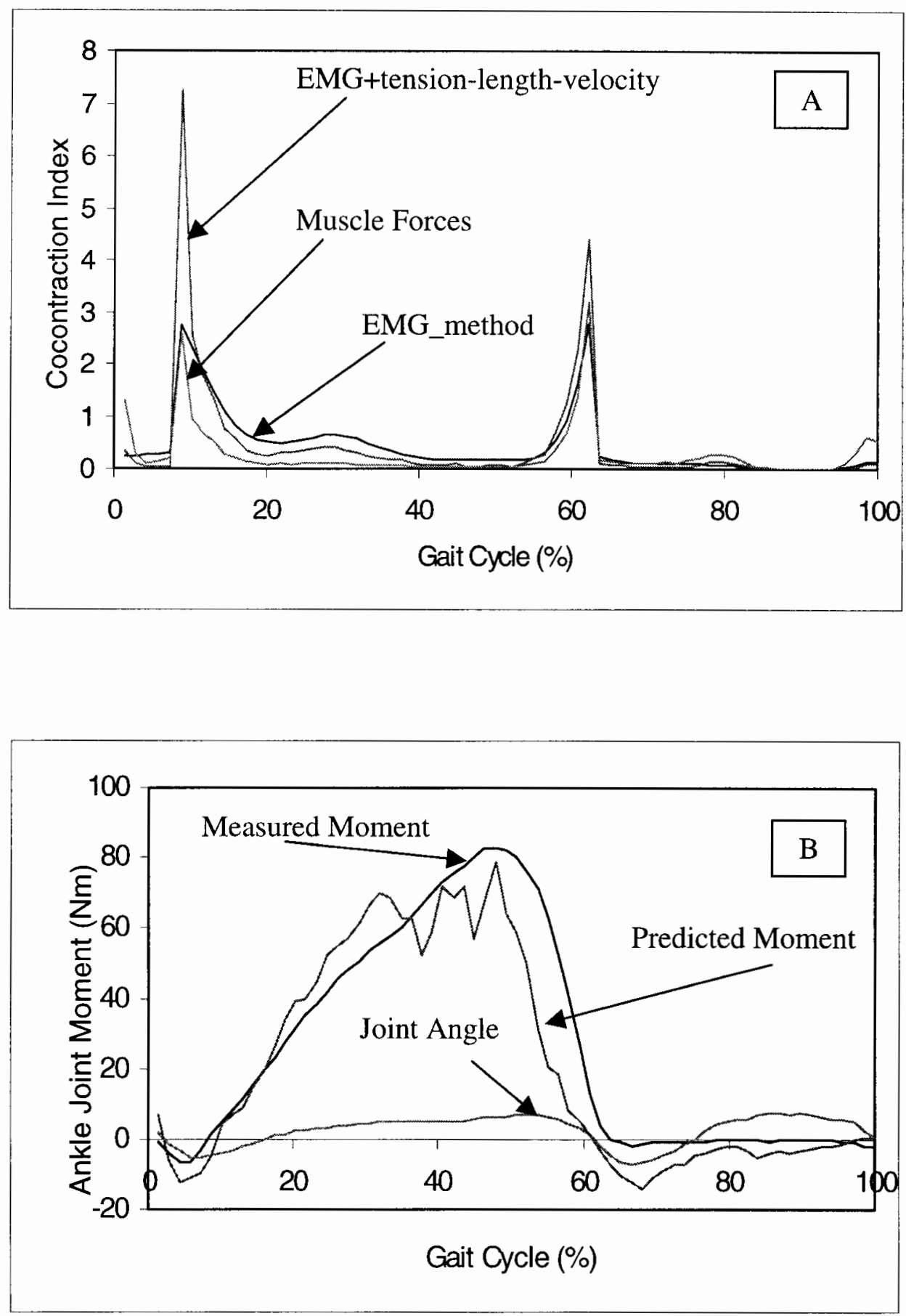

Figure 6.14. Cocontraction index (for SA004) at the ankle during a full gait cycle using three different cocontraction index algorithms (A). The respective kinetic and kinematic data, along with the predicted moment are also shown (B). 

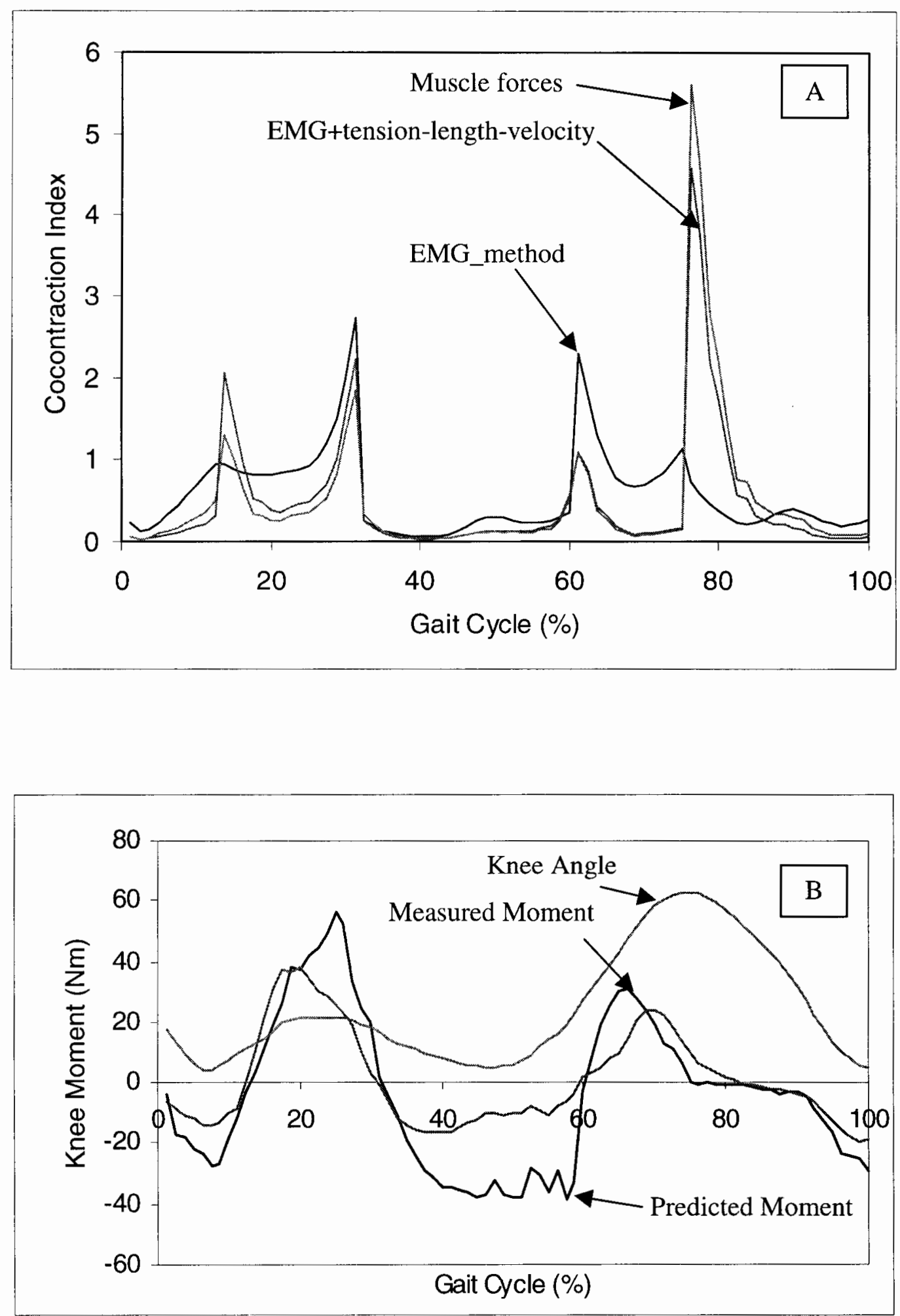

Figure 6.15. Cocontraction index (for SA004) at the knee during a full gait cycle using three different cocontraction index algorithms (A). The respective kinetic and kinematic data, along with the predicted moment are also shown (B). 
the knee and ankle joints in the sagittal plane. Externally measured moments, corrected for the contribution of the passive joint structures, provide the force magnitude at the joint to be modeled. The relative tension forces generated by each musculotendon unit acting at the knee or ankle joint are determined by the processed electormyographic histories. The same musculotendon unit forces, then, used for predicting the externally measured moments are used for the computation of the cocontraction index. Since the model appears to be a good predictor of the dynamic net joint moments acting at the knee and ankle joints, it is reasonable to assume that it may be a relatively good quantification tool for the measurement of cocontraction as a function of the individual musculotendon units at the respective lower extremity joints.

\section{Model Parameters}

\section{Muscle Architectural Inputs}

The muscle architectural inputs that were used in SIMM to derive the tensionlength-velocity relationships for each muscle of interest were adopted from the musculoskeletal model of Delp (1990). Because these values, especially the optimum fiber length and tendon slack length were already optimized such that the software force output matched experimental data available in the literature (Delp, 1990; Hoy et al., 1990), there was no basis for comparison or implementation of alternative values from the literature. The same was true for the computation of the physiological cross sectional areas. Rather than adopting them from the literature, they were computed from the anthropometric information, already optimized, in the musculoskeletal model of Delp (1990).

However, although this approach appeared to be reasonable, the experimental results of the isokinetic trials that were done at very low angular velocities, such that they could be considered almost isometric, did not confirm its appropriateness. With respect to the occurrence of the maximum moment, while for all plantar-dorsi flexors and knee extensors appeared to occur at a similar location as reported in the literature (for a review see Delp, (1990)) and as simulated by SIMM, this was not the case for the knee flexors.

SIMM predicted that, with a five degrees per second knee flexion, maximum knee flexor moment would be achieved with the ankle at a dorsi flexed position of ten degrees 
(for the purposes of this study). However, the experimental results showed that for all subjects maximum knee flexor moment was achieved with the ankle in a plantar flexed position (ten degrees). Furthermore, while SIMM predicted that maximum knee flexion moment would occur close to 50 degrees, for the subjects of this study maximum knee flexor moment occurred at 14, 12 and 30 degrees of knee flexion (for the last two subjects similar maximum moments occurred for both at 22 degrees. These values were similar to the SIMM output as if the person was performing with a hip kept in neutral position, i.e., as if the hip angle were zero. However, none of the calibration trials involved a hip kept in neutral.

It appears that other researchers had similar experiences and, thus, attempts have been made in the past to perform adjustments in the muscle architectural inputs, such as the tendon slack length, to allow the model match the experimental data for minimizing the error (Lloyd and Buchanan, 1996). Although the results were encouraging, i.e., the error was minimized, given the principles underlying Zajac's musculotendon model (1998), altering a musculotendon unit's tendon slack length, would result in a completely different mechanical performance of that musculotendon unit. At the same time variation between the actual and experimental moments, strictly on a modeling level could be a function of the moment arms predicted by the model, the muscle representation in the model and the corresponding estimated musculotendon unit lengths, as well as the EMG to muscle activation transfer function.

With regard to the EMG to muscle activation transfer function, recent evidence from dynamic and static optimization studies indicate that activation dynamics and the implementation of a time-independent performance criterion may not be a limitation when one solves for the muscle indeterminate problem (Anderson and Pandy, 2001). Such an observation makes EMG-driven modeling even more attractive as it would eliminate one of the potential sources for error. At the same time it would also eliminate a potential error for the simulations output from SIMM, and it would provide some direction about other, more viable sources of potential error associated with the musculoskeletal modeling implemented in SIMM. For example, the simulation kinetic output is based on the optimum muscle fiber length. However, that length is a function of 
the sarcomeres in series (Williams, 1988). However, immobilization or passive stretch can decrease or increase respectively the number of sarcomeres, and, thus affect the optimum fiber length (Lloyd and Buchanan, 1996). Exercise, or lack thereof, can affect the number of sarcomeres in series. Consequently, the optimum fiber length may be variable across individuals as a function of their activity level. One solution that would account for the individual musculoskeletal variability would be to implement the musculoskeletal parameters for each subject from respective MRI data.

\section{EMG patterns}

The fact that some muscles, such as the two heads of the gastrocnemius, were selectively recruited in different ways for similar contractions has been supported by Freund (1983) who suggested that synergistic recruitment is really a function of the position of the effected limb. He went on to speculate that different sensory inputs to the muscle can override the fundamental recruitment patterns allowing a certain flexibility for multifunctional muscles. Furthermore, he had suggested that the firing frequency of the synergistic muscles involved in similar movements is a function of the speed of the movement. Consequently, muscles, such as the soleus and the gastrocnemius, which have different distribution of fast and slow twitch types of motor units may have been selectively controlled differently under the various calibration joint velocities and joint interactions. On the basis of these suggestions then, the calibration approach of incorporating gait-specific joint interactions was effective in allowing for the multifunctionality of the muscles and the different recruitment patterns to be captured. That multifunctionality then was reflected in the average gain and the respective standard deviation.

When the recorded dynamic EMG signal was compared to the calibration EMG maximum for normalization, some normalized EMG values were greater than one. Damiano and her collaborators (2000), and White and Winter (1993) also provided normalized EMG values that were larger than one. The latter reported greater than one normalized EMG for the soleus muscle only. However, normalized EMG values, relative to a maximum, greater than one indicates that the selected EMG maxima, used to normalize the dynamic EMG signals, i.e., the normalization factors, were not the true 
EMG maxima for gait. Through the calibration procedure gait specific joint interactions were simulated and tested ranges of motion that corresponded to those joint interactions. The EMG maximum value was identified using the principle used by White and Winter (1993) and Olney and Winter (1985), i.e., the EMG maximum value corresponded to the EMG value at the time that maximum external moment was measured.

DeLuca (1997) has alluded to the problem of capturing the maximum EMG during a maximum voluntary contraction (MVC). Factors such as subject motivation may play a role. In this study the entire testing procedure lasted approximately 8 to 10 hours. Thus, although during the calibration component of the testing procedure physical fatigue was accounted for according to the recommendations by DeLuca (1997), mental fatigue might had been difficult to avoid.

Another factor that may have resulted in larger than one normalized EMGs might be related to the calibration procedure itself. The data from Appendix N where the RMS of the error was normalized relative to the maximum moment recorded during gait (NRMS_MOT) and to the maximum moment recorded on the isokinetic dynamometer (NRMS_KC) during the calibration procedure for each respective joint show that the NRMS_KC values for the ankle are larger than the NRMS_MOT values for the same trial. The implication is that the subjects generated larger ankle moments during gait than during the calibration. It was then hypothesized that the reason of the lower ankle joint moments might be related to the calibration and strapping procedure used. The straping procedure, however, was invented out of necessity to maintain each subject's heel on the supporting platform of the isokinetic dynamometer for the purposes of controlling with accuracy the excursion angle of the joint.

To test this observation three subjects, unrelated to the study, were tested on the isokinetic dynamometer under the strapping method implemented for the purposes of this study and under the method recommended by the manufacturer of the dynamometer, which had been previously verified to allow the heal of the subject to come off the supporting platform and thus would not allow an accurate representation of the forcejoint angle relationship. Representative results for the plantar flexions of one of the subjects are shown in figure 6.16. All subjects demonstrated the same pattern. 

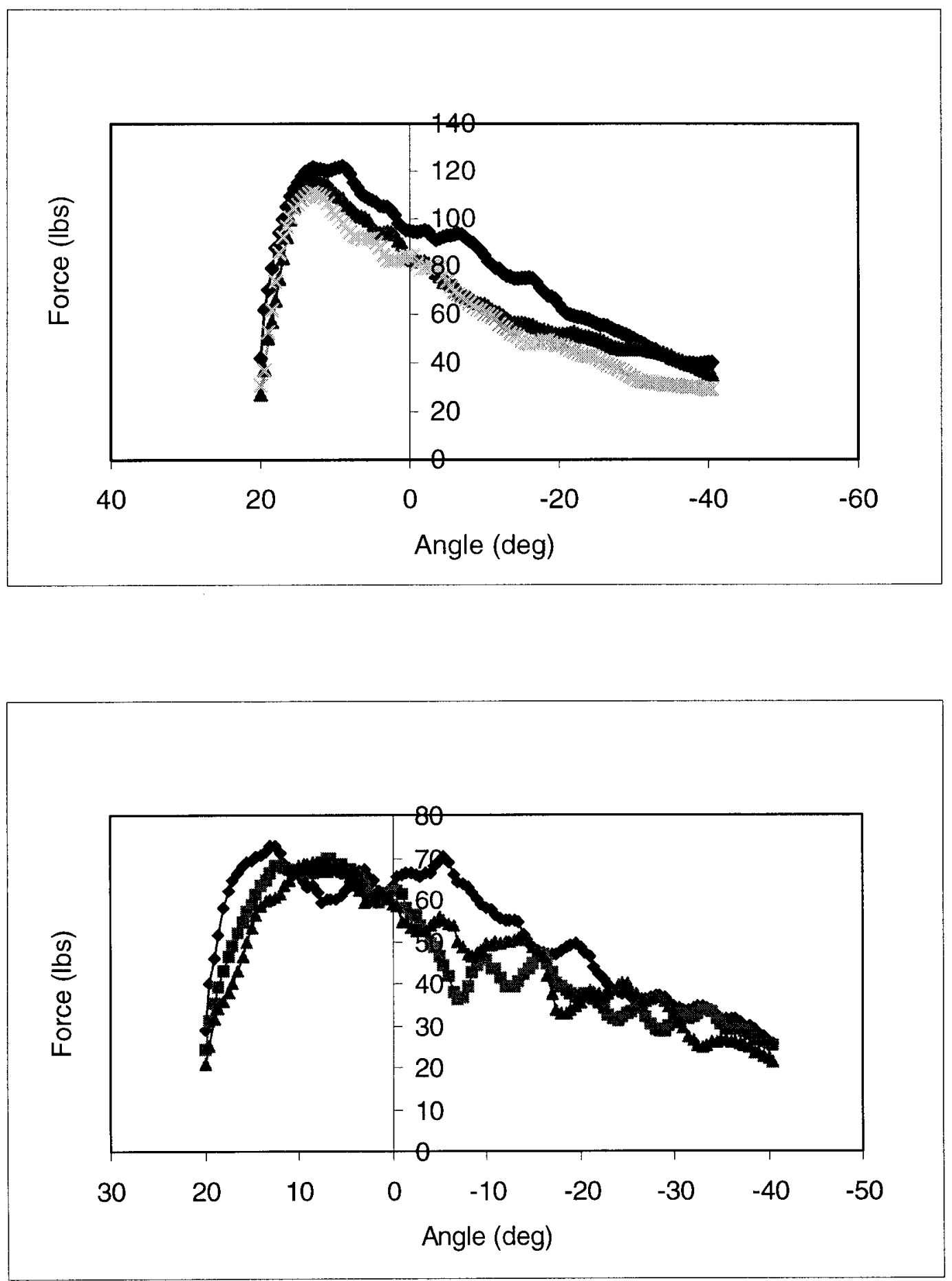

Figure 6.16. Two different strapping techniques for the ankle joint tested on the isokinetic dynamometer. On the top, the person's (subject SV) ankle was strapped according to the manufacturer's specifications allowing the heel to come off the supporting platform. On the bottom the ankle was stabilized such that the heel could not come off. 
The results of this testing indicate that the strapping technique used for the purposes of this study did not allow for the maximum ankle joint moment to be generated. Consequently, the corresponding ankle EMG recorded, could not have been the maximum EMG for gait which would appropriately scale the dynamic EMGs. Thus, one could infer that either submaximal contractions were performed during the ankle calibration or that maximum excitation with two different boundary conditions resulted in two different outcomes.

Another factor that may have affected the normalized EMG value is related to the type of isokinetic contractions performed relative to the specific contractions one implements during ambulation. Gage (1995) contended that gait involves "gravity control" contractions, i.e., eccentric contractions. However, eccentric contractions were not represented in the calibration procedure. On the other hand, Karakostas (1995) has demonstrated that eccentric contractions are represented by higher signal amplitudes compared to concentric contractions, which, given the tension-length-velocity relationship of the muscle fiber, may be expected to result in higher tensile forces. Consequently, the issue of greater than one normalized EMG values might have been resolved if the signals were normalized to EMG maxima obtained by eccentric calibration efforts. Nevertheless, all the normalized electormyographic results of this study, from a temporal and magnitude perspective, agree with the results presented by Rab (1994), and by Winter and Yack (1986) for the muscles recorded.

Finally, the EMG signal histories were used to predict moments in the sagittal plane only. However, to avoid musculotendon unit length prediction errors all kinematic data in all three planes of motion were used. White and Winter (1993), attempted to use their EMG-assisted model to predict joint moments in the coronal and transverse planes. However, they found it to be not feasible due to the noise and very low EMG recordings. They also had problems with the hip joint as they were able to monitor only sixty four percent of the musculature involved at the hip joint with surface electromyography.

\section{$\underline{\text { Gain }}$}

The validity of the EMG-driven model was assessed by evaluating the gain, correlation coefficients between the predicted and measured moments and the root mean 
square of the error between the respective moments. The necessity to employ all these parameters lies on the fact that each represents different validation aspects of the model. Gains within physiological margins imply that the model has physiological compatibility. The correlation coefficients are a means of assessing the temporal performance of the model and its ability to predict joint moments that are externally determined via inverse dynamics (Marras et al., 1999). The root mean square of the error represents an assessment of the model's performance to match the magnitude of the externally predicted joint moments.

The average gains estimated during the calibration procedure were within physiological limits. Unfortunately, to the best of this investigator's knowledge, there are no other studies in the literature that have reported gains used or computed as a result of EMG-assisted modeling of the lower extremity for the purpose of computing musculotendon unit forces. Consequently, this study is the first to provide a limited database of gains for future comparisons.

Granata and Marras (1995) and Marras (1997) have recognized the need for the intra-subject gain to be a constant, i.e., independent of condition since it represents the muscle force generated per cross sectional unit area. The gain can be variable across subjects due to variations in conditioning and natural strength. However, in the present study the gain from the calibration isokinetic exertions appeared to be variable. In an effort to identify the reason for the intra-subject variability, the gains were plotted against velocity and against muscle length which, for the monoarticular muscles, the respective lengths were a function of the testing joint angle, and, for the biarticular musculotendon units, were affected by the testing angle at the angle of the adjacent joint.

The results indicated that there may be a muscle length and velocity of contraction effect. Hasler and his coworkers (1994) have demonstrated the dependency of the EMG and of the EMG maxima on the joint angle. Since the estimation of gain is a function of the normalized EMG, and the EMGs are a function of the joint angle, then the gain estimation is affected by the artifact of the joint angle, or of the muscle length, on the EMG. Hof and Van Den Berg (1981b) and White and Winter (1993) also found variable gains from their calibration procedures. To maintain then a constant gain, polynomial 
equations were developed to account for the variability (Granata, 1993). The difference between the approach of Granata and that of White and Winter was that the latter modeled the gain as a third degree polynomial where the polynomial represented only the effect of change of muscle length on the gain while implementing Hill's model with the tension-length and tension-velocity relationships. Granata on the other hand, modeled the error of both the muscle length and velocity into two separate polynomials, lumping in each respective polynomial the tension-length and the tension-velocity relationships. While the velocity artifact polynomial did not appear to affect the gain estimation significantly, it did affect the coefficient of determination values.

In the present model the muscle length and velocity artifacts were not accounted for. The model employed Hill's tension-length and tension-velocity relationships along with other physiological parameters and its response in computing physiological gains was observed. The calibration procedure implemented a variety of isokinetic, gait specific joint interactions to account for the muscle multifunctionality and, consequently, the average gain encapsulated the variation. It is the goal, therefore, in the future, to model the variation as a function of the muscle length and muscle velocity of contraction by introducing another term in the model. The improvement, however, relative to other EMG-assisted modeling approaches is that now the model has laid the foundation for a means to investigate the muscle length and velocity of contraction errors independently, i.e., without lumping them in the tension-length-velocity relationship of the muscle while, at the same time accounting for the combined effect of biarticularity and muscle velocity of contraction.

In addition, variability of the gain during the isokinetic testing may, in part, be attributed to the calibration device used. Granata (1993) found very poor model validity with results from the isokinetic dynamometer. However, the results were excellent when the kinematic information was allowed to be obtained with a device, the lumbar monitor, that did not pose any restrictions on the movement of interest. Then, the movement of interest and the calibration movement for the identification of the parameters of the model were the same. Thus, there exists a need for identifying a calibration that would allow the implementation of an activity-specific calibration procedure. Such a device is 
not currently available for the purposes of gait. The present study, however, while maintaining the simplicity of the EMG-driven model, devised and employed a procedure which would allow the simulation of gait-specific joint interactions.

It can be argued that such a calibration procedure approach can provide upper and lower bounds for gains. Then gait related activities could be used to fine-tune these gains and adaptively reduce the error in the system.

In addition, such a procedure has been able to account for the effect of the musclesubcutaneous tissue-electrode relationship. White and Winter (1993) and Olney and Winter (1985) attributed some of the errors in their experimental results to the variability introduced by the muscle-electrode pick up volume especially for the knee flexors because the subjects were sitting on the electrodes during the calibration. In the present study the calibration was such that there was no such issue.

Furthermore, DeLuca (1997) has expressed concerns when EMG from calibration obtained type contractions are used for extrapolating to the muscle activation during dynamic activities due to the changes and the effects on the signal as a function of the dynamic relationship between muscle and recording electrodes. In fact, the fixed geometric relationship between electrode pick up volume and muscle may be one of the limitations in isometric contractions that aim in representing dynamic conditions. Furthermore, some recent evidence suggest that muscle length-tension isometric curves may not be able to predict dynamic angle-torque relationships involved in dynamic movements (Gillard et al., 2000). In the present study, by simulating gait specific joint interactions on the isokinetic dynamometer, the potential dynamic geometrical relationships during gait between the recording electrode and the muscle were accounted for. The effect of this dynamic relationship is also reflected in the gain and partly explains the gain variability observed among different dynamic conditions.

Model internal stability, on the other hand, was evaluated when isokinetic testing under the exact same conditions was performed. Then, the estimated gains for the trials of the same conditions should be the same. In fact, that was the case in the present model. Estimated gains for the trials of the same conditions were, most of the times almost the same (see Appendix M, bolded cases). Furthermore, during gait, when the 
optimum gain, using least squares, was computed, the intra-subject variability was very small, within experimental error. Consequently, the model appears to be stable under similar conditions.

Moreover, even if the joint excursions are similar, when the effect of contraction velocity is not included in the model, variability in the gain may be expected under different isokinetic velocities since constant isokinetic angular velocity does not necessarily imply a constant rate of muscular contraction, especially for the biarticular muscles (Osterning et al., 1983). To the best of this author's knowledge there exists no study that has assessed experimentally the effect of muscle biarticularity and muscle velocity of contraction under similar isokinetic conditions on the computation of gain for EMG-assisted models constructed for gait. Granata (1993) has found that muscle gain was independent of the inclusion of the muscle velocity artifact in an EMG-assisted model for the spine. However, the results of this study cannot be extrapolated to gait since biarticularity was not an issue and only one type of activity, lifting, was been investigated. When the same model was applied to a lowering of weight activity it was found that, to maintain a constant gain the muscle tension-length-velocity polynomial coefficients that accounted for the respective artifacts needed to be adjusted. In a gait cycle, however, one has to account for continuous knee flexion and extension and ankle plantar dorsi flexion under both concentric and eccentric conditions.

Thus, perhaps a more appropriate approach in the future would be to implement different gains for the flexors and different gains for the extensors of each joint of interest. Another alternative would be to represent different muscles with different gain values (Marras et al., 1999). Nevertheless, it was encouraging to observe that all optimum gains computed with least squares fell within the standard deviations of the average gains computed during the calibration procedure.

\section{Correlation coefficients}

The fact that the model gave very reasonable temporal predictions despite the cocontraction activity among the muscles was very encouraging. In addition, the shape of each two pairs of curves, i.e., predicted and measured, matched for both joints for all trials very well. Both of these observations are very encouraging when one puts into 
perspective the assumptions and limitations of the model, as well as the complex interaction of agonist and antagonist muscles throughout motion.

The correlation coefficient and the coefficient of determination, as parameters are independent of gain (Marras et al., 1999). The independence of the correlation coefficients and of the coefficients of determination from gain is verified from the results of this investigation also. In the process of implementing different gains to assess model performance in terms of the RMS error the correlation coefficients and coefficients of determination remained the same (see Appendix N and Appendix O, e.g. SA004_GlobK versus SA004_MOT for the knee). In addition, they are independent of the magnitude of the relative EMG. Thus, they allow the exploration of other sources of potential error (Marras et al., 1999), such as the effect of the electromechanical delay.

Temporal inconsistencies between predicted and measured moments can be attributed to a variety of factors such as:

a) The correct temporal prediction of the foot strike occurrence on the force plate. Error in identifying this parameter results in temporal inconsistencies with regard to the application of the ground reaction forces to the kinematic data, as well as it affects the temporal estimation of the center of pressure location, which, in turn, determines the relationship of the applied ground reaction forces with respect to the estimated joint center of rotation for the purposes of computing the net joint moments via the inverse dynamics method (Cappozzo et al., 1975). However, temporal inconsistencies at the onset of the joint moment were not an issue, based on the results of this study. Temporal inconsistencies did appear toward the end of the predicted moment for the knee for some trials, i.e., the predicted moments ended before the measured moments for some trials for the knee joint.

b) There exists the possibility that for the two trials that this happened, the reason may be related to the EMG-electrode relationship. Since the electrode is fixed on the skin and, therefore, its geometric pick up volume is constant, under dynamic conditions the muscle underneath the subcutaneous tissue is not fixed with respect to the electrode. Thus, temporal variations may be expected (De Luca, 1997). The temporal consistency of the model is more obvious when one takes into account the 
swing phase of the knee motion data. At that time there is a lot of cocontraction from both agonists and antagonists, reducing, therefore, the predicted flexor moment, and resulting at a somewhat delayed predicted moment from extensor to flexor during the swing. However, from a kinetic point of view, it appears that the flexor/extensor knee moments may be under or over estimated depending on which gain implementation procedure one selects to adopt. The same was true for the ankle too. Referring back to the calibration procedure, when the subjects were sitting during the calibration, the muscle-subcutaneous tissue-electrode relative distribution and pick up volume, may have been different than during standing and walking (De Luca, 1997; White and Winter, 1993), resulting in a signal with potentially different temporal and amplitude characteristics between the two cases.

c) Thus, one could speculate that a good calibration procedure is one where the calibration is activity specific by allowing the exact free activity of interest to be investigated with the calibration equipment. Such has been the case in the EMGassisted models for estimating loads in the spine (Granata, 1993; Granata and Marras, 1995; Marras and Granata, 1997). In fact in a comparison between the KinCom isokinetic dynamometer and the lumbar monitor for performing a calibration procedure to estimate EMG-assisted model parameters, Granata (1993) found that the isokinetic dynamometer performed very poorly compared to the lumbar monitor that allowed investigation of the activity of interest without posing any movement constraints. In fact, I believe that this is part of the success in the EMG-assisted models implemented by Dr. Marras's group, i.e., that the calibration movement to estimate the model parameters is exactly the same as the activity of interest. However, for the present and previous EMG-driven models for gait one has to extrapolate the physiological parameters estimated via isokinetic dynamometry calibration to the activity of walking gait.

Consequently, any number and combination of factors may have a potential effect on the temporal relation between the moment curves. Differences may even be due to the way the tension-length-velocity relationships for each musculotendon unit of interest 
were implemented. The present study estimated these relationships via simulations assuming maximum muscle activation and then performed a linear scaling. In doing so:

a) The effect of the passive element of the muscle was minimized. However, in vivo, during the calibration, this element could not be eliminated. Thus, its effect was embedded in the EMG signal recorded, and it could be expected to be more prominent at the ankle joint where maximum voluntary contraction efforts were not accomplished. The effect of the joint passive structures was accounted for, but not for the muscle passive structures. The presence of this effect would also result in a lag between the measured and predicted moments, with the measured moment lagging the predicted. In fact, this was the case with regard to the timing of the peak moments for almost all trials consistently (see Figure 6.17 and Figure 6.18). This effect might have been more prominent if the sample points in the EMG signal were not averaged.

b) The response/curve of the simulated muscle was dominated by the tendon slack length and thus a linear scaling of the tension-length-velocity response may not be appropriate (Karakostas et al., 1999).

In general, the correlation coefficients from this study for the knee are better than those reported from White and Winter (1993). Unfortunately, they reported results for one trial from each of two subjects. The correlation coefficients for the ankle joint were similar for both studies.

\section{$\underline{\text { Root Mean Square Error }}$}

The results for this parameter have been presented earlier and they need to be seen in the same context as the gain. One factor that may have affected the results, for the knee joint was fatigue. Fatigue would result in a decreased force per unit EMG relationship in the muscle (Bigland-Ritchie, 1981). Although such decrease has been suggested to be linear (Stephens and Taylor, 1972), the EMG-driven model presented in this study did not account for fatigue. Consequently, gain would have been overpredicted, resulting in a subsequent overprediction of the individual musculotendon unit forces. Given the fact that for each joint the predicted and measured moment pairs 


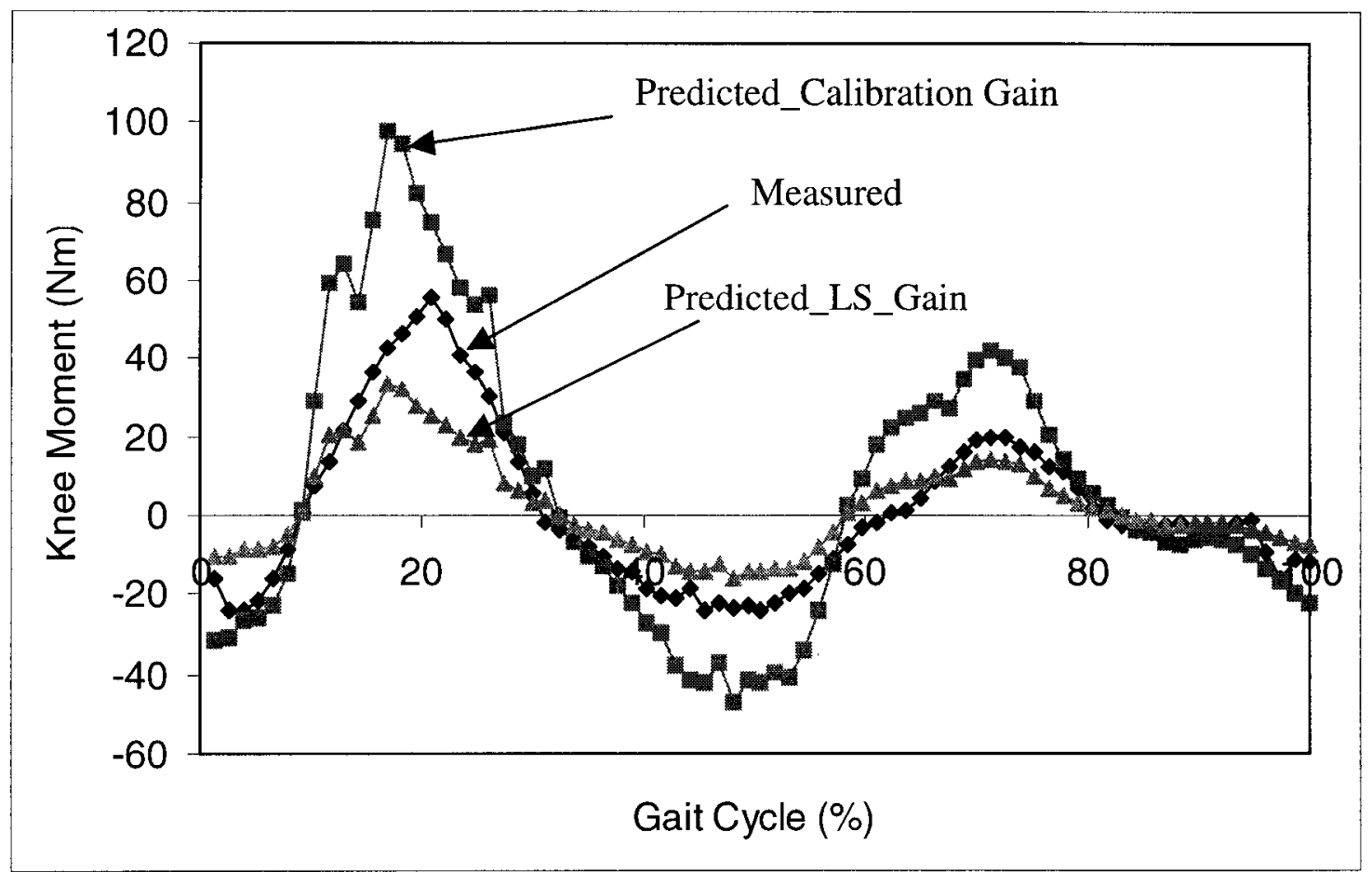

Figure 6.17. Temporal effect among the predicted and measured moments for the knee. Predicted moments were determined under two different kinds of gain implementation procedures.

are in good agreement with each other, it may be speculated that it is unlikely that other components of the model are in error.

Also, the exclusion of the series passive elastic component may have resulted in some of the underpredictions, and, therefore, in an increased RMS . Furthermore, the fact that the simulated tension-length-velocities of each musculotendon unit of interest, by virtue of maximum activation, minimized the effect of the parallel passive elastic component of the muscle, may also have resulted in an underprediction of the joint moment during the eccentric parts of the walking gait. During the eccentric phases of gait both of these elements are stretched, providing, thereby, additional resistance, i.e., force, to the already eccentrically contracting musculotendon units. Furthermore, during the shortening phase, following the eccentric contraction, the recoil of the two passive elements might contribute to the shortening muscle force. However, during the stance 


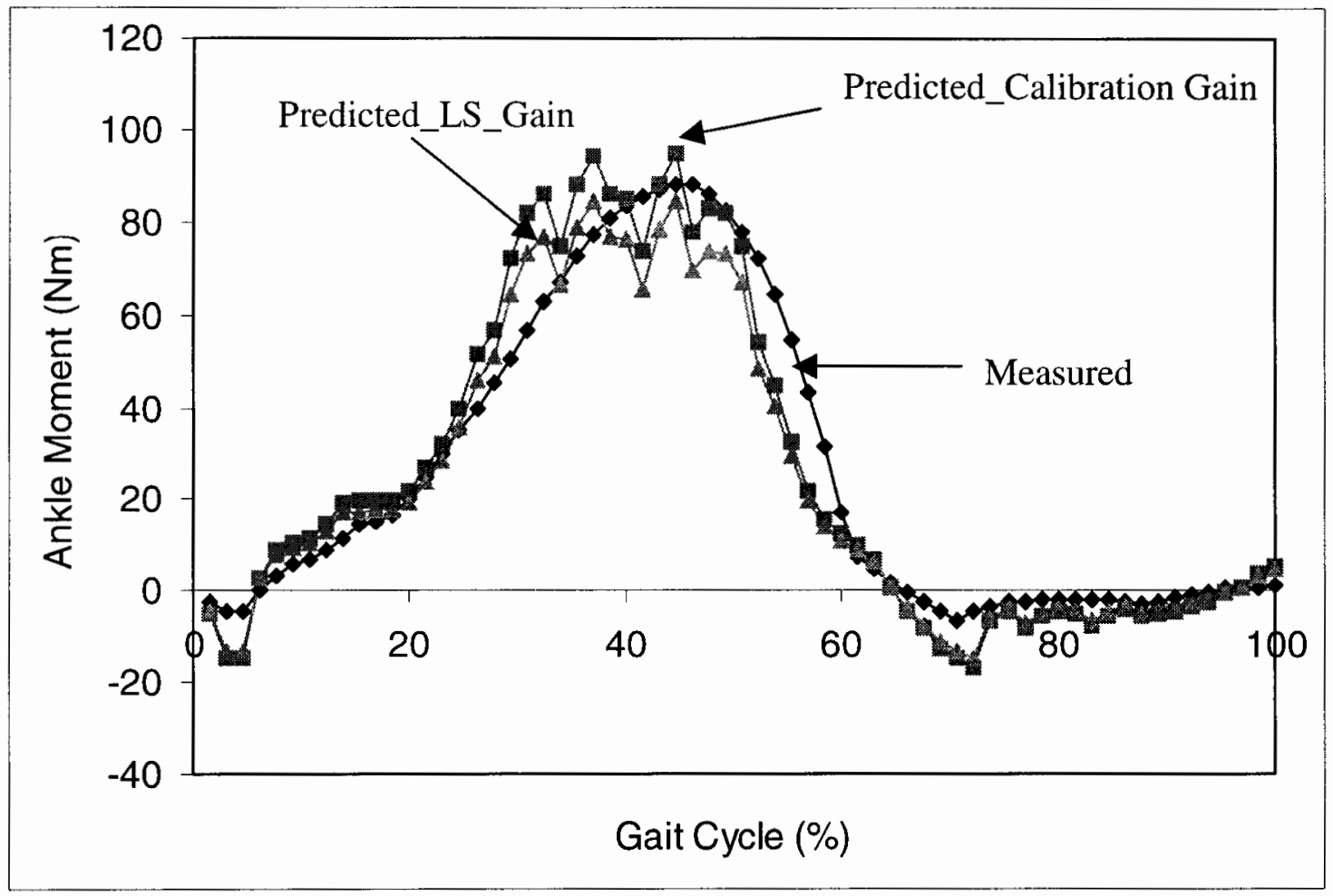

Figure 6.18. Temporal effect among the predicted and measured moments for the ankle. Predicted moments were determined under two different kinds of gain implementation procedures.

phase of gait the potential contribution of the parallel passive elastic component appears to be minimum, i.e., approximately 3 to $4 \mathrm{Nm}$ (Hof and Berg, 1981b).

A factor that may have contributed to increased RMS error might also be that some muscles were excluded from the model since their monitoring was not possible. These muscles include the deep plantar flexors. Excluding their contribution might have added to the RMS error. Finally, the soleus, a very powerful muscle, that is, however, dominated by slow twitch muscle fibers, during the simulations appeared to not be able to contribute much to the ankle joint moment by virtue of its high content of slow twitch muscle fibers, reflected in the maximum velocity of contraction. Thus, it may be that the fiber content in the soleus muscles of the subjects is different and, therefore, its contribution, and consequently the overall moment at the ankle was underpredicted. However, the results are consistent with those reported by Spector et al. (1980) and, with 
respect to the ankle joint, the predicted and measured moments were in much better agreement than the moments at the knee. Thus the error at the ankle was reduced.

Olney and Winter (1985) have shown the importance of including the tensionvelocity effects for the prediction of the measured joint moment, if the RMS values are to be reduced. In the present model the effect of muscle length and velocity on gain was not accounted for. Furthermore, no muscle simplification was performed. Consequently, the muscle to moment results represent a direct muscle force to moment conversion. All muscle moments were summed across time to arrive at the predicted net moment. Thus, an increased RMS error might be expected. In previous studies, cross talk has been an issue with respect to the RMS, but in this study the phasic activity of all muscles at all trials appeared to be consistent with what has been reported in the literature (Rab, 1994). Also, the knee joint model implemented in this study allowed for gliding and sliding but required the two articular surfaces to be always in contact, which had a direct affect on the estimation of the lengths of the musculotendon units crossing the joint and, consequently, on the estimation of the respective moment arms. Thus, the estimation of moment arms might be another potential source of error.

There are very few studies to which the validation results can be compared. The absolute RMS differences of the present study compare very well with the RMS differences reported by White and Winter (1993), except for the ankle RMSs of SA and $\mathrm{SN}$ where the calibration-based global gain and the calibration-based joint-specific gains were used to compute the predicted moments for the ankle. Normalized RMS values to the maximum moment for each respective joint computed in the gait envelope compare even better. Values, however, reported from Olney and Winter (1985) were much lower than those computed from the present model. For six subjects their RMS differences ranged from 3.2 to $9.5 \mathrm{~N}$ m during stance. In the model of Hof et al. (1983) the RMS difference was approximately $23 \mathrm{Nm}$ at the ankle. Olney and Winter (1985) and White and Winter (1993) were the only investigators to present data for the knee. For the stance phase of gait, RMS differences ranged from 4.7 to $13.0 \mathrm{Nm}$ and 11.2 and 17.9 respectively. Our results compared to those of White and Winter (1993) were a little higher. 
It can be argued that the results of the present study reflect, overall, better predictions compared to previous attempts. More specifically, in the study of Olney and Winter (1985), parameters were optimized for actual walking trials in order to give a best fit to their measured moment curves. White and Winter (1993) optimized their variable gain by accounting for the muscle length artifact without providing any information about the predicted muscle gains to verify that they were within physiological limits. However, the results presented here were achieved without optimizing the model parameters except when the gains were been estimated with a least squares approach. Thus a better understanding of their interactions may be achieved.

In the present model, biarticularity was addressed by estimating a global gain. White and Winter (1993) plotted in their calibration the relationship F/EMG versus muscle length $\left(\mathrm{L}^{\mathrm{m}}\right)$. To address biarticularity, they used only the data from the calibration that intersected and which were not many because of the selective recruitment of the muscles, i.e., of the gastrocnemius.

The cocontractions, from a temporal point of view were similar to those reported by others (Falconer and Winter, 1985). Three methods were used to compute the cocontraction index. When the respective cocontraction indexes were compared they were not found to be statistically significantly different consistently across methods. It can be speculated that the reason was that the t-tests were performed on the entire model output versus instead of only the time that the cocontraction occurred. However, the fact that most times the results were indeed statistically significant in terms of their differences would seem to suggest that accurate determination and assessment of cocontraction might be preferably done using the individual musculotendon unit forces.

\section{Methodology Limitations}

i) Inverse dynamics computed moments were estimated about fixed joint centers, whereas moment arms estimated by SIMM were computed with respect to instantaneous centers of rotation, as a function of the joint kinematics.

ii) Principal moments of inertia for the skeletal segments were estimated from cadaveric data, and each segment was considered to be a symmetrical geometric object. 
iii) Muscle architecture input in SIMM was not subject specific. Thus, the resulting tension-length and tension-velocity relationships were not subject specific either, unless it is assumed that the subjects that were used in the present study, by virtue of being normal, had similar muscle architectural characteristics with those implemented in SIMM, and, therefore, making these relationships invariable for implementation in the EMG-driven model was appropriate.

iv) The effect of muscle length and its respective rate of change on the estimation of gains was not considered in this study. Thus, intra-subject gain variability was not accounted for.

v) Only one gain was computed for all muscles that crossed a joint. Perhaps, a more appropriate approach could have been computing a separate gain for the flexors and separate gain for the extensors at each joint.

vi) Only concentric type of contractions were used in the present calibration procedure. However, gait is, primarily, a gravity-control activity, i.e., it involves primarily eccentric contractions.

vii) Dynamic normalized EMG values greater than one and the normalized RMS errors indicate that the normalization factors may not represent true electromyographic maxima, especially at the ankle.

viii) Previous reports suggest that more appropriate calibration devices for the purposes of EMG-assisted modeling involve instrumentation that mimics and allows the movement of interest to take place (Granata, 1993). For example, ankle plantar flexion during walking gait may occur in combination with foot internal rotation and inversion. The calibration device used in the present investigation did not allow joint movements to occur in their natural form.

ix) A consequence of the calibration device limitation is the issue of the accurate mapping of the electrode-muscle spatial relationship. In the present study, calibration of the knee flexors and extensors took place under sitting conditions. However, the walking gait is an activity that an individual performs adopting an upright posture. Thus, it can be speculated that there may be an issue with regard to 
the estimation of EMG-based model parameters in sitting for an activity that requires an upright posture.

$\mathrm{x}$ ) The calibration procedure was very long and fatigue, physical, but more importantly, mental, may have been a factor in the estimation of the gain parameters. 


\section{CHAPTER 7}

\section{CONCLUSIONS}

Previous researchers who have developed alternative EMG-assisted gait models to predict individual musculotendon unit forces have validated their models by reporting only the $r$ value along with the error RMS and the RMS relative to the maximum moment computed in the gait moment envelope for the respective gait (Olney, 1985; White, 1993). However, internal consistency and validity of the model requires that the computed gains all fall within physiologically acceptable limits to be valid.

Consequently, for the purposes of assessing the model performance and for comparing it with the performance of previous gait EMG-assisted models the same validation parameters have been presented as for previous studies. Additionally, the computed gains were presented along with $r^{2}$. Furthermore, model performance has been assessed via a distribution analysis of the correlation coefficients. It should be noted that the previous attempt closest to this model has reported model output only for one trial from each of two subjects tested, thereby, making the assessment of the model and a determination about its intra-consistency very difficult to make. The present study included three subjects and reported all data from multiple calibration trials and all data from three gait validation trials for each subject, thereby allowing an assessment of the intra-consistency of the model.

Since EMG-assisted models for the spine tend to include a distribution analysis of the coefficient of determination we have computed the parameter for the purposes of comparison, if desired. The performance is not as good as theirs, but one has to realize that gait involves biarticular muscles looking at results at two joints simultaneously whereas spine research focuses on only one joint. 
Furthermore, we have not modeled any error terms that would modulate the EMG signal with the strength-length and force-velocity polynomials. White and Winter (1993) also accounted for the EMG artifact encapsulated in the gain as a function of the muscle length. The same approach was used by Hof (1987).

We assessed the efficacy, fidelity and the strength of the model and contributed to the determination of its potential feasibility in investigating pathological cases by determining its physiological parameters and then allowing their interaction and their potential for producing reasonable, physiologically and statistically acceptable results. And it is the belief of this author that the performance of the model appears to be satisfactory.

The model allows the incorporation of all the benefits of Dr. Zajac's and his group's findings over the past two decades. It allows the incorporation of SIMM strengths in estimating individual musculotendon moment arms, and lengths accurately, thereby fulfilling Dr. Perry's 2000 suggestion (Perry, 2000), for identifying ways to incorporate SIMM related research into the clinical settings, and via the procedure for attaining specific model parameters it incorporates most of findings of Dr. Winter's group over the past two decades.

However, this model by no means has achieved its full potential. In the future, accounting for the muscle length and contraction velocity artifacts on the gain, thereby closing the loop and minimizing the error between the predicted and measured joint moments (see Figure 7.1), accounting for the eccentric aspects encapsulated in the electromyogram and letting these parameters be reflected in the normalization of the EMG, identifying a calibration procedure that will permit a more reliable activity-specific estimation of model parameters, computing different gains for each flexor or extensor group at the knee or ankle, involving an optimum muscle apportioning procedure by incorporating some recent research findings (Anderson and Pandy, 2001) such that muscles not monitored can be represented in the model, and finally incorporating subjectspecific anthropometric parameters taking advantage of the most recent developments in the field of imaging are all options and aspects of the model to be explored and improve its performance. 


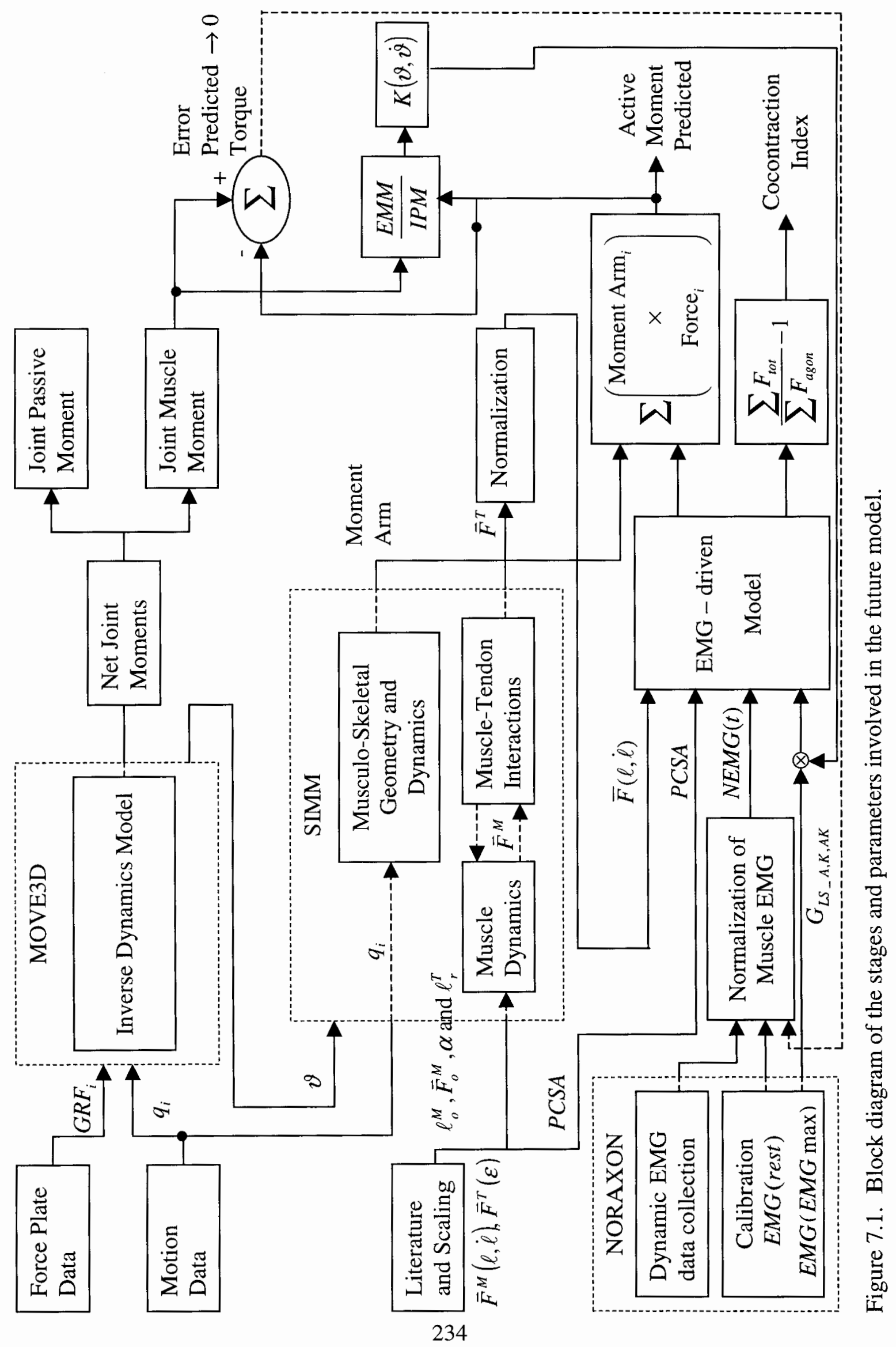


This author in the present study has acknowledged and incorporated many of the advances made possible by preceding investigators while at the same time the field of EMG-driven modeling fro gait has been advanced by developing a more physiologically specific model that allows a better investigation of the interactions among the physiological parameters, thereby allowing the potential of modeling the errors involved and therefore making the model a feasible option for clinical use for the purposes of investigating pathologies, assisting intervention decisions and monitoring rehabilitation outcomes. 


\section{LIST OF REFERENCES}

Abbot, B.C. and Aubert, X.M. (1952). The force exerted by active striated muscle durnig and after change of length. Journal of Physiology, 117, 77-86.

Abbot, B.C. and Wilkie, D.R. (1953). The relation between velocity of shortening and the tension-length curve of skeletal muscle. Journal of Physiology, 120, 214-223.

Acierno, S.P., Baratta, R.V. and Solomonow, M. (1998). A practical guide to electromyography. Paper presented at the North American Society of Gait and Clinical Movement Analysis: 3nd Annual Meeting., San Diego, CA.

Alexander, R.M. and Goldspink, G. (1977). Mechanics and energetics of animal locomotion. London: Chapman \& Hall.

Allison, G.T., Marshall, R.N. and Singer, K.P. (1993). EMG signal amplitude normalization technique in stretch-shortening cycle movements. Journal of Electromyography and Kinesiology, 3, 236-244.

An, K.N., Takahashi, K., Harrigan, T.P., and Chao, E.Y. (1984). Determination of muscle orientations and moment arms. Transactions of the American Society for Mechanical Engineers: Journal of Biomechanical Engineering, 106, 280-282.

An, K.N., Ueba, Y., Chao, E.Y. and Cooney, W.P. (1983). Tendon excursion and moment arm of index finger muscles. Journal of Biomechanics, 16, 419-425.

Anderson, F.C. and Pandy, M.G. (2001). Static and dynamic optimization solutions for gait are practically equivalent. Journal of Biomechanics, 34, 153-161.

Andriacchi, T.P., (1999). Discussion on the marker sets used for motion analysis. Personal Communication. Nashville, TN

Antonsson, E.K. and Mann, R.W. (1985). The frequency content of gait. Journal of Biomechanics, 18, 39-47.

Apkarian, J., Naumann, S. and Cairns, B. (1989). A three dimensional kinematic and dynamic model of the lower limb. Journal of Biomechanics, 22, 143-155. 
Ariano, M.A., Armstrong, R.B. and Edgerton, V.R. (1973). Hindlimb muscle fiber populations of five mammals. Journal of Histochemistry and Cytochemistry, 21, 51-55.

Arnold, A.S., (1996). Constructing a musculoskeletal model to study femoral derotational osteotomies. Personal Communication. Columbus, $\mathrm{OH}$

Arnold, A.S., Komatu, A.V. and Delp, S.L. (1997). Internal rotation gait: A compensatory mechanism to restore abduction capacity decreased by bone deformity? Developmental Medicine and Child Neurology, 39, 40-44.

Audu, M.L. and Davy, D.T. (1985). The influence of muscle model complexity in musculoskeletal motion modeling. Journal of Biomechanical Engineering, 107, $147-157$.

Ayoub, M.A., Ayoub, M.M. and Ramsey, J.D. (1970). A stereometric system for measuring human motion. Human Factors, 12, 523-535.

Bahler, A.S., Fales, J.T. and Zierler, K.L. (1967). The active state of mamalian skeletal muscle. Journal of General Physiology., 55, 2239-2253.

Bahler, A.S., Fales, J.T. and Zierler, K.L. (1968). The dynamic properties of mammalian skeletal muscle. Journal of General Physiology, 51, 369-384.

Baildon, R.W.A. and Chapman, A.E. (1983a). A new approach to the human muscle model. Journal of Biomechanics, 16, 803-809.

Baildon, R.W.A. and Chapman, A.E. (1983b). Mechanical properties of a single equivalent muscle producing forearm supination. Journal of Biomechanics, 16, 811-819.

Baratta, R. and Solomonow, M. (1991). The effect of tendon viscoelastic stiffness on the dynamic perforance of isometric muscle. Journal of Biomechanics, 24, 109-116.

Basmajian, J.V. (1976). The human bicycle. In P.V. Komi (Ed.), Biomechanics V-A. Baltimore, MD.: University Park Press.

Bawa, P. and Stein, R.B. (1976). Frequency response of human soleus muscle. Journal of Neurophysiology, 39, 768-793.

Bergman, B.W. (1974). Three dimensional cinematography: A flexible approach. Research Quarterly, 45, 303-309. 
Bigland, B. and Lippold, O.C.J. (1954). The relation between force, velocity and integrated electrical activity in human muscle. Journal of Physiology., 123, 214224.

Bigland-Ritchie, B. (1981). EMG/Force relations and fatigue of human voluntary contractions. ( Vol. 9). Baltimore: The Franklin Institute Press.

Binkhorst, R.A., Hoofd, L. and Vissers, A.C.A. (1977). Temperature and force-velocity relationship on human muscles. Journal of Applied Physiology, 42, 471-475.

Bobbert, M.F., Huijing, P.A. and Van Ingen Schenau, G.J. (1986). A model of the human triceps surae muscle-tendon complex applied to jumping. Journal of Biomechanics, 19, 887-898.

Bobet, J. and Norman, R.W. (1984). Effects of load placement on back muscle activity in load carriage. European Journal of Applied Physiology, 53, 7-75.

Bouisset, S. (1973). EMG and muscle force in normal motor activities. In J.E. Desmedt (Ed.), New Developments in EMG and Clinical Neurophysiology (Vol. 1, pp. 547583). Karger, Basel.

Bouisset, S. and Goubel, F. (1971). Interdependence of relations between integrated EMG and diverse biomechanical quantities in normal voluntary movements. Activitas Nervosa Superior, 13, 23-31.

Brand, R.A., Crowninshield, R.D., Wittstock, C.E., Pedersen, D.R., Clark, C.R. and Van Krieken, F.M. (1982). A model of the lower extremity muscular anatomy. Journal of Biomechanical Engineering, 104, 304-310.

Brand, R.A., Pedersen, D.R., and Friederich, J.A. (1986). The sensitivity of muscle force predictions to changes in physiologic cross-sectional area. Journal of Biomechanics, 19, 589-596.

Buchthal, F. and Schmalbruch, H. (1970). Contraction times and fibre types in intact human muscle. Act Physiologica Scandinavica., 79, 435-452.

Burden, A. and Bartlett, R. (1999). Normalization of EMG amplitude: an evaluation and comparison of old and new methods. Medical Engineering and Physics, 21, 247257.

Calderale, P.M. and Scheflo, G. (1987). A mathematical model of the locomotor apparatus. Engineering in Medicine, 16, 147-161.

Cappozzo, A. (1983). Stereophotogrammetric system for kinesiological studies. Medical and Biological Engineering and Computing, 21, 217-223. 
Cappozzo, A. (1984). Gait analysis methodology. Human Movement Science, 3, 27-50.

Cappozzo, A., Catani, F., Della Croce, U. and Leardini, A. (1995). Position and orientation in space of bones during movement: anatomical frame definition and determination. Clinical Biomechanics, 10, 171-178.

Cappozzo, A., Leo, T. and Pedotti, A. (1975). A general computing method for the analysis of human locomotion. Journal of Biomechanics, 8, 307-320.

Carlstedt, C.A. and Nordin, M. (1989). Tendons and Ligaments. In R.N.a.F. Nordin, V.H. (Ed.), Basic Biomechanics of the Musculoskeletal System (2nd ed.). Philadelphia, PA.: Lea \& Febiger.

Cavagna, G.A. and Kaneke, M. (1977). Mechanical work and efficiency in level walking and running. Journal of Physiology, 268, 467-481.

Cavanagh, P.R. (1974). Electromyography: its use and misuse in physical education. Journal of Health Physical Education and Recreation, 45, 61-64.

Cavanagh, P.R. (1981). Forces and pressures between the foot and the floor during normal walking and running. Paper presented at the Proceedings of the Biomechanics Symposium, Indiana University, Bloomington.

Chaffin, D.B., Myun, L. and Freivalds, A. (1980). Muscle strength assessment from EMG analysis. Medicine and Science in Sports and Exercise, 12, 205-211.

Chapman, A.E. (Ed.). (1978). The relation between length and the force-velocity curve of a single equivalent linear muscle during flexion of the elbow. (Vol. VI-B). Baltimore: University Park Press.

Chapman, A.E. (1985). The mechanical properties of human muscle. In R.L. Terjung (Ed.), Exercise and Sport Sciences Reviews (Vol. 13). New York: MacMillian Publishing Company.

Claflin, D.R. and Faulkner, J.A. (1985). Shortening velocity extrapolated to zero load and unloaded shortening velocity of whole rat skeletal muscle. Journal of Physiology., $359,357-363$.

Clarkson, H.M. and Gilewich, G.B. (1989). Musculoskeletal assessment: Joint range of motion and manual muscle strength. Baltimore, MD: Williams \& Wilkins.

Close, J.R., Nickel, E.D. and Todd, F.N. (1960). Motor unit action potential couns. Journal of Bone and Joint Surgery, 42, 1207-1222. 
Close, R.I. (1972). Dynamic properties of mammalian skeletal muscle. Physiological Reviews, 52, 129-199.

Coggshall, J.C. and Bekey, G.A. (1970). EMG-force dynamics in human skeletal muscle. Medical and Biological Engineering, 8, 265-270.

Collins, J.J. and O'Connor, J.J. (1991). Muscle-ligament interactions at the knee during walking. Proceedings Mechanical Engineers, 205, 11-18.

Corcos, D.M., Gotlieb, G.L. and Agarwal, G.C. (1989). Organizing principles for singlejoint movements II. A speed sensitive strategy. Journal of Neurophysiology, 62, 358-368.

Crosby, P.A. (1978). Use of surface electromyogram as a measure of dynamic force in human limb muscles. Medical and Biologicval Engineering and Computing., 16, 519-524.

Crowninshield, R.D. and Brand, R.A. (1981a). The prediction of forces in joint structures: Distribution of intersegmental resultants. In D.T. Miller (Ed.), Exercise and Sports Science Reviews (Vol. 9, pp. 159-181).

Damiano, D.L., Martellotta, T.L., Sullivan, T.J. and Granata, K.P. (2000). Muscle force production and functional performance in spastic cerebral palcy: relationship of cocontraction. Archives of Physical Medicine and Rehabilitation, 81, 895-900.

Dapena, J., Harman, E.A. and Miller, J.A. (1982). Three dimensional cinematography with control object of unknown shape. Journal of Biomechanics, 15, 11-19.

Davis, B., (1996). Discussion on the COP. Personal Communication. Cleveland, OH

Davis, R., Ounpuu, S., Tyburski, D. and Gage, J. (1991). A gait analysis data collection and reduction technique. Human Movement Science, 10, 575-587.

Davy, D.T. and Audu, M.L. (1987). A dynamic optimization technique for predicting muscle forces in the swing phase of gait. Journal of Biomechanics, 20, 793-801., 20, 793-801.

De Luca, C.J. (1979). Physiology and mathematics of myoelectric signals. IEEE Transactions On Biomedical Engineering, BME-26, 313-325.

De Luca, C.J. (1997). The use of surface electromyography in biomechanics. Journal of Applied Biomechanics, 13, 135-163.

Delagi, E.F. and Perotto, A. (1981). Anatomical Guide for the Electromyographer. (2nd ed.). Springfield, IL.: Charles C. Thomas. 
Delp, S.L. (1990). Surgery simulation: A computer graphics system to analyze and design musculoskeletal reconstructions of the lower limb. Unpublished Ph.D. dissertation, Stanford University, Stanford, CA.

Delp, S.L., and Loan, P.J. (1995). A graphics-based software system to develop and analyze models of musculoskeletal structures. Computers in Biology and Medicine, 25, 21-34.

Delp, S.L., Loan, P.J., Hoy, M.G., Zajac, F.E., Topp, E.L. and Rosen, J.M. (1990b). An interactive graphics-based model of the lower extremity to study orthopaedic surgical procedures. IEEE Transactions in Biomedical Engineering, 37, 757-767.

DeLuca, C.J. and Van Dyke, E.J. (1975). Derivation of some parameters of myoelectric signals recorded during sustained constant force isometric contractions. Biophysiology Journal, 15, 1167-1180.

Dempster, W.T. (1955). Space requirements of the seated operator (WADC TR 55-159). Ohio: Wright Patterson Air Force Base.

DeVries, H.A. (1968). "Efficiency of electrical activity" as a physiological measure of the functional state of muscle tissue. American Journal of Physical Medicine, 47, 1022.

Dvir, Z. (1996). An isokinetic study of combined activity of the hip and knee extensors. Clinical Biomechanics, 11, 135-138.

Eberstein, A. and Goodgold, J. (1968). Slow and fast twitch fibres in human skeletal muscle. American Journal of Physiology, 215, 535-541.

Edman, K.A.P. (1979). The velocity of unloaded shortening and its relation to sarcomere length and isometric force in vertebrate muscle fibers. Journal of Physiology, 291, 143-159.

Edman, K.A.P., Caputo, C. and Lou, F. (1993). Depression of tetanic force induced by loaded shortening of frog muscle fibers. Journal of Physiology, 466, 535-552.

Edman, K.A.P., Elzinga, G. and Noble, M.I.M. (1978). Enhancement of mechanical performance by stretch during tetanic contrations of vertebrate skeletal muscle fibres. Journal of Physiology, 281, 139-155.

Engin, A.E. and Tumer, S.T. (1993). Improved dynamic model of the human knee joint and its response to impact loading on the lower leg. Transactions of ASME Journal of Biomechanical Engineering, 115, 137-143. 
Ettema, G.J. and Huijing, P.A. (1989). Properties of tendinous structures and series elastic component of EDL muscle-tendon complex of the rat. Journal of Biomechanics, 22, 1209-1215.

Evetovich, T.K., Housh, T.J., Weir, J.P., Johnson, G.O., Smith, D.B. and Ebersole, K.T. (1999). Mean power frequency and amplitude of the mechanomyographic signal during maximal eccentric isokinetic muscle actions. Electromyography and Clinical Neurophysiology, 39, 123-127.

Falconer, K. and Winter, D.A. (1985). Quantitative assessment of co-contraction at the ankle joint in walking. Electromyography and Clinical Neurophysiology, 25, 135149.

Faulkner, J.A., Jones, D.A., Round, J.M. and Edwards, R.H.T. (1980). Dynamics of energetic process in human muscle. In P.C.a.B. Whipp (Ed.), Bioenergetics and Gas Exchange.: Elsevier/North-Holland Biomedical Press.

Fidelus, K. (1968). Some biomechanical principles of muscle cooperation in the upper extremities. Paper presented at the 1st International Seminar, Zurich.

Ford, L.E., Huxley, A.F. and Simmons, R.M. (1981). The relation between stiffness and filament overlap in stimulated frog muscles. Journal of Physiology, 311, 219-249.

Freivalds, A., Chaffin, D.B., Garg, A. and Lee, K.S. (1984). A dynamic biomechanical evaluation of lifting maximum acceptable loads. Journal of biomechanics, 17(4), 251-262.

Freund, H.-J. (1983). Motor unit and muscle activity in voluntary muscle control. Physiological Reviews., 63, 387-436.

Friederich, J.A. and Brand, R.A. (1990). Muscle fiber architecture in the human lower limb. Journal of Biomechanics, 23, 91-95.

Fuglevand, A.J., Winter, D.A. and Patla, A.E. (1993). Models of recruitment and rate coding organization in motor unit pools. Journal of Neurophysiology, 70, 24702488 .

Fukunaga, T., Kawakami, Y., Kuno, S., Funato, K. and Fukashiro, S. (1997). Muscle architecture and function in humans. Journal of Biomechanics, 30, 457-463.

Fung, Y.C.B. (1981). Biomechanics: Mechanical properties of living tissues. New York, NY.: Springer Verlag.

Gage, J.R. (1991). Gait Analysis in Cerebral Palsy. London: Mac Keith Press. 
Gage, J.R. and Koop, S.E. (1995). Clinical gait analysis: Application to management of cerebral palsy. In P. Allard, Stokes I.A.F., and Blanchi J-P. (Ed.), ThreeDimensional Analysis of Human Movement. Champaign, IL.: Human Kinetics.

Galea, V. and Norman, R.W. (Eds.). (1983). Bone-on-bone forces at the ankle joint during a rapid dynamic movement. ( Vol. XI-A). Waterloo: Human Kinetics Publishers Inc., Champaign, Ill.

Gerleman, D.G. and Cook, T.M. (1992). Instrumentation, Selected topics in surface electromyography for use in occupational setting: Expert perspectives.: U.S. Department of Health and Human Services, Public Health Service, DHHS (NIOSH) Publication No. 91-100.

Ghori, G.M.U., Donne, B. and Luckwill, R.G. (1995). Relationship between torque and EMG activity of a knee extensor muscle during isokinetic concentric and eccentric actions. Journal of Electromyography and Kinesiology, 5, 109-115.

Gillard, D.M., Yakovenko, S., Cameron, T. and Prochazka, A. (2000). Isometric muscle length-tension curves do not predict angle-torque curves of human wrist in continuous active movements. Journal of Biomechanics, 33, 1341-1348.

Gleeson, N.P. and Mercer, T.H. (1996). The utility of isokinetic dynamometry in the assessment of human muscle function. Sports Medicine, 21, 18-33.

Goldstein, H. (1980). Classical Mechanics ( 2nd ed.). Don Mills, Ontario: AddisonWesley Publishing Company.

Gordon, A.M., Huxley, A.F. and Julian, F.J. (1964). The length-tension diagram of single vertebrae striated muscle fibres. Proceedings of the Physiological Society., 171, 28-30.

Gordon, A.M., Huxley, A.F. and Julian, F.J. (1966b). The variation in isometric tension with sarcomere length in vertebrate muscle fibres. Journal of Physiology., 184, 170-192.

Gordon, C.C., Chumlea Cameron, W. and Roche, A.F. (1988). Stature, recumbent length, and weight. In T.G. Lohman, Roche, A.F., and Martorell, R. (Ed.), Anthropometric Standardization Reference Manual. Champaign, IL.: Human Kinetics.

Goslin, B.R. and Charteris, J. (1979). Isokinetic dynamometry: Normative data for clinical use in lower extremity (knee) cases. Scandinavica Journal of Rehabilitation Medicine, 11, 105-109. 
Gottlieb, G.L. and Agarwall, G.C. (1971). Dynamic relationship between isometric muscle tension and the electromyogram in man. Journal of Applied Physiology, $30,345-351$.

Gottlieb, G.L., Latash, M.L., Corcos, D.M., Liubinskas, T.J. and Agarwall, G.C. (1992). Organizing principles for single joint movements: Agonist - antagonist interactions. Journal of Neurophysiology, 67, 1417-1427.

Granata, K.P. (1993). An EMG-assisted model of biomechanical trunk loading during fre-dynamic lifting. Unpublished Ph.D., The Ohio State University, Columbus, $\mathrm{OH}$.

Granata, K.P., and Marras, W.S. (1995). An EMG-assisted model of trunk loading during free-dynamic lifting. Journal of Biomechanics, 28(11), 1309-1317.

Granata, K.P. and Marras, W.S. (1993). An EMG-assisted model of loads on the lumbar spine during asymmetric trunk extensions. Journal of Biomechanics, 26, 14291438 .

Granata, K.P. and Marras, W.S. (1994). The inluence of trunk muscle coactivity upon dynamic spinal loads. Spine.

Granata, K.P. and Marras, W.S. (1995a). An EMG-assisted model of trunk loading during free-dynamic lifting. Journal of Biomechanics, 28(11), 1309-1317.

Granata, K.P. and Marras, W.S. (1995b). The influence of trunk muscle coactivity upon dynamic spinal loads. Spine, 20, 913-919.

Gransberg, L. and Knuttson, E. (1983). Determination of dynamic muscle strength in man with acceleration controlled isokinetic movements. Acta Physiologica Scandinavica, 119, 317-320.

Green, J.I. (1992). Individualized dynamic musculoskeletal modeling of cerebral palsy patients with lower extremity spasticity in gait. Unpublished MSc., Arizona State University, Tempe, Arizona.

Grieves, D.W. and Pheasant, S.T. (1967). Myoelectric activity, posture and isometric torque in man. Electromyography and Clinical Neurophysiology, 16, 3-21.

Grood, E.S. and Suntay, W.J. (1983). A joint coordinate system for the clinical description of three-dimensional motions: Application to the knee. Journal of Biomechanical Engineering, 105, 136-144. 
Grood, E.S., Suntay, W.J., Noyes, F.R. and Butler, D.L. (1984). Biomechanics of the knee-extension exercise. Effect of cutting the anterior cruciate ligament. Journal of Bone and Joint Surgery, 66A, 725-734.

Halvorsen, K., Lesser, M. and Lundberg, A. (1999). A new method for estimating the axis of rotation and the center of rotation. Journal of Biomechanics, 32, 12211227.

Hardt, D.E. (1978). Determining muscle forces in the leg during normal human walking an application and evaluation of optimization methods. Journal of Biomedical Engineering, 100, 72-78.

Hardt, D.E. and Mann, R.W. (1980). A five body - three dimensional dynamic analysis of walking. Technical Note, Journal of Biomechanics, 13, 455-457. Technical Note, Journal of Biomechanics, 13, 455-457.

Harrison, A. and Kruze, R. (1987). Perturbation of a skilled action. II: Normalizing the responses of cerebral palsied individuals. Human Movement Science, 6, 133-159.

Hasler, E.M., Denoth, J., Stacoff, A. and Herzog, W. (1994). Influence of hip and knee joint angles on excitation of knee extensor muscles. Electromyography and Clinical Neurophysiology, 34, 355-361.

Hatze, H. (1980b). A mathematical model for the computational determination of parameter values of anthropometric segments. Journal of Biomechanics, 13, 833843.

Hatze, H. (1981a). Myocybernetic Control Models of Skeletal Muscle., University of South Africa.

Hatze, H. (1981b). A comprehensive model for human motion simulation and its application to the take-off phase of the long jump. IEEE Transactions on Automatic Control, 25, 375-385.

Hatze, H. (1981c). Estimation of myodynamic parameter values from observations on isometrically contracting muscle groups. European Journal of Applied Physiology, 46, 325-338.

Haut, R.C. (1991). Lectures on Tissue Mechanics: Theory of Tissue Mechanics. Michigan State University, East Lansing, MI.

Hawkins, D. and Bey, M. (1997). Muscle and tendon force-length properties and their interactions in vivo. Journal of Biomechanics, 30, 63-70. 
Herzog, W. (1988). The relationship between the resultant moments measured by an isokinetic dynamometer. Journal of Biomechanics, 21, 5-12.

Herzog, W., Guimaraes, A.C.S. and Zhang, Y.T. (1995). EMG. In B.M.a.H. Nigg, W. (Ed.), Biomechanics of the musculoskeletal system. NY.: Wiley \& Sons.

Herzog, W. and Leonard, T.R. (1997). Depression of cat soleus forces following isokinetic shortening. Journal of Biomechanics, 30, 865-872.

Herzog, W. and Leonard, T.R. (2000). The history dependence of force production in mamalian skeletal muscle following stretch-shortening and shortening-stretch cycles. Journal of Biomechanics, 33, 531-542.

Hill, A.V. (1922). The maximum work and mechanical efficiency of human muscles, and their most economical speed. Journal of Physiology, 56, 19-41.

Hill, A.V. (1938). The heat of shortening and the dynamic constants of muscle. Paper presented at the Proceeding of the Royal Society of Biology, London.

Hill, A.V. (1950). The series elastic component of muscle. Paper presented at the Proceedings of the Royal Society B., London.

Hill, A.V. (1953). The mechanics of active muscle. Paper presented at the Proceeding of the Royal Society B., London.

Hof, A.L. (1984). EMG and muscle force: An introduction. Human Movement Science., $3,119-153$.

Hof, A.L., (1999a). SEMG electrode placement. Biomech_L Discussion Forum.

Hof, A.L., (1999b). Summary: EMG normalization during walking. Biomech_L Discussion Forum.

Hof, A.L. and Berg, J.v.d. (1977). Linearity between the weighted sum of the EMGs of the human triceps surae and the total torque. Journal of Biomechanics, 10, 529539.

Hof, A.L. and Berg, J.v.d. (1981a). EMG to force processing I. An electrical analogue of the Hill muscle model. Journal of Biomechanics, 14, 747-758.

Hof, A.L. and Berg, J.v.d. (1981b). EMG to force processing II. Estimation of parameters of the Hill muscle model for the human triceps surae by means of a Calfergometer. Journal of Biomechanics, 14, 759-770. 
Hof, A.L. and Berg, J.v.d. (1981c). EMG to force processing III. Estimation of model parameters for the human triceps surae muscle and assessment of the accuracy by means of a torque plate. Journal of Biomechanics, 14, 771--785.

Hof, A.L. and Berg, J.v.d. (1981d). EMG to force processing IV. Eccentric concentric contraction on a spring-fly wheel set up. Journal of Biomechanics, 14, 787-792.

Hof, A.L., Green, B.A. and Berg, J.v.d. (1983). Calf muscle moment, work and efficiency in level walking; role of series elasticity. Journal of Biomechanics, 16, 523-537.

Hof, A.L., Pronk, C.N.A. and van Best, J.A. (1987). Comparison between EMG to force processing and kinetic analysis for the calf muscle moment in walking and stepping. Journal of Biomechanics, 20, 167-178.

Holden, J.P., Stanhope, S.J. and Orsini, J.A. (1994). Skeletal motion estimates: effect of surface taget techniques. Paper presented at the Proceedings of the Second World Congress of Biomechanics, Amsterdam, The Netherlands.

Hoy, M.G., Zajac, F.E. and Gordon, M.E. (1990). A musculoskeletal model of the human lower extremity: the effect of muscle, tendon, and moment arm on the momentangle relationship of musculotendon actuators at the hip, knee, and ankle. Journal of Biomechanics, 23, 157-169.

Hughes, R.E. (1991). Empirical evaluation of optimization-based lumbar muscle force prediction model. Unpublished Ph.D. dissertation, The University of Michigan.

Huxley, A.F. (1957). Muscle structure and theories of contraction. Progress in Biophysics., 7, 255-318.

Huxley, A.F. (1974). Muscular contraction. Journal of Physiology, 243, 1-43.

Inman, V.T., Ralstonon, J.H. and Saunders, J.B. (1952). Relation of human electromyogram to muscular tension. Electroencephalography and Clinical Neurophysiology, 4, 187-194.

Ismail, H.M. and Ranatunga, K.W. (1978). Isometric tension development in human skeletal muscle in relation to its working range of movement: The length-tension relationship of biceps brachii muscle. Experimental Neurology., 62, 595-604.

Johnson, M.A., Polgar, J., Weightman, D. and Appleton, D. (1973). Data on the distribution of fibre types in thirty-six human muscles. An autopsy study. Journal of Neurological Sciences, 18, 111-129. 
Jorgensen, K. and Bankov, S. (1971). Maximum strength of elbow flexors with pronated and supinated forearms. In a.J.W. J. Vredenbregt (Ed.), Medicine and Sport (Vol. 6: Biomechanics II, pp. 174-180). Karger: Basel.

Ju, M.S. and Mansour, J.M. (1988). Simulation of the double limb support phase of human gait. Journal of Biomechanical Engineering, 110, 223-229.

Kadaba, M.P., Ramakrishnan, H.K., Wooten, M.E., Gainey, J., Gorton, G. and Cochran, G.V.B. (1989). Repeatability of kinematic, kinetic, and electromyographic data in normal adult gait. Journal of Orthopaedic Research, 7, 849-860.

Kadaba, M.P., Ramakrishnan, and H.K., Wooten, M.E. (1990). Measurement of lower extremity kinematics during level walking. Journal of Orthopaedic Research, 8, 383-392.

Karakostas, T., Parnianpour, M., Berme, N., Pease, W.S., Quesada, P.M. and Simon, S.R. (1999). On the decoupling of the passive and active components of muscle force. In ASME (Ed.), Advances in Bioengineering (Vol. 16, pp. 125-126). Nashville: ASME.

Karakostas, T., Quesada, P.M. and Simon, S.R. (1995). Towards the quantification of the threshold level of spasticity. American Academy for Cerebral Palsy and Developmental Medicine Annual Meeting, Philadelphia, PA . Paper presented at the American Academy for Cerebral Palsy and Developmental Medicine Annual Meeting, Philadelphia, PA.

Katz, B. (1939). The relation between force and speed in muscular contraction. Journal of Physiology, 96, 45-64.

Kaufman, K.R., An, K.-N., Litchy, W.J. and Chao, E.Y.S. (1991a). Physiological predictions of muscle forces - I. Theoretical formulation. Neuroscience, 40, 781792.

Kaufman, K.R., An, K.-N., Litchy, W.J. and Chao, E.Y.S. (1991b). Physiological prediction of muscle forces-II. Application to isokinetic exercise. Neuroscience, $40,793-804$.

Kaufman, K.R., An, K-N and Chao, E.Y.S. (1995). A comparison of intersegmental joint dynamics to isokinetic dynamometer measurements. Journal of Biomechanics, 28, 1243-1256.

Kaufman, K.R., An, K-N, Litchy, W.J. and Chao, E.Y.S. (1991b). Physiological prediction of muscle forces-II. Application to isokinetic exercise. Neuroscience, 40, 793-804. 
Kellis, E. and Baltzopoulos, V. (1996). The effects of normalization method on antagonistic activity patterns during eccentric and concentric isokinetic knee extension and flexion. Journal of Electromyography and Kinesiology, 6, 235-245.

Kepple, T.M., (1997). Discussion on the body segment parameters using the MOVE3D software. Personal Communication. Columbus, $\mathrm{OH}$.

Khalaf, K.A., Parnianpour, M. and Karakostas, T. (2000). A normative surface response database of isokinetic lower extremity joint strength: Towards the evaluation of dynamic human performance. Accepted by the Journal of Applied Biomechanics.

Kinzel, G.I., Hall, A.S. and Hillberry, B.M. (1972). Measurement of the total motion between two body segments-I. Analytical development. Journal of Biomechanics, 5, 93-102.

Kirchberger, M.A. (1990). Excitation and contruction of skeletal muscle. In J.B. West (Ed.), Best and Taylor's Physiological Basis of Medical Practice. Baltimore, MD: Williams \& Wilkins.

Klein, P., S., M. and Rooze, M. (1996). Moment arm length variations of selected muscles acting on talocrural and subtalar joints during movement: an in vitro study. Journal of Biomechanics, 29, 21-30.

Knutson, L.M., Soderberg, G.L., Ballantyne, B.T. and Clarke, W.R. (1994). A study of various normalization procedures for within day electromyographic data. Journal of Electromyography and Kinesiology, 4, 47-59.

Komi, P.V. (1973a). Measurement of force-velocity relationship in human muscle under concentric and eccentric contractions. Medicine in Sport, Biomechanics III, 8, 224-229.

Komi, P.V. (Ed.). (1973b). Relationship between muscle tension, EMG and velocity of contraction under concentric and eccentric work. (Vol. 1).

Komi, P.V., Salomen, M. and Jarvinen, M. (1987). In vivo registration of Achilles tendon forces in man: I. Methodological development. International Journal of Sports Medicine, 8 (Suppl. 1), 3-8.

Kovanen, V., Suominen, H. and Heikkinen, E. (1984). Mechanical properties of fast and slow skeletal muscle with special reference to collagen and endurance training. Journal of Biomechanics, 17, 725-735.

Lamb, R. and Hobart, D. (1992). Anatomic and physiologic basis for surface electromyography. Selected Topics in Surface Electromyography for Use in the 
Occupational Setting: Expert Perspectives.: U.S. Department of Health and Human Services, Public Health Service, DHHS (NIOSH) Publication No. 91-100.

Lawrence, J.H. and De Luca, C.J. (1983). Myoelectric signal versus force relationship in different human muscles. Journal of Applied Physiology., 54, 1653-1659.

Lawrence, J.H. and DeLuca, C.J.H. (1983). Myoelectric signal versus force relationship in different human muscles. Journal of Applied Physiology, 54, 1653-1659.

LeVeau, B. and Andersson, G. (1992). Output forms: Data analysis and applications., Selected topics in surface electromyography for use in occupational setting: Expert perspectives.: U.S. Department of Health and Human Services, Public Health Service, DHHS (NIOSH) Publication No. 91-100.

Lieber, R.L. (1992). Skeletal muscle anatomy. In R.L. Lieber (Ed.), Skeletal muscle structure and function - Implications for Rehabilitation and Sports Medicine. Baltimore, MD: Williams \& Wilkins.

Lieber, R.L. (1994). Experimental design and statistical analysis. In S.R. Simon (Ed.), Orthopaedic Basic Science. Rosemont, IL: American Academy of Orthopaedic Surgeons.

Lieber, R.L., Roos, K.P., Lubell, B.A., Cline, J.W. and Baskin, R.J. (1983). High speed digital data acquisition of sarcomere length from isolated skeletal and cardiac muscle cells. IEEE Transactions On Biomedical Engineering, 50-57.

Lippold, O.C.J. (1952). The relation between integrated action potentials in a human muscle and its isometric tension. Journal of Physiology., 177, 492-499.

Lloyd, D.G. and Buchanan, T.S. (1996). A model of load sharing between muscles and soft tissues at the human knee during static tasks. Journal of Biomechanical Engineering, 118, 367-376.

Loan, P.J., (1998). On the estimation of maximum velocity of contraction for the purposes of SIMM simulations. Personal Communication. ColumbusS

Lyons, K., Perry, J. and Gronely, J.K. (1983). Timing and relative intensity of hip extensor and abductor muscle action during level and stair ambulation. Physical Therapy, 63, 1597-1159.

Marechal, G. and Plaghki, L. (1979). The deficit of the isometric tetanic tension redeveloped after a release of frog muscle at a constant velocity. Journal of General Physiology, 73, 453-467. 
Marras, W.S. and G.A., M. (1990). Muscle activities during asymmetric trunk angular accelerations. Journal of Orthopedic Research, 8, 824-832.

Marras, W.S. and G.A., M. (1993). Electromyographic studies of the lumbar trunk musculature during the generation of low-level trunk acceleration. Journal of Bone and Joint Surgery, 11, 811-817.

Marras, W.S. and Granata, K.P. (1997). The development of an EMG-assisted model to assess spine loading during whole-body free-dynamic lifting. Journal of Electromyography and Kinesiology, 7, 259-268.

Marras, W.S., Granata, K.P. and Davis, K.G. (1999). Variability in spine loading model performance. Clinical Biomechanics, 14, 505-514.

Marras, W.S. and Mirka, G.A. (1992). A comprehensive evaluation of trunk response to asymmetric trunk motion. Spine, 17(3), 318-326.

Marras, W.S. and Sommerich, C.M. (1991a). A three-dimensional motion model of loads on the lumbar spine - I. Model structure. Human Factors, 33(2), 123-137.

Marras, W.S., Wongsam, P.E. and Rangarajulu, S.L. (1986). Trunk motion during lifting: The relative cost. International Journal of Industrial Ergonomics, 1, 103-113.

Marsh, E., Sale, D., McComas, A.J. and Quinlan, J. (1981). Influence of joint position on ankle dorsiflexion in humans. Journal of Applied Physiology, 51, 160-167.

Mashima, H., Akazawa, K., Kushima, H. and Fujii, K. (1972). The force-load-velocity relation and the viscous-like force in the frog skeletal muscle. Japanese Journal of Physiology, 22, 103-120.

Matsumoto, Y. (1967). Validity of the force-velocity relation for muscle contraction in the length region, 1<lo. Journal of General Physiology., 50, 1125-1137.

McConville, J.T., Churchill, T.D., Kaleps, I., Clauser, C.E. and Cuzzi, J. (1980). Anthropometric relationships of body and body segment moments of inertia. (AFAMRL-TR-80-119). Dayton, OH: Wright-Patterson Air Force Base.

McCubin, J.A. and Shasby, G.B. (1985). Effects of isokinetic exercise on adolescents with cerebral palsy. Adapted Physical Activity Quarterly, 2, 56-64.

McGill, S.M. (1992). A myoelectrically based dynamic three dimensional model to predict loads on lumbar spine tissues during lateral bending. Journal of Biomechanics, 25, 395-414. 
McGill, S.M. and Norman, R. (1986). Partitioning the L4-L5 dynamic moment into disc, ligamentous, and muscular components during lifting. Spine, 11, 666-678.

Meglan, D. and Todd, F. (1994). Kinetics of human locomotion. In J.a.G. Rose, J.G. (Ed.), Human Wlaking (2 ed.). Baltmore: Williams \& Wilkins.

Meijer, K., Grootenboer, H.J., Koopman, B.F.J.M. and Huijing, P. (1997). Fully isometric length-force curves of rat muscle differ from those during and after concentric contractions. Journal of Applied Biomechanics, 13, 164-181.

Mena, D., Mansour, J.M. and Simon, S.R. (1981). Analysis and synthesis of human swing leg motion during gait and its clinical applications. Journal of Biomechanics, 14, 823-832.

Merletti, R. and Lo Conte, L.R. (1997). Surface EMG signal processing during isometric contractions. Journal of Electromyography and Kinesiology, 7, 241-250.

Milner-Brown, H.S. and Stein, R.B. (1975). The relation between the surface electromyogram and muscular force. Journal of Physiology., 246, 549-569.

Milner-Brown, H.S., Stein, R.B. and Yemm, R. (1973a). The contractile properties of human motor units during voluntary isometric contractions. Journal of Physiology., 228, 285-336.

Milner-Brown, H.S., Stein, R.B., and Yemm, R. (1973b). The orderly recruitmentof human motor units during voluntary isometric contrations. Journal of Physiology., $230,359-370$.

Milner-Brown, H.S., Stein, R.B., and Yemm, R. (1973c). Changes in firing rate of human motor units during linearly changing voluntary contractions. Journal of Physiology, 230, 371-390.

Mirka, G.A. (1991). The quantification of EMG normalizationerror. Ergonomics, 34, 343-352.

Mochon, S. and McMahon, T.A. (1980). Ballistic walking. Journal of Biomechanics, 13, 49-57.

Moffroid, M., Whipple, R., Hofkosh, J., Lowman, E. and Thistle, H. (1969). A study of isokinetic exercise. Physical Therapy, 49, 735-747.

Moore, K.L. (1992). Clinically oriented anatomy (3rd ed.). Philadelphia, PA: Williams \& Wilkins. 
Moran, D.W. (1994). Effects of orthopedic surgery on musculoskeletal control strategies in children with cerebral palsy. Unpublished Ph.D. dissertation, Arizona State University, Tempe, Arizona.

Murray, D.A., Harrison, E. and Wood, G.A. (1982). Cybex II reliability: An appraisal. Medicine and Science in Sport and Exercise, 14, 153.

Nemeth, G. and Ohlsen, H. (1985). In vivo moment arm lengths for hip extensor muscles at different angles of hip flexion. Journal of Biomechanics, 18, 129-140.

Nordin, R.N. and Frankel, V.H. (1989). Biomechanics of the knee. In R.N. Nordin, and Frankel, V.H. (Ed.), Basic Biomechanics of the Musculoskeletal System (2nd ed.). Philadelphia, PA.: Lea and Febiger.

Nussbaum, M.A. and Chaffin, D.B. (1998). Lumbar muscle force estimation using a subject-invariant 5-parameter EMG-based model. Journal of Biomechanics, 31, 667-672.

Olney, S.J. (1982). Prediction of knee and ankle moments of force in walking from EMG and kinematica data. Unpublished Ph.D., University of Waterloo, Ontario.

Olney, S.J. and Winter, D.A. (1985). Predictions of knee and ankle moments of force in walking from EMG and kinematic data. Journal of Biomechanics, 18, 9-20.

Onyshko, S. and Winter, D.A. (1980). A mathematical model for the dynamics of human locomotion. Journal of Biomechanics, 13, 361-368.

Osterning, L.R., Hamill, J., Sawmill, J.A. and Bates, B.T. (1983). Influence of torque and limb speed on power production in isokinetic exercise. American Journal of Physical Medicine, 62, 163-171.

Otten, E. (1988). Concepts and models of functional architecture in skeletal muscle. Exercise Sports Science Reviewws, 16, 89-137.

Ounpuu, S. and Wyatt, M.P. (2000). Interpretation of gait analysis data for informed treatment decision -making and evaluation. Paper presented at the Gait and Clinical Movement Analysis, Rochester, Minnesota.

Pandy, M.G. and Berme, N. (1987a). Synthesis of human walking: a three dimensional model for single support. Part 1: Normal gait. Paper presented at the Biomechanics of Normal and Prosthetic Gait, ASME Winter Annual Meeting in Boston.

Pandy, M.G. and Berme, N. (1987b). Synthesis of human walking: a three dimensional model for single support. Part 2: Normal gait. Paper presented at the 
Biomechanics of Normal and Prosthetic Gait., ASME Winter Annual Meeting in Boston.

Pandy, M.G. and Berme, N. (1988a). A numerical method for simulating the dynamics of human walking. Journal of Biomechanics, 21, 1043-1051.

Pandy, M.G. and Berme, N. (1988b). Synthesis of human walking: a planar model for single support. Journal of Biomechanics, 21, 1053-1060.

Pandy, M.G. and Zajac, F.E. (1989). Dependence of jumping performance on muscle strength, muscle fiber speed, and tendon compliance. Paper presented at the Issues in the Modeling and Control of Biomechanical Systems., ASME Winter Annual Meeting in San Francisco.

Parnianpour, M., (1995). Lecture series in Occupational Biomechanics. Columbus, Ohio

Parnianpour, M., Nordin, M. and Sheikhzadeh, A. (1990). The relationship of torque, velocity, and power with constant resistive load during sagittal trunk movement. Spine, 15, 639-643.

Parnianpour, M., Sparto, P.J. and Chen, M.C. (1997). Validation of electrolytic-liquid tilt sensors for human motion measurement. Paper presented at the 34th Annual Rocky Mountain Bioengineering Symposium. Instrument Society of America., Rocky Mountain.

Partridge, L.D. and Benton, L.A. (1981). Muscle the motor. In V.B. Brooks (Ed.), Handook of Physiology. Section I: The Nervous System, Volume II: Motor Control, Part I (Vol. Chapter 3, pp. 43-106). Bethesda, Maryland: American Physiological Society.

Patla, A.E., Hudgins, B.S., Parker, P.A. and Scott, R.N. (1982). Myoelectric signal as a quantitative measure of muscle mechanical output. Medical and Biological Engineering Computing, 20, 319-328.

Patriarco, A.G., Mann, R.W., Simon, S.R. and Mansour, J.M. (1981). An evaluation of the approaches of optimization models in the prediction of muscle forces during human gait. Journal of Biomechanics, 14, 513-525.

Pedotti, A., Krishnan, V.V. and Stark, L. (1978). Optimization of muscle-force sequencing in human locomotion. Mathematical Bioscience, 38, 57-76.

Perry, J. (1992). Gait Analysis: Normal and Pathological Functions. New York, NY: McGraw-Hill.

Perry, J., (2000). Keynote speaker lecture. Rochester, MN 
Perry, J. and Bekey, G.A. (1981). EMG-Force relationships in skeletal muscle. Critical Reviews in Biomedical Engineering, 7, 1-22.

Perry, J., Boyd, L.A., Rao, S.S. and Mulroy, S.J. (1997). Prosthetic weight acceptance mechanics in transtibial amputees wearing the Single Axis, Seattle Lite, and Flex Foot. IEEE Transactions in Rehabilitation Engineering, 5, 283-289.

Person, R. and Libkin, M. (1970). Simulation of electromyograms showing interference patterns. Electromyography and Clinical Neurophysiology, 28, 625-632.

Petrofsky, J.S. and Lind, A.R. (1975). The relationship of body fat content to deel muscle temperature and isometric endurance in man. Clinical Science and Molecular Medicine, 48, 405-412.

Petrofsky, J.S. and Phillips, C.A. (1981). The influence of temperature, initial length and electrical activity on the force-velocity relationship of the medial gastrocnemius muscle of the cat. Journal of Biomechanics, 14, 297-306.

Pheasant, S. (1996). Bodyspace : Anthropometry, Ergonomics and the Design of the Work. New York: Taylor \& Francis.

Phillips, C.A. and Petrofsky, J.S. (1980). Velocity of contraction of skeletal muscle as a function of activation and fibre composition: A mathematical model. Journal of Biomechanics., 13, 549-558.

Phillips, C.A. and Petrofsky, J.S. (1981). The passive elastic force-velocity relationship of cat skeletal muscle influence upon maximal contractile element velocity. Journal of Biomechanics, 14, 399-401.

Pierrynowski, M.R. (1982). A physiological model for the solution of individual muscle forces during normal human walking. Unpublished Ph.D. Dissertation, Simon Fraser University.

Pierrynowski, M.R. (1995). Analytic representation of muscle line of action and geometry. In P. Allard, Stokes, I.A.F., Blanchi, J-P. (Ed.), Three dimensional analysis of human movement (pp. 215-256). Champaign: Human Kinetics.

Pierrynowski, M.R. and Morrison, J.B. (1985). Estimating the muscle forces generated in human lower extremity when walking. A physiological solution. Mathematical Biosciences, 75, 43-68.

Potvin, J.R., Norman, R.W. and McGill, S.M. (1996). Mechanically corrected EMG for teh continuous estimation of errector spinae muscle loading during repetitive lifting. European Journal of Applied Physiology, 74, 119-132. 
Powell, P.L., Roland, R.R., Kanim, P., Bello, M.A. and Edgerton, V.R. (1984). Predictability of skeletal muscle tension from architectural determinations in guinea pig hindlimbs. Journal of Applied Physiology: Respiration, Environment, and Exercise Physiology, 57, 1715-1721.

Proske, U. and Morgan, D.L. (1987). Tendon stiffness: methods of measurement and significance for the control of movement. A review. Journal of Biomechanics, $20,75-82$.

Rab, G.T. (1994). Muscle. In J. Rose and J.G. Gamble (Eds.), Human Wlaking (2 ed.). Baltmore: Williams \& Wilkins.

Rack, P.M.H. and Westbury, D.R. (1969). The effects of length and stimulus rate on tension in the isometric cat soleus muscle. Journal of Physiology, 204, 443-460.

Ralston, H.J., Todd, F.N. and Inman, V.T. (1976). Comparison of electrical activity and duration of tension in the human rectus femoris muscle. Electroencephalography and Clinical Neurophysiology, 16, 271-279.

Redferen, M.S. (1992). Functional muscle: effects on electromyographic output., Selected topics in surface electromyography for use in occupational setting: Expert perspectives. (pp. 104-121): U.S. Department of Health and Human Services, Public Health Service, DHHS (NIOSH) Publication No. 91-100.

Redfern, M.S. (1988). Electromyographic (EMG) signal processing and biomechanical modeling of lower leg muscles. Unpublished Doctoral Dissertation, University of Michigan, Ann Arbor, MI.

Roeleveld, K., Baratta, R.V., Solomonow, M., Van Soest, A.J. and Huijing, P.A. (1993). Role of tendon properties on the dynamic performance of different isometric muscles. Journal of Applied Physiology, 74, 1348-1355.

Rothstein, J.M. (1987). Clinical uses of isokinetic measurements: Critical issues. Physical Therapy, 67, 1840-1844.

Rugg, S.G., Gregor, R.J., Mandelbaum, B.R. and Chiu, L. (1990). In vivo moment arm calculations at the ankle using magnetic resonance imaging (RMN). Journal of Biomechanics, 23, 495-501.

Sacks, R.D. and Roy, R.R. (1982). Architecture of the hind limb muscles of cats: Functional significance. Journal of Morphology, 173, 185-195. 
Sale, D., Quinlan, J., Marsh, E., McComas, A.J. and Belanger, A.Y. (1982). Influence of joint positions on ankle plantar flexion in humans. Journal of Applied Physiology, 52(6), 1636-1642.

Sapega, A.A., Nicholas, J.A., Sokolow, D. and Saraniti, A. (1982). The nature of 'overshoot' in Cybex isokinetic dynamometry. Medicine and Science in Sport and Exercise, 14, 368-375.

Saunders, J.B., Inman, V.T. and Eberhart, H.D. (1953). The major determinants in normal and pathological gait. Journal of Bone and Joint Surgery, 35A, 543-559.

Schipplein, O.D. and Andriacchi, T.P. (1991). Interaction between active and passive knee stabilizers during level walking. Journal of Orthopaedic Research, 9, 113119.

Scudder, G.N. (1980). Torque curves produced at the knee during isometric and isokinetic exercise. Archives of Physical Medicine and Rehabilitation, 61, 68-72.

Seger, J.Y. and Thorstensson, A. (1994). Muscle strength and myoelectric activity in prepubertal and adult males and females. European Journal of Applied Physiology, 69, 81-87.

Seireg, A. and Arvicar, R.J. (1975). The prediction of muscular load sharing and joint forces in the lower extremities during walking. Journal of Biomechanics, 8, 89102.

Seireg, A. and Arvikar, R.J. (1973). A mathematical model for evaluation of forces in lower extremities of the musculo-skeletal systems. Journal of Biomechanics, 6 , 313-326.

Shapiro, R. (1978). Direct linear transformation method for three-dimensional cinematography. Research Quarterly, 49, 197-205.

Sheehan, F.T., Zajac, F.E. and Drace, J.E. (1996). An in vivo study of knee joint \& muscle dynamics, using cine phase contrast magnetic resonance (PCMR) imaging. Paper presented at the Engineering Foundation Conferences: Biomechanics \& neural control movement, Deer Creek Resort \& Conference Center, Mt. Sterling, Ohio.

Shimba, T. (1984). An estimation of center of gravity from force platform data. Journal of Biomechanics, 17, 53-60.

Siegler, S., Seliktar, R. and Hyman, W. (1982). Simulation of human gait with the aid of a simple mechanical model. Journal of Biomechanics, 15, 415-425. 
Simon, S.R., (1997). Discussion on the joint angle at the hip for the purposes of knee testing on the isokinetic dynamometer if gait-like situations are to be simulated. Personal Communication. Columbus, $\mathrm{OH}$.

Simon, S.R. and Ryan, A.W. (1992). Biomechanical/Neurophysiologic factors related to surgical correction of equinus deformity. In M.D. Sussman (Ed.), The Diplegic Child, Evaluation and Management. Rosemont, IL.: American Academy of Orthopedic Surgeons.

Smidt, G. (1973). Biomechanical analysis of knee flexion and extension. Journal of Biomechanics, 6, 79-92.

Soderberg, G.L. (1992). Recording techniques, Selected topics in surface electromyography for use in occupational setting: Expert perspectives.: U.S. Department of Health and Human Services, Public Health Service, DHHS (NIOSH) Publication No. 91-100.

Soderberg, G.L. and Knutson, L.M. (2000). Physical therapists are among the most common users of electromyography as a method of understanding function and dysfunction of the neuromuscular system. Physical Therapy, 80, 486-498.

Solomonow, M., Baratta, R., Shoji, H. and D'Ambrosia, R. (1990). The EMG-force relationships of skeletal muscle; dependence on contraction rate, and motor units control strategy. Electromyography and Clinical Neurophysiology, 30, 141-152.

Solomonow, M., Baratta, R. and Zhou, B.H. (1986). Historical update and new developments on the EMG-force relationships of skeletal muscles. Orthopedics, 9, 154-1543.

Soudan, K. and Audekercke, R.V. (1979). Methods, difficulties and inaccuracies in the study of human joint kinematics and pathokinematics by the instant axis concept. Example: The knee joint. Journal of Biomechanics, 12, 27-33.

Soutas-Little, R.W. (1991). Lectures on Biodynamics: Analytical methods of Biomechanics. Michigan State University. East Lansing, MI.

Soutas-Little, R.W. and Inman, D.J. (1999). Engineering mechanics: Dynamics. New Jersey, NJ: Prentice-Hall, Inc.

Soutas-Little, R.W. and Verstrate, M.C. (1990). Use of a joint coordinate system between the foot and shank for gait analysis. In N.a.C. Berme, A. (Ed.), Biomechanics and Human Movement: Applications in Rehabilitation, Sports and Ergonomics. (pp. 274-278). Worthington, OH.: Bertec Corporation. 
Sparto, P.J. (1998). Trunk muscle electromyographic responses, spinal loading, and wavelet detection of fatigue during repetitive trunk extension. Unpublished Ph.D., The Ohio State University, Columbus, Ohio.

Sparto, P.J. and Parnianpour, M. (1998). Estimation of trunk muscle forces and spinal loads during fatiguing repetitive trunk exertions. Spine, 23, 2563-2573.

Sparto, P.J. and Parnianpour, M. (1999). An electromyography-assisted model to estimate trunk muscle forces during fatiguing repetitive trunk exertions. Journal of Spinal Disorders, 12, 509-518.

Sparto, P.J., Parnianpour, M., Marras, W.S., Granata, K.P., Reinsel, T.E. and S., S. (1998). Effect of electromyogram-force relationships and method of gain estimation on the predictions of an electromyogram-driven model of spinal loading. Spine, 23, 423-429.

Spector, S.A., Gardiner, P.F., Zernicke, R.F., Roy, R.R. and Edgerton, V.R. (1980). Muscle architecture and force-velocity characteristics of cat soleus and medial gastrocnemius: Implications for motor control. Journal of Neurophysiology, 44, 951-960.

Spiegelman, J.J. and Woo, S.L.-Y. (1987). Arigid body method for finding centers of rotation and angular displacements of planar joint motion. Journal of Biomechanics, 20, 715-721.

Spoor, C.W., Leeuwen, J.L., van, M., C.G.M., Titulaer, A.F. and Huson, A. (1990). Moment arm length variations of selected muscles acting on talocrural and subtalar joints during movement: An invitro stuby. Journal of Biomechanical Engineering, 29, 21-30.

Spoor, C.W., Leeuwen, J.L.v., Meskers, C.G.M., Titulaer, A.F. and Huson, A. (1990). Moment arm length variations of selected muscles acting on talocrural and subtalar joints during movement: An invitro stuby. Journal of Biomechanical Engineering, 29, 21-30.

Stephens, J.A. and Taylor, A. (1972). Fatigue of maintained voluntary muscle contractions in man. Journal of Physiology., 220, 1-18.

Stevens, J.C., Dickinson, V. and Jones, N.B. (1980). Mechanical properties of human skeletal muscle from in vitro studies of biopsies. Medical and Biological Engineering and Computing., 18, 1-9.

Sugi, H. and Tsuchiya, T. (1988). Stiffness changes during enhancement and deficit of isometric force by slow length changes in frog skeletal muscle fibers. Journal of Physiology, 407, 215-229. 
Sutherland, D.H., Kaufman, K.R. and Moitoza, J. (1994). Kinematics of normal human gait. In J.a.G. Rose, J.G. (Ed.), Human Walking. (pp. 23-44). Baltimore, MD: Williams and Wilkins.

Tabary, J.C., Tardieu, C., Tardieu, G., Tabary, C. and Gagnard, L. (1976). Functional adaptation of sarcomere number of normal cat muscle. Journal of Physiology, 72, 277-291.

Thorstensson, A., Grimby, G. and Karlsson, J. (1976). Force-velocity relations and fibre composition in human knee extensor muscles. Journal of Applied Physiology, 40, $12-16$.

Thunnissen, J.G.M. (1993). Muscle force prediction during human gait. Unpublished Ph.D., University of Twente, Nederlands.

Tredinnick, T. and Duncan, P. (1988). Reliability of measurements of concentric and eccentric isokinetic loading. Physical Therapy, 68, 656-659.

Tumer, T.S. and Engin, A.E. (1993). Three-body segment dynamic model of the human knee. Transactions of ASME Journal of Biomechanical Engineering, 115, 350356.

Tumer, T.S., Wnag, X. and Akkas, N. (1995). A planar dynamic anatomical model of the human lower limb. Biomedical Engineering - Applications, Basis \& Communications, 7, 5-17.

Van Dieen, J.H. and Visser, B. (1999). Estimating net lumbar sagittal plane moments from EMG data. The validity of calibration procedures. Journal of Electromyography and Kinesiology, 9, 309-315.

Van Gheluwe, B. (1974). A new three-dimensional filming technique involving simplified alignment and measurement procedures. Paper presented at the Biomechanics IV. Proceedings for the fourth International Society for Biomechanics, University Park, Pennsylvania.

Van Soest, A.J. and Bobbert, M.F. (1993). The contribution of muscle properties in the control of explosive movements. Biological Cybernetics, 69, 195-204.

Van Soest, A.J., Huijing, P.A. and Solomonow, M. (1995). The effect of tendon on muscle force in dynamic isometric contractions: A Simulation study. Journal of Biomechanics, 28, 801-807.

Vicon, O.M.L. (1997a). Vicon 370: User's Manual. Oxford, UK. 
Vicon, O.M.L. (1997b). Vicon Clinical Manager: User Manual. Oxford, UK.

Vicon, O.M.L. (1998). Vicon Clinical Manager: User Manual. Oxford, UK.

Viitasalo, J.H.T. and Komi, P.V. (1975). Signal characteristics of EMG with special reference to reproducibility of measurements. Acta Physiologica Scandinavia, 93, 531-539.

Vrendenbregt, J. and Rau, G. (1973). Surface electromyography in relation to force, muscle length and endurance. In J.E. Desmedt (Ed.), New Developments in Electromyography and Clinical Neurophysiology. (Vol. 1, pp. 607-622). Karger, Basil.

Webster, J.G. (1992). Medical instrumentation. Application and Design. Boston, MA: Houghton Mifflin Company.

Westing, S.H., Cresswell, A.G. and Thorstensson, A. (1991). Muscle activation during maximal voluntary eccentric and concentric knee extension. European Journal of Applied Physiology, 62, 104-108.

Westing, S.H., Seger, J.Y. and Thorstensson, A. (1990). Effects of electrical stimulation on eccentric and concentric torque-velocity relationships during knee extension in man. Acta Physiologica Scandinavica, 140, 17-22.

White, S.C. (1997a). Discussion on the targeting of the biceps femoris, short head, for the purposes of collecting surface EMG. Personal Communication. Chicago, IL.

White, S.C. (1997b). Discussion on the targeting of the biceps femoris, short head, for the purposes of collecting surface EMG, and on the effectiveness of a calibration procedure. Chicago, IL.

White, S.C. and Winter, D.A. (1993). Predicting muscle forces in gait from EMG signals and musculotendon kinematics. Journal of Electromyography and Kinesiology, 2(4), 217-231.

White, S.C., Yack, H.J. and Winter, D.A. (1989). A three-dimensional musculoskeletal model for gait analysis. Anatomical variability estimates. Journal of Biomechanics, 22, 885-893.

White, S.C., Yack, H.J. and Winter, D.A. (1989). A three-dimensional musculoskeletal model for gait analysis. Anatomical variability estimates. Journal of

Biomechanics, 22, 885-893. 
Wickiewicz, T.L., Roy, R.R., Powell, P.L. and Edgerton, V.R. (1983). Muscle architecture of the human lower limb. Clinical Orthopaedics and related research., 179, 275-283.

Wilkie, D.R. (1950). The relation between force and velocity in human muscle. Journal of Physiology, 110, 249-280.

Wilkie, D.R. (1956). The mechanical properties of muscle. British Medical Bulletin, 12, 177-182.

Williams, P.E. (1988). Effect of intermittent stretch on immobilized muscle. Annals of Rheumatory Disease, 47, 1014-1016.

Winkel, J. and Bendix, T. (1986). Muscular performance during seated work evaluated by two different EMG methods. European Journal of Applied Physiology, 55, $167-173$.

Winter, D.A. (1976). Biomechanical model relating to changing isometric tension. Paper presented at the Digest of the 11th International Conference on Medical and Biological Engineering., Ottawa, Canada.

Winter, D.A. (1987). The Biomechanics and Motor Control of Human Gait. Waterloo, Ontario, Canada: University of Waterloo Press.

Winter, D.A. (1990). Biomechanics and motor control of human movement. ( 2 nd ed.). New York, NY: John Wiley \& Sons, Inc.

Winter, D.A. (1997). Future directions in gait analysis -- A wish list (invited speaker's talk). Paper presented at the North American Society of Gait and Clinical Movement Analysis: 2nd Annual Meeting., Chicago, IL.

Winter, D.A., Eng, J.J. and Ishac, M.G. (1995). A review of kinetic parameters in human walking. In R.L.a.O. Craik, C.A. (Ed.), Gait analysis: Theory and application. Baltimore: Mosby.

Winter, D.A. and Patla, A.E. (1997). Signal processing and linear systems for the movement sciences. Waterloo, Ont.: Waterloo Biomechanics.

Winter, D.A., Rau, G., Kadefors, R., Broman, H. and De Luca, C.J. (1980). Units, terms and standards in the reporting of EMG research.: Report by the Ad Hoc Committee of the International Society of Electrophysiological Kinesiology.

Winter, D.A., Wells, R.P. and Orr, G.W. (1981). Errors in the use of isokinetic dynamometers. European Journal of Applied Physiology, 46, 397-408. 
Winter, D.A., Wells, R.P. and Orr, G.W. (1981). Errors in the use of isokinetic dynamometers. European Journal of Applied Physiology, 46, 397-408.

Winters, J.M. (1995). Concepts in neuromuscular modeling. In P. Allard, Stokes, I.A.F. and Blanchi, J-P. (Ed.), Three-Dimensional Analysis of Human Movement. Champaign, IL.: Human Kinetics.

Woltring, H.J. (1980). Planar control in multi-camera calibration for 3-D gait studies. Journal of Biomechanics, 13, 39-48.

Woltring, H.J. (1986). On instantaneous centres and axes of rotation for defining functional joint pivots in gait analysis. (Unpublished manuscript. ).

Woltring, H.J. (1990). Data processing and error estimation. In N. Berme and A. Cappozzo (Eds.), Biomechanics of Human Movement. Worthington, OH: Bertec Corporation.

Wongchaisuwat, C., Hemami, H. and Buchner, H.J. (1984). Control of sliding and rolling at natural joints. Journal of Biomechanical Engineering, 106, 368-375.

Woods, J.J. and Bigland-Ritchie, B. (1983). Linear and non-linear surface EMG/Force relations in human muscles. American Journal of Physical Medicine., 62, 287 299.

Wu, G. (1995). A review of body segmental displacement, velocity and acceleration in human gait. In R.L.a.O. Craik, C.A. (Ed.), Gait Analysis: Theory and Application. St. Louis, Missouri: Mosby - Year Book, Inc.

Yamaguchi, G.T. (1989a). Feasibility and conceptual design of functional neuromuscular stimulation systems for the restoration of natural gait to paraplegics based on dynamic musculoskeletal models. Unpublished Ph.D. dissertation, Stanford University, Stanford, CA.

Yamaguchi, G.T. (1997). Estimation of maximum isometric muscle force from physiological cross sectional area data. Personal Communication. Columbus, Ohio

Yamaguchi, G.T. and Zajac, F.E. (1989). A planar model of the knee joint to characterize the knee extensor mechanism. Journal of Biomechanics, 22, 1-10.

Yamaguchi, G.T. and Zajac, F.E. (1989b). A planar model of the knee joint to characterize the knee extensor mechanism. Journal of Biomechanics, 22, 1-10. 
Yang, J.F., Stein, R.B. and James, K.P. (1991). Contribution of peripheral afferents to the activation of the soleus muscle during walking. Experimental Brain Research, 87(3), 679-687.

Yang, J.F. and Winter, D.A. (1982). The effect of EMG normalization methods on intersubject variability. Paper presented at the Procedings of the Canadian Society for Biomechanics, Kingston, Ontario.

Yang, J.F. and Winter, D.A. (1983). Electromyography reliability in maximal and submaximal contractions. Archives of Physical Medicine and Rehabilitation, 64, 417-420.

Yang, J.F. and Winter, D.A. (1984). Electromyographic amplitude methods: Improving their sensitivity as diagnostic tools in gait analysis. Archives of Physical Medicine and Rehabilitation, 65, 517-521.

Yen, V.N., M.L. (1987). Biomechanics of normal and prosthetic gait. Paper presented at the ASME Winter Annual Meeting, Boston, MA.

Zajac, F. (1992). How musculotendon architecture and joint geometry affect the capacity of muscles to move and exert force on objects: A review with application to arm and forearm tendon transfer design. Journal of Hand Surgery, 17, 799-804.

Zajac, F. and Winters, J.M. (1990). Modeling musculoskeletal movement systems: Joint and body-segment dynamics, musculotendinous actuation, and neuromuscular control. In W.J.M.a.W. S.L-Y. (Ed.), Multiple Muscle Systems. New York, NY.: Springer-Verlag.

Zajac, F.E. (1989). Muscle and tendon properties: models, scaling, and application to biomechanics and motor control. CRC Critical Reviews in Biomedical Engineering, 17, 359-411.

Zajac, F.E. (1989a). Muscle and tendon properties: models, scaling, and application to biomechanics and motor control. CRC Critical Reviews in Biomedical Engineering, 17, 359-411.

Zajac, F.E. and Gordon, M.E. (Eds.). (1989). Determining muscle's force and action in multi-articular movement. Baltimore, MD: Williams \& Wilkins.

Zajac, F.E. and Gordon, M.E. (Eds.). (1989b). Determining muscle's force and action in multi-articular movement. Baltimore, MD: Williams \& Wilkins.

Zajac, F.E., Stevenson, P.J. and Topp, E.L. (1986b). A dimensionless musculotendon actuator model for use in computer simulations of body coordination: 
staticproperties. Paper presented at the Proceedings of the North American Congress on Biomechanics, Montreal.

Zatsiorsky, V. and Seluyanov, V. (1985). Estimation of the mass and inertia characteristics of human body by means of the best predictive regression equations. Paper presented at the Biomechanics IX. Proceedings of the ninth International Congress of Biomechanics, Waterloo.

Zatsiorsky, V.a.S., V. (1983). The mass and inertia characteristics of main segments of human body. In H.M.K. Kobayashi. (Ed.), Biomechanics VIII. Proceedings of the Eighth International Congerss of Biomechanics, Nagoya, Japan. (Vol. VIII-B). Champaign, Ill: Human Kinetics Pub. Inc.

Zuurbier, C.J. and Huijing, P.A. (1992). Influence of muscle geometry on shortening speed of fibre, aponeurosis and muscle. Journal of Biomechanics, 25, 1017-1026. 


\section{APPENDICES}


APPENDIX A

PROPERTIES OF EACH BODY SEGMENT 
1) For a cone, the location of the center of mass and the principal moments of inertia:

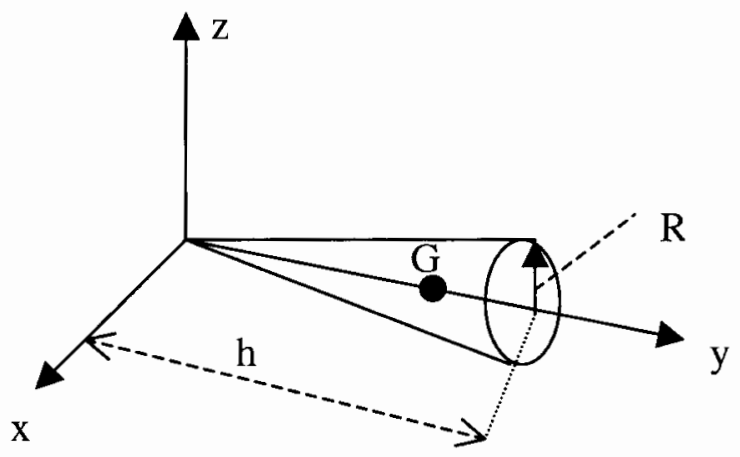

$$
\begin{aligned}
& y_{c m}=\frac{3}{4} h \\
& I_{y}=\frac{3}{10} m R^{2} \\
& I_{z}=I_{x}=\frac{3}{20} m\left(R^{2}+4 h^{2}\right) \\
& I_{c m z}=I_{c m x}=\frac{3}{80} m\left(4 R^{2}+h^{2}\right)
\end{aligned}
$$

where $m$ represents the mass.

2) For a cylinder, the principal moments of inertia:

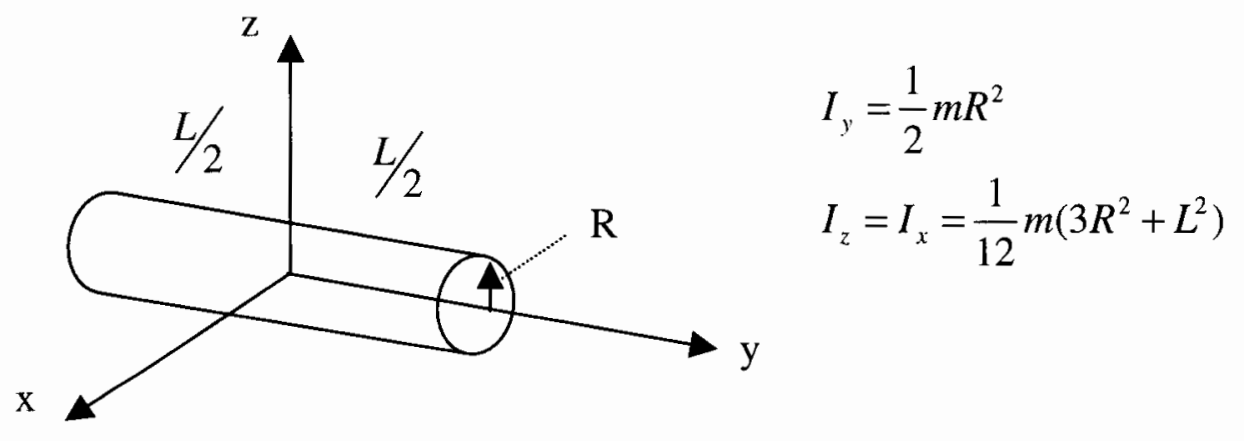

where $m$ represents the mass. 
APPENDIX B

SAMPLE INPUT MODEL FILE TO MOVE3D 


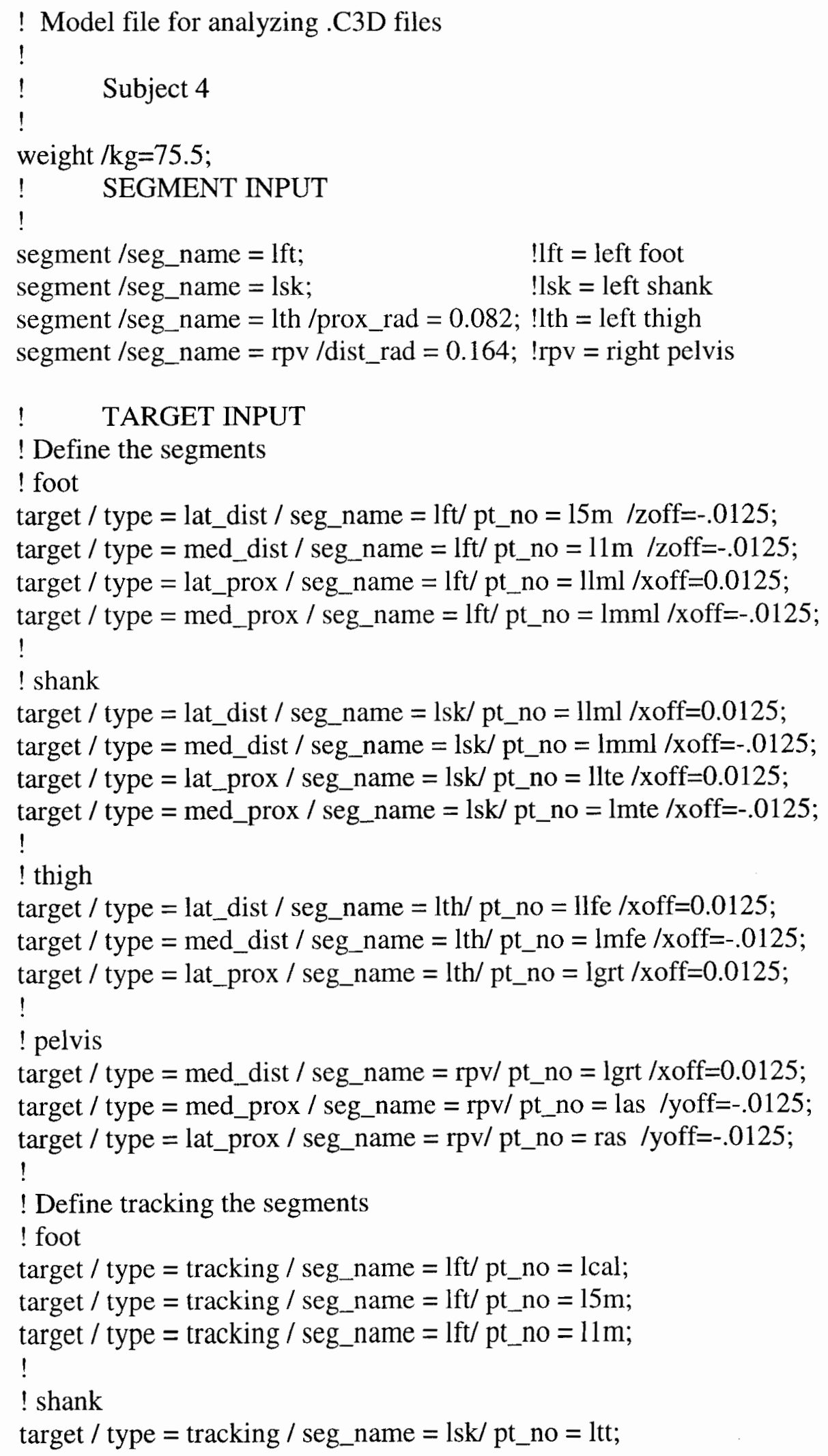




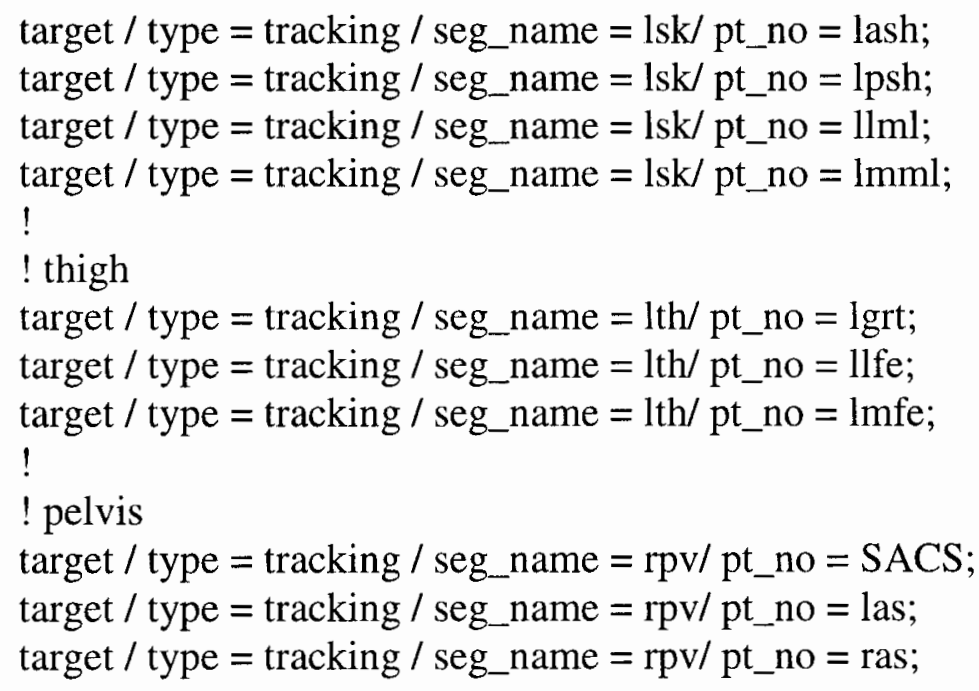


APPENDIX C

SAMPLE ANTHROPOMETRIC OUTPUT FROM MOVE3D 


\section{APPENDIX D}

INSTANTANEOUS JOINT CENTER DEFINITION 
The author of MOVE3D, to the best of my knowledge, has provided no documentation about the computation of the instantaneous helical axis. Consequently, the purpose of this appendix is to demonstrate the computation of the screw axis and subsequently the location of the instantaneous center of rotation, as defined by the author of MOVE3D. The author of MOVE3D defined the instantaneous joint center of rotation as the intercept of the helical axis with the sagittal plane. It should be mentioned that a general formulation for computing the screw axis can be found in the literature (SoutasLittle, 1999).

Considering the ankle as an example first and the targeting scheme for the shank and foot in Figure D.1:
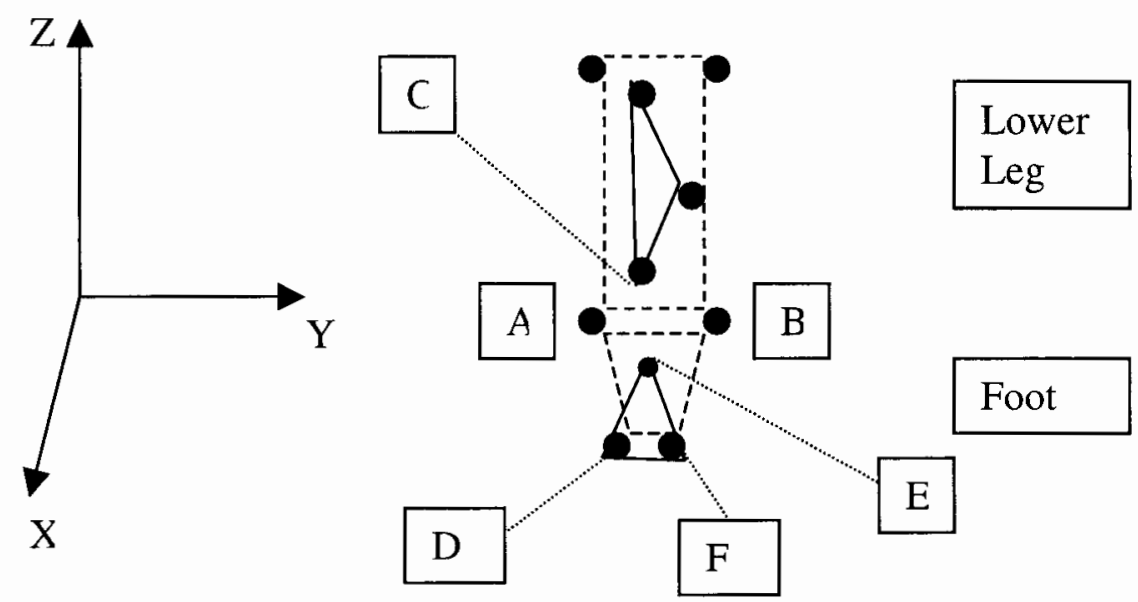

Figure D.1. Part of the lower extremity rigid link segment model representing the left lower leg (shank) and foot (anterior view). Marker A represents the medial maleolus, marker B represents the lateral maleolus, $\mathrm{C}$ represents the anterior shank marker, D is the first metatarsal head, and marker $\mathrm{E}$ represents the calcaneus.

Assuming that the positions of $\mathrm{A}, \mathrm{B}, \mathrm{C}, \mathrm{D}, \mathrm{E}$, and $\mathrm{F}$ are known from the motion analysis system, and the time interval between frames is known, then the velocities of these points are known, i.e., the $\vec{v}_{A}, \vec{v}_{B}, \vec{v}_{C}, \vec{v}_{D}, \bar{v}_{E}, \vec{v}_{F}$ are known. Then:

A) For the shank:

define a position vector of $\mathrm{B}$ relative to $\mathrm{A}$

$$
\vec{P}_{B / A}=\vec{P}_{B}-\vec{P}_{A}
$$

and a position vector of $\mathrm{C}$ relative to $\mathrm{A}$

$$
\vec{P}_{C / A}=\vec{P}_{C}-\vec{P}_{A}
$$

the relationships of the velocities of $\mathrm{B}$ and $\mathrm{C}$ relative to $\mathrm{A}$ are:

$$
\vec{v}_{B}=\vec{v}_{A}+\bar{\omega} \times \vec{P}_{B / A} \Rightarrow \vec{v}_{B}-\vec{v}_{A}=\vec{\omega} \times \vec{P}_{B / A}
$$




$$
\vec{v}_{C}=\bar{v}_{A}+\vec{\omega} \times \vec{P}_{C / A} \Rightarrow \bar{v}_{C}-\vec{v}_{A}=\bar{\omega} \times \bar{P}_{C / A}
$$

from those two relationships the components of $\vec{\omega}_{s}$ (shank angular velocity) are known.

B) Similarly, the angular velocity of the foot, $\vec{\omega}_{f}$, is defined from the D, E, and F markers

C) Then the angular velocity at the ankle joint, $\vec{\omega}_{J}$ is:

$$
\bar{\omega}_{j}=\bar{\omega}_{s}-\bar{\omega}_{f}
$$

D) The effective velocity, $\vec{v}_{\text {eff }}$, between the shank and foot is:

first we need a position vector $\bar{P}_{A / E}=\vec{P}_{A}-\vec{P}_{E}$

then the effective velocity is:

$$
\vec{v}_{e f f}=\left(\vec{v}_{E}+\vec{\omega}_{f} \times \vec{P}_{A / E}\right)-\vec{v}_{A}
$$

where $\bar{v}_{E}+\bar{\omega}_{f} \times \bar{P}_{A / E}$ can be considered as the velocity of point $\mathrm{A}$ if it were on the foot.

E) To determine the location of the instantaneous helical axis relative to a point $\mathrm{A}$, a unit vector along the direction of $\bar{\omega}_{J}$ is formed:

$$
\hat{i}_{\omega_{J}}=\frac{\vec{\omega}_{J}}{\left|\vec{\omega}_{J}\right|}
$$

F) The effective velocity has two components, one parallel to the helical axis, and one perpendicular to the helical axis:

$$
\vec{v}_{\text {eff }}=\vec{v}_{\text {eff }}+\vec{v}_{\text {patallet }}+\text { eff perpendicular }
$$

a) the parallel component is:

$$
\bar{v}_{\text {eff } f_{\text {paraltel }}}=\vec{v}_{/ / \omega_{J}}=\left(\vec{v}_{e f f} \bullet \hat{i}_{\omega_{J}}\right) \hat{i}_{\omega_{J}}
$$

b) the perpendicular component is:

$$
\vec{v}_{\text {eff perpenicular }}=\vec{v}_{\perp \omega_{J}}=\vec{v}_{\text {eff }}-\bar{v}_{\text {eff }} \text { paratel }
$$

$\mathrm{G}$ A point $\mathrm{H}$ on the helical axis where the velocity of the point is $\bar{v}_{H}=\vec{v}_{\text {eff parallel }}$ is:

$$
\vec{v}_{H}=\vec{v}_{/ / \omega_{J}}=\vec{v}_{\omega_{1}}+\vec{\omega} \times \vec{P}_{H / A}
$$

therefore, the location of $\mathrm{H}$ relative to $\mathrm{A}$ is:

$$
\begin{gathered}
\vec{\omega}_{J} \times \vec{P}_{H / A}=-\vec{v}_{\perp A} \\
\omega_{y} z_{H / A}-\omega_{z} y_{H / A}=-v_{A \perp x} \\
\omega_{z} x_{H / A}-\omega_{x} z_{H / A}=-v_{A \perp y} \\
\omega_{x} y_{H / A}-\omega_{y} x_{H / A}=-v_{A \perp z}
\end{gathered}
$$

The intercept of the helical axis with the sagittal plane (z,x plane) can be found by setting $y_{H / A}=0$.

The same procedure can be followed for the knee to determine the instantaneous center of rotation. 
APPENDIX E

MOVE3D INVERSE DYNAMICS ANALYSIS 
Since for the purposes of this investigation the joint moments at the knee and ankle were of interest, this discussion focuses on these two joints. The subsections "Determination of joint angles", " Parameters of the body segments", "Estimation of the Joint Centers", "Force plate analysis", and "Net joint moments determination" along with their associated Appendices describe how to obtain the parameters involved in the inverse dynamics approach. Considering the foot and shank at the position shown and with the specific targeting scheme (see Figure E.1), the inverse dynamics analysis can be initiated by considering the foot segment first (see Figure E.2).
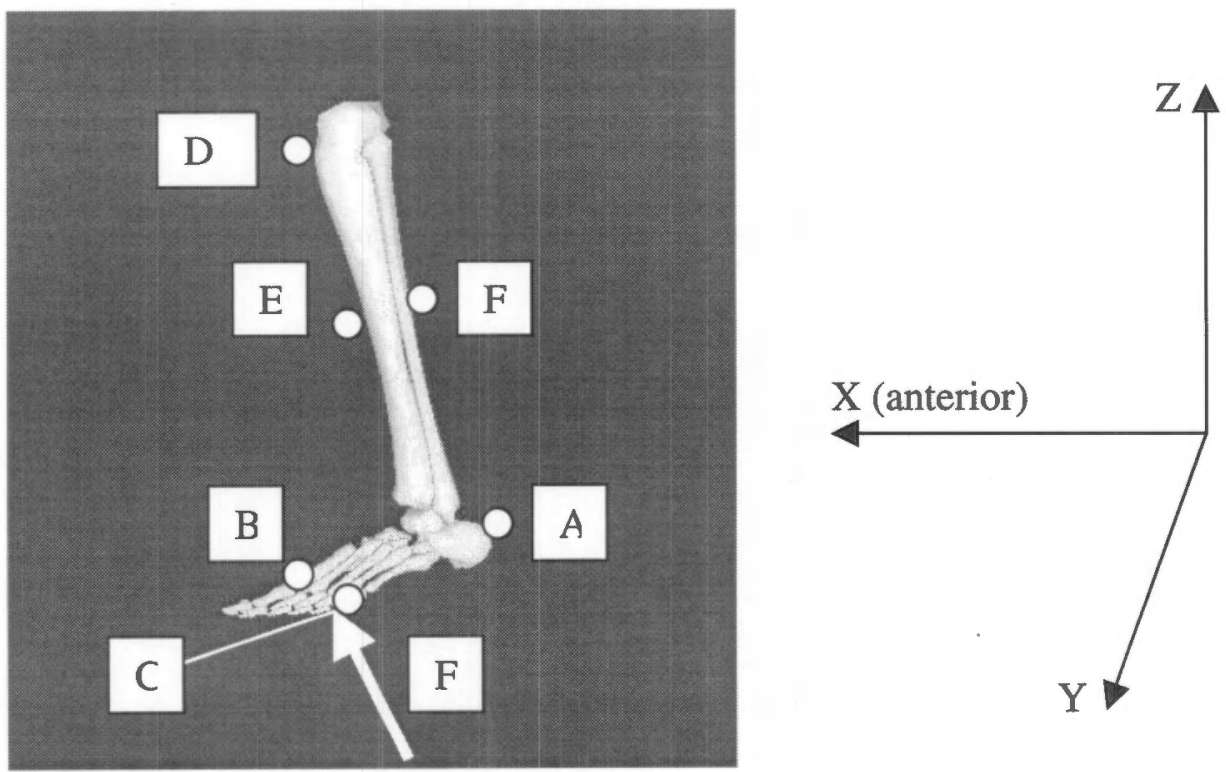

Figure E.1 The foot and shank during the push of phase of a gait cycle for the left leg. The ground reaction force is represented by $\mathrm{F}$. The targets that were placed on the foot and shank to track their motion can also be seen. Target A was placed on the calcaneus, B was placed on the first metatarsal head, C was placed on the fifth metatarsal, D was placed on the tibial tuberosity, target $\mathrm{E}$ was placed on the shank, and $\mathrm{F}$ was placed on the posterior shank in line with $\mathrm{E}$.

From the motion analysis data the location, velocity, and acceleration of the targets A, B, and C are known $\left(\vec{P}_{A}, \vec{P}_{B}\right.$, and $\vec{P}_{C}, \vec{v}_{A}, \vec{v}_{B}$, and $\vec{v}_{C}, \vec{a}_{A}, \vec{a}_{B}$, and $\vec{a}_{C}$ respectively) relative to the global coordinate system (GCS) (see Figure E.2a). From previous analysis, a segment coordinate system (SCS), has been established for each segment at the respective centers of mass $(\mathrm{cm})$, to determine the orientation of the segments in space and relative to each other (see Figure E.2b, and Figure E.3a). From the same data, the absolute angular velocity and angular acceleration of the segments can be determined. 
The axes of the SCS are coincident with the principal axes of the segment. The location of the instantaneous joint center of rotation relative to a target (here target $\mathrm{A}$ on the foot) has also been established (see Figure E.2b for the ankle, and Figure E.3a for the knee). The location of the point of application of the ground reaction force $(\mathrm{F})$, external to the body, has been determined too (see Figure E.2c). Finally, the segment parameters of the foot are known also.
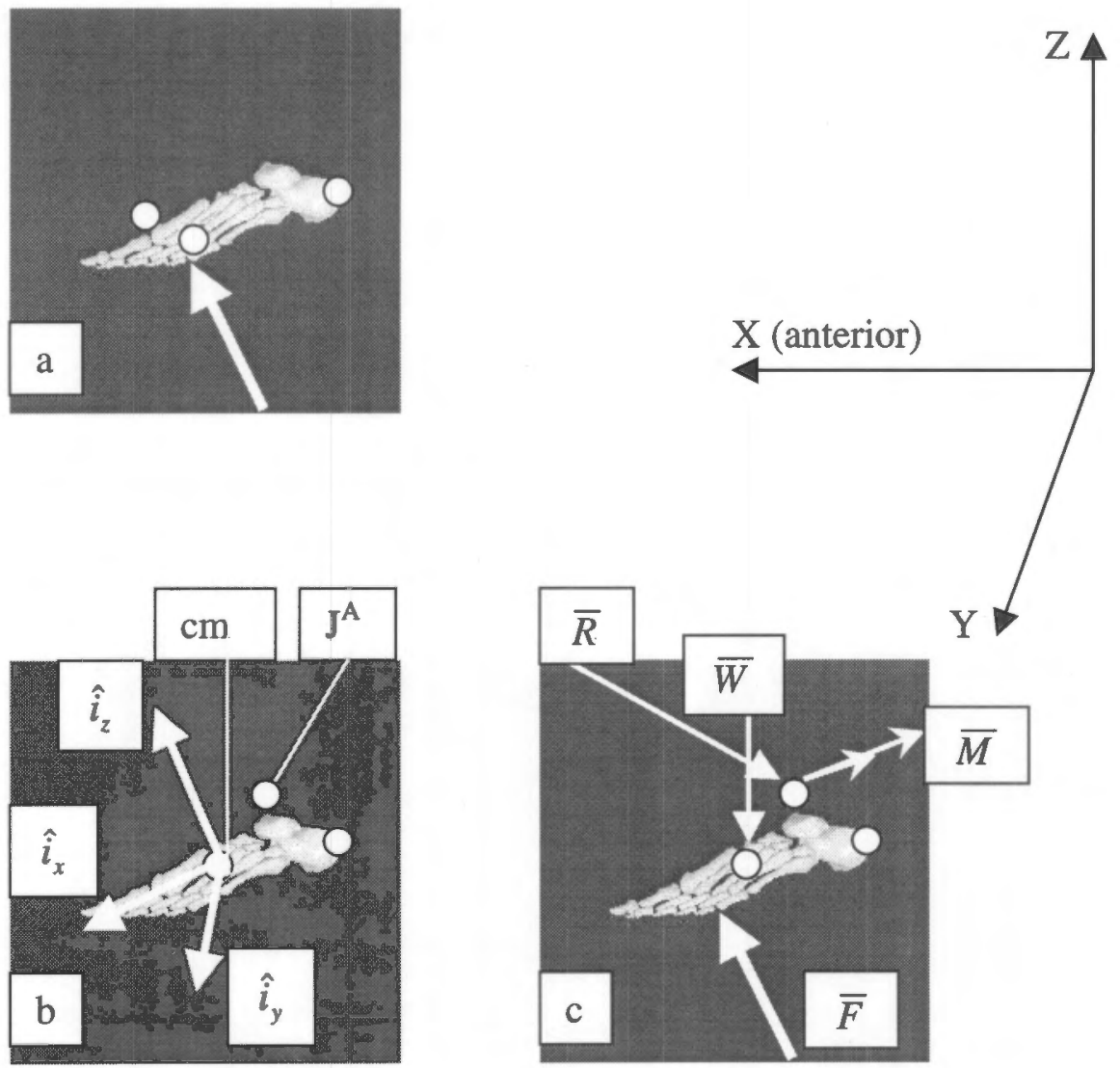

Figure E.2. a) The foot and the targets placed on it along with the resultant ground reaction force; $b$ ) the SCS located at the $\mathrm{cm}$ of the segment and the instantaneous joint center of rotation for the ankle $\left(\mathrm{J}^{\mathrm{A}}\right)$ has been determined; $\left.\mathrm{c}\right)$ a free body diagram with the kinetic information at the foot ( $\vec{F}$ is the external ground reaction force, $\vec{R}$ is the reaction force acting at $\mathrm{J}^{\mathrm{A}}, \bar{W}$ represents the weight of the foot, and $\vec{M}$ is the joint moment).

The location of the $\mathrm{cm}$ can be expressed in the GCS if the location of a target, e.g. A, is known:

$$
\vec{P}_{c m}=\vec{P}_{A}+c m_{x} \hat{i}_{x}^{f}+c m_{y} \hat{i}_{y}^{j f}+c m_{z} \hat{i}_{z}^{f}
$$


where $f$ indicates the foot SCS. In the same manner the location of the instantaneous joint center of rotation can be expressed in the GCS:

$$
\vec{P}_{J^{A}}=\vec{P}_{A}+J_{x}^{A} \hat{i}_{x}^{f}+J_{y}^{A} \hat{i}_{y}^{f}+J_{z}^{A} \hat{i}_{z}^{f}
$$

where $J^{A}$ is the instantaneous joint center of rotation for the ankle. Consequently, everything, except the moments can be expressed in the GCS.

Thus, when the Newton-Euler equations are solved:

$$
\begin{aligned}
& \sum F_{X}=m a_{c m_{X}} \\
& \sum F_{Y}=m a_{c m_{Y}} \\
& \sum F_{Z}=m a_{c m_{z}} \\
\sum M_{x}= & I_{x x} \alpha_{x}+\left(I_{z z}-I_{y y}\right) \omega_{y} \omega_{x} \\
\sum M_{y}= & I_{y y} \alpha_{y}+\left(I_{x x}-I_{z z}\right) \omega_{x} \omega_{z} \\
\sum M_{z}= & I_{z z} \alpha_{z}+\left(I_{y y}-I_{x x}\right) \omega_{x} \omega_{y}
\end{aligned}
$$

The forces $F_{X}, F_{Y}, F_{Z}$ (Figure \#c) and $a_{c m_{X}}, a_{c m_{Y}}, a_{c m_{Z}}$ were expressed in the three orthogonal axes of the GCS $(X, Y, Z)$. However, for the purposes of computing the moments, the forces and, therefore, the acceleration of the segment's center of $\mathrm{cm}$, should be expressed in the specific SCS, here the foot. Thus, the force equations become:

$$
\begin{aligned}
& \sum \vec{F} \bullet \hat{i}_{x}^{f}=m\left(\vec{a}_{c m} \bullet \hat{i}_{x}^{f}\right) \\
& \sum \vec{F} \bullet \hat{i}_{y}^{f}=m\left(\vec{a}_{c m} \bullet \hat{i}_{y}^{f}\right) \\
& \sum \bar{F} \bullet \hat{i}_{z}^{f}=m\left(\vec{a}_{c m} \bullet \hat{i}_{z}^{f}\right)
\end{aligned}
$$

where $\hat{i}_{x}^{f}, \hat{i}_{y}^{f}, \hat{i}_{z}^{f}$ are the base unit vectors of the foot SCS. Unknown forces determined from these equations, such as the reaction force $\vec{R}$ at the joint can be expressed back into the GCS:

$$
\vec{R}=R_{x}^{f} \hat{i}_{x}^{f}+R_{y}^{f} \hat{i}_{y}^{f}+R_{z}^{f} \hat{i}_{z}^{f}
$$

The moments (see Figure \#c) were determined in SCS. The moment vectors $M_{x}, M_{y}, M_{z}$ were coincident with the principal axes of the segment $(x, y, z)$. Consequently, the moment equations could be expressed in their simplest form where $I_{x x}, I_{y y}, I_{z z}$ would be the principal centroidal moments of inertia of the segment about the $\mathrm{cm}$ (Soutas-Little, 1999). For the purposes of the inverse dynamics analysis, i.e., to estimate joint moments in a sequence along the link segments (here the ankle and knee), each joint moment, of the ankle, was expressed in the GCS prior to including its effect on the succeeding link segment, the knee:

$$
\vec{M}_{A}=M_{A x}^{f} \hat{i}_{x}^{f}+M_{A y}^{f} \hat{i}_{y}^{f}+M_{A z}^{f} \hat{i}_{z}^{f}
$$

where $\vec{M}_{A}$ represents the moment at the ankle joint.

For the estimation of joint moments at the succeeding link segment, the polarities of the forces and moments were reversed at the joint center according to Newton's third law (see Figure E.3b). The reversed moments and forces were applied at the distal end of the succeeding link, the shank (see Figure E.3b). These moments and forces were also 
expressed in the knee SCS for the purposes of the inverse dynamics analysis by using the same methods as described previously. Thus, the resultant moments were again calculated along the principal axes of the segment before they were expressed back to the GCS.
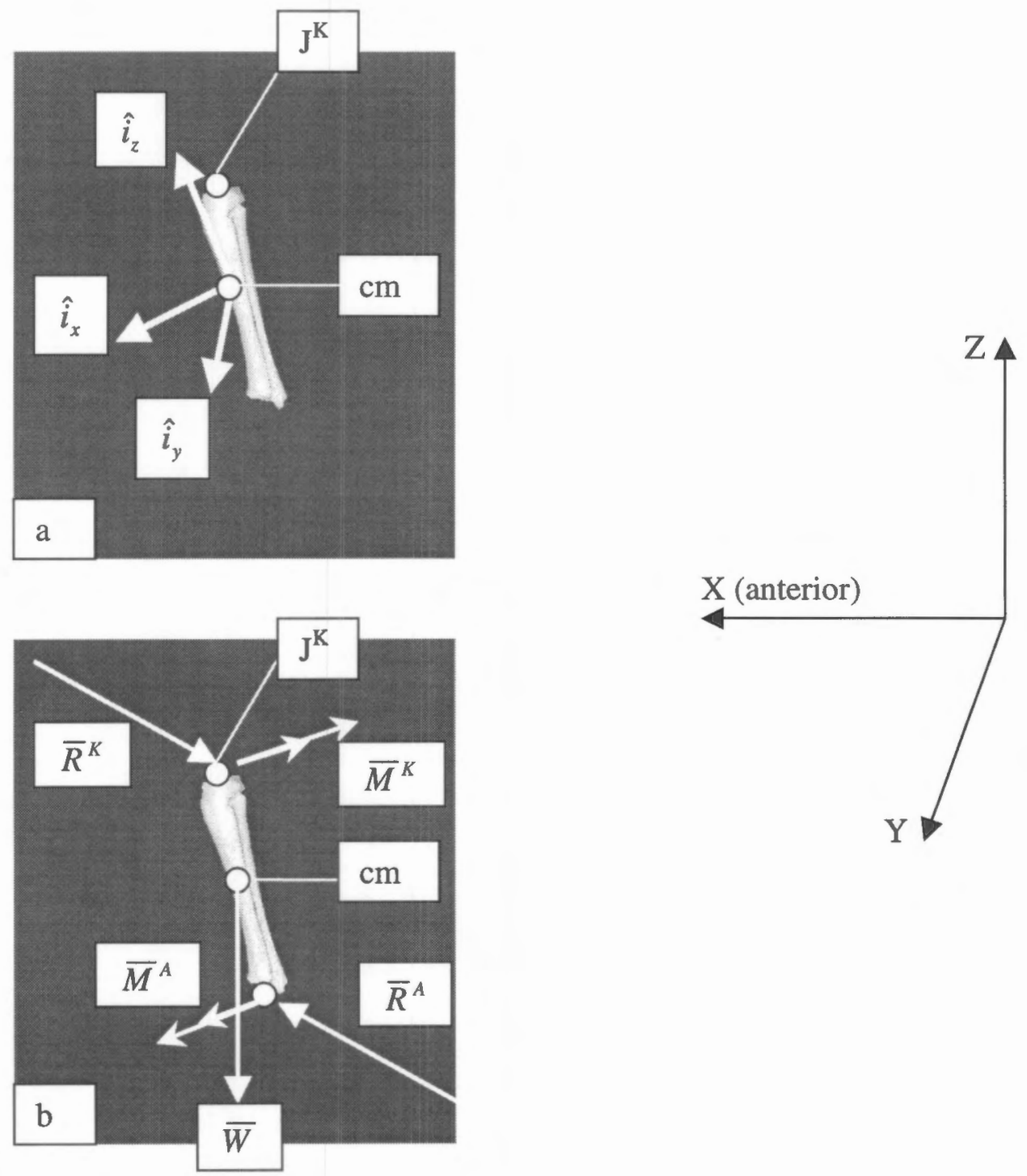

Figure E.3. a) The shank along with the SCS located at the center of mass $(\mathrm{cm})$ of the segment and the instantaneous joint center of rotation for the knee joint $\left(J^{\mathrm{K}}\right)$ have been determined; b) a free body diagram with the kinetic information at the shank $\left(\vec{R}^{A}\right)$ and $\vec{R}^{K}$ are the reaction forces acting at the ankle joint, applied at the distal end of the shank segment, and at the $\mathrm{J}^{\mathrm{K}}$, respectively, $\vec{W}$ represents the weight of the shank, $\vec{M}^{A}$ and $\vec{M}^{K}$ are the reaction joint moment at the ankle, applied at the distal end of the shank, and the joint moment at the knee, respectively). 
APPENDIX F

INFORMED CONSENT 


\title{
THE OHIO STATE UNIVERSITY
}

\author{
An EMG-assisted Model \\ to Investigate Lower Extremity Cocontraction during Normal Gait.
}

\section{CONSENT TO INVESTIGATIONAL PROCEDURE}

I, hereby authorize or direct Sheldon Simon MD or associates or assistants of his choosing, to perform the following treatment or procedure.

I am asked to participate in a research project to study the motion of the body, the forces the body exerts on the ground and the muscular activity as I walk. Various pieces of equipment will be used to accurately and permanently record my body's movements as I walk.

upon

(name of subject)

\section{The experimental (research) portion of the treatment is:}

I will be asked to wear shorts so that my legs may be viewed while I am walking. Harmless one inch square patches of adhesive tape will be placed on my skin at several positions on the body to define points on the body which will be studied while walking. Light weight, wooden rods will be placed on the skin of my lower leg and lower back to magnify smaller motions in these areas. Muscle activity will be detected by the placement of a special disk on the skin in the area of the muscle. The special disk will lead to a transmitter located on a belt on my waist. The transmitter will send the muscle signal to an antenna connected to recording equipment. In addition, during some strength measurements, three 1.5 volt tilt sensors will be placed on my leg to measure leg angle.

First Part: I will be asked to perform multiple leg flexions/extensions while sitting on a Kin/Com isokinetic dynamometer. The flexions/extensions will involve the ankle and knee joints.

Second Part: I will be asked to walk along a 40 foot walkway. I will be asked to complete 5 walks. Six cameras that can only "see" the positions of the wooden rods in space will record each walk. The special disks will record my muscle activity while walking. The force of my step on a special section of the walkway will be measured during each walk.

All records and data obtained will be analyzed and maintained in a file at the gait analysis laboratory. These records and data may be used for research and teaching purposes. In all cases my name will remain confidential.

This is done as a part of an investigation entitled: An EMG-assisted Model to Investigate Lower Extremity Cocontraction during Normal Gait. 
1. Purpose of the procedure or treatment: To record the activity of the muscles and the motion of the legs during walking, and during strength measurements. This information will then be used to estimate muscle forces in normal or abnormal walking.

2. Possible appropriate alternative procedures or treatment (not to participate in the study is always an option): Subjects may choose not to participate.

3. Discomforts and risks reasonably to be expected: Risks and discomforts associated with this study include physical fatigue, muscle soreness, and minimal risk of muscle strain from the use of isokinetic dynamometer.

4. Possible benefits for subjects/society: This study will help us to assess the feasibility of a model to understand individual normal walking muscle loading although there may be no direct benefit to you for your participation in this study. The information obtained will be made available to your physician for diagnosis and planning a treatment program if any abnormality is found.

5. Anticipated duration of subject's participation: One visit lasting approximately four hours.

I hereby acknowledge that Dr. Sheldon Simon or his designate has provided information about the procedure described above, about my rights as a subject, and he/she answered all questions to my satisfaction. I understand that I may contact Dr. Simon at 293-8710 should I have additional questions. He/she has explained the risks described above and I understand them; he/she has also offered to explain all possible risks or complications.

I understand that, where appropriate, the US Food and Drug Administration may inspect records pertaining to this study. I understand further that records obtained during my participation in this study may be made available to the sponsor of this study. Beyond this, I understand that my participation will remain confidential.

I understand that I am free to withdraw my consent and participation in this project at any time after notifying the project director without prejudicing future care. No guarantee has been given to me concerning this treatment or procedure.

I understand in signing this form that beyond giving consent, I am not waiving any legal rights that I might otherwise have, and I am not releasing the investigator, the sponsor, the institution, or its agents from any legal liability for damages that might otherwise have.

In the event of injury resulting from participation in this study, I understand that immediate medical treatment is available at University Hospital of The Ohio State University and that the costs of such treatment will be at my expense; financial compensation beyond that required by law is not available. Questions about this should be directed to the Office of Research Risks, 2925958.

I have read and fully understand the consent form. I sign it freely and voluntarily. A copy has been given to me.

Date: Time a.m./p.m.

Signed:

(subject) 
or

(person authorized to consent for subject if required)

Witness(es)

if

Required

I certify that I have personally completed all blanks in this form and explained them to the subject or his/her representative before requesting the subject or his/her representative to sign it.

Signed

(Signature of Project Director, or

his/her authorized Representative) 


\section{APPENDIX G}

TILT SENSORS CALIBRATION 
Figure G.1 shows the set up connections for the tilt sensor and signal conditioner pair. Each pair was calibrated at the manufacturer site for its full range of motion, i.e., $360^{\circ}$ circular motion, and then the output was verified at the Ohio State University (OSU).

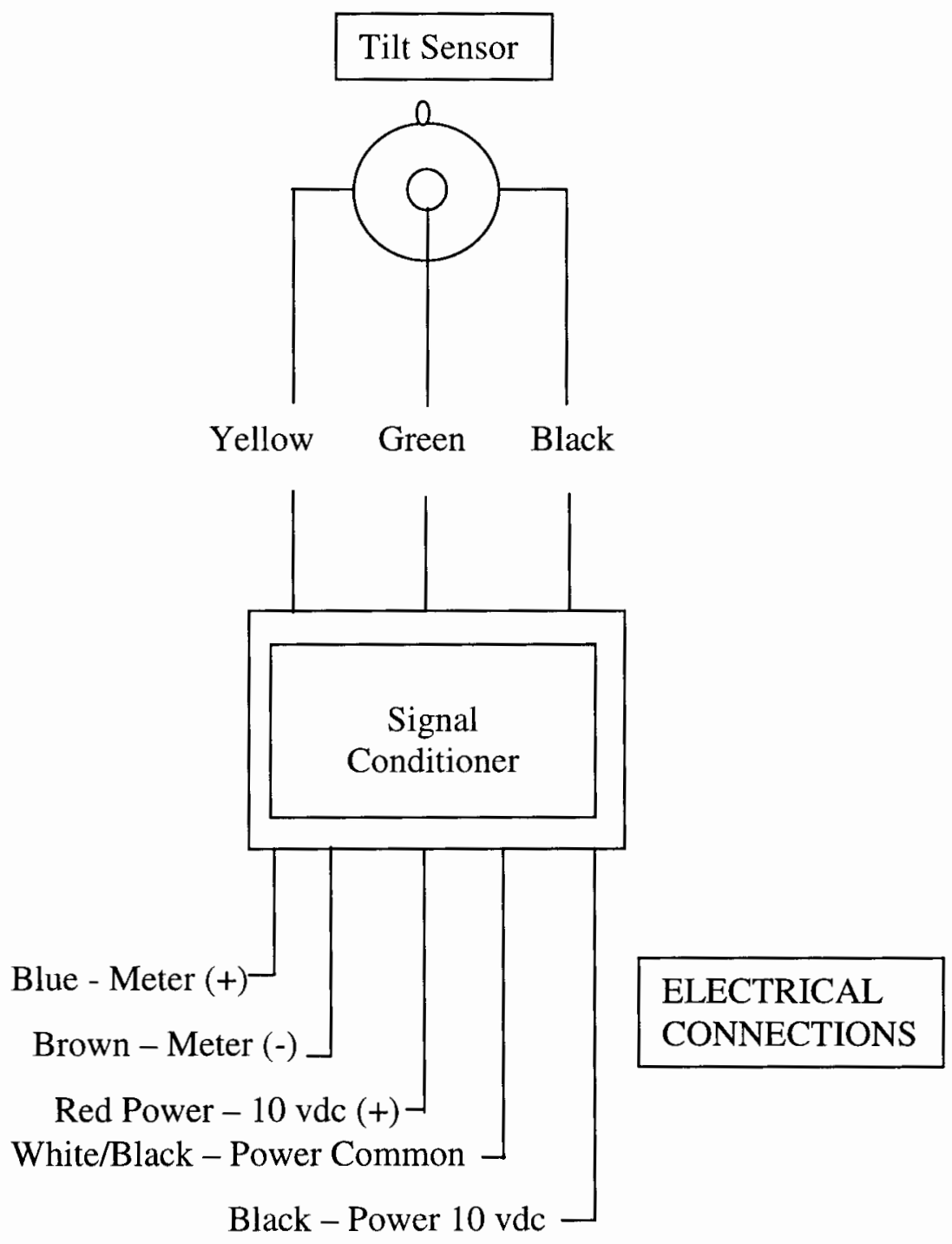

Figure G.1. Set up connections for the tilt sensor - signal conditioner pair

At the vendor's site, the manufacturer had build a compact module with standard surface-mounted electrical components for reliability and a socket for the placement of the sensor. The module converted the sensor output into a \pm 2.5 voltage full-scale linear output digital signal. Their experiments have determined that the average life span of a 
tilt sensor is over 10,000 hours. At the OSU testing site the data from the manufacturer's calibration procedure were verified to the extend that the hardware allowed (see Table G.1).

\begin{tabular}{|c|c|c|c|c|}
\hline $\begin{array}{l}\text { Tilt Angle } \\
\text { (Deg) }\end{array}$ & $\begin{array}{l}\text { Manuf_Output } \\
\text { (mV.SC3-S8) }\end{array}$ & $\begin{array}{l}\text { Manuf_Output } \\
\text { (mV.SC8-S4) }\end{array}$ & $\begin{array}{c}\text { Lab_Output } \\
\text { (V.SC8 - S4) }\end{array}$ & $\begin{array}{l}\text { Lab_Output } \\
\text { (V.SC3-S8) }\end{array}$ \\
\hline & & & & \\
\hline-180 & 34.31 & -117.32 & & \\
\hline-175 & -53.66 & -197.88 & -0.104 & -0.187 \\
\hline-165 & -228.43 & -360.16 & -0.26 & -0.322 \\
\hline-155 & -404.91 & -528.16 & -0.431 & -0.488 \\
\hline-145 & -585.45 & -699.75 & -0.622 & -0.643 \\
\hline-135 & -769.79 & -867.85 & -0.827 & -0.816 \\
\hline-125 & -951.62 & -1029.73 & -1.021 & -0.978 \\
\hline-115 & -1125.42 & -1177.12 & -1.202 & -1.135 \\
\hline-105 & -1286.45 & -1288.44 & -1.369 & -1.27 \\
\hline-95 & -1390.56 & -1313.17 & -1.483 & -1.338 \\
\hline-85 & -1410.25 & -1289.74 & -1.504 & -1.345 \\
\hline-75 & -1334.32 & -1195.04 & -1.452 & -1.328 \\
\hline-65 & -1196.54 & -1042.79 & -1.326 & -1.239 \\
\hline-55 & -994.12 & -880.3 & -1.13 & -1.098 \\
\hline-45 & -810.64 & -719.67 & -0.93 & -0.929 \\
\hline-35 & -625.05 & -559.19 & -0.742 & -0.764 \\
\hline-25 & -440.73 & -399.58 & -0.545 & -0.602 \\
\hline-15 & -262.71 & -240.65 & -0.368 & -0.446 \\
\hline-5 & -86.76 & -81.54 & -0.204 & -0.275 \\
\hline 5 & 87.42 & 78.5 & -0.039 & -0.144 \\
\hline 15 & 262.19 & 242.28 & 0.106 & 0.006 \\
\hline 25 & 439.57 & 410.39 & 0.279 & 0.161 \\
\hline 35 & 619.57 & 579.65 & 0.454 & 0.32 \\
\hline 45 & 808.87 & 762.96 & 0.638 & 0.493 \\
\hline 55 & 995.76 & 934.13 & 0.819 & 0.674 \\
\hline 65 & 1183.69 & 1117.33 & 1.008 & 0.843 \\
\hline 75 & 1374.05 & 1234.99 & 1.213 & 1.026 \\
\hline 85 & 1481.62 & 1270.38 & 1.328 & 1.159 \\
\hline 95 & 1509.87 & 1248.73 & 1.401 & 1.215 \\
\hline 105 & 1412.48 & 1154.5 & 1.374 & 1.204 \\
\hline 115 & 1247.55 & 999.32 & 1.256 & 1.13 \\
\hline 125 & 1062.54 & 831.12 & 1.088 & 0.979 \\
\hline 135 & 868.41 & 653.33 & 0.902 & 0.814 \\
\hline
\end{tabular}

Table G.1 (continued) 
Table G.1 (continued)

\begin{tabular}{|l|c|c|c|c|}
\hline 145 & 669.93 & 472.2 & 0.747 & 0.641 \\
\hline 155 & 481.51 & 295.96 & & \\
\hline 165 & 300.24 & 128.52 & & \\
\hline 175 & 122.58 & 34.79 & & \\
\hline
\end{tabular}

Table G.1.

Voltage output data as a function of the tilt angle. The data reported by the manufacturer of the inclinometers were reported in millivolts $(\mathrm{mV})$, whereas the data at the OSU testing site are reported in volts (V). The signal conditioner (SC) - tilt sensor (S) pairs that were used are reported also.

To collect the corresponding data at OSU a goniometer was attached on the isokinetic dynamometer such that the pointer to the angle side was free and was acting under gravity (see Figure G.2). The tilt sensor of interest was attached on the goniometer. The lever arm of the KinCom was moved through its full range of motion and the pointer of the goniometer always pointed to the angle with respect to vertical functioning in the same way as the tilt sensor (see Figure G.2). The angle for

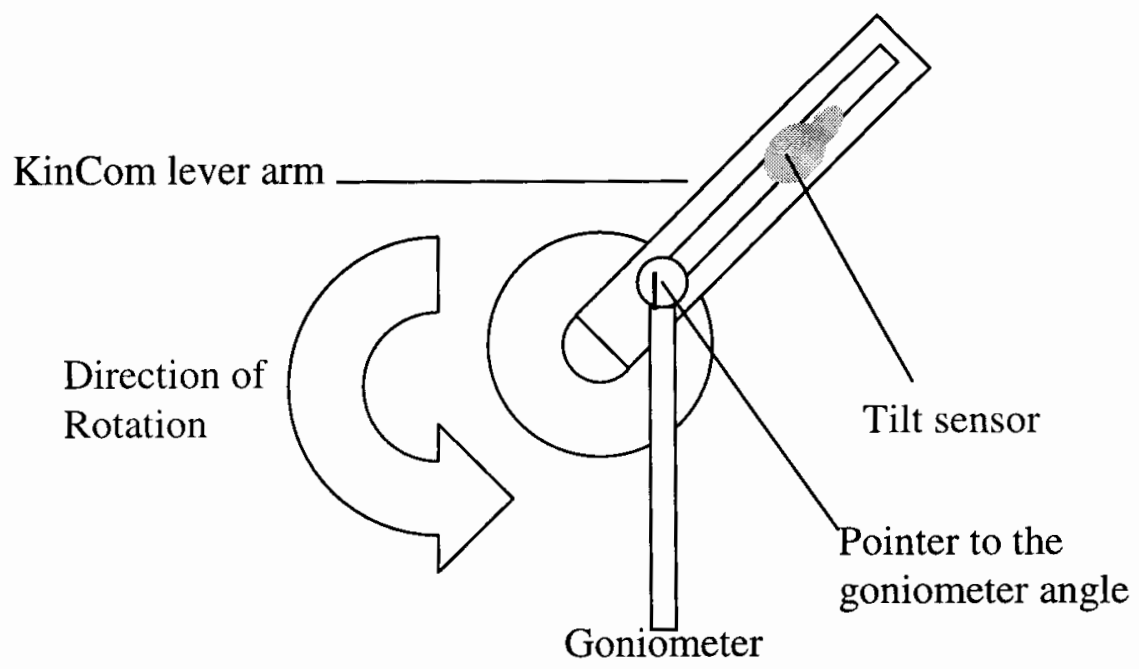

Figure G.2. The set up with the isokinetic dynamometer and the tilt sensor to verify the voltage as a function of the tilt angle. 
every $10^{\circ}$ and the respective voltage from the paired tilt sensor and signal conditioner was recorded. However, the motion of the lever arm of the isokinetic dynamometer was limited by the mechanical locks of the isokinetic device. Thus, the full range of motion of the lever arm was from $-175^{\circ}$ to $145^{\circ}$ where at $0^{\circ}$ the sensor was perpendicular to the horizontal pointing up such that the electrolyte in the sensor was level and, therefore, the impedance at the three coplanar electrodes was the same. The limited range of motion of the KinCom lever arm was not a limited factor since the focus was on identifying the linear response range of the tilt sensor to be used during the actual data collection procedure with the subjects who participated in the present project.

The results of the verification process can be seen in Figure G.3 (A and B), and Figure G.4 (A and B). It can be observed that, although the pattern of voltage response was similar for the manufacturer and OSU, the actual voltage values were different for the same angles. In other words, although there is an implicit assumption of linearity, i.e., that the inclinometer produces voltage values that are linear to the angles measured, a zero millivolt measurement, however, may not correspond to a zero degree angle measured. A zero angle might correspond to several or several hundred millivolts. Consequently, regression equations relating the voltage to the angle for the linear response range of each tilt sensor - signal conditioner pair were established. 

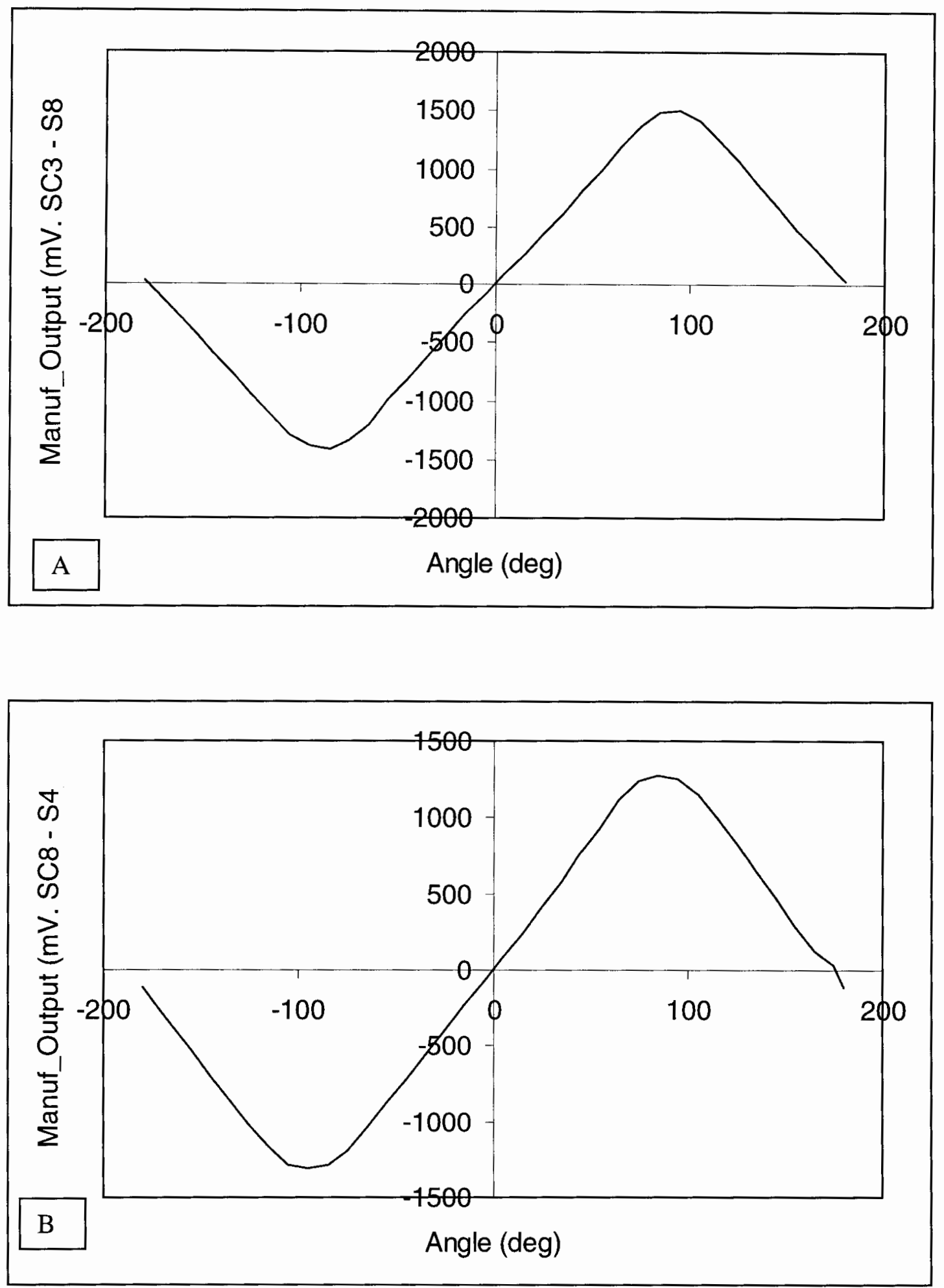

Figure G.3 (A and B). Manufacturer output of the tilt sensor (S) with its paired signal conditioner $(\mathrm{SC})$, in millivolts $(\mathrm{mV})$ as a function of the tilt angle. 

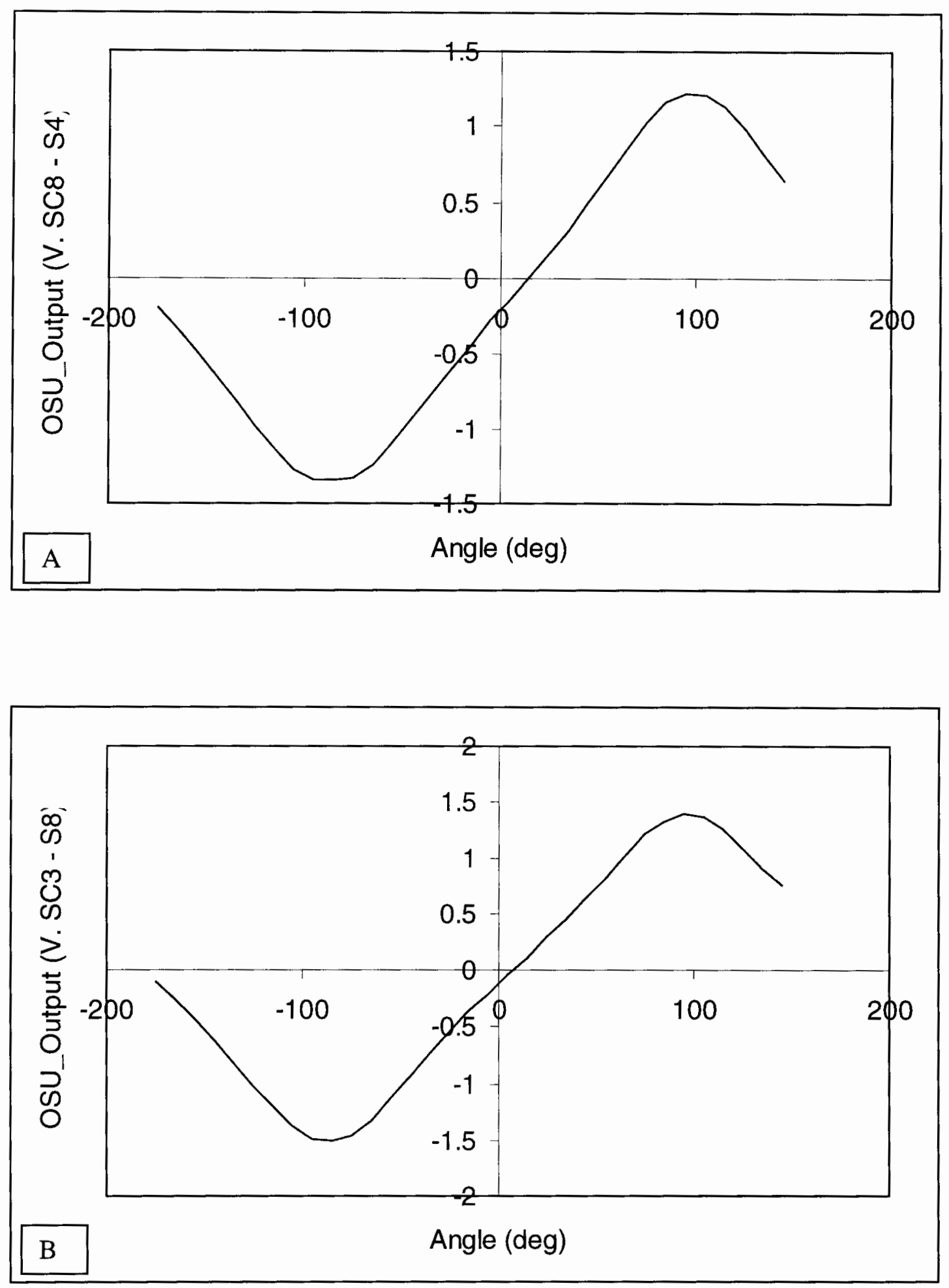

Figure G.4 (A and B). Output of the tilt sensors (S) with its paired signal conditioner $(\mathrm{SC})$, in volts $(\mathrm{V})$ as a function of the tilt angle when the testing took place at OSU. 


\section{APPENDIX H}

\section{ANTHROPOMETRIC INFORMATION FORM}


Name:

Date of Birth:

Address:

Telephone Number:

(home)

(work)

Height (in):

Weight(lb):

Inter-ASIS distance $(\mathrm{cm})$ :

ASIS-to-trochanter distance $(\mathrm{cm})$ :

Leg Length $(\mathrm{cm})$ :

Knee Width $(\mathrm{cm})$ :

Ankle Width (cm):

Skinfold $\quad(\mathrm{mm})$ :

Max. Dorsi Flexion:

Max. Plantar Flexion: 
APPENDIX I

SIMULATION RESULTS FROM SIMM 


\begin{tabular}{|c|c|}
\hline $\begin{array}{c}\text { andf } \\
\text { degrees })\end{array}$ & $\begin{array}{c}\text { AnkDF_Sum } \\
(\mathrm{Nm})\end{array}$ \\
\hline-30 & 37 \\
\hline-28 & 37 \\
\hline-26 & 38 \\
\hline-24 & 38 \\
\hline-22 & 39 \\
\hline-20 & 39 \\
\hline-18 & 40 \\
\hline-16 & 40 \\
\hline-14 & 40 \\
\hline-12 & 40 \\
\hline-10 & $\mathbf{4 1}$ \\
\hline-8 & $\mathbf{4 1}$ \\
\hline-6 & $\mathbf{4 1}$ \\
\hline-4 & $\mathbf{4 1}$ \\
\hline-2 & $\mathbf{4 1}$ \\
\hline 0 & $\mathbf{4 1}$ \\
\hline 2 & $\mathbf{4 1}$ \\
\hline 4 & $\mathbf{4 1}$ \\
\hline 6 & 40 \\
\hline 8 & 40 \\
\hline 10 & 39 \\
\hline 12 & 38 \\
\hline 14 & 37 \\
\hline 16 & 36 \\
\hline 18 & 35 \\
\hline 20 & 33 \\
\hline & \\
\hline
\end{tabular}

Table I.1.

The ankle dorsi flexor muscle group moment (AnkDF_Sum) output as a function of the dorsi flexion joint angle (andf) using SIMM. The bolded values indicate the range of angles at which maximum muscle moment occurred. No plantar flexion cocontraction has been included in this table because the result would be a plantar flexor moment. No joint interaction data are being presented either, because the dorsi flexors are all monoarticular muscles. 


\begin{tabular}{|c|c|c|c|c|c|c|c|c|}
\hline$\overline{\text { anpf }}$ & \begin{tabular}{|c|} 
AnkPF \\
K0 \\
\end{tabular} & $\begin{array}{c}\text { AnkPF } \\
\text { K7 } \\
\end{array}$ & $\begin{array}{c}\text { AnkPF } \\
\mathrm{K} 20\end{array}$ & $\begin{array}{c}\text { AnkPF } \\
\text { K55 } \\
\end{array}$ & $\begin{array}{c}\text { AnkPF } \\
\text { K0CoCon } \\
\end{array}$ & \begin{tabular}{|c|} 
AnkPF \\
K7CoCon \\
\end{tabular} & $\begin{array}{c}\text { AnkPF } \\
\text { K20CoCon } \\
\end{array}$ & $\begin{array}{c}\text { AnkPF } \\
\text { K55CoCon } \\
\end{array}$ \\
\hline 20 & -167 & -167 & -165 & -161 & -133 & -133 & -131 & -127 \\
\hline 18 & -170 & -170 & -168 & -163 & -135 & -134 & -132 & -127 \\
\hline 16 & -172 & -171 & -169 & -164 & -136 & -135 & -133 & -127 \\
\hline 14 & -173 & -172 & -170 & -163 & -135 & -134 & -132 & -125 \\
\hline 12 & -174 & -173 & -170 & -162 & -135 & -134 & -132 & -123 \\
\hline 10 & -174 & -172 & -170 & -160 & -134 & -133 & -131 & -121 \\
\hline 8 & -173 & -172 & .170 & -158 & -133 & -132 & -130 & -118 \\
\hline 6 & -172 & -171 & -168 & -154 & -132 & -131 & -128 & -114 \\
\hline 4 & -170 & -169 & -165 & -150 & -129 & -128 & -124 & -108 \\
\hline 2 & -167 & -165 & -161 & -143 & -125 & -124 & -119 & -102 \\
\hline 0 & -162 & -160 & -154 & -135 & -120 & -118 & -112 & -93 \\
\hline-2 & -155 & -152 & -145 & -125 & -113 & -111 & -104 & -83 \\
\hline-4 & -146 & -143 & -135 & -113 & -105 & -102 & -94 & -72 \\
\hline-6 & -136 & -132 & -124 & -101 & -95 & -91 & -82 & -60 \\
\hline-8 & -125 & -121 & -111 & -89 & -83 & -79 & -70 & -47 \\
\hline-10 & -113 & -108 & -98 & -76 & -72 & -67 & -57 & -35 \\
\hline-12 & -101 & -96 & -85 & -65 & -60 & -55 & -44 & -24 \\
\hline-14 & -90 & -85 & -74 & -55 & -49 & -44 & -33 & -14 \\
\hline-16 & -80 & -75 & -64 & -47 & -40 & -34 & -23 & -6 \\
\hline-18 & -72 & -66 & -55 & -40 & -32 & -26 & -15 & -0 \\
\hline-20 & -64 & -58 & -48 & -36 & -25 & -19 & -9 & 3 \\
\hline-22 & -58 & -52 & -43 & -32 & -18 & -13 & -3 & 6 \\
\hline \begin{tabular}{|l|}
-24 \\
\end{tabular} & -52 & -46 & -38 & -30 & -13 & -8 & 0 & 8 \\
\hline-26 & -47 & -42 & -35 & -29 & -8 & -4 & 2 & 9 \\
\hline-28 & -43 & -38 & -32 & -28 & -5 & -1 & 5 & 9 \\
\hline-30 & -39 & -35 & -30 & -27 & -2 & 1 & 6 & 10 \\
\hline
\end{tabular}

Table I.2.

The ankle plantar flexor muscle group moment (AnkPF) SIMM output during plantar flexion as a function of the joint angle (anpf) and joint interactions with the angle at the knee. The knee positions tested were: neutral (K0), knee flexion at seven degrees (K7), knee flexion at twenty degrees (K20) and knee flexion at fifty five degrees (K55). The ankle plantar flexor muscle group moment output was obtained for the same conditions while assuming maximum antagonistic cocontraction (CoCon) from the dorsi flexors. Bolded values indicate the range of angles at which maximum muscle moment occurred. The hip was kept at 45 degrees. 


\begin{tabular}{|c|c|c|c|c|c|}
\hline knee & Mkke & $\begin{array}{c}\text { Mkke } \\
\text { A0CoCon }\end{array}$ & $\begin{array}{c}\text { Mkke } \\
\text { A10PCoCon }\end{array}$ & $\begin{array}{c}\text { Mkke } \\
\text { A20PCoCon }\end{array}$ & $\begin{array}{c}\text { Mkke } \\
\text { 10DCoCon }\end{array}$ \\
\hline 70 & -184 & -87 & -92 & -94 & -81 \\
\hline 68 & -187 & -88 & -93 & -95 & -82 \\
\hline 66 & -190 & -89 & -94 & -96 & -82 \\
\hline 64 & -193 & -89 & -94 & -96 & -82 \\
\hline 62 & -195 & -89 & .94 & -97 & -82 \\
\hline 60 & -197 & -88 & -94 & -97 & -82 \\
\hline 58 & -198 & -88 & -94 & -97 & -81 \\
\hline 56 & -199 & -87 & -93 & -96 & -81 \\
\hline 54 & -199 & -86 & -93 & -96 & -80 \\
\hline 52 & -199 & -85 & -92 & -95 & -79 \\
\hline 50 & -199 & -84 & -91 & -95 & -79 \\
\hline 48 & -198 & -84 & -90 & -94 & -78 \\
\hline 46 & -197 & -83 & -89 & -93 & -77 \\
\hline 44 & -196 & -82 & -89 & -93 & -77 \\
\hline 42 & -195 & -82 & -88 & -93 & -76 \\
\hline 40 & -194 & -81 & -88 & -93 & -76 \\
\hline 38 & -192 & -80 & -87 & -92 & -75 \\
\hline 36 & -190 & -79 & -86 & -92 & -74 \\
\hline 34 & -188 & -78 & -85 & -91 & -73 \\
\hline 32 & -185 & -76 & -84 & -90 & -72 \\
\hline 30 & -182 & -74 & -81 & -88 & -70 \\
\hline 28 & -178 & -71 & -79 & -86 & -67 \\
\hline 26 & -174 & -68 & -76 & -83 & -64 \\
\hline 24 & -168 & -64 & -72 & -79 & -60 \\
\hline 22 & -162 & -60 & -67 & -75 & -56 \\
\hline 20 & -154 & -55 & -62 & -70 & -51 \\
\hline 18 & -146 & -49 & -56 & -64 & -46 \\
\hline 16 & -137 & -42 & -48 & -57 & -39 \\
\hline 14 & -126 & -34 & -40 & -49 & -31 \\
\hline 12 & -114 & -25 & -31 & -39 & -22 \\
\hline 10 & -101 & -15 & -21 & -29 & -13 \\
\hline 8 & -88 & -5 & -10 & -18 & -3 \\
\hline 6 & -75 & 4 & 0 & -8 & 6 \\
\hline 4 & -62 & 13 & 8 & 0 & 15 \\
\hline 2 & -50 & 21 & 16 & 9 & 23 \\
\hline 0 & -39 & 28 & 23 & 16 & 29 \\
\hline
\end{tabular}

Table I.3.

Knee extensor muscle group moment (Mkke) SIMM output during knee extension (knex) and joint interactions with the ankle. Maximum knee flexor muscle group cocontraction (CoCon) was assumed. Ankle positions were: neutral (A0), 10 degrees plantar flexion (A10P), 20 degrees plantar flexion (A20P), and 10 degrees dorsi flexion (A10D). Bolded values indicate where maximum moment occurred. The hip was kept at 45 degrees. 


\begin{tabular}{|c|c|c|c|c|}
\hline knfx & MkkfA0 & MkkfA10P & Mkkf A20P & Mkkf10D \\
\hline 0 & 67 & 63 & 56 & 69 \\
\hline 2 & 71 & 67 & 60 & 73 \\
\hline 4 & 75 & 71 & 63 & 77 \\
\hline 6 & 79 & 74 & 66 & 81 \\
\hline 8 & 83 & 77 & 69 & 85 \\
\hline 10 & 86 & 80 & 72 & 88 \\
\hline 12 & 89 & 83 & 74 & 91 \\
\hline 14 & 92 & 85 & 77 & 94 \\
\hline 16 & 94 & 88 & 79 & 97 \\
\hline 18 & 97 & 90 & 82 & 100 \\
\hline 20 & 99 & 92 & 84 & 103 \\
\hline 22 & 101 & 94 & 86 & 105 \\
\hline 24 & 103 & 96 & 88 & 107 \\
\hline 26 & 105 & 98 & 90 & 109 \\
\hline 28 & 106 & 99 & 92 & 111 \\
\hline 30 & 107 & 100 & 94 & 112 \\
\hline 32 & 109 & 101 & 95 & 113 \\
\hline 34 & 110 & 103 & 97 & 114 \\
\hline 36 & 111 & 104 & 98 & 116 \\
\hline 38 & 112 & 105 & 99 & 117 \\
\hline 40 & 112 & 105 & 101 & 117 \\
\hline 42 & 113 & 106 & 102 & 118 \\
\hline 44 & 113 & $\mathbf{1 0 7}$ & 103 & 119 \\
\hline 46 & $\mathbf{1 1 4}$ & $\mathbf{1 0 7}$ & 103 & 119 \\
\hline 48 & $\mathbf{1 1 4}$ & $\mathbf{1 0 7}$ & $\mathbf{1 0 4}$ & $\mathbf{1 2 0}$ \\
\hline 50 & $\mathbf{1 1 4}$ & $\mathbf{1 0 7}$ & $\mathbf{1 0 4}$ & $\mathbf{1 2 0}$ \\
\hline 52 & 113 & $\mathbf{1 0 7}$ & 103 & 119 \\
\hline 54 & 112 & 106 & 103 & 119 \\
\hline 56 & 111 & 105 & 102 & 118 \\
\hline 58 & 110 & 104 & 101 & 116 \\
\hline 60 & 108 & 102 & 99 & 114 \\
\hline 62 & 106 & 100 & 98 & 112 \\
\hline 64 & 104 & 98 & 96 & 110 \\
\hline 66 & 101 & 96 & 94 & 108 \\
\hline 68 & 99 & 94 & 92 & 105 \\
\hline & & & & \\
\hline
\end{tabular}

Table I.4.

The knee flexor muscle group moment (MkkfA0) output as a function of the knee flexion angle $(\mathrm{knfx})$ and joint interactions with the angle at the ankle using SIMM. Bolded values indicate the range of angles at which maximum group muscle moment occurred. No maximum knee extensor cocontraction has been included in this table because the result would be a knee extensor moment. The hip was kept at 45 degrees of flexion. 


\section{APPENDIX J}

GAIT KNEE AND ANKLE JOINT VELOCITIES 


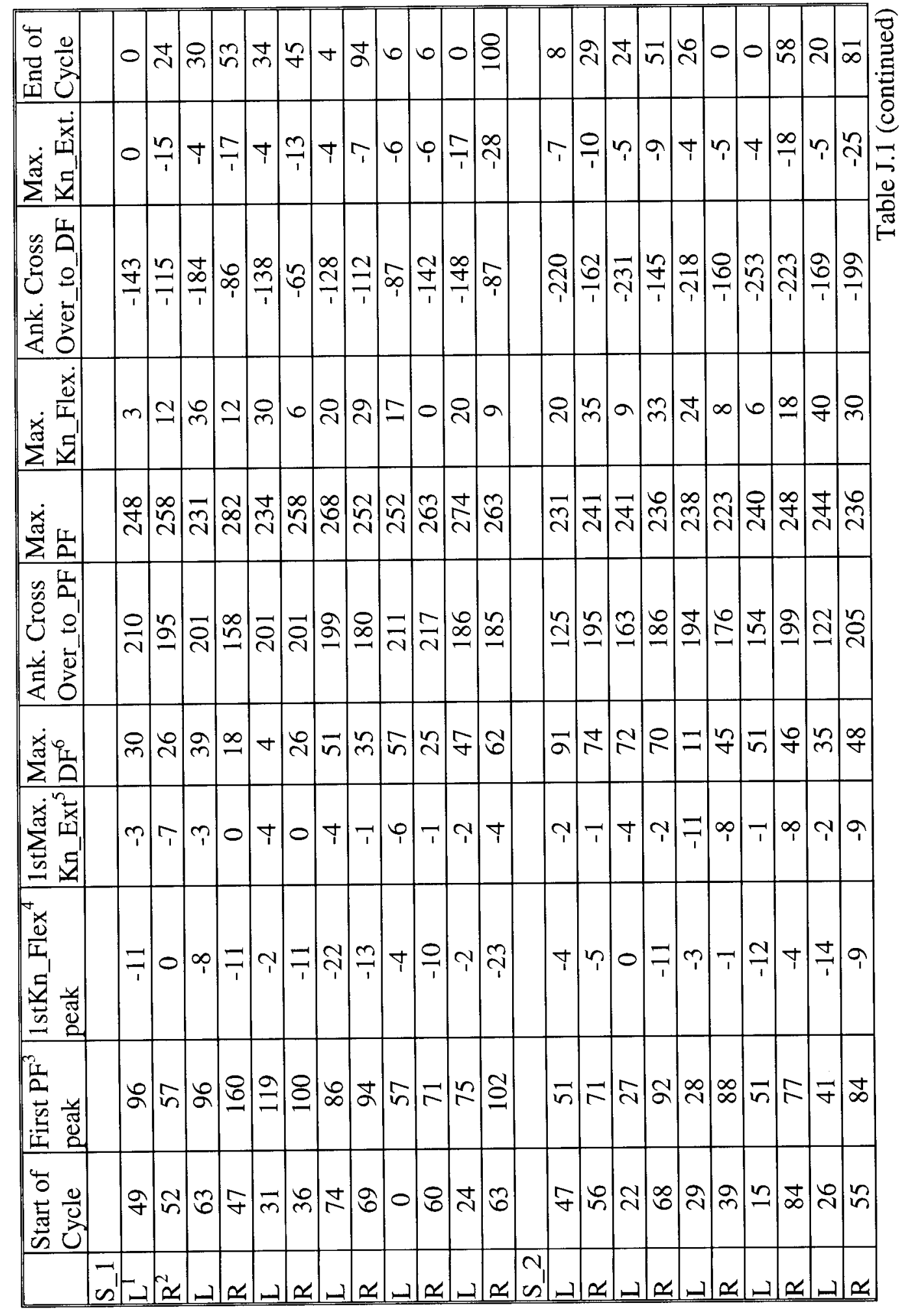




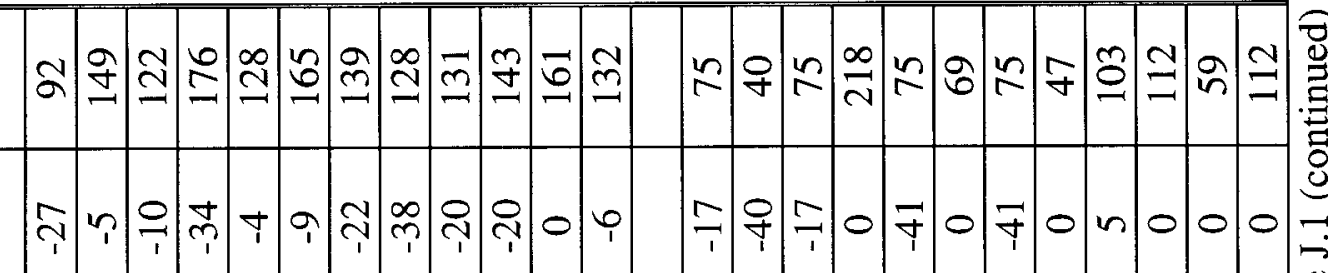

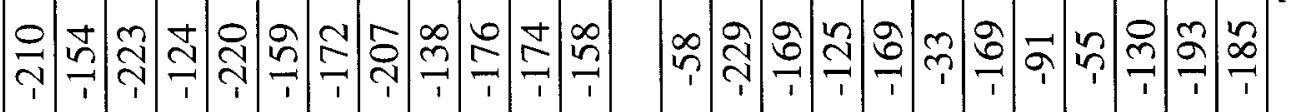

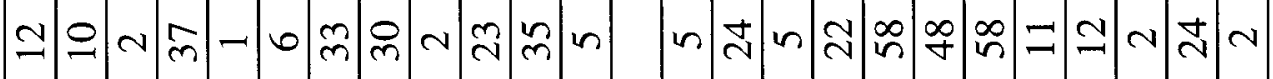

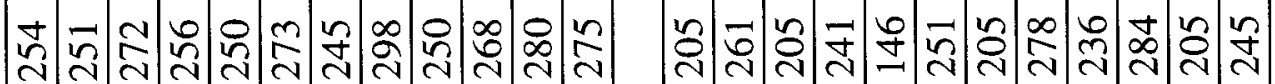

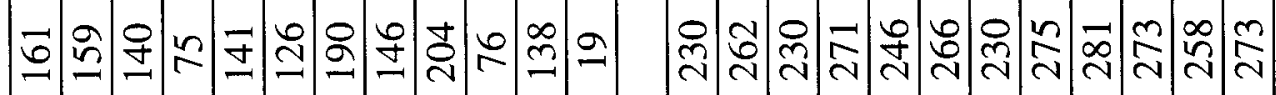

n் 0.00000 m.

this

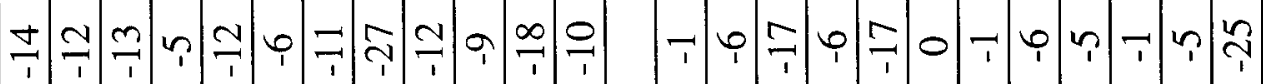

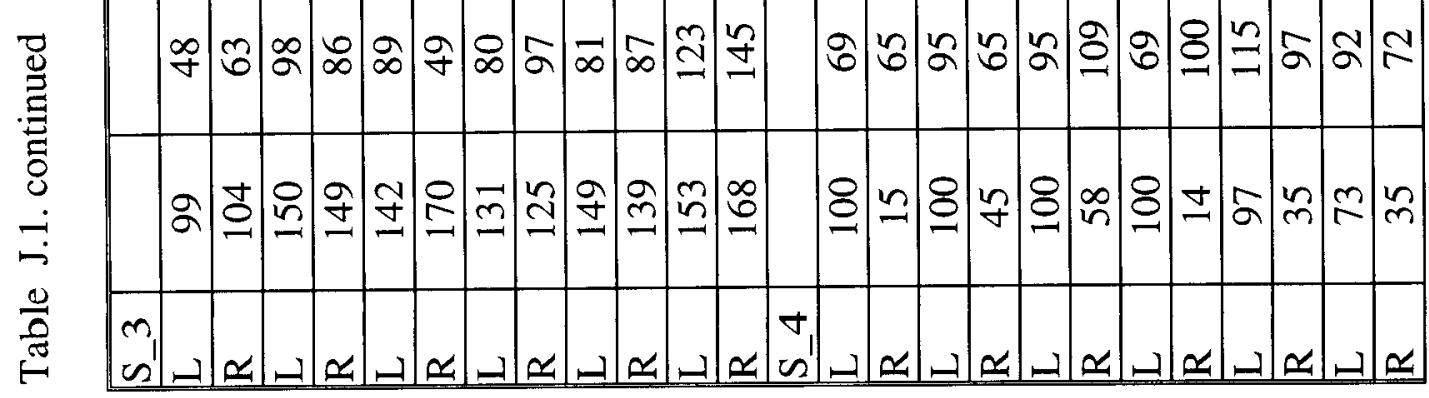




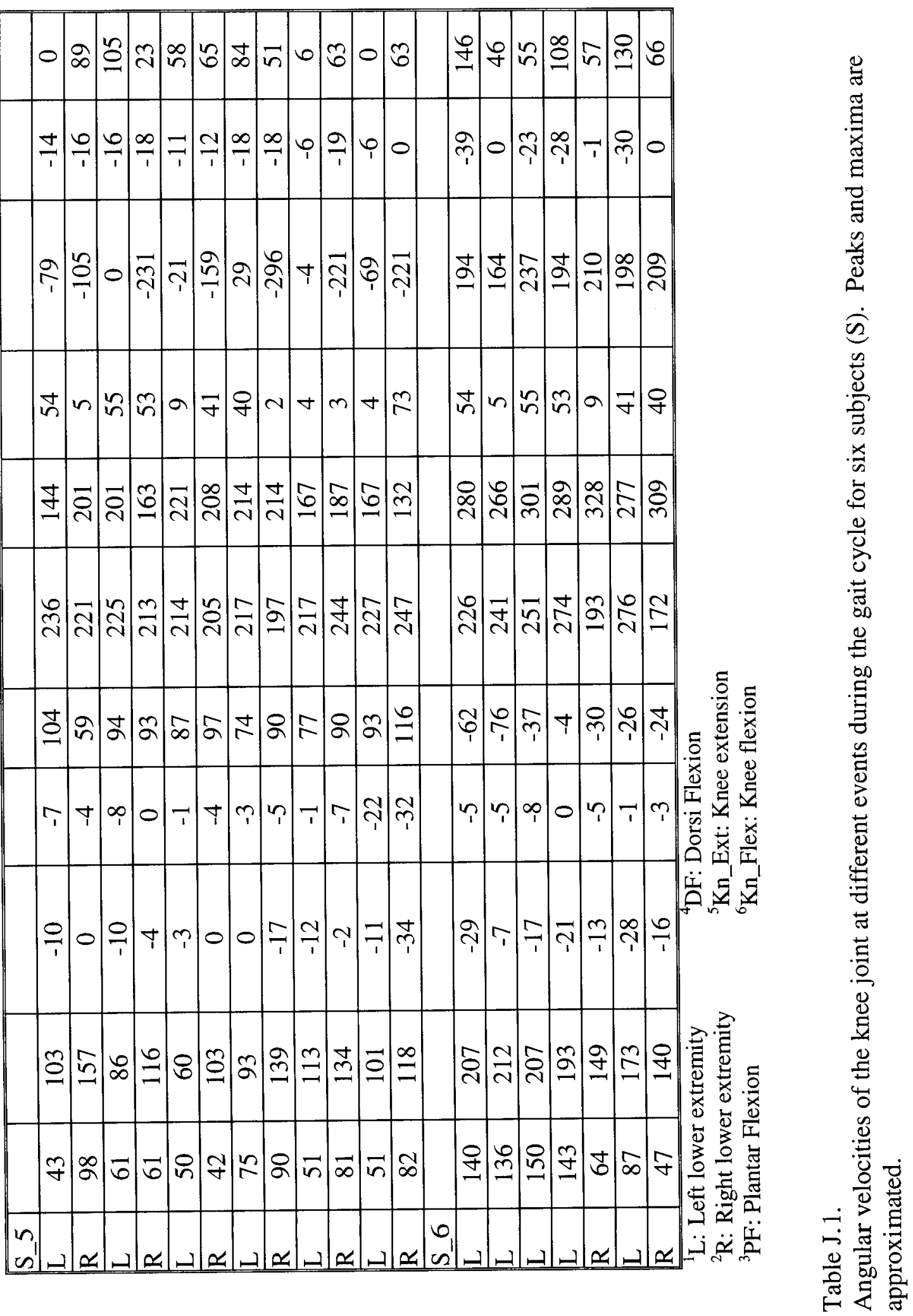




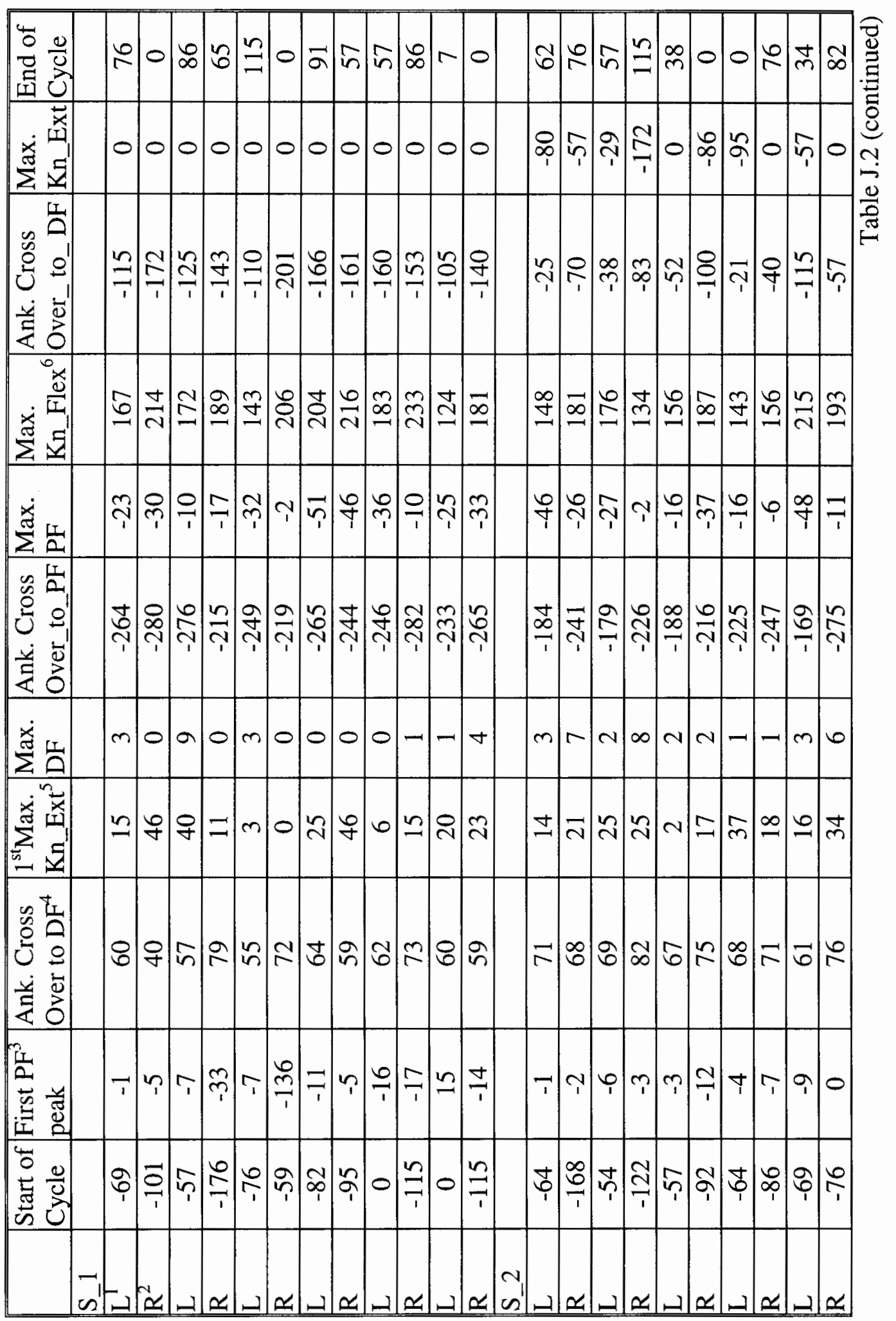




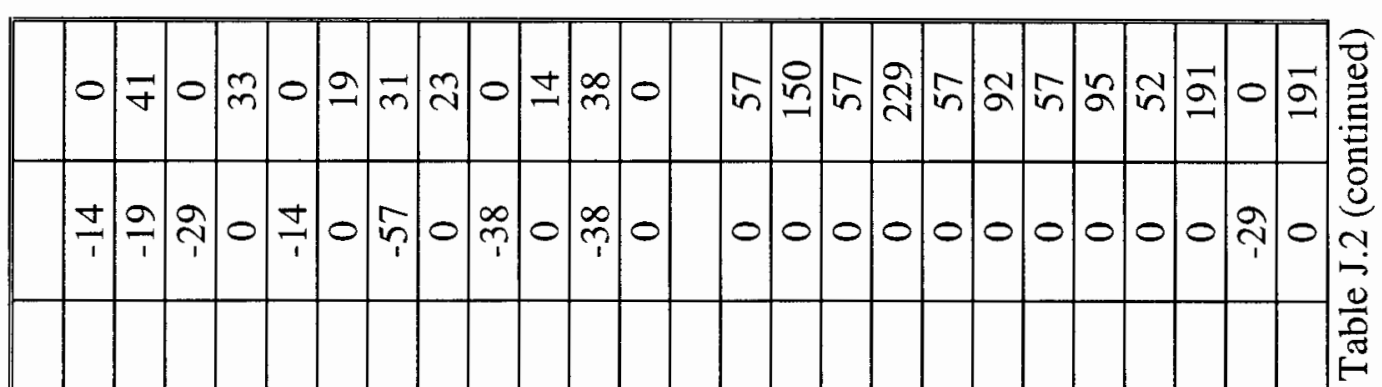

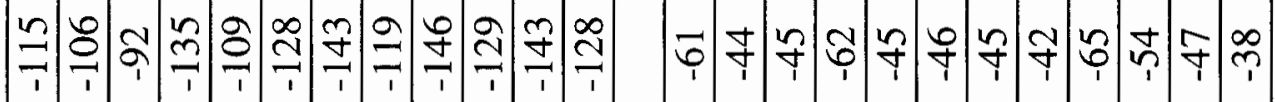

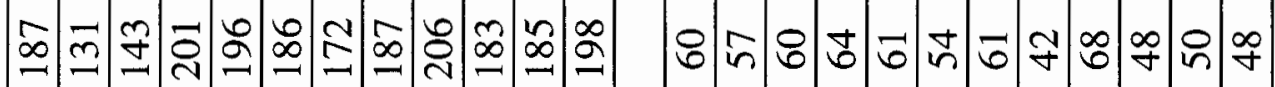



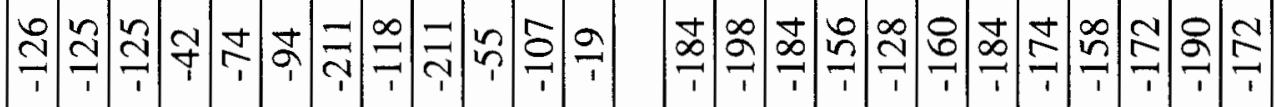

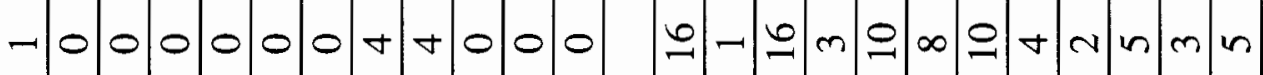

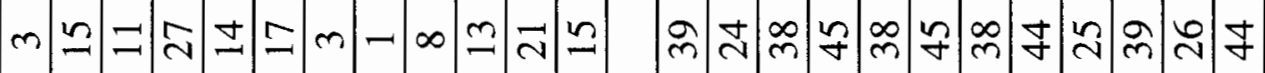

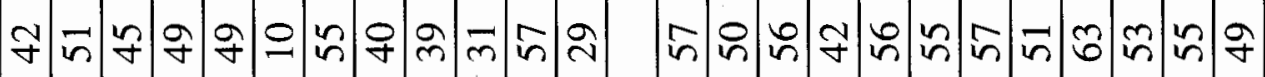

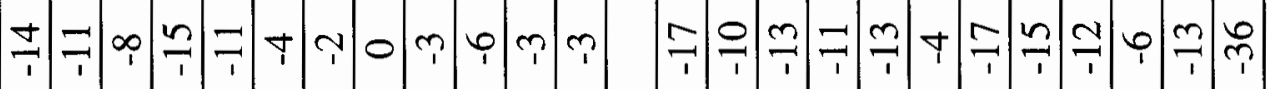

范

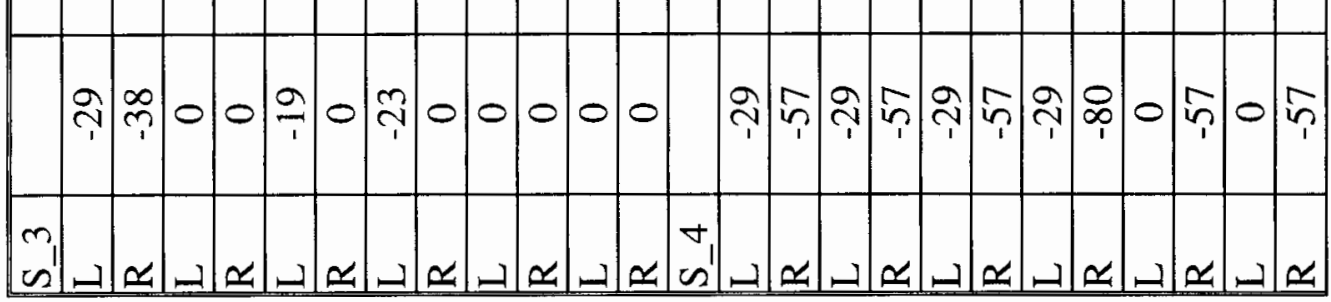




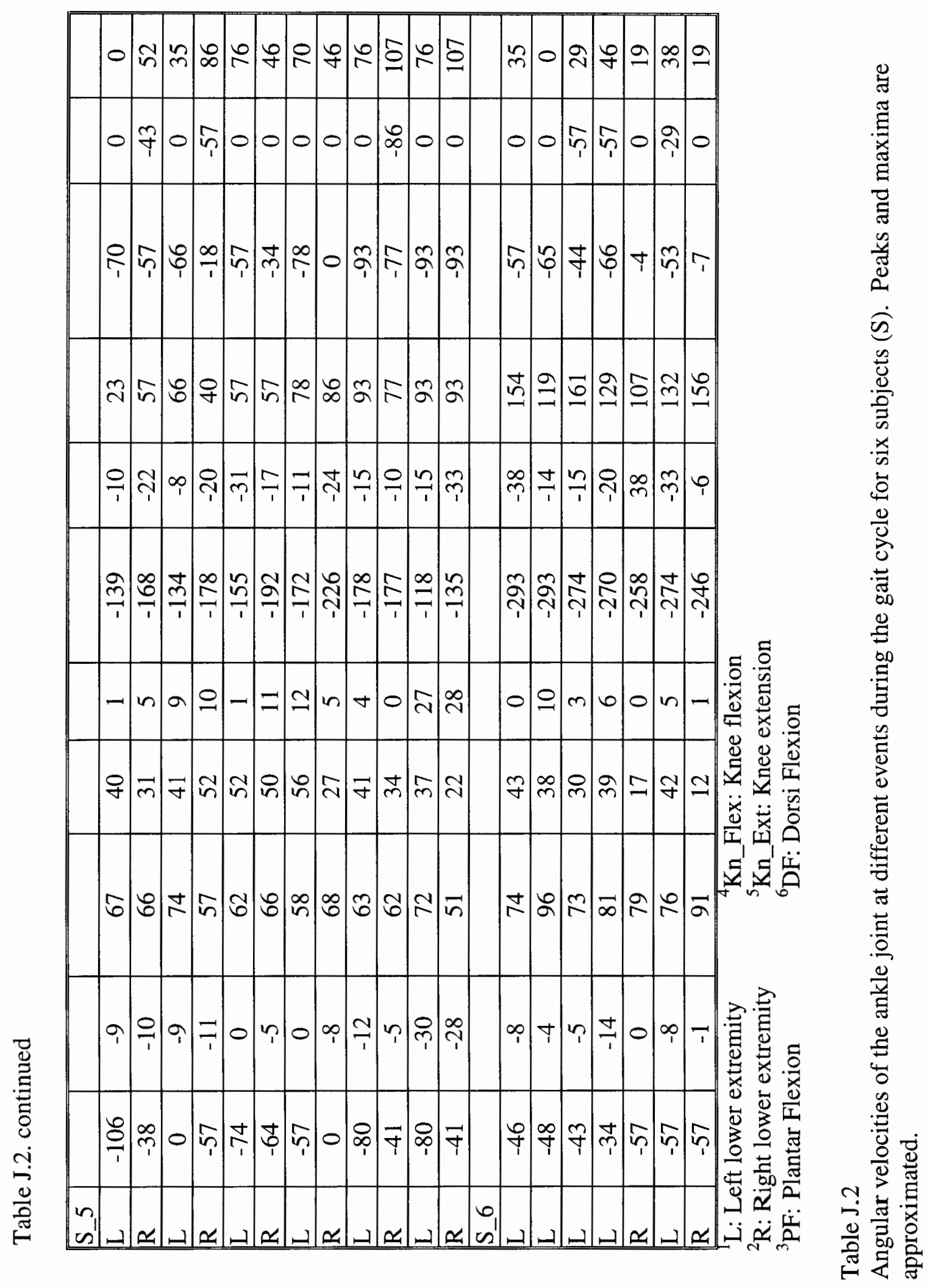




\section{APPENDIX K}

GAIT KNEE AND ANKLE JOINT RANGES OF MOTION 

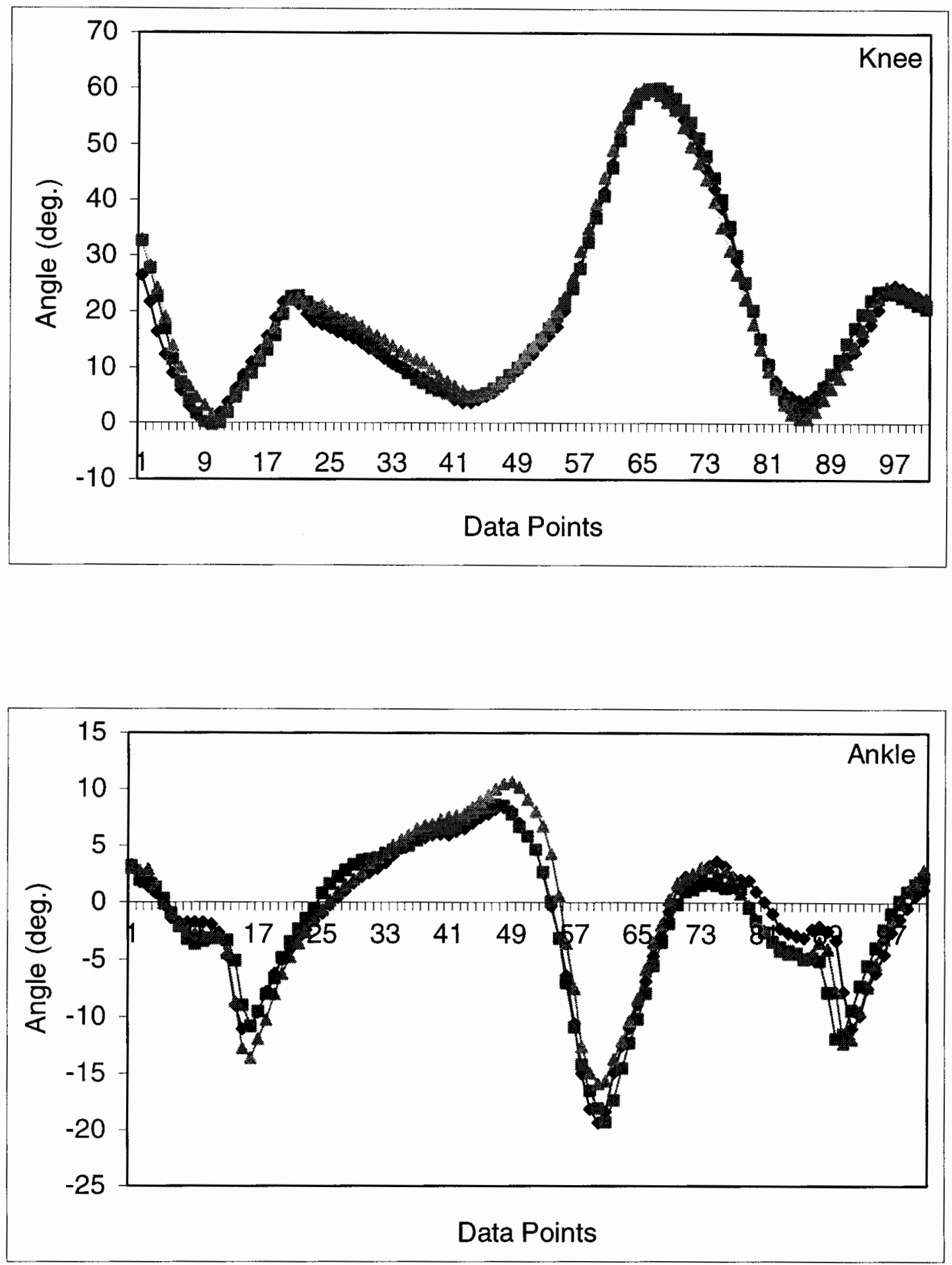

Figure K.1. Kinematic data for all three trials of subject SN for the knee and ankle joints. The data are consistent. 

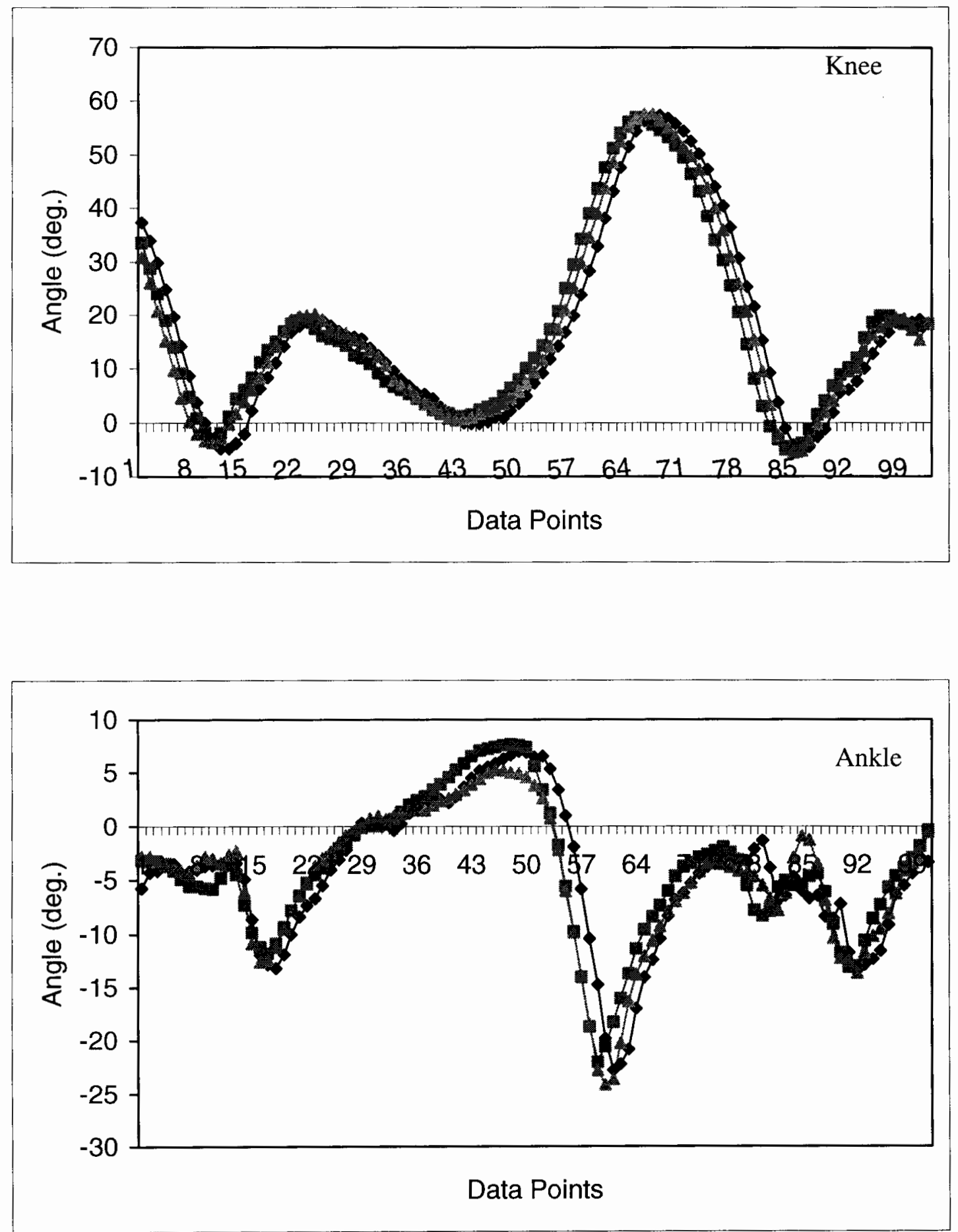

Figure K.2 Kinematic data for all three trials of subject SC for the knee and ankle joints. The data are consistent. 

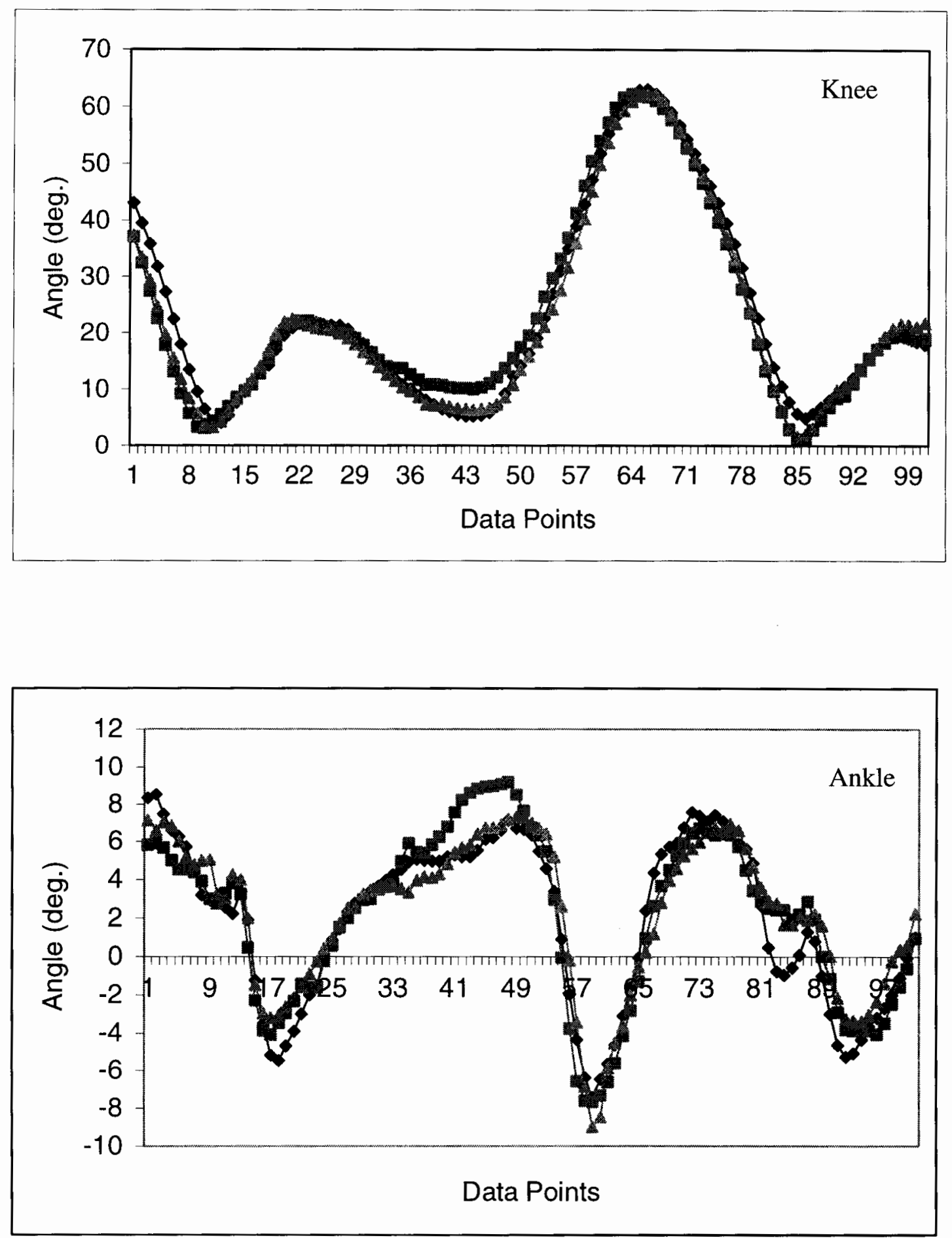

Figure K.3 Kinematic data for all three trials of subject SA for the knee and ankle joints. The data are consistent. 


\section{APPENDIX L}

GAIT KNEE AND ANKLE JOINT MOMENTS 

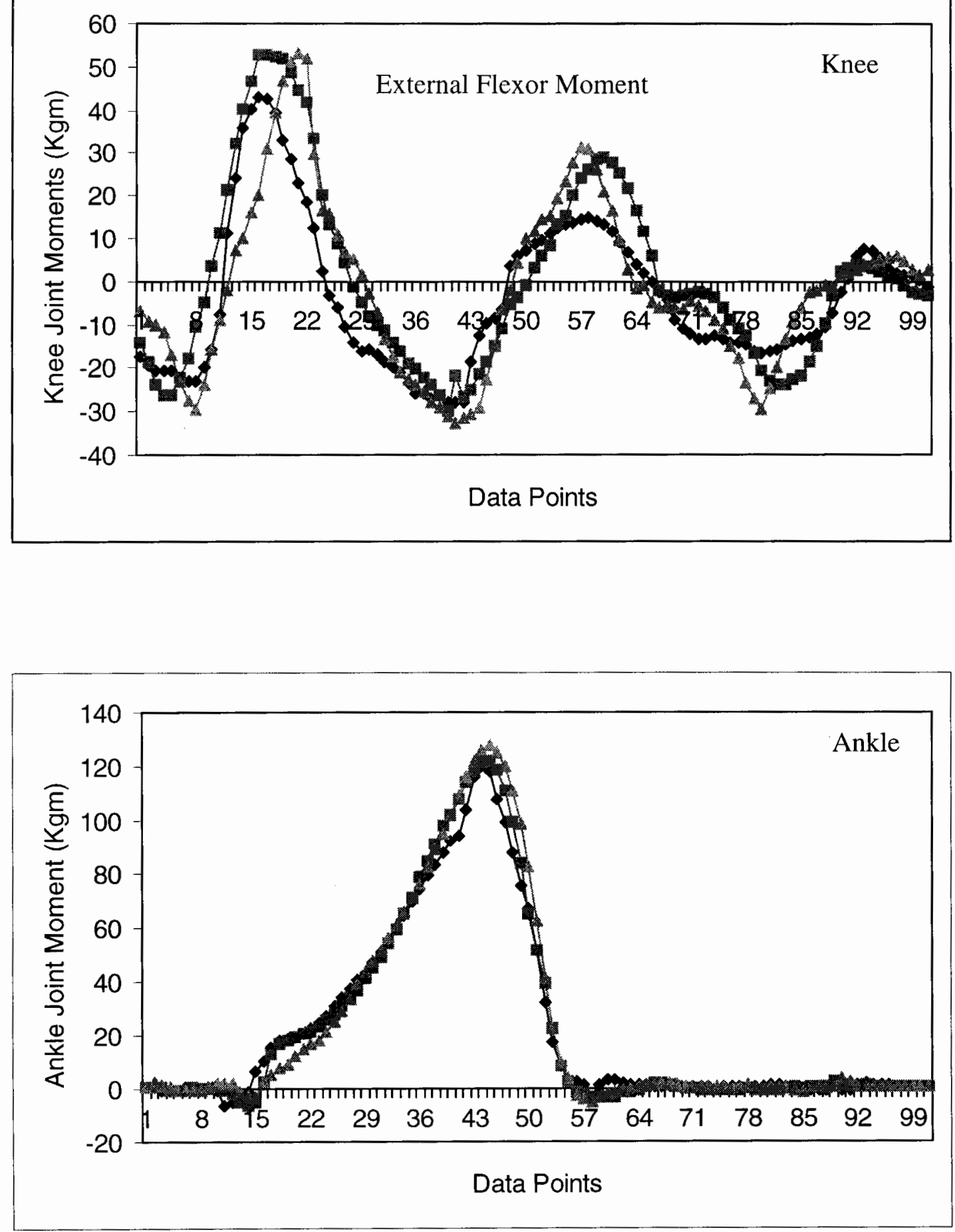

Figure L.1 Joint moment for all three trials of subject SN for the knee and ankle. The data are consistent with a little variability at the knee. The information is expressed in percent of the data points processed. 

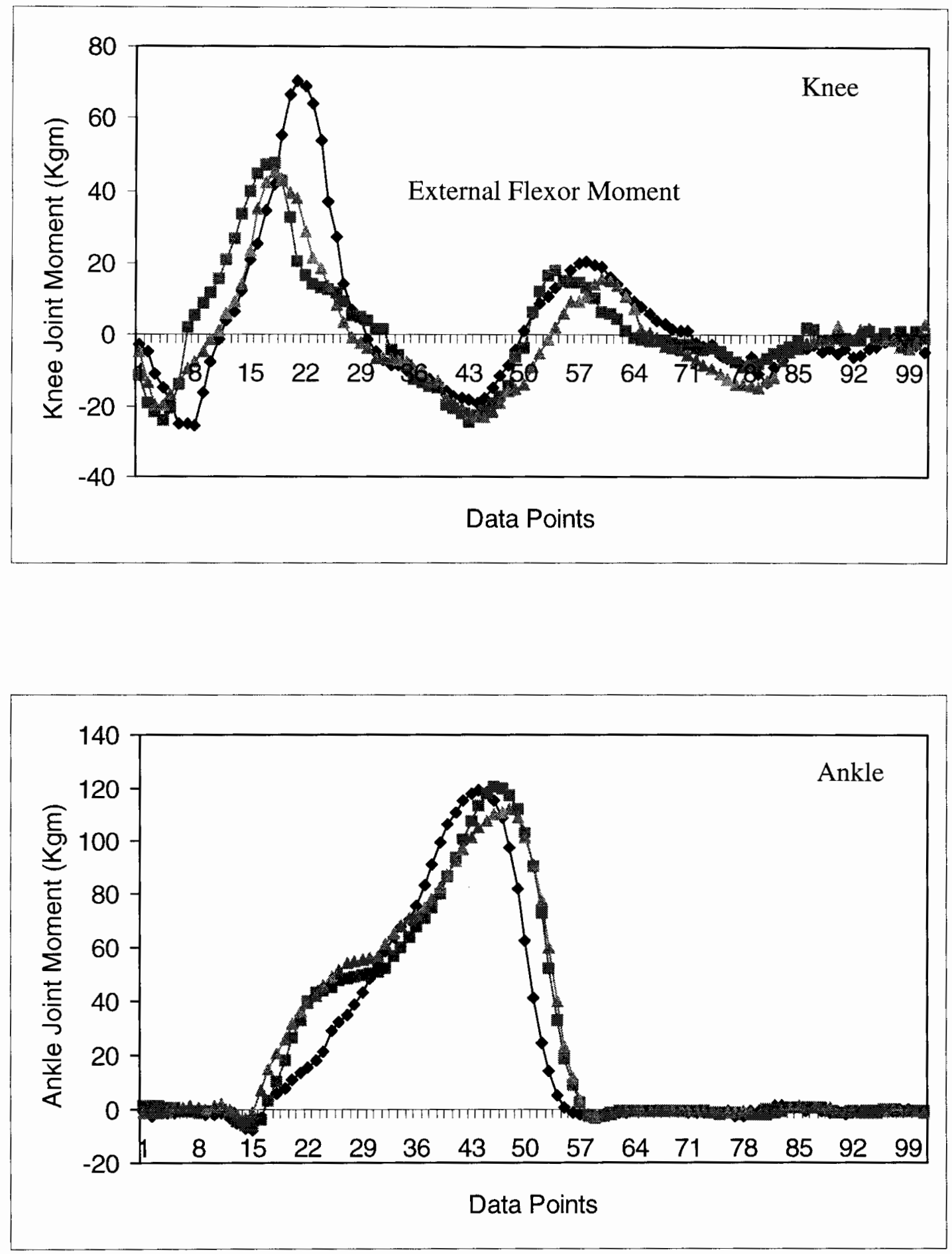

Figure L.2 Joint moments for all three trials of subject SC for the knee and ankle. The data are mostly consistent. The information is expressed in percent of the data points processed. 

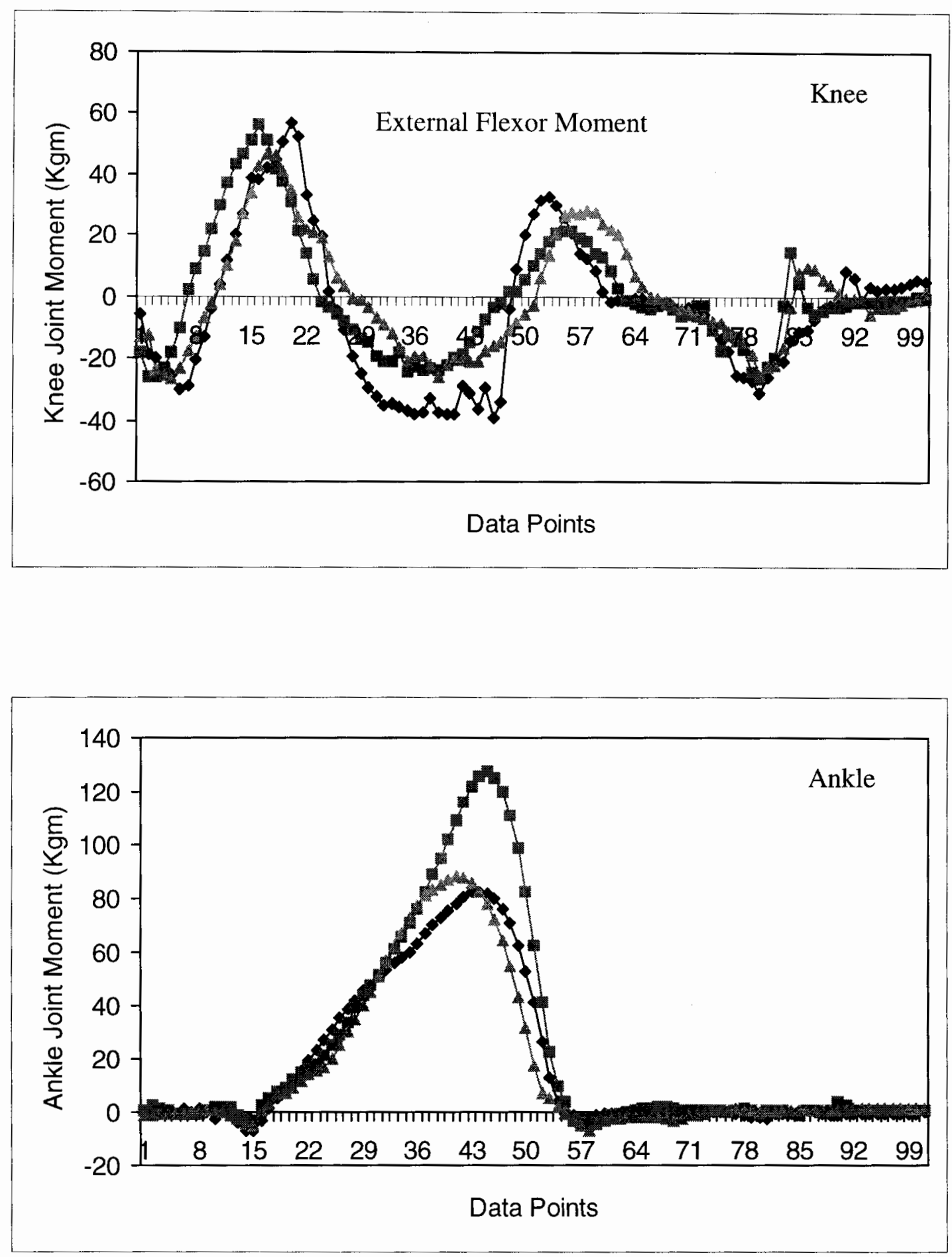

Figure L.3 Joint moment data for all three trials of subject SA for the knee and ankle. The data are consistent with a little variability at the knee joint. The information is expressed in percent of the data points processed. 


\section{APPENDIX M}

ISOKINETIC KNEE AND ANKLE RESULTS 


\begin{tabular}{|c|c|c|c|c|c|c|}
\hline Trial & Gain & $\mathrm{R}$ & $\mathrm{R}^{2}$ & RMS & NRMS_EnvMax & NRMS_MAX \\
\hline SNDF5CK0 ${ }^{1}$ & 13.16 & 0.24 & 0.06 & 4.26 & 29.30 & 29.30 \\
\hline SNDF5CK7 & 19.79 & 0.81 & 0.66 & 2.06 & 14.39 & 14.14 \\
\hline SNDF5CK20 & 17.25 & 0.79 & 0.62 & 1.55 & 13.28 & 10.63 \\
\hline SNDF5CK52 & 13.32 & 0.88 & 0.77 & 1.84 & 17.44 & 12.68 \\
\hline SNDF60CK52 & 19.46 & 0.80 & 0.64 & 1.57 & 20.23 & 10.82 \\
\hline SNDF120CK52 & 25.01 & 0.78 & 0.61 & 2.67 & 19.07 & 18.32 \\
\hline SNPF5CK0 & 14.36 & 0.42 & 0.18 & 10.60 & 20.09 & 20.09 \\
\hline SNPF5CK8 & 11.58 & 0.82 & 0.68 & 5.38 & 12.72 & 10.20 \\
\hline SNPF30CK8 & 13.56 & 0.01 & 0.00 & 9.25 & 24.37 & 17.54 \\
\hline SNPF60CK8 & 10.53 & 0.81 & 0.65 & 2.97 & 11.19 & 5.63 \\
\hline SNPF90CK8 & 10.26 & 0.87 & 0.76 & 2.48 & 8.26 & 4.70 \\
\hline SNPF5CK20 & 11.66 & 0.67 & 0.45 & 4.48 & 19.61 & 8.50 \\
\hline SNPF30CK20 & 16.31 & 0.19 & 0.04 & 5.45 & 26.91 & 10.33 \\
\hline SNPF60CK20 & 10.35 & 0.71 & 0.50 & 2.20 & 11.72 & 4.18 \\
\hline SNPF90CK20 & 10.02 & 0.95 & 0.89 & 1.38 & 7.93 & 2.61 \\
\hline SNPF5CK52 & 8.57 & 0.97 & 0.94 & 0.86 & 9.58 & 1.64 \\
\hline SNPF60CK52 & 11.45 & 0.94 & 0.88 & 1.06 & 13.31 & 2.01 \\
\hline Average & 13.92 & 0.69 & 0.55 & 3.53 & 16.43 & 10.78 \\
\hline $\begin{array}{c}\text { Standard } \\
\text { Deviation }\end{array}$ & 4.36 & 0.29 & 0.30 & 2.80 & 6.41 & 7.45 \\
\hline Median & 13.16 & 0.80 & 0.64 & 2.48 & 14.39 & 10.33 \\
\hline
\end{tabular}

1: An example of how an ankle trial should be read is "Subject $\mathrm{N}$, performing Dorsi Flexions at an angular velocity of 5 degrees per second Concentrically with the Knee flexed at 0 degrees" ("PF" would indicate plantar flexion).

Table M.1

Isokinetic trials data for the ankle (subject SN) under different conditions (the various isokinetic conditions have been explained in the text) to identify the gain. Other parameters that have been estimated are: a) the correlation coefficient (R); b) the coefficient of determination $\left(\mathbf{R}^{2}\right)$; c) the root mean square of the error (RMS); d) the normalized root mean square of the error with respect to the maximum moment in the trial (NRMS_EnvMax); and e) the normalized root mean square of the error with respect to the maximum moment of all trials (NRMS_EnvMax). The average values along with the standard deviations and the medians for each of the parameters have also been computed. 


\begin{tabular}{|l|c|c|c|c|c|c|}
\hline Trial & Gain & $\mathrm{R}$ & $\mathrm{R}^{2}$ & $\mathrm{RMS}$ & NRMS_EnvMax & NRMS_MAX \\
\hline SNKF5CA0 & 24.08 & 0.97 & 0.94 & 4.87 & 8.94 & 3.46 \\
\hline SNKF60CA0 & 61.14 & 0.90 & 0.81 & 4.48 & 5.16 & 3.18 \\
\hline SNKF5CA10P & 72.47 & 0.44 & 0.19 & 12.33 & 8.76 & 8.76 \\
\hline SNKF60CA10P & 76.69 & 0.77 & 0.59 & 9.76 & 9.82 & 6.93 \\
\hline SNKF120CA10P & 59.59 & 0.91 & 0.83 & 15.46 & 17.50 & 10.99 \\
\hline SNKF5CA20P & 64.62 & 0.90 & 0.82 & 12.47 & 10.48 & 8.86 \\
\hline SNKF5CA10D & 61.10 & 0.52 & 0.27 & 10.27 & 8.83 & 7.30 \\
\hline SNKE5CA0 & 46.11 & 0.93 & 0.86 & 14.86 & 11.18 & 7.50 \\
\hline SNKE60CA0 & 45.53 & 0.89 & 0.80 & 17.14 & 19.64 & 8.66 \\
\hline SNKE120CA0 & 38.72 & 0.83 & 0.69 & 19.03 & 24.71 & 9.61 \\
\hline SNKE190CA0 & 24.73 & 0.54 & 0.29 & 18.11 & 36.66 & 9.15 \\
\hline SNKE250CA0 & 40.62 & 0.68 & 0.46 & 16.49 & 28.83 & 8.33 \\
\hline SNKE5CA10P & 52.60 & 0.85 & 0.72 & 32.04 & 16.18 & 16.18 \\
\hline SNKE60CA10P & 32.90 & 0.87 & 0.76 & 20.87 & 16.62 & 10.54 \\
\hline SNKE120CA10P & 32.07 & 0.84 & 0.70 & 24.00 & 18.83 & 12.12 \\
\hline SNKE190CA10P & 26.90 & 0.38 & 0.15 & 24.76 & 26.83 & 12.50 \\
\hline SNKE250CA10P & 17.63 & 0.39 & 0.15 & 15.86 & 25.57 & 8.01 \\
\hline SNKE5CA10D & 44.77 & 0.92 & 0.85 & 7.94 & 12.33 & 4.01 \\
\hline SNKE60CA10D & 40.39 & 0.95 & 0.91 & 4.45 & 12.84 & 2.25 \\
\hline SNKE120CA10D & 31.01 & 0.89 & 0.79 & 7.30 & 18.26 & 3.69 \\
\hline SNKE190CA10D & 23.04 & 0.89 & 0.80 & 13.35 & 21.76 & 6.74 \\
\hline SNKE250CA10D & 19.27 & 0.94 & 0.89 & 4.67 & 9.78 & 2.36 \\
\hline & & & & & & \\
\hline Average & 42.54 & 0.78 & 0.65 & 14.11 & 16.80 & 7.78 \\
\hline Standard & 24.74 & 0.19 & 0.27 & 7.32 & 8.02 & 3.59 \\
\hline Deviation & & & & & & 8.17 \\
\hline Median & 40.50 & 0.88 & 0.77 & 14.10 & 16.40 & \\
\hline 1: An exam0 & 6 & & & & & \\
\hline
\end{tabular}

1: An example of how a knee trial should be read is "Subject $\mathrm{N}$, performing Knee Flexions at an angular velocity of 5 degrees per second Concentrically with the Ankle at 0 degrees" ("P" would indicate plantar flexed, while "D" would indicate dorsi flexed).

Table M.2

Isokinetic trials data for the knee (subject SN) under different conditions (the various isokinetic conditions have been explained in the text) to identify the gain. Other parameters that have been estimated are: a) the correlation coefficient $(\mathbf{R})$; b) the coefficient of determination $\left(\mathbf{R}^{2}\right)$; c) the root mean square of the error (RMS); d) the normalized root mean square of the error with respect to the maximum moment in the trial (NRMS_EnvMax); and e) the normalized root mean square of the error with respect to the maximum moment of all trials (NRMS_EnvMax). The average values along with the standard deviations and the medians for each of the parameters have also been computed. 


\begin{tabular}{|c|c|c|c|c|c|c|}
\hline TRIAL & GAIN & $\mathrm{R}$ & $\overline{\mathrm{R}^{2}}$ & RMS & NRMS_EnvMax & $\overline{\overline{\text { NRMS_MAX }}}$ \\
\hline SADF5CK0 ${ }^{1}$ & 18.45 & 0.80 & 0.64 & 7.48 & 33.12 & 33.12 \\
\hline SADF5CK7 & 10.58 & 0.85 & 0.72 & 4.81 & 32.99 & 21.30 \\
\hline SADF5CK20 & 10.00 & 0.80 & 0.64 & 7.18 & 49.14 & 31.83 \\
\hline SADF5CK51 & 8.08 & 0.45 & 0.20 & 7.13 & 72.26 & 31.59 \\
\hline SADF60CK51 & 8.35 & 0.63 & 0.40 & 7.20 & 62.93 & 31.88 \\
\hline SADF120CK51 & 12.37 & 0.89 & 0.79 & 4.11 & 53.49 & 18.20 \\
\hline SAPF5CK0 & 14.90 & 0.67 & 0.45 & 10.46 & 21.20 & 21.20 \\
\hline SAPF5CK7 & 16.12 & 0.65 & 0.42 & 9.79 & 29.06 & 19.84 \\
\hline SAPF60CK7 & 17.93 & 0.47 & 0.22 & 7.75 & 19.54 & 15.71 \\
\hline $\begin{array}{l}\text { SAPF120CK7 } \\
\end{array}$ & 19.56 & 0.16 & 0.03 & 2.20 & 6.11 & 4.45 \\
\hline SAPF5CK20 & 8.42 & 0.93 & 0.86 & 2.40 & 10.23 & 4.86 \\
\hline SAPF5CK20_2 & 8.77 & 0.87 & 0.76 & 3.35 & 11.13 & 6.79 \\
\hline SAPF30CK20 & 7.37 & 0.55 & 0.30 & 2.64 & 12.01 & 5.36 \\
\hline SAPF60CK20 & 5.81 & 0.39 & 0.15 & 1.43 & 14.17 & 2.89 \\
\hline SAPF90CK20 & 5.97 & 0.95 & 0.90 & 0.51 & 3.84 & 1.04 \\
\hline SAPF120CK20 & 6.14 & 1.00 & 1.00 & 0.56 & 4.05 & 1.13 \\
\hline SAPF5CK51 & 5.83 & 0.85 & 0.72 & 6.34 & 28.74 & 12.86 \\
\hline SAPF60CK51 & 6.97 & 0.83 & 0.69 & 21.04 & 88.03 & 42.66 \\
\hline Average & 10.65 & 0.71 & 0.55 & 5.91 & 30.67 & 17.04 \\
\hline $\begin{array}{c}\text { Standard } \\
\text { Deviation }\end{array}$ & 4.72 & 0.23 & 0.29 & 4.86 & 24.98 & 13.03 \\
\hline Median & 8.60 & 0.80 & 0.64 & 5.58 & 24.97 & 16.96 \\
\hline
\end{tabular}

1: An example of how an ankle trial should be read is "Subject A, performing Dorsi Flexions at an angular velocity of 5 degrees per second Concentrically with the Knee flexed at 0 degrees" ("PF" would indicate plantar flexion). Trial labels that end in _2 indicate that this is the second recorded effort for the specific condition.

Table M.3

Isokinetic trials data for the ankle (subject SA) under different conditions (the various isokinetic conditions have been explained in the text) to identify the gain. Other parameters that have been estimated are: a) the correlation coefficient $(\mathbf{R}) ;$ b) the coefficient of determination $\left(\mathbf{R}^{2}\right)$; c) the root mean square of the error (RMS); d) the normalized root mean square of the error with respect to the maximum moment in the trial (NRMS_EnvMax); e) and the normalized root mean square of the error with respect to the maximum moment of all trials (NRMS_EnvMax). The average values along with the standard deviations and the medians for each of the parameters have also been computed. 


\begin{tabular}{|l|c|c|c|c|c|c|}
\hline TRIAL & GAIN & $\mathrm{R}$ & $\mathrm{R}^{2}$ & RMS & NRMS_EnvMax & NRMS_MAX \\
\hline SAKE5CA0 & 18.27 & 0.86 & 0.73 & 11.02 & 18.25 & 9.52 \\
\hline SAKE60CA0 & $\mathbf{3 5 . 2 7}$ & 0.76 & 0.58 & 15.98 & 16.74 & 13.80 \\
\hline SAKE60CA0_2 & $\mathbf{3 2 . 0 2}$ & 0.44 & 0.20 & 9.52 & 16.89 & 8.22 \\
\hline SAKE120CA0 & 32.84 & 0.82 & 0.66 & 20.37 & 41.70 & 17.59 \\
\hline SAKE190CA0 & $\mathbf{3 9 . 0 5}$ & 0.76 & 0.58 & 2.91 & 6.41 & 2.52 \\
\hline SAKE190CA0_2 & $\mathbf{3 7 . 3 6}$ & 0.98 & 0.96 & 5.09 & 10.85 & 4.39 \\
\hline SAKE190CA0_3 & $\mathbf{4 2 . 3 1}$ & 0.93 & 0.86 & 11.56 & 19.94 & 9.99 \\
\hline SAKE5CA10P & 22.31 & 0.63 & 0.40 & 20.48 & 17.70 & 17.69 \\
\hline SAKE60CA10P & $\mathbf{2 2 . 8 7}$ & 0.38 & 0.14 & 34.52 & 33.48 & 29.82 \\
\hline SAKE60CA10P_2 & $\mathbf{2 2 . 0 9}$ & 0.57 & 0.32 & 29.83 & 25.78 & 25.76 \\
\hline SAKE120CA10P & 20.17 & 0.84 & 0.70 & 12.35 & 12.05 & 10.67 \\
\hline SAKE190CA10P & 18.64 & 0.95 & 0.91 & 4.70 & 7.18 & 4.06 \\
\hline SAKE250CA10P & $\mathbf{2 2 . 4 8}$ & 0.97 & 0.94 & 1.52 & 2.71 & 1.31 \\
\hline SAKE250CA10P_2 & $\mathbf{2 2 . 4 1}$ & 0.97 & 0.94 & 1.52 & 2.70 & 1.31 \\
\hline P2KF5CA0 & 61.79 & 0.80 & 0.64 & 9.93 & 24.79 & 13.79 \\
\hline SAKF120CA0 & 33.99 & 0.75 & 0.57 & 18.86 & 35.10 & 26.20 \\
\hline SAKF5CA10P & 10.66 & 0.26 & 0.07 & 24.44 & 34.00 & 33.95 \\
\hline SAKF120CA10P & 38.70 & 0.33 & 0.11 & 5.96 & 19.88 & 8.28 \\
\hline SAKF190CA10P & 59.30 & 0.73 & 0.53 & 11.39 & 30.06 & 15.82 \\
\hline SAKF5CA20P & 21.93 & 0.60 & 0.36 & 13.47 & 18.97 & 18.70 \\
\hline SAKF5CA10D & 33.89 & 0.40 & 0.16 & 13.38 & 18.78 & 18.59 \\
\hline SAKF60CA10D & 53.22 & 0.81 & 0.66 & 5.43 & 8.45 & 7.55 \\
\hline & & & & & & \\
\hline Average & 35.06 & 0.71 & 0.54 & 12.92 & 19.58 & 13.81 \\
\hline Standard & 21.50 & 0.22 & 0.28 & 8.70 & 10.77 & 9.11 \\
\hline Deviation & & & & & & \\
\hline Median & 32.84 & 0.76 & 0.58 & 11.56 & 18.78 & 13.80 \\
\hline
\end{tabular}

1: An example of reading a knee trial: "Subject A, performing Knee Flexions with angular velocity 5 degrees per second Concentrically with the Ankle at 0 degrees" ("P" and " $\mathrm{D}$ " indicate plantar and dorsi flexed respectively). Trial labels that end in _2 or _3 indicate that this is the second (_2) or third (_3) recorded effort for the specific condition.

Table M.4

Knee isokinetic trials data (subject SA) under different conditions (the various isokinetic conditions have been explained in the text) to identify the gain. Other parameters estimated: a) the correlation coefficient (R); b) the coefficient of determination $\left(\mathbf{R}^{2}\right)$; c) the root mean square of the error (RMS); d) the normalized root mean square of the error with respect to the maximum moment in the trial (NRMS_EnvMax); and e) the normalized root mean square of the error with respect to the maximum moment of all trials (NRMS_EnvMax). The average values along with the standard deviations and the medians for each of the parameters have also been computed. 


\begin{tabular}{|l|c|c|c|c|c|c|}
\hline TRIAL & GAIN & $\mathrm{R}$ & $\mathrm{R}^{2}$ & $\mathrm{RMS}$ & NRMS_EnvMax & NRMS_MAX \\
\hline SCDF5CK0_2 & $\mathbf{3 7 . 8 3}$ & 0.89 & 0.80 & 2.32 & 18.87 & 18.87 \\
\hline SCDF5CK0_3 & $\mathbf{4 3 . 2 6}$ & 0.75 & 0.57 & 4.17 & 38.23 & 33.93 \\
\hline SCDF5CK7 & 64.17 & 0.94 & 0.88 & 1.03 & 9.79 & 8.38 \\
\hline SCDF5CK20 & $\mathbf{2 2 . 9 1}$ & 0.66 & 0.43 & 8.56 & 69.91 & 69.64 \\
\hline SCDF5CK20_2 & $\mathbf{2 3 . 3 1}$ & 0.66 & 0.43 & 4.49 & 37.22 & 36.53 \\
\hline SCDF5CK50 & 12.22 & 0.47 & 0.22 & 1.83 & 35.23 & 14.91 \\
\hline SCDF120CK50 & 15.95 & 0.55 & 0.30 & 0.35 & 27.59 & 2.87 \\
\hline SCPF5CK0 & 15.81 & 0.89 & 0.80 & 6.45 & 11.73 & 11.73 \\
\hline SCPF5CK10 & 23.44 & 0.81 & 0.66 & 8.54 & 24.86 & 15.53 \\
\hline SCPF30CK10 & 20.95 & 0.22 & 0.05 & 12.92 & 23.50 & 23.51 \\
\hline SCPF60CK10 & 18.38 & 0.49 & 0.24 & 3.15 & 7.98 & 5.73 \\
\hline SCPF90CK10 & 20.89 & 0.23 & 0.05 & 3.05 & 8.51 & 5.55 \\
\hline SCPF120CK10 & 23.26 & 0.38 & 0.14 & 1.43 & 3.35 & 2.60 \\
\hline SCPF5CK20 & 24.14 & 0.48 & 0.23 & 6.51 & 17.14 & 11.85 \\
\hline SCPF30CK20 & 19.31 & 0.63 & 0.40 & 4.09 & 15.29 & 7.44 \\
\hline SCPF60CK20 & 15.56 & 0.96 & 0.91 & 1.66 & 4.48 & 3.02 \\
\hline SCPF90CK20 & $\mathbf{1 5 . 7 1}$ & 0.98 & 0.96 & 2.30 & 7.39 & 4.18 \\
\hline SCPF90CK20_2 & $\mathbf{1 3 . 1 9}$ & 0.88 & 0.77 & 1.31 & 5.81 & 2.38 \\
\hline SCPF120CK20 & 16.50 & 1.00 & 1.00 & 0.86 & 3.22 & 1.56 \\
\hline SCPF5CK50 & 18.36 & 0.95 & 0.89 & 4.41 & 12.94 & 8.03 \\
\hline SCPF60CK50 & $\mathbf{2 5 . 0 1}$ & 0.99 & 0.97 & 3.62 & 9.93 & 6.58 \\
\hline SCPF60CK50_2 & $\mathbf{2 2 . 1 2}$ & 0.98 & 0.96 & 3.49 & 9.40 & 6.35 \\
\hline SCPF120CK50 & 20.89 & 0.68 & 0.46 & 2.66 & 16.54 & 4.84 \\
\hline & & & & & & \\
\hline Average & 23.18 & 0.72 & 0.57 & 3.88 & 18.21 & 13.31 \\
\hline $\begin{array}{l}\text { Standard } \\
\text { Deviation }\end{array}$ & 11.41 & 0.25 & 0.33 & 3.01 & 15.50 & 15.56 \\
\hline Median & 20.89 & 0.75 & 0.57 & 3.15 & 12.94 & 7.44 \\
\hline 1:An_xa & & & & & & \\
\hline
\end{tabular}

1: An example of reading an ankle trial: "Subject C, performing Dorsi Flexions with angular velocity 5 degrees per second Concentrically with the Knee flexed at 0 degrees" ("PF" would indicate plantar flexion). Trial labels that end in _2 indicate that this is the second recorded effort for the specific condition.

Table M.5

Ankle isokinetic trials data (subject SC) under different conditions (see text) to identify the gain. Other parameters estimated: a) correlation coefficient $(\mathbf{R})$; b) coefficient of determination $\left(\mathbf{R}^{2}\right)$; c) root mean square of the error (RMS); d) normalized root mean square of the error with respect to the maximum moment in the trial (NRMS_EnvMax); e) and normalized root mean square of the error with respect to the maximum moment of all trials (NRMS_EnvMax). The average values along with the standard deviations and the medians for each of the parameters have also been computed. 


\begin{tabular}{|l|c|c|c|c|c|c|}
\hline TRIAL & GAIN & $\mathrm{R}$ & $\mathrm{R}^{2}$ & RMS & NRMS_EnvMax & NRMS_MAX \\
\hline SCKE5CA0 & 13.29 & 0.96 & 0.92 & 9.42 & 8.43 & 7.38 \\
\hline SCKE60CA0 & 16.68 & 0.94 & 0.88 & 14.55 & 13.16 & 11.39 \\
\hline SCKE120CA0 & 17.50 & 0.96 & 0.93 & 2.59 & 4.52 & 2.03 \\
\hline SCKE5CA10P & 66.98 & 0.72 & 0.52 & 10.21 & 7.99 & 7.99 \\
\hline SCKE60CA10P & 15.04 & 0.39 & 0.15 & 29.83 & 24.17 & 23.36 \\
\hline SCKE120CA10P & 15.29 & 0.30 & 0.09 & 33.65 & 26.56 & 26.35 \\
\hline SCKE190CA10P & 14.22 & 0.07 & 0.00 & 18.24 & 21.22 & 14.29 \\
\hline SCKE250CA10P & 15.24 & 0.95 & 0.91 & 2.69 & 4.10 & 2.10 \\
\hline SCKE5CA10D & 54.54 & 0.85 & 0.72 & 11.62 & 12.97 & 9.10 \\
\hline SCKE60CA10D & 30.97 & 0.12 & 0.02 & 5.45 & 7.94 & 4.27 \\
\hline SCKE190CA10D & 20.94 & 0.82 & 0.68 & 20.98 & 29.91 & 16.43 \\
\hline SCKF5CA0 & $\mathbf{3 3 . 9 8}$ & 0.73 & 0.54 & 8.38 & 8.31 & 6.84 \\
\hline SCKF5CA10P_2 & $\mathbf{3 5 . 6 3}$ & 0.92 & 0.85 & 5.59 & 4.56 & 4.56 \\
\hline SCKF60CA10P & 63.64 & 0.24 & 0.06 & 10.71 & 9.06 & 8.74 \\
\hline SCKF5CA20P & 39.02 & 0.66 & 0.44 & 6.60 & 6.18 & 5.38 \\
\hline SCKF60CA20P & 45.45 & 0.95 & 0.90 & 1.99 & 1.63 & 1.63 \\
\hline SCKF5CA10D & 43.36 & 0.65 & 0.42 & 14.43 & 15.23 & 11.78 \\
\hline SCKF60CA10D & 45.41 & 0.87 & 0.76 & 3.04 & 2.78 & 2.48 \\
\hline & & & & & & \\
\hline Average & 32.62 & 0.67 & 0.54 & 11.67 & 11.59 & 9.23 \\
\hline $\begin{array}{c}\text { Standard } \\
\text { Deviation }\end{array}$ & 20.76 & 0.31 & 0.35 & 9.11 & 8.55 & 7.11 \\
\hline & & & & & & \\
\hline Median & 32.48 & 0.78 & 0.61 & 9.81 & 8.37 & 7.69 \\
\hline
\end{tabular}

1: An example of how a knee trial should be read is "Subject C, performing Knee Flexions at an angular velocity of 5 degrees per second Concentrically with the Ankle at 0 degrees" ("P" would indicate plantar flexed, while " $\mathrm{D}$ " would indicate dorsi flexed). Trial labels that end in _ 2 or $\_3$ indicate that this is the second recorded effort for the specific condition.

\section{Table M.6}

Isokinetic trials data for the knee (subject SC) under different conditions (the various isokinetic conditions have been explained in the text) to identify the gain. Other parameters that have been estimated are: a) the correlation coefficient (R); b) the coefficient of determination $\left(\mathbf{R}^{2}\right)$; c) the root mean square of the error (RMS); d) the normalized root mean square of the error with respect to the maximum moment in the trial (NRMS_EnvMax); e) and the normalized root mean square of the error with respect to the maximum moment of all trials (NRMS_EnvMax). The average values along with the standard deviations and the medians for each of the parameters have also been computed. 


\section{APPENDIX N}

GAIT KNEE AND ANKLE RESULTS 


\begin{tabular}{|l|c|c|c|c|c|c|}
\hline \multicolumn{1}{|c|}{ Trial } & Gain & $\mathrm{R}$ & $\mathrm{R}^{2}$ & RMS & NRMS_MOT & NRMS_KC \\
\hline SA004_GLOB & 23.00 & 0.85 & 0.72 & 44.11 & & \\
\hline SA005_GLOB & 23.00 & 0.84 & 0.70 & 40.48 & & \\
\hline SA008_GLOB & 23.00 & 0.92 & 0.84 & 38.23 & & \\
\hline SA004_GLOBK & 23.00 & 0.90 & 0.80 & 12.74 & 22.76 & 11.01 \\
\hline SA005_GLOBK & 23.00 & 0.96 & 0.92 & 7.29 & 13.06 & 6.29 \\
\hline SA008_GLOBK & 23.00 & 0.94 & 0.88 & 9.92 & 21.62 & 8.57 \\
\hline SA004_GLOBA & 23.00 & 0.93 & 0.87 & 61.06 & 73.75 & 123.79 \\
\hline SA005_GLOBA & 23.00 & 0.87 & 0.75 & 58.09 & 67.28 & 117.77 \\
\hline SA008_GLOBA & 23.00 & 0.96 & 0.92 & 53.57 & 60.67 & 108.60 \\
\hline & & & & & & \\
\hline Average & 23.00 & 0.91 & 0.82 & 36.17 & 43.19 & 62.67 \\
\hline Standard Deviation & 0.00 & 0.05 & 0.08 & 21.10 & 26.87 & 59.42 \\
\hline
\end{tabular}

Table N.1

Model validation results for the EMG-driven model for subject SA. The validation parameters included the global gain identified from the calibration trials, the correlation coefficients $(\mathbf{R})$, the coefficient of determination $\left(\mathbf{R}^{2}\right)$, the root mean square of the error (RMS), the RMS expressed relative to the respective joint maximum measured moment in the envelope (NRMS_MOT), and the RMS expressed relative to the respective joint maximum measured moment from the calibration trials (NRMS_KC).

\begin{tabular}{|c|c|c|c|c|c|c|}
\hline Trial & GainK & RK & $\mathrm{R}^{2} \mathrm{~K}$ & RMSK & NRMSK_Mot & NRMSK_KC \\
\hline SA004_MOT & 35.06 & 0.90 & 0.80 & 20.94 & 37.40 & 18.09 \\
\hline SA005_MOT & 35.06 & 0.96 & 0.91 & 17.37 & 31.13 & 15.00 \\
\hline SA008_MOT & 35.06 & 0.94 & 0.88 & 22.27 & 48.52 & 19.24 \\
\hline & & & & & & \\
\hline Average & 35.06 & 0.93 & 0.87 & 20.20 & 39.02 & 17.44 \\
\hline Standard Deviation & 0.00 & 0.03 & 0.06 & 2.53 & 8.81 & 2.19 \\
\hline
\end{tabular}

Table N.2

Model validation results for the EMG-driven model for subject SA. The validation parameters included the correlation coefficients $(\mathbf{R})$, the coefficient of determination $\left(\mathbf{R}^{2}\right)$, the root mean square of the error (RMSK), the RMSK expressed relative to the respective joint maximum measured moment in the envelope (NRMSK_Mot), and the RMSK expressed relative to the respective joint maximum measured moment from the calibration trials (NRMSK_KC). 


\begin{tabular}{|c|c|c|c|c|c|c|}
\hline Trial & GainA & RA & R $^{2}$ A & RMSA & NRMSA_Mot & NRMSA_KC \\
\hline SA004_MOT & 10.65 & 0.93 & 0.87 & 13.11 & 15.83 & 26.58 \\
\hline SA005_MOT & 10.65 & 0.87 & 0.75 & 17.05 & 19.75 & 34.57 \\
\hline SA008_MOT & 10.65 & 0.96 & 0.92 & 9.18 & 10.40 & 18.62 \\
\hline & & & & & & \\
\hline Average & 10.00 & 0.92 & 0.85 & 13.11 & 15.33 & 26.59 \\
\hline Standard Deviation & 0.00 & 0.05 & 0.09 & 3.93 & 4.69 & 7.98 \\
\hline
\end{tabular}

Table N.3

Model validation results for the EMG-driven model for subject SA. The validation parameters included the correlation coefficients $(\mathbf{R})$, the coefficient of determination $\left(\mathbf{R}^{2}\right)$, the root mean square of the error (RMSA), the RMSA expressed relative to the respective joint maximum measured moment in the envelope (NRMSA_Mot), and the RMSA expressed relative to the respective joint maximum measured moment from the calibration trials (NRMSA_KC). The gain implemented was identified from the calibration trials.

\begin{tabular}{|l|c|c|c|c|c|c|}
\hline \multicolumn{1}{|c|}{ Trial } & Gain & $\mathrm{R}$ & $\mathrm{R}^{2}$ & $\mathrm{RMS}$ & NRMS_MOT & NRMS_KC \\
\hline SC003_GLOB & 27.50 & 0.89 & 0.79 & 16.17 & & \\
\hline SC004_GLOB & 27.50 & 0.80 & 0.64 & 20.39 & & \\
\hline SC005_GLOB & 27.50 & 0.80 & 0.64 & 20.20 & & \\
\hline SC003_GLOBK & 27.50 & 0.82 & 0.68 & 12.66 & 18.13 & 9.91 \\
\hline SC004_GLOBK & 27.50 & 0.93 & 0.87 & 7.81 & 16.73 & 6.12 \\
\hline SC005_GLOBK & 27.50 & 0.90 & 0.81 & 10.95 & 24.78 & 8.58 \\
\hline SC003_GLOBA & 27.50 & 0.97 & 0.94 & 7.52 & 6.30 & 13.69 \\
\hline SC004_GLOBA & 27.50 & 0.88 & 0.78 & 20.94 & 17.38 & 38.10 \\
\hline SC005_GLOBA & 27.50 & 0.89 & 0.79 & 19.98 & 17.84 & 36.35 \\
\hline \multicolumn{1}{|c|}{} & & & & & & 18.79 \\
\hline Average & 27.50 & 0.88 & 0.77 & 15.18 & 16.86 & 14.50 \\
\hline Standard Deviation & 0.00 & 0.06 & 0.10 & 5.55 & 5.95 & 19 \\
\hline
\end{tabular}

Table N.4

Model validation results for the EMG-driven model for subject SC. The validation parameters included the global gain identified from the calibration trials, the correlation coefficients (R), the coefficient of determination $\left(\mathbf{R}^{2}\right)$, the root mean square of the error (RMS), the RMS expressed relative to the respective joint maximum measured moment in the envelope (NRMS_MOT), and the RMS expressed relative to the respective joint maximum measured moment from the calibration trials (NRMS_KC). 


\begin{tabular}{|c|c|c|c|c|c|c|}
\hline Trial & GainK & RK & $\mathrm{R}^{2} \mathrm{~K}$ & RMSK & NRMSK_Mot & NRMSK_KC \\
\hline SC003_MOT & 32.17 & 0.82 & 0.68 & 14.51 & 20.78 & 11.36 \\
\hline SC004_MOT & 32.17 & 0.93 & 0.87 & 10.64 & 22.79 & 8.33 \\
\hline SC005_MOT & 32.17 & 0.90 & 0.81 & 13.81 & 31.27 & 10.82 \\
\hline & & & & & & \\
\hline Average & 32.17 & 0.89 & 0.79 & 12.99 & 24.95 & 10.17 \\
\hline Standard Deviation & 0.00 & 0.06 & 0.10 & 2.06 & 5.57 & 1.61 \\
\hline
\end{tabular}

Table N.5

Model validation results for the EMG-driven model for subject SC. The validation parameters included the correlation coefficients $(\mathbf{R})$, the coefficient of determination $\left(\mathbf{R}^{2}\right)$, the root mean square of the error (RMSK), the RMSK expressed relative to the respective joint maximum measured moment in the envelope (NRMSK_Mot), and the RMSK expressed relative to the respective joint maximum measured moment from the calibration trials (NRMSK_KC). The gain implemented was identified from the calibration trials.

\begin{tabular}{|c|c|c|c|c|c|c|}
\hline Trial & GainA & RA & $\mathrm{R}^{2}$ A & RMSA & NRMSA_Mot & NRMSA_KC \\
\hline SC003_MOT & 23.18 & 0.97 & 0.94 & 6.98 & 5.85 & 12.70 \\
\hline SC004_MOT & 23.18 & 0.88 & 0.78 & 21.48 & 17.83 & 39.08 \\
\hline SC005_MOT & 23.18 & 0.89 & 0.79 & 20.67 & 18.45 & 37.60 \\
\hline & & & & & & \\
\hline Average & 23.18 & 0.91 & 0.84 & 16.38 & 14.04 & 29.79 \\
\hline Standard Deviation & 0.00 & 0.05 & 0.09 & 8.15 & 7.10 & 14.82 \\
\hline
\end{tabular}

Table N.6

Model validation results for the EMG-driven model for subject SC. The validation parameters included the correlation coefficients $(\mathbf{R})$, the coefficient of determination $\left(\mathbf{R}^{2}\right)$, the root mean square of the error (RMSA), the RMSA expressed relative to the respective joint maximum measured moment in the envelope (NRMSA_Mot), and the RMSA expressed relative to the respective joint maximum measured moment from the calibration trials (NRMSA_KC). The gain implemented was identified from the calibration trials. 


\begin{tabular}{|l|c|c|c|c|c|c|}
\hline \multicolumn{1}{|c|}{ Trial } & Gain & $\mathrm{R}$ & $\mathrm{R}^{2}$ & RMS & NRMS_MOT & NRMS_KC \\
\hline SNO02_GLOB & 28.00 & 0.90 & 0.81 & 47.17 & & \\
\hline SN004_GLOB & 28.00 & 0.84 & 0.71 & 50.72 & & \\
\hline SN005_GLOB & 28.00 & 0.86 & 0.75 & 36.27 & & \\
\hline SN002_GLOBK & 28.00 & 0.81 & 0.66 & 12.33 & 29.28 & 6.23 \\
\hline SN004_GLOBK & 28.00 & 0.79 & 0.62 & 19.12 & 36.78 & 9.66 \\
\hline SN005_GLOBK & 28.00 & 0.88 & 0.77 & 13.76 & 25.96 & 6.95 \\
\hline SN002_GLOBA & 28.00 & 0.93 & 0.86 & 68.04 & 56.70 & 128.99 \\
\hline SN004_GLOBA & 28.00 & 0.89 & 0.78 & 71.39 & 58.52 & 135.36 \\
\hline SN005_GLOBA & 28.00 & 0.90 & 0.82 & 49.42 & 38.61 & 93.69 \\
\hline & & & & & & \\
\hline Average & 28.00 & 0.87 & 0.75 & 40.91 & 40.97 & 63.48 \\
\hline Standard Deviation & 0.00 & 0.04 & 0.08 & 22.15 & 13.71 & 62.84 \\
\hline
\end{tabular}

Table N.7

Model validation results for the EMG-driven model for subject SN. The validation parameters included the global gain identified from the calibration trials, the correlation coefficients $(\mathbf{R})$, the coefficient of determination $\left(\mathbf{R}^{2}\right)$, the root mean square of the error (RMS), the RMS expressed relative to the respective joint maximum measured moment in the envelope (NRMS_MOT), and the RMS expressed relative to the respective joint maximum measured moment from the calibration trials (NRMS_KC).

\begin{tabular}{|c|c|c|c|c|c|c|}
\hline Trial & GainK & RK & $\mathrm{R}^{2} \mathrm{~K}$ & RMSK & NRMSK_Mot & NRMSK_KC \\
\hline SN002_MOT & 42.00 & 0.81 & 0.66 & 20.18 & 47.92 & 10.19 \\
\hline SN004_MOT & 42.00 & 0.78 & 0.61 & 29.04 & 55.84 & 14.66 \\
\hline SN005_MOT & 42.00 & 0.88 & 0.77 & 17.48 & 32.98 & 8.83 \\
\hline & & & & & & \\
\hline Average & 42.00 & 0.82 & 0.68 & 22.23 & 45.58 & 11.23 \\
\hline Standard Deviation & 0.00 & 0.05 & 0.08 & 6.05 & 11.61 & 3.05 \\
\hline
\end{tabular}

Table N.8

Model validation results for the EMG-driven model for subject SN. The validation parameters included the correlation coefficients (R), the coefficient of determination $\left(\mathbf{R}^{2}\right)$, the root mean square of the error (RMSK), the RMSK expressed relative to the respective joint maximum measured moment in the envelope (NRMSK_Mot), and the RMSK expressed relative to the respective joint maximum measured moment from the calibration trials (NRMSK_KC). The gain implemented was identified from the calibration trials. 


\begin{tabular}{|c|c|c|c|c|c|c|}
\hline Trial & GainA & RA & R $^{2} \mathrm{~A}$ & RMSA & NRMSA_Mot & NRMSA_KC \\
\hline SN002_MOT & 14.00 & 0.93 & 0.86 & 18.20 & 15.17 & 34.51 \\
\hline SN004_MOT & 14.00 & 0.89 & 0.78 & 22.15 & 18.16 & 42.00 \\
\hline SN005_MOT & 14.00 & 0.90 & 0.82 & 17.55 & 13.71 & 33.28 \\
\hline & & & & & & \\
\hline Average & 14.00 & 0.91 & 0.82 & 19.30 & 15.68 & 36.60 \\
\hline Standard Deviation & 0.00 & 0.02 & 0.04 & 2.49 & 2.27 & 4.72 \\
\hline
\end{tabular}

Table N.9

Model validation results for the EMG-driven model for subject SN. The validation parameters included the correlation coefficients $(\mathbf{R})$, the coefficient of determination $\left(\mathbf{R}^{2}\right)$, the root mean square of the error (RMSA), the RMSA expressed relative to the respective joint maximum measured moment in the envelope (NRMSA_Mot), and the RMSA expressed relative to the respective joint maximum measured moment from the calibration trials (NRMSA_KC). The gain implemented was identified from the calibration trials. 


\section{APPENDIX O}

OPTIMUM GAIT KNEE AND ANKLE RESULTS 


\begin{tabular}{|l|c|c|c|c|c|c|}
\hline \multicolumn{1}{|c|}{ Trial } & Gain & $\mathrm{R}$ & $\mathrm{R}^{2}$ & RMS & NRMS_MOT & NRMS_KC \\
\hline SA004_GLOB & 8.51 & 0.85 & 0.72 & 26.29 & & \\
\hline SA005_GLOB & 8.81 & 0.84 & 0.70 & 25.28 & & \\
\hline SA008_GLOB & 9.20 & 0.92 & 0.84 & 24.19 & & \\
\hline SA004_GLOBK & 8.51 & 0.90 & 0.80 & 17.31 & 30.92 & 14.95 \\
\hline SA005_GLOBK & 8.81 & 0.96 & 0.92 & 12.97 & 23.24 & 11.20 \\
\hline SA008_GLOBK & 9.20 & 0.94 & 0.88 & 10.79 & 23.51 & 9.32 \\
\hline SA004_GLOBA & 8.51 & 0.93 & 0.87 & 12.70 & 15.34 & 25.76 \\
\hline SA005_GLOBA & 8.81 & 0.87 & 0.75 & 15.58 & 18.05 & 31.59 \\
\hline SA008_GLOBA & 9.20 & 0.96 & 0.92 & 9.88 & 11.19 & 20.03 \\
\hline & & & & & & \\
\hline Average & 8.84 & 0.91 & 0.82 & 17.22 & 20.37 & 18.81 \\
\hline Standard Deviation & 0.30 & 0.05 & 0.08 & 6.44 & 6.99 & 8.67 \\
\hline
\end{tabular}

Table O.1

Model validation results for the EMG-driven model for subject SA. The validation parameters included the optimum global gain, the correlation coefficients $(\mathbf{R})$, the coefficient of determination $\left(\mathbf{R}^{2}\right)$, the root mean square of the error (RMS), the RMS expressed relative to the respective joint maximum measured moment in the envelope (NRMS_MOT), and the RMS expressed relative to the respective joint maximum measured moment from the calibration trials (NRMS_KC).

\begin{tabular}{|c|c|c|c|c|c|c|}
\hline Trial & GainK & RK & R $^{2} \mathrm{~K}$ & RMSK & NRMSK_Mot & NRMSK_KC \\
\hline SA004_MOT & 14.17 & 0.90 & 0.80 & 15.94 & 28.46 & 13.76 \\
\hline SA005_MOT & 12.20 & 0.96 & 0.91 & 12.41 & 22.23 & 10.71 \\
\hline SA008_MOT & 11.71 & 0.94 & 0.88 & 10.38 & 22.62 & 8.97 \\
\hline & & & & & & \\
\hline Average & 12.69 & 0.93 & 0.87 & 12.91 & 24.44 & 11.15 \\
\hline Standard Deviation & 1.30 & 0.03 & 0.06 & 2.81 & 3.49 & 2.43 \\
\hline
\end{tabular}

Table 0.2

Model validation results for the EMG-driven model for subject SA. The validation parameters included the optimum knee gain, the correlation coefficients $(\mathbf{R})$, the coefficient of determination $\left(\mathbf{R}^{2}\right)$, the root mean square of the error (RMSK), the RMSK expressed relative to the respective joint maximum measured moment in the envelope (NRMSK_Mot), and the RMSK expressed relative to the respective joint maximum measured moment from the calibration trials (NRMSK_KC). 


\begin{tabular}{|c|c|c|c|c|c|c|}
\hline Trial & GainA & RA & $\mathrm{R}^{2} \mathrm{~A}$ & RMSA & NRMSA_Mot & NRMSA_KC \\
\hline SA004_MOT & 7.97 & 0.93 & 0.87 & 12.53 & 15.13 & 25.40 \\
\hline SA005_MOT & 8.49 & 0.87 & 0.75 & 15.54 & 17.99 & 31.50 \\
\hline SA008_MOT & 8.93 & 0.96 & 0.92 & 9.84 & 11.14 & 19.94 \\
\hline & & & & & & \\
\hline Average & 8.46 & 0.92 & 0.85 & 12.63 & 14.76 & 25.61 \\
\hline Standard Deviation & 0.48 & 0.05 & 0.09 & 2.85 & 3.44 & 5.78 \\
\hline
\end{tabular}

Table 0.3

Model validation results for the EMG-driven model for subject SA. The validation parameters included the optimum ankle gain, the correlation coefficients (R), the coefficient of determination $\left(\mathbf{R}^{2}\right)$, the root mean square of the error (RMSA), the RMSA expressed relative to the respective joint maximum measured moment in the envelope (NRMSA_Mot), and the RMSA expressed relative to the respective joint maximum measured moment from the calibration trials (NRMSA_KC).

\begin{tabular}{|l|c|c|c|c|c|c|}
\hline \multicolumn{1}{|c|}{ Trial } & Gain & $\mathrm{R}$ & $\mathrm{R}^{2}$ & RMS & NRMS_MOT & NRMS_KC \\
\hline SC003_GLOB & 21.89 & 0.89 & 0.79 & 32.22 & & \\
\hline SC004_GLOB & 21.39 & 0.80 & 0.64 & 32.67 & & \\
\hline SC005_GLOB & 21.43 & 0.80 & 0.64 & 32.56 & & \\
\hline SC003_GLOBK & 21.89 & 0.82 & 0.68 & 17.70 & 25.36 & 13.86 \\
\hline SC004_GLOBK & 21.39 & 0.93 & 0.87 & 14.76 & 31.61 & 11.56 \\
\hline SC005_GLOBK & 21.43 & 0.90 & 0.81 & 14.94 & 33.82 & 11.70 \\
\hline SC003_GLOBA & 21.89 & 0.97 & 0.94 & 9.46 & 7.93 & 17.22 \\
\hline SC004_GLOBA & 21.39 & 0.88 & 0.78 & 22.05 & 18.30 & 40.11 \\
\hline SC005_GLOBA & 21.43 & 0.89 & 0.79 & 21.61 & 19.29 & 39.32 \\
\hline & & & & & & \\
\hline Average & 21.57 & 0.88 & 0.77 & 22.00 & 22.72 & 22.30 \\
\hline Standard Deviation & 0.24 & 0.06 & 0.10 & 8.72 & 9.58 & 13.65 \\
\hline
\end{tabular}

Table 0.4

Model validation results for the EMG-driven model for subject SC. The validation parameters included the optimum global gain, the correlation coefficients $(\mathbf{R})$, the coefficient of determination $\left(\mathbf{R}^{2}\right)$, the root mean square of the error (RMS), the RMS expressed relative to the respective joint maximum measured moment in the envelope (NRMS_MOT), and the RMS expressed relative to the respective joint maximum measured moment from the calibration trials (NRMS_KC). 


\begin{tabular}{|c|c|c|c|c|c|c|}
\hline Trial & GainK & RK & $\mathrm{R}^{2} \mathrm{~K}$ & RMSK & NRMSK_Mot & NRMSK_KC \\
\hline SC003_MOT & 12.81 & 0.82 & 0.68 & 15.55 & 22.28 & 12.18 \\
\hline SC004_MOT & 10.83 & 0.93 & 0.87 & 11.07 & 23.70 & 8.67 \\
\hline SC005_MOT & 10.12 & 0.90 & 0.81 & 10.98 & 24.85 & 8.60 \\
\hline & & & & & & \\
\hline Average & 11.25 & 0.89 & 0.79 & 12.53 & 23.61 & 9.82 \\
\hline Standard Deviation & 1.40 & 0.06 & 0.10 & 2.62 & 1.28 & 2.05 \\
\hline
\end{tabular}

Table 0.5

Model validation results for the EMG-driven model for subject SC. The validation parameters included the optimum knee gain, the correlation coefficients $(\mathbf{R})$, the coefficient of determination $\left(\mathbf{R}^{2}\right)$, the root mean square of the error (RMSK), the RMSK expressed relative to the respective joint maximum measured moment in the envelope (NRMSK_Mot), and the RMSK expressed relative to the respective joint maximum measured moment from the calibration trials (NRMSK_KC).

\begin{tabular}{|c|c|c|c|c|c|c|}
\hline Trial & GainA & RA & $\mathrm{R}^{2} \mathrm{~A}$ & RMSA & NRMSA_Mot & NRMSA_KC \\
\hline SC003_MOT & 24.57 & 0.97 & 0.94 & 8.27 & 6.93 & 15.04 \\
\hline SC004_MOT & 24.88 & 0.88 & 0.78 & 21.32 & 17.70 & 38.80 \\
\hline SC005_MOT & 24.89 & 0.89 & 0.79 & 20.87 & 18.63 & 37.97 \\
\hline & & & & & & \\
\hline Average & 24.78 & 0.91 & 0.84 & 16.82 & 14.42 & 30.60 \\
\hline Standard Deviation & 0.18 & 0.05 & 0.09 & 7.41 & 6.51 & 13.48 \\
\hline
\end{tabular}

Table 0.6

Model validation results for the EMG-driven model for subject SC. The validation parameters included the optimum ankle gain, the correlation coefficients $(\mathbf{R})$, the coefficient of determination $\left(\mathbf{R}^{2}\right)$, the root mean square of the error (RMSA), the RMSA expressed relative to the respective joint maximum measured moment in the envelope (NRMSA_Mot), and the RMSA expressed relative to the respective joint maximum measured moment from the calibration trials (NRMSA_KC). 


\begin{tabular}{|l|c|c|c|c|c|c|}
\hline \multicolumn{1}{|c|}{ Trial } & Gain & $\mathrm{R}$ & $\mathrm{R}^{2}$ & RMS & NRMS_MOT & NRMS_KC \\
\hline SN002_GLOB & 10.81 & 0.90 & 0.81 & 29.04 & & \\
\hline SN004_GLOB & 10.67 & 0.84 & 0.71 & 31.70 & & \\
\hline SNO05_GLOB & 13.60 & 0.86 & 0.75 & 32.76 & & \\
\hline SN002_GLOBK & 10.81 & 0.81 & 0.66 & 11.85 & 28.15 & 5.98 \\
\hline SN004_GLOBK & 10.67 & 0.79 & 0.62 & 15.62 & 30.03 & 7.89 \\
\hline SN005_GLOBK & 13.60 & 0.88 & 0.77 & 12.57 & 23.72 & 6.35 \\
\hline SN002_GLOBA & 10.81 & 0.93 & 0.86 & 14.49 & 12.07 & 27.47 \\
\hline SN004_GLOBA & 10.67 & 0.89 & 0.78 & 18.70 & 15.33 & 35.46 \\
\hline SNO05_GLOBA & 13.60 & 0.90 & 0.82 & 19.86 & 15.52 & 37.66 \\
\hline & & & & & & \\
\hline Average & 11.70 & 0.87 & 0.75 & 20.73 & 20.80 & 20.13 \\
\hline Standard Deviation & 1.43 & 0.04 & 0.08 & 8.29 & 7.51 & 15.07 \\
\hline
\end{tabular}

Table 0.7

Model validation results for the EMG-driven model for subject SN. The validation parameters included the optimum global gain, the correlation coefficients $(\mathbf{R})$, the coefficient of determination $\left(\mathbf{R}^{2}\right)$, the root mean square of the error (RMS), the RMS expressed relative to the respective joint maximum measured moment in the envelope (NRMS_MOT), and the RMS expressed relative to the respective joint maximum measured moment from the calibration trials (NRMS_KC).

\begin{tabular}{|c|c|c|c|c|c|c|}
\hline Trial & GainK & RK & $\mathrm{R}^{2} \mathrm{~K}$ & RMSK & NRMSK_Mot & NRMSK_KC \\
\hline SN002_MOT & 16.50 & 0.81 & 0.66 & 11.02 & 26.19 & 5.57 \\
\hline SN004_MOT & 15.31 & 0.78 & 0.61 & 15.03 & 28.90 & 7.59 \\
\hline SN005_MOT & 19.05 & 0.88 & 0.77 & 11.82 & 22.29 & 5.97 \\
\hline & & & & & & \\
\hline Average & 16.96 & 0.82 & 0.68 & 12.62 & 25.79 & 6.37 \\
\hline Standard Deviation & 1.91 & 0.05 & 0.08 & 2.12 & 3.32 & 1.07 \\
\hline
\end{tabular}

Table 0.8

Model validation results for the EMG-driven model for subject SN. The validation parameters included the optimum knee gain, the correlation coefficients $(\mathbf{R})$, the coefficient of determination $\left(\mathbf{R}^{2}\right)$, the root mean square of the error (RMS), the RMS expressed relative to the respective joint maximum measured moment in the envelope (NRMS_MOT), and the RMS expressed relative to the respective joint maximum measured moment from the calibration trials (NRMS_KC). 


\begin{tabular}{|c|c|c|c|c|c|c|}
\hline Trial & GainA & RA & $\mathrm{R}^{2} \mathrm{~A}$ & RMSA & NRMSA_Mot & NRMSA_KC \\
\hline SN002_MOT & 10.56 & 0.93 & 0.86 & 14.46 & 12.05 & 27.42 \\
\hline SN004_MOT & 10.39 & 0.89 & 0.78 & 18.67 & 15.31 & 35.40 \\
\hline SN005_MOT & 13.25 & 0.90 & 0.82 & 19.83 & 15.49 & 37.60 \\
\hline & & & & & & \\
\hline Average & 11.40 & 0.91 & 0.82 & 17.65 & 14.28 & 33.47 \\
\hline Standard Deviation & 1.60 & 0.02 & 0.04 & 2.83 & 1.94 & 5.36 \\
\hline
\end{tabular}

Table 0.9

Model validation results for the EMG-driven model for subject SN. The validation parameters included the optimum ankle gain, the correlation coefficients (R), the coefficient of determination $\left(\mathbf{R}^{2}\right)$, the root mean square of the error (RMS), the RMS expressed relative to the respective joint maximum measured moment in the envelope (NRMS_MOT), and the RMS expressed relative to the respective joint maximum measured moment from the calibration trials (NRMS_KC). 
APPENDIX P

COMPARISON OF THE COCONTRACTION METHODS 


\begin{tabular}{|c|c|c|c|c|c|c|}
\hline ANKLE & I vs. II & I vs. II & I vs. III & I vs. III & II vs. III & II vs. III \\
\hline MOTION & $\mathrm{t}$ & $p$ & $\mathrm{t}$ & $\mathrm{p}$ & $\mathrm{t}$ & $p$ \\
\hline SA004 & 1.15 & 0.25 & 0.05 & 0.96 & 0.32 & 0.75 \\
\hline SA005 & 2.09 & 0.04 & 0.18 & 0.86 & 0.83 & 0.41 \\
\hline SA008 & 1.00 & 0.32 & 3.76 & 0.00 & 3.71 & 0.00 \\
\hline \multicolumn{7}{|l|}{ KIN_COM } \\
\hline $\mathrm{d} 5 \mathrm{c} 0^{1}$ & 13.38 & 0.00 & 12.32 & 0.00 & 12.09 & 0.00 \\
\hline $\mathrm{d} 5 \mathrm{c} 7$ & 11.63 & 0.00 & 10.97 & 0.00 & 10.80 & 0.00 \\
\hline $\mathrm{d} 5 \mathrm{c} 20$ & 9.34 & 0.00 & 6.36 & 0.00 & 5.81 & 0.00 \\
\hline $\mathrm{d} 5 \mathrm{c} 51$ & 11.64 & 0.00 & 0.67 & 0.51 & 4.39 & 0.00 \\
\hline $\mathrm{d} 60 \mathrm{c} 51$ & 5.12 & 0.00 & 2.55 & 0.02 & 4.15 & 0.00 \\
\hline d120c51 & 13.86 & 0.00 & 5.31 & 0.00 & 3.46 & 0.00 \\
\hline $\mathrm{p} 5 \mathrm{c} 0$ & 4.09 & 0.00 & 23.45 & 0.00 & 16.56 & 0.00 \\
\hline $\mathrm{p} 5 \mathrm{c} 7$ & 4.91 & 0.00 & 16.88 & 0.00 & 12.00 & 0.00 \\
\hline p60c7 & 12.76 & 0.00 & 23.15 & 0.00 & 15.65 & 0.00 \\
\hline $\mathrm{p} 120 \mathrm{c} 7$ & 34.56 & 0.00 & 4.99 & 0.00 & 75.00 & 0.00 \\
\hline $\mathrm{p} 5 \mathrm{c} 20$ & 5.38 & 0.00 & 3.55 & 0.00 & 9.50 & 0.00 \\
\hline p5c20_2 & 5.66 & 0.00 & 4.35 & 0.00 & 9.58 & 0.00 \\
\hline $\mathrm{p} 30 \mathrm{c} 20$ & 1.49 & 0.15 & 0.81 & 0.43 & 1.61 & 0.12 \\
\hline p60c20 & 10.13 & 0.00 & 3.27 & 0.01 & 15.40 & 0.00 \\
\hline $\mathrm{p} 90 \mathrm{c} 20$ & 34.56 & 0.00 & 4.99 & 0.00 & 75.00 & 0.00 \\
\hline $\mathrm{p} 120 \mathrm{c} 20$ & 23.40 & 0.00 & 0.18 & 0.86 & 33.38 & 0.00 \\
\hline $\mathrm{p} 5 \mathrm{c} 51$ & 8.41 & 0.00 & 4.41 & 0.00 & 2.52 & 0.02 \\
\hline p60c51 & 0.00 & 0.00 & 0.00 & 0.00 & 0.00 & 0.00 \\
\hline
\end{tabular}

1. indicates Dorsi Flexion at five degrees per second concentrically with the knee at 0 degrees of flexion/extension ( $\_2$ means second trial for the same condition).

Table P.1

Results from t-tests for pairwise comparisons. The three cocontraction estimation methods were compared (I. cocontraction on the basis of the normalized EMG; II. cocontraction on the basis the normalized EMG and the tension-length-velocity for each musculotendon unit; III. cocontraction on the basis of the individual musculotendon units). Bolded and italicized indicates significant differences at $\mathrm{p}<0.01666$, with $\mathrm{df}=$ 100 and $t=2.43$. These results are for the ankle joint of subject SA during the motion and KinCom calibration trials. 


\begin{tabular}{|c|c|c|c|c|c|c|}
\hline KNEE & I vs. II & I vs. II & I vs. III & I vs. III & II vs. III & II vs. III \\
\hline MOTION & $\mathrm{t}$ & $p$ & $\mathrm{t}$ & $\mathrm{p}$ & $t$ & $p$ \\
\hline SA004 & 1.53 & 0.13 & 0.67 & 0.50 & 1.75 & 0.08 \\
\hline SA005 & 1.15 & 0.25 & 0.31 & 0.76 & 1.56 & 0.12 \\
\hline SA008 & 2.41 & 0.02 & 1.61 & 0.11 & 0.73 & 0.47 \\
\hline \multicolumn{7}{|l|}{ KIN_COM } \\
\hline $\mathrm{e} 5 \mathrm{c} 0^{1}$ & 2.98 & 0.01 & 3.13 & 0.00 & 0.53 & 0.60 \\
\hline $\mathrm{e} 60 \mathrm{c} 0$ & 7.90 & 0.00 & 9.00 & 0.00 & 3.72 & 0.00 \\
\hline e60c0_2 & 6.89 & 0.00 & 7.17 & 0.00 & 6.09 & 0.00 \\
\hline $\mathrm{e} 120 \mathrm{c} 0$ & 12.05 & 0.00 & 13.11 & 0.00 & 9.89 & 0.00 \\
\hline $\mathrm{e} 190 \mathrm{c0}$ & 365.50 & 0.00 & 186.58 & 0.00 & 73.34 & 0.00 \\
\hline e190c0_2 & 36.56 & 0.00 & 39.45 & 0.00 & 30.27 & 0.00 \\
\hline e190c0_3 & 24.91 & 0.00 & 23.69 & 0.00 & 31.64 & 0.00 \\
\hline $\mathrm{e} 5 \mathrm{c} 10 \mathrm{p} 2$ & 7.75 & 0.00 & 11.81 & 0.00 & 9.61 & 0.00 \\
\hline$e 60 c 10 p$ & 2.74 & 0.01 & 3.43 & 0.00 & 11.43 & 0.00 \\
\hline e60c10p_2 & 3.16 & 0.00 & 4.06 & 0.00 & 15.69 & 0.00 \\
\hline $\mathrm{e} 120 \mathrm{c} 10 \mathrm{p}$ & 3.96 & 0.00 & 4.72 & 0.00 & 9.24 & 0.00 \\
\hline e190c10p & 3.65 & 0.00 & 186.58 & 0.00 & 73.34 & 0.00 \\
\hline $\mathrm{e} 250 \mathrm{c} 10 \mathrm{p}$ & 8.19 & 0.00 & 1.32 & 0.21 & 63.82 & 0.00 \\
\hline $\mathrm{e} 250 \mathrm{c} 10 \mathrm{p} \_2$ & 8.16 & 0.00 & 1.33 & 0.20 & 60.79 & 0.00 \\
\hline $\mathrm{f} 5 \mathrm{c} 0$ & 3.25 & 0.00 & 4.58 & 0.00 & 13.66 & 0.00 \\
\hline $\mathrm{f} 120 \mathrm{c} 0$ & 3.03 & 0.02 & 9.90 & 0.00 & 20.93 & 0.00 \\
\hline $\mathrm{f} 5 \mathrm{c} 10 \mathrm{~d}$ & 10.07 & 0.00 & 0.87 & 0.40 & 8.42 & 0.00 \\
\hline $\mathrm{f} 60 \mathrm{c} 10 \mathrm{~d}$ & 4.81 & 0.00 & 0.07 & 0.95 & 20.22 & 0.00 \\
\hline $\mathrm{f} 5 \mathrm{c} 10 \mathrm{p}$ & 11.38 & 0.00 & 17.34 & 0.00 & 18.82 & 0.00 \\
\hline $\mathrm{f} 120 \mathrm{c} 10 \mathrm{p}$ & 24.78 & 0.00 & 23.66 & 0.00 & 21.53 & 0.00 \\
\hline f190c10p & 26.68 & 0.00 & 33.76 & 0.00 & 68.73 & 0.00 \\
\hline $\mathrm{f} 5 \mathrm{c} 20 \mathrm{p}$ & 15.90 & 0.00 & 20.28 & 0.00 & 24.55 & 0.00 \\
\hline
\end{tabular}

1. indicates Extension of the knee at five degrees per second concentrically with the ankle at zero degrees of dorsi and plantar flexion, i.e., at neutral (a 10d or 10p would indicate that the ankle is at 10 degrees of dorsi or plantar flexion respectively; _2 means second trial for the same condition).

Table P.2

Pairwise comparisons t-test results. Three cocontraction estimation methods were compared (I. cocontraction using normalized EMG; II. cocontraction using normalized EMG and the musculotendon unit tension-length-velocity; III. cocontraction using the individual musculotendon units forces). Bolded and italicized indicates significant differences at $p<0.01666$, with $\mathrm{df}=100$ and $t=2.43$. Results are for subject's SA knee joint during the motion and KinCom calibration trials. 


\begin{tabular}{|c|c|c|c|c|c|c|}
\hline ANKLE & I vs. II & I vs. II & I vs. III & I vs. III & II vs. III & II vs. III \\
\hline MOTION & $t$ & $p$ & $\mathrm{t}$ & $p$ & $\mathrm{t}$ & $\bar{p}$ \\
\hline SC003 & 1.87 & 0.06 & 2.16 & 0.03 & 0.50 & 0.62 \\
\hline $\mathrm{SC004}$ & 2.61 & 0.01 & 2.23 & 0.03 & 1.30 & 0.20 \\
\hline SC005 & 2.86 & 0.01 & 1.91 & 0.06 & 0.36 & 0.72 \\
\hline \multicolumn{7}{|c|}{ KIN_COM } \\
\hline $\mathrm{d} 5 \mathrm{c} 0 \_2^{1}$ & 7.37 & 0.00 & 6.89 & 0.00 & 3.15 & 0.00 \\
\hline $\mathrm{d} 5 \mathrm{c} \mathrm{c}_{-} 3$ & 7.16 & 0.00 & 6.79 & 0.00 & 6.42 & 0.00 \\
\hline $\mathrm{d} 5 \mathrm{c} 7$ & 4.14 & 0.00 & 4.18 & 0.00 & 3.90 & 0.00 \\
\hline $\mathrm{d} 5 \mathrm{c} 20$ & 16.84 & 0.00 & 11.27 & 0.00 & 11.37 & 0.00 \\
\hline $\mathrm{d} 5 \mathrm{c} 20 \_2$ & 17.83 & 0.00 & 20.84 & 0.00 & 8.49 & 0.00 \\
\hline $\mathrm{d} 5 \mathrm{c} 50$ & 8.05 & 0.00 & 8.04 & 0.00 & 7.01 & 0.00 \\
\hline $\mathrm{d} 120 \mathrm{c} 50$ & 13.18 & 0.00 & 13.82 & 0.00 & 12.14 & 0.00 \\
\hline $\mathrm{p} 5 \mathrm{co}$ & 2.57 & 0.02 & 3.52 & 0.00 & 3.75 & 0.00 \\
\hline $\mathrm{p} 5 \mathrm{c} 10$ & 1.60 & 0.12 & 2.51 & 0.02 & 2.63 & 0.01 \\
\hline p30c10 & 5.27 & 0.00 & 5.20 & 0.00 & 5.25 & 0.00 \\
\hline $\mathrm{p} 60 \mathrm{c} 10$ & 32.37 & 0.00 & 12.46 & 0.00 & 41.87 & 0.00 \\
\hline $\mathrm{p} 90 \mathrm{c} 10$ & 6.93 & 0.00 & 6.43 & 0.00 & 6.86 & 0.00 \\
\hline $\mathrm{p} 120 \mathrm{c} 10$ & 119.54 & 0.00 & 120.47 & 0.00 & 96.16 & 0.00 \\
\hline $\mathrm{p} 5 \mathrm{c} 20$ & 4.22 & 0.00 & 1.06 & 0.30 & 0.32 & 0.75 \\
\hline $\mathrm{p} 30 \mathrm{c} 20$ & 11.39 & 0.00 & 0.11 & 0.91 & 4.09 & 0.00 \\
\hline $\mathrm{p} 60 \mathrm{c} 20$ & 29.01 & 0.00 & 13.99 & 0.00 & 2.18 & 0.05 \\
\hline $\mathrm{p} 90 \mathrm{c} 20$ & 3.68 & 0.00 & 3.56 & 0.01 & 3.66 & 0.00 \\
\hline p90c20_2 & 46.53 & 0.00 & 20.33 & 0.00 & 9.28 & 0.00 \\
\hline $\mathrm{p} 120 \mathrm{c} 20$ & 92.43 & 0.00 & 188.06 & 0.00 & 10.83 & 0.00 \\
\hline $\mathrm{p} 5 \mathrm{c} 50$ & 2.01 & 0.05 & 1.97 & 0.06 & 1.94 & 0.06 \\
\hline p60c50 & 16.00 & 0.00 & 7.77 & 0.00 & 3.67 & 0.00 \\
\hline p60c50_2 & 53.66 & 0.00 & 15.76 & 0.00 & 3.38 & 0.00 \\
\hline $\mathrm{p} 120 \mathrm{c} 50$ & 97.07 & 0.00 & 23.02 & 0.00 & 0.16 & 0.88 \\
\hline
\end{tabular}

1. indicates Dorsi Flexion at five degrees per second concentrically with the knee at 0 degrees of flexion/extension ( $\_2$ means second trial for the same condition).

Table P.3.

Pairwise comparison t-test results. Three cocontraction estimation methods were compared (I. cocontraction on the basis of the normalized EMG; II. cocontraction on the basis the normalized EMG and the tension-length-velocity for each musculotendon unit; III. cocontraction on the basis of the individual musculotendon units). Bolded and italicized indicates significant differences at $p<0.01666$, with $\mathrm{df}=100$ and $t=2.43$. Results for subject's SA ankle joint during the motion and KinCom calibration trials. 


\begin{tabular}{|c|c|c|c|c|c|c|}
\hline KNEE & I vs. II & I vs. II & I vs. III & I vs. III & II vs. III & II vs. III \\
\hline MOTION & $t$ & $p$ & $t$ & $p$ & $\mathrm{t}$ & $\mathrm{p}$ \\
\hline $\mathrm{SCO03}$ & 1.58 & 0.12 & 0.23 & 0.82 & 2.37 & 0.02 \\
\hline SC004 & 0.55 & 0.58 & 1.47 & 0.14 & 2.32 & 0.02 \\
\hline SC005 & 1.38 & 0.17 & 0.85 & 0.40 & 2.84 & 0.01 \\
\hline \multicolumn{7}{|c|}{ KIN_COM } \\
\hline $\mathrm{e} 5 \mathrm{c} 0^{1}$ & 2.81 & 0.01 & 1.25 & 0.22 & 9.45 & 0.00 \\
\hline $\mathrm{e} 60 \mathrm{c} 0$ & 5.33 & 0.00 & 2.33 & 0.03 & 9.85 & 0.00 \\
\hline $\mathrm{e} 120 \mathrm{c} 0$ & 11.67 & 0.00 & 9.34 & 0.00 & 13.28 & 0.00 \\
\hline e5c10d & 5.23 & 0.00 & 2.52 & 0.02 & 8.42 & 0.00 \\
\hline e60c10d & 13.40 & 0.00 & 9.87 & 0.00 & 22.35 & 0.00 \\
\hline e190c10d & 15.46 & 0.00 & 14.12 & 0.00 & 18.28 & 0.00 \\
\hline$e 5 c 10 p$ & 11.58 & 0.00 & 17.14 & 0.00 & 18.12 & 0.00 \\
\hline e60c10p & 6.15 & 0.00 & 17.08 & 0.00 & 11.87 & 0.00 \\
\hline e120c10p & 1.57 & 0.12 & 10.13 & 0.00 & 16.89 & 0.00 \\
\hline e190c10p & 13.43 & 0.00 & 1.06 & 0.30 & 52.39 & 0.00 \\
\hline $\mathrm{e} 250 \mathrm{c} 10 \mathrm{p}$ & 8.73 & 0.00 & 8.83 & 0.00 & 8.68 & 0.00 \\
\hline $\mathrm{f} 5 \mathrm{c} 0$ & 0.00 & 0.00 & 0.00 & 0.00 & 0.00 & 0.00 \\
\hline $\mathrm{f} 5 \mathrm{c} 10 \mathrm{~d}$ & 1.21 & 0.24 & 0.58 & 0.57 & 8.88 & 0.00 \\
\hline f60c $10 \mathrm{~d}$ & 1.12 & 0.29 & 5.08 & 0.00 & 49.08 & 0.00 \\
\hline $\mathrm{f} 5 \mathrm{c} 10 \mathrm{p} \_2$ & 9.93 & 0.00 & 11.46 & 0.00 & 13.00 & 0.00 \\
\hline$f 60 \mathrm{c} 10 p$ & 12.80 & 0.00 & 13.66 & 0.00 & 14.70 & 0.00 \\
\hline $\mathrm{f} 5 \mathrm{c} 20 \mathrm{p}$ & 16.16 & 0.00 & 20.37 & 0.00 & 24.64 & 0.00 \\
\hline $660 \mathrm{c} 20 \mathrm{p}$ & 10.23 & 0.00 & 12.01 & 0.00 & 12.59 & 0.00 \\
\hline
\end{tabular}

1. indicates Extension of the knee at five degrees per second concentrically with the ankle at zero degrees of dorsi and plantar flexion, i.e., at neutral (a 10d or 10p would indicate that the ankle is at 10 degrees of dorsi or plantar flexion respectively; _2 means second trial for the same condition).

Table P.4

Results from t-tests for pairwise comparisons. The three cocontraction estimation methods were compared (I. cocontraction on the basis of the normalized EMG; II. cocontraction on the basis the normalized EMG and the tension-length-velocity for each musculotendon unit; III. cocontraction on the basis of the individual musculotendon units). Bolded and italicized indicates significant differences at $\mathrm{p}<0.01666$, with $\mathrm{df}=$ 100 and $t=2.43$. These results are for the knee joint of subject SA during the motion and KinCom calibration trials. 


\begin{tabular}{|c|c|c|c|c|c|c|}
\hline ANKLE & I vs. II & I vs. II & I vs. III & I vs. III & II vs. III & II vs. III \\
\hline MOTION & $\mathrm{t}$ & $\mathrm{p}$ & $\mathrm{t}$ & $p$ & $t$ & $p$ \\
\hline SNO02 & 0.56 & 0.58 & 1.98 & 0.05 & 2.42 & 0.02 \\
\hline SNO04 & 3.21 & 0.00 & 1.34 & 0.18 & 2.84 & 0.01 \\
\hline SN005 & 2.49 & 0.01 & 0.11 & 0.91 & 1.32 & 0.19 \\
\hline \multicolumn{7}{|c|}{$\mathrm{KIN} \_\mathrm{COM}$} \\
\hline $\mathrm{d} 5 \mathrm{co}$ & 1.75 & 0.09 & 5.31 & 0.00 & 18.86 & 0.00 \\
\hline $\mathrm{d} 5 \mathrm{c7}$ & 0.16 & 0.87 & 11.39 & 0.00 & 13.04 & 0.00 \\
\hline $\mathrm{d} 5 \mathrm{c} 20$ & 5.92 & 0.00 & 4.76 & 0.00 & 7.93 & 0.00 \\
\hline $\mathrm{d} 5 \mathrm{c} 52$ & 7.68 & 0.00 & 2.17 & 0.04 & 5.49 & 0.00 \\
\hline $\mathrm{d} 60 \mathrm{c52}$ & 4.02 & 0.03 & 6.88 & 0.01 & 13.76 & 0.00 \\
\hline $\mathrm{d} 120 \mathrm{c} 52$ & 0.63 & 0.54 & 4.94 & 0.00 & 6.61 & 0.00 \\
\hline $\mathrm{p} 5 \mathrm{co}$ & 0.22 & 0.83 & 4.72 & 0.00 & 7.23 & 0.00 \\
\hline $\mathrm{p} 5 \mathrm{c} 8$ & 3.68 & 0.00 & 3.56 & 0.01 & 3.66 & 0.00 \\
\hline $\mathrm{p} 30 \mathrm{c} 8$ & 8.67 & 0.00 & 12.49 & 0.00 & 13.47 & 0.00 \\
\hline p60c8 & 8.83 & 0.00 & 3.12 & 0.00 & 6.78 & 0.00 \\
\hline $\mathrm{p} 90 \mathrm{c} 8$ & 8.15 & 0.00 & 9.67 & 0.00 & 13.42 & 0.00 \\
\hline p5c20 & 6.74 & 0.00 & 0.89 & 0.38 & 8.15 & 0.00 \\
\hline p30c20 & 6.40 & 0.00 & 8.17 & 0.00 & 12.11 & 0.00 \\
\hline $\mathrm{p} 60 \mathrm{c} 20$ & 6.60 & 0.00 & 8.13 & 0.00 & 14.64 & 0.00 \\
\hline p90c20. & 5.83 & 0.00 & 7.50 & 0.00 & 5.13 & 0.00 \\
\hline $\mathrm{p} 5 \mathrm{c} 52$ & 8.70 & 0.00 & 9.17 & 0.00 & 8.37 & 0.00 \\
\hline p60c52 & 5.38 & 0.00 & 5.73 & 0.00 & 3.06 & 0.01 \\
\hline
\end{tabular}

1. indicates Dorsi Flexion at five degrees per second concentrically with the knee at 0 degrees of flexion/extension.

Table P.5

Results from t-tests for pairwise comparisons. The three cocontraction estimation methods were compared (I. cocontraction on the basis of the normalized EMG; II. cocontraction on the basis the normalized EMG and the tension-length-velocity for each musculotendon unit; III. cocontraction on the basis of the individual musculotendon units). Bolded and italicized indicates significant differences at $p<0.01666$, with $d f=$ 100 and $t=2.43$. These results are for the ankle joint of subject $\mathrm{SA}$ during the motion and KinCom calibration trials. 


\begin{tabular}{|c|c|c|c|c|c|c|}
\hline ANKLE & I vs. II & I vs. II & I vs. III & I vs. III & II vs. III & II vs. III \\
\hline MOTION & $\mathrm{t}$ & $\mathrm{p}$ & $t$ & $\mathrm{p}$ & $\mathrm{t}$ & $\mathrm{p}$ \\
\hline SNO02 & 5.86 & 0.00 & 5.58 & 0.00 & 1.40 & 0.16 \\
\hline SNO04 & 4.20 & 0.00 & 3.44 & 0.00 & 2.21 & 0.03 \\
\hline SN005 & 5.88 & 0.00 & 4.43 & 0.00 & 2.22 & 0.03 \\
\hline \multicolumn{7}{|c|}{ KIN_COM } \\
\hline$e 5 \mathrm{co}^{1}$ & 4.30 & 0.00 & 4.30 & 0.00 & 4.04 & 0.00 \\
\hline e60a0 & 12.38 & 0.00 & 2.72 & 0.01 & 11.15 & 0.00 \\
\hline $\mathrm{e} 120 \mathrm{c} 0$ & 7.98 & 0.00 & 5.84 & 0.00 & 8.80 & 0.00 \\
\hline \multicolumn{7}{|l|}{ e190c0 } \\
\hline $\mathrm{e} 250 \mathrm{c} 0$ & 9.58 & 0.00 & 4.60 & 0.00 & 7.10 & 0.00 \\
\hline $\mathrm{e} 5 \mathrm{c} 10 \mathrm{~d}$ & 4.23 & 0.00 & 4.05 & 0.00 & 0.59 & 0.56 \\
\hline e60c10d & 5.67 & 0.00 & 5.48 & 0.00 & 3.71 & 0.00 \\
\hline e120c10d & 6.61 & 0.00 & 5.87 & 0.00 & 6.79 & 0.00 \\
\hline e190c10d & 1.46 & 0.15 & 1.13 & 0.26 & 0.41 & 0.68 \\
\hline $\mathrm{e} 25010 \mathrm{~d}$ & 1.95 & 0.06 & 1.86 & 0.07 & 1.69 & 0.10 \\
\hline $\mathrm{e} 5 \mathrm{c} 10 \mathrm{p}$ & 4.53 & 0.00 & 4.54 & 0.00 & $3 . \overline{72}$ & 0.00 \\
\hline e60c10p & 2.35 & 0.02 & 6.58 & 0.00 & 13.18 & 0.00 \\
\hline e120c10p & 2.67 & 0.01 & 0.48 & 0.63 & 11.37 & 0.00 \\
\hline e190c10p & 0.96 & 0.34 & 1.51 & 0.14 & 1.89 & 0.06 \\
\hline $\mathrm{e} 250 \mathrm{c} 10 \mathrm{p}$ & 24.93 & 0.00 & 20.90 & 0.00 & 3.53 & 0.00 \\
\hline $\mathrm{f} 5 \mathrm{c} 0$ & 0.75 & 0.46 & 5.94 & 0.00 & 7.73 & 0.00 \\
\hline $\mathrm{f} 60 \mathrm{c} 0$ & 1.75 & 0.09 & 5.31 & 0.00 & 18.86 & 0.00 \\
\hline $\mathrm{f} 5 \mathrm{c} 10 \mathrm{p}$ & 2.74 & 0.01 & 3.43 & 0.00 & 11.43 & 0.00 \\
\hline $\mathrm{f} 60 \mathrm{c} 10 \mathrm{p}$ & 5.62 & 0.00 & 10.97 & 0.00 & 50.06 & 0.00 \\
\hline f120c10p & 13.97 & 0.00 & 19.39 & 0.00 & 37.99 & 0.00 \\
\hline $\mathrm{f} 5 \mathrm{c} 20 \mathrm{p}$ & 1.21 & 0.24 & 0.58 & 0.57 & 8.88 & 0.00 \\
\hline $\mathrm{f} 5 \mathrm{c} 10 \mathrm{~d}$ & 0.93 & 0.36 & 3.35 & 0.00 & 13.61 & 0.00 \\
\hline
\end{tabular}

1. indicates Extension of the knee at five degrees per second concentrically with the ankle at zero degrees of dorsi and plantar flexion, i.e., at neutral (a $10 \mathrm{~d}$ or $10 \mathrm{p}$ would indicate that the ankle is at 10 degrees of dorsi or plantar flexion respectively).

Table P.6.

Results from t-tests for pairwise comparisons. The three cocontraction estimation methods were compared (I. cocontraction on the basis of the normalized EMG; II. cocontraction on the basis the normalized EMG and the tension-length-velocity for each musculotendon unit; III. cocontraction on the basis of the individual musculotendon units). Bolded and italicized indicates significant differences at $\mathrm{p}<0.01666$, with $\mathrm{df}=$ 100 and $t=2.43$. These results are for the knee joint of subject SA during the motion and KinCom calibration trials. 